$Q H$

91

A1566

Fishes

\title{
Nile Delta Drill Core and Sample Database for 1985-1994: Mediterranean Basin (MEDIBA) Program
}

DANIEL JEAN STANLEY,

JAMES E. MCREA, JR.

and

JOHN C. WALDRON

SMITHSONIAN CONTRIBUTIONS TO THE MARINE SCIENCES • NUMBER 37 


\title{
SERIES PUBLICATIONS OF THE SMITHSONIAN INSTITUTION
}

Emphasis upon publication as a means of "diffusing knowledge" was expressed by the first Secretary of the Smithsonian. In his formal plan for the institution, Joseph Henry outlined a program that included the following statement: "It is proposed to publish a series of reports, giving an account of the new discoveries in science, and of the changes made from year to year in all branches of knowledge." This theme of basic research has been adhered to through the years by thousands of titles issued in series publications under the Smithsonian imprint, commencing with Smithsonian Contributions to Knowledge in 1848 and continuing with the following active series:

\author{
Smithsonian Contributions to Anthropology \\ Smithsonian Contributions to Botany \\ Smithsonian Contributions to the Earth Sciences \\ Smithsonian Contributions to the Marine Sciences \\ Smithsonian Contributions to Paleobiology \\ Smithsonian Contributions to Zoology \\ Smithsonian Folklife Studies \\ Smithsonian Studies in Air and Space \\ Smithsonian Studies in History and Technology
}

In these series, the Institution publishes small papers and full-scale monographs that report the research and collections of its various museums and bureaux or of professional colleagues in the world of science and scholarship. The publications are distributed by mailing lists to libraries, universities, and similar institutions throughout the world.

Papers or monographs submitted for series publication are received by the Smithsonian Institution Press, subject to its own review for format and style, only through departments of the various Smithsonian museums or bureaux, where the manuscripts are given substantive review. Press requirements for manuscript and art preparation are outlined on the inside back cover.

\author{
I. Michael Heyman \\ Secretary \\ Smithsonian Institution
}




\section{Nile Delta Drill Core and Sample Database for 1985-1994: Mediterranean Basin (MEDIBA) Program}

Daniel Jean Stanley, James E. McRea, Jr., and John C. Waldron

\section{ISSUED}

DEC 61996

\section{SMITHSUINIAN INSTITUTION}

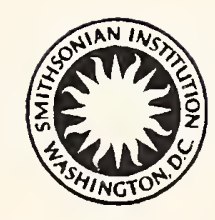

SMITHSONIAN INSTITUTION PRESS

Washington, D.C. 


\section{A B S T R A C T}

Stanley, Daniel Jean, James E. McRea, Jr., and John C. Waldron. Nile Delta Drill Core and Sample Database for 1985-1994: Mediterranean Basin (MEDIBA) Program. Smithsonian Contributions to the Marine Sciences, number 37, 428 pages, 10 figures, 2 tables, 1996.-This document is designed to serve as the catalog for a complete set of lithologic logs of 87 sediment borings drilled in the northern Nile delta of Egypt in the course of the Nile Delta Project, from 1985 to 1994. The project, part of the Mediterranean Basin (MEDIBA) Program, was initiated to interpret the recent geological evolution of this depocenter, from the time of its formation about 8000 years ago to the present. The data set includes the major petrologic attributes of these borings, which range in length from -20 to $60 \mathrm{~m}$. The results of textural and sand-sized compositional analyses of 2500 core samples are provided, as well as the ages of 358 radiocarbon-dated samples to as old as $-35,000$ years before present. These data constitute the foundation of the Nile Delta Project's investigation. A review of the methods employed in the field and laboratory and an inventory of published articles and theses completed through 1994 as part of this multidisciplinary and multinational effort also are presented. This database facilitates the distinction between anthropogenic and natural factors that determine the evolution of the delta. It is intended to provide a comprehensive record of subsurface deposits in the northern delta, accumulating in late Pleistocene to Holocene time, to be used by those agencies and specialists responsible for monitoring the rapidly changing Nile delta depocenter.

The information published in this document is accessible electronically on the Internet from the Smithsonian Institution's National Museum of Natural History Gopher Server at URL "gopher://nmnhgoph.si.edu/11/.paleo" or via hypertext document (http) at "http://nmnhwww.si.edu/gopher-menus/." Further information can be obtained from the National Museum of Natural History's Collection and Research Information System (CRIS) Program, Washington, D.C. 20560.

OfFICIAL PUBLICATION DATE is handstamped in a limited number of initial copies and is recorded in the Institution's annual report, Smithsonian Year. SERIES COVER DESIGN: Seascape along the Atlantic Coast of eastern North America.

Library of Congress Cataloging-in-Publication Data

Stanley, Daniel J.

Nile Delta drill core and sample database for 1985-1994: Mediterranean Basin (MEDIBA) Program / Daniel Jean

Stanley, James E. McRea, Jr., and John C. Waldron.

p. cm. - (Smithsonian contributions to the marine sciences ; no. 37)

Includes bibliographical references (p. 426-428).

1. Borings-Egypt-Nile River Delta-Catalogs. 2. Geology, Stratigraphic-Quaternary-Catalogs.

3. Geology-Egypt-Nile River Delta-Catalogs. I. McRea, James E. II. Waldron, John C.

III. Title. IV. Series.

QE328.S73 $1996 \quad 551.7^{\prime} 9^{\prime} 09621-d c 20 \quad 96-17239$

(6) The paper used in this publication meets the minimum requirements of the American National Standard for Permanence of Paper for Printed Library Materials Z39.48-1984. 


\section{Contents}

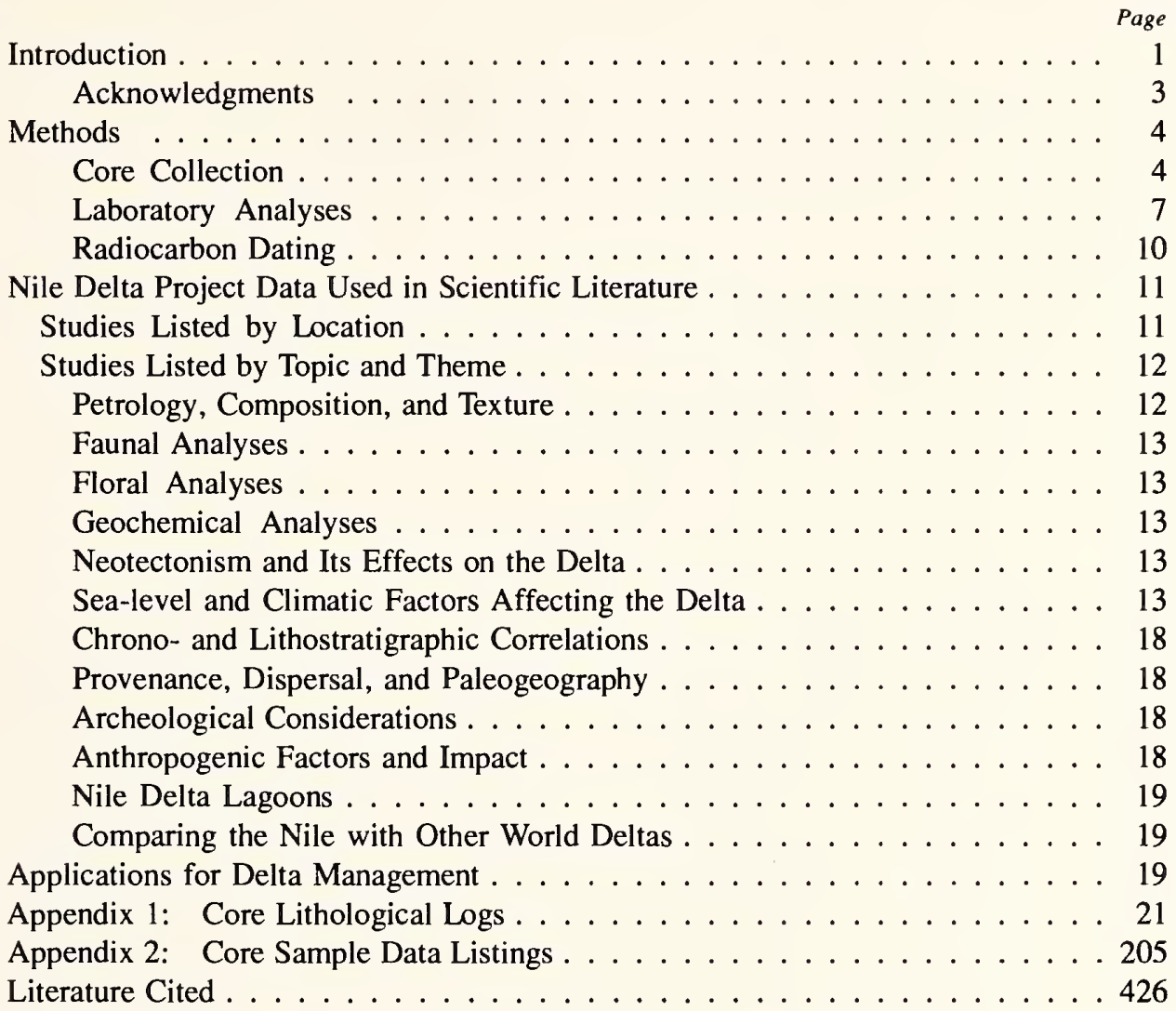





\title{
Nile Delta Drill Core and Sample Database for 1985-1994: Mediterranean Basin (MEDIBA) Program
}

\author{
Daniel Jean Stanley, James E. McRea, Jr., \\ and John C. Waldron
}

\section{Introduction}

Modern marine deltas are vital agricultural and aquacultural resources for the world's rapidly growing population. These coastal depocenters are generally low-lying and thus highly vulnerable to natural environmental changes, such as global sea-level oscillations and vertical displacement of land relative to sea level. Most of the world's large deltas are subsiding, largely as an isostatic response to loading by thick depositional sequences and their compaction. Thus, even if global sea level were not to rise in the future, the lower plains and coasts of deltas are particularly prone to incursion by the sea, which will induce land loss and reduce agricultural productivity at a time when it is most needed. The situation will be substantially aggravated if global sea level should rise, as predicted by some for the next century (Wigley and Raper, 1992).

Until recently, surprisingly little research pertaining to deltas has focused on differentiating the effects of global rise in sea level from those of lowering of land by isostasy, tectonism, and sediment compaction. This problem is of considerable concern,

Daniel Jean Stanley, Deltas-Global Change Program, Department of Paleobiology, National Museum of Natural History, Smithsonian Institution, Washington, D.C. 20560. James E. McRea, Jr., Division of Paleobotany, Department of Paleobiology, National Museum of Natural History, Smithsonian Institution, Washington, D.C. 20560. John C. Waldron, Deltas-Global Change Program, Department of Paleobiology, National Museum of Natural History, Smithsonian Institution, Washington, D.C. 20560.

Review Chairman: William A. DiMichele, Department of Paleobiology, National Museum of Natural History, Smithsonian Institution. Reviewers: Terry A. Nelsen, Atlantic Oceanographic and Meteorological Laboratory, National Oceanic and Atmospheric Administration, 4301 Rickenbacker Causeway, Miami, Florida 33149; and Donald J.P. Swift, Department of Oceanography, Old Dominion University, Norfolk, Virginia 23529. particularly in view of the increased effects of humans on world river and coastal systems. For example, emplacement of dams, diversion and dredging of river channels, intensification of agricultural projects, construction of increasingly complex and dense irrigation systems, and modification of coastlines are producing unexpected and frequently deleterious side-effects in deltaic areas. Coastal management reports on deltas all concur that this interaction of natural and anthropogenic factors is presently inducing accelerated changes in delta plains and coasts (Kay, 1993), and that these environments now require more active monitoring by scientists and engineers. Geologists can play a valuable role in this type of environmental monitoring in that they are trained to map and evaluate changes in time and space. Moreover, they are adept in using a multidisciplinary approach that integrates stratal geometry and petrologic, biological, and chemical information (Broussard, 1975; Coleman, 1982; Posamentier and Vail, 1987; Stanley and Warne, 1993a).

It is recalled that the Nile delta, positioned in a desert environment on the northeastern African margin, was one of the first such depocenters to attract the attention of scholars interested in recording deltaic phenomena. In the mid-fifth century B.C., the Greek historian Herodotus called attention to some general sedimentological aspects of the Nile delta, and to its triangular shape giving rise to the term "delta" to denote this type of geographical feature. Despite this early interest, no systematic, comprehensive geological and environmental study of the Nile delta had been undertaken prior to the end of this century.

A project to define the late Quaternary geological evolution of the lower Nile delta plain of northern Egypt, taking into account both natural and anthropogenic factors, was thus initiated in 1985 at the National Museum of Natural History, 


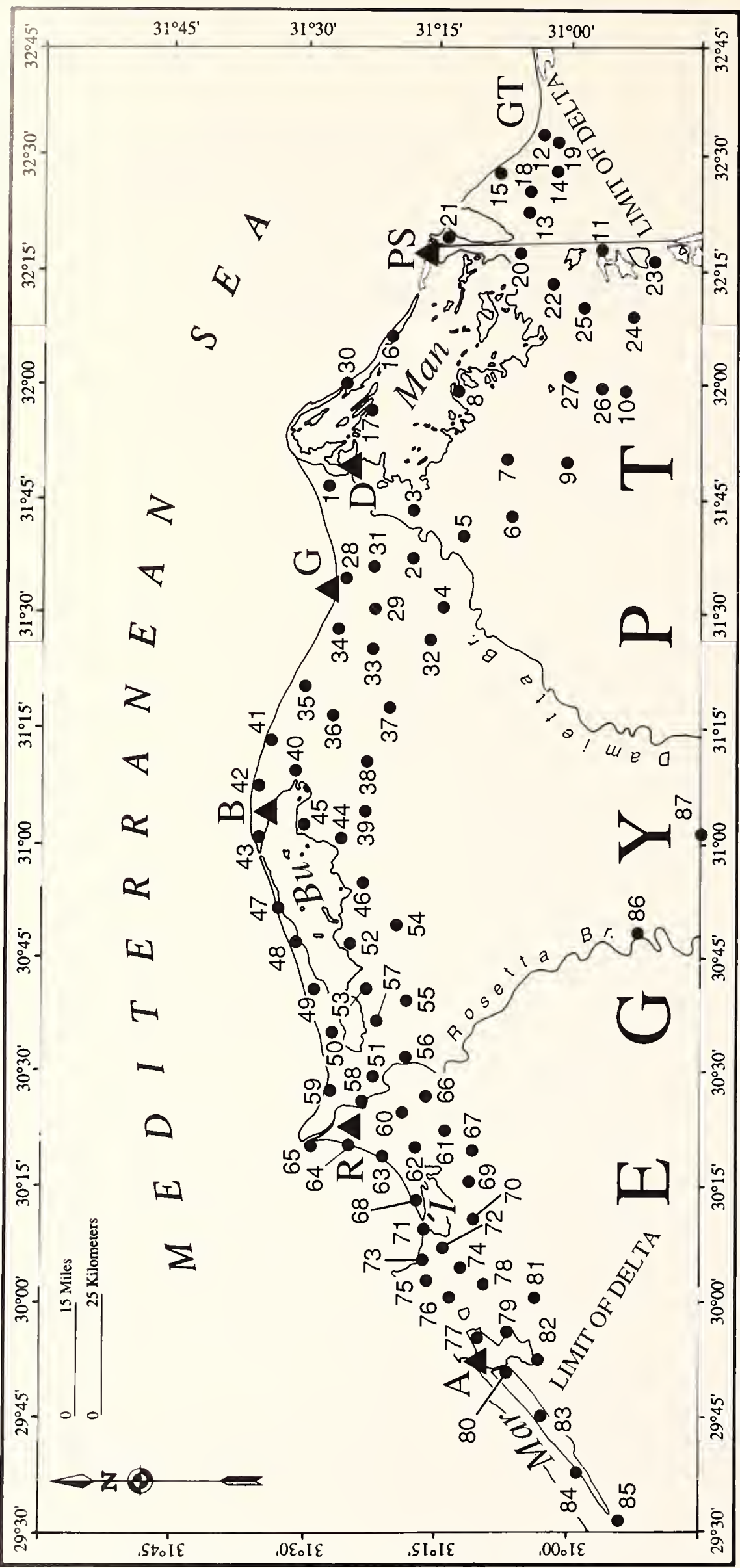

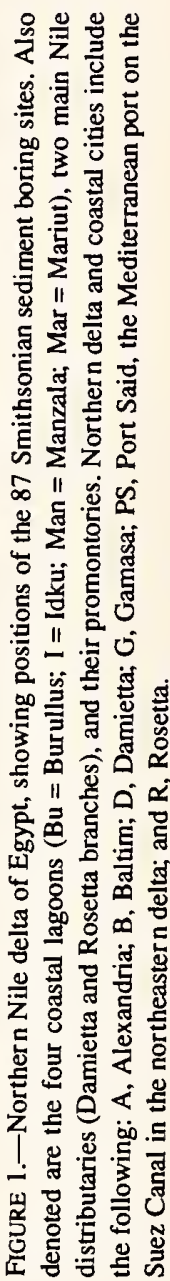


Smithsonian Institution. The Nile delta depocenter was specifically selected for several reasons: (1) this delta is the major breadbasket for Egypt, where the population is now approaching 60 million; (2) Egypt's already limited percapita arable land has declined steadily to about 0.06 ha, now the lowest figure of any country in Africa (Biswas, 1993); (3) the delta's northern sector is near (elevation of little more than $1 \mathrm{~m}$ ) and in some areas below sea level, and thus it is particularly vulnerable to even small changes of sea level; (4) the delta has long been occupied, cultivated, and modified by humans (Butzer, 1976; Stanley and Warne, 1993b); and (5) its fluvial regime has been completely altered since the beginning of the century by intensified irrigation projects and emplacement of two dams at Aswan and also a series of barrages along the Nile from upper Egypt to near the Mediterranean coast (Waterbury, 1979).

Following closure of the High Aswan Dam in 1964, numerous studies have focused on increased problems related to land reclamation (Waterbury, 1979; Biswas, 1993) and erosion of the Nile delta coast (UNDP/UNESCO, 1976, 1977, 1978; Abdel-Kader, 1982; Frihy, 1988; Smith and AbdelKader, 1988). Recent changes offshore (Stanley and Maldonado, 1977; Maldonado and Stanley, 1978; Coleman et al., 1981; Frihy and Lotfy, 1994), including those in the northern sector of the Suez Canal that crosses the northeastern delta (Morcos and Messieh, 1973; Stanley et al., 1982; Gerges and Stanley, 1985), have also been considered. It is of note, however, that as recently as the early 1980 s, no systematic analyses had been made of the recent geological history of the landward part of the delta.

In 1983, the senior author was invited to Egypt to assess the possibility of initiating a long-term geological study of the Nile delta and its evolution from latest Pleistocene to Holocene time. The Nile Delta Project was formalized and officially initiated in early 1985, and for ten years it became the major activity of staff and visiting scientists participating in the Mediterranean Basin (MEDIBA) Program. Throughout this period, the project was directed from the United States' National Museum of Natural History, Smithsonian Institution, in Washington, D.C., and involved the cooperation of Egyptian scientists at the Coastal Research Institute in Alexandria, the Ain Shams University in Abassiya, Cairo, and the University of Cairo. More than 40 scientists from North America, Egypt, Europe, and Asia have been part of the Nile Delta Project team.

To interpret the late Pleistocene and Holocene history of the northern Nile delta, including its coastal plain, lagoons, marshes, and strandline, the project emphasized study of a series of radiocarbon-dated sediment cores. A large number of borings were recovered from drill sites established across the northern delta (Figure 1), enabling us to interpret sedimentary facies and evaluate their changes in time and space by study of the petrology, geochemistry, fauna, and flora of approximately 3500 core samples. Remote sensing and archeological data were also used in this project. As a direct result of this joint effort, 52 articles have been published in scientific journals, 7 theses have been completed, and 25 presentations have been made at scientific meetings by the end of 1994 .

A synthesis article summarizing the salient aspects of the late Quaternary history (the past $\sim 30,000$ years are considered) of the northern Nile delta, based in large part on the study of the numerous core samples, was recently published (Stanley and Warne, 1993a). The present monograph serves as a companion document and detailed data source to this synthesis and also to earlier project publications. Its main purpose is to present a complete set of simplified lithologic logs of the 87 sediment cores (Appendix 1) and the results of textural and sand-size compositional analyses of 2496 core samples (Appendix 2) that constitute the foundation of Nile Delta Project investigations. We also provide herein a brief review of the various methods employed, and an inventory of specific topics and interpretations published in scientific journals and as theses derived from these and other data collected as part of the project during the past 10 years. The present document is thus intended to provide a comprehensive database for the northern Nile delta during the late Pleistocene to Holocene that may be used by those responsible for monitoring changes in the rapidly evolving Nile delta depocenter.

To facilitate data computer exchange and distribution, all the information published in this document ( $\sim 75$ megabytes) is accessible to users of the Internet from the Smithsonian Institution's National Museum of Natural History Gopher Server at URL "gopher://nmnhgoph.si.edu/11/.paleo" or via hypertext document (http) at "http://nmnhwww.si.edu/gopher-menus/." Further information can be obtained from the National Museum of Natural History's Collections and Research Information System (CRIS) Program, Washington, D.C. 20560.

ACKNOWledgments.-The Nile Delta Project within the Mediterranean Basin (MEDIBA) Program has been a team effort in every sense, involving the valued cooperation of many specialists from diverse organizations. The research has benefited greatly from their collaboration, input, and interaction during the past 10 years. Most came to the Smithsonian Institution, some for extended research visits of up to two years. We are indebted to the following for active contributions to various aspects of the research (listed in alphabetical order): H.S. El D. Abdel Wahab (Ain Shams University, Cairo), M. Abu-Zeid (Ain Shams University, Cairo), D. Arbouille (PetroConsultants, Geneva), D. Arnold (Metropolitan Museum of Art, New York), M.P. Bernasconi (University of Calabria, Cosenza), Z. Chen (East China Normal University, Shanghai), V. Coutellier (Laboratoire de Géodynamique, Villefranche-surMer), H.R. Davis (Environmental Protection Agency, Washington), J. Dominik (University of Geneva, Versoix), G. Drapeau (University of Quebec, Rimouski), A. Foucault (Muséum National d'Histoire Naturelle, Paris), G.L. Freeland (Freeland and Associates, Miami), O.E. Frihy (Coastal Research Institute, Alexandria), C.D. Gerber (Woodward-Clyde Consultants, Washington), M.A. Gerges (UNDP/UNESCO, Nairobi), H. Goedicke (The Johns Hopkins University, Baltimore), G.A. Goodfriend (Carnegie Geophysical Laboratory, Washington), N. Gupta (Ohio State University, Colum- 
bus), H.A. Hamroush (University of Cairo, Cairo), F.H. Hamza (Ain Shams University, Cairo), H.L. Howa (University of Bordeaux, Talence), M. Kontrovitz (Northeast Louisiana University, Monroe), V.A. Kulyk (The George Washington University, Washington), S. Leroy (Université Catholique de Louvain, Louvain-la-Neuve), N. Liyanage (Air Liquide Laboratories, Tsukuba), J.-L. Loizeau (University of Geneva, Versoix), F. Longo (University of Calabria, Cosenza), M. Morsi (Ain Shams University, Cairo), Y. Pan (Wuhan College of Geology, Hubei), A. Pimmel (Texas A \& M, College Station), N. Pugliese (University of Trieste, Trieste), G. Randazzo (University of Catania, Catania), J. Schneiderman (Pomona College, Claremont), H. Sheng (National Museum of Natural History, Washington), B.S. Shergill (University of Kentucky, Lexington), F.R. Siegel (The George Washington University, Washington), M.L. Slaboda (The George Washington University, Washington), J.M. Slack (Bossier Parish Community College, Bossier City, Louisiana), B. Thomas (Université Pierre et Marie Curie, Paris), and A.G. Warne (Waterways Experiment Station, Vicksburg).

Our most special appreciation is expressed to our good friend, Dr. Bahay Issawi, Assistant Secretary for State in Cairo, for his strong support of our project. His recognition of the potential value of our research for Egypt, as well as his firm backing and persuasive initiatives to facilitate our field work in the delta, made all the difference. Dr. A. Bassiouni, Chairman of the Department of Geology, Ain Shams University, Cairo, encouraged the Faculty of Science and some of his staff to become involved in Nile delta research. We are indebted to Engineer A. Madi of MISR Raymond International in Cairo for maintaining technical interest in our project, with friendship, flexibility, and good will, and to Mr. M. Hamzawi, MISR Raymond supervisor in Ismailia, for his gentle and even humor, ingenuity, and human qualities that kept the work on schedule during those five drilling expeditions. We will also remember the long hours in the field shared with the drilling teams, which in $1985,1987,1988,1989$, and 1990 were so ably led by field supervisors Awad, Saleh, Moustafa, and El Moati.

We express our gratitude to numerous persons who provided valuable assistance at the Smithsonian Institution: W. Boykins, H. Sheng, and J. Wingerath for processing samples in the Sedimentology Laboratory; R.L. Stuckenrath, Radiobiological Laboratory, for radiocarbon analyses made at the beginning of the Project; D.A. Dean, for thin section preparation in the Department of Paleobiology; M. Parrish, for drafting graphs and plates, at the Department of Paleobiology Illustration Laboratory; and V. Krantz, L. Thomas, and M.E. McCaffrey, for assistance at the Smithsonian's Department of Photographic Services.

Funding for the Nile Delta Project during the period 1985-1994 was provided primarily by numerous Smithsonian Institution awards and grants, including those from the Scholarly Studies Program, Office of the Assistant Secretary for Science, Office of Fellowships and Grants, Director of the
National Museum of Natural History, and the Museum's Department of Paleobiology. A large part of the field expenses, including drilling, were generously borne by the Research and Exploration Committee of the National Geographic Society. We also gratefully acknowledge the much needed support to meet field, laboratory, and postdoctoral research expenses given by AMOCO, ARCO, ELF-Aquitaine (Washington), IEOC-Cairo, and TEXACO-USA.

This monograph was kindly reviewed by T.A. Nelsen and D.J.P. Swift, and also by Z. Chen and A.G. Warne.

\section{Methods}

CORE COLlection.-The northern Nile delta plain is characterized by low gentle relief and dense vegetal cover, and by the general absence of surface exposures of older Holocene and late Pleistocene deposits. These attributes, plus the variable thickness $(<10 \mathrm{~m}$ to $>50 \mathrm{~m})$ and gentle inclination of subsurface Holocene strata, thus required that drilling be used to study the late Quaternary geological evolution of the Nile delta. The basis of the drilling strategy was to systematically recover complete sections of Holocene marine, brackish, and alluvial deposits of the modern Nile delta and portions of the underlying late Pleistocene alluvial deposits. Our preliminary surveys indicated that the area of major interest should extend across the entire deltaic arc, from the east at the Gulf of Tineh to the outskirts of Alexandria as far as Burg el Arab in the west (a distance of $\sim 225 \mathrm{~km}$ ). The study area in the low-lying northern third of the delta also extends as far south as $\sim 30 \mathrm{~km}$ from the present Mediterranean coast (Figure 1) to ensure that long drill cores collected in this sector would allow reasonably detailed stratigraphic correlations and paleogeographic interpretations of former interfingering fluvial, deltaic, and marine sections to be made. It was anticipated that the region selected for drilling would help define those areas of the delta most susceptible to rising sea level and land subsidence.

A total of 87 Smithsonian cores (S1-S87) ranging in depth from -20 to $60 \mathrm{~m}$ (lithologic logs in Appendix 1) and relatively evenly spaced $(\sim 10 \mathrm{~km})$ across the northern Nile delta were recovered (Figure 1). These were collected during five field seasons: cores S1-S17 in September-October 1985; cores S18-S30 in April-May 1987; cores S31-S46 in AugustSeptember 1988; cores S47-S65 in September 1989; and cores S66-S87 in August-September 1990. Drilling of the cores was made progressively from east to west across the northern Nile delta. Positioning of drill sites in the field was determined using recent detailed contour maps (scale 1:50,000) compiled since the 1970 s by the U.S. Defense Mapping Agency Hydrographic/Topographic Center in Washington, D.C. (DMA Map Series P 773 and 1501 NH 36), and also diverse sets of satellite images. Core number, total core length, date of core recovery, latitude and longitude, and approximate geographic position of each of the 87 core sites is listed in Table 1. Accuracy of core site positions in most cases is to 
TABLE 1.-Data pertaining to Smithsonian boring sites S1-S87 collected as a primary base for the Nile Delta Project in 1985, 1987, 1988, 1989, and 1990. General information lists the total length of core, date of recovery, latitude, longitude, and approximate location of the drill site (see Figure 1). U.S. Defense Mapping Agency chart series $\mathrm{P} 773$ and $1501 \mathrm{NH} 36$ served as a control for latitude and longitude, which provides accuracy to within 6 seconds, or $-200 \mathrm{~m}$.

\begin{tabular}{|c|c|c|c|c|c|}
\hline $\begin{array}{l}\text { Borehole } \\
\text { number }\end{array}$ & $\begin{array}{c}\text { Total } \\
\text { length }(m)\end{array}$ & $\begin{array}{c}\text { Date } \\
\text { recovered }\end{array}$ & Latitude & Longitude & $\begin{array}{c}\text { Approximate } \\
\text { location }\end{array}$ \\
\hline S1 & 28.96 & $9 / 25 / 1985$ & $31^{\circ} 26^{\prime} 54^{\prime \prime} \mathrm{N}$ & $31^{\circ} 46^{\prime} 42^{\prime \prime} \mathrm{E}$ & In Abbas Zahir \\
\hline S2 & 19.81 & $9 / 29 / 1985$ & $31^{\circ} 18^{\prime} 18^{\prime \prime} \mathrm{N}$ & $31^{\circ} 36^{\prime} 18^{\prime \prime} \mathrm{E}$ & $1.2 \mathrm{~km}$ ENE Abu Hammuda \\
\hline S3 & 29.87 & $9 / 23 / 1985$ & $31^{\circ} 17^{\prime} 48^{\prime \prime} \mathrm{N}$ & $31^{\circ} 43^{\prime} 24^{\prime \prime} \mathrm{E}$ & 0.9 km SW El Ghuneimiya \\
\hline S4 & 32.46 & $9 / 30 / 1985$ & $31^{\circ} 13^{\prime} 42^{\prime \prime} \mathrm{N}$ & $31^{\circ} 30^{\prime} 54^{\prime \prime} \mathrm{E}$ & 1.5 km NNW El Hisas \\
\hline S5 & 27.43 & $9 / 19 / 1985$ & $31^{\circ} 11^{\prime} 36^{\prime \prime} \mathrm{N}$ & $31^{\circ} 39^{\prime} 30^{\prime \prime} \mathrm{E}$ & 1 km NW Nasl Ez. Hasan Shakir \\
\hline S6 & 26.37 & $9 / 16 / 1985$ & $31^{\circ} 6^{\prime} 30^{\prime \prime} \mathrm{N}$ & $31^{\circ} 42^{\prime} 36^{\prime \prime} \mathrm{E}$ & $1.5 \mathrm{~km}$ E El Gineina \\
\hline S7 & 24.38 & $9 / 12 / 1985$ & $31^{\circ} 7^{\prime} 48^{\prime \prime} \mathrm{N}$ & $31^{\circ} 52^{\prime} 18^{\prime \prime} \mathrm{E}$ & $6.5 \mathrm{~km} \mathrm{SW}$ Manzalah \\
\hline S8 & 41.30 & $9 / 8 / 1985$ & $31^{\circ} 12^{\prime} 48^{\prime \prime} \mathrm{N}$ & $32^{\circ} 2^{\prime} 18^{\prime \prime} E$ & $2.5 \mathrm{~km} \mathrm{~N}$ El Matariya \\
\hline S9 & 15.70 & $9 / 4 / 1985$ & $30^{\circ} 58^{\prime} 42^{\prime \prime} \mathrm{N}$ & $31^{\circ} 52^{\prime} 42^{\prime \prime} \mathrm{E}$ & 1 km E San El Hagar El Qibliya \\
\hline S10 & 24.38 & $9 / 2 / 1985$ & $30^{\circ} 51^{\prime} 24^{\prime \prime} \mathrm{N}$ & $32^{\circ} 1^{\prime} 12^{\prime \prime} \mathrm{E}$ & 2 km NNE El Munagat El Kubra \\
\hline S11 & 29.87 & $9 / 8 / 1985$ & $30^{\circ} 55^{\prime} 12^{\prime \prime} \mathrm{N}$ & $32^{\circ} 18^{\prime} 24^{\prime \prime} \mathrm{E}$ & 3.5 km SE Ez. El Cop \\
\hline S12 & 23.77 & $9 / 12 / 1985$ & $31^{\circ} 3^{\prime} 48^{\prime \prime} \mathrm{N}$ & $32^{\circ} 33^{\prime} 18^{\prime \prime} \mathrm{E}$ & $2.5 \mathrm{~km} \mathrm{NE} \mathrm{Tel} \mathrm{El} \mathrm{Farama}$ \\
\hline S13 & 30.48 & $9 / 15 / 1985$ & $31^{\circ} 4^{\prime} 42^{\prime \prime} \mathrm{N}$ & $32^{\circ} 23^{\prime} 36^{\prime \prime} \mathrm{E}$ & Israeli Rd. $7.8 \mathrm{~km} \mathrm{E}$ of Suez Canal \\
\hline S14 & 23.16 & $9 / 18 / 1985$ & $30^{\circ} 59^{\prime} 54^{\prime \prime} \mathrm{N}$ & $32^{\circ} 28^{\prime} 12^{\prime \prime} \mathrm{E}$ & 7.5 km WSW Baluza \\
\hline S15 & 35.36 & $9 / 22 / 1985$ & $31^{\circ} 7^{\prime} 30^{\prime \prime} \mathrm{N}$ & $32^{\circ} 30^{\prime} 18^{\prime \prime} \mathrm{E}$ & 9 km NW Tel El Farama \\
\hline S16 & 28.04 & $9 / 30 / 1985$ & $31^{\circ} 21^{\prime} 36^{\prime \prime} \mathrm{N}$ & $32^{\circ} 3^{\prime} 48^{\prime \prime} \mathrm{E}$ & 1 km WSW Ez. Shalabi El Rudi \\
\hline S17 & 43.28 & $10 / 1 / 1985$ & $31^{\circ} 22^{\prime} 42^{\prime \prime} \mathrm{N}$ & $31^{\circ} 57^{\prime} 54^{\prime \prime} \mathrm{E}$ & $0.7 \mathrm{~km}$ NE Geziret Umm Abdalla \\
\hline S18 & 53.19 & $4 / 26 / 1987$ & $31^{\circ} 4^{\prime} 42^{\prime \prime} \mathrm{N}$ & $32^{\circ} 20^{\prime} 30^{\prime \prime} \mathrm{E}$ & 11.5 km WNW Tel Farama \\
\hline S19 & 12.19 & $5 / 2 / 1987$ & $31^{\circ} 2^{\prime} 54^{\prime \prime} \mathrm{N}$ & $32^{\circ} 33^{\prime} 0^{\prime \prime} \mathrm{E}$ & At Tel Farama \\
\hline S20 & 50.29 & $5 / 4 / 1987$ & $31^{\circ} 6^{\prime} 36^{\prime \prime} \mathrm{N}$ & $32^{\circ} 18^{\prime} 6^{\prime \prime} \mathrm{E}$ & $1 \mathrm{~km}$ W Suez Canal NE Extension \\
\hline S21 & 49.38 & $5 / 17 / 1987$ & $31^{\circ} 13^{\prime} 48^{\prime \prime} \mathrm{N}$ & $32^{\circ} 20^{\prime} 30^{\prime \prime} \mathrm{E}$ & $3 \mathrm{~km}$ SE Port Fouad \\
\hline S22 & 37.80 & $5 / 12 / 1987$ & $31^{\circ} 1^{\prime} 18^{\prime \prime} \mathrm{N}$ & $32^{\circ} 12^{\prime} 30^{\prime \prime} \mathrm{E}$ & $2 \mathrm{~km}$ W Ushash Arab Zeidan \\
\hline S23 & 13.72 & $5 / 10 / 1987$ & $30^{\circ} 49^{\prime} 48^{\prime \prime} \mathrm{N}$ & $32^{\circ} 15^{\prime} 12^{\prime \prime} \mathrm{E}$ & In Alawi Umm El Rish \\
\hline S24 & 10.97 & $5 / 9 / 1987$ & $30^{\circ} 51^{\prime} 6^{\prime \prime} \mathrm{N}$ & $32^{\circ} 10^{\prime} 18^{\prime \prime} \mathrm{E}$ & $5.5 \mathrm{~km} \mathrm{SW}$ Ushash Ibrahim Abu Muh \\
\hline S25 & 14.33 & $5 / 13 / 1987$ & $30^{\circ} 57^{\prime} 42^{\prime \prime} \mathrm{N}$ & $32^{\circ} 10^{\prime} 48^{\prime \prime} \mathrm{E}$ & 4 km SW Ushash Arab El Gadadia \\
\hline S26 & 13.72 & $5 / 12 / 1987$ & $30^{\circ} 54^{\prime} 24^{\prime \prime} \mathrm{N}$ & $32^{\circ} 1^{\prime} 48^{\prime \prime} \mathrm{E}$ & 0.2 km SE Minshat Abu Omar \\
\hline S27 & 15.24 & $5 / 16 / 1987$ & $31^{\circ} 0^{\prime} 24^{\prime \prime} \mathrm{N}$ & $32^{\circ} 1^{\prime} 54^{\prime \prime} \mathrm{E}$ & $3 \mathrm{~km} \mathrm{~S} \mathrm{Ubash} \mathrm{Mallaha}$ \\
\hline S28 & 36.58 & $5 / 24 / 1987$ & $31^{\circ} 26^{\prime} 30^{\prime \prime} \mathrm{N}$ & $31^{\circ} 33^{\prime} 18^{\prime \prime} \mathrm{E}$ & 0.7 km SSW Ez. El Gamasa E1 Shardya \\
\hline S29 & 39.62 & $5 / 25 / 1987$ & $31^{\circ} 21^{\prime} 42^{\prime \prime} \mathrm{N}$ & $31^{\circ} 27^{\prime} 6^{\prime \prime} \mathrm{E}$ & $0.5 \mathrm{~km}$ W Ez. El Mazia \\
\hline S30 & 42.67 & $5 / 22 / 1987$ & $31^{\circ} 24^{\prime} 30^{\prime \prime} \mathrm{N}$ & $32^{\circ} 0^{\prime} 42^{\prime \prime} \mathrm{E}$ & 4 km NW Ez. Shalabi Rudi \\
\hline S31 & 45.72 & $8 / 21 / 1988$ & $31^{\circ} 22^{\prime} 6^{\prime \prime} \mathrm{N}$ & $31^{\circ} 36^{\prime} 0^{\prime \prime} \mathrm{E}$ & 2.5 km E Kafr Wastani \\
\hline S32 & 21.34 & $9 / 1 / 1988$ & $31^{\circ} 16^{\prime} 48^{\prime \prime} \mathrm{N}$ & $31^{\circ} 24^{\prime} 30^{\prime \prime} \mathrm{E}$ & 0.3 km SW Hagg Shirbin Ez. Bahr El lsh \\
\hline S33 & 25.91 & $8 / 27 / 1988$ & $31^{\circ} 24^{\prime} 42^{\prime \prime} \mathrm{N}$ & $31^{\circ} 21^{\prime} 48^{\prime \prime} \mathrm{E}$ & 0.5 km N Ez. El Gezira \\
\hline S34 & 39.62 & $8 / 29 / 1988$ & $31^{\circ} 27^{\prime} 12^{\prime \prime} \mathrm{N}$ & $31^{\circ} 23^{\prime} 18^{\prime \prime} \mathrm{E}$ & $2 \mathrm{~km} \mathrm{~N} \mathrm{Abu} \mathrm{Madi}$ \\
\hline S35 & 35.05 & $8 / 24 / 1988$ & $31^{\circ} 31^{\prime} 42^{\prime \prime} \mathrm{N}$ & $31^{\circ} 18^{\prime} 30^{\prime \prime} \mathrm{E}$ & 1 km SE Qabr Sidi Durrgnam \\
\hline S36 & 45.72 & $9 / 5 / 1988$ & $31^{\circ} 27^{\prime} 48^{\prime \prime} \mathrm{N}$ & $31^{\circ} 15^{\prime} 24^{\prime \prime} \mathrm{E}$ & $0.5 \mathrm{~km}$ W ruins Kom Niqueza \\
\hline S37 & 21.34 & $8 / 31 / 1988$ & $31^{\circ} 22^{\prime} 12^{\prime \prime} \mathrm{N}$ & $31^{\circ} 16^{\prime} 48^{\prime \prime} \mathrm{E}$ & 3.3 km SE Kabira Gazireyet El Darfil \\
\hline S38 & 27.43 & $9 / 8 / 1988$ & $31^{\circ} 25^{\prime} 18^{\prime \prime} \mathrm{N}$ & $31^{\circ} 10^{\prime} 24^{\prime \prime} \mathrm{E}$ & In Ez. El Baralsa \\
\hline S39 & 18.29 & $8 / 29 / 1988$ & $31^{\circ} 25^{\prime} 12^{\prime \prime} \mathrm{N}$ & $31^{\circ} 4^{\prime} 24^{\prime \prime} \mathrm{E}$ & 0.75 km SE Kom El Masura \\
\hline $\mathrm{S} 40$ & 28.19 & $8 / 24 / 1988$ & $31^{\circ} 30^{\prime} 48^{\prime \prime} \mathrm{N}$ & $31^{\circ} 8^{\prime} 30^{\prime \prime} \mathrm{E}$ & 1.7 km NNE Hammad Mahattet El Kasha \\
\hline S41 & 51.82 & $9 / 14 / 1988$ & $31^{\circ} 34^{\prime} 30^{\prime \prime} \mathrm{N}$ & $31^{\circ} 12^{\prime} 18^{\prime \prime} \mathrm{E}$ & In Hammad Mahattet El Kasha \\
\hline S42 & 45.72 & $9 / 13 / 1988$ & $31^{\circ} 35^{\prime} 54^{\prime \prime} \mathrm{N}$ & $31^{\circ} 5^{\prime} 48^{\prime \prime} \mathrm{E}$ & 0.7 km E Baltim Resort Center \\
\hline S43 & 42.67 & $8 / 25 / 1988$ & $31^{\circ} 35^{\prime} 12^{\prime \prime} \mathrm{N}$ & $30^{\circ} 58^{\prime} 42^{\prime \prime} \mathrm{E}$ & ln El Burg \\
\hline S44 & 21.34 & $9 / 4 / 1988$ & $31^{\circ} 26^{\prime} 12^{\prime \prime} \mathrm{N}$ & $30^{\circ} 59^{\prime} 30^{\prime \prime} \mathrm{E}$ & $1.8 \mathrm{~km}$ SE Geziret El lsbiryas \\
\hline S45 & 30.48 & $8 / 30 / 1988$ & $31^{\circ} 30^{\prime} 30^{\prime \prime} \mathrm{N}$ & $31^{\circ} 1^{\prime} 54^{\prime \prime} \mathrm{E}$ & In Rsa El Bar \\
\hline S46 & 45.72 & $9 / 10 / 1988$ & $31^{\circ} 24^{\prime} 0^{\prime \prime} \mathrm{N}$ & $30^{\circ} 8^{\prime} 54^{\prime \prime} \mathrm{E}$ & 3.5 NNE Kom El Nashwein \\
\hline S47 & 42.67 & $9 / 3 / 1989$ & $31^{\circ} 32^{\prime} 24^{\prime \prime} \mathrm{N}$ & $30^{\circ} 50^{\prime} 12^{\prime \prime} \mathrm{E}$ & In Arab El Hanafi \\
\hline S 48 & 43.28 & $9 / 6 / 1989$ & $31^{\circ} 30^{\prime} 12^{\prime \prime} \mathrm{N}$ & $30^{\circ} 46^{\prime} 30^{\prime \prime} \mathrm{E}$ & $4.5 \mathrm{~km}$ SW Kiman El Saiyar \\
\hline S49 & 41.15 & $9 / 4 / 1989$ & $31^{\circ} 28^{\prime} 30^{\prime \prime} \mathrm{N}$ & $30^{\circ} 41^{\prime} 6^{\prime \prime} \mathrm{E}$ & $1 \mathrm{~km}$ SW Kom Mastaroh \\
\hline S50 & 41.15 & $9 / 5 / 1989$ & $31^{\circ} 26^{\prime} 6^{\prime \prime} \mathrm{N}$ & $30^{\circ} 34^{\prime} 54^{\prime \prime} \mathrm{E}$ & 3.5 km SSW Kom Mishtil \\
\hline S51 & 41.15 & $9 / 6 / 1989$ & $31^{\circ} 22^{\prime} 12^{\prime \prime} \mathrm{N}$ & $30^{\circ} 29^{\prime} 42^{\prime \prime} \mathrm{E}$ & $0.4 \mathrm{~km} \mathrm{~S}$ Ez. El Sakara \\
\hline S52 & 41.15 & $9 / 7 / 1989$ & $31^{\circ} 24^{\prime} 24^{\prime \prime} \mathrm{N}$ & $30^{\circ} 46^{\prime} 12^{\prime \prime} \mathrm{E}$ & 2 km NE Ras E1 Husan \\
\hline S53 & 27.43 & $9 / 8 / 1989$ & $31^{\circ} 23^{\prime} 42^{\prime \prime} \mathrm{N}$ & $30^{\circ} 40^{\prime} 36^{\prime \prime} \mathrm{E}$ & $0.4 \mathrm{~km}$ SW Atlet El Baqar \\
\hline S54 & 19.51 & $9 / 9 / 1989$ & $31^{\circ} 19^{\prime} 36^{\prime \prime} \mathrm{N}$ & $30^{\circ} 47^{\prime} 30^{\prime \prime} \mathrm{E}$ & $0.4 \mathrm{~km} \mathrm{~N} \mathrm{El} \mathrm{Haddadi}$ \\
\hline S55 & 19.81 & $9 / 9 / 1989$ & $31^{\circ} 18^{\prime} 54^{\prime \prime} \mathrm{N}$ & $30^{\circ} 40^{\prime} 18^{\prime \prime} \mathrm{E}$ & In Ez. El Saiyid Mansur \\
\hline S56 & 19.81 & $9 / 10 / 1989$ & $31^{\circ} 19^{\prime} 30^{\prime \prime} \mathrm{N}$ & $30^{\circ} 31^{\prime} 24^{\prime \prime} \mathrm{E}$ & $1 \mathrm{~km}$ SW Minyet El Murshid \\
\hline
\end{tabular}




\begin{tabular}{|c|c|c|c|c|c|}
\hline $\begin{array}{l}\text { Borehole } \\
\text { number }\end{array}$ & $\begin{array}{c}\text { Total } \\
\text { length }(m)\end{array}$ & $\begin{array}{c}\text { Date } \\
\text { recovered }\end{array}$ & Latitude & Longitude & $\begin{array}{l}\text { Approximate } \\
\text { location }\end{array}$ \\
\hline S57 & 19.81 & $9 / 10 / 1989$ & $31^{\circ} 22^{\prime} 24^{\prime \prime} \mathrm{N}$ & $30^{\circ} 35^{\prime} 42^{\prime \prime} \mathrm{E}$ & Fish market 1 km SE Gazayir El Minsirib \\
\hline S58 & 22.86 & 9/11/1989 & $31^{\circ} 23^{\prime} 6^{\prime \prime} \mathrm{N}$ & $30^{\circ} 25^{\prime} 48^{\prime \prime} \mathrm{E}$ & 0.7 km N Giddiya \\
\hline S59 & 41.15 & $9 / 12 / 1989$ & $31^{\circ} 27^{\prime} 42^{\prime \prime} \mathrm{N}$ & $30^{\circ} 26^{\prime} 30^{\prime \prime} \mathrm{E}$ & $3 \mathrm{~km}$ NE Abu Khashaba \\
\hline S60 & 30.48 & $9 / 12 / 1989$ & $31^{\circ} 19^{\prime} 42^{\prime \prime} \mathrm{N}$ & $30^{\circ} 24^{\prime} 36^{\prime \prime} \mathrm{E}$ & $0.4 \mathrm{~km}$ SE El Buseili Station \\
\hline S61 & 41.15 & $9 / 13 / 1989$ & $31^{\circ} 13^{\prime} 36^{\prime \prime} \mathrm{N}$ & $30^{\circ} 22^{\prime} 18^{\prime \prime} \mathrm{E}$ & $6 \mathrm{~km}$ WSW Hamad Dumeih \\
\hline S62 & 24.38 & $9 / 14 / 1989$ & $31^{\circ} 16^{\prime} 30^{\prime \prime} \mathrm{N}$ & $30^{\circ} 19^{\prime} 42^{\prime \prime} \mathrm{E}$ & $3.3 \mathrm{~km}$ SE Idku \\
\hline S63 & 22.25 & $9 / 14 / 1989$ & $31^{\circ} 21^{\prime} 0^{\prime \prime} \mathrm{N}$ & $30^{\circ} 18^{\prime} 54^{\prime \prime} \mathrm{E}$ & $1.6 \mathrm{~km} \mathrm{NE} \mathrm{El} \mathrm{Nawa} \mathrm{Fort}$ \\
\hline S64 & 41.15 & $9 / 16 / 1989$ & $31^{\circ} 24^{\prime} 30^{\prime \prime} \mathrm{N}$ & $30^{\circ} 20^{\prime} 42^{\prime \prime} \mathrm{E}$ & $0.2 \mathrm{~km} \mathrm{SW}$ El Farash Fort \\
\hline S65 & 48.62 & $9 / 15 / 1989$ & $31^{\circ} 28^{\prime} 36^{\prime \prime} \mathrm{N}$ & $30^{\circ} 21^{\prime} 30^{\prime \prime} \mathrm{E}$ & $0.6 \mathrm{~km}$ SSE Sidi Mansur \\
\hline S66 & 20.12 & $8 / 29 / 1990$ & $31^{\circ} 16^{\prime} 6^{\prime \prime} \mathrm{N}$ & $30^{\circ} 27^{\prime} 24^{\prime \prime} \mathrm{E}$ & $2.2 \mathrm{~km} \mathrm{~W} \mathrm{El} \mathrm{Faiza}$ \\
\hline S67 & 19.81 & $9 / 1 / 1990$ & $31^{\circ} 10^{\prime} 30^{\prime \prime} \mathrm{N}$ & $30^{\circ} 19^{\prime} 54^{\prime \prime} \mathrm{E}$ & $1.5 \mathrm{~km} \mathrm{~S} \mathrm{lbr} \mathrm{Zaiyat} \mathrm{Ez.} \mathrm{Kom} \mathrm{Aziza}$ \\
\hline S68 & 44.20 & $8 / 30 / 1990$ & $31^{\circ} 16^{\prime} 30^{\prime \prime} \mathrm{N}$ & $30^{\circ} 13^{\prime} 54^{\prime \prime} \mathrm{E}$ & 1.5 km NNE Gazayir El Tawila \\
\hline S69 & 19.81 & $10 / 1 / 1990$ & $31^{\circ} 11^{\prime} 36^{\prime \prime} \mathrm{N}$ & $30^{\circ} 16^{\prime} 30^{\prime \prime} \mathrm{E}$ & $1 \mathrm{~km}$ NNE Barsig Pumping Station \\
\hline S70 & 19.81 & $9 / 2 / 1990$ & $31^{\circ} 10^{\prime} 54^{\prime \prime} \mathrm{N}$ & $30^{\circ} 10^{\prime} 24^{\prime \prime} \mathrm{E}$ & $0.5 \mathrm{~km}$ SE Minshat Bulin \\
\hline S71 & 44.20 & $9 / 2 / 1990$ & $31^{\circ} 15^{\prime} 54^{\prime \prime} \mathrm{N}$ & $30^{\circ} 10^{\prime} 24^{\prime \prime} \mathrm{E}$ & $0.5 \mathrm{~km}$ SW El Miaddiya Outlet \\
\hline S72 & 19.81 & $9 / 3 / 1990$ & $31^{\circ} 13^{\prime} 54^{\prime \prime} \mathrm{N}$ & $30^{\circ} 8^{\prime} 0^{\prime \prime} \mathrm{E}$ & In Kom Tarfa \\
\hline S73 & 44.20 & $9 / 3 / 1990$ & $31^{\circ} 16^{\prime} 36^{\prime \prime} \mathrm{N}$ & $30^{\circ} 5^{\prime} 0^{\prime \prime} \mathrm{E}$ & 0.6 km N Ez. Hod \#4 \\
\hline S74 & 18.29 & $9 / 4 / 1990$ & $31^{\circ} 13^{\prime} 24^{\prime \prime} \mathrm{N}$ & $30^{\circ} 4^{\prime} 42^{\prime \prime} \mathrm{E}$ & $0.8 \mathrm{~km} \mathrm{NNE} \mathrm{El} \mathrm{Akhdar}$ \\
\hline S75 & 24.38 & $9 / 4 / 1990$ & $31^{\circ} 16^{\prime} 24^{\prime \prime} \mathrm{N}$ & $30^{\circ} 2^{\prime} 48^{\prime \prime} \mathrm{E}$ & In Ez. Maqnas \\
\hline S76 & 19.81 & $10 / 4 / 1990$ & $31^{\circ} 13^{\prime} 48^{\prime \prime} \mathrm{N}$ & $30^{\circ} 0^{\prime} 48^{\prime \prime} \mathrm{E}$ & $3 \mathrm{~km}$ SSE Ez. Farqon \\
\hline S77 & 40.54 & $10 / 6 / 1990$ & $31^{\circ} 10^{\prime} 48^{\prime \prime} \mathrm{N}$ & $29^{\circ} 55^{\prime} 54^{\prime \prime} \mathrm{E}$ & 1 km SW Fouad 1 Airport \\
\hline S78 & 19.81 & $10 / 8 / 1990$ & $31^{\circ} 9^{\prime} 0^{\prime \prime} \mathrm{N}$ & $30^{\circ} 2^{\prime} 24^{\prime \prime} \mathrm{E}$ & 1.5 km NE Kom Lunsan \\
\hline S79 & 42.67 & $9 / 5 / 1990$ & $31^{\circ} 6^{\prime} 12^{\prime \prime} \mathrm{N}$ & $29^{\circ} 56^{\prime} 54^{\prime \prime} \mathrm{E}$ & 3 km SW Prince Omar Tusan's kiosks \\
\hline$S 80$ & 45.72 & $9 / 7 / 1990$ & $31^{\circ} 6^{\prime} 6^{\prime \prime} \mathrm{N}$ & $29^{\circ} 51^{\prime} 12^{\prime \prime} \mathrm{E}$ & 1.8 km NW Kom El Shuran \\
\hline S81 & 21.34 & $10 / 10 / 1990$ & $31^{\circ} 3^{\prime} 12^{\prime \prime} \mathrm{N}$ & $29^{\circ} 59^{\prime} 30^{\prime \prime} \mathrm{E}$ & 1.8 km NE Prince Omar Tusan's house \\
\hline S82 & 30.48 & $9 / 8 / 1990$ & $31^{\circ} 3^{\prime} 0^{\prime \prime} \mathrm{N}$ & $29^{\circ} 52^{\prime} 24^{\prime \prime} \mathrm{E}$ & 2 km E Kom Mitauwh \\
\hline S83 & 45.72 & $9 / 9 / 1990$ & $31^{\circ} 3^{\prime} 24^{\prime \prime} \mathrm{N}$ & $29^{\circ} 46^{\prime} 12^{\prime \prime} \mathrm{E}$ & $5.5 \mathrm{~km} \mathrm{NW} \mathrm{El} \mathrm{Gamiriya}$ \\
\hline S84 & 22.86 & $9 / 11 / 1990$ & $30^{\circ} 59^{\prime} 36^{\prime \prime} \mathrm{N}$ & $29^{\circ} 37^{\prime} 6^{\prime \prime} \mathrm{E}$ & $0.8 \mathrm{~km}$ SSW Manaret fish market \\
\hline S85 & 10.21 & $9 / 10 / 1990$ & $30^{\circ} 55^{\prime} 24^{\prime \prime} \mathrm{N}$ & $29^{\circ} 31^{\prime} 36^{\prime \prime} \mathrm{E}$ & $1.5 \mathrm{~km} \mathrm{NW}$ Burg El Arab \\
\hline S86 & 41.15 & $10 / 11 / 1990$ & $30^{\circ} 51^{\prime} 18^{\prime \prime} \mathrm{N}$ & $30^{\circ} 47^{\prime} 48^{\prime \prime} \mathrm{E}$ & 3 km Kafr El Zaiyat \\
\hline S87 & 41.15 & $9 / 12 / 1990$ & $30^{\circ} 44^{\prime} 24^{\prime \prime} \mathrm{N}$ & $31^{\circ} 1^{\prime} 54^{\prime \prime} \mathrm{E}$ & $0.7 \mathrm{~km} \mathrm{~S}$ El Malwani Mosque \\
\hline
\end{tabular}

within $200 \mathrm{~m}$. More detailed notations made during the course of drilling, including more exact position of boring sites (to within $50 \mathrm{~m}$ ), are recorded in a series of 12 field books permanently archived at the National Museum of Natural History.

Two ACKER II trailer-mounted rigs were used concurrently by two drilling teams during each of the five expeditions (Figure 2). Casing was used at sites where thick subsurface sections of sand or soft mud prevailed (Figure 3). Sediment recovery at each drill site was continuous, by progressively connecting iron core tube barrels of either 5 foot $(1.52 \mathrm{~m})$ or 10 foot $(3.05 \mathrm{~m})$ lengths. Sediment core diameter ranged from 8 to $10 \mathrm{~cm}$. Recovery of moderate to well-indurated mud-rich sections was good to excellent, preserving original physical and biogenic structures. Extrusion of very stiff mud, usually highly consolidated clayey silts of late Pleistocene age, was usually accomplished by high-pressure pumping of circulated water (Figure 4). Collection of very soft (undersaturated) mud and thick sand mud sequences proved more difficult. Where sections were comprised essentially of sand, washings from pumped circulated water (rather than cores) were obtained from core tubes (Figure 5), usually at 1 to $2 \mathrm{~m}$ depth intervals. Original structures are not preserved in these washings. In the western part of the study area, between Alexandria and Burg el Arab, semiconsolidated to indurated carbonate sections were recovered (Figure 6) beneath thin Holocene sections.

Upon extrusion from the drill barrel, sediment core sections were cut into $\sim 1.5 \mathrm{~m}$ lengths (Figure 7), laid in plastic liners, described, photographed, and then wrapped and sealed with plastic sheeting and placed in specially prepared $1.5 \mathrm{~m}$-long wooden boxes (Figure 8 ). Washings were collected in plastic jars. Cores were assigned consecutive roman numerals, whereas washings received consecutive arabic numerals, down-boring. Cores and washings were then transported by air to the Smithsonian Institution in Washington, D.C., where they were stored in a refrigerated room prior to study. Upon recovery, representative core and washing samples $(30-40$ per core) were also selected from each boring and provided to our Egyptian counterpart organizations: cores S1-S17, to Dr. M. Khafagy at the Coastal Research Institute, Alexandria, in 1985; and cores S18-S87, to Dr. A. Bassiouni at the Department of Geology at Ain Shams University in Abbassia, Cairo, in 1987, 1988, 1989, and 1990.

Descriptions recorded in the field for each recovered core section include depth, length of drill barrel used, length of sediment section recovered, sediment color, gross texture and 
obvious sedimentary structures, biogenic features (such as shell and peat), and sediment density (hardness, consistency) using a pocket penetrometer. Color, texture, and unusual features were also recorded for sands collected as washings. Upon recovery, $35 \mathrm{~mm}$ color slide photographs were made of every core section at approximately $50 \mathrm{~cm}$ length intervals, with some overlap, and these include a metric scale to determine core length. These photographs and data notations in field books are maintained at the National Museum of Natural History.

In addition to the above, lithologic logs and representative samples from nine long drill cores collected earlier in the Manzala Lagoon area were provided by the Coastal Research Institute in Alexandria to the Nile Delta Project team for additional study in Washington (Stanley and Liyanage, 1986). We also consulted lithologic logs of northern Nile delta drill borings from various unpublished sources, such as engineering consulting firms, the Egyptian Ministry of Irrigation and Agriculture departments, the Suez Canal authority, and U.S. AID reports, and in publications including those of Attia (1954) and UNDP/UNESCO (1978). These valuable documents supplemented information from the five Smithsonian drilling surveys (published in, respectively, from east to west: Coutellier and Stanley, 1987; Stanley, Warne et al., 1992; Arbouille and Stanley, 1991; Chen et al., 1992; Warne and Stanley, 1993b). Two Smithsonian cores collected in the central delta near Kafr El-Zaiyat and Tanta (S86 and S87) are described by Chen and Stanley (1993).

In addition to the long drill cores cited above, a suite of about 100 short cores, for the most part less than $1 \mathrm{~m}$ in length (Figure 9), along with approximately 200 surficial samples, were also collected for more specific study of the Nile delta lagoons (Manzala, Burullus, and Idku), former Abu Qir Lagoon, and Lake Mariut. These sediment samples are not presented in this monograph, but they are described in archival field books and detailed in publications by Randazzo (1992), Loizeau and Stanley (1993), Bernasconi and Stanley (1994), Loizeau and Stanley (1994), and Siegel et al. (1994). Also described elsewhere in a series of publications are data on short cores and surficial samples collected seaward of the delta on the shelf and Nile Cone (Stanley and Maldonado, 1983; Anastasakis and Stanley, 1984, 1985; Stanley, 1985, 1988a; Frihy et al., 1995), in the Suez Canal (Stanley et al., 1982), and in the River Nile (Schneiderman, 1995).

LABORATORY ANALYSES.-Extensive petrological, geochemical, faunal, and floral studies of the 87 long drill cores (S1-S87) were made so as to define the major late Pleistocene and Holocene (to modern) lithofacies in the northern Nile delta and to more precisely distinguish among prodelta, delta-front, strandline, lagoon, and floodplain deposits. This information was then used to make lithostratigraphic correlations and paleogeographic maps of the northern delta, to calculate land subsidence rates, and to interpret sea-level and climate changes through time.

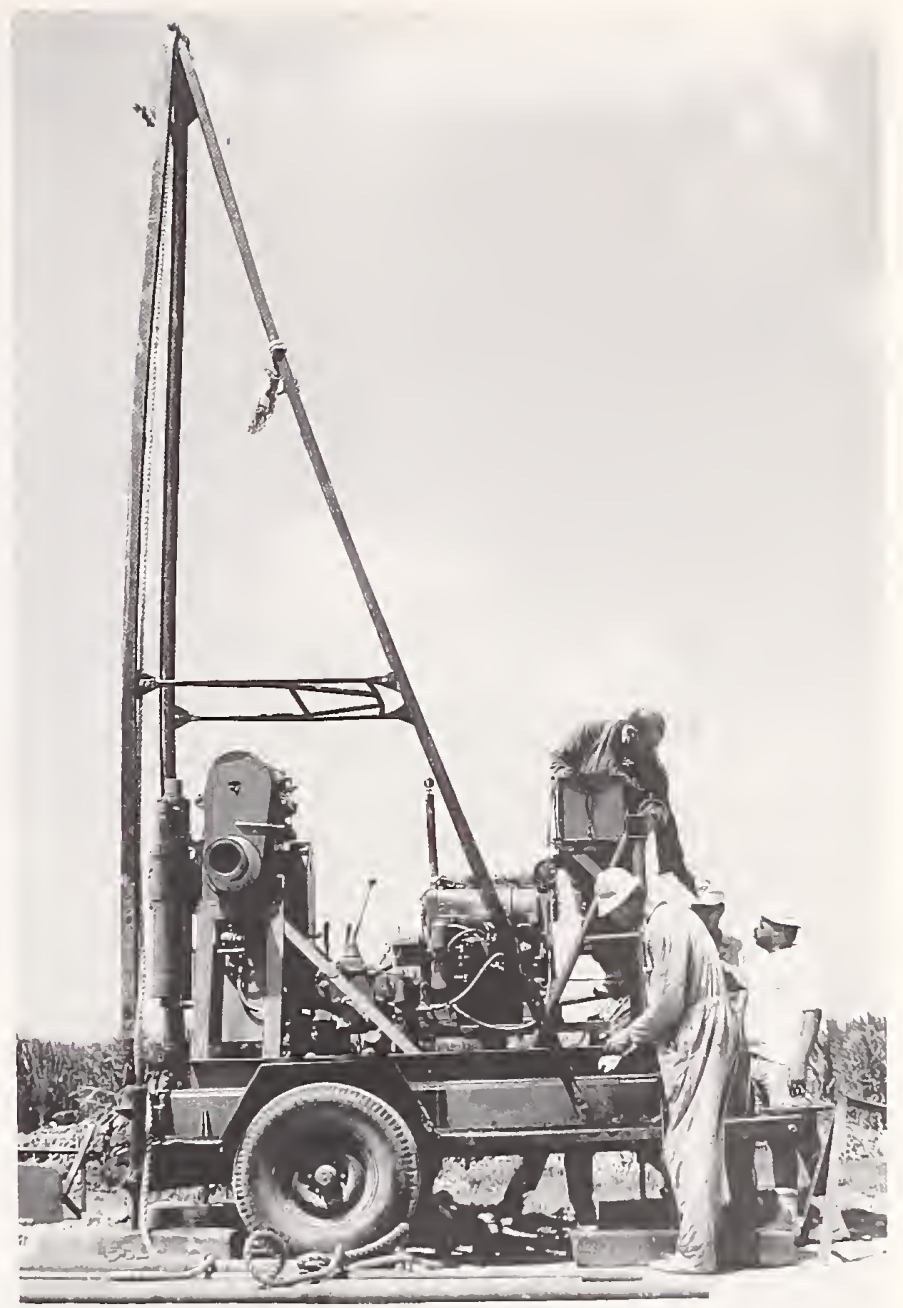

FIGURE 2.-Trailer-mounted ACKER II equipment of the type used during the five Nile delta drilling surveys described in this study. Photograph taken at site S69 in September 1990

The core sections were placed in a high humidity refrigerated room, with the temperature maintained at $4^{\circ} \mathrm{C}$, until they were ready for study. After the cores were split, all sections were $\mathrm{X}$-radiographed using $14 \times 17$ inch $(35.6 \times 43.2 \mathrm{~cm})$ industrial film, and positive prints (1:1 scale) were made from the $\mathrm{X}$-radiographs. Split core sections, while still moist, were then photographed, using $35 \mathrm{~mm}$ color slide film, at about $40 \mathrm{~cm}$ length intervals, with some overlap. A detailed lithological log was made of each Smithsonian core on the basis of visual observations, including color, details of sedimentary and biogenic structures in the strata, subtle features noted in $\mathrm{X}$-radiographs, and penetrometer sediment hardness readings of the split cores. These notations were compiled and recorded during the period 1985 to 1992 in laboratory books presently archived, along with the complete set of X-radiographs and 


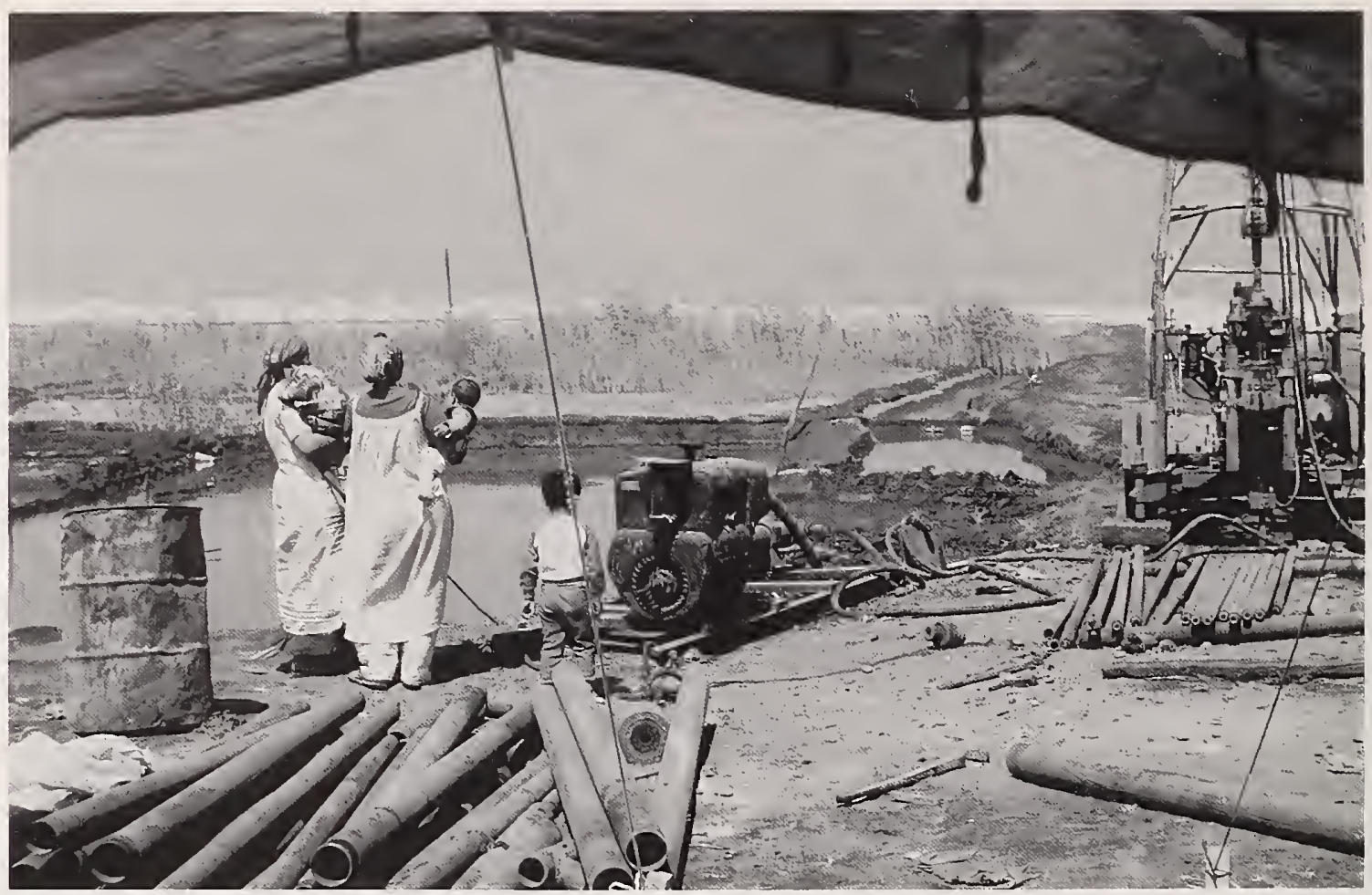

FIGURE 3.-Large-diameter pipes in foreground are used for casing, particularly when drilling in thick sections of sand and/or soft mud. Note 10 -foot $(3.05 \mathrm{~m})$ lengths of drill pipe assembled near the drilling equipment. Photograph taken at site S11 in September 1985.

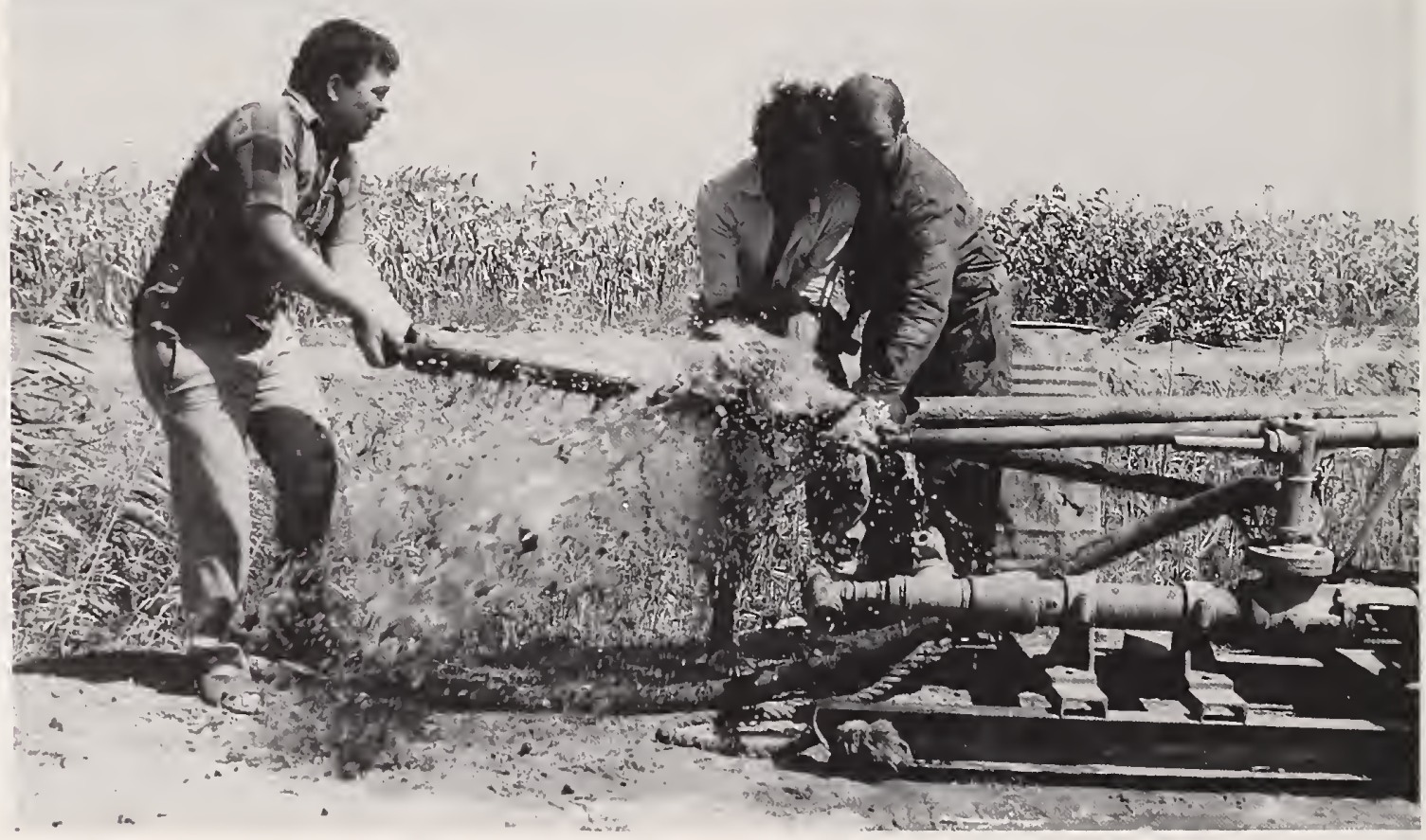

FIGURE 4.-Extrusion from drill pipe of stiff clayey silt of late Pleistocene age, using high pressure pumping of circulated water. Photograph taken at site S55 in September 1989. 


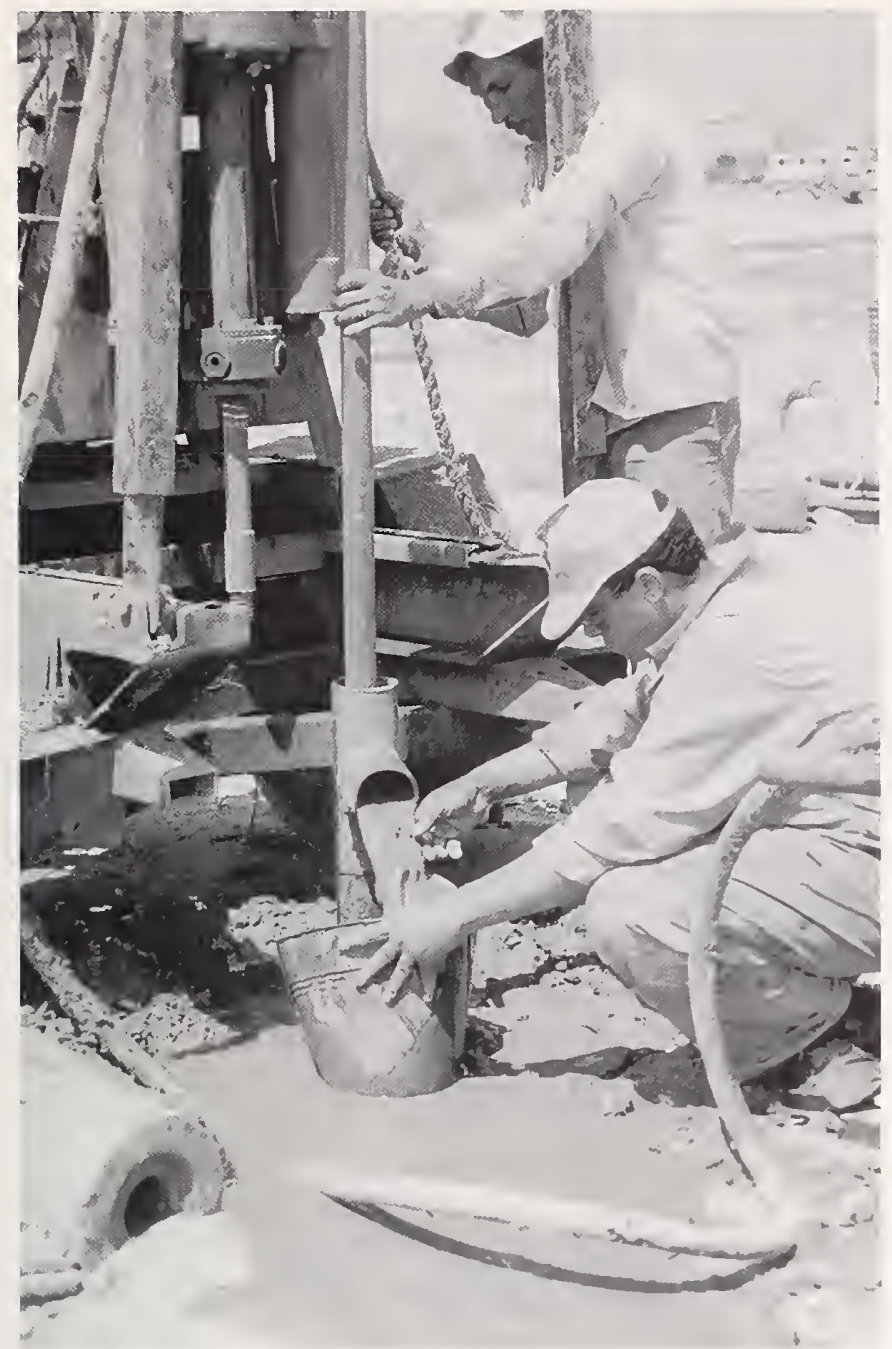

FIGURE 5.-Recovery of sediment from washings of circulated water in a thick sand sequence. Photograph taken at site S77 in September 1990.

color slides of split cores, at the National Museum of Natural History. Petrologic attributes for core sections and washings derived from these documents were used to draft detailed lithologic logs of the 87 borings. Simplified logs are presented in Appendix 1.

To obtain more detailed petrologic information, samples were collected down-boring at every change of lithology, or in the case of homogenous sections at a minimum of $50 \mathrm{~cm}$ intervals (except in the case of washings) along the entire length of the boring. More than 2500 samples were selected from the 87 borings, or an overall average of -30 samples per core, for standard textural and compositional analyses (data listed in Appendix 2). Core and washing sample numbers and depths in this listing correspond to those shown on the lithologic logs in Appendix 1.

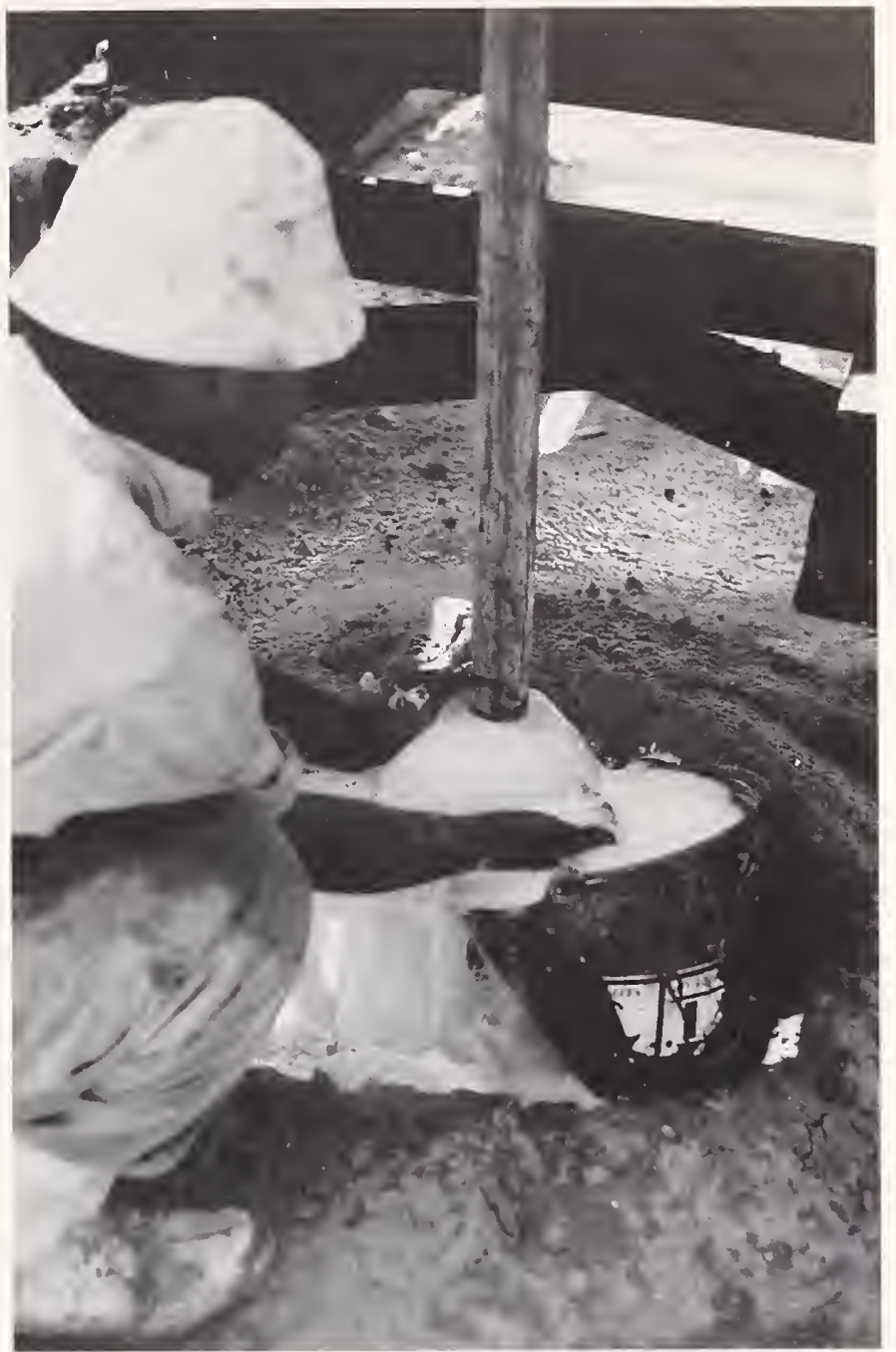

FIGURE 6.-Drilling through consolidated carbonate section of late Pleistocene age in the region west of Alexandria. Pumped water is typically white when circulated through drilled carbonate sequences. Photograph taken at site S85 in September 1990.

The proportions of sand $(>63 \mu \mathrm{m})$, silt $(2-63 \mu \mathrm{m})$, and clay $(<2 \mu \mathrm{m})$ fractions were determined by sieve and pipette analyses. A separate study of the relative percentages of components forming the sand-sized fraction in all samples was made using a binocular microscope, following the petrographic method of Coutellier and Stanley (1987), Frihy and Stanley (1988), and Stanley and Chen (1991). Relative percentages of major sand-sized components were calculated from point counts of $>300$ grains for all samples. The 16 components counted include 8 mineralogical (light and heavy minerals, mica, glauconite/verdine, pyrite, evaporite/gypsum, lithic fragments, aggregate), 6 faunal (indeterminate shell fragments, foraminifera, ostracod, gastropod, pelecypod, sponge), and 2 floral (plant fragments, including seed and fiber, and diatom). 


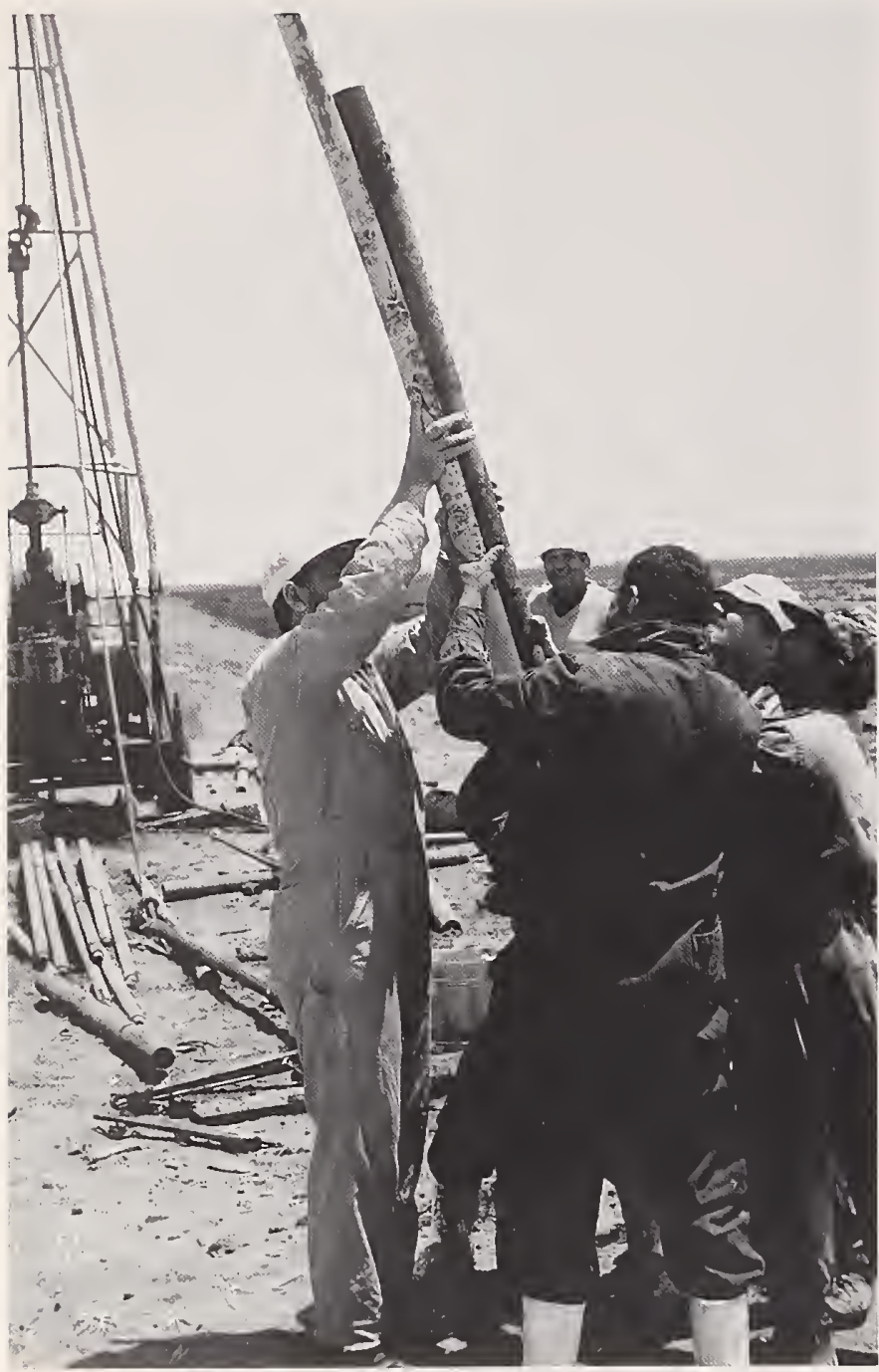

FIGURE 7.-Mud-rich sediment core of Holocene age placed in a plastic liner after extrusion from the drill barrel. Photograph taken at site SI 8 in April 1987.

Proportions of the various sand-sized compositional components and their positions along the core length (data in Appendix 2) are graphically depicted on each of the core lithologic logs (Appendix 1). All this information has been used in a series of geological studies of the northern delta, including the five regional surveys by Coutellier and Stanley (1987), Arbouille and Stanley (1991), Stanley, Warne et al. (1992), Chen et al. (1992), and Warne and Stanley (1993b).

In addition to the 2500 samples taken from the 87 long cores cited above, another -1000 core samples were selected for separate, more specific, petrographic and faunal study. These include those of the sand-sized fraction examined for glauconite/verdine (Pimmel and Stanley, 1989), surface features of quartz (Frihy and Stanley, 1987; Stanley and Chen, 1991), heavy minerals (Stanley et al., 1988; Foucault and Stanley, 1989; Stanley, 1989), and carbonates (Stanley and Hamza,
1992). Biogenic fractions of sand-sized material in numerous core samples also were examined: foraminifera (Kulyk, 1987); ostracods (Pugliese and Stanley, 1991); molluscs (Bernasconi et al., 1991; Longo, 1992; Bernasconi and Stanley, 1994); and plant matter (Howa and Stanley, 1991). Volcanic shards (Stanley and Sheng, 1986) and pollen (Leroy, 1992) were detailed in the silt-size fraction of some long core samples in the Manzala Lagoon area. Analyses of clay minerals also were made (Stanley and Liyanage, 1986; Abu-Zeid and Stanley, 1990; Abdel Wahab and Stanley, 1991). Geochemical analyses of long core samples were made in the northeastern Nile delta using either the sand fraction (Hamroush and Stanley, 1991; Allen et al., 1993), the silt and clay fractions (Dominik and Stanley, 1993), or primarily the clay fraction (Gerber, 1988; Gupta, 1989; Shergill, 1990; Siegel et al., 1995).

The petrology, including structures, petrography, and texture, of short core sections and surficial grab samples in different localities was also examined, using methods comparable to those employed for study of the long cores. These areas include the following: the Nile delta shelf and Nile Cone (Stanley and Maldonado, 1983; Anastasakis and Stanley, 1984, 1985; Stanley, 1985, 1988a, 1989; Frihy et al., 1995); Suez Canal (Stanley et al., 1982); delta lagoons (Randazzo, 1992; Longo, 1992; Loizeau and Stanley, 1993, 1994; Siegel et al., 1994); and the River Nile between Aswan and the Mediterranean coast (Schneiderman, 1995).

RADIOCARBON DATING.-An essential part of the Nile delta core study was to clearly distinguish subsurface Holocene from late Pleistocene sections and to subdivide Holocene sections into viable mappable stratigraphic sequences. It was anticipated that by obtaining a large set of radiocarbon dates we could establish a chronostratigraphic framework that would enhance regional litho- and biostratigraphic correlations. A total of 412 samples in 86 of the borings (all except core S65) were submitted for radiocarbon dating (Table 2); of these samples, 358 provided radiocarbon ages, and 54 had insufficient carbon for reliable dates. This constitutes a base of approximately four dates per boring. Sample positions and depth in the borings are shown in the lithologic logs in Appendix 1. Material selected for dating was obtained from split core sections $10-12 \mathrm{~cm}$ in length. Most of these dates were obtained using total carbon in dark olive organic-rich layers (for the most part lagoonal deposits) and peats (marsh deposits); shell matter was also used in a few instances. Most analyses were made by Beta Analytic Inc. $\odot$ of Miami, Florida; an additional 19 samples, selected from borings S7 and S8, were treated by the Smithsonian Institution's Radiobiology Laboratory. The dates are shown in Table 2 and in Appendices 1 and 2, and the permanent radiocarbon record numbers are also listed in Table 2 . Chronostratigraphic correlations based on these data are depicted in a series of published studies made across the northern delta, from east to west: Coutellier and Stanley (1987); Stanley, Warne et al. (1992); Arbouille and Stanley (1991); Chen et al. (1992); and Warne and Stanley (1993b). 


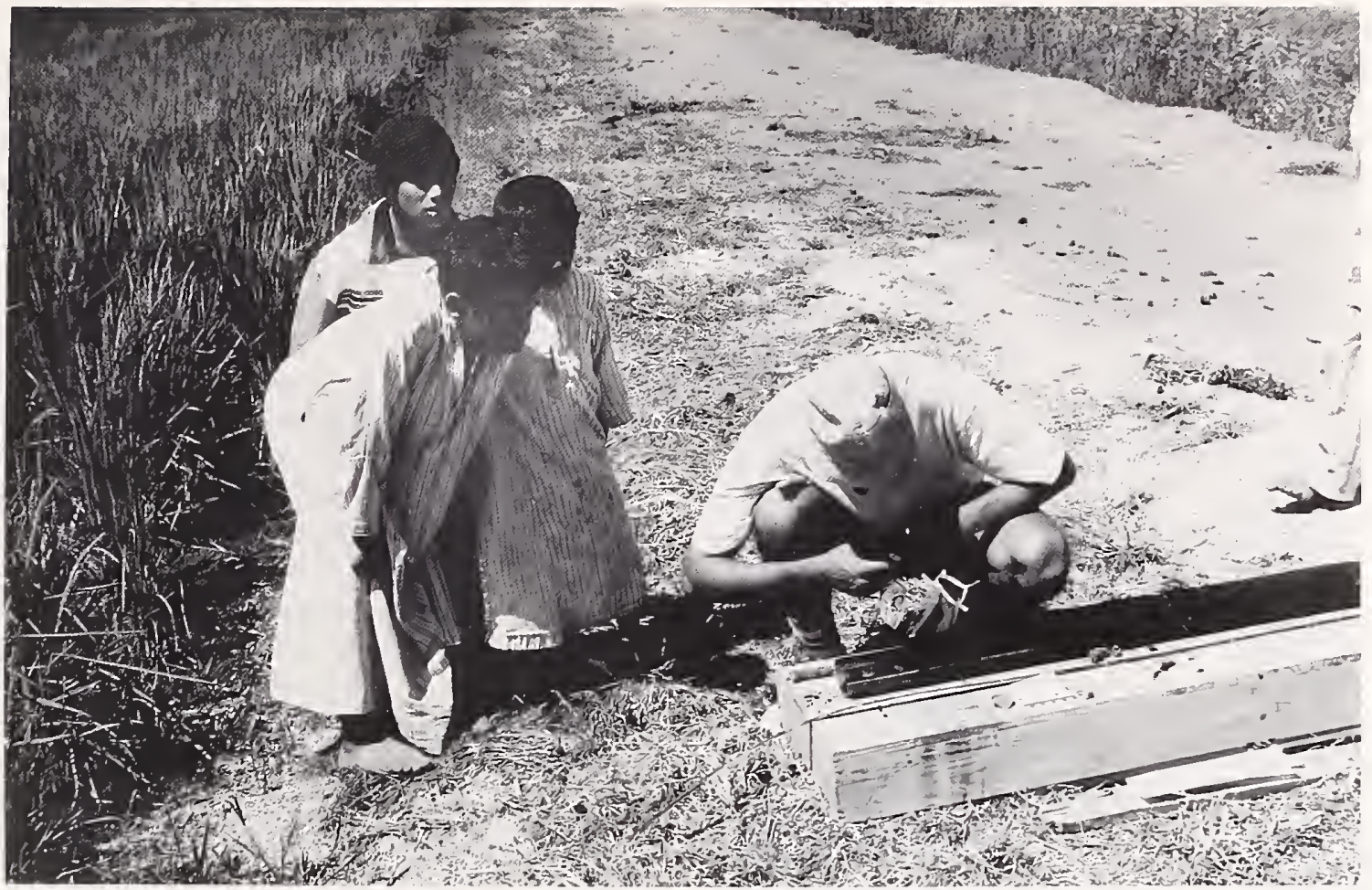

FIGURE 8.-After extraction from the drill barrel, mud-rich sediment cores are cut into 5-foot $(1.5 \mathrm{~m})$ lengths, placed in plastic liners, and described prior to storage in boxes. Photograph taken at site S55 in September 1989.

Short cores in other sectors have also been radiocarbon dated, and this information is available in several publications: in the northern Suez Canal (Stanley et al., 1982); and in the Nile delta shelf and Nile Cone (Stanley and Maldonado, 1983; Anastasakis and Stanley, 1984, 1985; Stanley, 1985, 1988a).

Identified archeological material preserved in cores (Stanley et al., 1992; Warne and Stanley, 1993a) and a dating study emphasizing the amino acid racemization methodology on several long cores (Goodfriend and Stanley, 1996) provide additional age information on the Holocene delta sections.

\section{Nile Delta Project Data Used in Scientific Literature}

\section{STUdIES LISTED BY LOCATION}

A recent bibliographic listing of all known publications through 1993 that pertain to the geology and geography of the Nile delta proper and its immediate vicinity, on land and offshore, recently has been compiled (Stanley et al., 1994). It is of note that during the past decade there have been over 50 articles published in the scientific literature and seven theses have been completed as a direct result of research undertaken in Egypt as sponsored by the Nile Delta Project. These describe in considerable detail the various methods used in the study of the
Nile materials cited above. Most of these documents include at least some of the S1-S87 core data referred to in the previous section and presented in Appendices 1 and 2.

A series of publications that draw upon the Smithsonian Institution's borings and core sample data focus on the late Pleistocene to Recent paleogeographic evolution of the northern delta plain. Many of these emphasize petrologic descriptions, lithofacies interpretations, chrono- and lithostratigraphic core-to-core correlations of late Quaternary sections, and the effects of sea-level change, climate, neotectonism, and sediment transport processes on Nile delta deposits. These investigations include studies of the northeastern delta (Coutellier and Stanley, 1987; cores S1-S17; Stanley, 1988b; cores S1-S36); the northern delta (Stanley, Warne et al., 1992; cores S1-S16, S28-S46); the north-northwestern delta (Arbouille and Stanley, 1991; cores S38-S59); and the northwestern delta (Chen et al., 1992; cores S51-S78; Stanley and Hamza, 1992; cores S74-S85; Warne and Stanley, 1993b; cores S72-S85). A study using selected borings in the central delta (cores S86 and S87) was made by Chen and Stanley (1993).

Other Nile Delta Project studies of the lower plain region, focusing on the late Holocene to present time, have primarily used short cores ( $<1 \mathrm{~m}$ length) and surficial sediment grab samples, rather than the long S-cores. Study areas include 


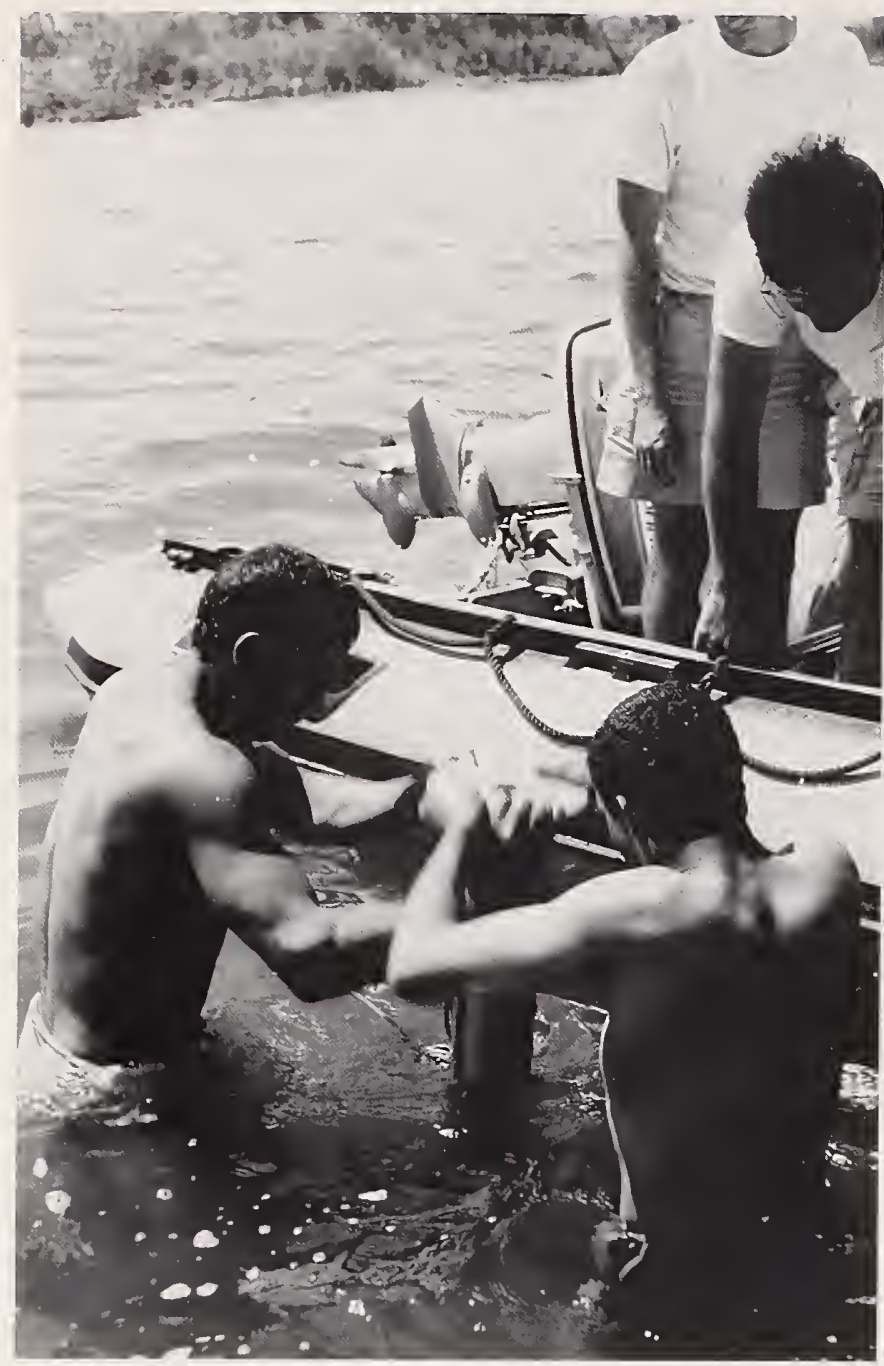

FIGURE 9.- Recovery of a short core $(-1 \mathrm{~m})$ in Manzala Lagoon. Photograph taken at short core site Man-IV and collected in September 1989.

Manzala Lagoon (Randazzo, 1992; Slaboda, 1993; Bernasconi and Stanley, 1994; Siegel et al., 1994), Burullus Lagoon (Bernasconi and Stanley, 1994), Idku Lagoon (Loizeau and Stanley, 1993; Bernasconi and Stanley, 1994), and Mariut Lake (Bernasconi and Stanley, 1994; Loizeau and Stanley, 1994). Analyses were also made on Suez Canal sediments, including those in its northern sector, positioned in the northeastern Nile delta (Stanley et al., 1982; Gerges and Stanley, 1985; Bernasconi and Stanley, in press). A petrologic investigation of lower Nile River deposits, between the Egypt-Sudan border and Cairo, has been initiated with a first study on heavy minerals completed by Schneiderman (1995). Studies of late Quaternary deposits seaward of the Nile delta include those on Abu Qir Bay (Frihy et al., 1995) and several on the Nile delta shelf and Nile Cone (Stanley and Maldonado, 1983; Anastasakis and Stanley, 1984, 1985; Stanley, 1985, 1988a, 1989).

\section{STUdIES LISTED BY TOPIC AND THEME}

In addition to geographic attribution, most of the studies listed above, along with other published scientific articles and theses completed during the course of the Nile Delta Project, can be listed by specific topic or theme. We recognize 12 major categories in which we can incorporate data collected as part of the project. As would be expected, most published articles and theses can be assigned to at least two or three of these. Each category comprises contributions listed chronologically, including year, last name of author(s), and abbreviated topic notation. For complete reference citations, the reader is directed to the Literature Cited in this publication.

Petrology, Composition, AND TeXture.-Most studies made during the course of the project consider lithologic attributes, such as sedimentary and biogenic structures, grain size, and composition of sand- and clay-size fractions. It is primarily on this basis that Nile delta facies of late Pleistocene and Holocene age are defined and interpreted, and their distribution mapped in time and space.

1982 Stanley, Freeland, and Sheng: Suez Canal sediments

1983 Stanley and Maldonado: Nile Cone sedimentation

1984 Anastasakis and Stanley: Sapropels on the Nile Cone

1985 Stanley: Mud redeposition on the Nile Cone

1985 Anastasakis and Stanley: Sapropels on the Nile Cone

1986 Stanley and Sheng: Volcanic shards in the delta

1986 Stanley and Liyanage: Clay minerals in the northeastern delta

1987 Frihy and Stanley: Quartz grain surface textures

1987 Coutellier and Stanley: Petrology and lithofacies, northeastern delta

1988 Frihy and Stanley: Texture and composition of delta deposits

1988 Stanley, Sheng, and Pan: Heavy minerals, northeastern delta

1988 Gerber: Clays and geochemistry, northeastern delta cores

1988a Stanley: Sedimentation on the Nile delta shelf

1989 Pimmel and Stanley: Verdinized fecal pellets in Holocene deposits

1989 Foucault and Stanley: Heavy minerals, northeastern delta

1989 Gupta: Clays and geochemistry, northeastem delta

1989 Stanley: Heavy minerals between the delta and Israeli margin

1990 Abu-Zeid and Stanley: Clay minerals, northeastern delta

1990 Shergill: Clays and geochemistry, northeastern delta

1991 Abdel Wahab and Stanley: Clay minerals, northern delta 
1991 Arbouille and Stanley: Petrology and lithofacies, northern delta

1991 Howa and Stanley: Petrology and plant matter across the delta

1991 Stanley and Chen: Stain-grained and sand-size composition of diverse modern delta facies

1992 Stanley and Hamza: Carbonate sediments, northwestern delta

1992 Stanley, Warne et al.: Petrology and lithofacies, northern delta

1992 Randazzo: Petrology of Manzala Lagoon sediments

1992 Chen, Warne, and Stanley: Petrology and lithofacies, northwestern delta

1993 Chen and Stanley: Alluvial stiff muds, late Pleistocene

1993b Warne and Stanley: Petrology and lithofacies, northwestern delta

1993 Loizeau and Stanley: Lithofacies, Idku Lagoon

1994 Loizeau and Stanley: Lithofacies, Mariut Lake

1995 Frihy, Moussa, and Stanley: Abu Qir Bay sediments

1995 Schneiderman: River Nile sands between Aswan and the delta

FAUNAL ANALYSES.-Studies in this category include micro- and macro-fossil analyses in long cores in the northern Nile delta, and also short core and grab samples collected in delta lagoons. These investigations provide ecological information on depositional environments.

1987 Kulyk: Foraminifera in the northeastern delta

1991 Bernasconi, Stanley, and DiGeronimo: Molluscan faunas in the northeastern delta

1991 Pugliese and Stanley: Ostracodes in the northeastern delta

1992 Longo: Molluscan faunas and palaeoecology in delta lagoons

1994 Bernasconi and Stanley: Molluscan biofacies in delta lagoons

FLORAL ANALYSES.-To date, only two floral studies in Nile delta sediments have been completed within the framework of this project. Plant matter of sand size and pollen in long cores provide information on paleoclimatological and paleoecological changes with time in the study area.

1991 Howa and Stanley: Plant matter distribution across the northern delta

1992 Leroy: Palynological assemblages, northeastern delta

GEOCHEMICAL ANALYSES.-Included in this category are investigations of trace and rare earth elements, which provide information on environmental and paleoclimatic changes through time, provenance, and dispersal, and the means to gauge increased pollution in northern delta sectors, including lagoons.

1988 Gerber: Trace elements, northeastern delta

1989 Gupta: Trace elements, northeastern delta

1990 Shergill: Trace elements, northeastern delta

1991 Hamroush and Stanley: Rare earth elements and paleoclimate oscillations

1993 Allen, Hamroush, and Stanley: Trace elements and archaeological implications

1993 Dominik and Stanley: Trace elements and peats

1993 Slaboda: Trace elements in recent Manzala Lagoon

1994 Siegel, Slaboda, and Stanley: Trace elements and pollution in Manzala Lagoon

1995 Siegel et al.: Trace elements in cores of the northeastern delta

NeOteCtonism and ITS EFFECTS ON THE DELTA.-Studies listed below emphasize the vertical motion of land, rates of subsidence, and evidence of tilting to the northeast of the Nile delta during the late Quaternary. Measurements involve displacement of radiocarbon-dated lithofacies, which were originally deposited at or near sea level and are now buried below the delta plain surface.

1985 Stanley and Wetzel: Structural displacement in the southeastern Mediterranean

1988b Stanley: Subsidence rates in the northeastern delta

1990 Stanley: Subsidence and tilting of the delta plain

1991 Arbouille and Stanley: Subsidence in the northern delta

1992 Stanley, Warne et al.: Subsidence in the northern delta

1992 Chen, Warne, and Stanley: Subsidence in the northwestern delta

1992 Stanley: Subsidence rates of the northern delta plain

1992 Stanley, Arnold, and Warne: Subsidence and burial of a Predynastic site

1993a Stanley and Warne: Synthesis of delta subsidence

1993a Warne and Stanley: Measuring subsidence rates using archeological data

1993b Warne and Stanley: Subsidence in the northwestern delta

Sea-level and Climatic Factors affecting the DELTA.-Investigations in this category emphasize the influences of global (eustatic) sea-level oscillations, excluding land motion, and effects of climate change, which controlled fluvial and sediment input and delta aggradation during the late Quaternary.

1987 Coutellier and Stanley: Northeastern delta

1991 Arbouille and Stanley: North-central delta

1991 Hamroush and Stanley: Paleoclimatic oscillations

1992 Stanley, Warne et al.: North-central delta 
TABLE 2.--Radiocarbon dates for samples from boreholes S1-S87, in uncorrected years before present (BP). Data listing includes depth from top of boring, Smithsonian sample letter code, and number code assigned by Beta Analytic Inc.@; the 19 samples analyzed by the Smithsonian's Radiobiology Laboratory are designated by the SI letter code prefix.

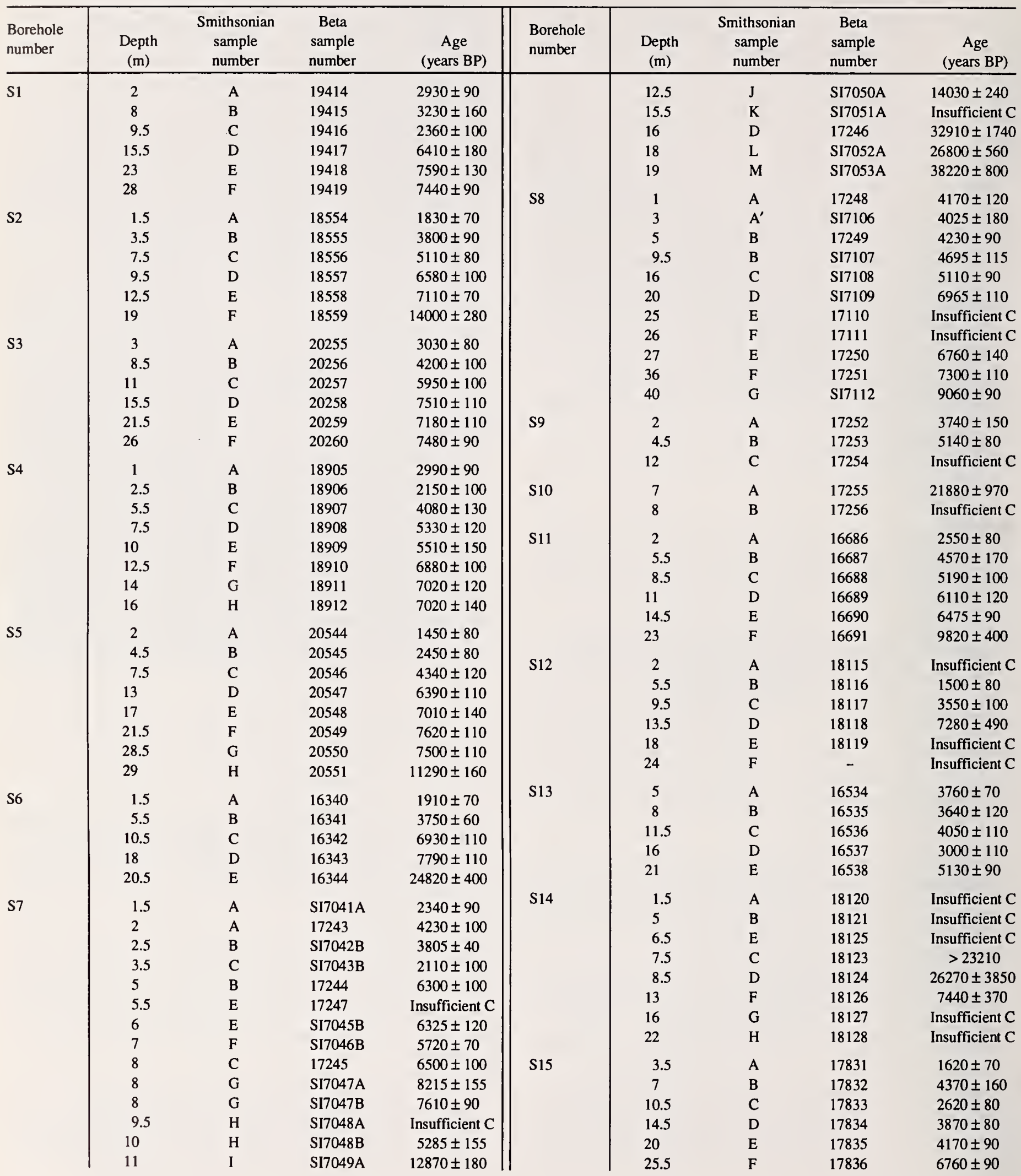




\begin{tabular}{|c|c|c|c|c|c|c|c|c|c|}
\hline $\begin{array}{l}\text { Borehole } \\
\text { number }\end{array}$ & $\begin{array}{c}\text { Depth } \\
(\mathrm{m})\end{array}$ & $\begin{array}{c}\text { Smithsonian } \\
\text { sample } \\
\text { number } \\
\end{array}$ & $\begin{array}{c}\text { Beta } \\
\text { sample } \\
\text { number }\end{array}$ & $\begin{array}{c}\text { Age } \\
\text { (years BP) } \\
\end{array}$ & $\begin{array}{l}\text { Borehole } \\
\text { number }\end{array}$ & $\begin{array}{c}\text { Depth } \\
\text { (m) }\end{array}$ & $\begin{array}{c}\text { Smithsonian } \\
\text { sample } \\
\text { number } \\
\end{array}$ & $\begin{array}{c}\text { Beta } \\
\text { sample } \\
\text { number } \\
\end{array}$ & $\begin{array}{c}\text { Age } \\
\text { (years BP) }\end{array}$ \\
\hline & 35 & $\mathrm{H}$ & 17838 & $7420 \pm 90$ & S25 & 3.5 & A & 25463 & $3860 \pm 90$ \\
\hline \multirow[t]{6}{*}{ S16 } & 3 & A & 16345 & $2360 \pm 90$ & & 8 & B & 25464 & $6630 \pm 110$ \\
\hline & 9 & B & 16346 & $1940 \pm 90$ & & 12.5 & C & 25465 & $6760 \pm 100$ \\
\hline & 14 & $\mathrm{C}$ & 16347 & $4500 \pm 90$ & & 14 & D & 25466 & $6210 \pm 100$ \\
\hline & 18 & D & 16348 & $4820 \pm 80$ & S26 & 1 & $\mathrm{~K}$ & 24895 & $2500 \pm 170$ \\
\hline & 23 & E & 16349 & $7700 \pm 110$ & & 2.5 & L & 24896 & $2820 \pm 120$ \\
\hline & 27.5 & $\mathrm{~F}$ & 16350 & $7340 \pm 90$ & & 5 & $\mathbf{M}$ & 24897 & $4370 \pm 170$ \\
\hline \multirow[t]{8}{*}{ S17 } & 2 & A & 20079 & $1420 \pm 80$ & & 5.5 & $N$ & 24898 & $4210 \pm 90$ \\
\hline & 8 & B & 20080 & $4200 \pm 120$ & & 6.5 & $\mathrm{O}$ & 24899 & Insufficient $\mathrm{C}$ \\
\hline & 12.5 & C & 20081 & $4480 \pm 110$ & S27 & 2 & A & 25537 & $3160 \pm 120$ \\
\hline & 19 & D & 20082 & $4890 \pm 110$ & & 6 & B & 25538 & $2520 \pm 90$ \\
\hline & 30 & E & 20083 & $7980 \pm 90$ & & 6.5 & C & 25539 & $2980 \pm 70$ \\
\hline & 35 & F & 20084 & $7850 \pm 100$ & & 9 & D & 25540 & $3330 \pm 90$ \\
\hline & 42.5 & G & 20085 & $8940 \pm 120$ & & 10.5 & E & 25541 & $6560 \pm 90$ \\
\hline & 30 & F & 16539 & $7150 \pm 110$ & S28 & 5 & $\mathrm{~F}$ & 26381 & $4500 \pm 120$ \\
\hline \multirow[t]{8}{*}{ S18 } & 1.5 & A & 24004 & $1400 \pm 80$ & & 18 & G & 26382 & $8640 \pm 110$ \\
\hline & 6.5 & B & 24005 & $4650 \pm 120$ & & 23.5 & $\mathrm{H}$ & 26383 & $7230 \pm 80$ \\
\hline & 11 & C & 24006 & $4100 \pm 70$ & & 26 & I & 26384 & Insufficient $\mathrm{C}$ \\
\hline & 17.5 & D & 24007 & $4480 \pm 110$ & & 28 & $\mathbf{J}$ & 26385 & $10950 \pm 90$ \\
\hline & 26 & E & 24008 & $7400 \pm 80$ & & 35 & K & 26386 & Insufficient $\mathrm{C}$ \\
\hline & 27.5 & $\mathrm{~F}$ & 24009 & $4040 \pm 100$ & S29 & 4 & A & 26387 & $3460 \pm 100$ \\
\hline & 41 & G & 24010 & $11530 \pm 80$ & $\$ 29$ & 6 & B & 26388 & $4580 \pm 100$ \\
\hline & 52.5 & $\mathrm{H}$ & 24011 & $12070 \pm 370$ & & 7 & $\mathrm{C}$ & 26389 & $5190 \pm 90$ \\
\hline \multirow[t]{4}{*}{ S19 } & 1 & F & 24890 & Insufficient $\mathrm{C}$ & & 9 & D & 26390 & $4910 \pm 90$ \\
\hline & 4 & G & 24891 & $3070 \pm 110$ & & 18 & $\mathrm{E}$ & 26391 & $8870 \pm 170$ \\
\hline & $\begin{array}{l}6.5 \\
8.5\end{array}$ & H & $\begin{array}{l}24892 \\
24893\end{array}$ & $\begin{array}{l}3960 \pm 100 \\
4080 \pm 90\end{array}$ & S30 & 18.5 & A & 26910 & $5270 \pm 90$ \\
\hline & 11.5 & I & $\begin{array}{l}24893 \\
24894\end{array}$ & $\begin{array}{l}4080 \pm 90 \\
\text { Insufficient } C\end{array}$ & & 22.5 & B & 26911 & $5020 \pm 110$ \\
\hline \multirow[t]{8}{*}{$\mathrm{S} 20$} & 2 & A & 25256 & $2890 \pm 130$ & & 26.5 & $\mathrm{C}$ & 26912 & $8090 \pm 120$ \\
\hline & 11 & B & 25257 & $4190 \pm 90$ & & 27.5 & D & 26913 & $8040 \pm 250$ \\
\hline & 22 & $\mathrm{C}$ & 25258 & $5110 \pm 110$ & & 28.5 & E & 26914 & Insufficient $\mathrm{C}$ \\
\hline & 27.5 & D & 25259 & $7460 \pm 80$ & & 34 & $\mathbf{F}$ & 26915 & $10770 \pm 120$ \\
\hline & 34 & E & 25260 & $7260 \pm 90$ & S31 & 4.5 & A & 32455 & $3260 \pm 90$ \\
\hline & 40 & F & 25261 & $7630 \pm 90$ & & 10.5 & B & 32456 & $5840 \pm 140$ \\
\hline & 42 & G & 25262 & $7360 \pm 90$ & & 15 & $\mathrm{C}$ & 32457 & $6590 \pm 110$ \\
\hline & 45 & H & 25263 & $15110 \pm 640$ & & 19.5 & D & 32458 & $7650 \pm 150$ \\
\hline \multirow[t]{8}{*}{ S21 } & 1 & A & 25937 & $3400 \pm 140$ & & 25 & E & 32459 & $7850 \pm 140$ \\
\hline & 9.5 & B & 25938 & $3530 \pm 90$ & & 27 & $\mathrm{~F}$ & 32460 & $6880 \pm 80$ \\
\hline & 16 & $\mathrm{C}$ & 25939 & $3870 \pm 100$ & & 28 & G & 32461 & Insufficient $C$ \\
\hline & 25 & D & 25940 & $4520 \pm 110$ & & 28.5 & $\mathrm{H}$ & 33115 & $>25670$ \\
\hline & 34 & E & 25941 & $5780 \pm 130$ & S32 & 7.5 & A & 33116 & $5880 \pm 170$ \\
\hline & 40.5 & F & 25942 & $7140 \pm 110$ & & 11.5 & B & 33117 & $7100 \pm 130$ \\
\hline & 46 & G & 25943 & $8190 \pm 110$ & & 12 & $\mathrm{C}$ & 33118 & $7960 \pm 150$ \\
\hline & 48 & H & 25944 & $8140 \pm 130$ & & 19 & D & 33119 & Insufficient $\mathrm{C}$ \\
\hline \multirow[t]{8}{*}{ S22 } & 0.5 & A & 23672 & $3630 \pm 110$ & S33 & 12 & A & 30599 & $5500 \pm 190$ \\
\hline & 3 & B & 23673 & $3770 \pm 90$ & & 12.5 & B & 30600 & $3560 \pm 150$ \\
\hline & 8 & $\mathrm{C}$ & 23674 & $4670 \pm 80$ & & 21 & C & 30601 & Insufficient $\mathrm{C}$ \\
\hline & 14 & D & 23675 & $6630 \pm 150$ & & 21.5 & D & 30602 & $34380 \pm 1740$ \\
\hline & 21.5 & E & 23676 & $7910 \pm 150$ & S34 & 13 & A & 30603 & $8370 \pm 180$ \\
\hline & 22 & $\mathrm{~F}$ & 23677 & $7540 \pm 70$ & & 13.5 & B & 30604 & $6710 \pm 190$ \\
\hline & 31 & G & 23678 & $32920 \pm 930$ & & 24 & $\mathrm{C}$ & 30605 & $19450 \pm 840$ \\
\hline & 37 & $\mathrm{H}$ & 23679 & $24320 \pm 2030$ & & 25 & D & 30606 & $21050 \pm 920$ \\
\hline \multirow[t]{2}{*}{ S23 } & 1.5 & A & 24885 & $2490 \pm 80$ & S35 & 10 & $\mathrm{~F}$ & 31486 & $6160 \pm 80$ \\
\hline & 5 & B & 24886 & Insufficient $\mathrm{C}$ & & 17.5 & $\mathrm{E}$ & 31485 & $7730 \pm 120$ \\
\hline \multirow[t]{3}{*}{ S24 } & 0.5 & $\mathrm{C}$ & $24887 \mathrm{~A}$ & $4130 \pm 180$ & & 21 & A & 30607 & $7260 \pm 110$ \\
\hline & 7.5 & D & 24887B & $9200 \pm 110$ & & 22.5 & B & 30608 & $4770 \pm 110$ \\
\hline & 10 & $\mathrm{E}$ & 24889 & $24240 \pm 1510$ & & 30 & $\mathrm{C}$ & 30609 & Insufficient $\mathrm{C}$ \\
\hline
\end{tabular}




\begin{tabular}{|c|c|c|c|c|c|c|c|c|c|}
\hline $\begin{array}{l}\text { Borehole } \\
\text { number }\end{array}$ & $\begin{array}{c}\text { Depth } \\
\text { (m) }\end{array}$ & $\begin{array}{c}\text { Smithsonian } \\
\text { sample } \\
\text { number } \\
\end{array}$ & $\begin{array}{c}\text { Beta } \\
\text { sample } \\
\text { number }\end{array}$ & $\begin{array}{c}\text { Age } \\
\text { (years BP) }\end{array}$ & $\begin{array}{l}\text { Borehole } \\
\text { number }\end{array}$ & $\begin{array}{l}\text { Depth } \\
\text { (m) }\end{array}$ & $\begin{array}{l}\text { Smithsonian } \\
\text { sample } \\
\text { number }\end{array}$ & $\begin{array}{c}\text { Beta } \\
\text { sample } \\
\text { number }\end{array}$ & $\begin{array}{c}\text { Age } \\
\text { (years BP) }\end{array}$ \\
\hline S36 & $\begin{array}{l}14 \\
14.5 \\
25 \\
34.5\end{array}$ & $\begin{array}{l}\text { A } \\
\text { B } \\
\text { C } \\
\text { D }\end{array}$ & $\begin{array}{l}30610 \\
30611 \\
30612 \\
30613\end{array}$ & $\begin{array}{c}7080 \pm 120 \\
\text { Insufficient } C \\
27720 \pm 670 \\
25570 \pm 720\end{array}$ & S46 & $\begin{array}{l}8 \\
10.5 \\
12 \\
18\end{array}$ & $\begin{array}{l}\text { A } \\
\text { B } \\
\text { C } \\
\text { D }\end{array}$ & $\begin{array}{l}34478 \\
34479 \\
34480 \\
34481\end{array}$ & $\begin{array}{c}7130 \pm 80 \\
6620 \pm 70 \\
12940 \pm 240 \\
\text { Insufficient C }\end{array}$ \\
\hline S37 & $\begin{array}{c}5 \\
12.5\end{array}$ & $\begin{array}{l}\text { A } \\
\text { B }\end{array}$ & $\begin{array}{l}33120 \\
33121\end{array}$ & $\begin{array}{l}3260 \pm 80 \\
6870 \pm 170\end{array}$ & S47 & $\begin{array}{c}5.5 \\
15\end{array}$ & $\begin{array}{l}\text { A } \\
\text { B }\end{array}$ & $\begin{array}{l}36764 \\
36765\end{array}$ & $\begin{array}{l}4270 \pm 110 \\
8050 \pm 140\end{array}$ \\
\hline S38 & $\begin{array}{l}12 \\
13 \\
14\end{array}$ & $\begin{array}{l}\text { G } \\
\text { A } \\
\text { B }\end{array}$ & $\begin{array}{l}31487 \\
30117 \\
30118\end{array}$ & $\begin{array}{l}7240 \pm 90 \\
7210 \pm 130 \\
3380 \pm 200\end{array}$ & & $\begin{array}{l}23 \\
30 \\
37\end{array}$ & $\begin{array}{l}\text { C } \\
\text { D } \\
\text { E }\end{array}$ & $\begin{array}{l}36767 \\
36766 \\
36768\end{array}$ & $\begin{array}{l}\text { Insufficient C } \\
\text { Insufficient } C \\
26820 \pm 1340\end{array}$ \\
\hline & $\begin{array}{l}18 \\
23 \\
29.5 \\
31\end{array}$ & $\begin{array}{l}\text { C } \\
D \\
E \\
F\end{array}$ & $\begin{array}{l}30119 \\
30120 \\
30121 \\
30122\end{array}$ & $\begin{array}{c}21090 \pm 600 \\
\text { Insufficient C } \\
\text { Insufficient C } \\
\quad>29260\end{array}$ & S48 & $\begin{array}{l}3 \\
4.5 \\
7.5 \\
14\end{array}$ & $\begin{array}{l}\text { A } \\
\text { B } \\
\text { C } \\
\text { D }\end{array}$ & $\begin{array}{l}37298 \\
37299 \\
37300 \\
37301\end{array}$ & $\begin{array}{l}1610 \pm 60 \\
1670 \pm 50 \\
4480 \pm 80 \\
6340 \pm 100\end{array}$ \\
\hline S39 & $\begin{array}{c}1.5 \\
2.5 \\
4 \\
13.5 \\
13.5\end{array}$ & $\begin{array}{l}\text { D } \\
\text { A } \\
\text { C } \\
\text { E } \\
\text { B }\end{array}$ & $\begin{array}{l}33122 \\
29858 \\
29860 \\
33123 \\
29859\end{array}$ & $\begin{array}{c}3840 \pm 100 \\
4540 \pm 290 \\
\text { Insufficient } C \\
21700 \pm 2460 \\
11320 \pm 290\end{array}$ & S49 & $\begin{array}{r}6 \\
11 \\
15 \\
26\end{array}$ & $\begin{array}{l}\text { A } \\
\text { B } \\
\text { C } \\
\text { D }\end{array}$ & $\begin{array}{l}36769 \\
36770 \\
36771 \\
36772\end{array}$ & $\begin{array}{c}3190 \pm 90 \\
5410 \pm 100 \\
7170 \pm 180 \\
\text { Insufficient C }\end{array}$ \\
\hline$S 40$ & $\begin{array}{c}3 \\
15.5 \\
16.5 \\
25.5\end{array}$ & $\begin{array}{l}\text { E } \\
\text { A } \\
\text { C } \\
\text { D }\end{array}$ & $\begin{array}{l}33124 \\
29861 \\
29863 \\
29864\end{array}$ & $\begin{array}{l}3430 \pm 110 \\
7450 \pm 120 \\
3540 \pm 150 \\
6050 \pm 140\end{array}$ & S50 & \begin{tabular}{l}
\multicolumn{1}{c}{4.5} \\
16 \\
24 \\
25.5
\end{tabular} & $\begin{array}{l}\text { A } \\
\text { B } \\
\text { C } \\
\text { D }\end{array}$ & $\begin{array}{l}37302 \\
37303 \\
37304 \\
37305\end{array}$ & $\begin{array}{c}2870 \pm 80 \\
6600 \pm 80 \\
7950 \pm 90 \\
11040 \pm 330\end{array}$ \\
\hline & $\begin{array}{l}27.5 \\
26.5\end{array}$ & $\begin{array}{l}\text { F } \\
\text { B }\end{array}$ & $\begin{array}{l}33125 \\
29862\end{array}$ & $\begin{array}{l}19350 \pm 950 \\
\text { Insufficient } C\end{array}$ & S51 & $\begin{array}{l}5.5 \\
8\end{array}$ & A & $\begin{array}{l}37747 \\
37748\end{array}$ & $\begin{array}{l}3800 \pm 60 \\
5930 \pm 130\end{array}$ \\
\hline S41 & $\begin{array}{l}8.5 \\
9 \\
18 \\
20 \\
20.5 \\
24\end{array}$ & $\begin{array}{l}\text { D } \\
\text { F } \\
\text { A } \\
\text { B } \\
\text { E } \\
\text { C }\end{array}$ & $\begin{array}{l}29867 \\
31489 \\
31488 \\
29865 \\
29868 \\
29866\end{array}$ & $\begin{array}{c}\text { Insufficient C } \\
3060 \pm 70 \\
6630 \pm 250 \\
6330 \pm 100 \\
3490 \pm 100 \\
\text { Insufficient C }\end{array}$ & S52 & $\begin{array}{l}11.5 \\
2 \\
6 \\
7.5 \\
11.5 \\
12\end{array}$ & $\begin{array}{l}\text { C } \\
\text { A } \\
\text { B } \\
\text { C } \\
\text { D } \\
\text { E }\end{array}$ & $\begin{array}{l}37749 \\
37750 \\
37751 \\
37752 \\
37753 \\
37754\end{array}$ & $\begin{array}{c}6580 \pm 110 \\
1670 \pm 60 \\
3250 \pm 100 \\
4790 \pm 70 \\
6550 \pm 80 \\
10510 \pm 130\end{array}$ \\
\hline & $\begin{array}{l}25 \\
25.5 \\
14.5\end{array}$ & $\begin{array}{l}\text { D } \\
\text { G } \\
\text { G }\end{array}$ & $\begin{array}{l}29867 \\
33126 \\
31490\end{array}$ & $\begin{array}{c}\text { Insufficient C } \\
\text { Insufficient } C \\
3870 \pm 110\end{array}$ & S53 & $\begin{array}{r}4 \\
10 \\
11\end{array}$ & $\begin{array}{l}\text { A } \\
\text { B } \\
\text { C }\end{array}$ & $\begin{array}{l}37755 \\
37756 \\
37757\end{array}$ & $\begin{array}{c}2470 \pm 60 \\
5820 \pm 100 \\
11930 \pm 170\end{array}$ \\
\hline S42 & $\begin{array}{r}5 \\
11 \\
21 \\
23 \\
24 \\
28\end{array}$ & $\begin{array}{l}\text { E } \\
\text { F } \\
\text { D } \\
\text { A } \\
\text { B } \\
\text { C }\end{array}$ & $\begin{array}{l}31492 \\
31493 \\
31491 \\
29869 \\
29870 \\
29871\end{array}$ & $\begin{array}{l}3610 \pm 110 \\
4890 \pm 100 \\
7410 \pm 100 \\
8290 \pm 120 \\
6730 \pm 150 \\
7860 \pm 90\end{array}$ & S54 & $\begin{array}{l}14.5 \\
5 \\
9.5 \\
10 \\
14.5\end{array}$ & $\begin{array}{l}\text { D } \\
\text { A } \\
\text { B } \\
\text { C } \\
\text { D }\end{array}$ & $\begin{array}{l}37758 \\
38098 \\
38099 \\
38100 \\
38101\end{array}$ & $\begin{array}{c}\text { Insufficient } C \\
3080 \pm 70 \\
5990 \pm 100 \\
12310 \pm 120 \\
22820 \pm 770\end{array}$ \\
\hline S43 & $\begin{array}{c}4 \\
7 \\
14 \\
14.5\end{array}$ & $\begin{array}{l}\text { E } \\
\text { F } \\
\text { A } \\
\text { D }\end{array}$ & $\begin{array}{l}31494 \\
31495 \\
29872 \\
29875\end{array}$ & $\begin{array}{c}4620 \pm 130 \\
5610 \pm 110 \\
6970 \pm 110 \\
\text { Insufficient C }\end{array}$ & S55 & $\begin{array}{l}3.5 \\
6 \\
7 \\
9.5\end{array}$ & $\begin{array}{l}\text { A } \\
\text { B } \\
\text { C } \\
\text { D }\end{array}$ & $\begin{array}{l}37759 \\
37760 \\
37761 \\
37762\end{array}$ & $\begin{array}{c}2420 \pm 110 \\
3400 \pm 100 \\
4170 \pm 90 \\
14120 \pm 160\end{array}$ \\
\hline & $\begin{array}{l}18 \\
18.5\end{array}$ & $\begin{array}{l}\text { B } \\
\text { E }\end{array}$ & $\begin{array}{l}29873 \\
33127\end{array}$ & $\begin{array}{l}\text { Insufficient C } \\
\text { Insufficient C }\end{array}$ & S56 & 8.5 & A & 37763 & $1490 \pm 80$ \\
\hline & $\begin{array}{l}18.5 \\
25 \\
24.5\end{array}$ & $\begin{array}{l}\mathrm{E} \\
\mathrm{F} \\
\mathrm{C}\end{array}$ & $\begin{array}{l}33128 \\
29874\end{array}$ & $\begin{array}{l}\text { Insufficient C } \\
\text { Insufficient C } \\
\text { Insuficient C }\end{array}$ & S57 & $\begin{array}{l}6 \\
12 \\
13.5\end{array}$ & $\begin{array}{l}\text { A } \\
\text { B } \\
\text { C }\end{array}$ & $\begin{array}{l}38102 \\
38103 \\
38104\end{array}$ & $\begin{array}{c}3630 \pm 70 \\
6310 \pm 90 \\
13630 \pm 100\end{array}$ \\
\hline S44 & $\begin{array}{c}2 \\
5 \\
6.5 \\
10.5 \\
14\end{array}$ & $\begin{array}{l}\text { A } \\
\text { B } \\
\text { C } \\
\text { A } \\
\text { B }\end{array}$ & $\begin{array}{l}46010 \\
46011 \\
46012 \\
30123 \\
30124\end{array}$ & $\begin{array}{c}2570 \pm 70 \\
3980 \pm 80 \\
3260 \pm 90 \\
6370 \pm 180 \\
\text { Insufficient C }\end{array}$ & S58 & $\begin{array}{l}3 \\
5.5 \\
11.5 \\
16\end{array}$ & $\begin{array}{l}\text { A } \\
\text { B } \\
\text { C } \\
\text { D }\end{array}$ & $\begin{array}{l}38091 \\
38092 \\
38093 \\
38094\end{array}$ & $\begin{array}{l}3020 \pm 80 \\
3770 \pm 80 \\
4890 \pm 80 \\
7500 \pm 70\end{array}$ \\
\hline & 14.5 & C & 33129 & $15600 \pm 290$ & S59 & 11.5 & A & 37306 & $3140 \pm 100$ \\
\hline S45 & $\begin{array}{l}12.5 \\
13\end{array}$ & $\begin{array}{l}\text { A } \\
\text { B }\end{array}$ & $\begin{array}{l}30125 \\
30126\end{array}$ & $\begin{array}{c}7100 \pm 160 \\
\text { Insufficient } C\end{array}$ & & $\begin{array}{l}17 \\
27.5\end{array}$ & $\begin{array}{l}\mathrm{B} \\
\mathrm{C}\end{array}$ & $\begin{array}{l}37307 \\
37308\end{array}$ & $\begin{array}{l}3560 \pm 80 \\
9110 \pm 120\end{array}$ \\
\hline & $\begin{array}{l}19 \\
24\end{array}$ & $\begin{array}{l}\mathrm{C} \\
\mathrm{D}\end{array}$ & $\begin{array}{l}30127 \\
30128\end{array}$ & $\begin{array}{l}24320 \pm 1080 \\
29000 \pm 1380\end{array}$ & $S 60$ & $\begin{array}{c}8.5 \\
11\end{array}$ & $\begin{array}{l}\text { A } \\
\text { C }\end{array}$ & $\begin{array}{l}36773 \\
36774\end{array}$ & $\begin{array}{l}4760 \pm 110 \\
5020 \pm 90\end{array}$ \\
\hline
\end{tabular}




\begin{tabular}{|c|c|c|c|c|c|c|c|c|c|}
\hline $\begin{array}{l}\text { Borehole } \\
\text { number }\end{array}$ & $\begin{array}{l}\text { Depth } \\
\text { (m) }\end{array}$ & $\begin{array}{c}\text { Smithsonian } \\
\text { sample } \\
\text { number }\end{array}$ & $\begin{array}{c}\text { Beta } \\
\text { sample } \\
\text { number }\end{array}$ & $\begin{array}{c}\text { Age } \\
\text { (years BP) }\end{array}$ & $\begin{array}{l}\text { Borehole } \\
\text { number }\end{array}$ & $\begin{array}{c}\text { Depth } \\
\text { (m) }\end{array}$ & $\begin{array}{c}\text { Smithsonian } \\
\text { sample } \\
\text { number }\end{array}$ & $\begin{array}{c}\text { Beta } \\
\text { sample } \\
\text { number }\end{array}$ & $\begin{array}{c}\text { Age } \\
\text { (years BP) } \\
\end{array}$ \\
\hline S61 & $\begin{array}{l}1.5 \\
6 \\
13 \\
16.5\end{array}$ & $\begin{array}{l}\text { A } \\
\text { B } \\
\text { C } \\
\text { D }\end{array}$ & $\begin{array}{l}37309 \\
37310 \\
37311 \\
37312\end{array}$ & $\begin{array}{l}2990 \pm 80 \\
4220 \pm 100 \\
3430 \pm 110 \\
7310 \pm 100\end{array}$ & S77 & $\begin{array}{r}8 \\
5.5 \\
8.5 \\
10.5\end{array}$ & $\begin{array}{l}\text { B } \\
\text { A } \\
\text { B } \\
\text { E }\end{array}$ & $\begin{array}{l}45642 \\
46283 \\
46284 \\
48367\end{array}$ & $\begin{array}{c}6680 \pm 100 \\
6430 \pm 90 \\
6170 \pm 110 \\
>28000\end{array}$ \\
\hline S62 & $\begin{array}{r}6 \\
13 \\
\end{array}$ & A & $\begin{array}{l}38095 \\
38096\end{array}$ & $\begin{array}{l}3660 \pm 70 \\
6220 \pm 100\end{array}$ & \multirow[b]{2}{*}{ S78 } & $\begin{array}{l}11.5 \\
17\end{array}$ & $\begin{array}{l}\text { C } \\
\mathrm{D}\end{array}$ & $\begin{array}{l}46285 \\
46286\end{array}$ & $\begin{array}{l}\text { Insufficient } C \\
\text { Insufficient } C\end{array}$ \\
\hline $\begin{array}{l}S 63 \\
S 64\end{array}$ & $\begin{array}{l}17.5 \\
19 \\
135\end{array}$ & $\begin{array}{l}\mathrm{C} \\
\mathrm{A}\end{array}$ & $\begin{array}{l}38097 \\
36775 \\
37313\end{array}$ & $\begin{array}{l}7160 \pm 70 \\
6590 \pm 110 \\
2250+100\end{array}$ & & $\begin{array}{l}4.5 \\
6.5 \\
7.5\end{array}$ & $\begin{array}{l}\text { A } \\
\text { B } \\
\text { C }\end{array}$ & $\begin{array}{l}46287 \\
46288 \\
46289\end{array}$ & $\begin{array}{c}3250 \pm 60 \\
6730 \pm 70 \\
15920 \pm 140\end{array}$ \\
\hline S65 & $\begin{array}{l}13.5 \\
19.5 \\
-\end{array}$ & $\begin{array}{l}\text { A } \\
\text { B } \\
-\end{array}$ & $\begin{array}{l}37313 \\
37314 \\
\text { None }\end{array}$ & $\begin{array}{c}2250 \pm 100 \\
3780 \pm 120 \\
-\end{array}$ & S79 & $\begin{array}{l}4 \\
4.5 \\
5\end{array}$ & $\begin{array}{l}\text { D } \\
\text { A } \\
\text { B }\end{array}$ & $\begin{array}{l}48368 \\
47331 \\
47332\end{array}$ & $\begin{array}{c}3850 \pm 80 \\
4480 \pm 70 \\
17900 \pm 220\end{array}$ \\
\hline S66 & $\begin{array}{l}6.5 \\
9 \\
12 \\
14.5\end{array}$ & $\begin{array}{l}\text { A } \\
\text { B } \\
\text { C } \\
\text { D }\end{array}$ & $\begin{array}{l}45078 \\
45079 \\
45080 \\
45081\end{array}$ & $\begin{array}{l}4020 \pm 100 \\
3950 \pm 70 \\
5480 \pm 80 \\
7230 \pm 70\end{array}$ & S80 & $\begin{array}{l}6.5 \\
3 \\
3.5 \\
7.5\end{array}$ & $\begin{array}{l}\text { C } \\
\text { A } \\
\text { C } \\
\text { D }\end{array}$ & $\begin{array}{l}47333 \\
46290 \\
48369 \\
48370\end{array}$ & $\begin{array}{l}\text { Insufficient } C \\
\text { Insufficient } C \\
3530 \pm 60 \\
23070 \pm 1880\end{array}$ \\
\hline S67 & 5 & A & 45633 & $11890 \pm 380$ & \multirow[b]{2}{*}{ S81 } & 8 & B & 46291 & Insufficient $\mathrm{C}$ \\
\hline S68 & \begin{tabular}{l}
\multicolumn{1}{c}{5} \\
6.5 \\
13.5 \\
14
\end{tabular} & $\begin{array}{l}\text { A } \\
\text { B } \\
\text { C } \\
\text { D }\end{array}$ & $\begin{array}{l}45082 \\
45083 \\
45084 \\
45085\end{array}$ & $\begin{array}{l}2730 \pm 80 \\
4980 \pm 70 \\
6830 \pm 80 \\
7170 \pm 70\end{array}$ & & $\begin{array}{l}1.5 \\
2.5 \\
3 \\
5.5\end{array}$ & $\begin{array}{l}\text { A } \\
\text { D } \\
\text { B } \\
\text { E } \\
\text { F }\end{array}$ & $\begin{array}{l}48371 \\
48372 \\
47334 \\
48373\end{array}$ & $\begin{array}{c}1350 \pm 80 \\
19630 \pm 140 \\
28200 \pm 460 \\
29480 \pm 330\end{array}$ \\
\hline S69 & $\begin{array}{l}3.5 \\
7.5\end{array}$ & $\begin{array}{l}\text { A } \\
\text { C }\end{array}$ & $\begin{array}{l}45634 \\
46282\end{array}$ & $\begin{array}{c}4410 \pm 80 \\
23670 \pm 370\end{array}$ & \multirow[b]{2}{*}{ S82 } & $\begin{array}{l}11 \\
12.5 \\
3\end{array}$ & $\begin{array}{l}\mathrm{F} \\
\mathrm{C}^{\prime}\end{array}$ & $\begin{array}{l}48374 \\
47335 \\
48375\end{array}$ & $\begin{array}{c}>38000 \\
\text { Insufficient } \mathrm{C} \\
5050+70\end{array}$ \\
\hline$\$ 70$ & $\begin{array}{l}12.5 \\
2.5 \\
4\end{array}$ & $\begin{array}{l}\text { B } \\
\text { A } \\
\text { B }\end{array}$ & $\begin{array}{l}45635 \\
45086 \\
45087\end{array}$ & $\begin{array}{r}35260 \pm 610 \\
3220 \pm 120 \\
3690 \pm 140\end{array}$ & & $\begin{array}{l}3 \\
3.5 \\
4 \\
4.5\end{array}$ & $\begin{array}{l}\text { C } \\
\text { A } \\
\text { D } \\
\text { B }\end{array}$ & $\begin{array}{l}48375 \\
46292 \\
48376 \\
46293\end{array}$ & $\begin{array}{c}5050 \pm 70 \\
5850 \pm 150 \\
16540 \pm 220 \\
\text { Insufficient C }\end{array}$ \\
\hline S71 & $\begin{array}{l}2 \\
5.5 \\
12 \\
12.5\end{array}$ & $\begin{array}{l}\text { C } \\
\text { A } \\
\text { D } \\
\text { B }\end{array}$ & $\begin{array}{l}48364 \\
45636 \\
48365 \\
45637\end{array}$ & $\begin{array}{l}3030 \pm 90 \\
4660 \pm 80 \\
6860 \pm 50 \\
7250 \pm 100\end{array}$ & S83 & $\begin{array}{l}3 \\
3.5 \\
4 \\
4.5\end{array}$ & $\begin{array}{l}\text { E } \\
\text { D } \\
\text { A } \\
\text { B }\end{array}$ & $\begin{array}{l}58280 \\
48379 \\
47336 \\
48377\end{array}$ & $\begin{array}{r}5410 \pm 120 \\
8350 \pm 140 \\
8860 \pm 130 \\
14990 \pm 100\end{array}$ \\
\hline S72 & $\begin{array}{l}4.5 \\
8.5\end{array}$ & $\begin{array}{l}\text { A } \\
\text { B }\end{array}$ & $\begin{array}{l}46014 \\
46015\end{array}$ & $\begin{array}{l}2900 \pm 70 \\
6420 \pm 80\end{array}$ & \multirow[t]{2}{*}{ S84 } & $\begin{array}{l}5 \\
1\end{array}$ & $\begin{array}{l}\mathrm{C} \\
\mathrm{C}\end{array}$ & $\begin{array}{l}48378 \\
48380\end{array}$ & $\begin{array}{c}24770 \pm 240 \\
2890 \pm 60\end{array}$ \\
\hline S73 & $\begin{array}{l}4.5 \\
12.5 \\
12\end{array}$ & $\begin{array}{l}\text { A } \\
\text { B } \\
\text { D }\end{array}$ & $\begin{array}{l}45638 \\
45639 \\
48366\end{array}$ & $\begin{array}{c}3990 \pm 90 \\
7590 \pm 90 \\
12760 \pm 110\end{array}$ & & $\begin{array}{l}2 \\
2.5 \\
5.5\end{array}$ & $\begin{array}{l}\text { A } \\
\text { D } \\
\text { B }\end{array}$ & $\begin{array}{l}46294 \\
48381 \\
46295\end{array}$ & $\begin{array}{l}16760 \pm 120 \\
23510 \pm 260 \\
39350 \pm 800\end{array}$ \\
\hline & 14.5 & C & 45640 & Insufficient $\mathrm{C}$ & S85 & 2.5 & B & 48382 & $20330 \pm 270$ \\
\hline S74 & $\begin{array}{l}3.5 \\
8.5\end{array}$ & $\begin{array}{l}\text { A } \\
\text { B }\end{array}$ & $\begin{array}{l}45088 \\
45089\end{array}$ & $\begin{array}{l}6290 \pm 140 \\
6420 \pm 90\end{array}$ & \multirow[t]{3}{*}{ S86 } & $\begin{array}{l}6.5 \\
1.5\end{array}$ & $\begin{array}{l}\mathrm{A} \\
\mathrm{A}\end{array}$ & $\begin{array}{l}46296 \\
51454\end{array}$ & $\begin{array}{r}>39730 \\
1690 \pm 80\end{array}$ \\
\hline S75 & $\begin{array}{l}2.5 \\
7\end{array}$ & $\begin{array}{l}\text { A } \\
\text { B }\end{array}$ & $\begin{array}{l}46016 \\
46017\end{array}$ & $\begin{array}{l}2900 \pm 60 \\
5830 \pm 90\end{array}$ & & $\begin{array}{c}7 \\
16.5\end{array}$ & $\begin{array}{l}\mathrm{B} \\
\mathrm{C}\end{array}$ & $\begin{array}{l}51455 \\
51456\end{array}$ & $\begin{array}{l}4910 \pm 100 \\
6430 \pm 110\end{array}$ \\
\hline S76 & $\begin{array}{c}13.5 \\
5\end{array}$ & $\begin{array}{l}\mathrm{C} \\
\mathrm{A}\end{array}$ & $\begin{array}{l}46018 \\
45641\end{array}$ & $\begin{array}{l}6960 \pm 110 \\
4950 \pm 130\end{array}$ & & $\begin{array}{l}0.5 \\
9\end{array}$ & $\begin{array}{l}\mathrm{A} \\
\mathrm{B}\end{array}$ & $\begin{array}{l}51457 \\
51458\end{array}$ & $\begin{array}{l}1720 \pm 80 \\
7030 \pm 130\end{array}$ \\
\hline
\end{tabular}


1992 Chen, Warne, and Stanley: Northwestern delta

1992 Leroy: Palynology and climate change

1993 Dominik and Stanley: Trace elements and climate change

1993a Stanley and Warne: Synthesis of effects on the delta

1993b Stanley and Warne: Sea-level effects and archeology

1993 Stanley: Severe climatic effects in winter of 1992

1993b Warne and Stanley: Northwestern delta

1994 Stanley and Warne: Sea level and its role in Holocene delta initiation

1995 Warne and Stanley: World deltas concurrently affected by sea level

CHRONO- AND LITHOSTRATIGRAPHIC CORRELATIONS.Many of the project studies depict core-to-core correlations, most involving well-defined radiocarbon-dated lithofacies in the delta proper and sectors seaward of the coast to the Nile Cone.

1983 Stanley and Maldonado: Outer Nile shelf and Nile Cone

1985 Stanley and Wetzel: Nile Cone and southeastern Mediterranean

1985 Anastasakis and Stanley: Nile Cone

1987 Coutellier and Stanley: Northeastern delta

1987 Kulyk: Using foraminifera, northeastern delta

1988 Frihy and Stanley: Methods using petrology

1988a Stanley: Nile delta shelf

1988 Gerber: Using geochemistry, northeastern delta

1989 Pimmel and Stanley: Delta-front and prodelta facies

1989 Gupta: Using trace elements, northeastern delta

1990 Stanley: Correlations to measure subsidence of the delta plain

1990 Shergill: Using geochemistry, northeastern delta

1991 Arbouille and Stanley: North-central delta

1991 Howa and Stanley: Using plant biofacies, across the northern delta

1991 Pugliese and Stanley: Biofacies correlations, northeastern delta

1992 Stanley, Warne et al.: North-central delta

1992 Chen, Warne, and Stanley: Northwestern delta

1993b Warne and Stanley: Northwestern delta

1993a Stanley and Warne: Synthesis of delta correlations

Provenance, Dispersal, AND Paleogeography.-This group of investigations includes topics pertaining to the origin and dispersal of sediments, displacement of depositional environments, and paleogeographic changes through time in the Nile delta. This category is based on correlation of well-defined, radiocarbon-dated lithofacies of late Pleistocene to Holocene age.
1983 Stanley and Maldonado: Outer Nile shelf and Nile Cone

1985 Gerges and Stanley: Northem Suez Canal

1985 Stanley and Wetzel: Nile Cone and southeastern Mediterranean

1985 Anastasakis and Stanley: Outer Nile shelf and Nile Cone

1987 Coutellier and Stanley: Northeastern delta

1989 Foucault and Stanley: Upper River Nile system to the northern delta

1989 Stanley: Nile delta to Israeli margin, based on heavy minerals

1990 Abu-Zeid and Stanley: Northeast delta, based on clays

1991 Abdel Wahab and Stanley: North-central delta, based on clays

1991 Arbouille and Stanley: North-central delta

1992 Stanley, Warne et al.: North-central delta

1992 Chen, Warne, and Stanley: Northwestern delta

1993 Chen and Stanley: Central delta plain

1993b Warne and Stanley: Northwestern delta

1993a Stanley and Warne: Synthesis of lower delta plain

1993 Slaboda: Trace elements in Manzala Lagoon

1995 Frihy, Moussa, and Stanley: Abu Qir Bay

1995 Schneiderman: Lower River Nile system, based on mineralogy

ARCheological Considerations.-Publications in this category involve the results of petrological, sedimentological, and stratigraphic analyses in archaeological investigations.

1986 Stanley and Sheng: Santorini volcanic shards, Manzala Lagoon region, and possible Biblical exodus

1992 Stanley, Arnold, and Wame: Discovery of oldest Pharaonic site in the northern delta

1993 Allen, Hamroush, and Stanley: Egyptian civilization, environmental change, and geochemistry

1993b Stanley and Warne: Predynastic culture as related to sea level

1993a Warne and Stanley: Northern delta archaeological site and subsidence rates

ANTHROPOGENIC FACTORS AND IMPACT.-A series of studies takes into account the growing influence of humans on the Nile delta, including the much altered River Nile system and pollution.

1985 Gerges and Stanley: Human influence on the northern Suez Canal

1993 Loizeau and Stanley: Altered Idku Lagoon environment

1993a Stanley and Warne: Effects of altered River Nile system and predictions for the future 
1993 Stanley: Some recent anthropogenic effects and responses in the Alexandria region

1994 Loizeau and Stanley: Altered Mariut Lagoon subenvironment

1994 Siegel, Slaboda, and Stanley: Recent increased pollution in Manzala Lagoon

Nile Delta LAGOONS.-Increased attention is being paid to the recent evolution of the shallow Manzala, Burullus, Idku, and Mariut water bodies in the northern Nile delta and the sedimentary and faunal facies therein.

1992 Randazzo: Petrology of recent Manzala Lagoon deposits

1992 Longo: Molluscan faunas and palaeoecology in modern lagoons

1993 Loizeau and Stanley: Changing lithofacies, Idku Lagoon

1994 Loizeau and Stanley: Changing lithofacies, Mariut Lagoon

1994 Bernasconi and Stanley: Changes in molluscan biofacies

1994 Siegel, Slaboda, and Stanley: Recently increased pollution in Manzala Lagoon

COMPARING THE Nile WITH OTHER WORLD DelTAS.-A more recent research effort considers attributes of the Holocene Nile delta, which enable it to be compared with other such depocenters elsewhere in the world. Of special consideration are the timing and factors responsible for initiation of modern world deltas.

1986 Stanley: Mediterranean deltas, fans, and cones

1994 Stanley and Warne: Timing of delta initiation and the role of sea level

1995 Warne and Stanley: Comparing factors controlling the development of world deltas

\section{Applications For Delta Management}

The northern Nile delta is presently undergoing rapid environmental deformation and ecological decline. Most serious are the combined effects of natural factors, such as land subsidence and rising sea level, with anthropogenic factors, such as irrigation and damming. This results in, among other changes, seawater incursion into the delta's water table and coastal erosion (Stanley and Warne, 1993a). Salination has increased substantially since closure of the Aswan High Dam in 1964, reducing agricultural productivity (Biswas, 1993) and altering the chemistry of the delta's lagoon and lake waters (Kerambrun, 1986). The dam now controls the flood cycle, which previously flushed the delta plain and prevented substantial accumulation of salts in this evaporitic setting. Also significant is the trapping of sediments at Lake Nasser behind the dam, reducing nutrients formerly carried downstream in the flow to the delta and offshore. At the same time, the rapidly increasing population has necessitated intensified agricultural development, unprecedented municipal expansion, accelerated diversion of Nile waters through a dense and complex irrigation system, and land reclamation of vital delta water bodies, such as lagoons and marshes. These activities, particularly the much-reduced sediment discharge, have also contributed to heightened coastal erosion by Mediterranean nearshore currents (Figure 10).

It is our hope that the information collected in this document can be of assistance to geologists, ecologists, agronomists, and engineers having the responsibility of maintaining and improving environmental conditions in the lower Nile delta plain. With the available database, specialists will be able to distinguish and measure changes, both natural- and humaninduced, over time. Our work, focusing on dated subsurface sedimentary sections, serves this purpose for the lower Nile delta from the time of its initiation in the early Holocene ( $\sim 8000$ years ago) to the 1990 s. Of primary value are the data serving to compile paleogeographic reconstructions of the northern delta, including positions of earlier Nile channels, strandplains, lagoons, and marshes, prior to and during the early phases of human settlement.

Data gathered from the borings provide a means to distinguish the effects of natural from anthropogenic factors. In essence, we have compiled a temporal and spatial record of change from the time when human impact was minimal, prior to $\sim 7000$ years ago (Stanley and Warne, 1993b), when sea-level and climate oscillations, neotectonism, and effects of fluvial and coastal processes were dominant in controlling the configuration of the lower Nile delta plain. Although our data indicate that fluctuations of these natural factors continue to modify the delta, the record shows that the escalating role of people began to overtake the influence of natural factors as early as the Dynastic period (Butzer, 1976). Since the beginning of Egypt's industrialization, and particularly since the latter part of the nineteenth and the beginning of the twentieth century (when the first dam at Aswan was completed, along with a series of barrages and River Nile channelization projects), our records show that human influence on the delta has expanded by several orders of magnitude. The dated borings thus serve as a gauge against which present changes can be compared. For example, rates and amounts of land subsidence and delta tilting (Stanley, 1990) and recent increases in pollutants in lagoon and marsh areas can be measured accurately against the long-term record (Siegel et al., 1994).

As in the case of many of the world's major deltas, the Nile is low-lying and highly vulnerable to even minor changes in sea level and subsidence. Inasmuch as the sediment supply has now been artificially curtailed, this depocenter has become increasingly subject to marine incursion, which further reduces its 


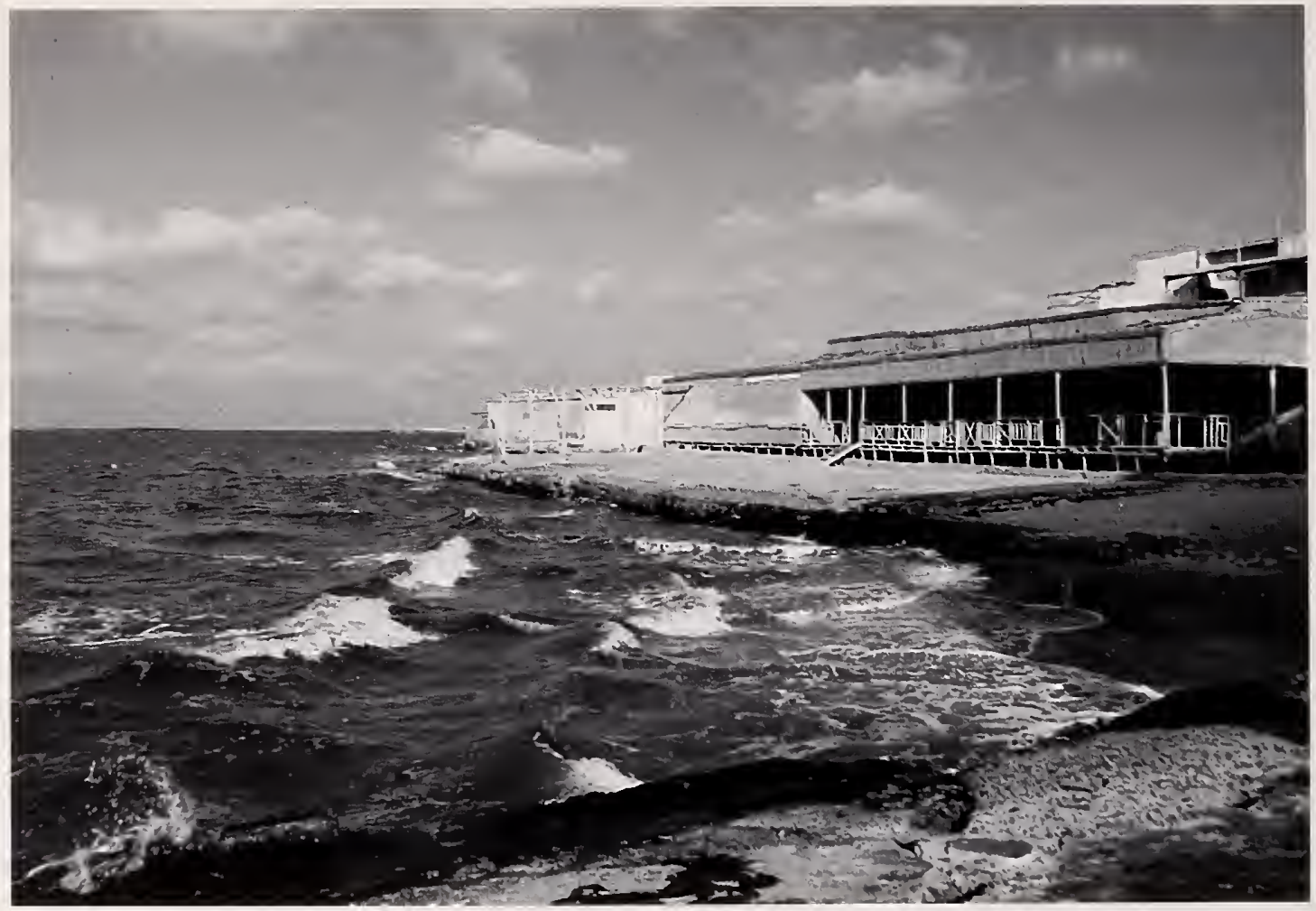

FIGURE 10.-Section of the town of Ras El-Barr at the Damietta promontory (north of Damietta, D on Figure 1), recently abandoned and undergoing destruction by intense coastal current erosion. Relatively rapid incursion of the sea in this region is due to concurrent subsidence of land and rise in sea level as indicated by analyses of radiocarbon-dated drill cores. Photograph taken in October 1992. (Courtesy of G. Drapeau.)

ability to sustain a dense and burgeoning population. Given this rapidly changing dynamic of interplay among human and natural factors, the availabilty of a comprehensive database becomes essential for the implementation of effective protection measures in a region where dependency on agricultural production has reached a critical stage. 


\section{Appendix 1: Core Lithological Logs}

\section{Legend for core logs S1-S87}

\begin{tabular}{|c|c|}
\hline$\Leftrightarrow$ & Plant Fossil \\
\hline q & Root \\
\hline ¿ & Shell Fragments \\
\hline 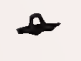 & Potsherd \\
\hline$\wedge$ & Gypsum \\
\hline $\begin{array}{l}69 \\
69\end{array}$ & Whole Shell \\
\hline$\odot$ & Organic Matter \\
\hline$m$ & Mica \\
\hline$N$ & Nodule \\
\hline- & Pebbles \\
\hline$\infty$ & Sand Pocket \\
\hline 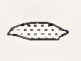 & Clay Pocket \\
\hline$\Phi$ & Limestone Pocket \\
\hline$T$ & Limestone \\
\hline
\end{tabular}

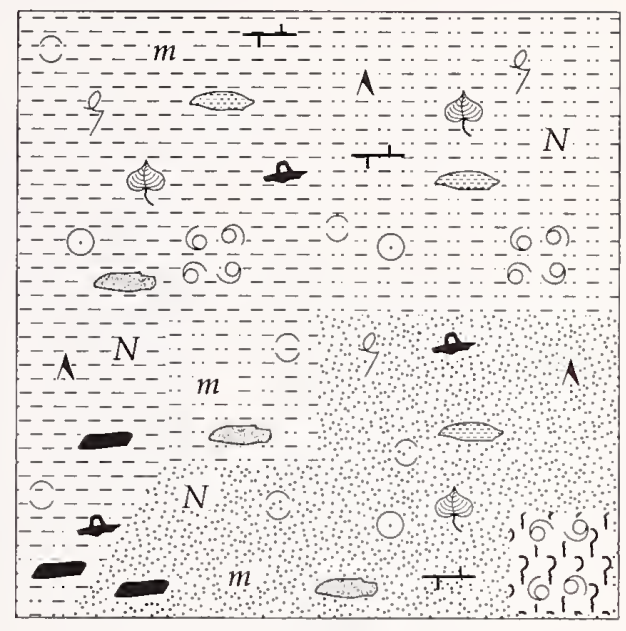

$$
\begin{array}{cl}
\text { । } & \text { Core } \\
W_{1} & \text { Washing } \\
3 & \text { Sample } \\
\text { I } & \text { Spoon Sample } \\
\text { A } & \text { Radiocarbon Sample }
\end{array}
$$

Lithologic Patterns
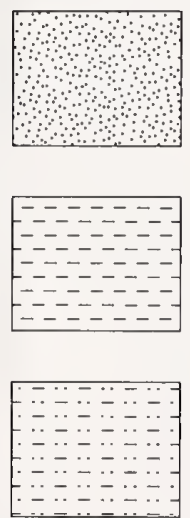

Silt/Mud

Peat

Clay

\section{Structural Patterns}
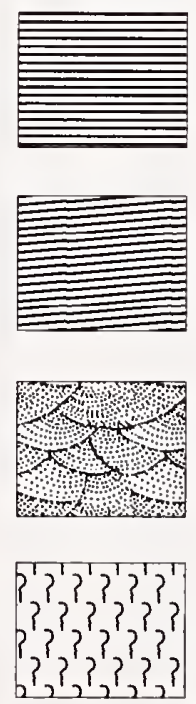

Planar

Laminae

Non-

Planar

Beds

Cross-

Bedded

Bio-

turbated

Peat
Textural Patterns

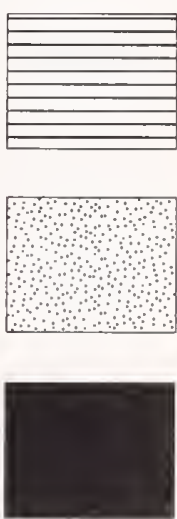

Silt

Sand

Clay

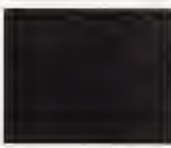


APPENDIX 1.-Continued.

CORE NUMBER S1I
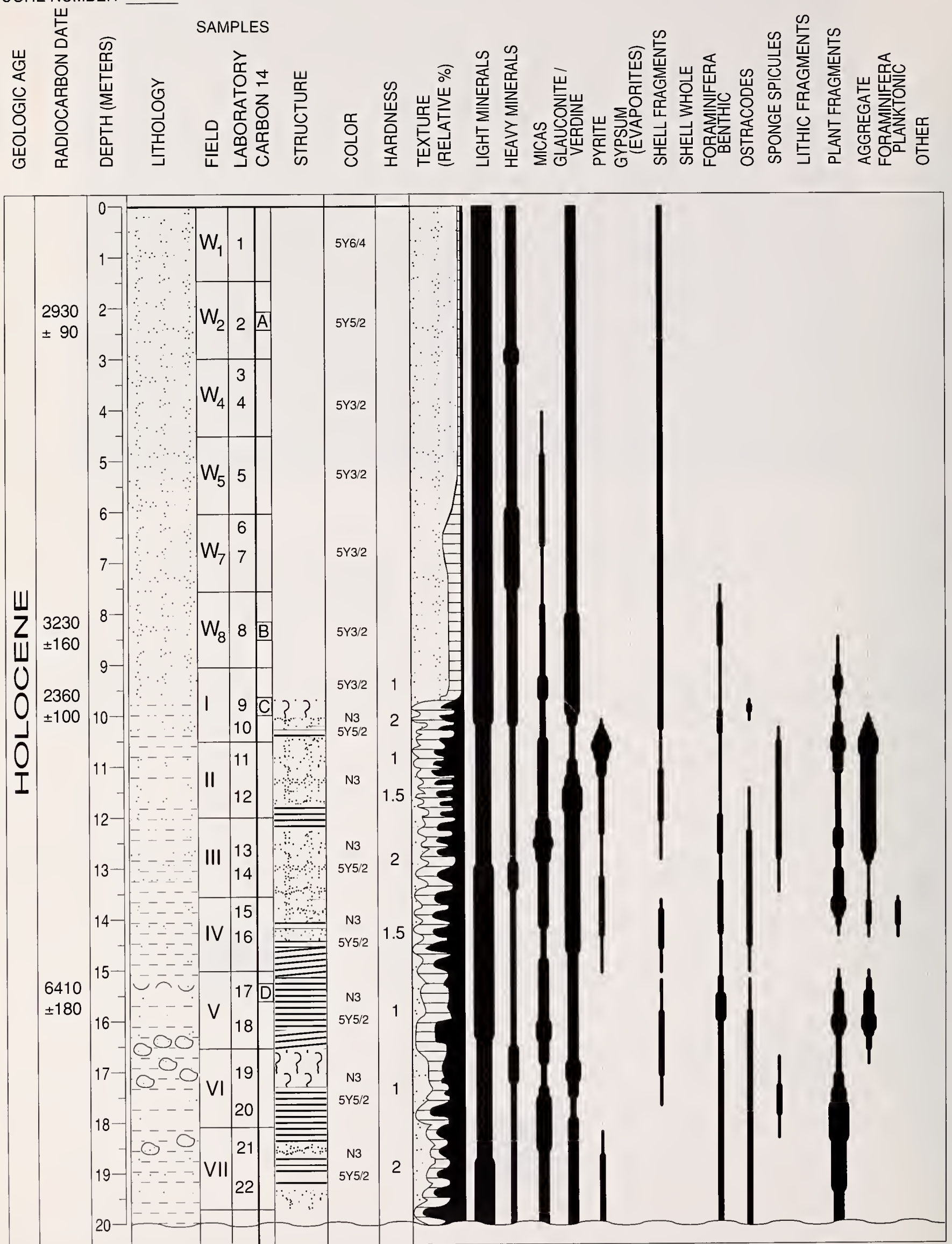
APPENDIX 1.-Continued.

CORE NUMBER S1II

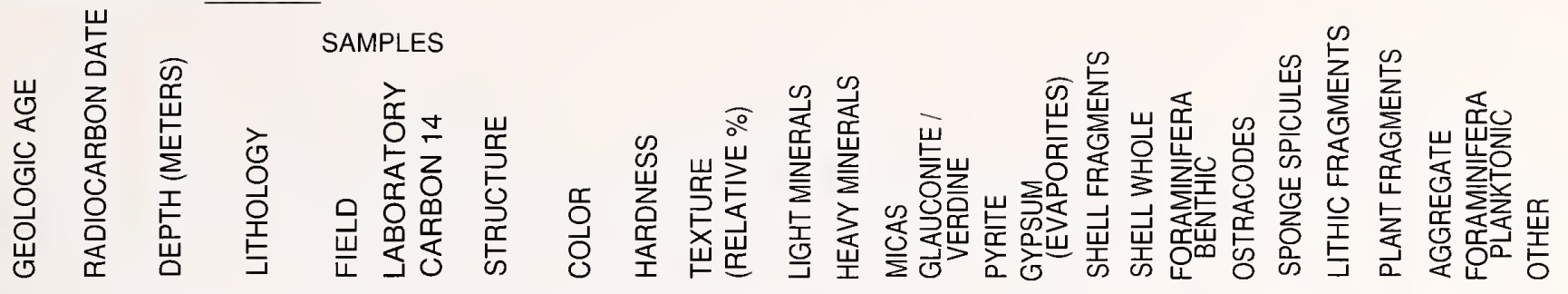

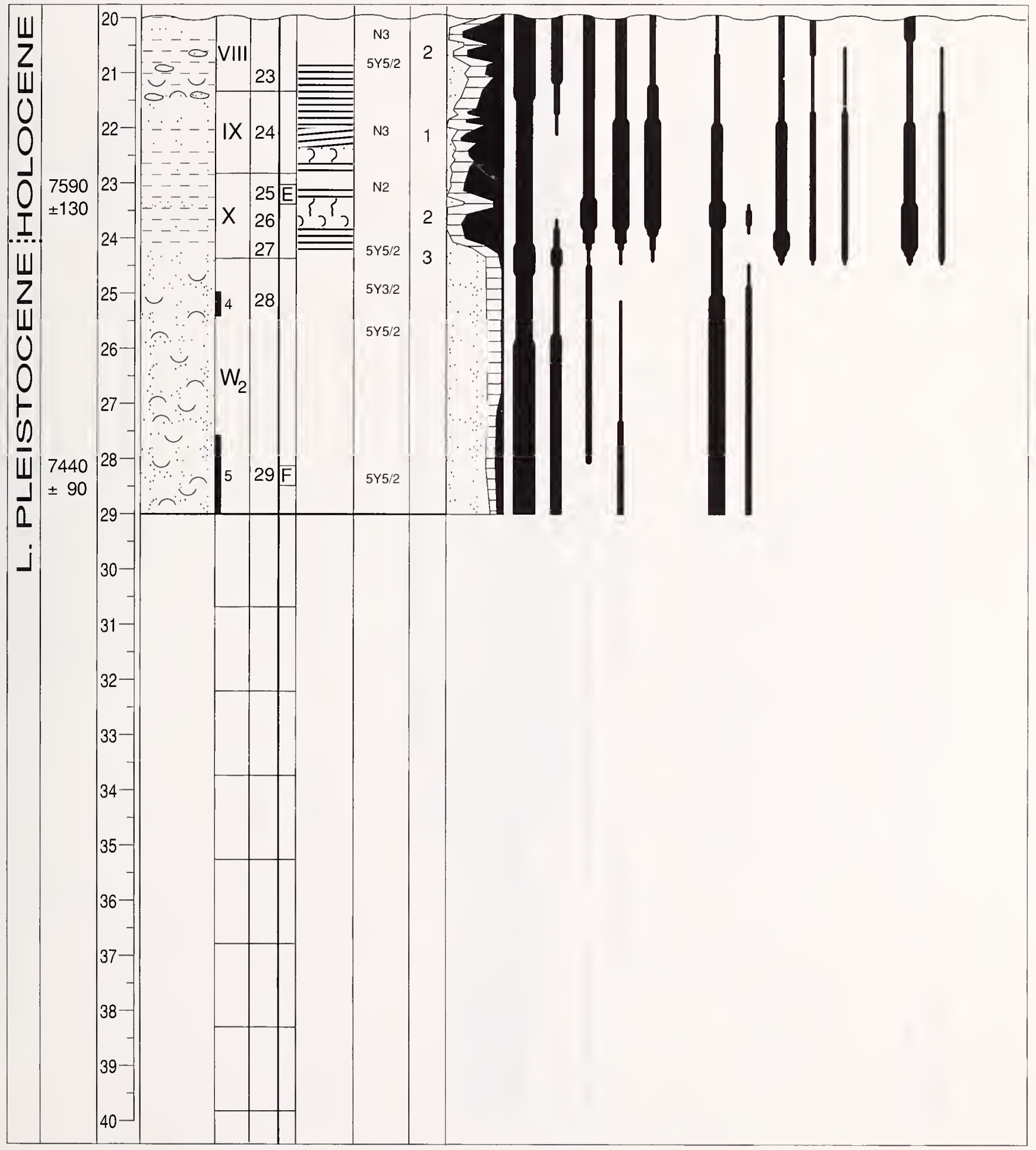


APPENDIX 1.-Continued.

CORE NUMBER S2
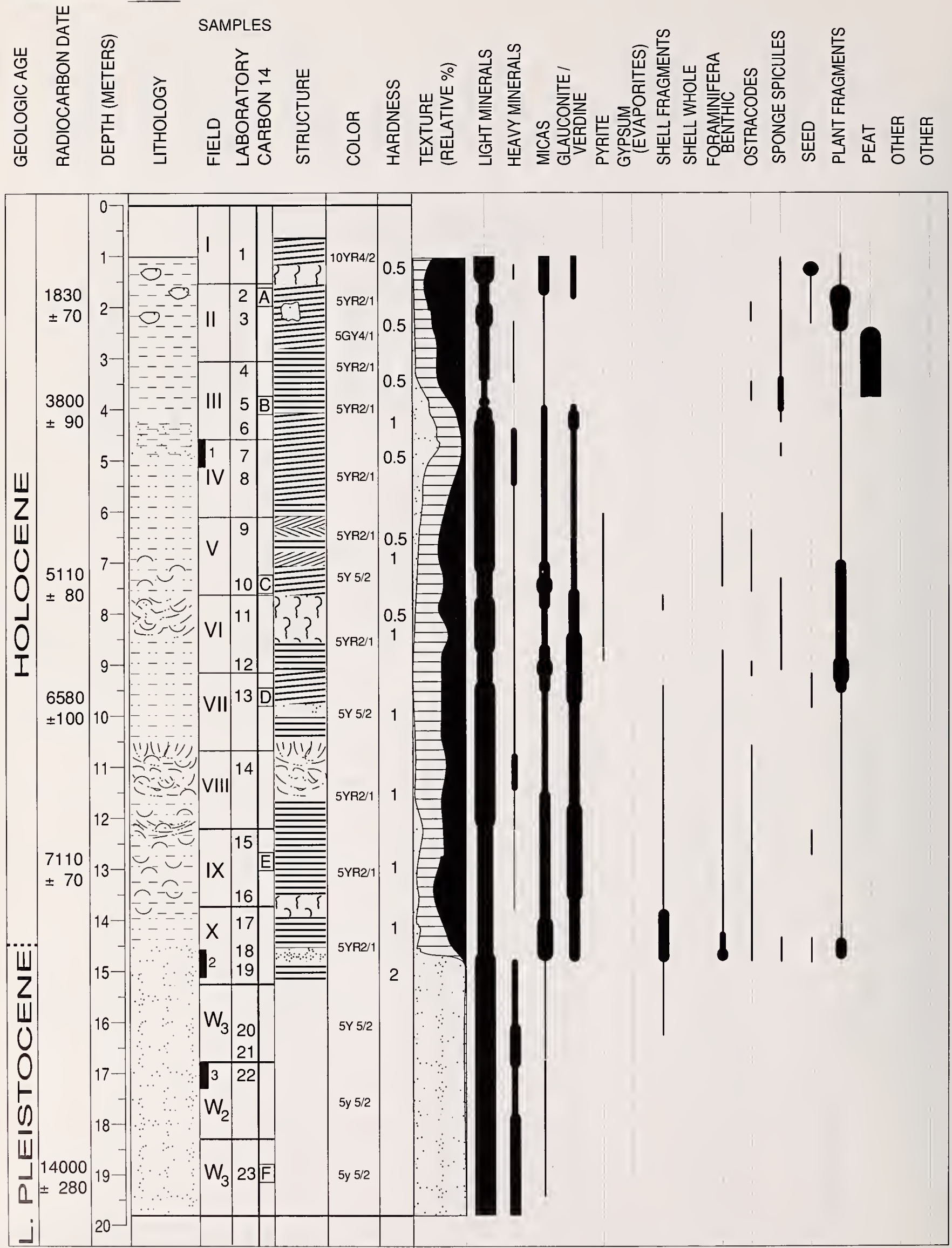
APPENDIX 1.-Continued.

CORE NUMBER S3 I
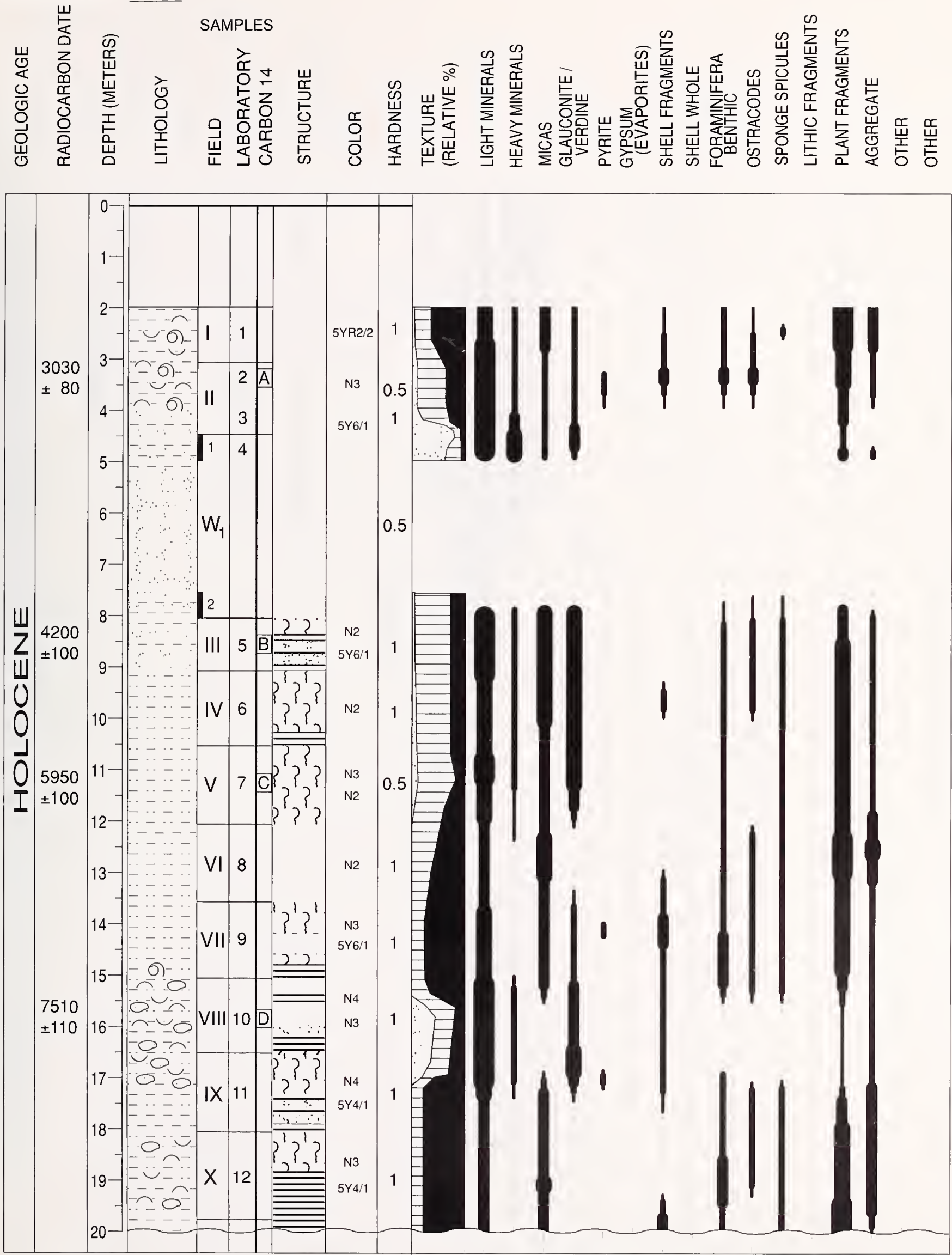
APPENDIX 1.-Continued.

CORE NUMBER S3 II

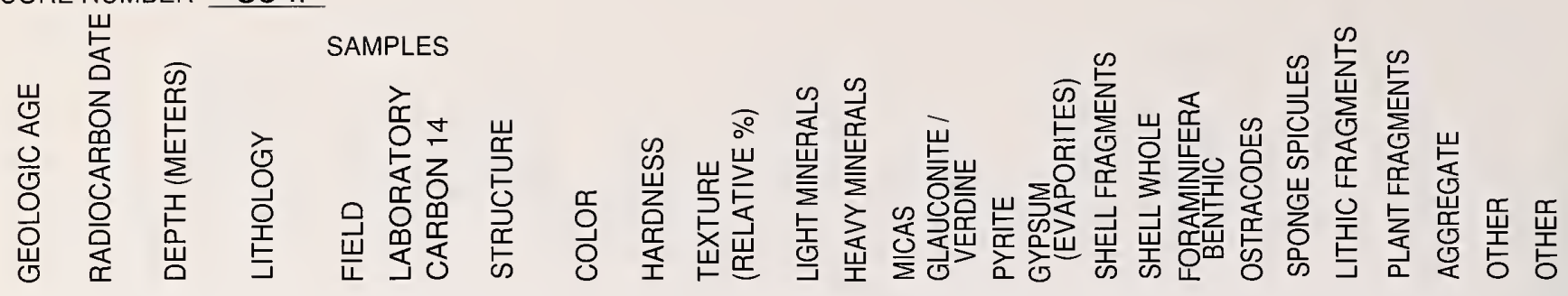

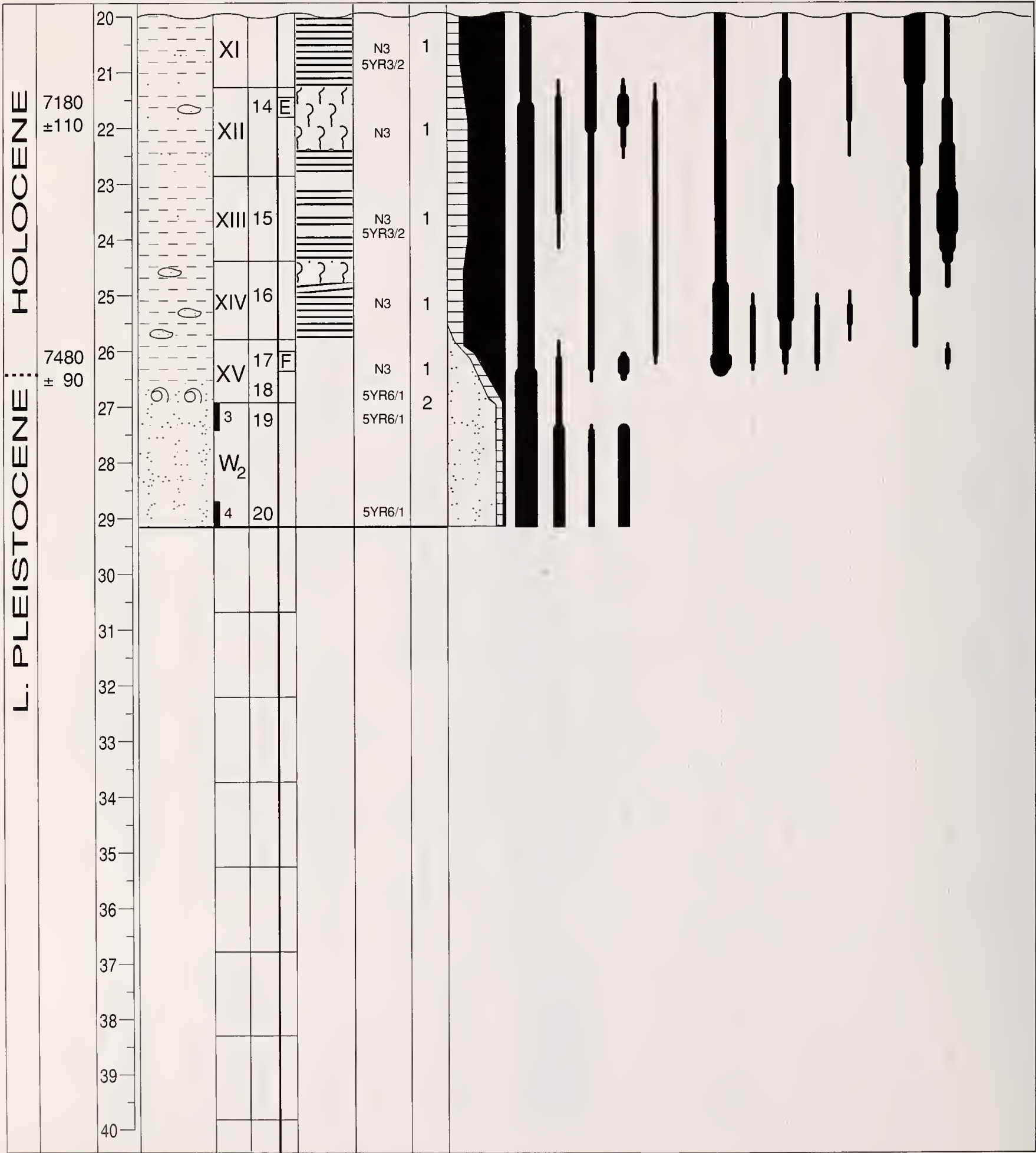


APPENDIX 1.-Continued.

CORE NUMBER S4

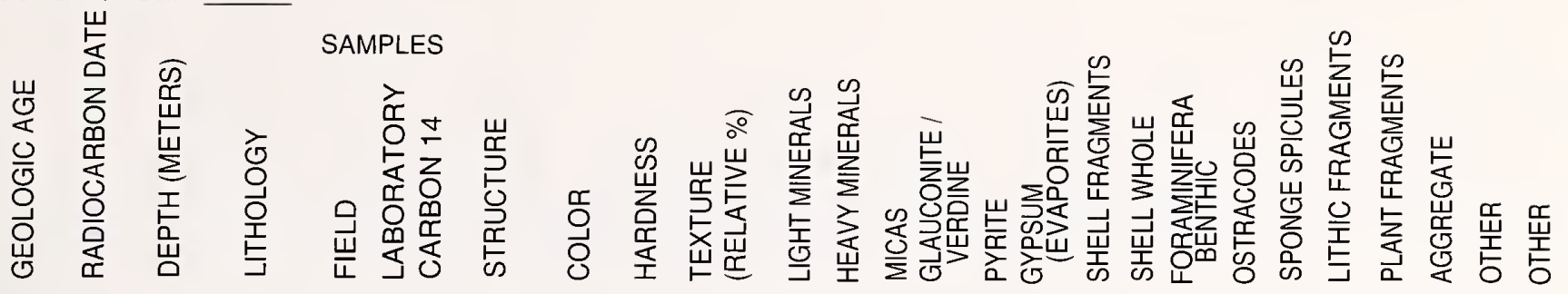

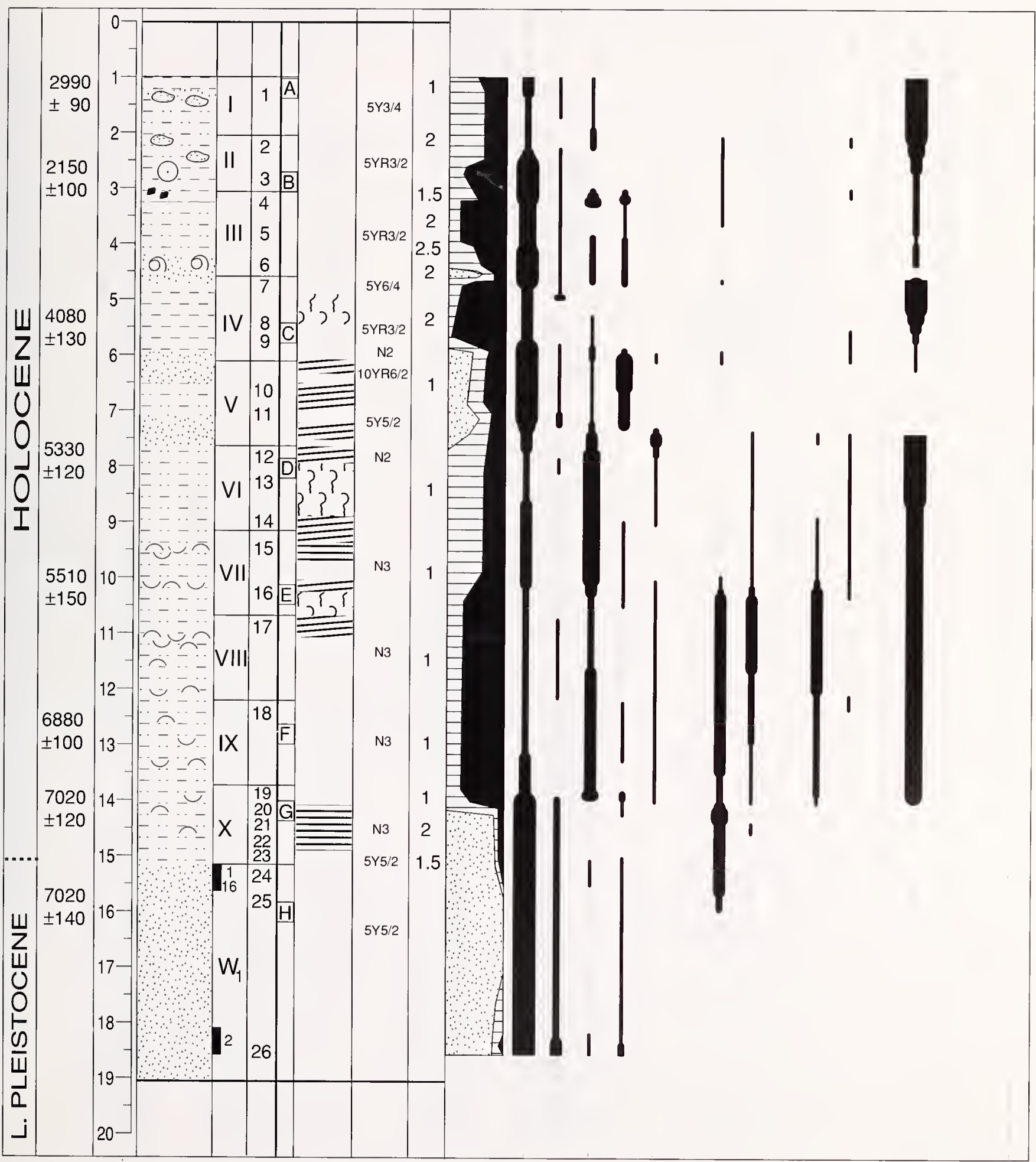


APPENDIX 1.-Continued.

CORE NUMBER S5I
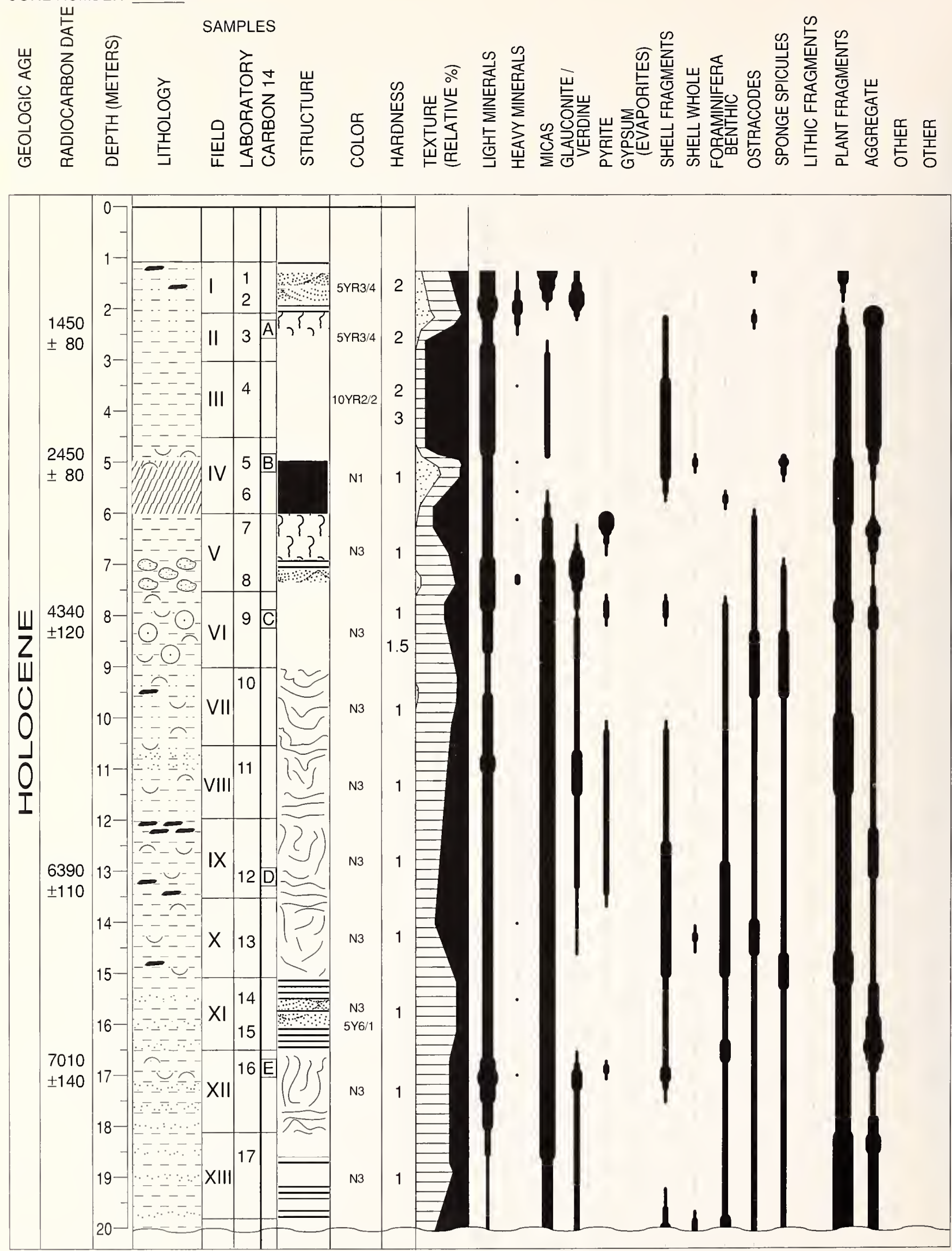
APPENDIX 1.-Continued.
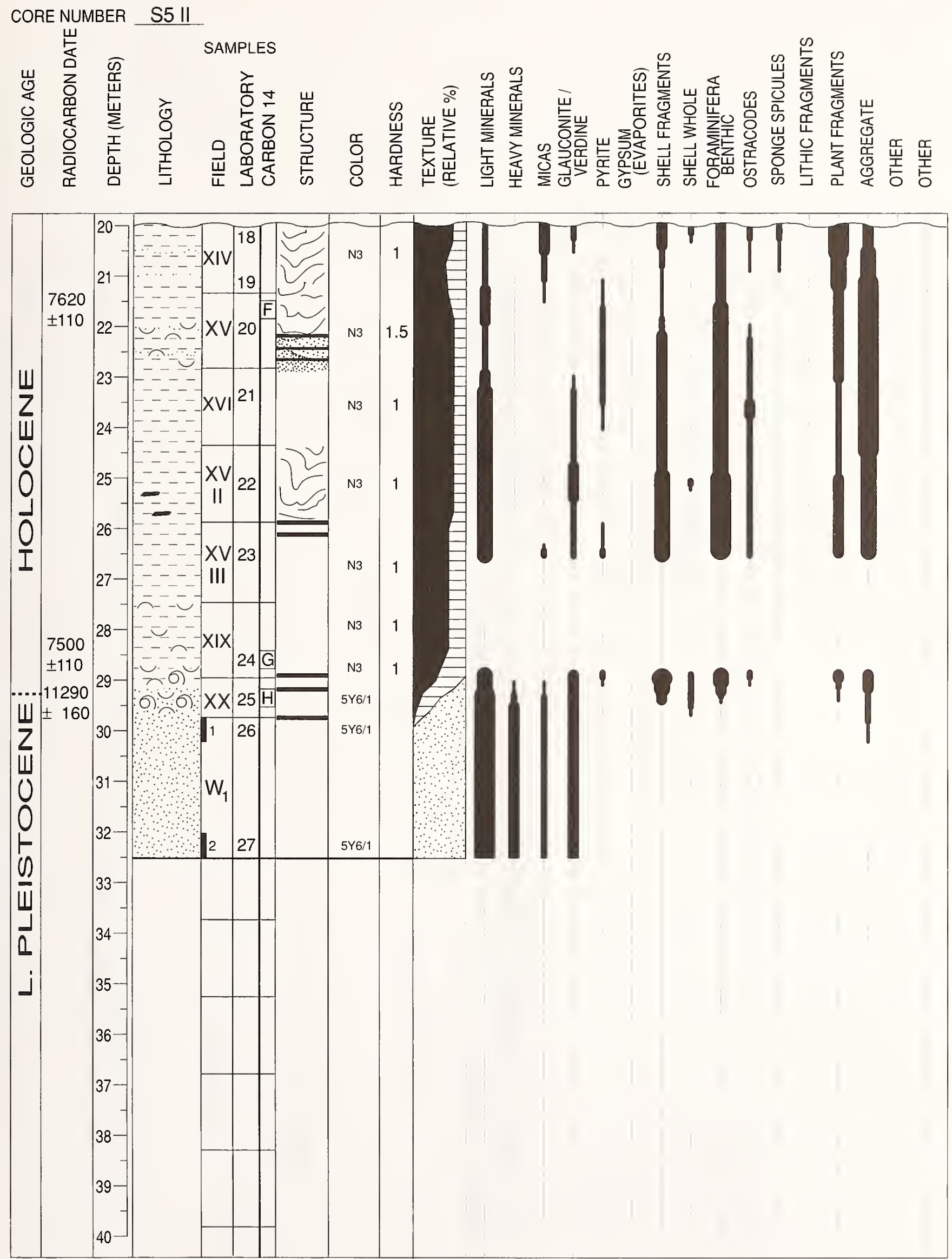
APPENDIX 1.-Continued.

CORE NUMBER S6I
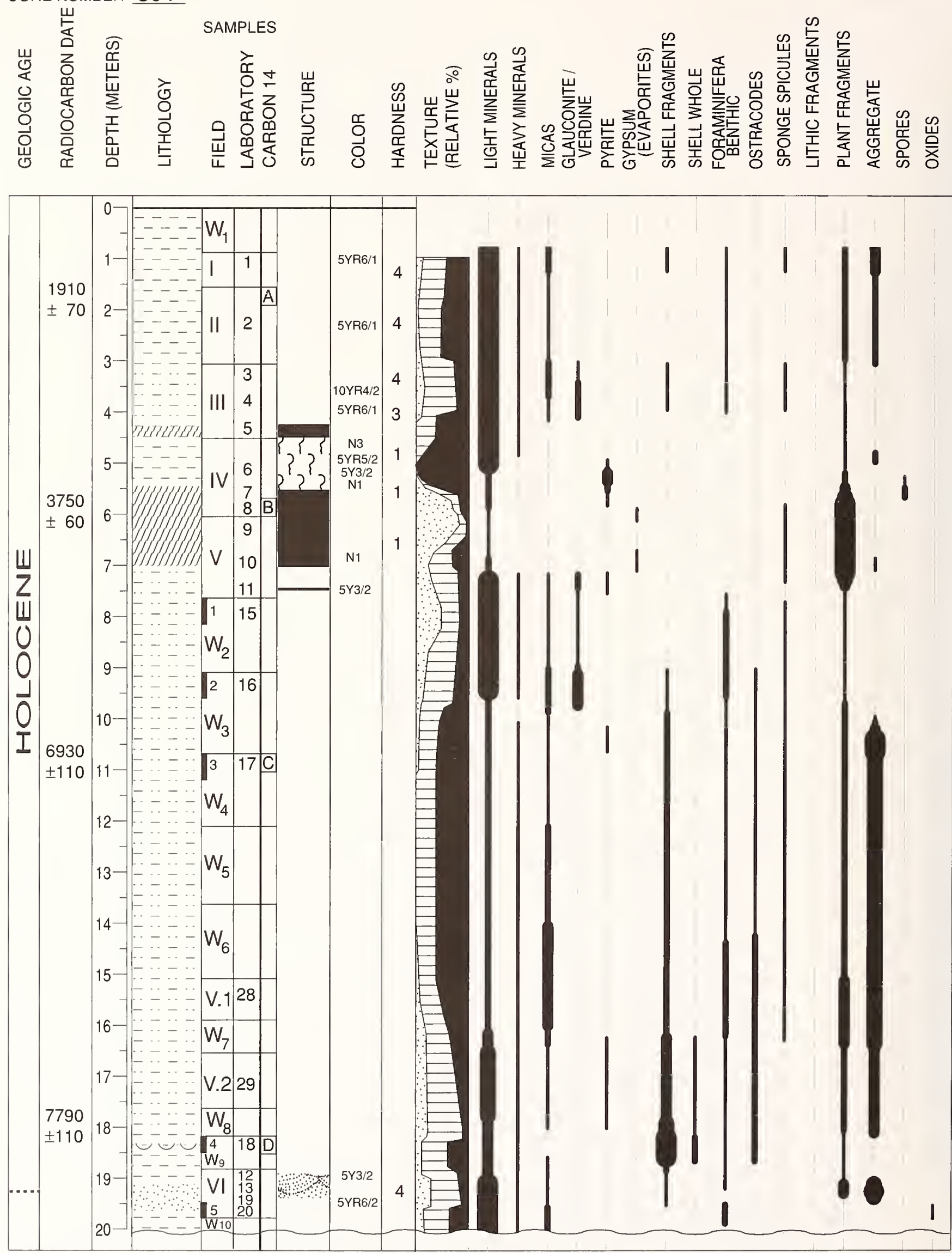
APPENDIX 1.-Continued.
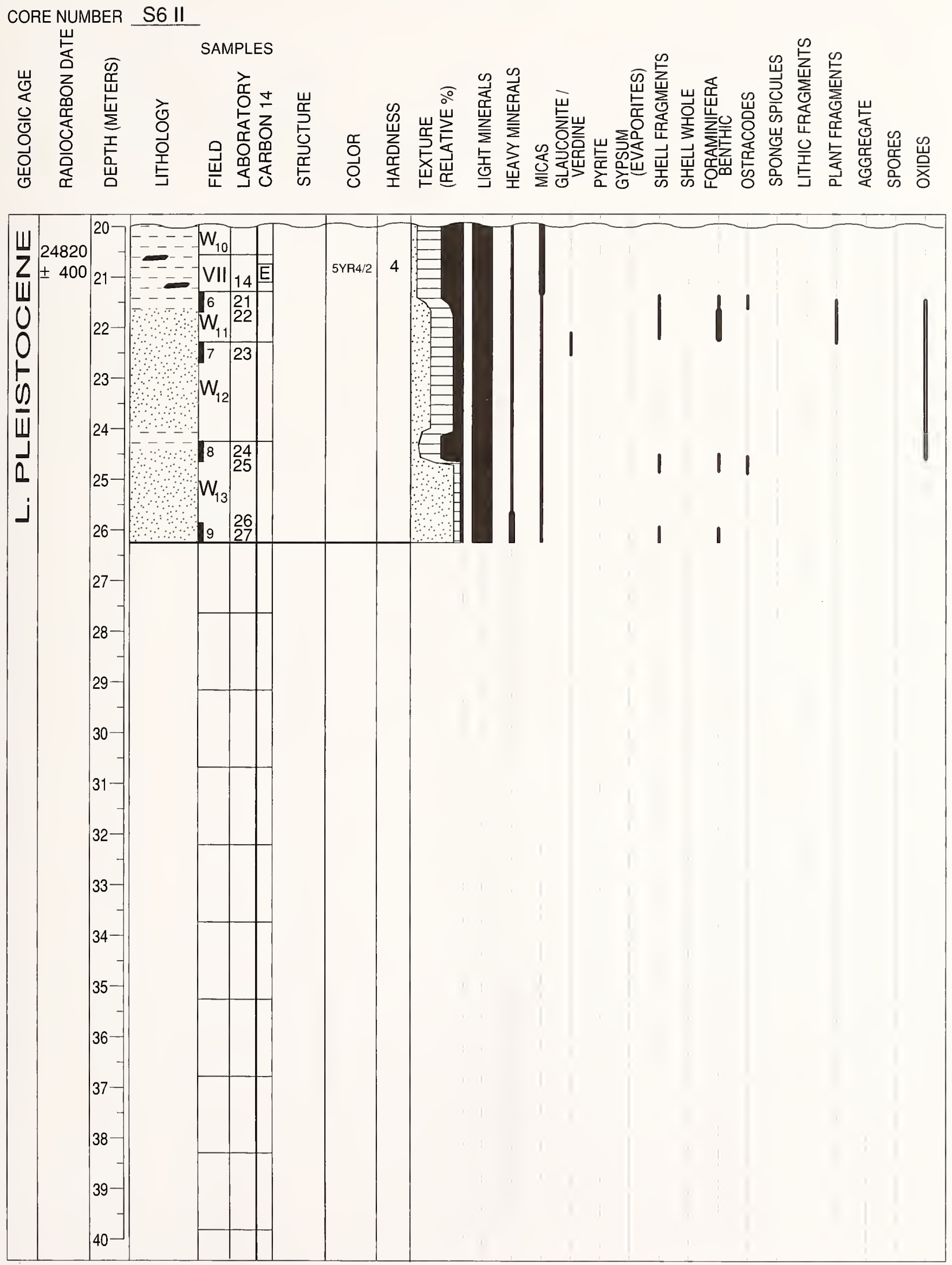
APPENDIX 1.-Continued.

CORE NUMBER S7I
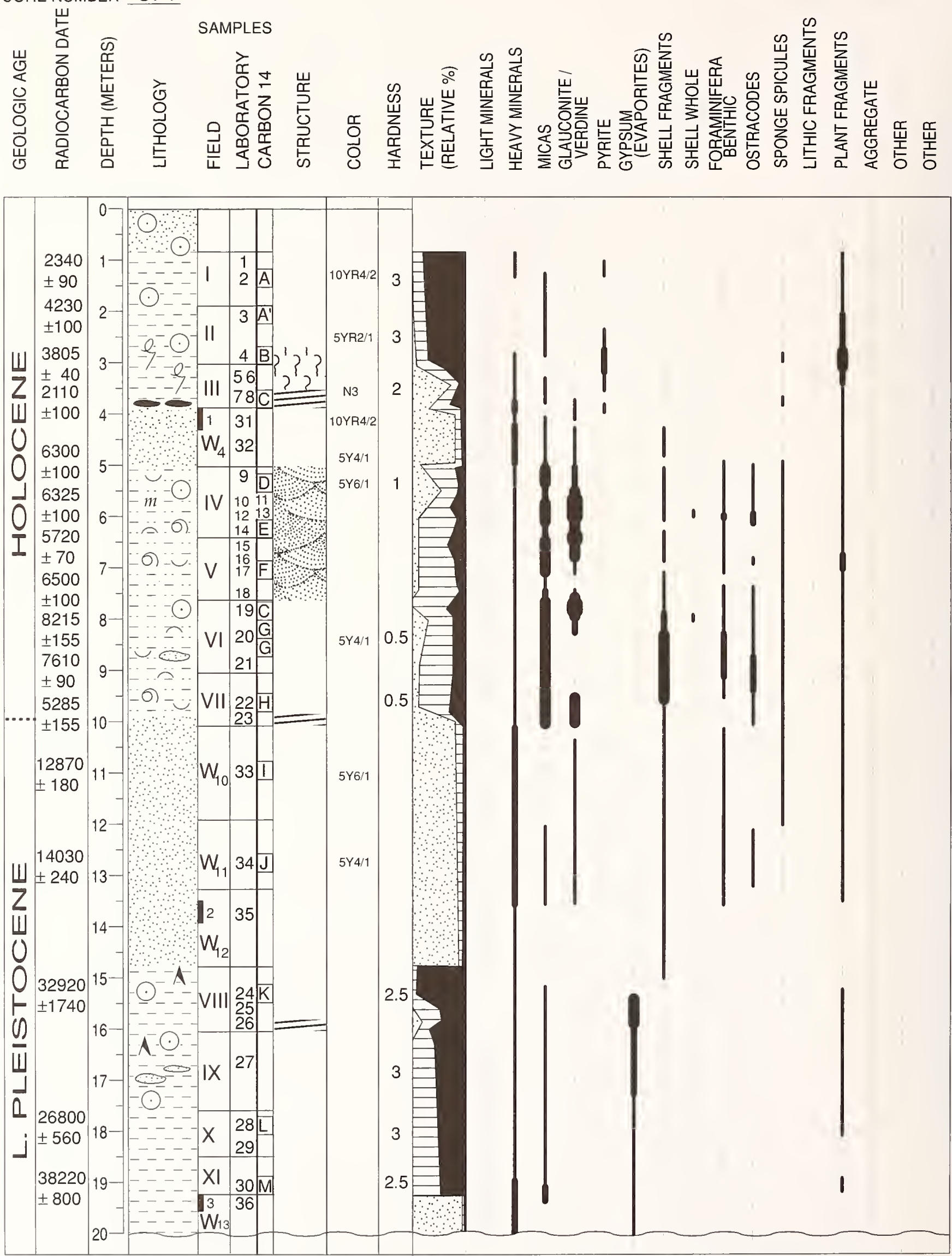
APPENDIX 1.--Continued.

CORE NUMBER S7 II

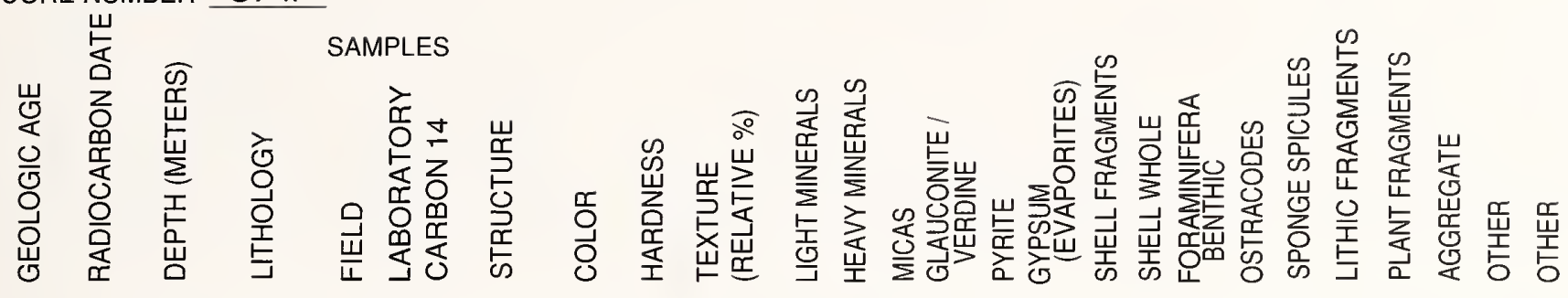

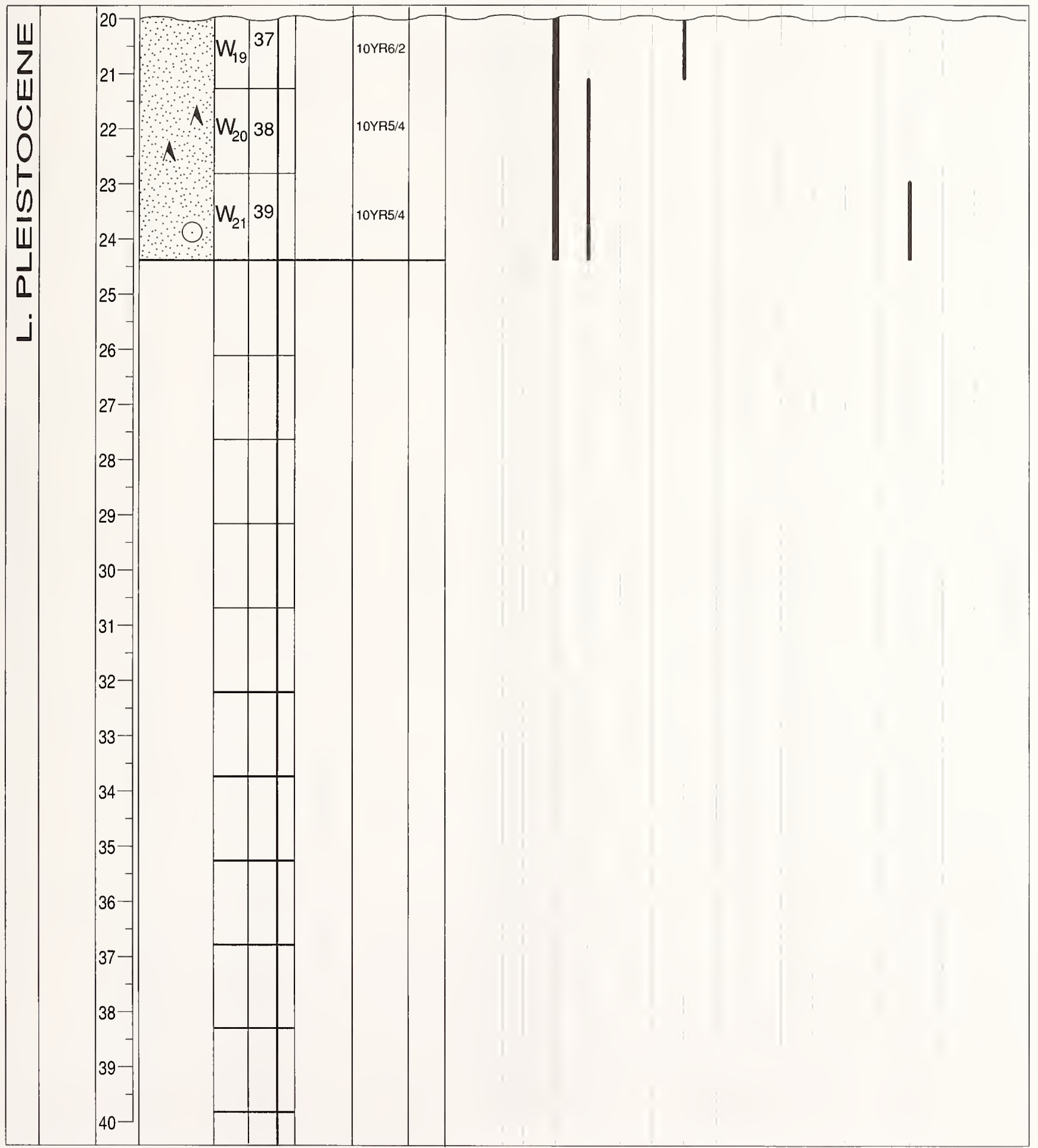


APPENDIX 1.-Continued.

CORE NUMBER S8I

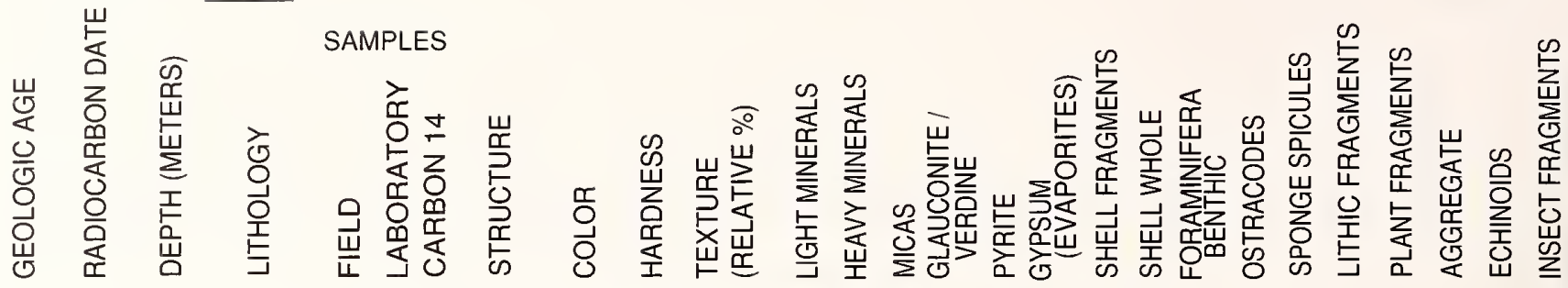

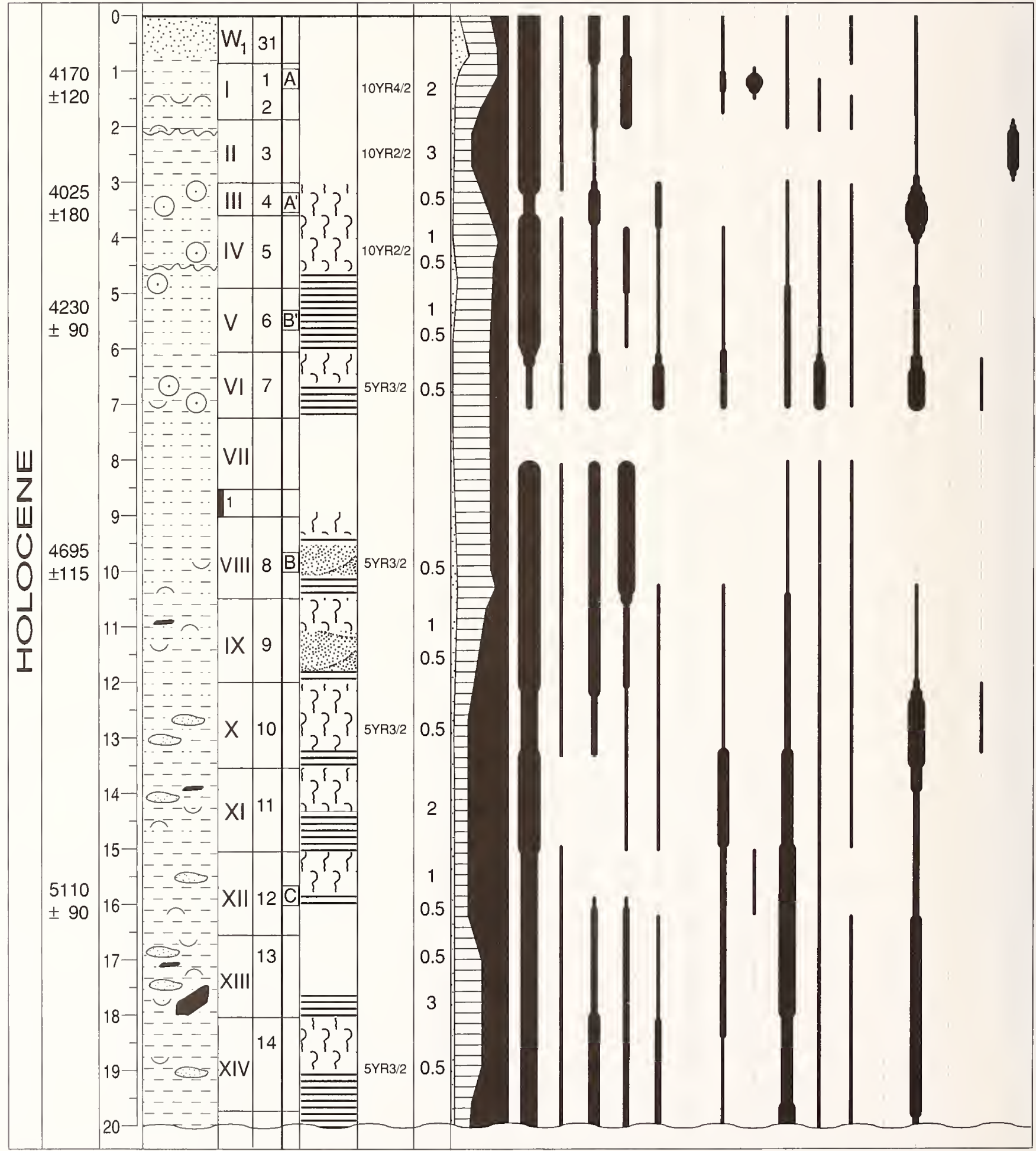


APPENDIX 1.--Continued.

CORE NUMBER S8 II

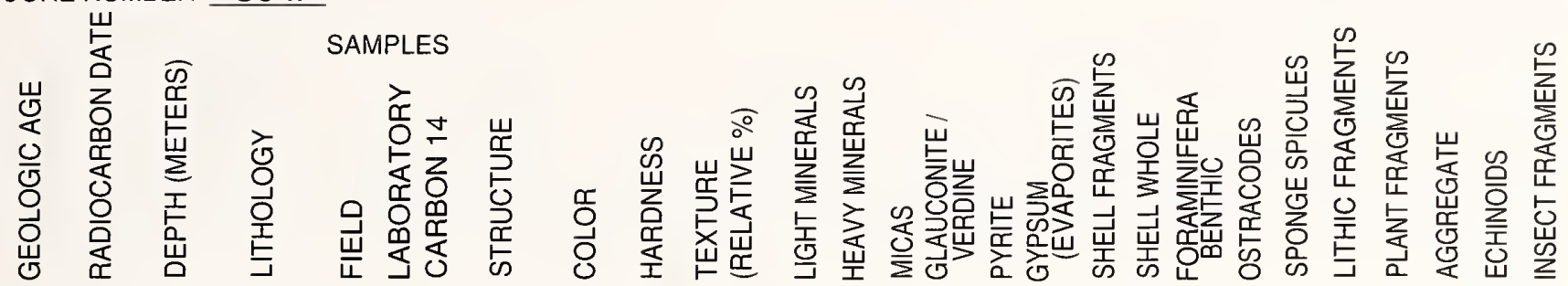

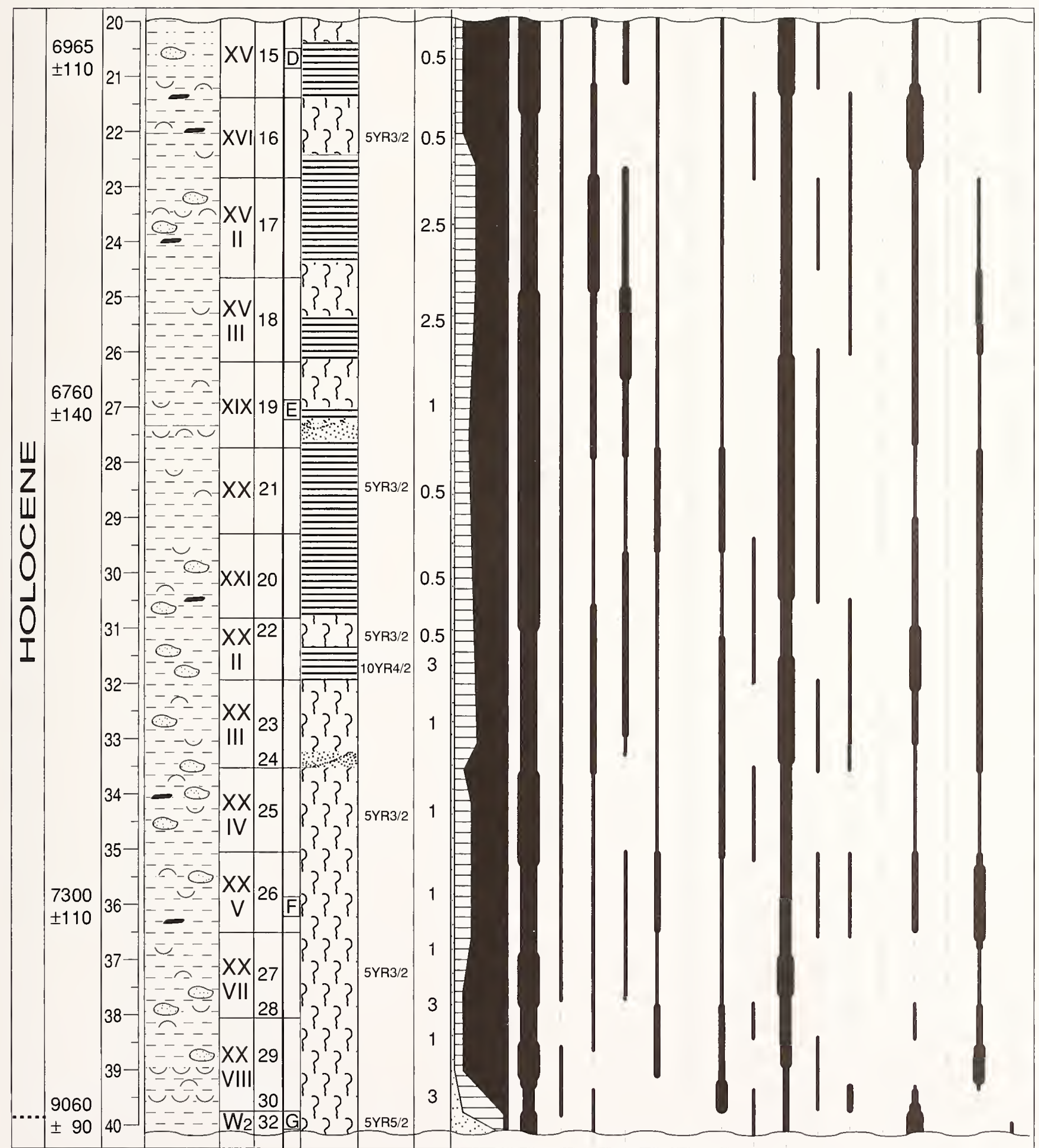


APPENDIX 1.-Continued.

CORE NUMBER S8 III

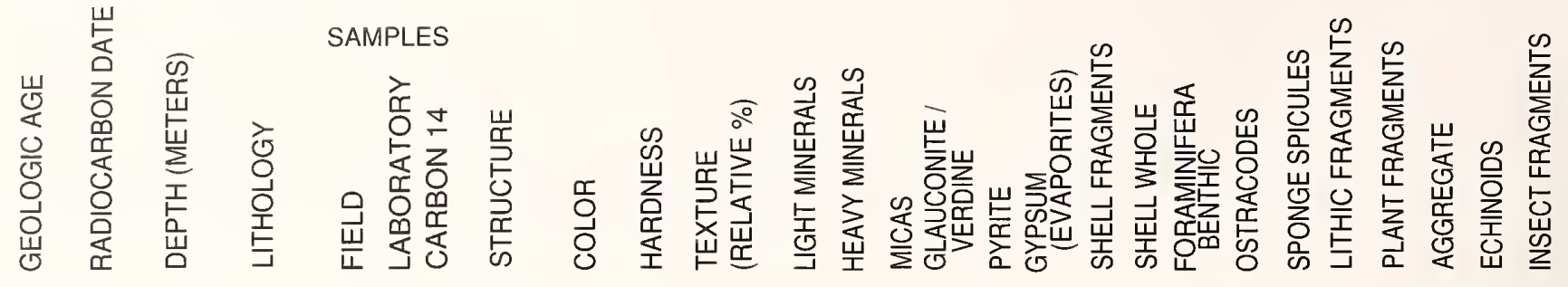

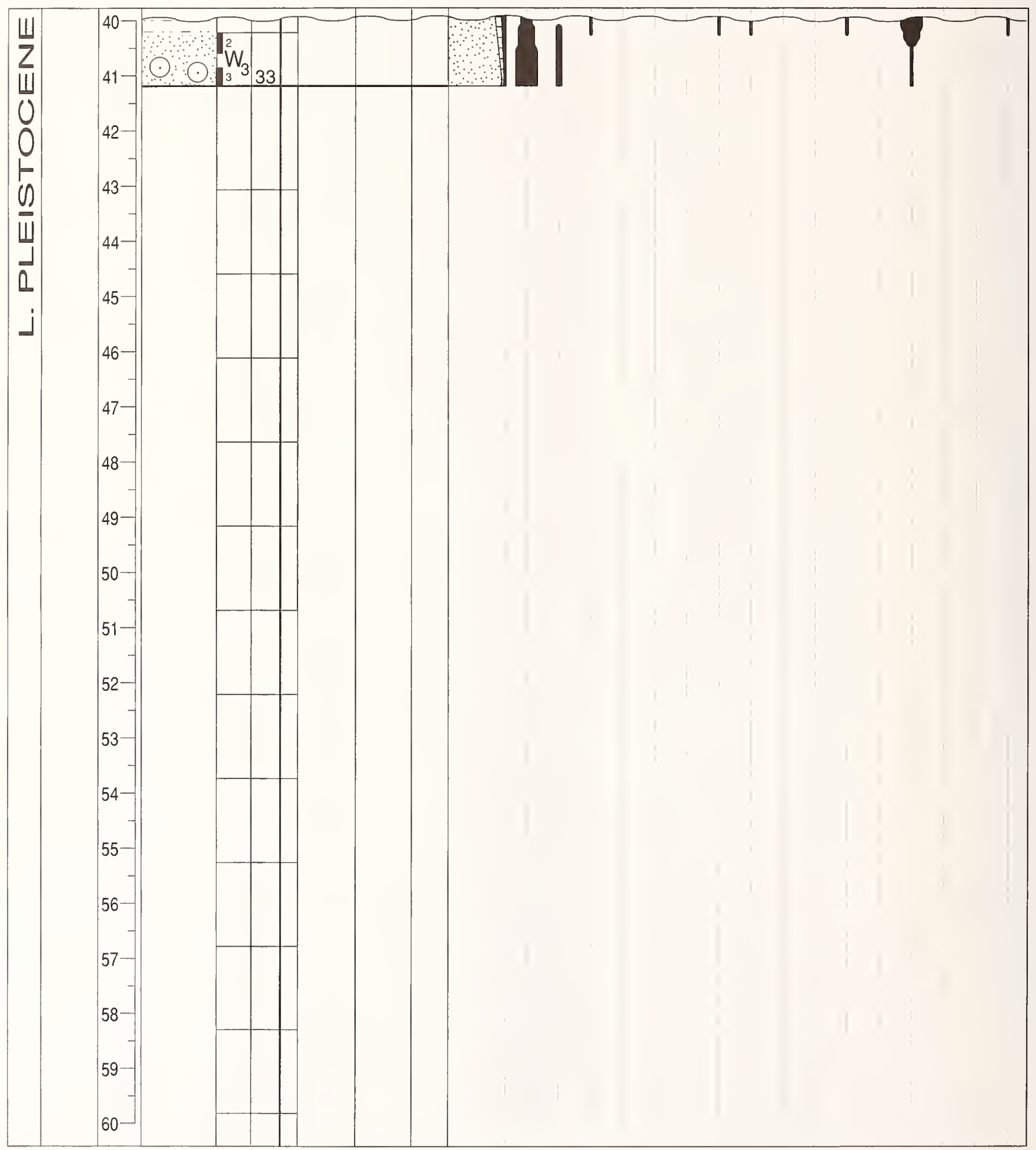


APPENDIX 1.-Continued.

CORE NUMBER S9

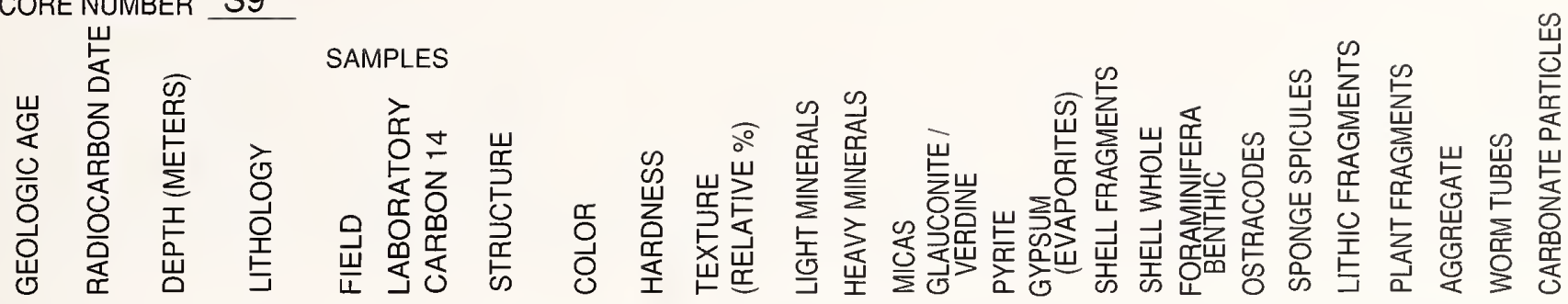

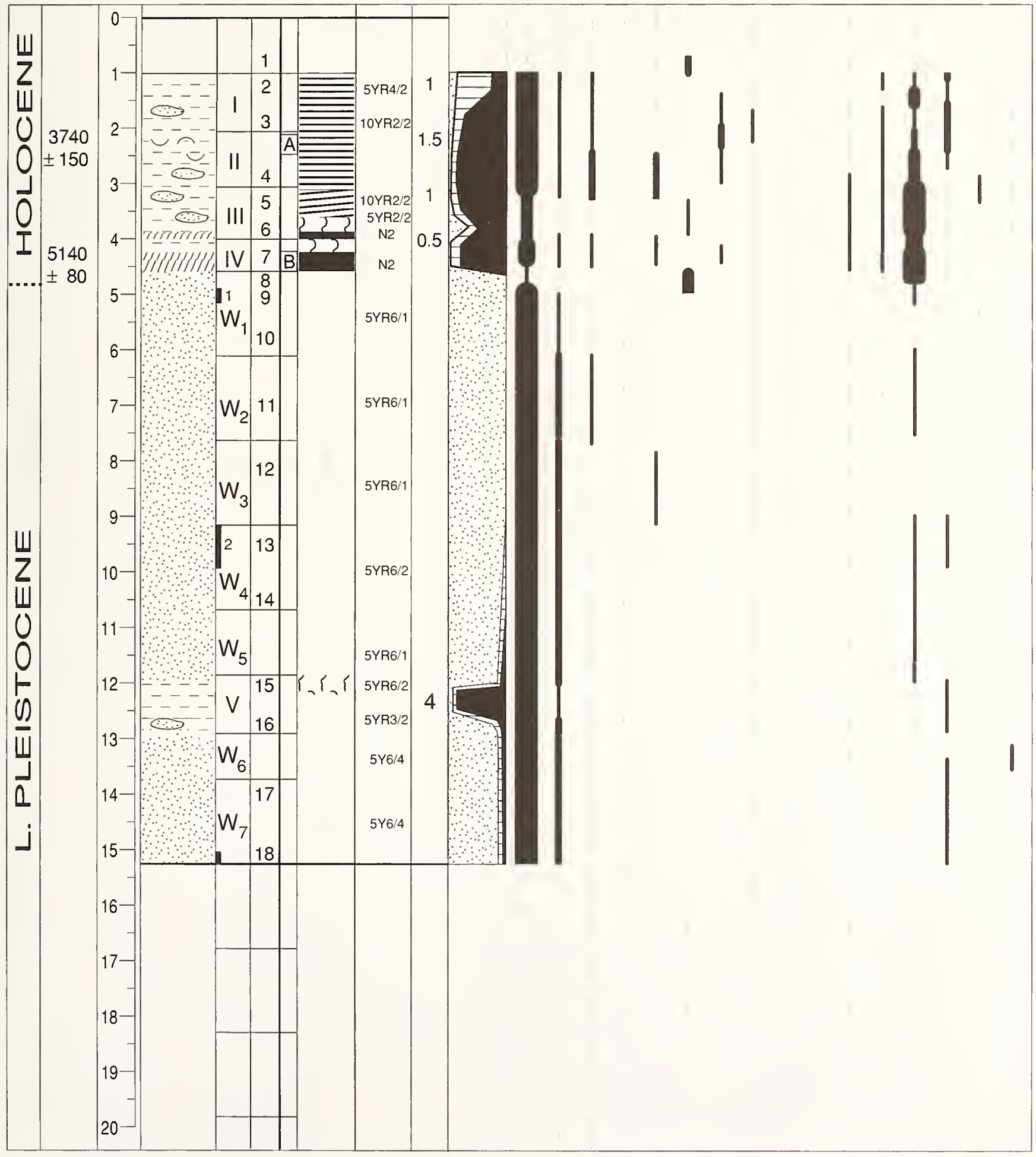


APPENDIX 1.-Continued
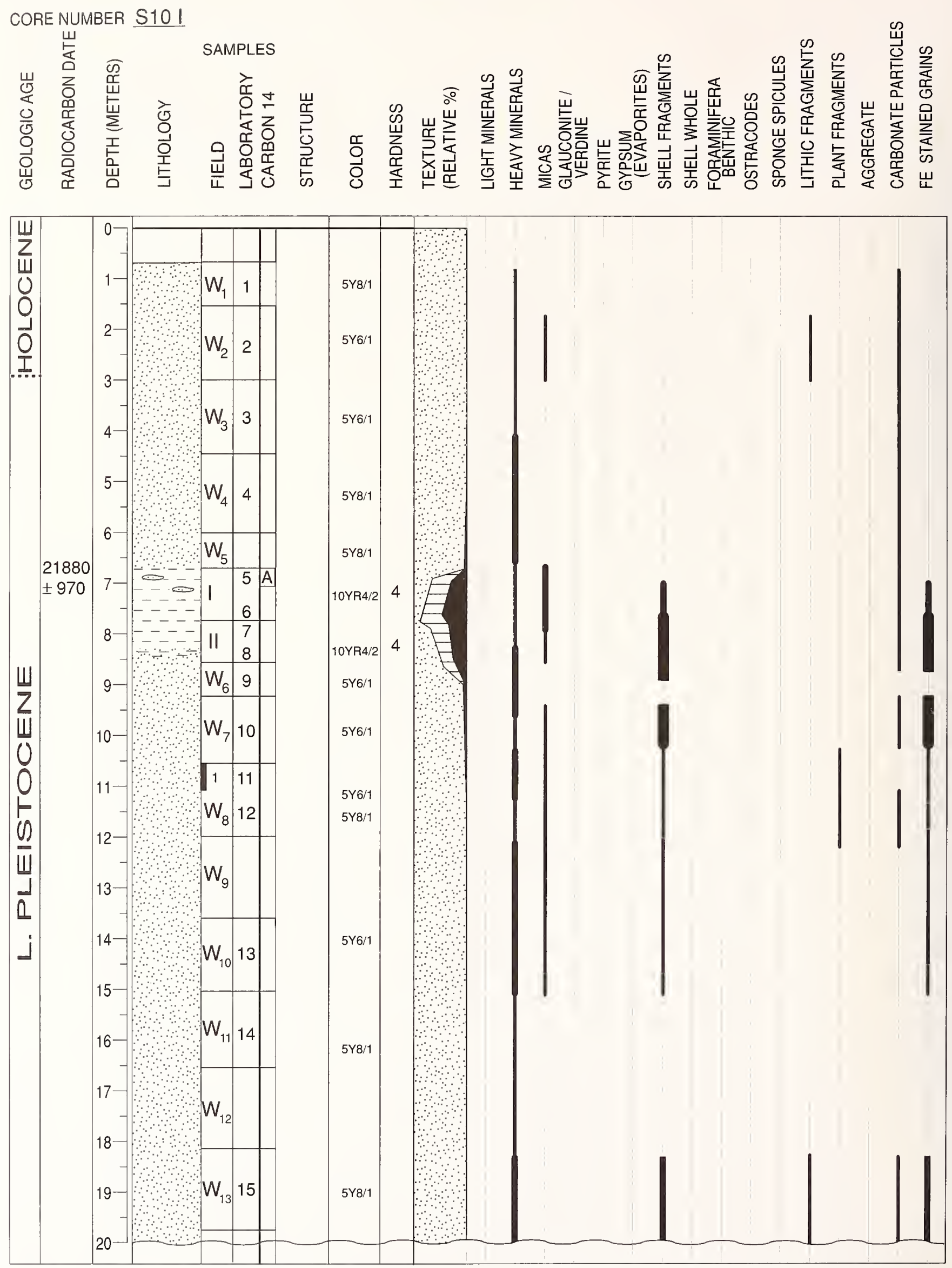
APPENDIX 1.-Continued.

CORE NUMBER S10 II

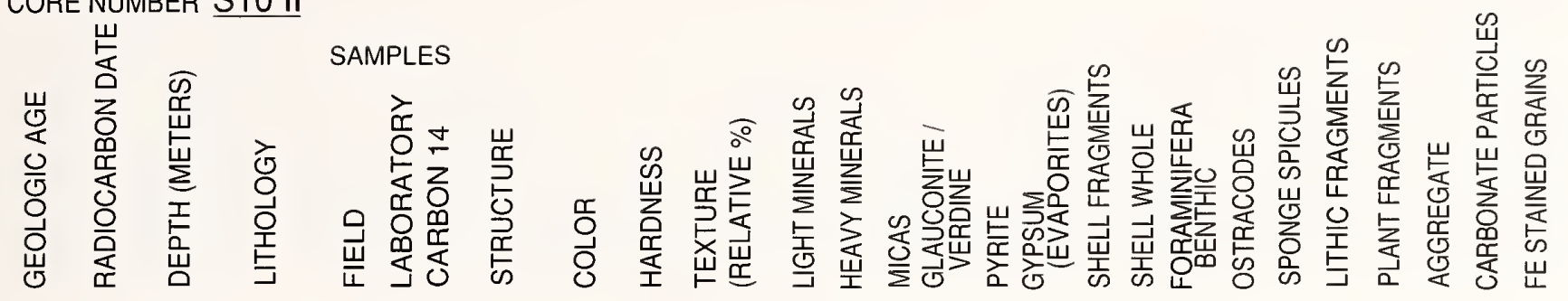

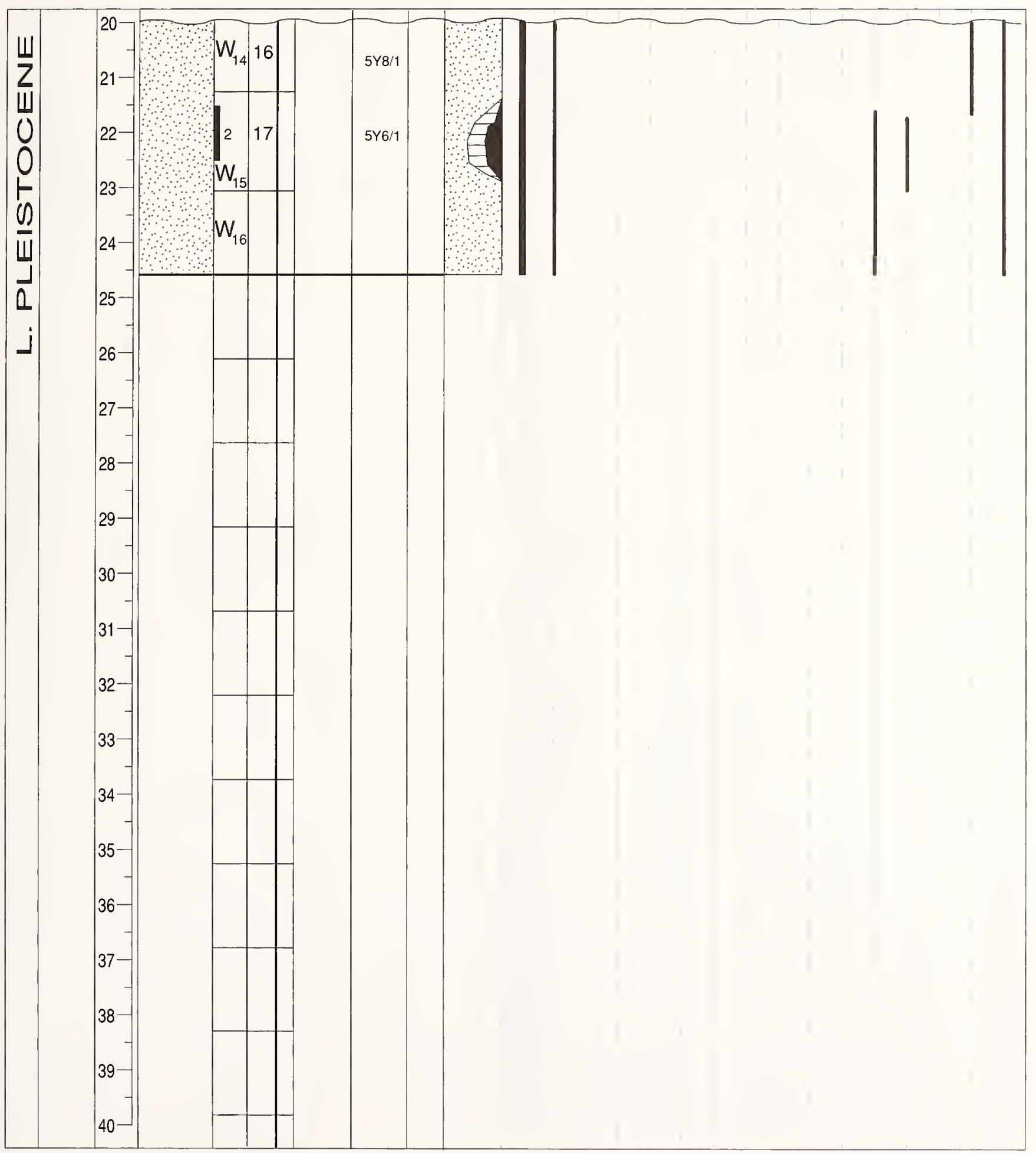


APPENDIX 1.-Continued.

CORE NUMBER S111

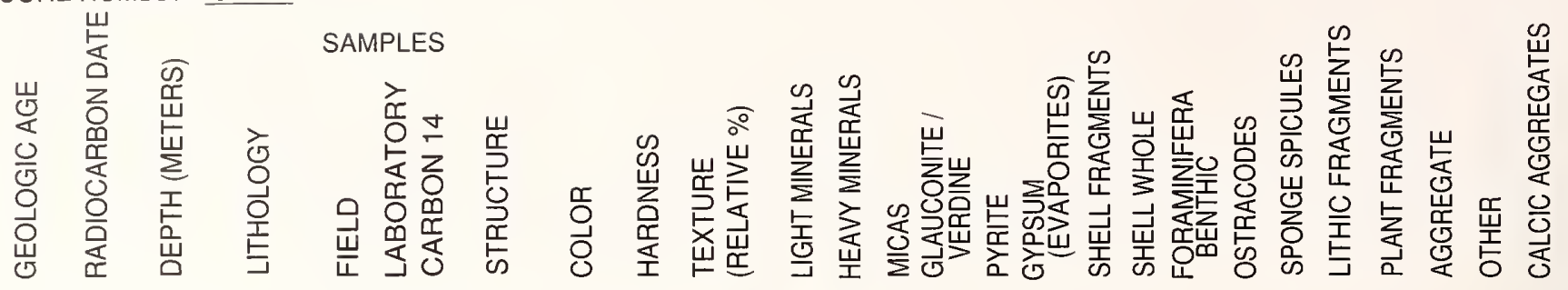

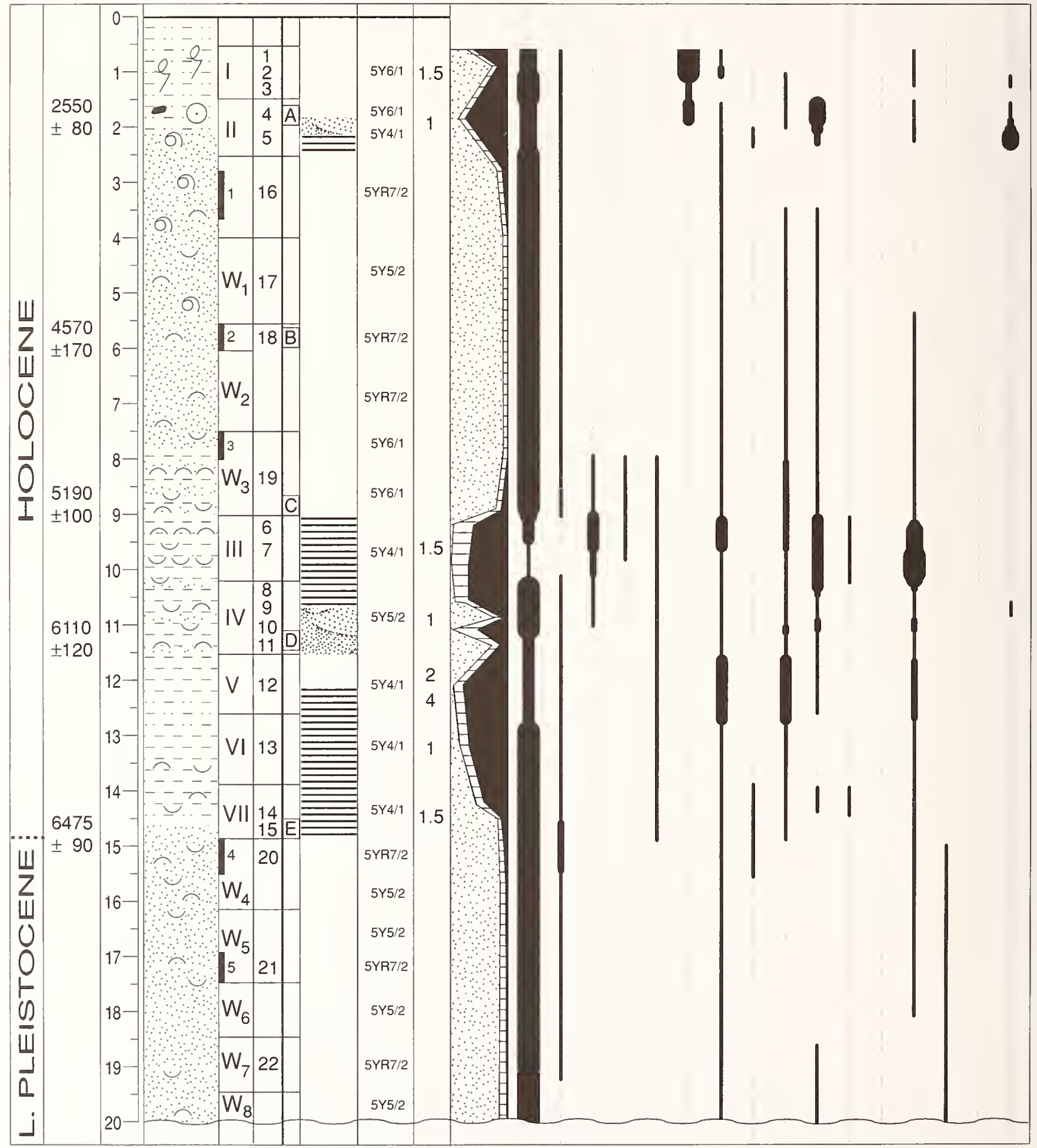


APPENDIX 1.-Continued.

CORE NUMBER S11II

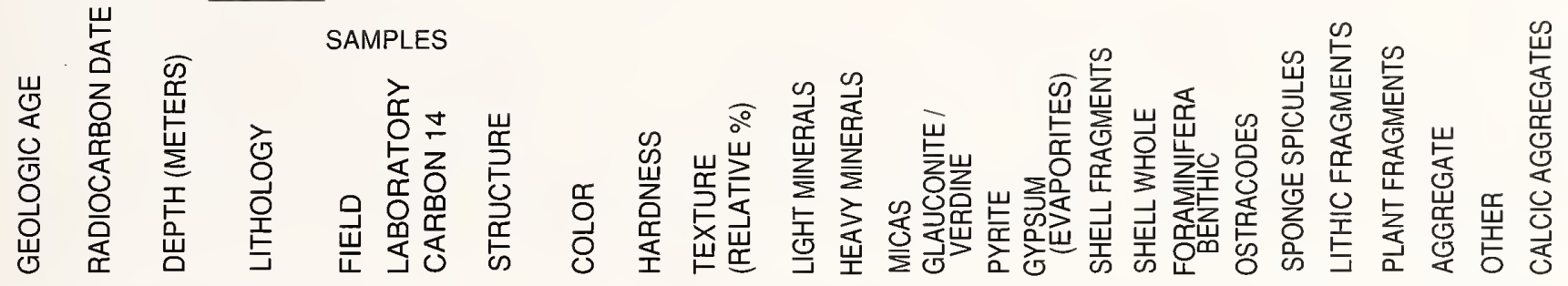

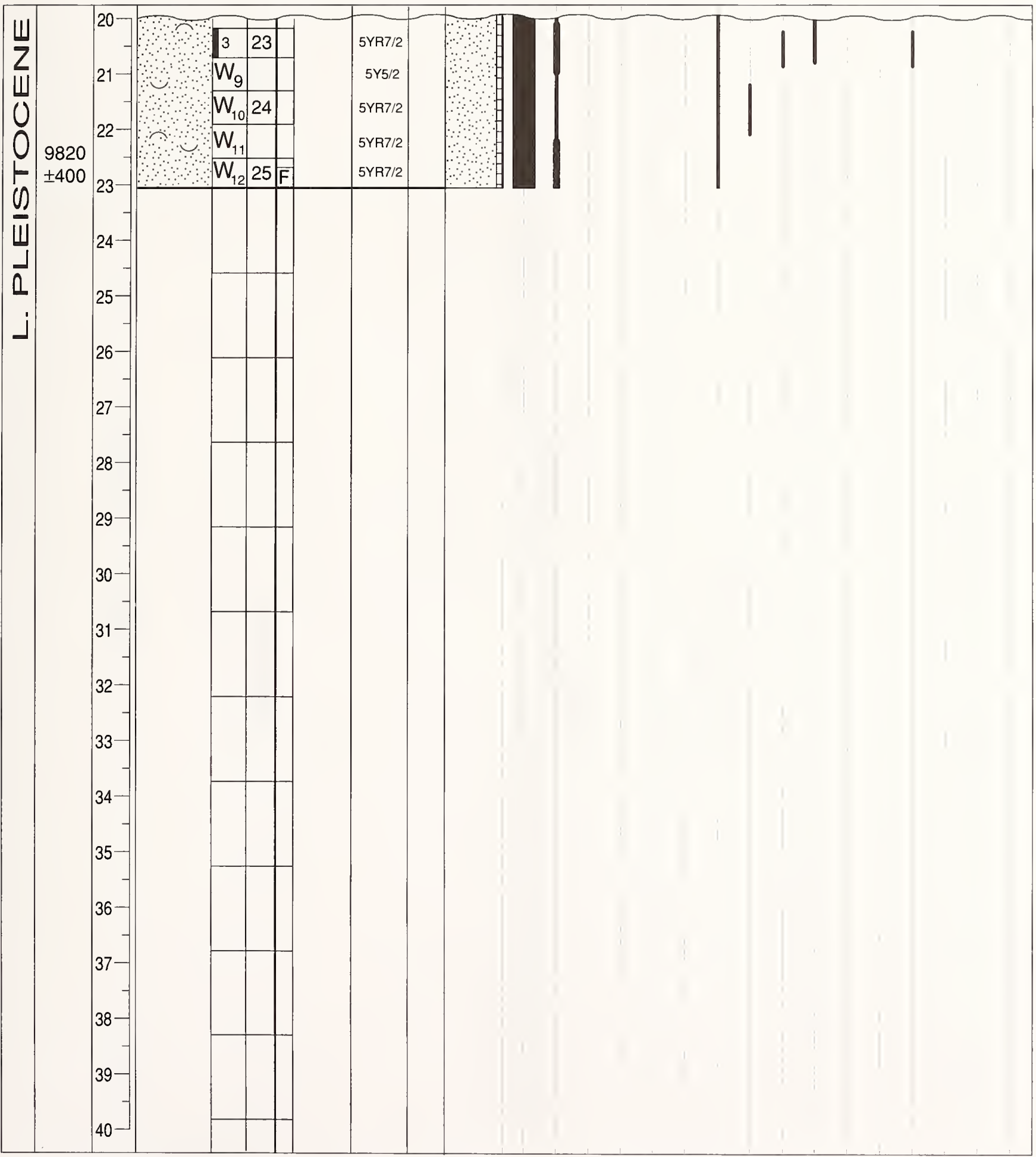


APPENDIX 1.-Continued.

CORE NUMBER S12I
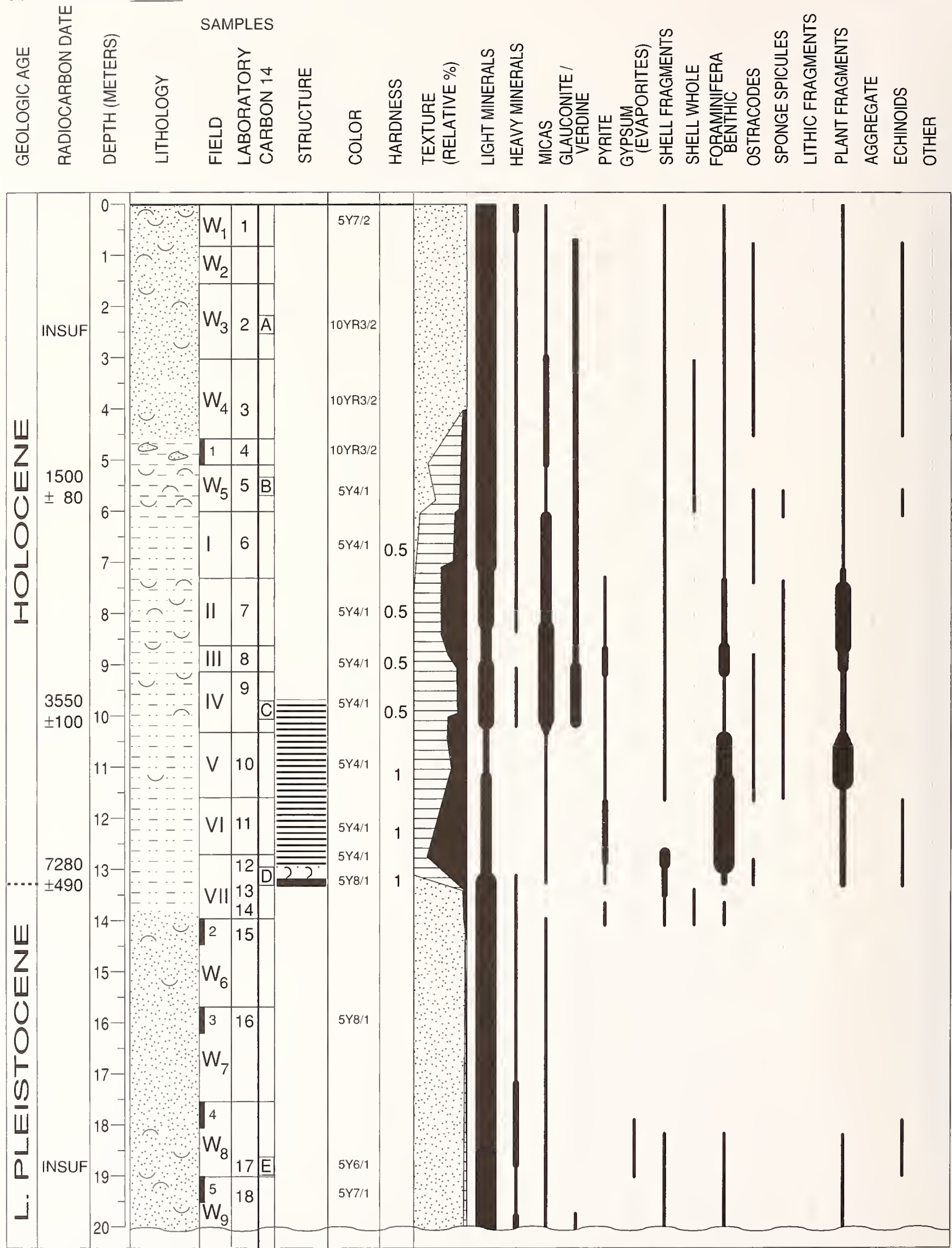
APPENDIX 1.-Continued.

CORE NUMBER S12 II

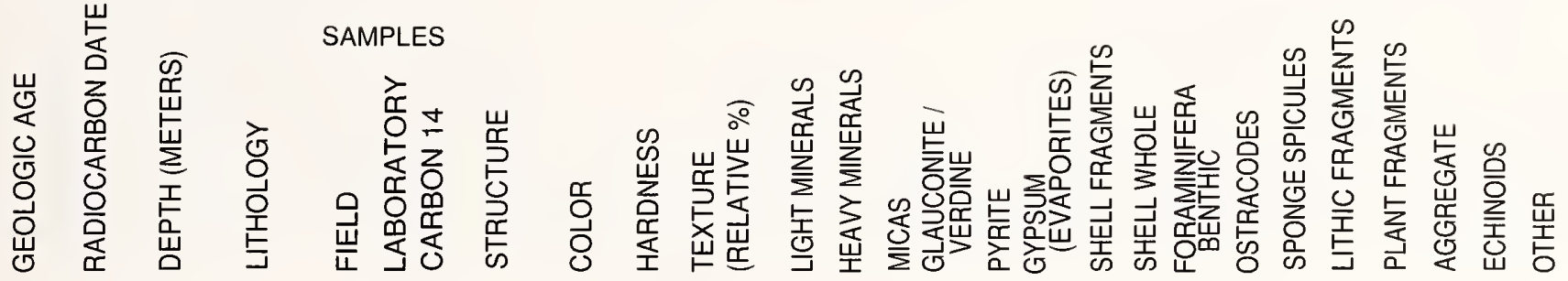

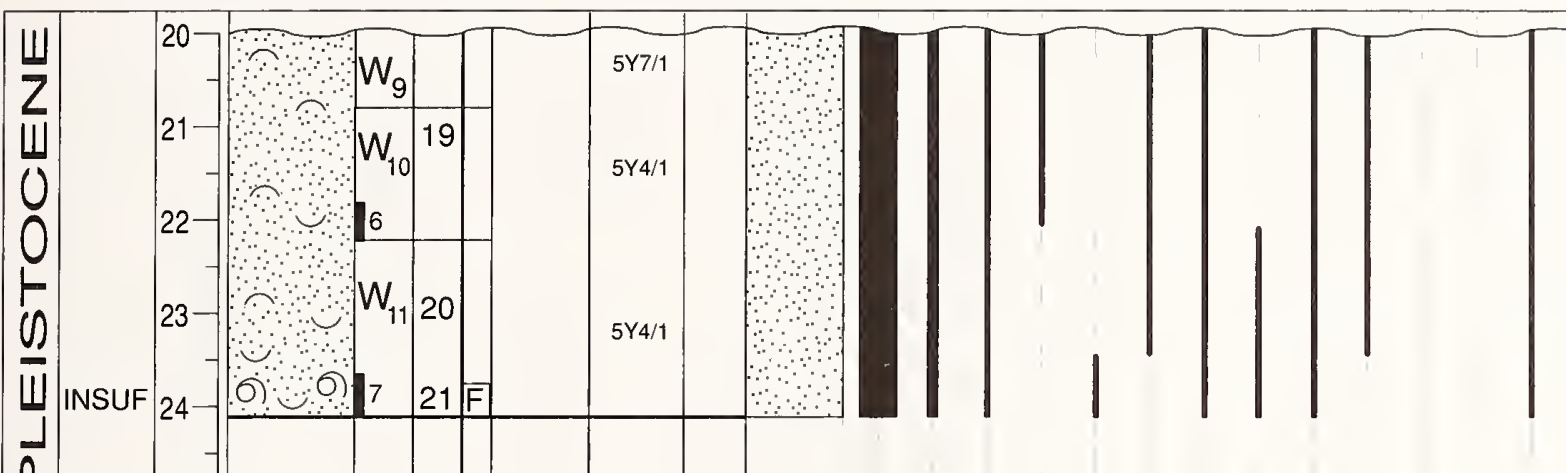


APPENDIX 1.-Continued.

CORE NUMBER S13I
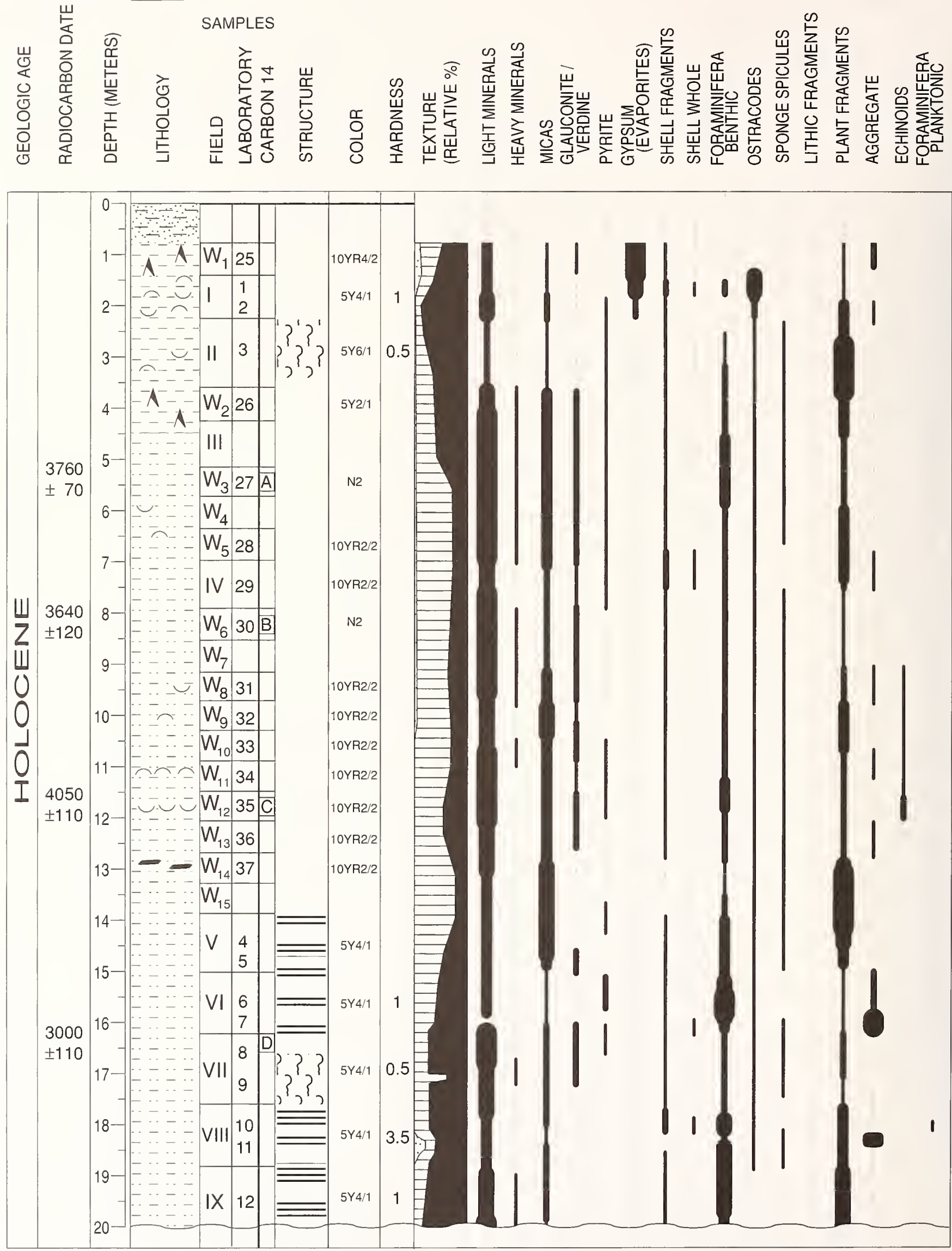
APPENDIX 1.-Continued.

CORE NUMBER S13 II
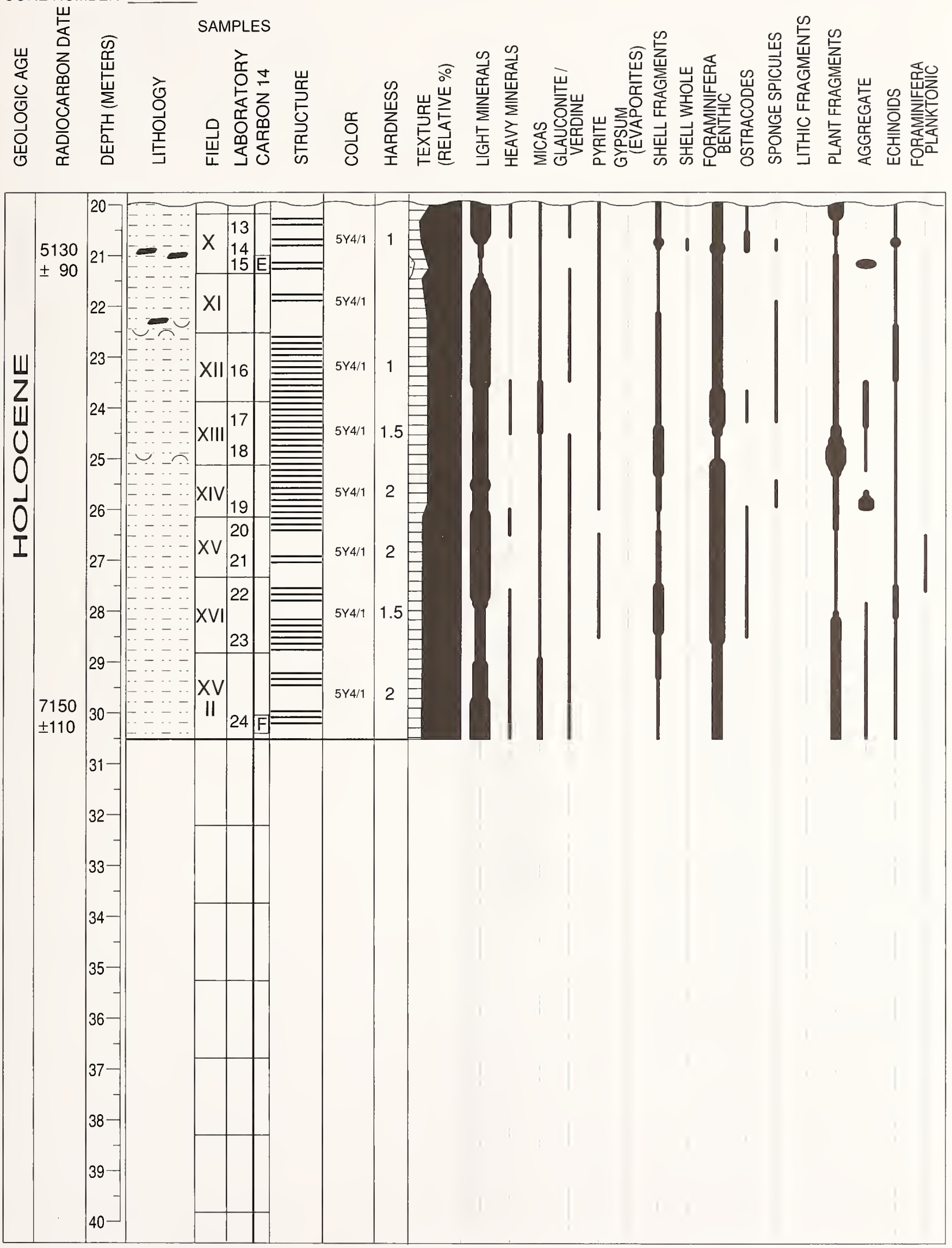
APPENDIX 1.-Continued.

CORE NUMBER S14I
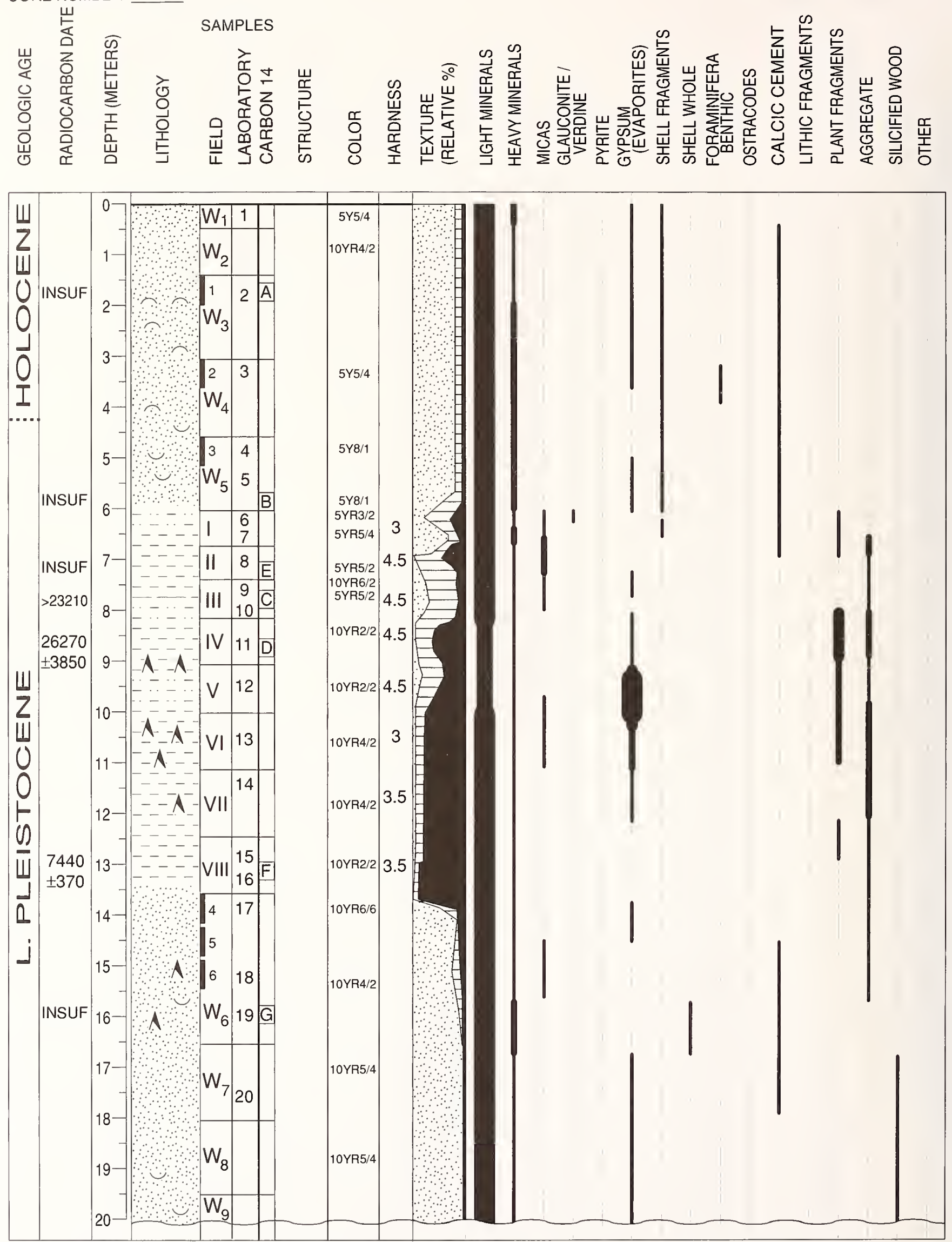
APPENDIX 1.-Continued.

CORE NUMBER S14 II

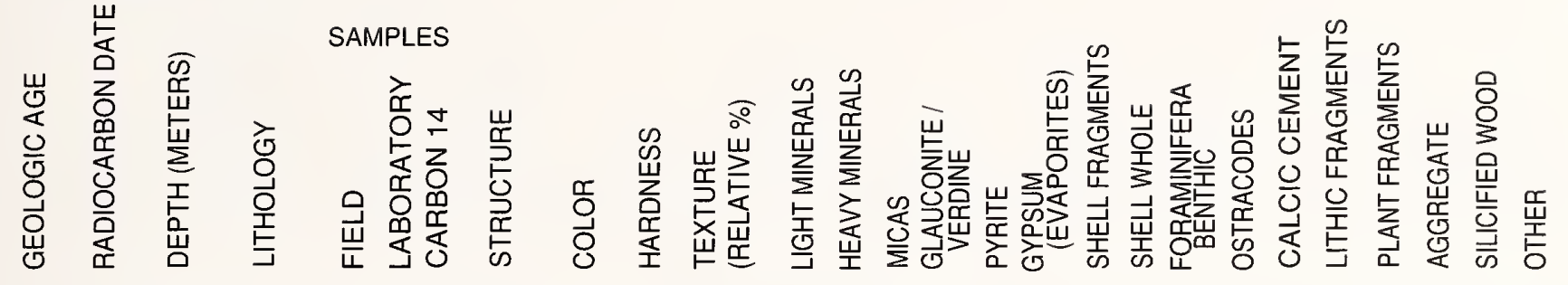

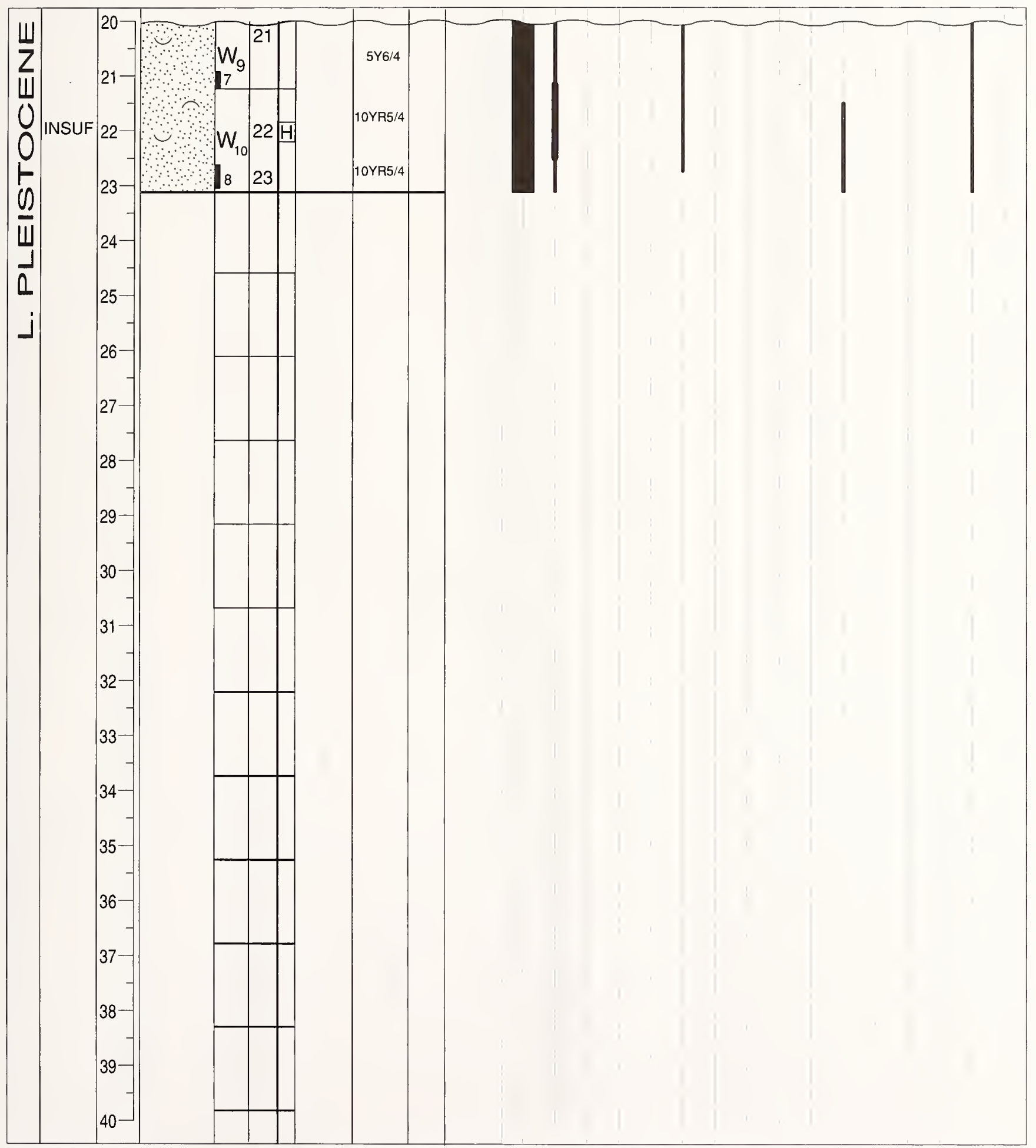


APPENDIX 1.-Continued.

CORE NUMBER S15I
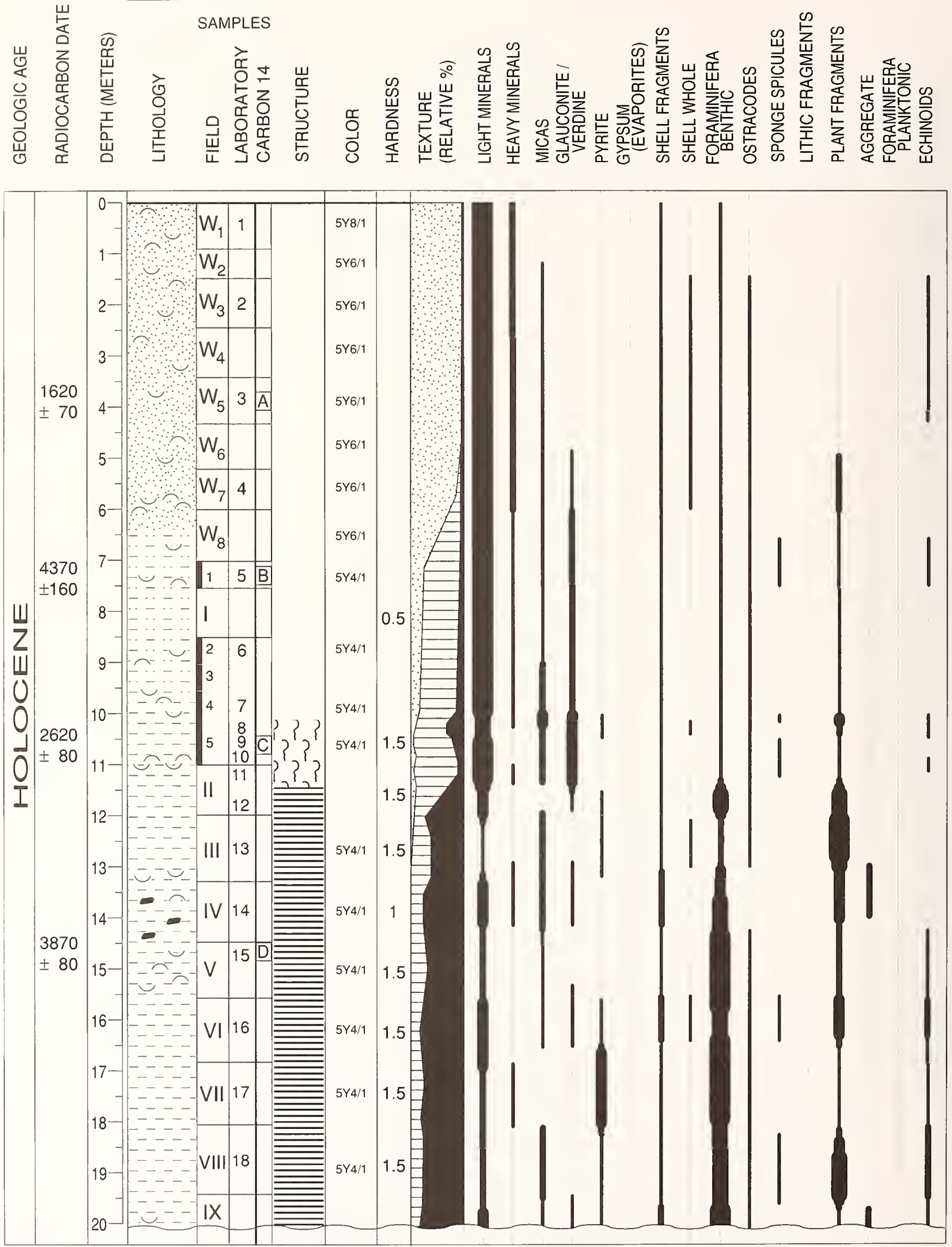
APPENDIX 1.-Continued.

CORE NUMBER S15 II
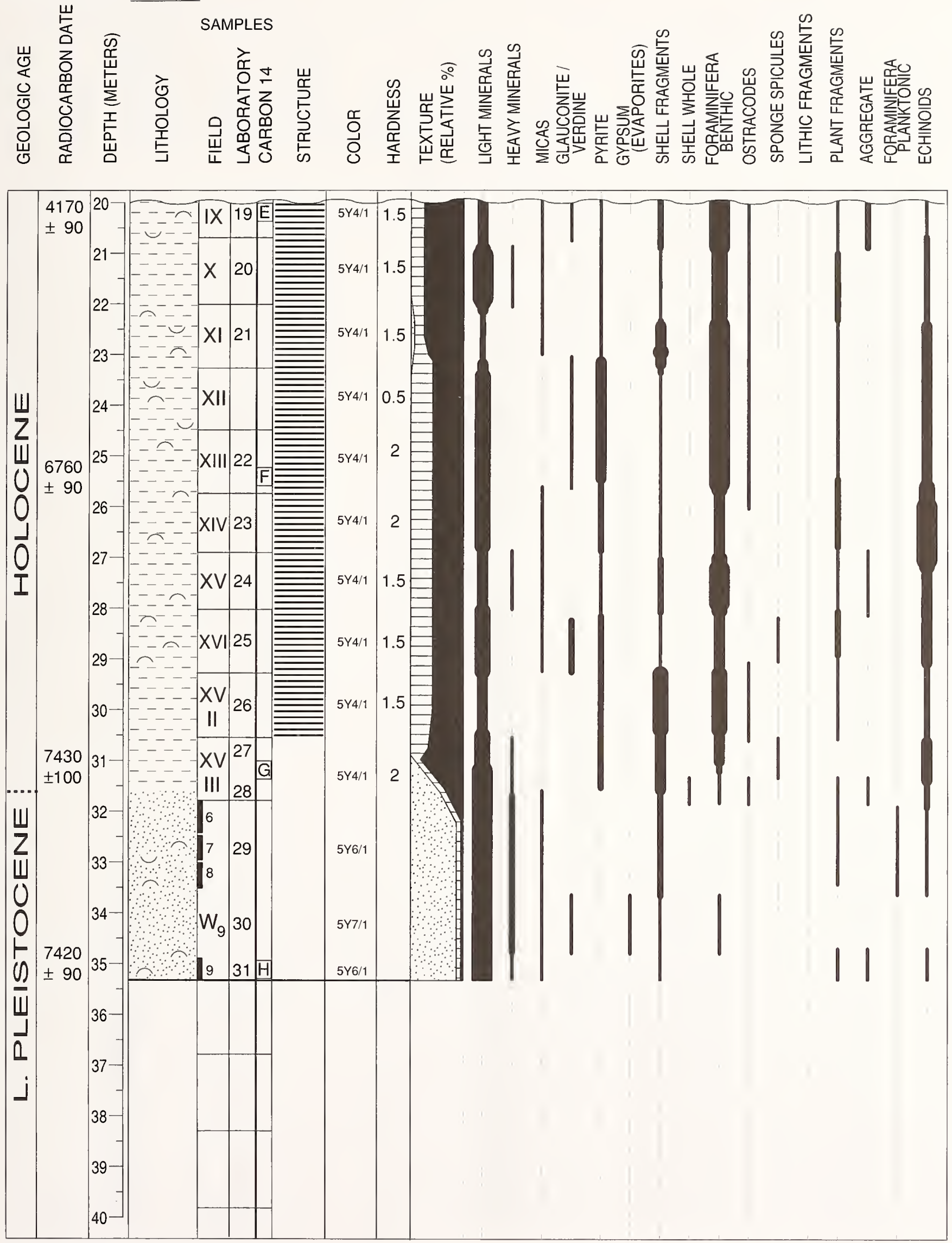
APPENDIX 1.-Continued.

CORE NUMBER S16I
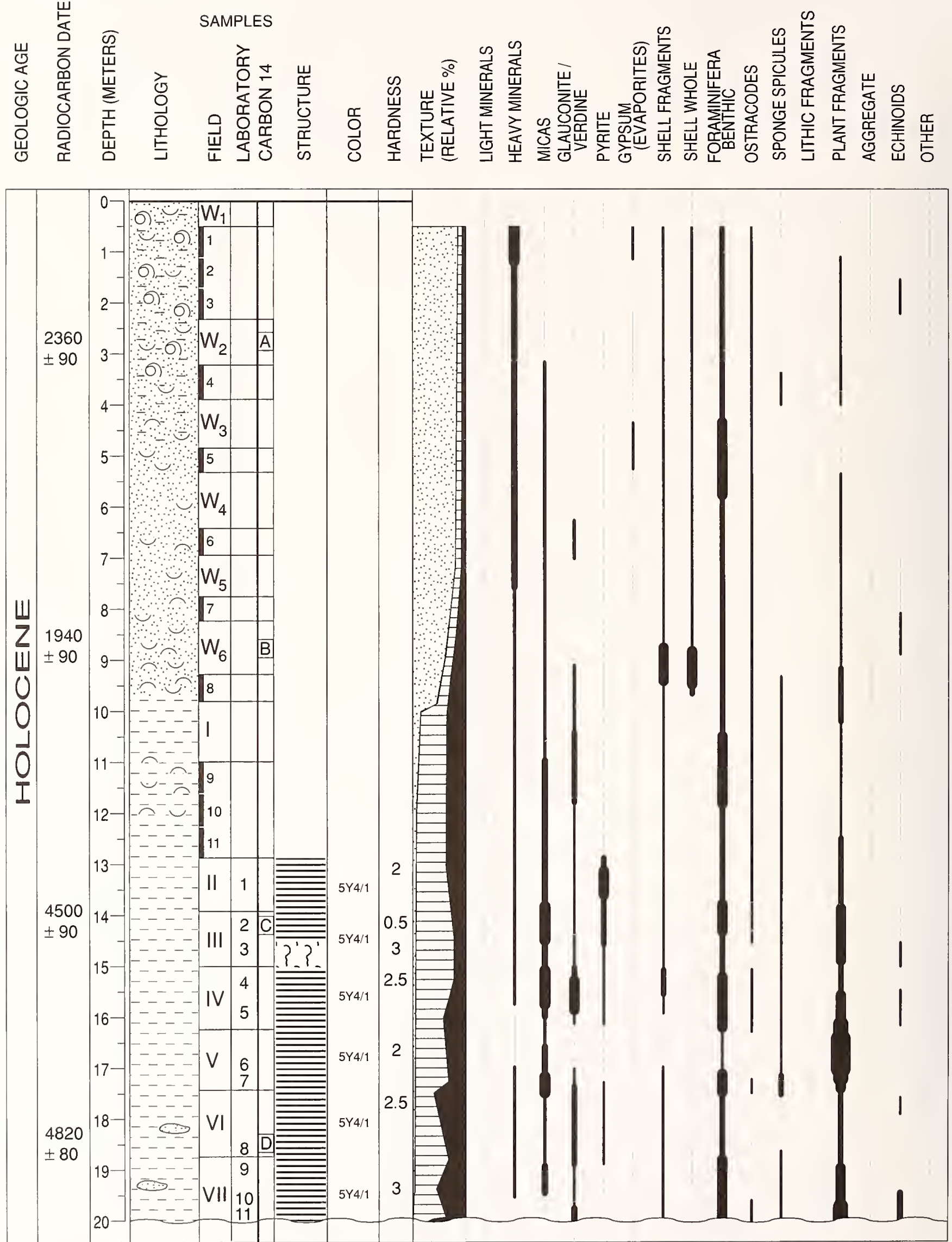
APPENDIX 1.-Continued.

CORE NUMBER S16 II

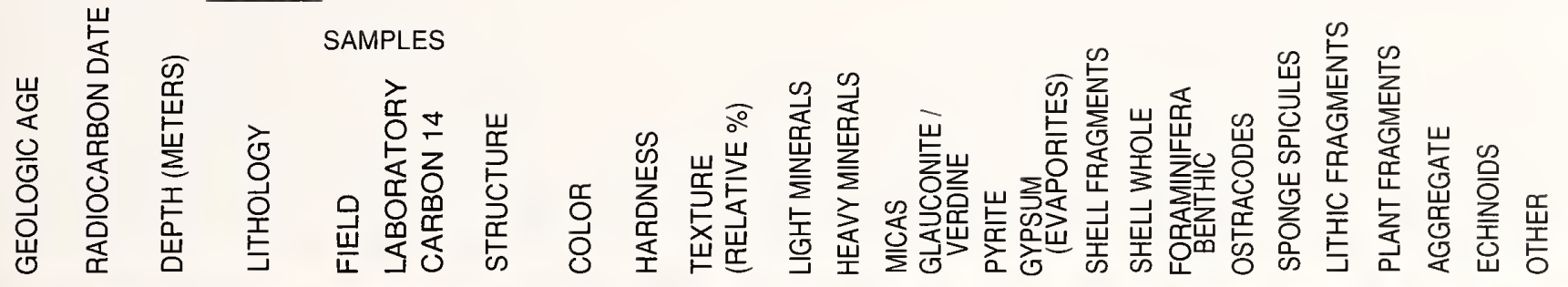

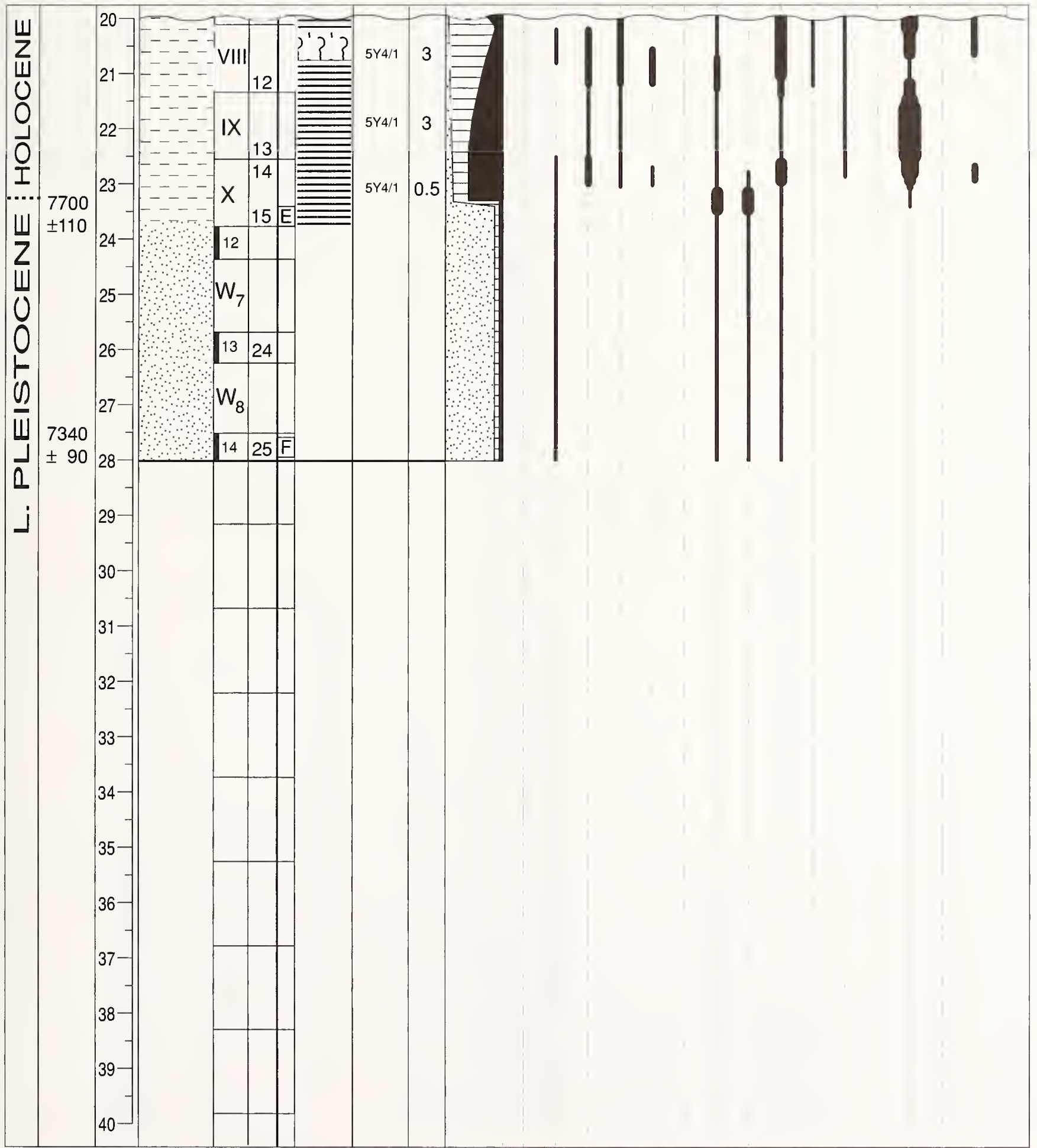


APPENDIX 1.-Continued.

CORE NUMBER S17I
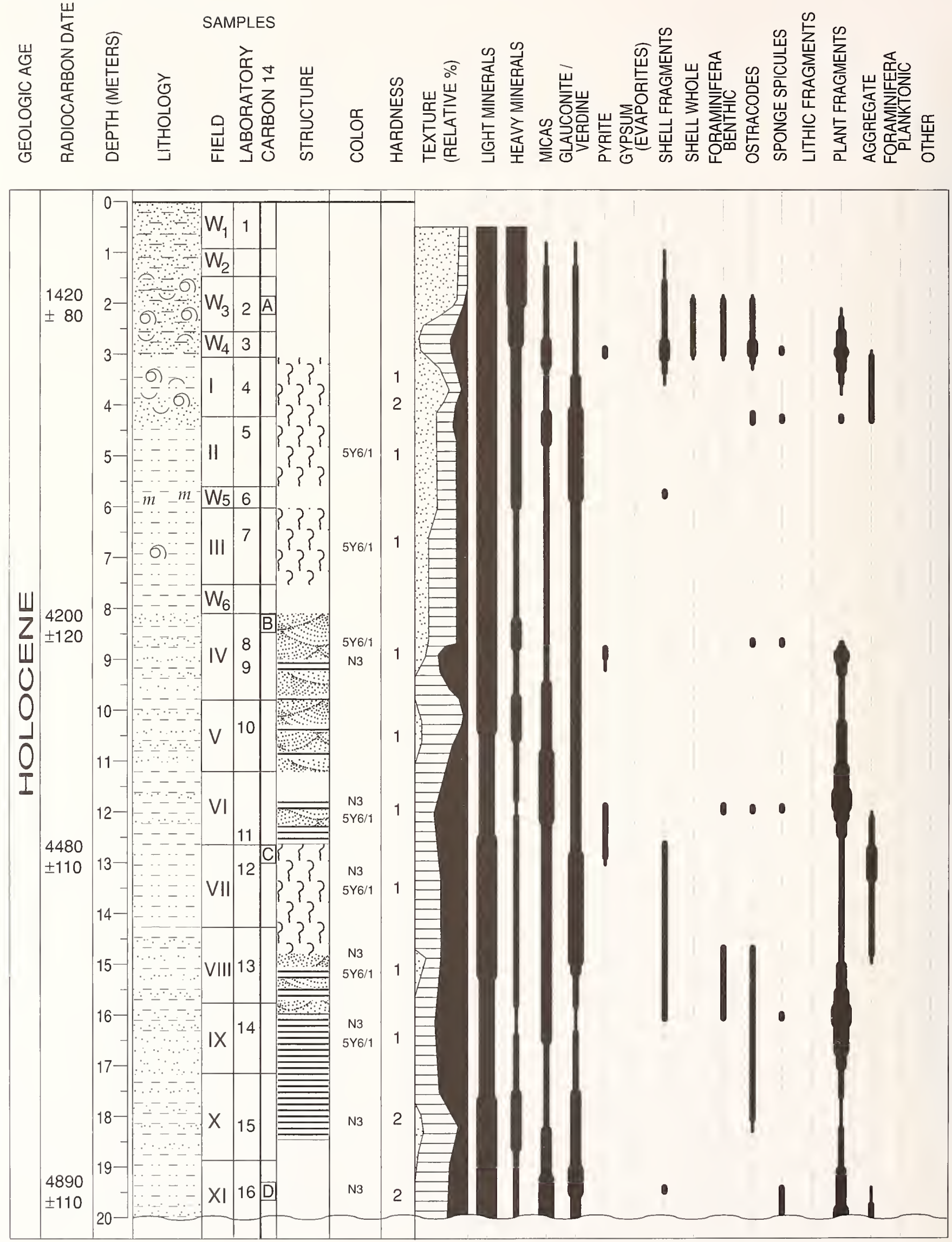
APPENDIX 1.-Continued.

CORE NUMBER S17॥

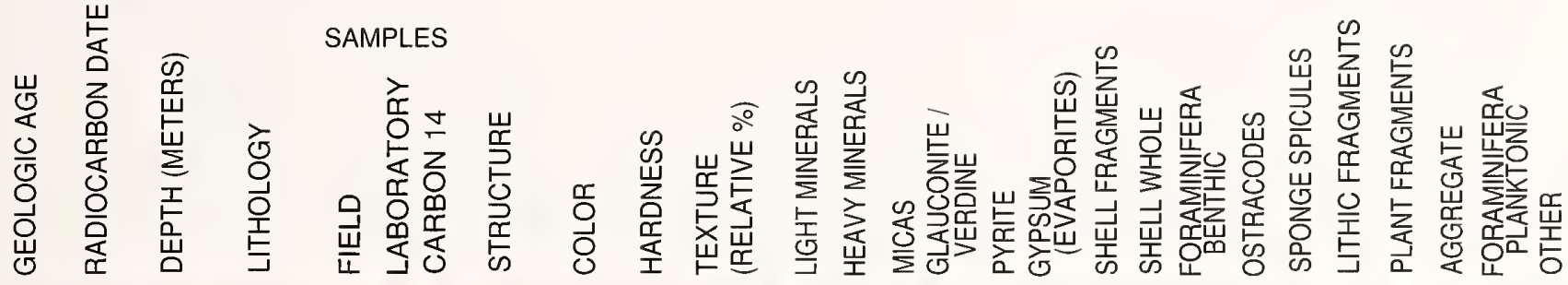

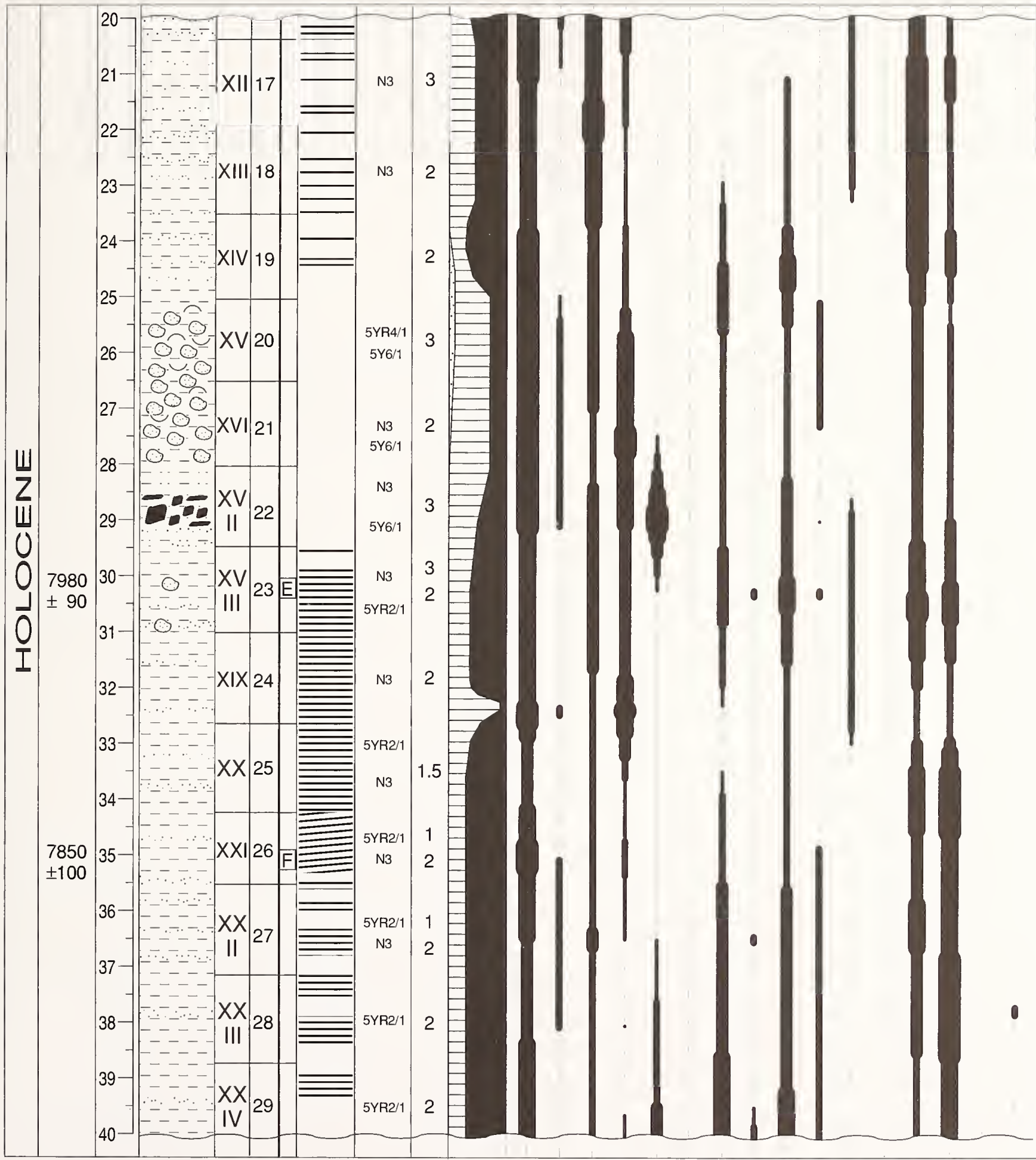


APPENDIX 1.-Continued.

CORE NUMBER S17 III

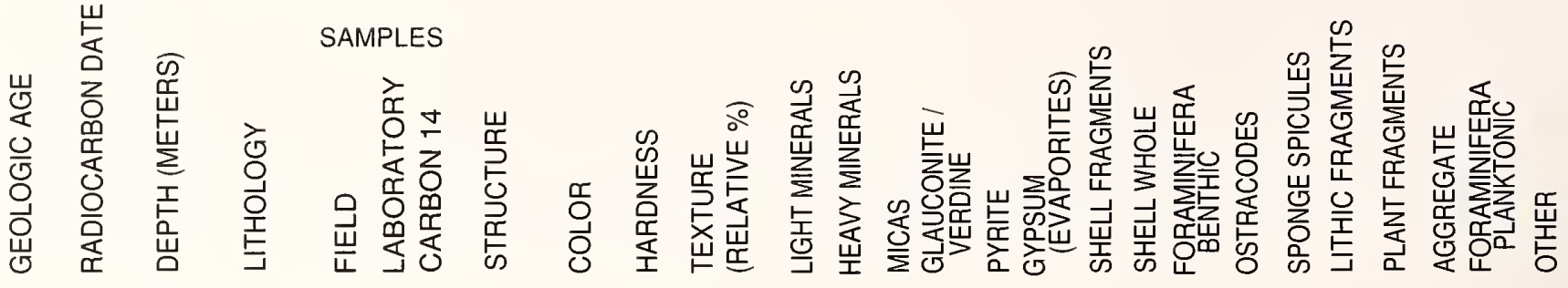

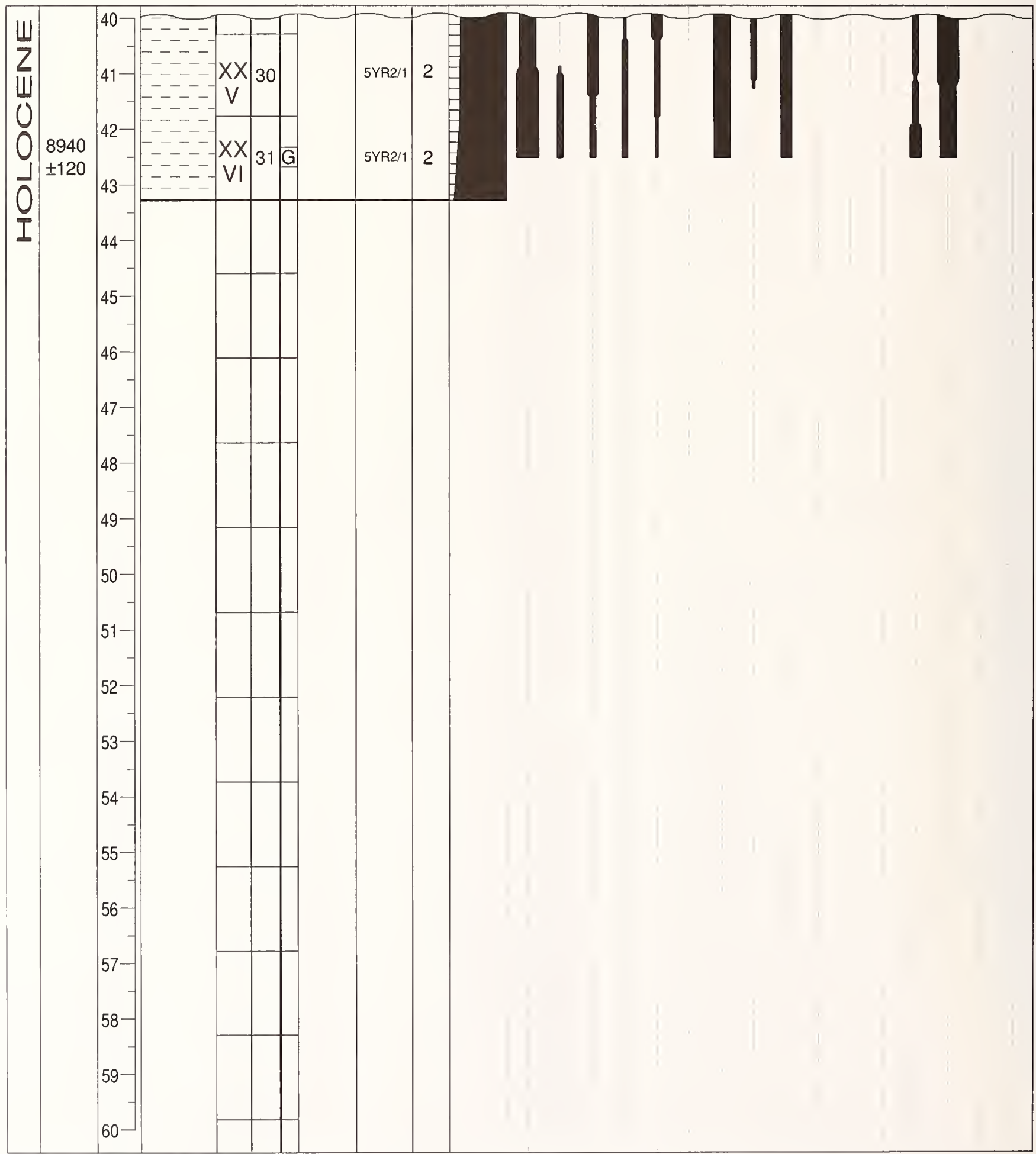


APPENDIX 1.-Continued.

CORE NUMBER S18I
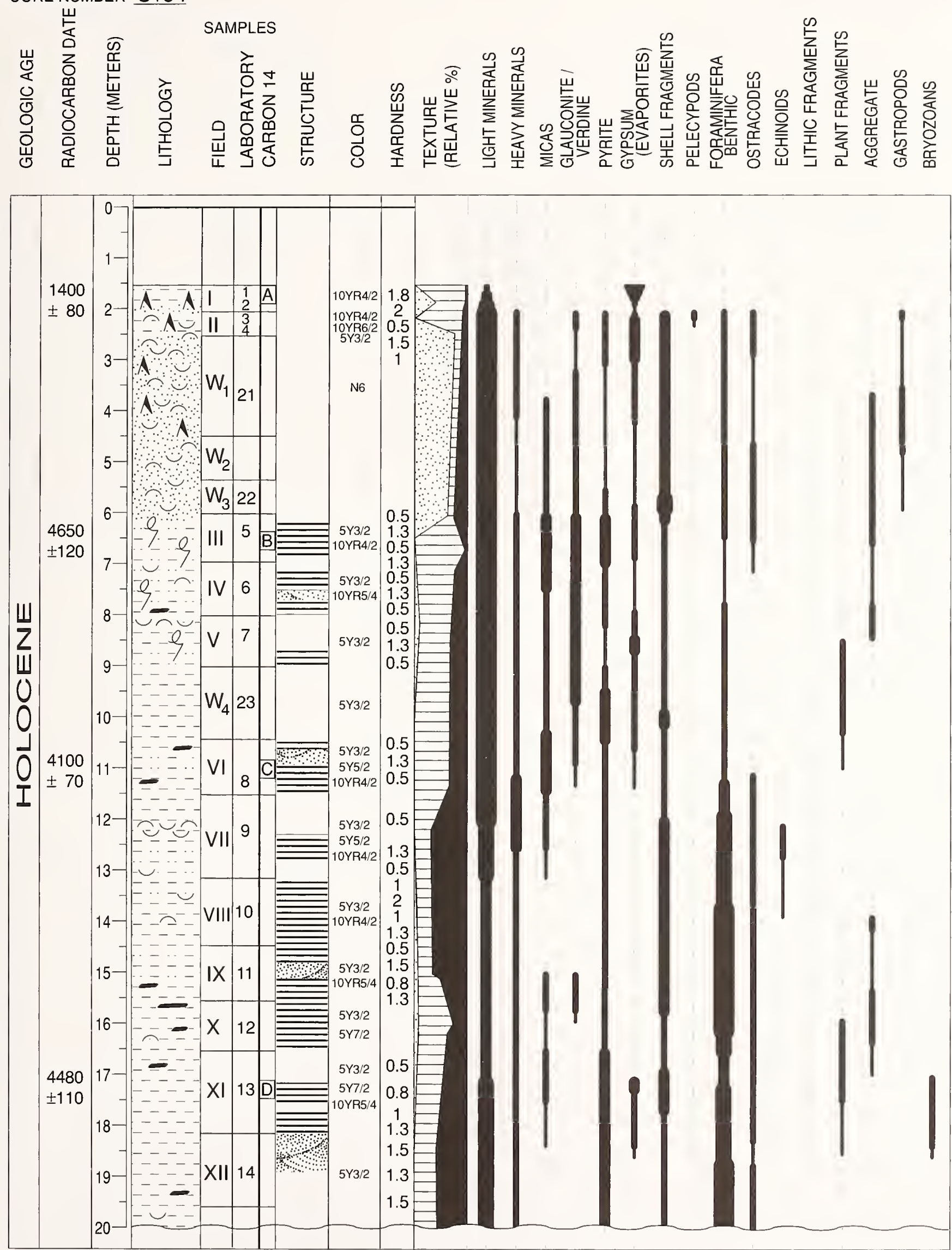
APPENDIX 1.-Continued.
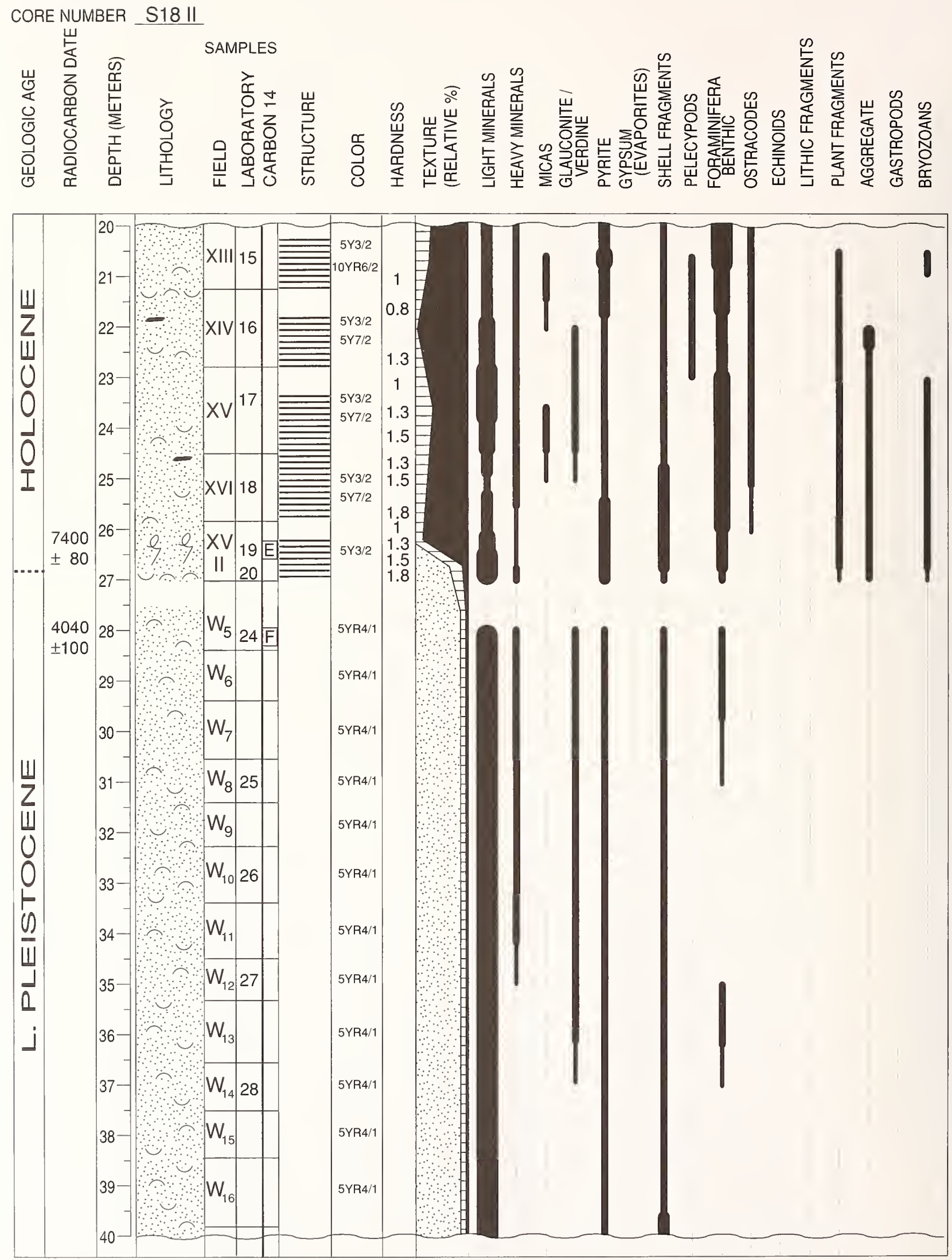
APPENDIX 1.-Continued.

CORE NUMBER S18 III
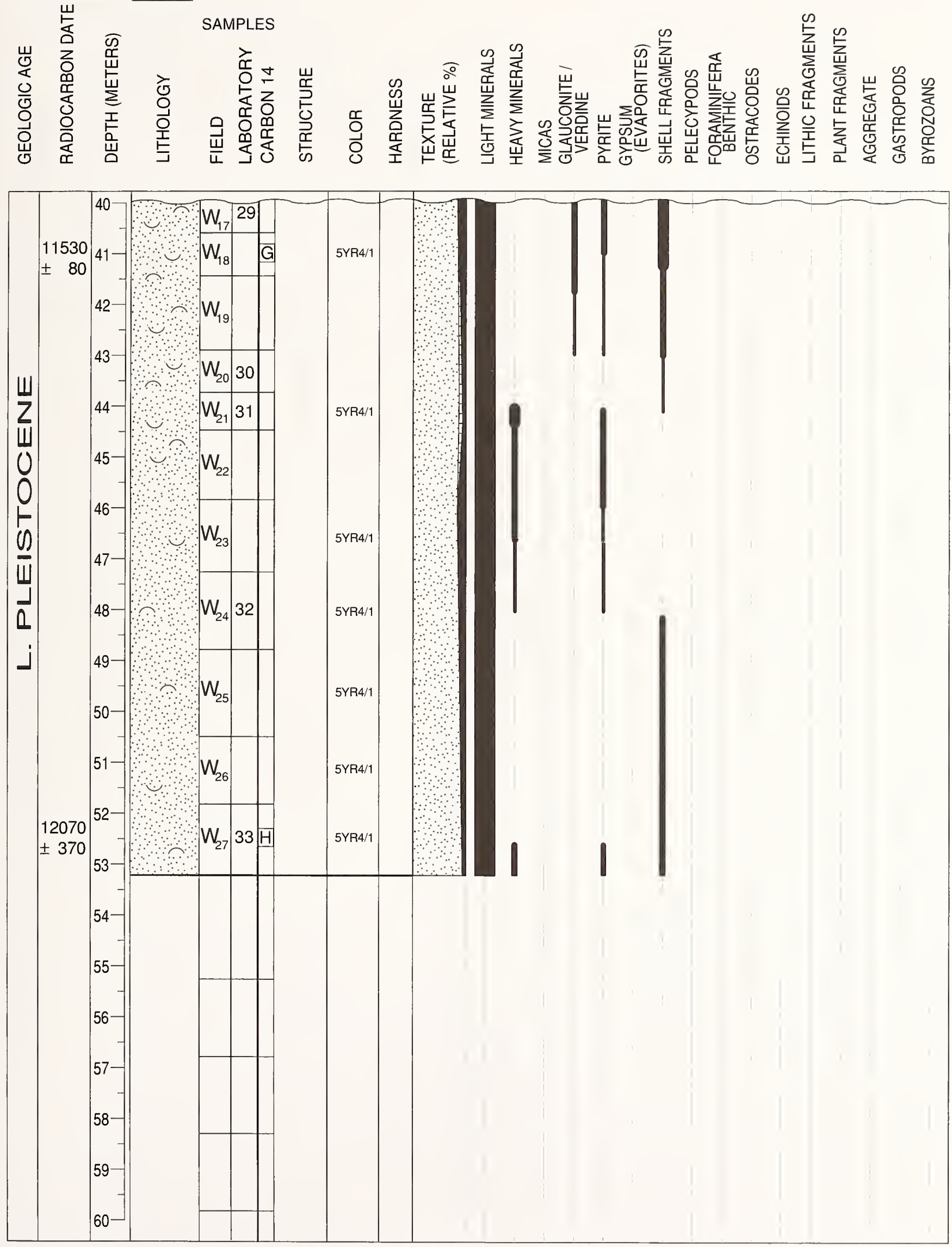
APPENDIX 1.-Continued.

CORE NUMBER S19

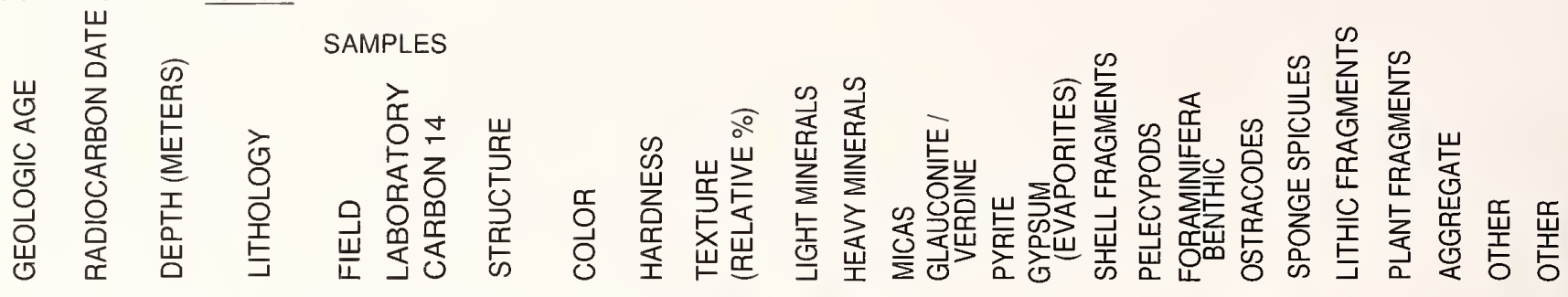

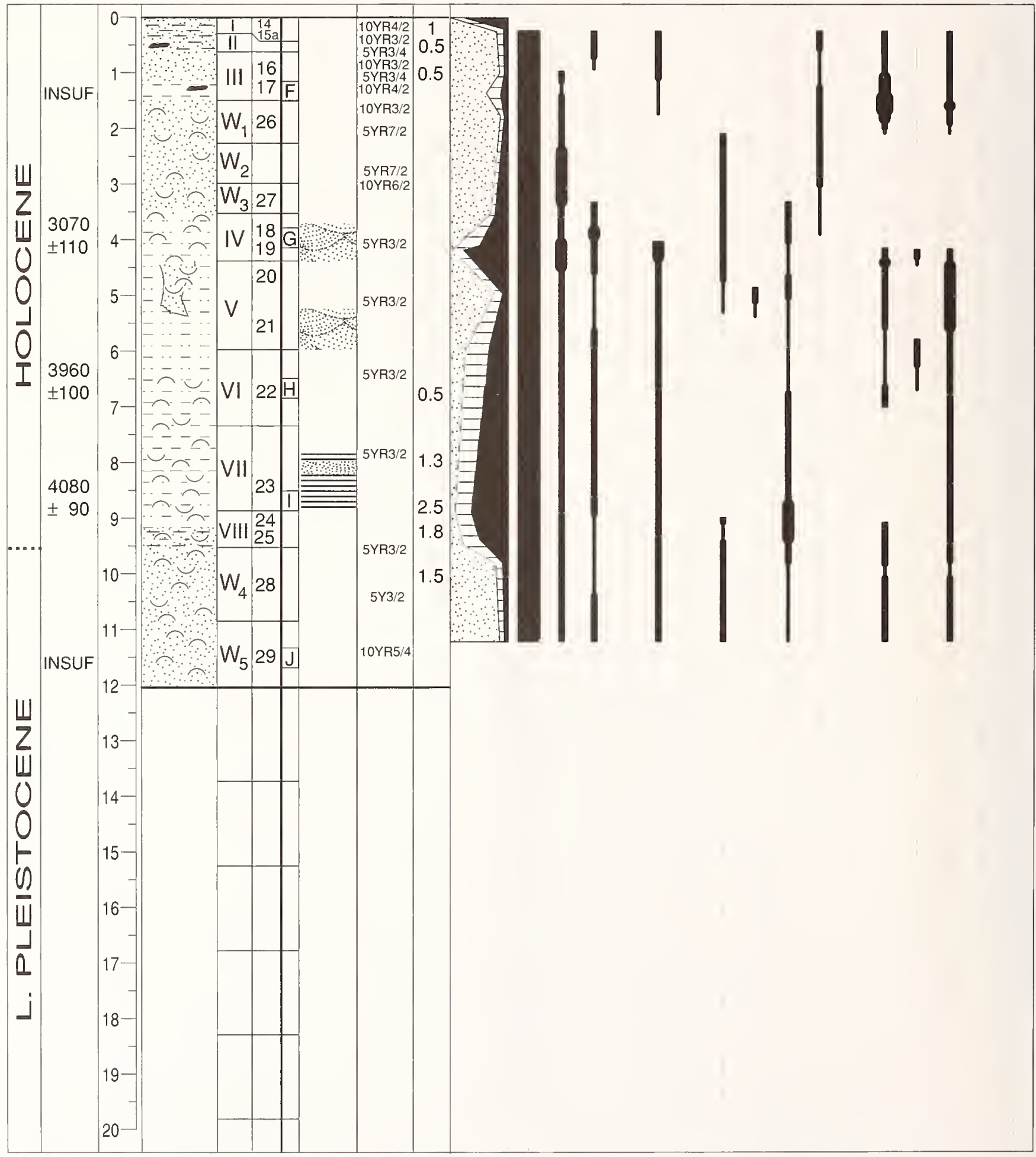


APPENDIX 1.-Continued.

CORE NUMBER S20 I
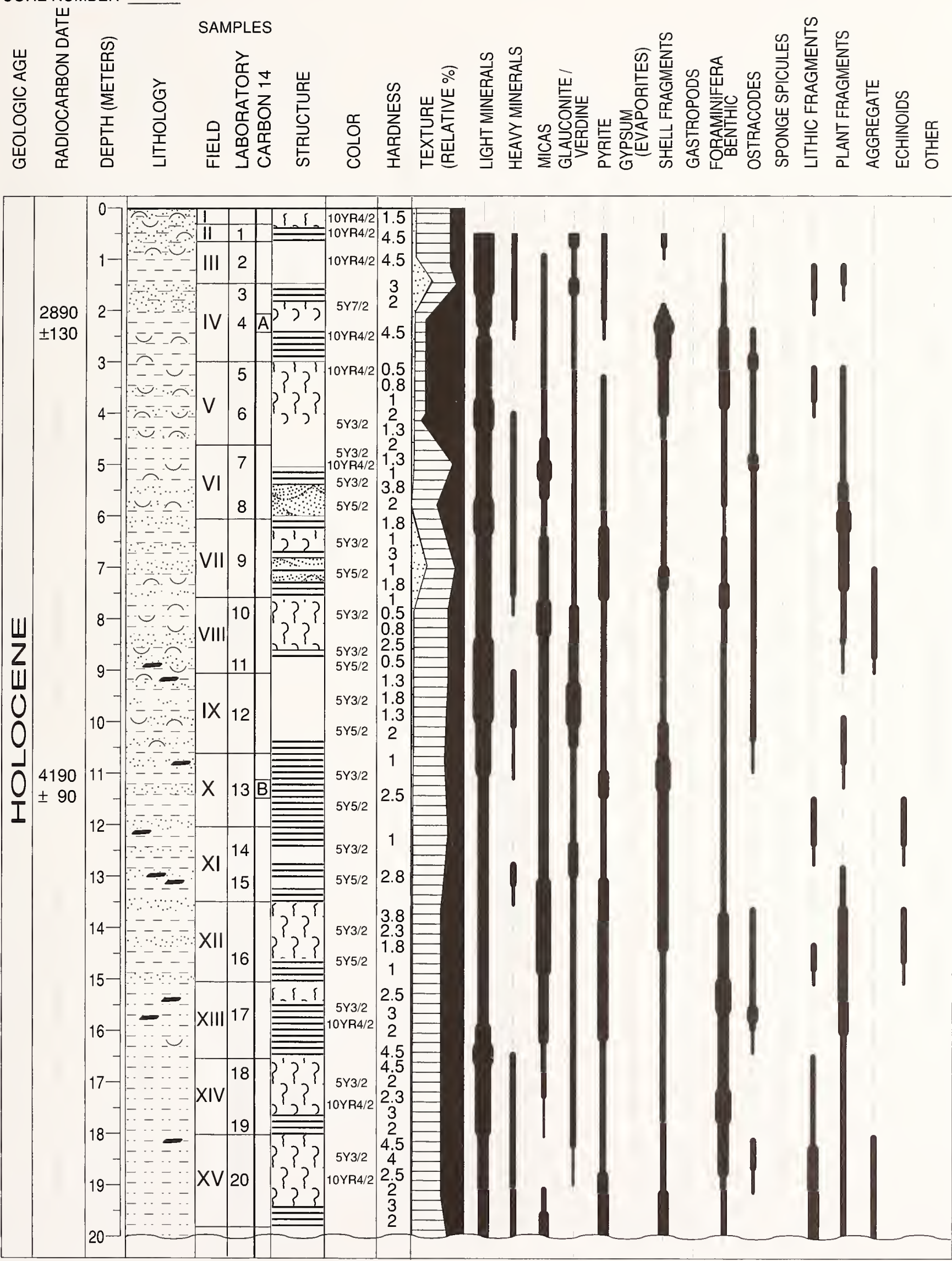
APPENDIX 1.-Continued.

CORE NUMBER S20 II
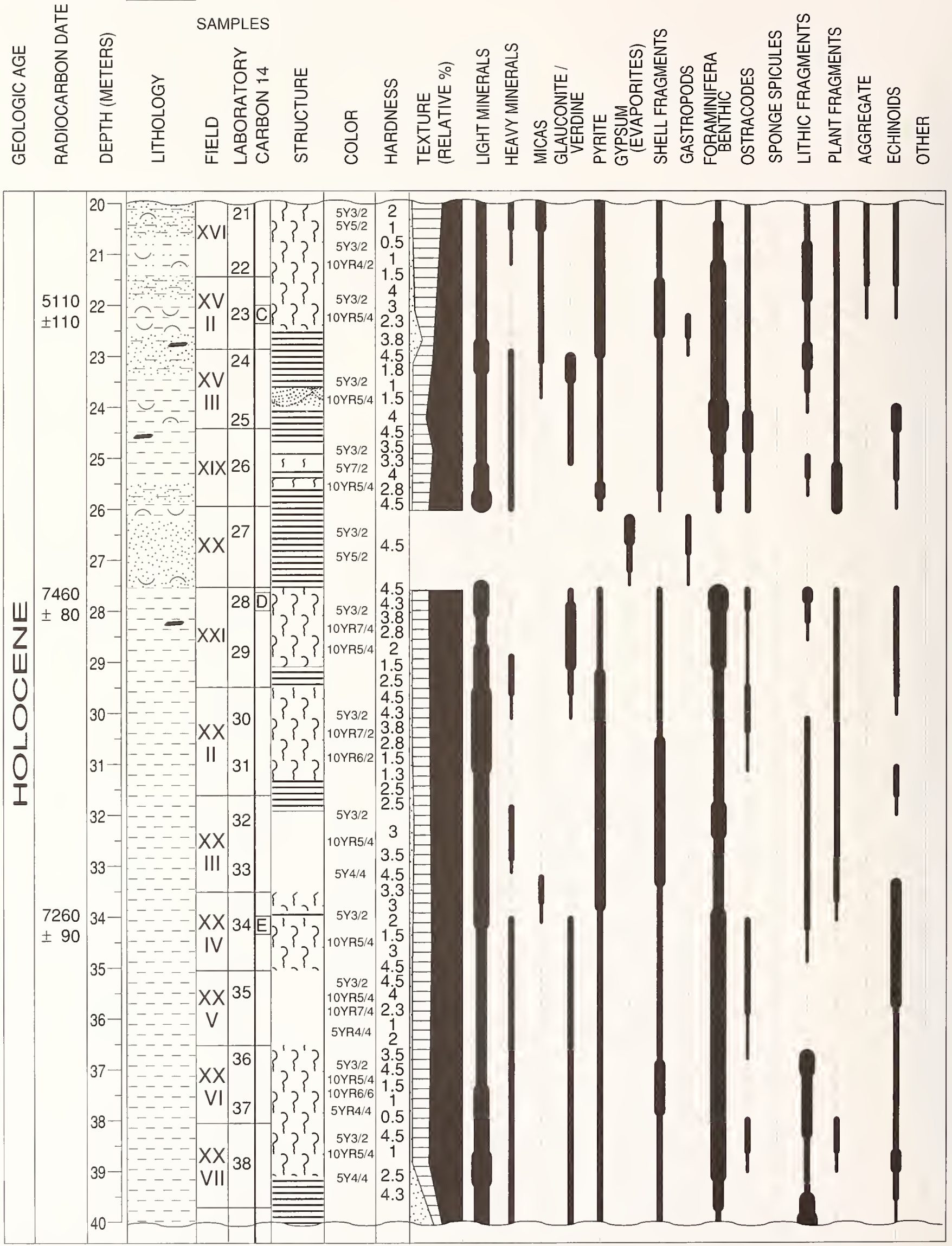
APPENDIX 1.-Continued.

CORE NUMBER S2O III

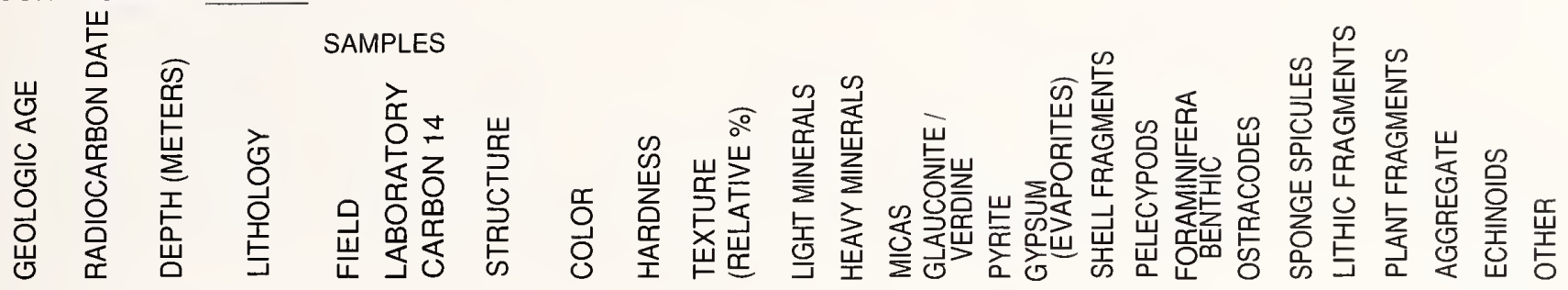

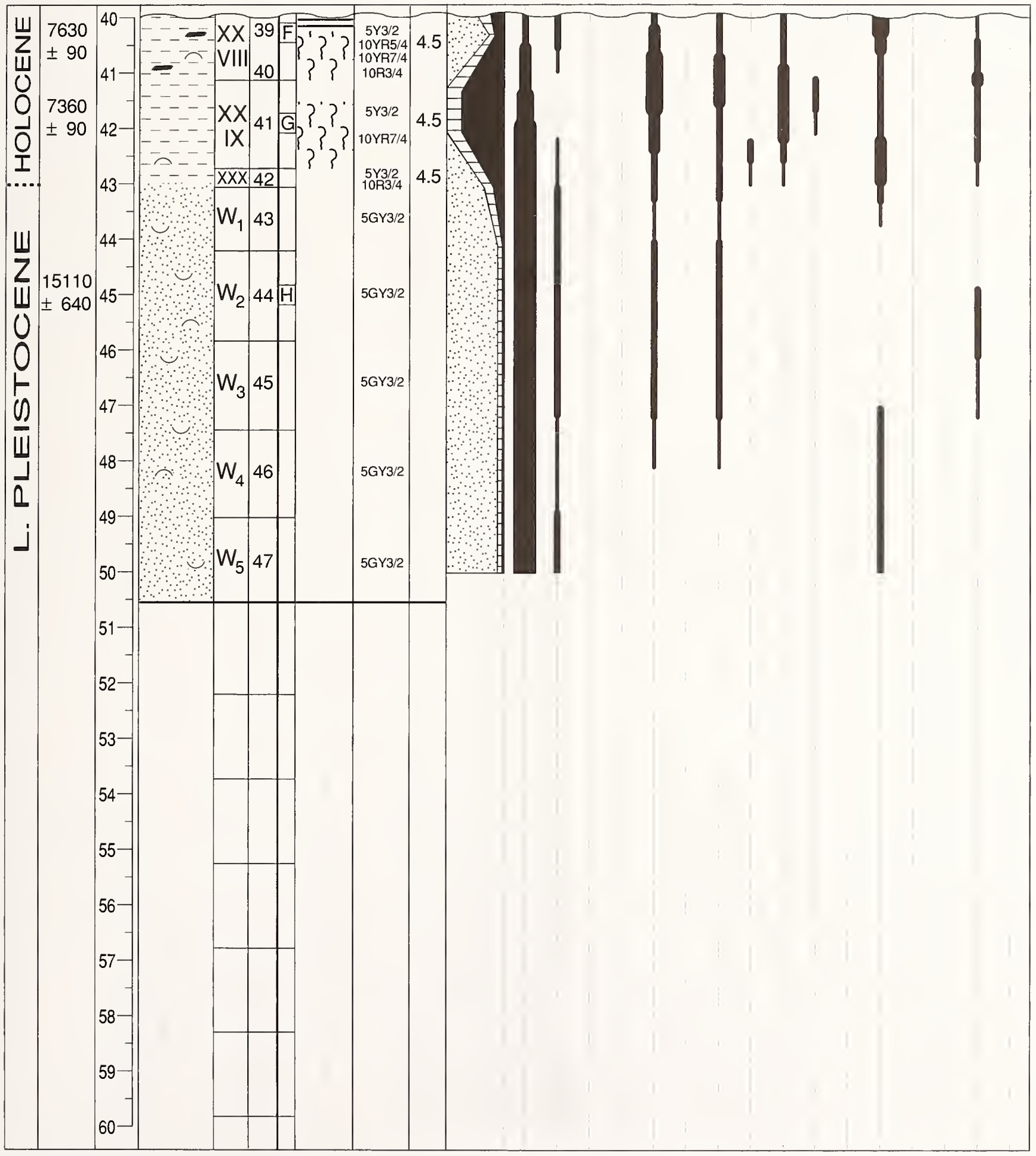


APPENDIX 1.-Continued.

CORE NUMBER S21I

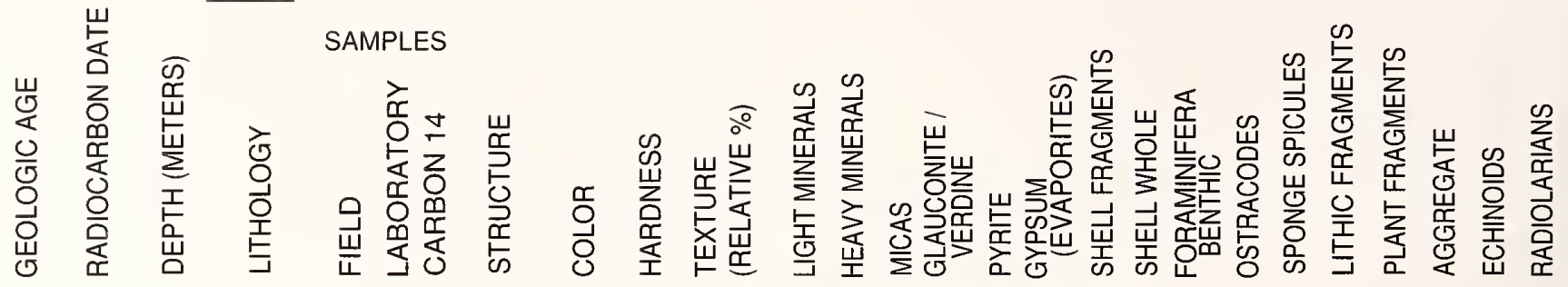

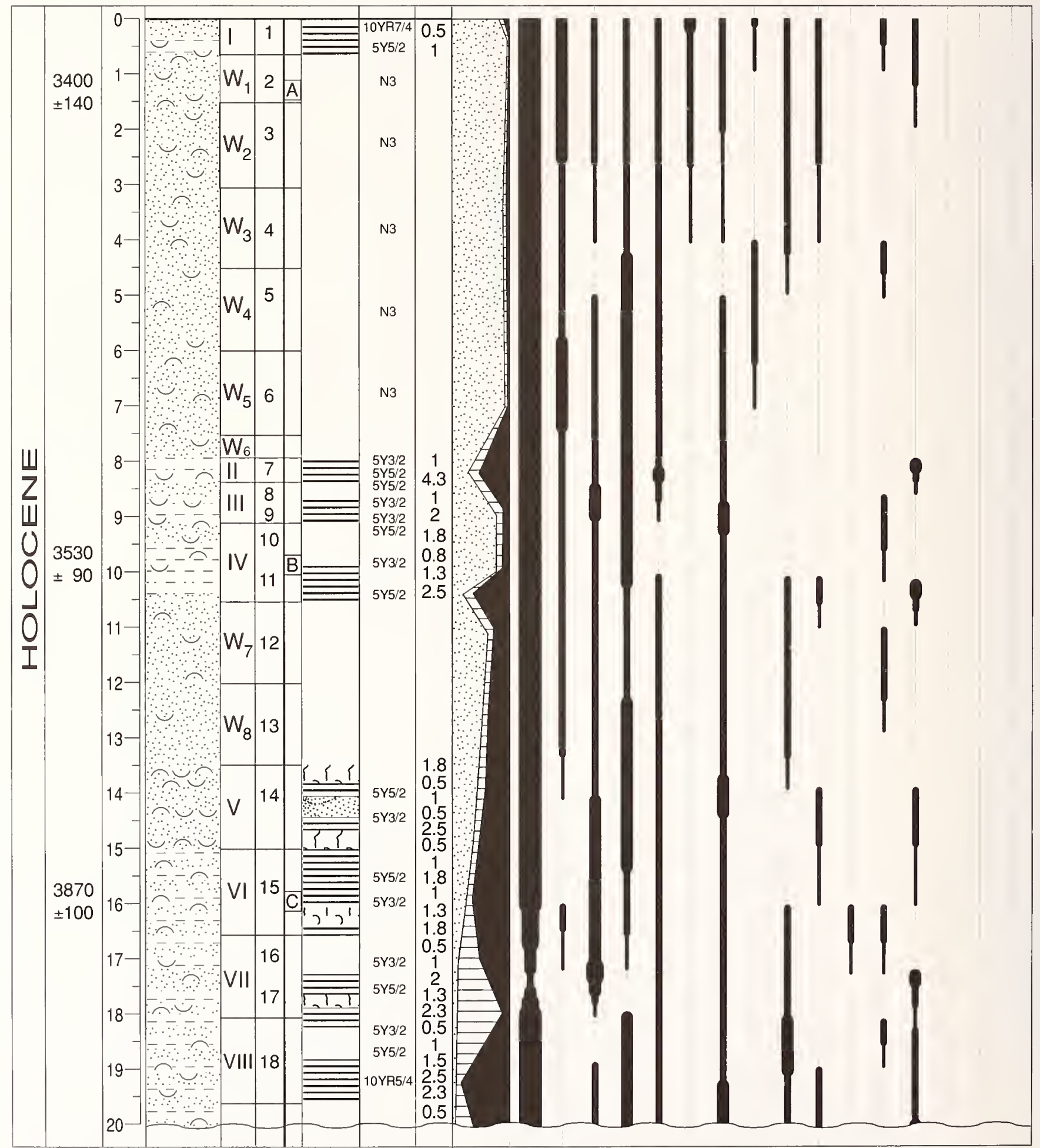


APPENDIX 1.-Continued.

CORE NUMBER S21 II
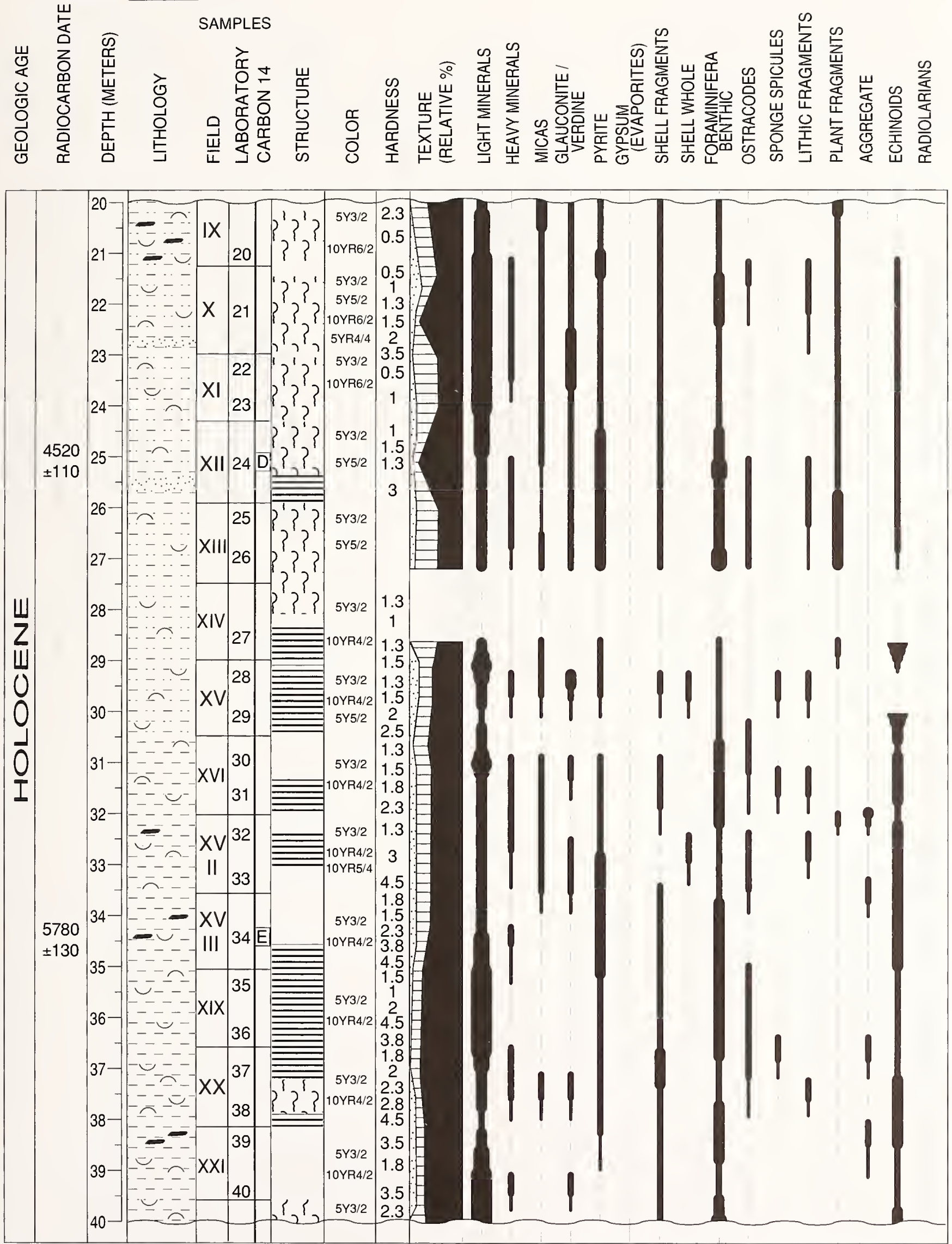
APPENDIX 1.-Continued.

CORE NUMBER S21 III
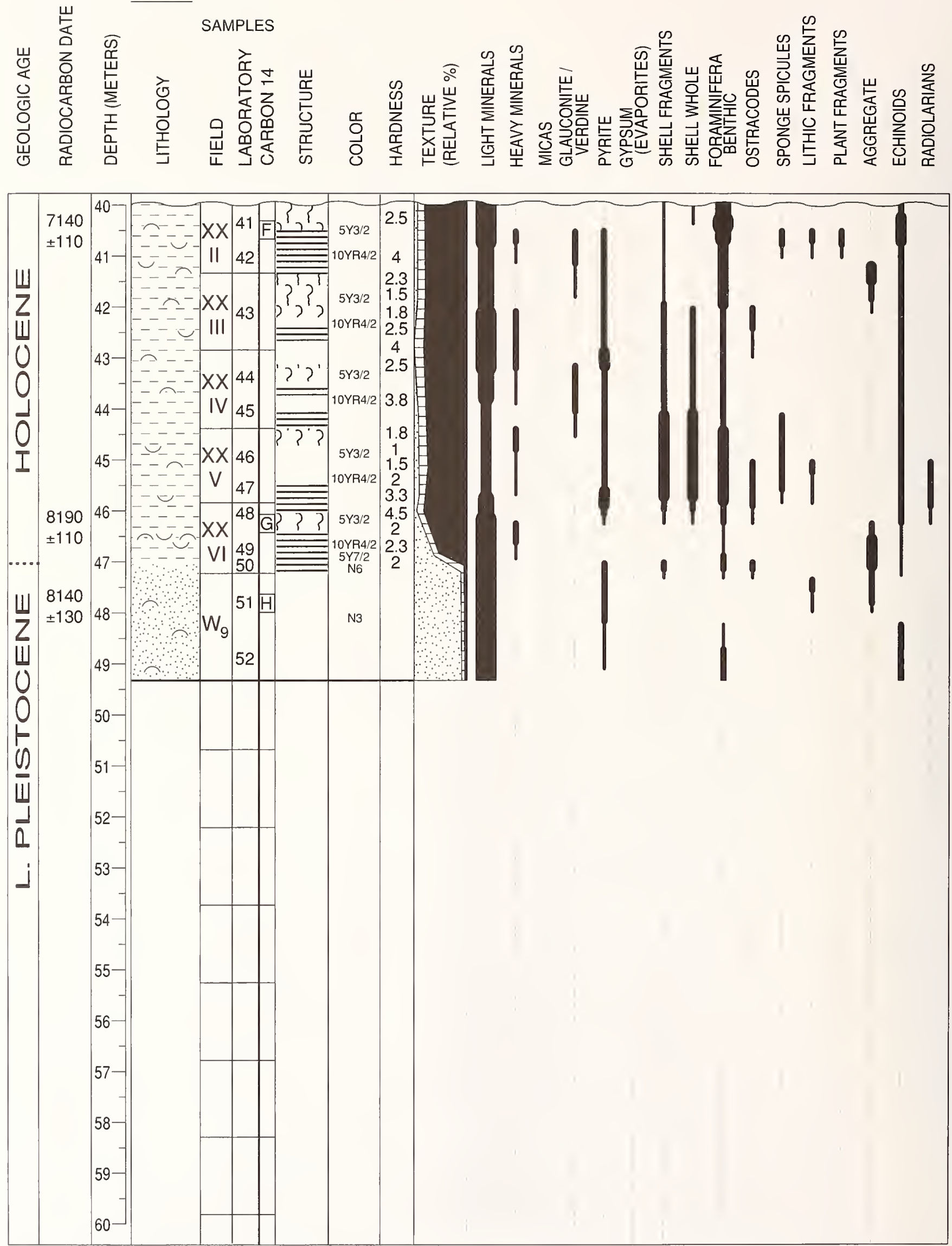
APPENDIX 1.-Continued.

CORE NUMBER S22 I

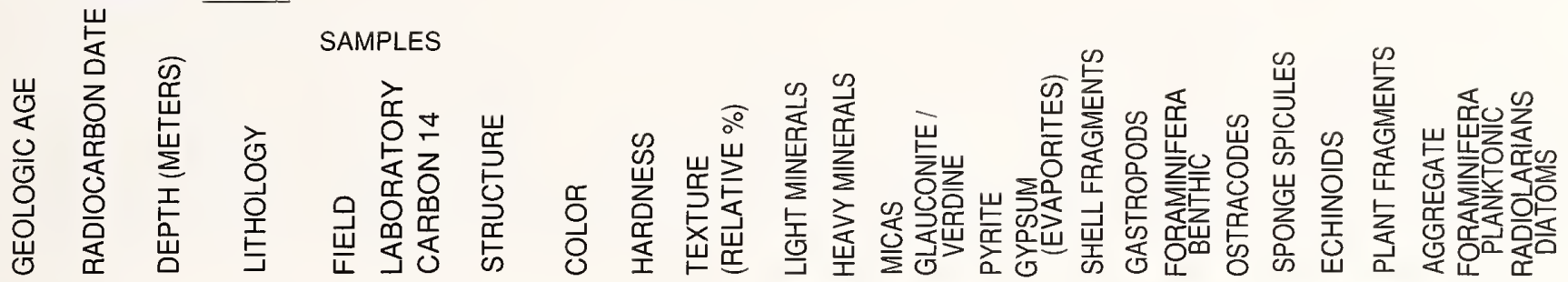

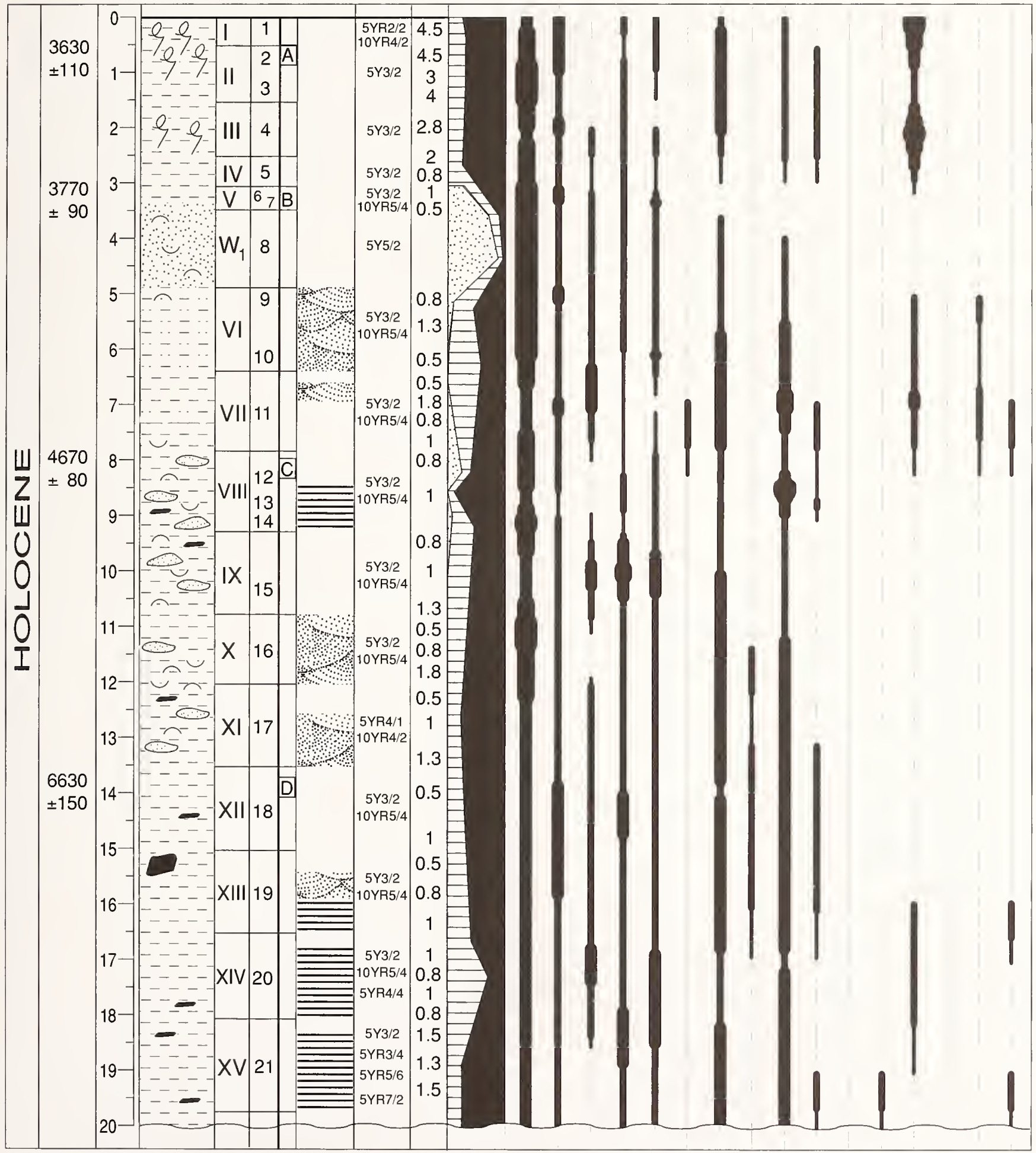


APPENDIX 1.-Continued.

CORE NUMBER S22 II
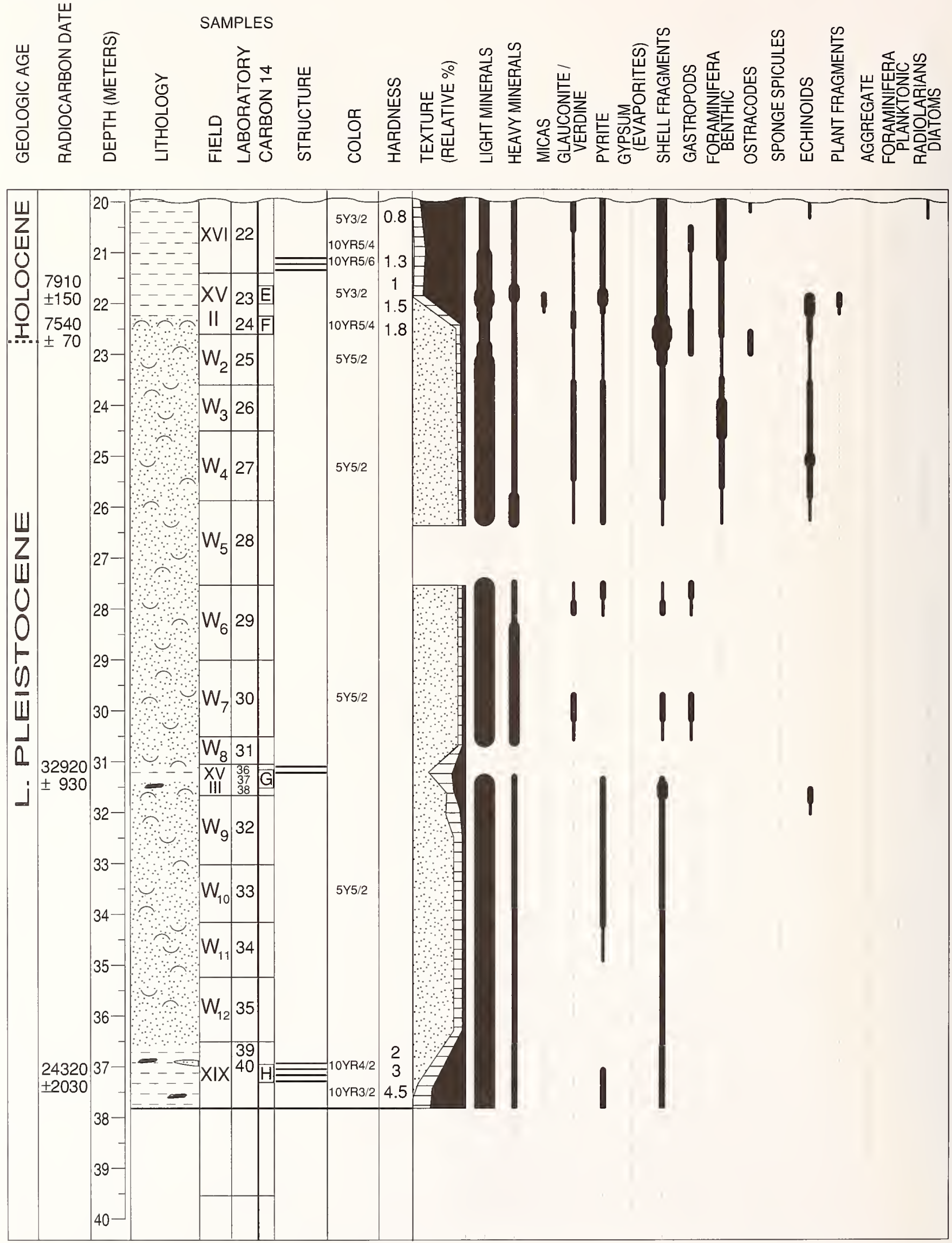
APPENDIX 1.-Continued.
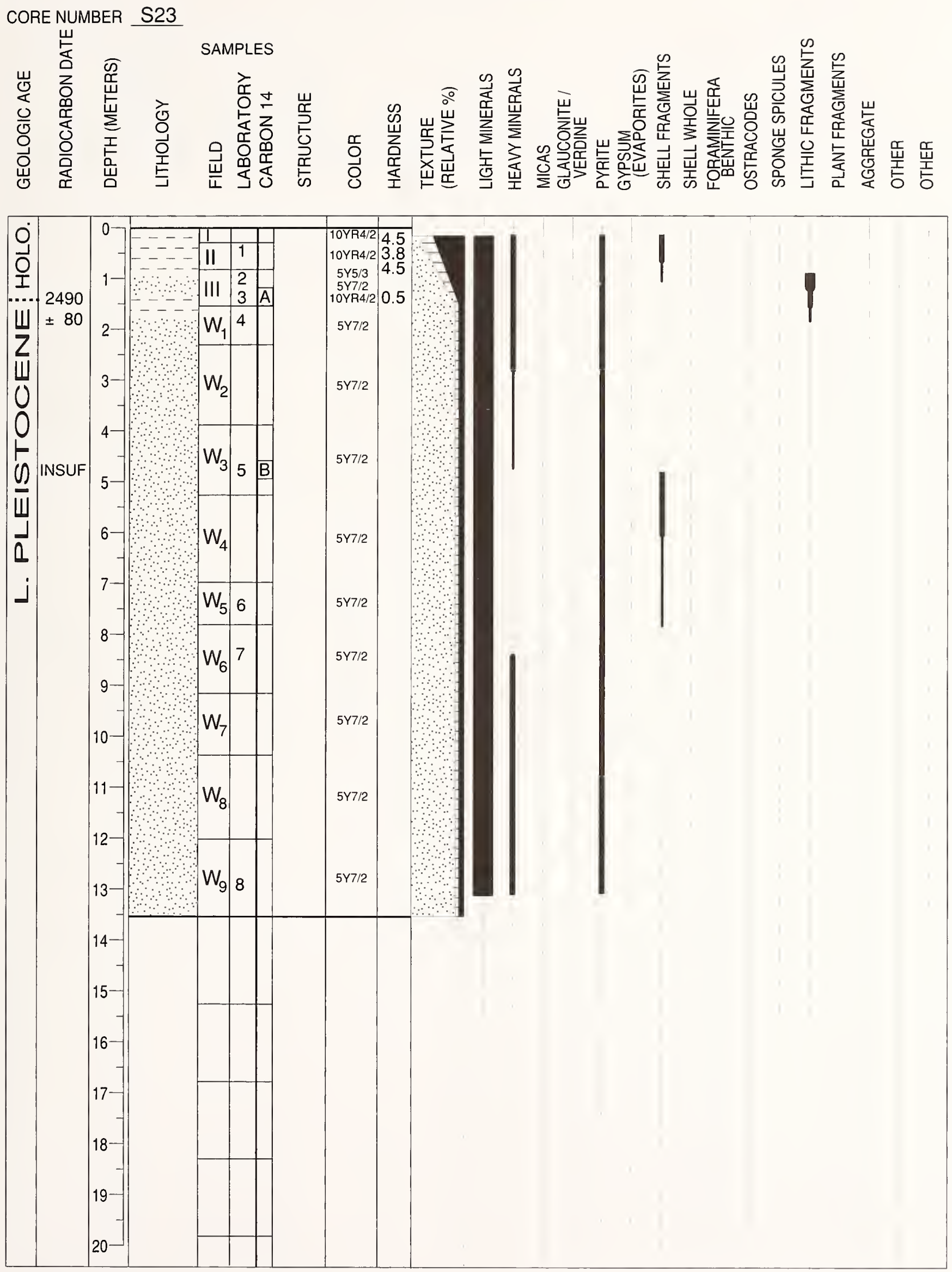
APPENDIX 1.-Continued.
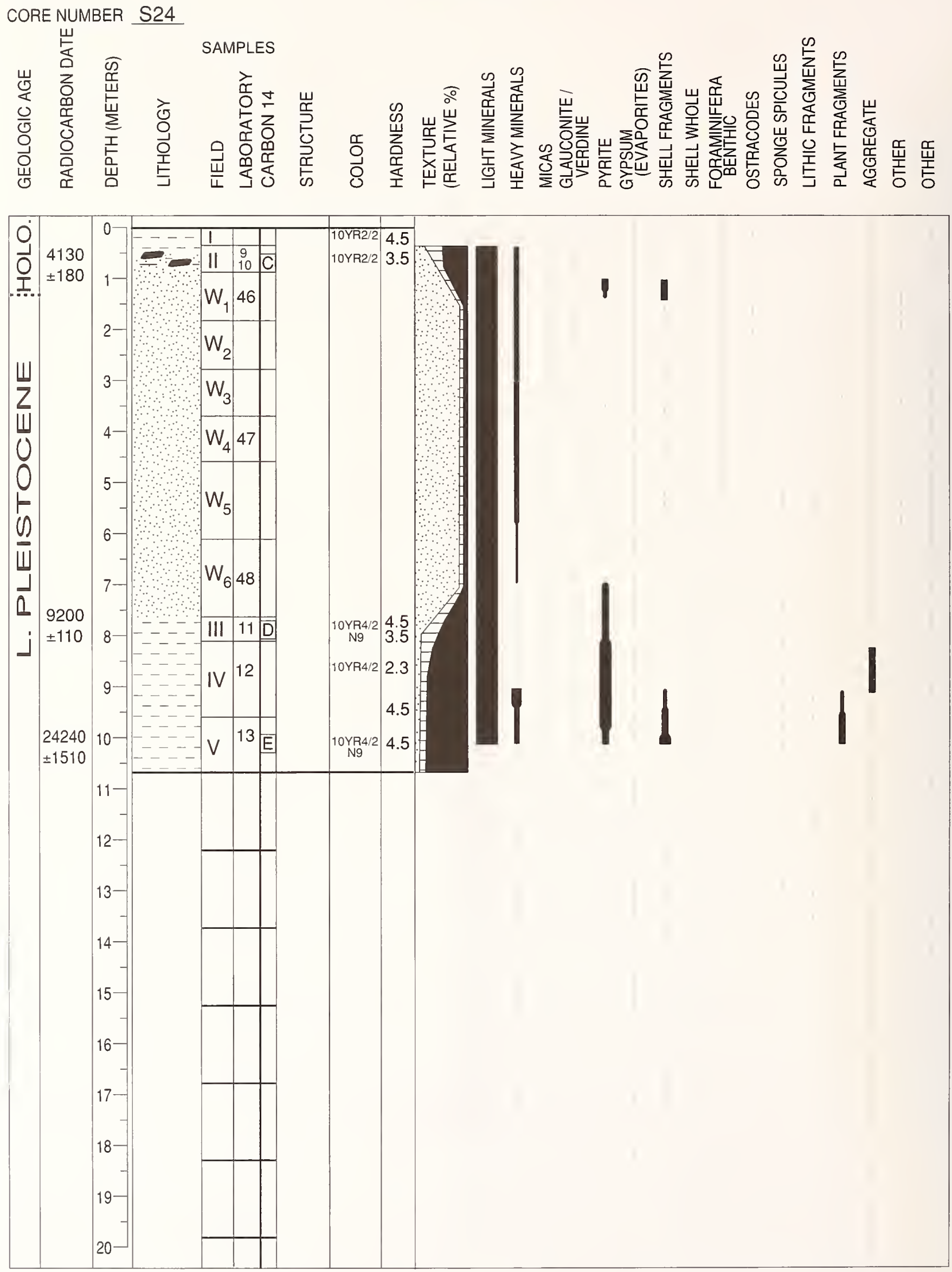
APPENDIX 1.-Continued.

CORE NUMBER S25
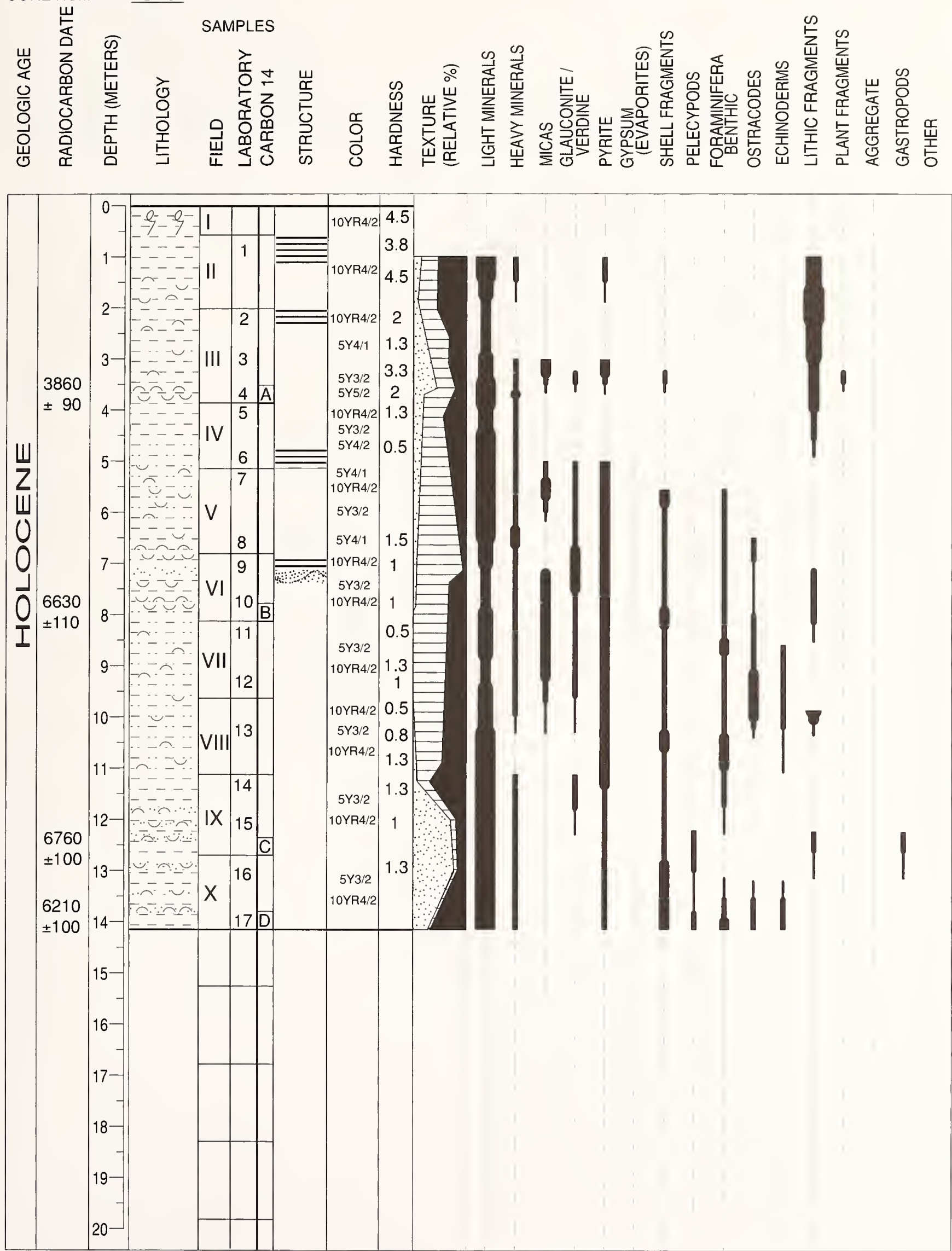
APPENDIX 1.-Continued.

CORE NUMBER S26

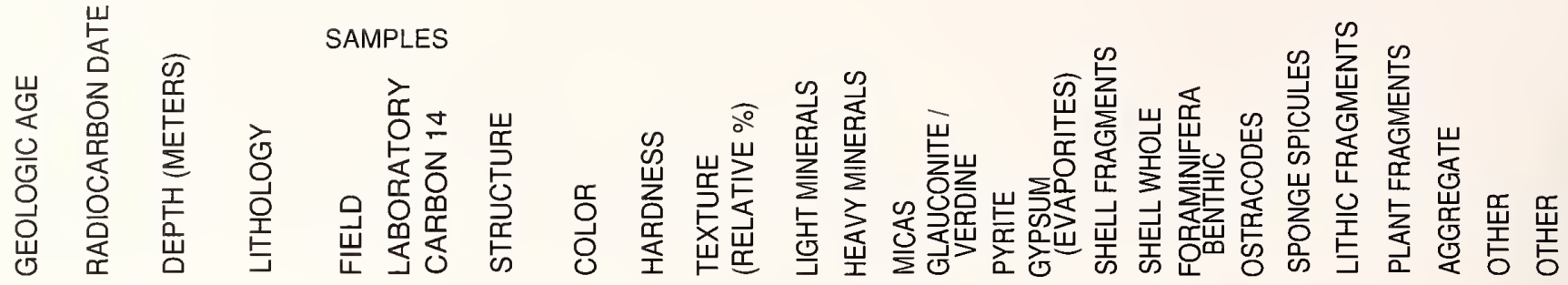

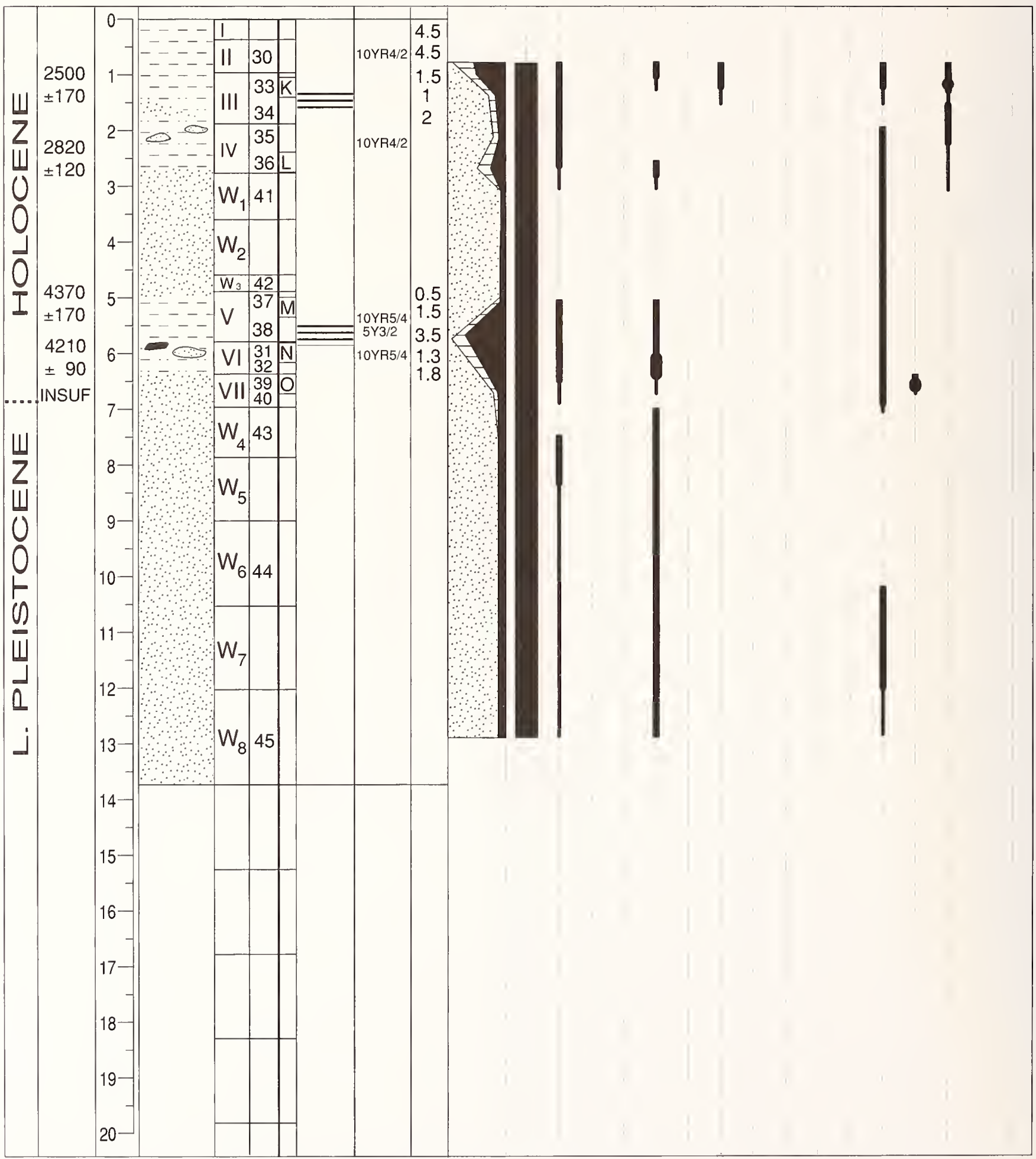


APPENDIX 1.-Continued.

CORE NUMBER S27
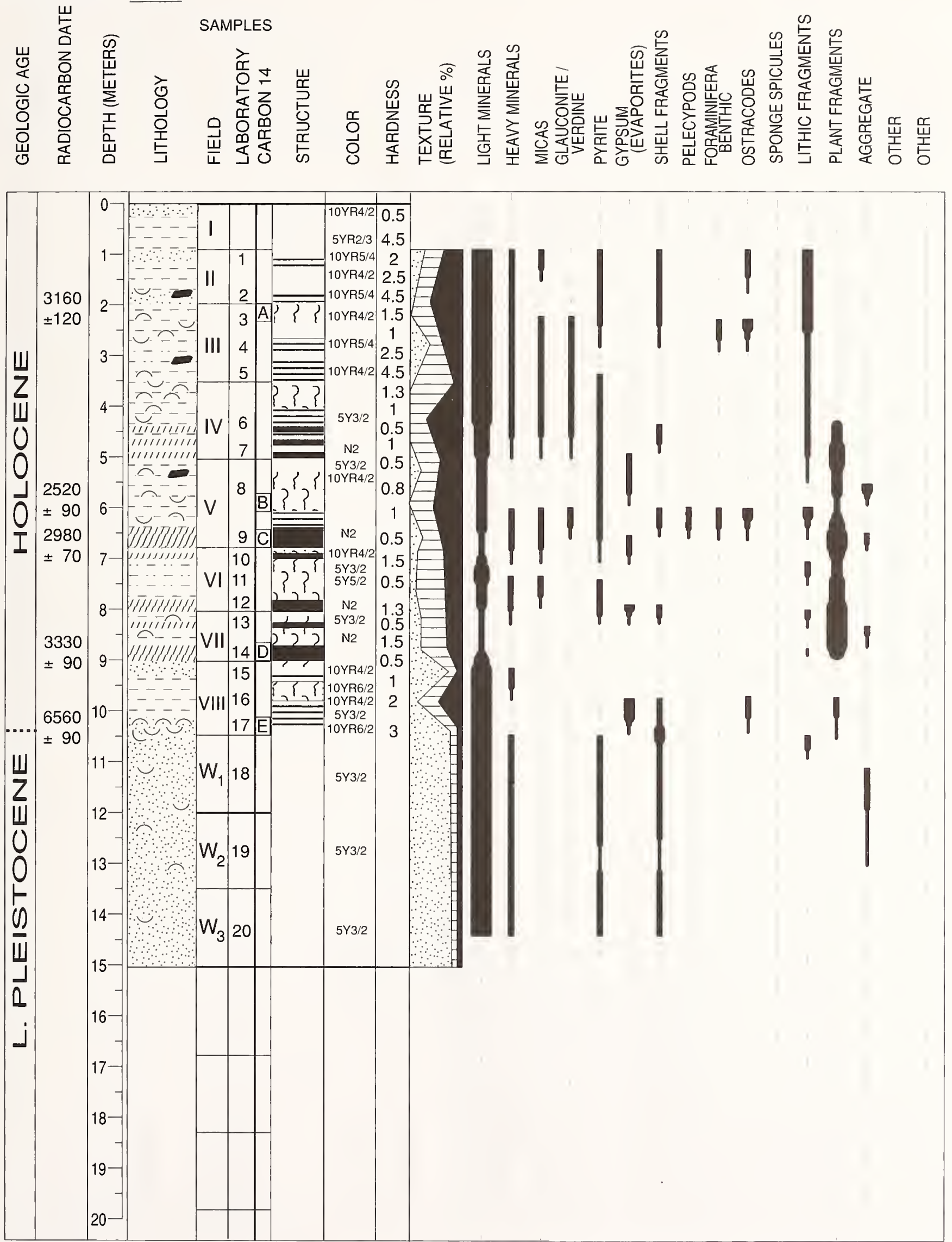
APPENDIX 1.-Continued.

CORE NUMBER S28 I
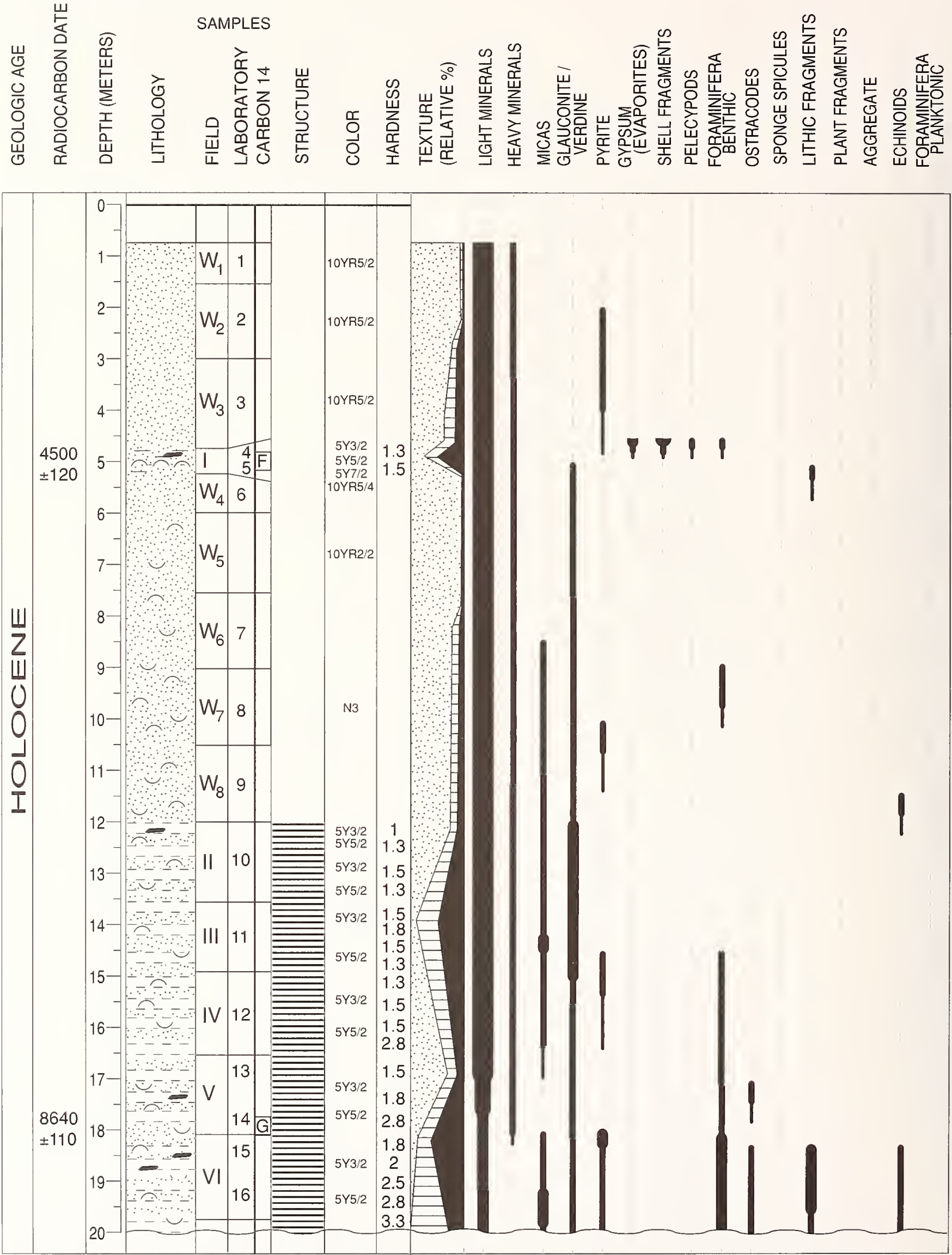
APPENDIX 1.-Continued.

CORE NUMBER S28 II

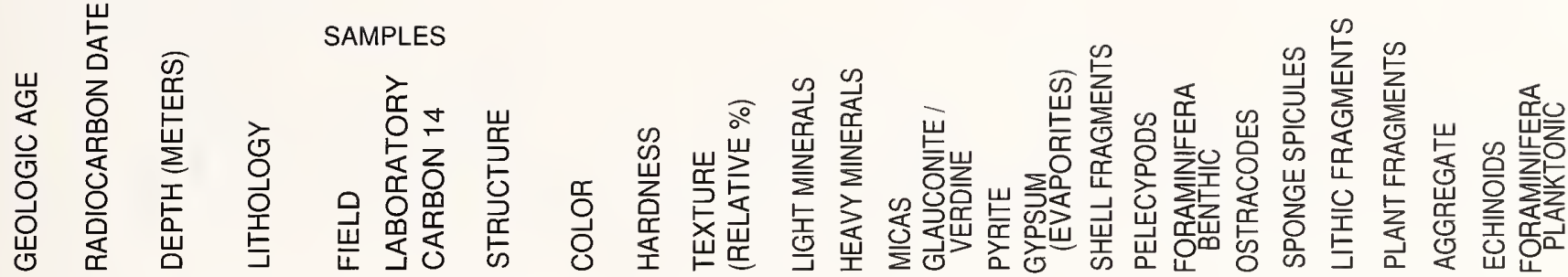

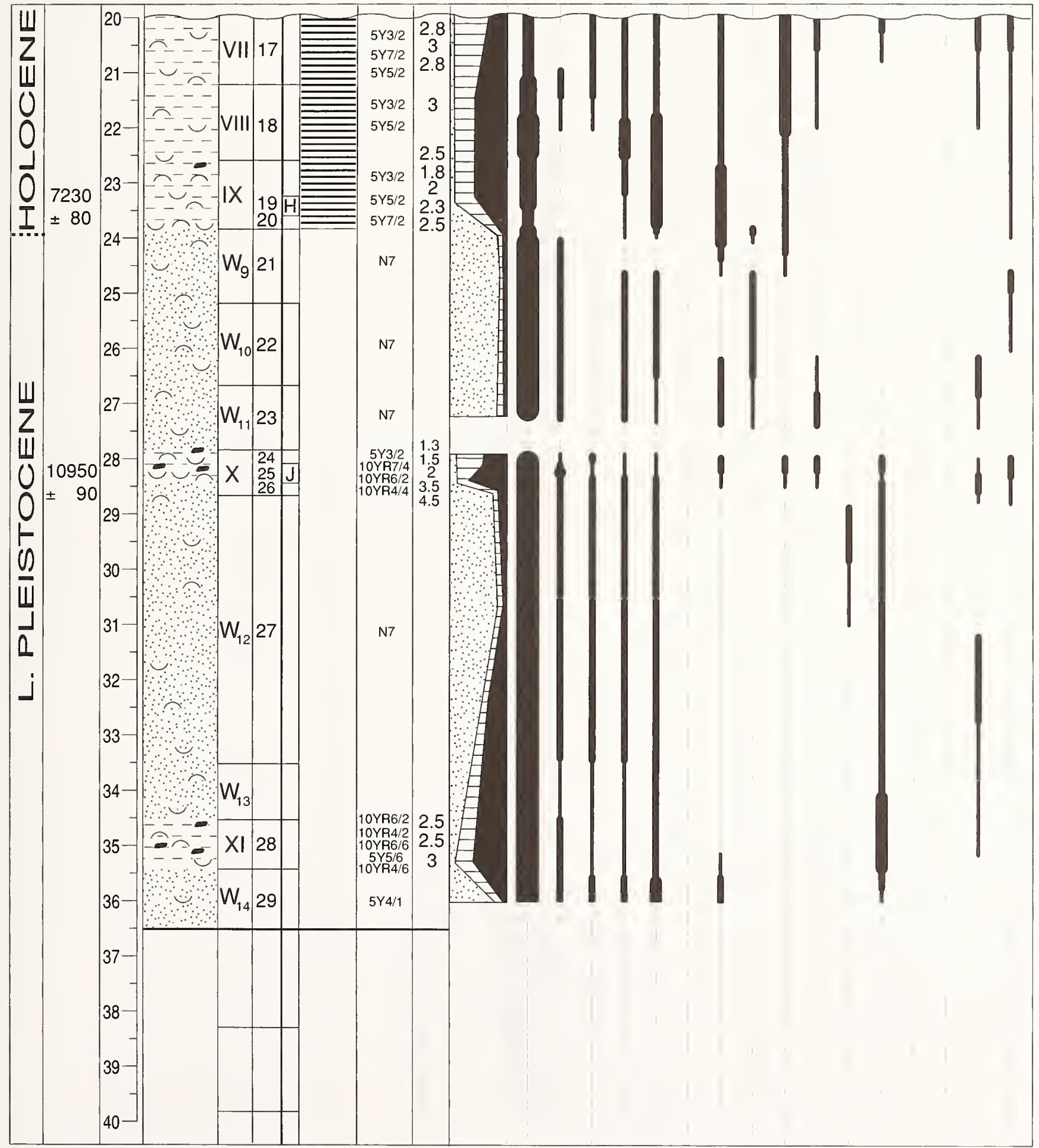


APPENDIX 1.-Continued.
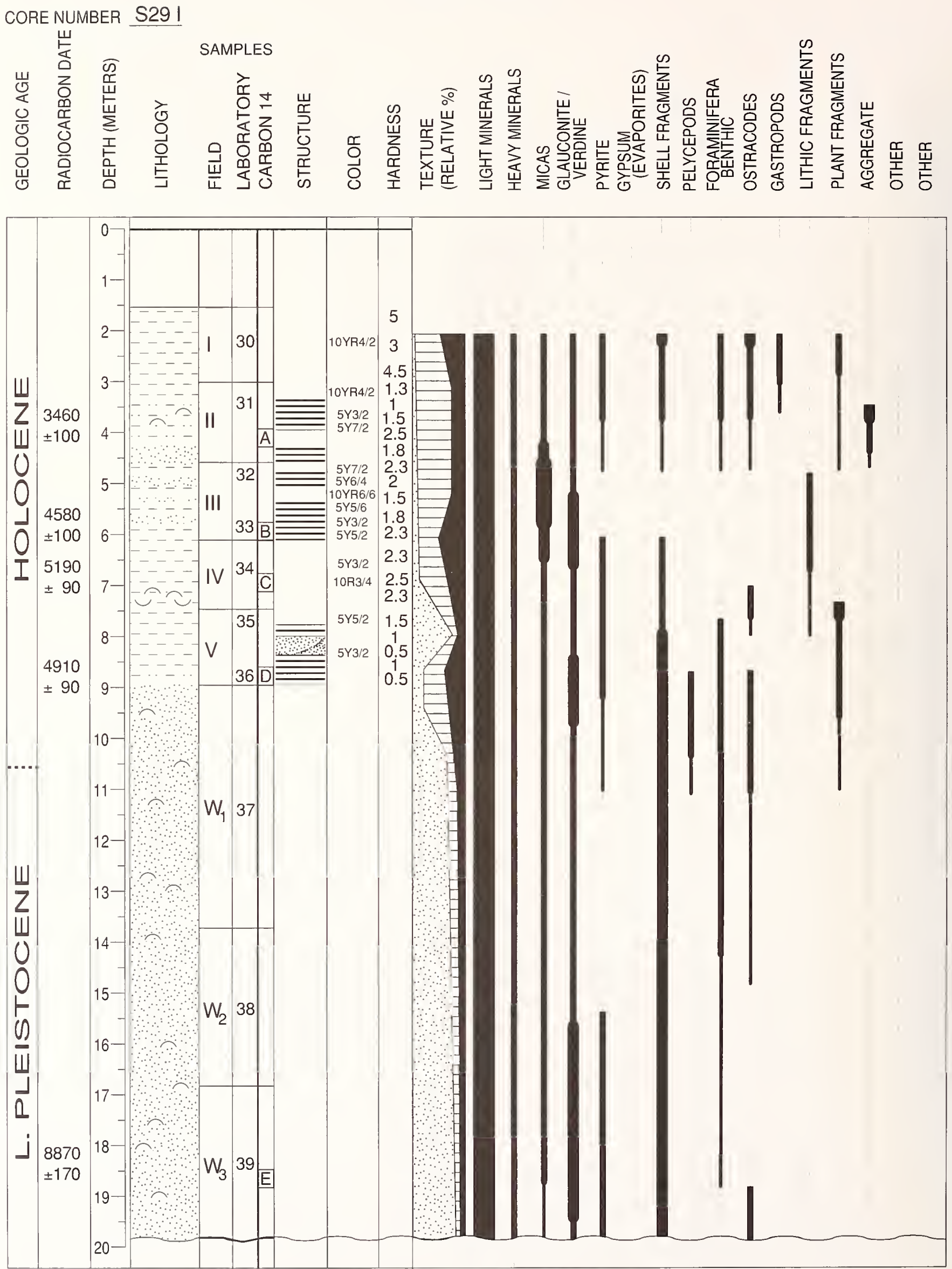
APPENDIX 1.-Continued.

CORE NUMBER S29 II

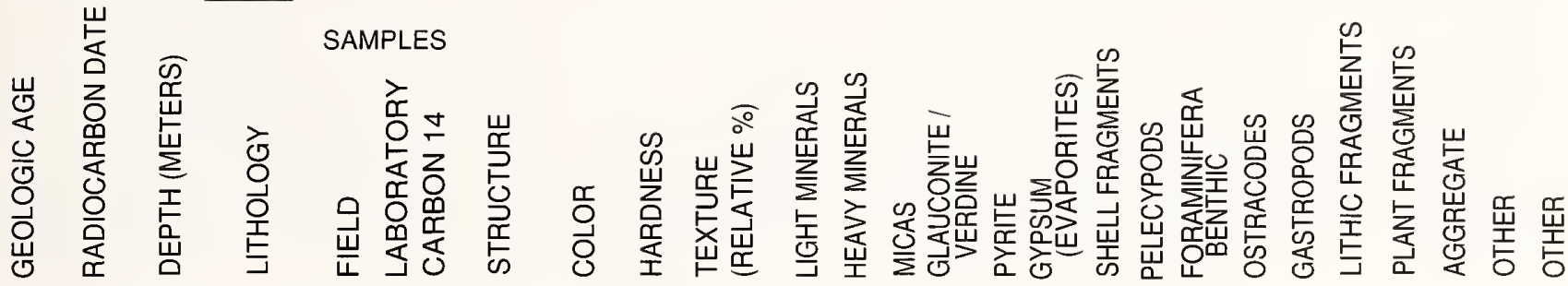

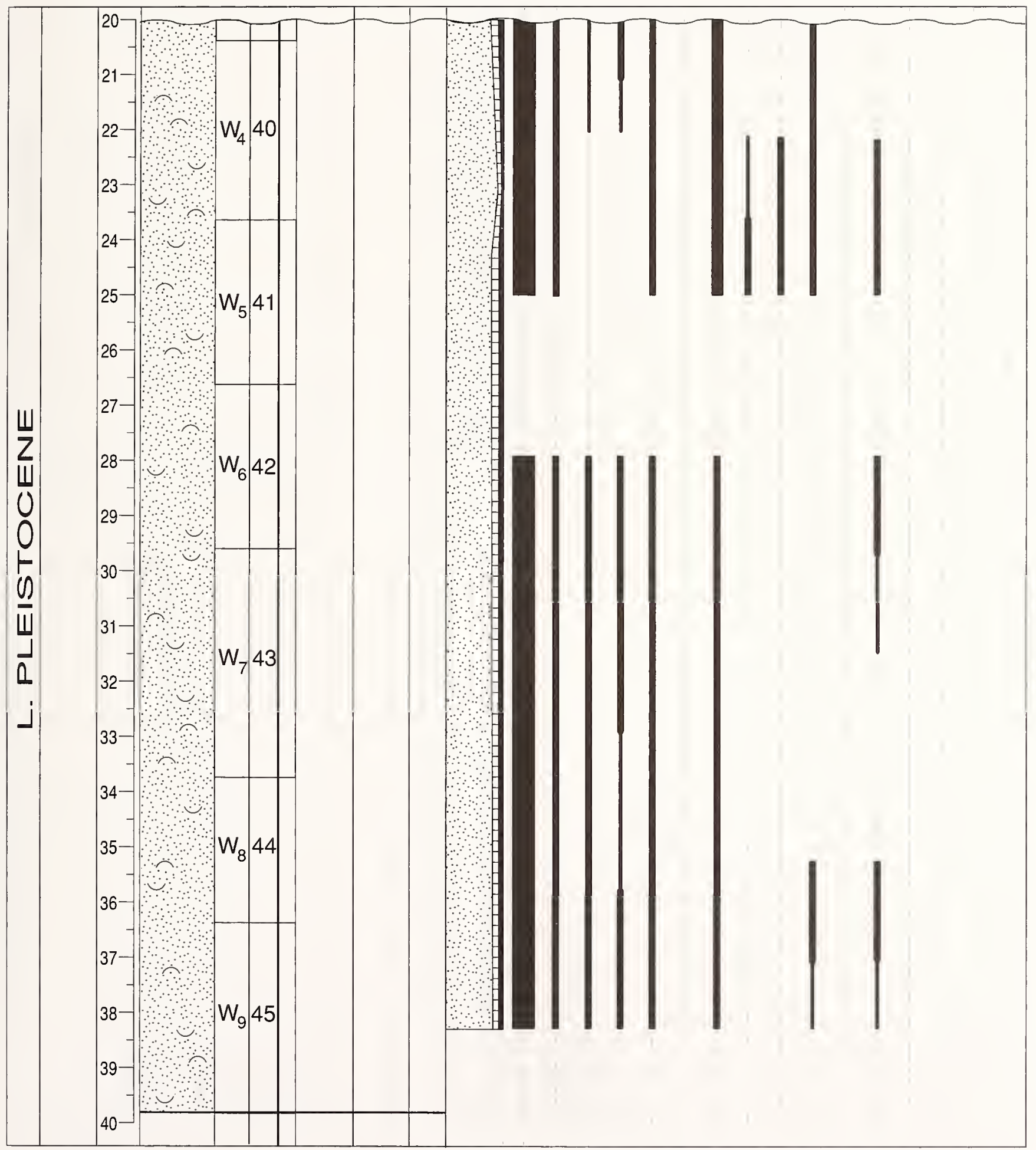


APPENDIX 1.--Continued.

CORE NUMBER S3OI

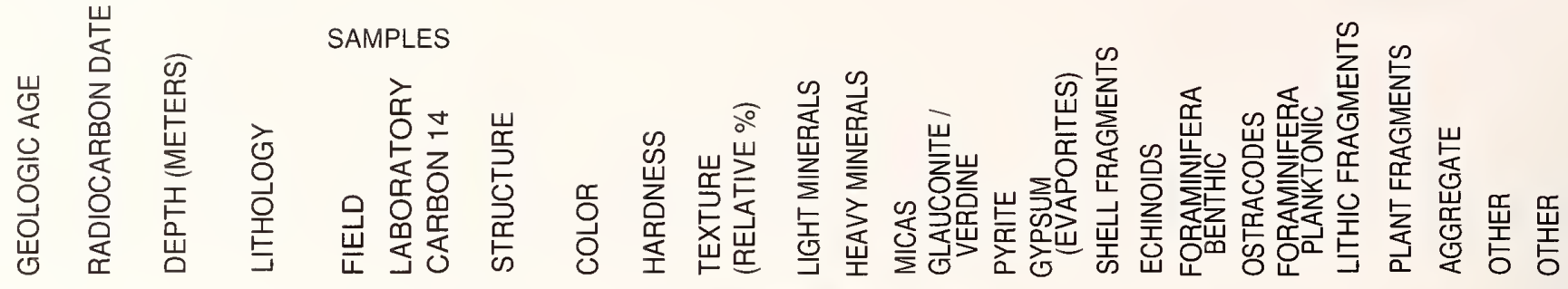

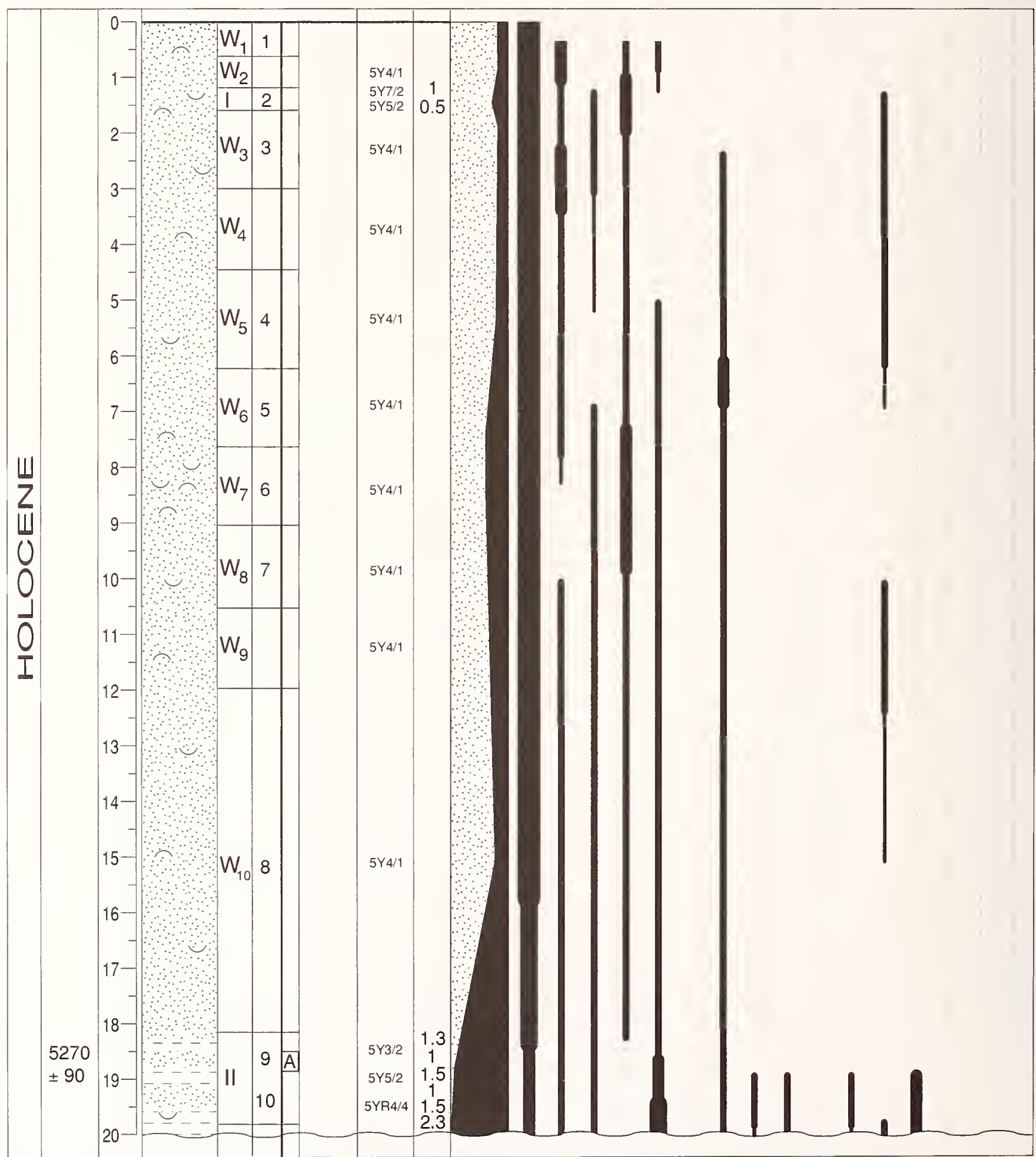


APPENDIX 1.-Continued.

CORE NUMBER S30 II
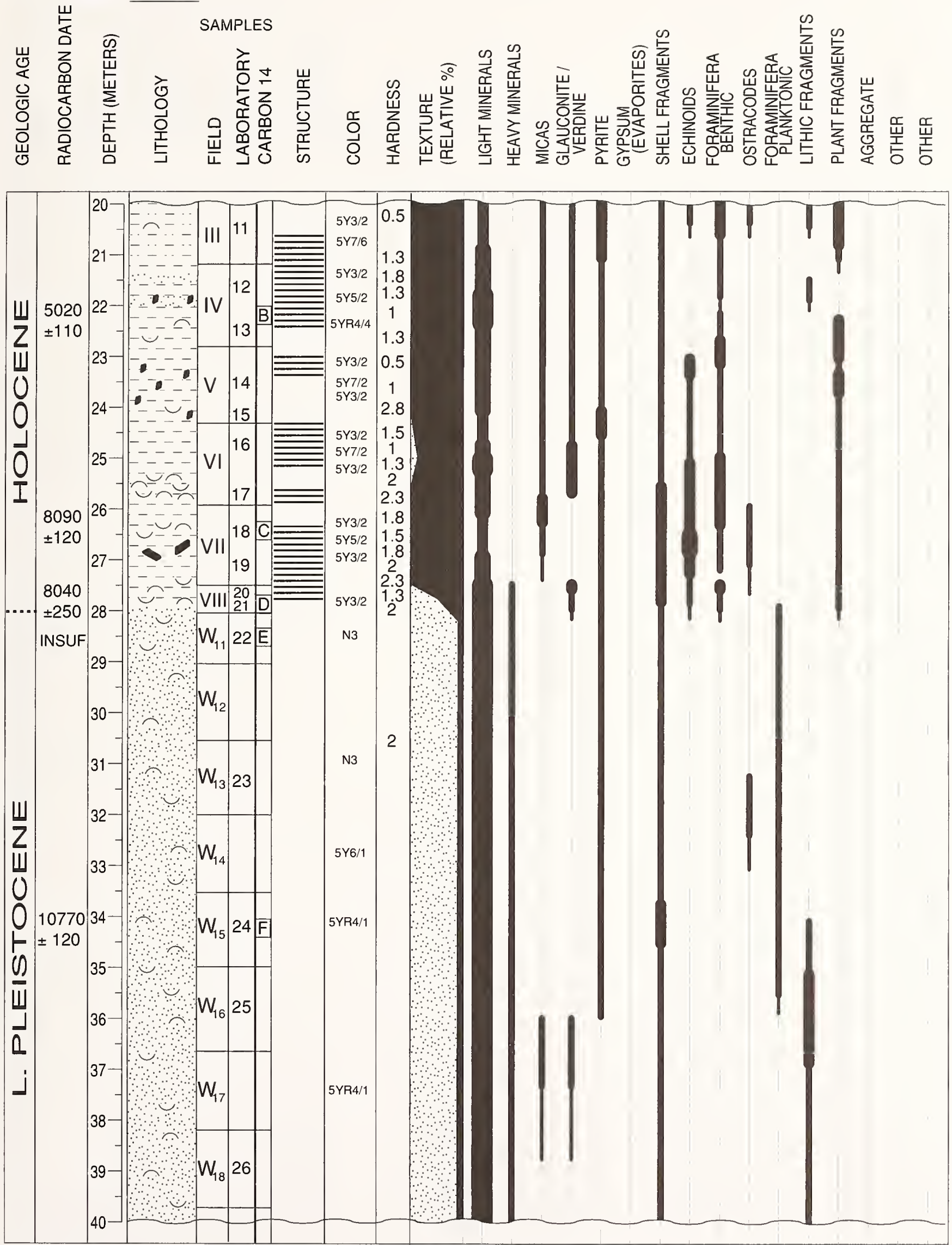
APPENDIX 1.-Continued.

CORE NUMBER S30 III

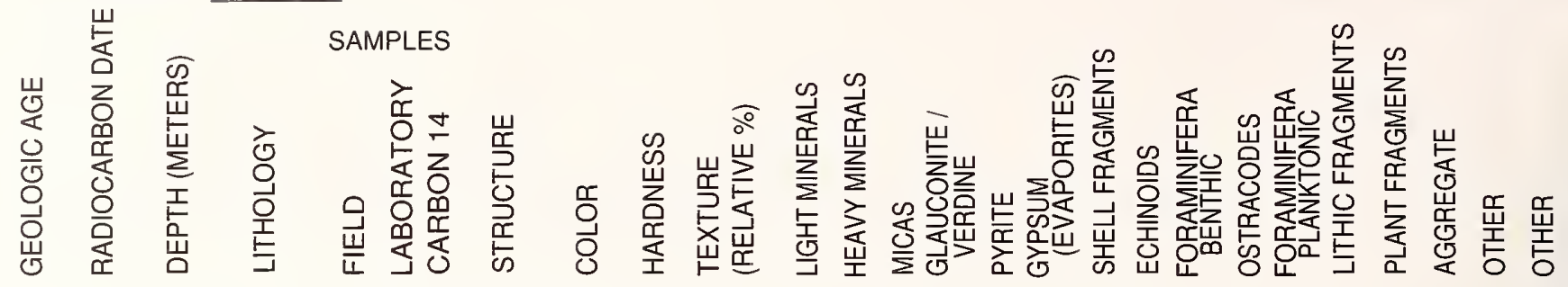

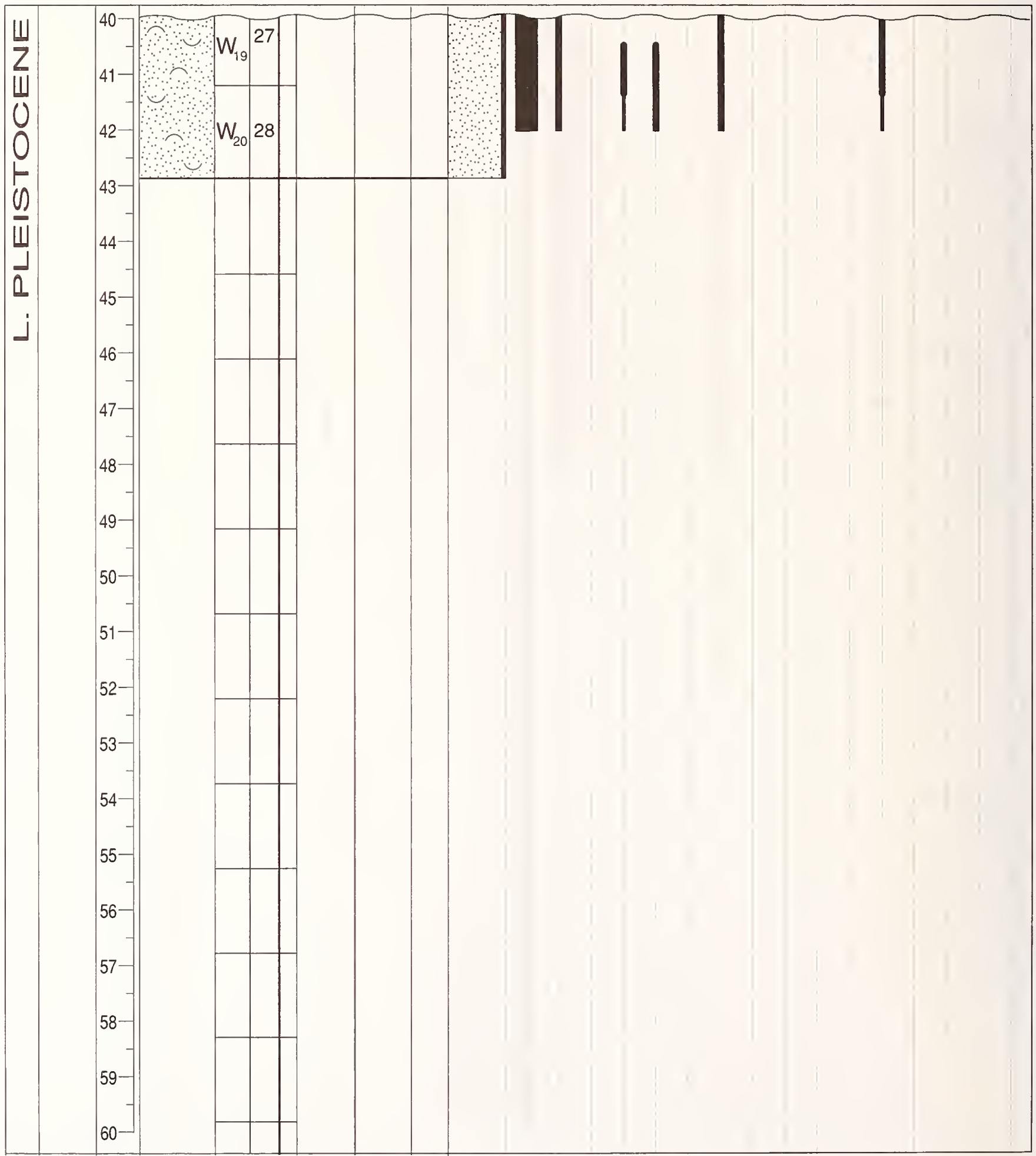


APPENDIX 1.-Continued.

CORE NUMBER S31I

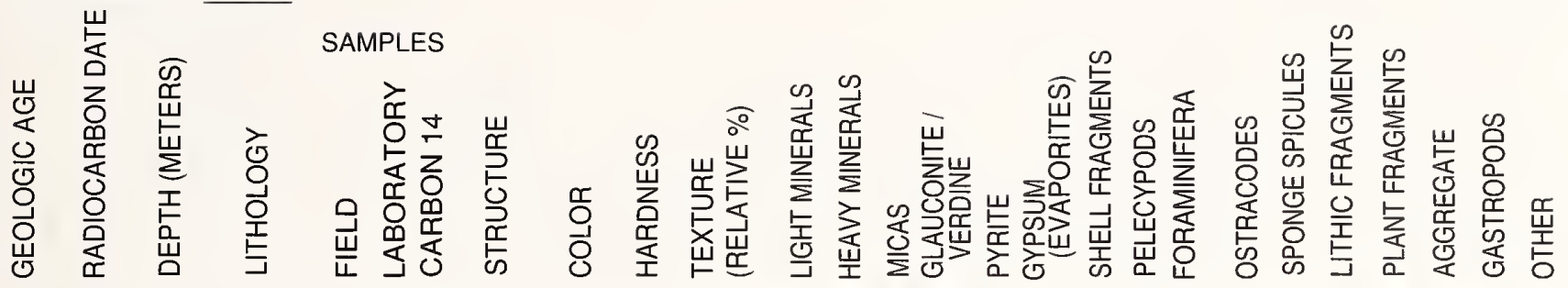

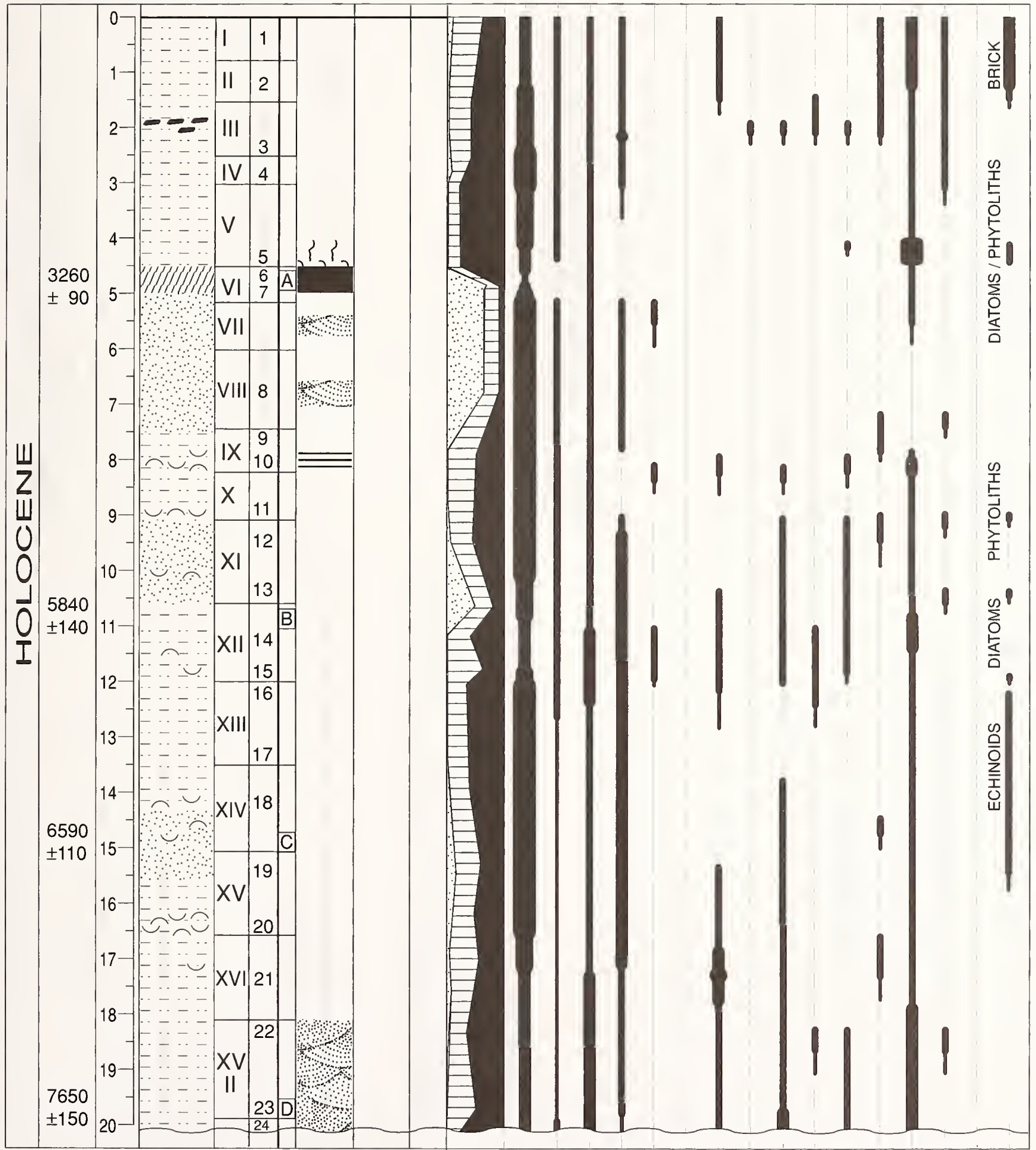


APPENDIX 1.-Continued.

CORE NUMBER S31 II

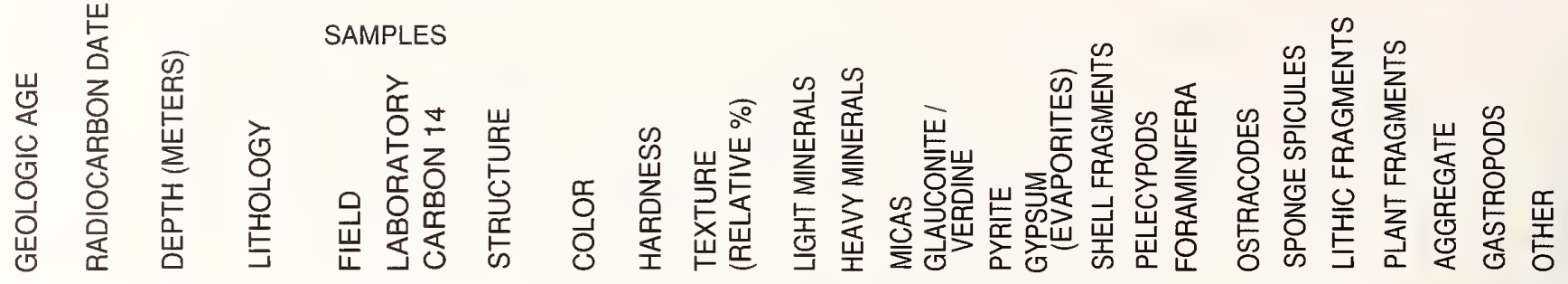

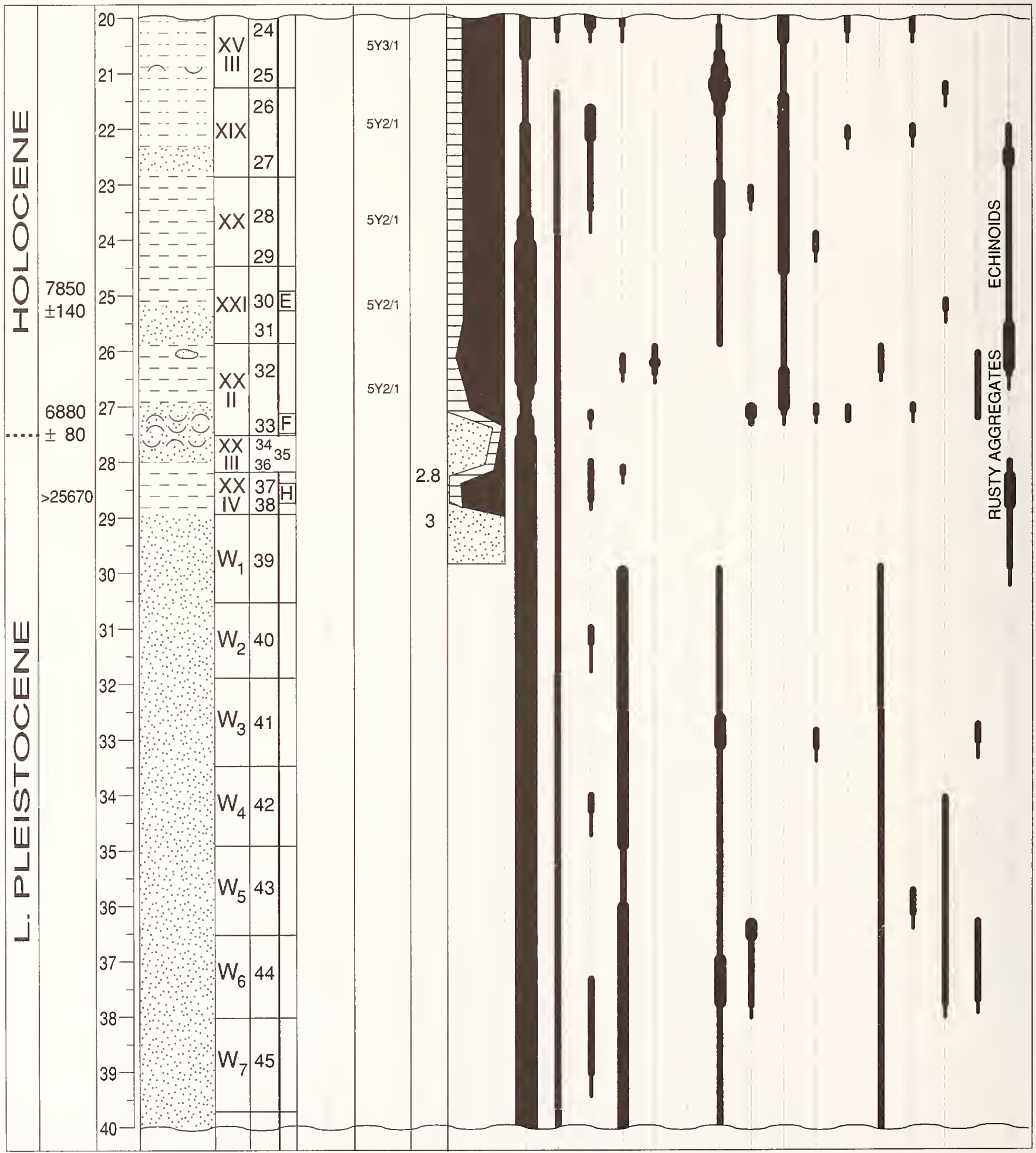


APPENDIX 1.-Continued.

CORE NUMBER S31 III

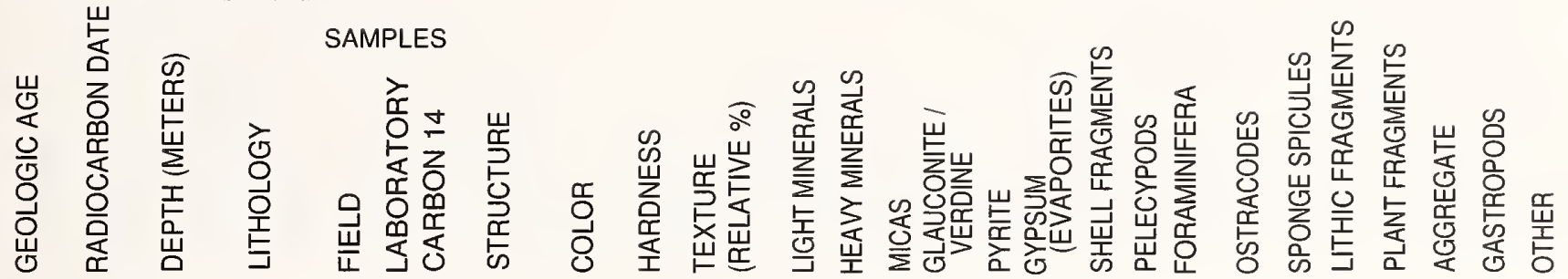

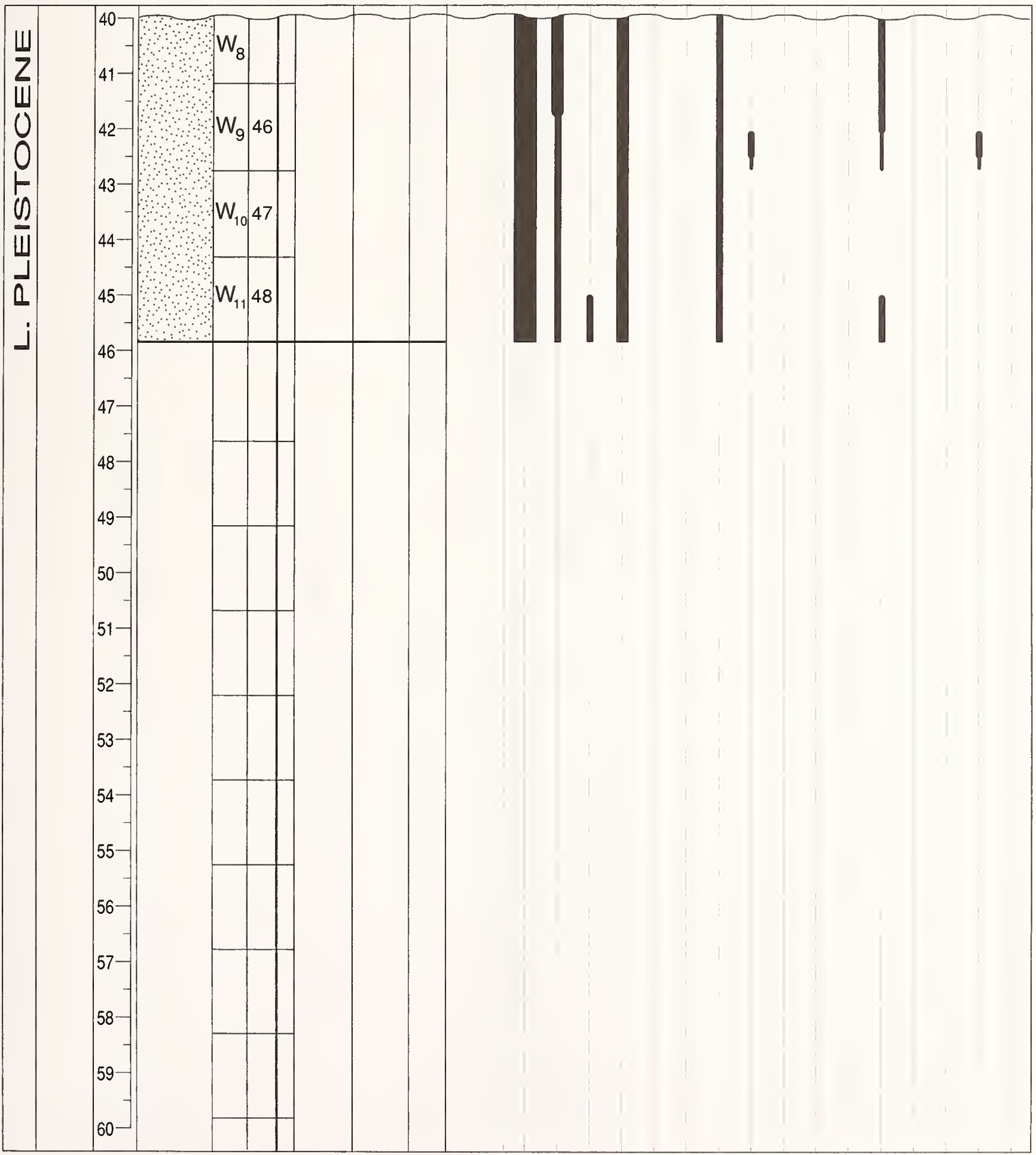


APPENDIX 1.-Continued.

CORE NUMBER S32 I
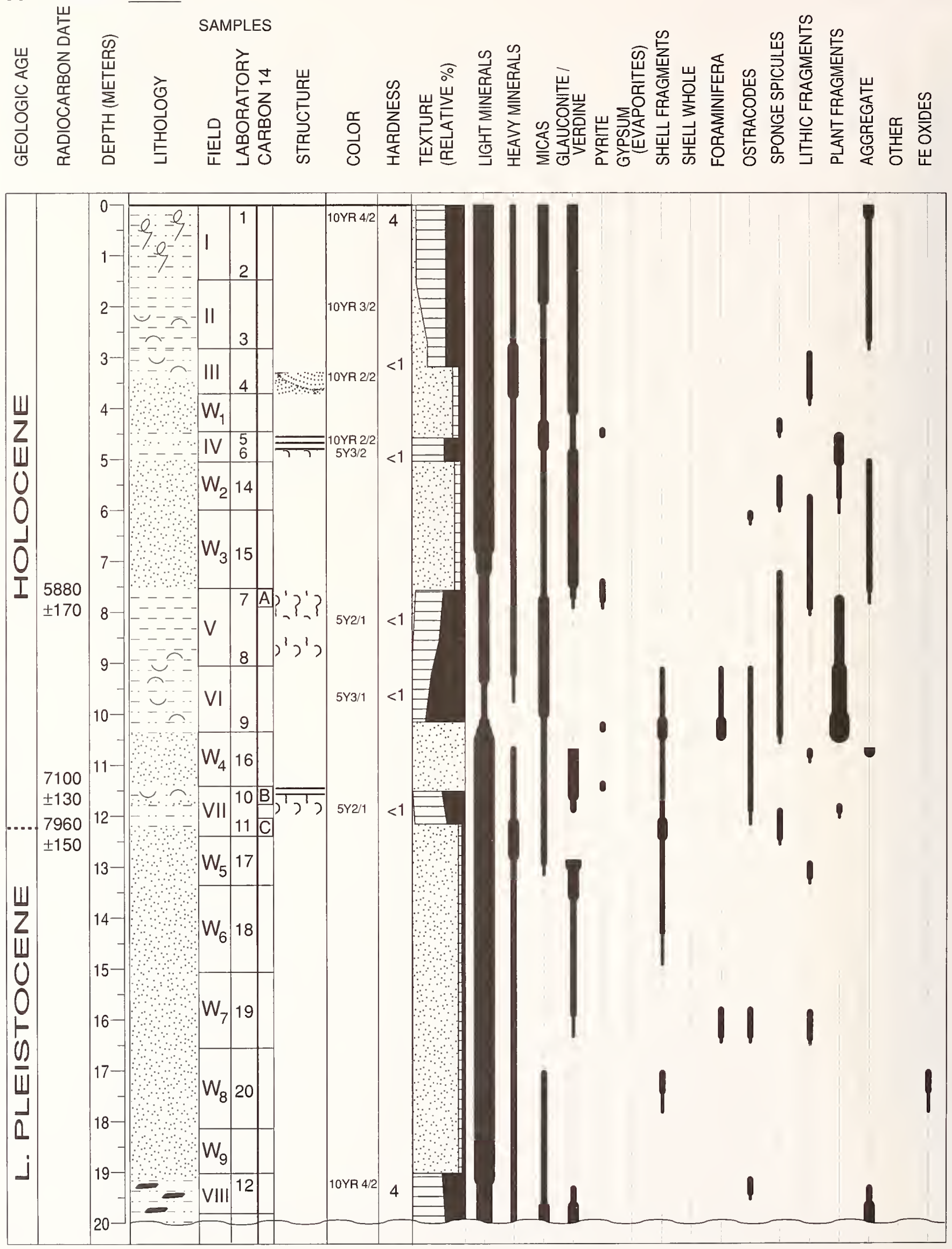
APPENDIX 1.-Continued.

CORE NUMBER S32 II

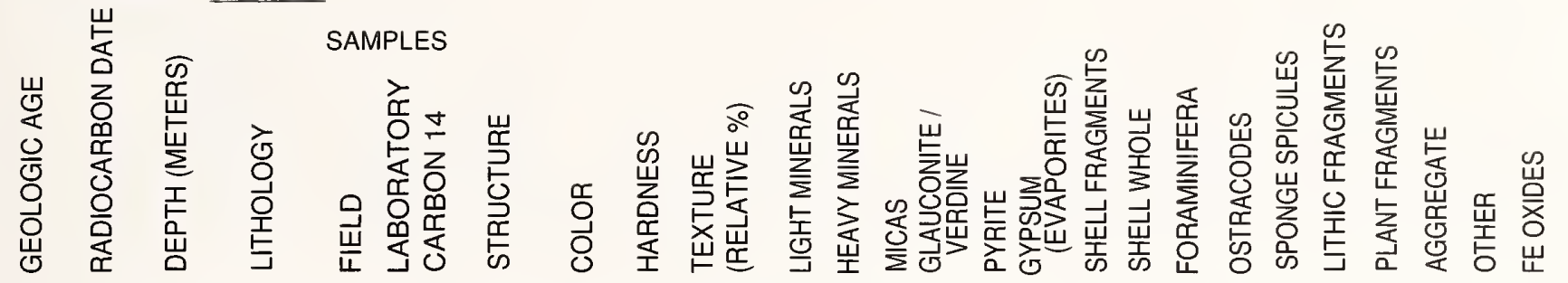

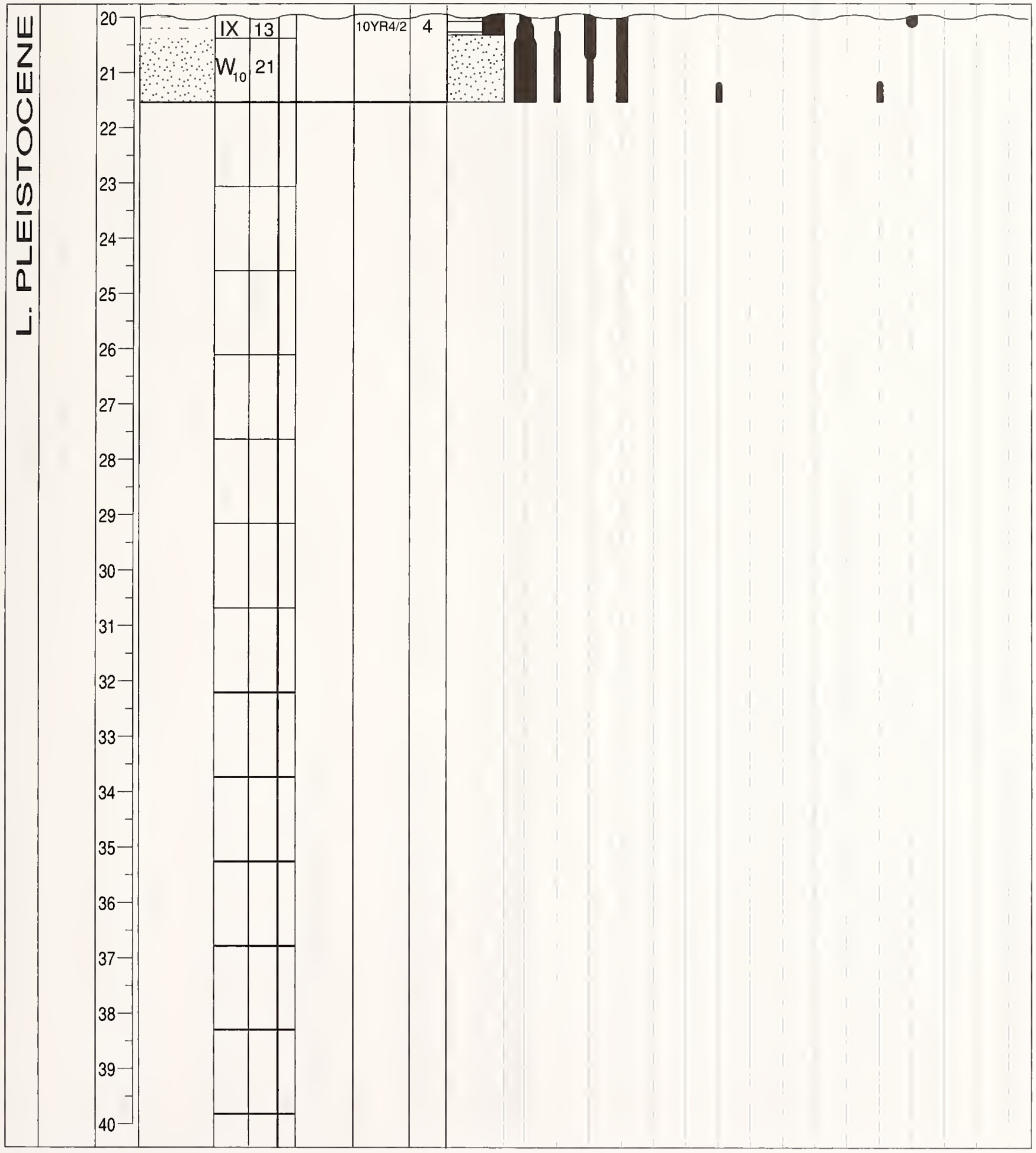


APPENDIX 1.-Continued.

CORE NUMBER S33I

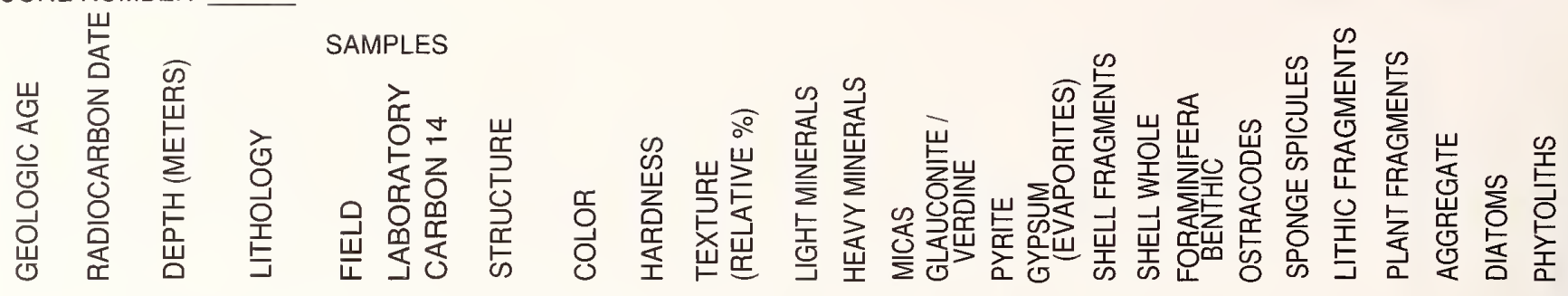

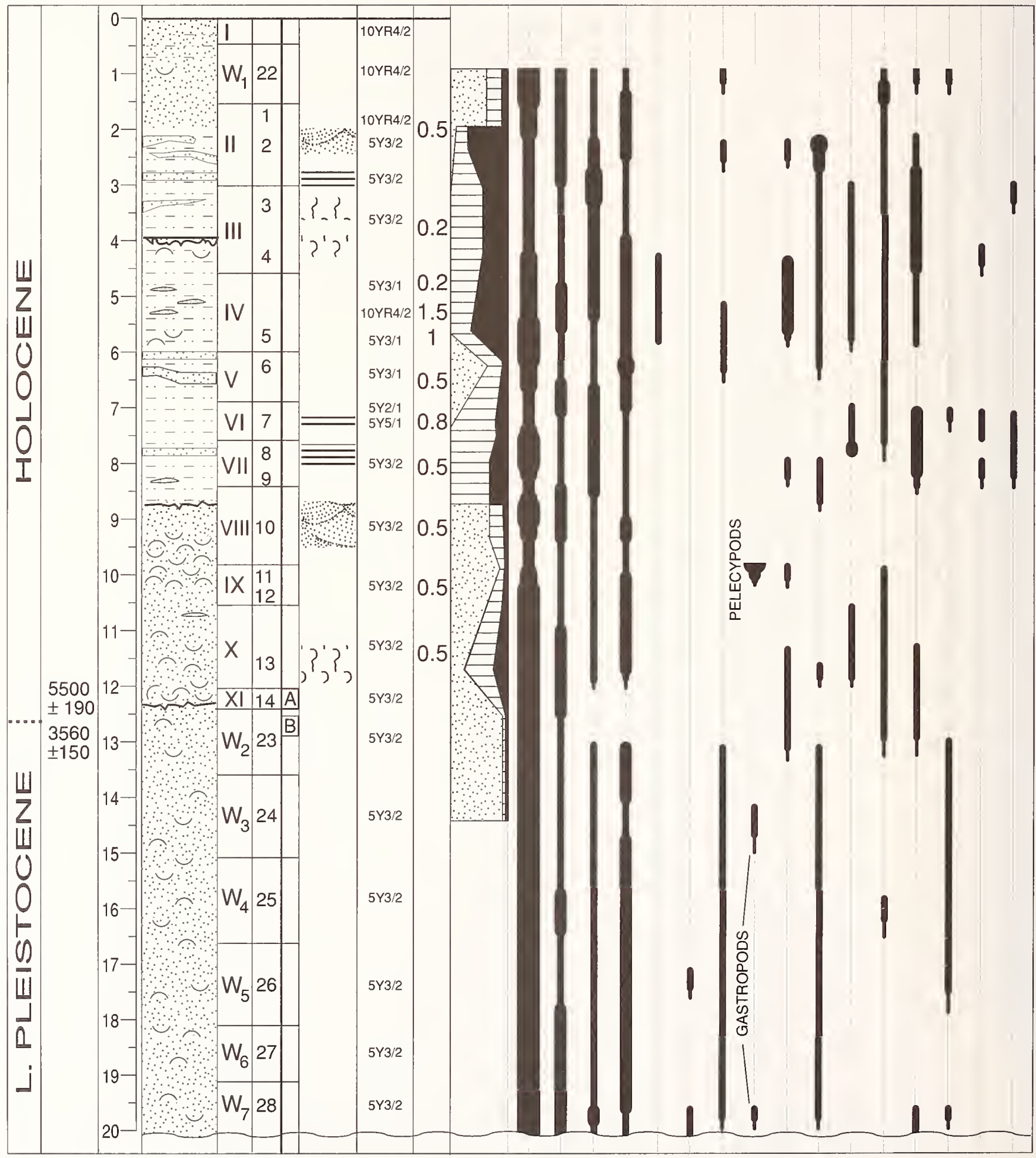


APPENDIX 1.-Continued.

CORE NUMBER S33 $\|$

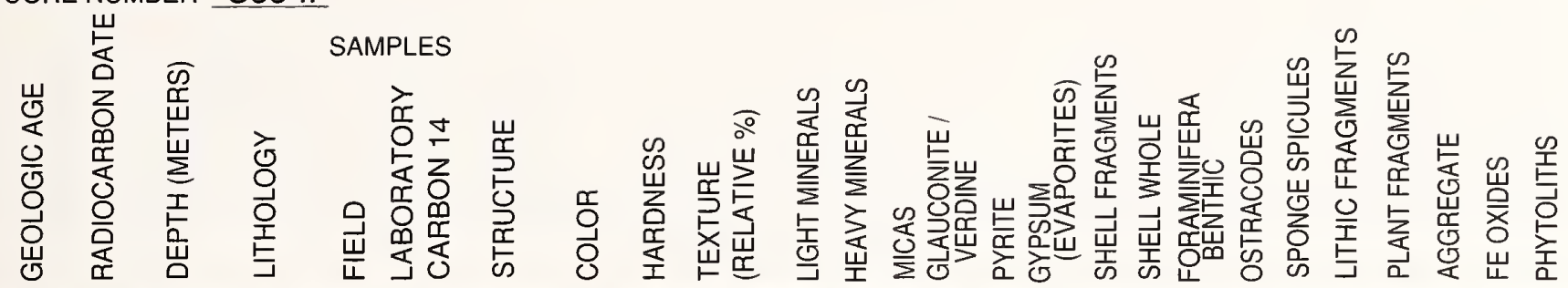

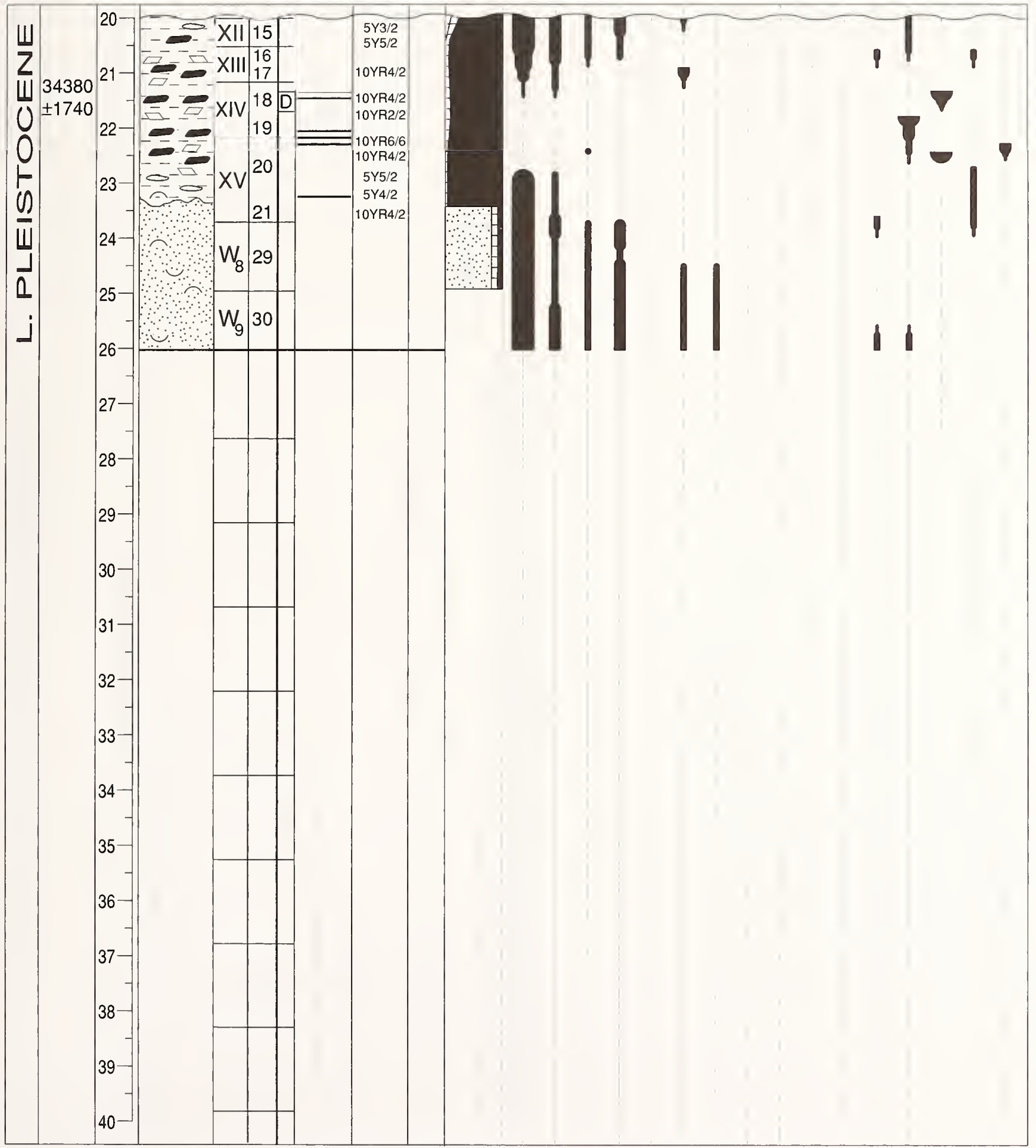


APPENDIX 1.-Continued.

CORE NUMBER S34 I

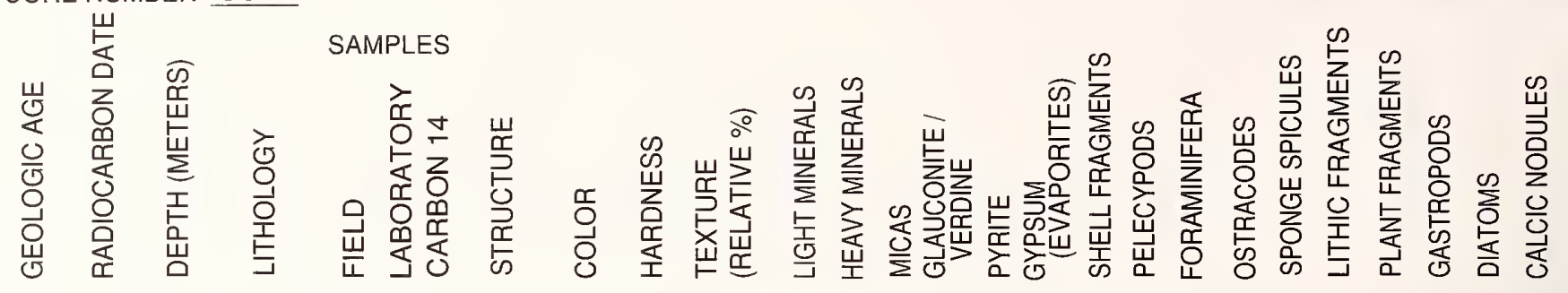

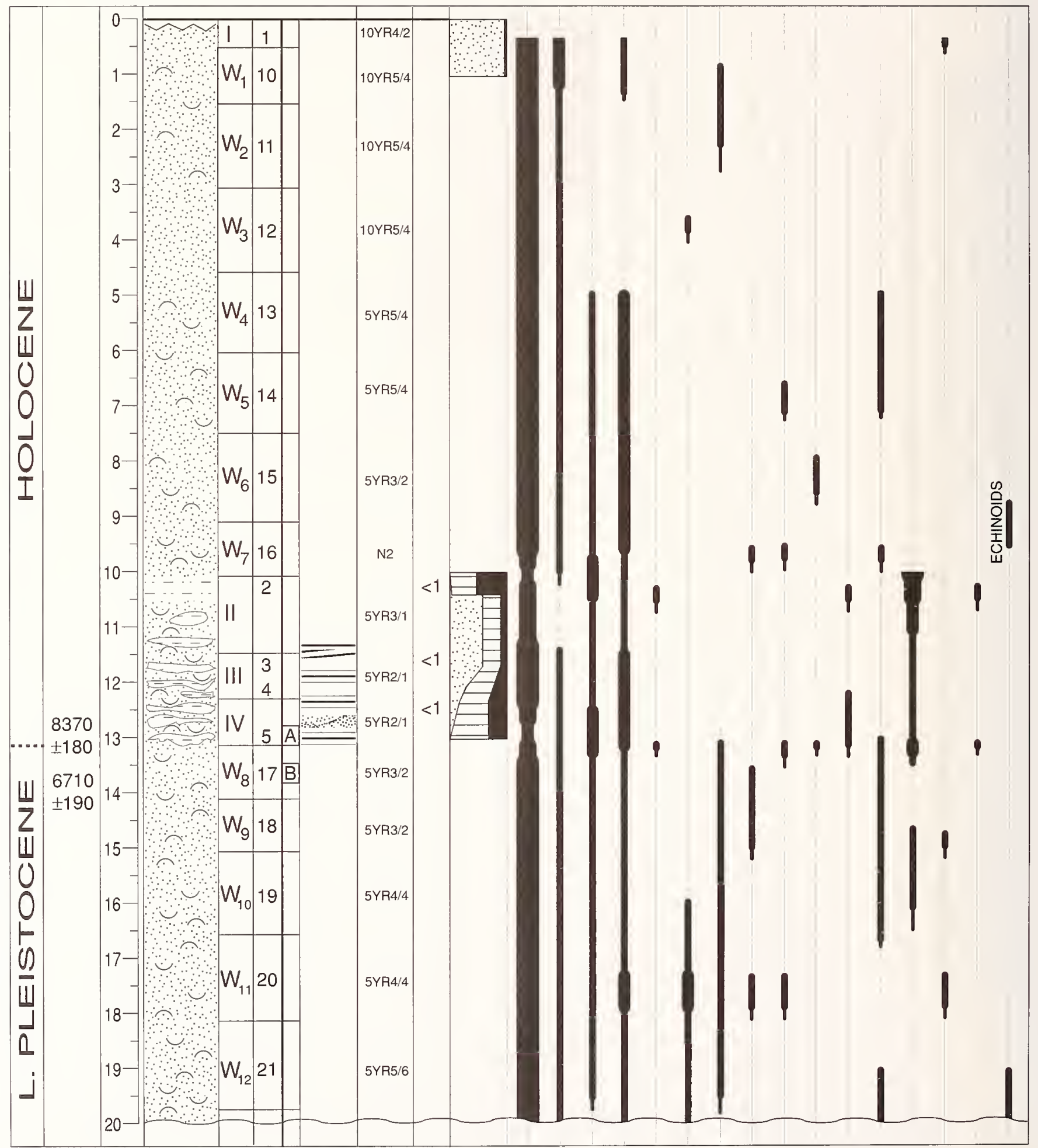


APPENDIX 1.-Continued.

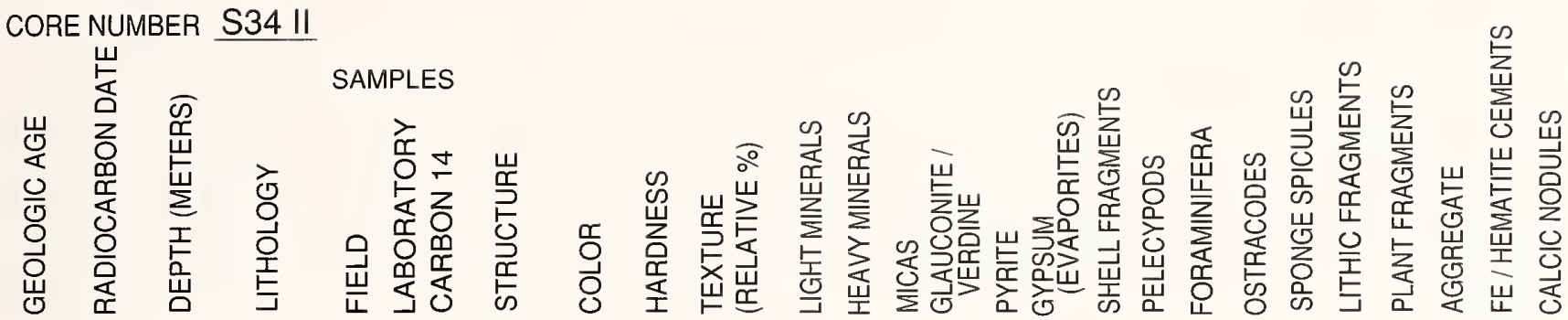

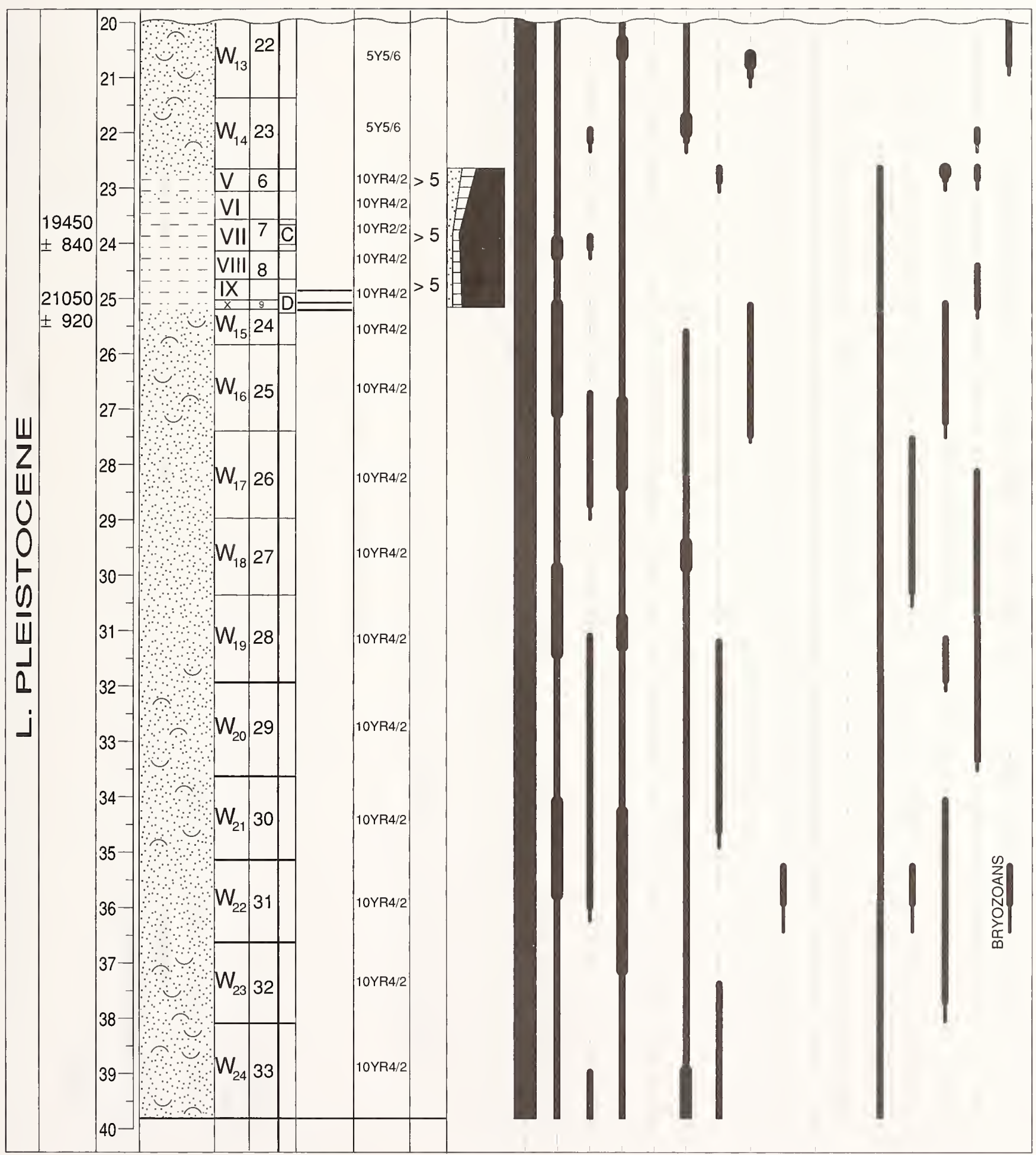


APPENDIX 1.-Continued.

CORE NUMBER S35 I

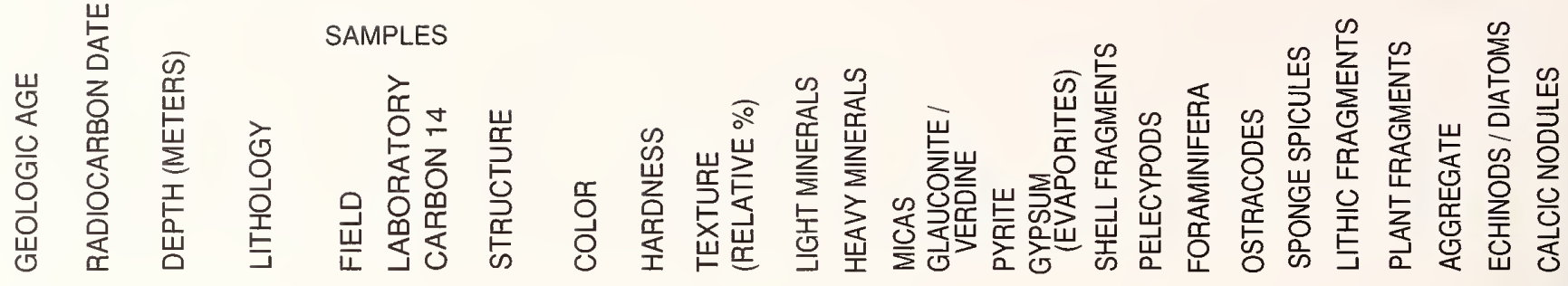

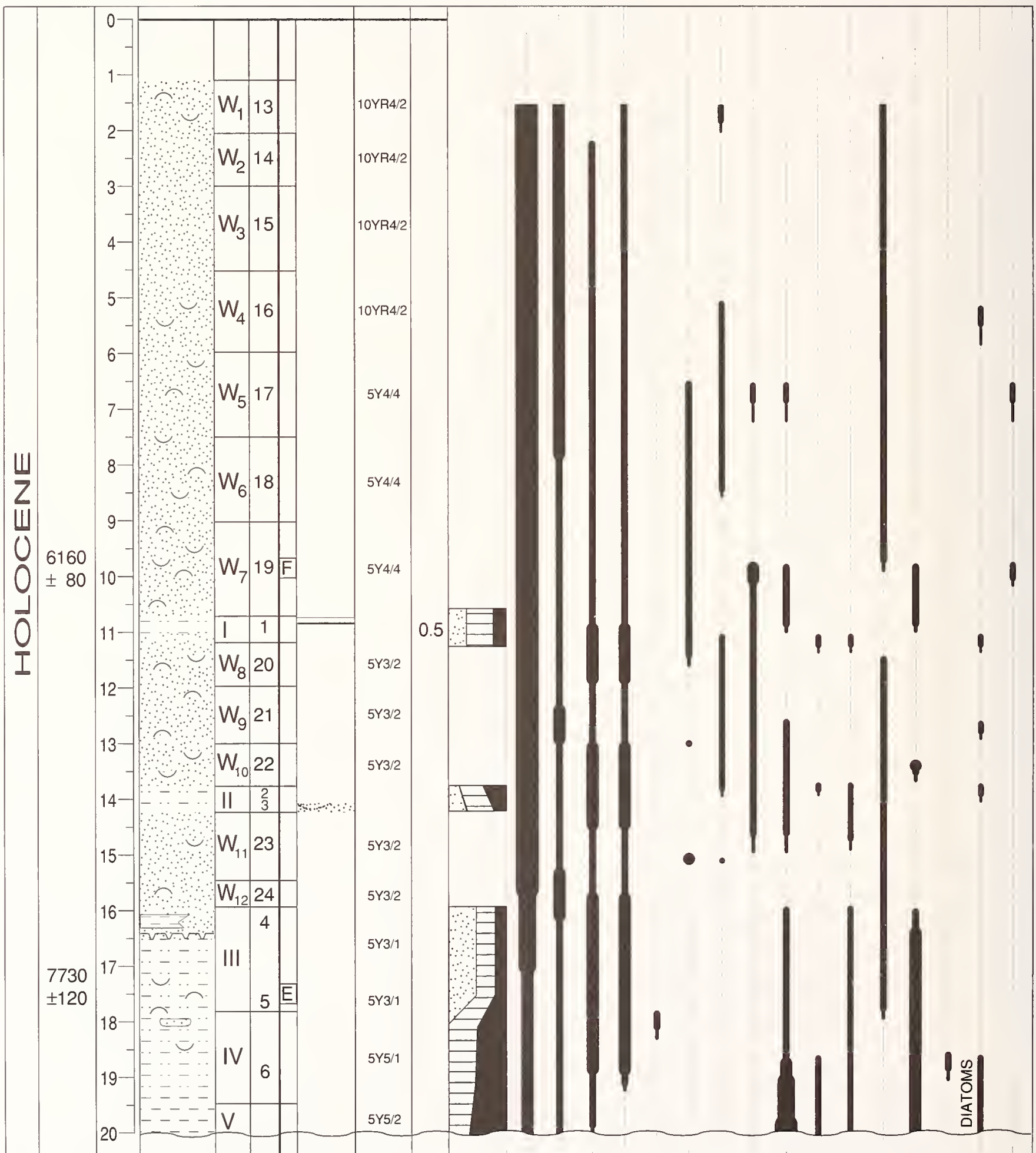


APPENDIX 1.-Continued.

CORE NUMBER S35॥

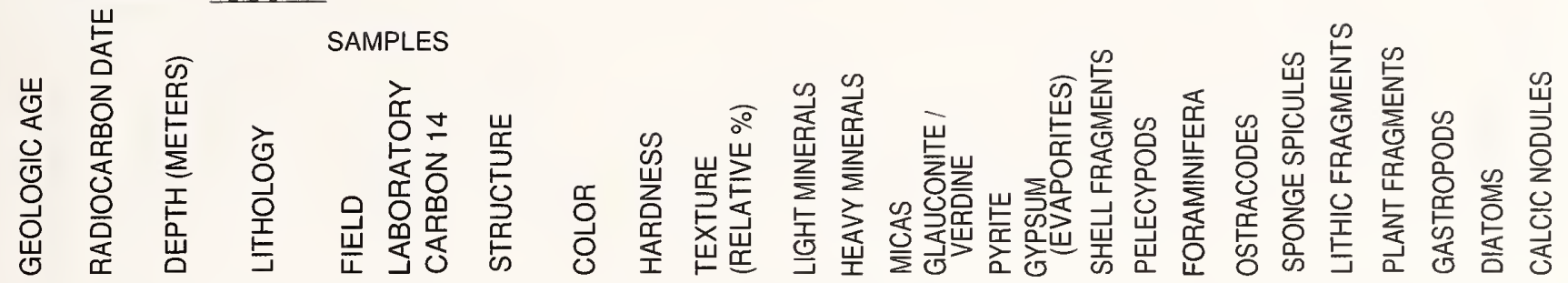

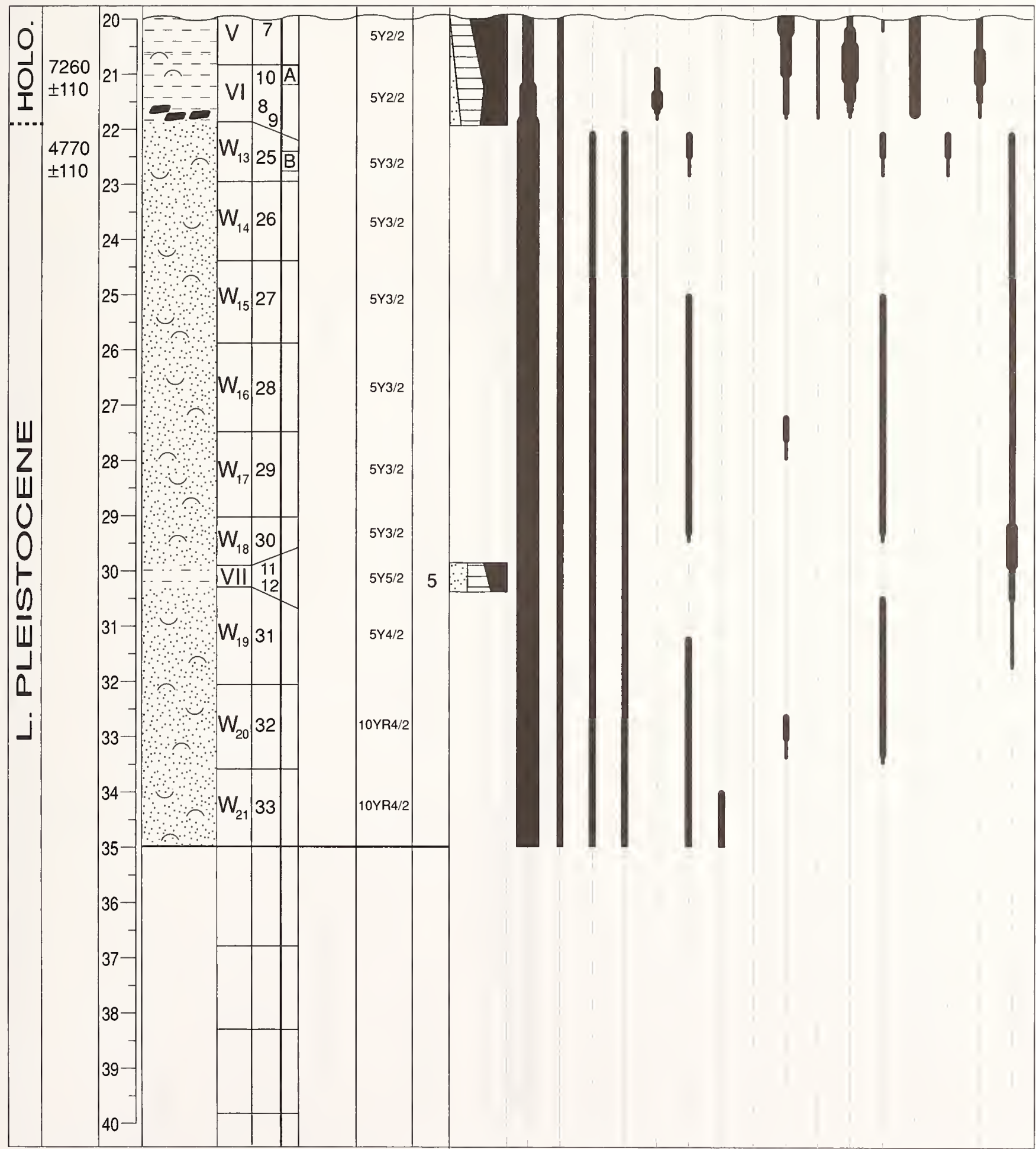


APPENDIX 1.-Continued.

CORE NUMBER S36 I

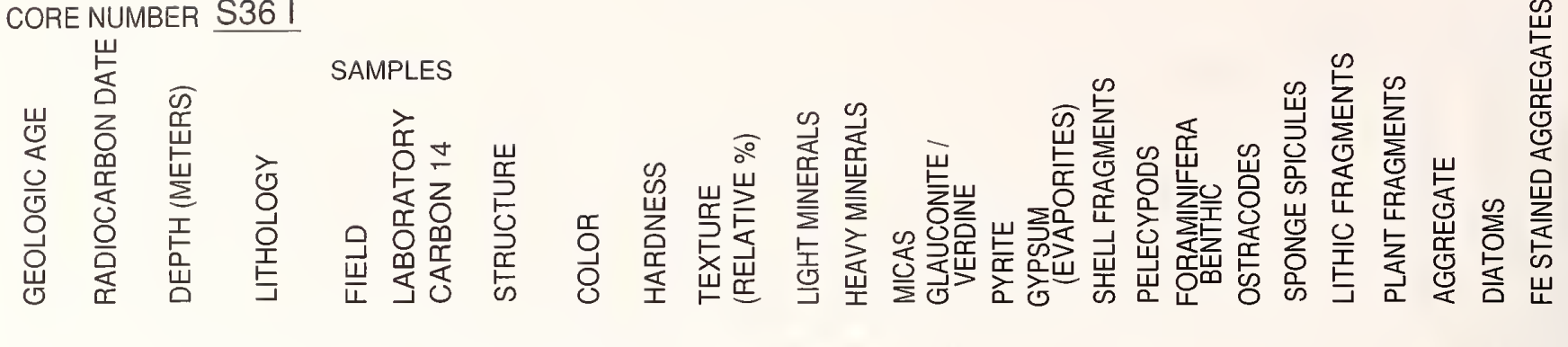

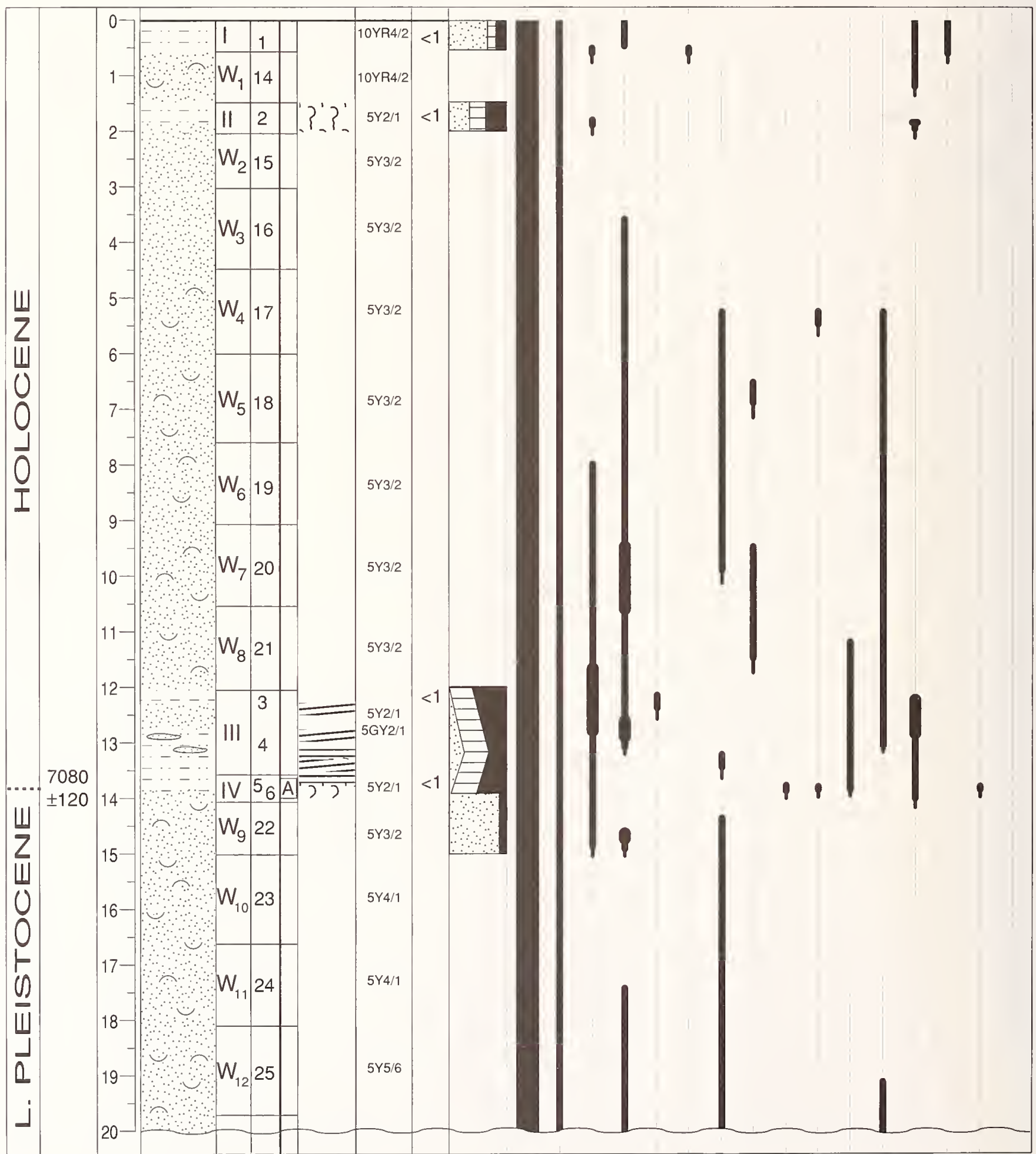


APPENDIX 1.-Continued.
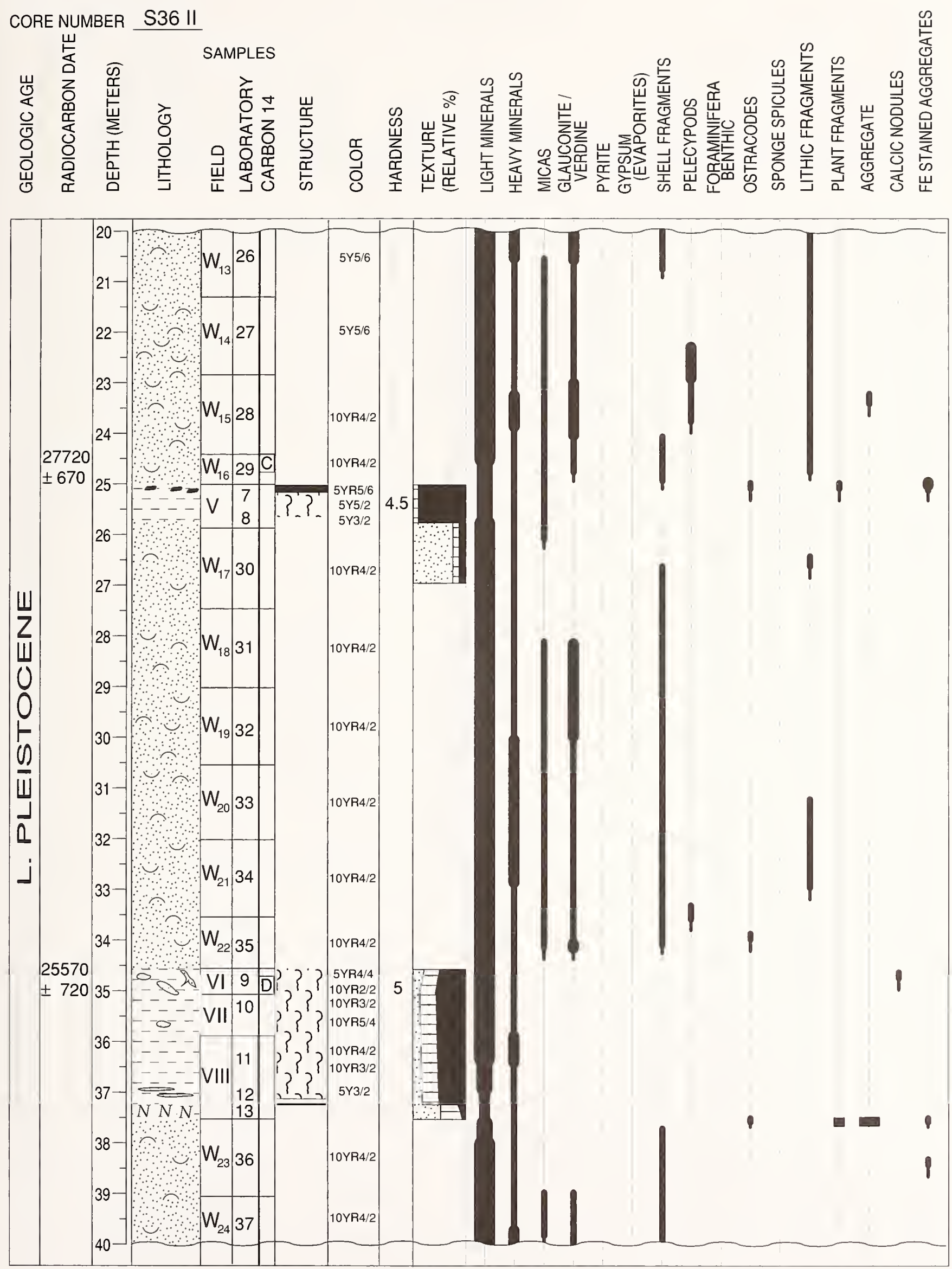
APPENDIX 1.-Continued.
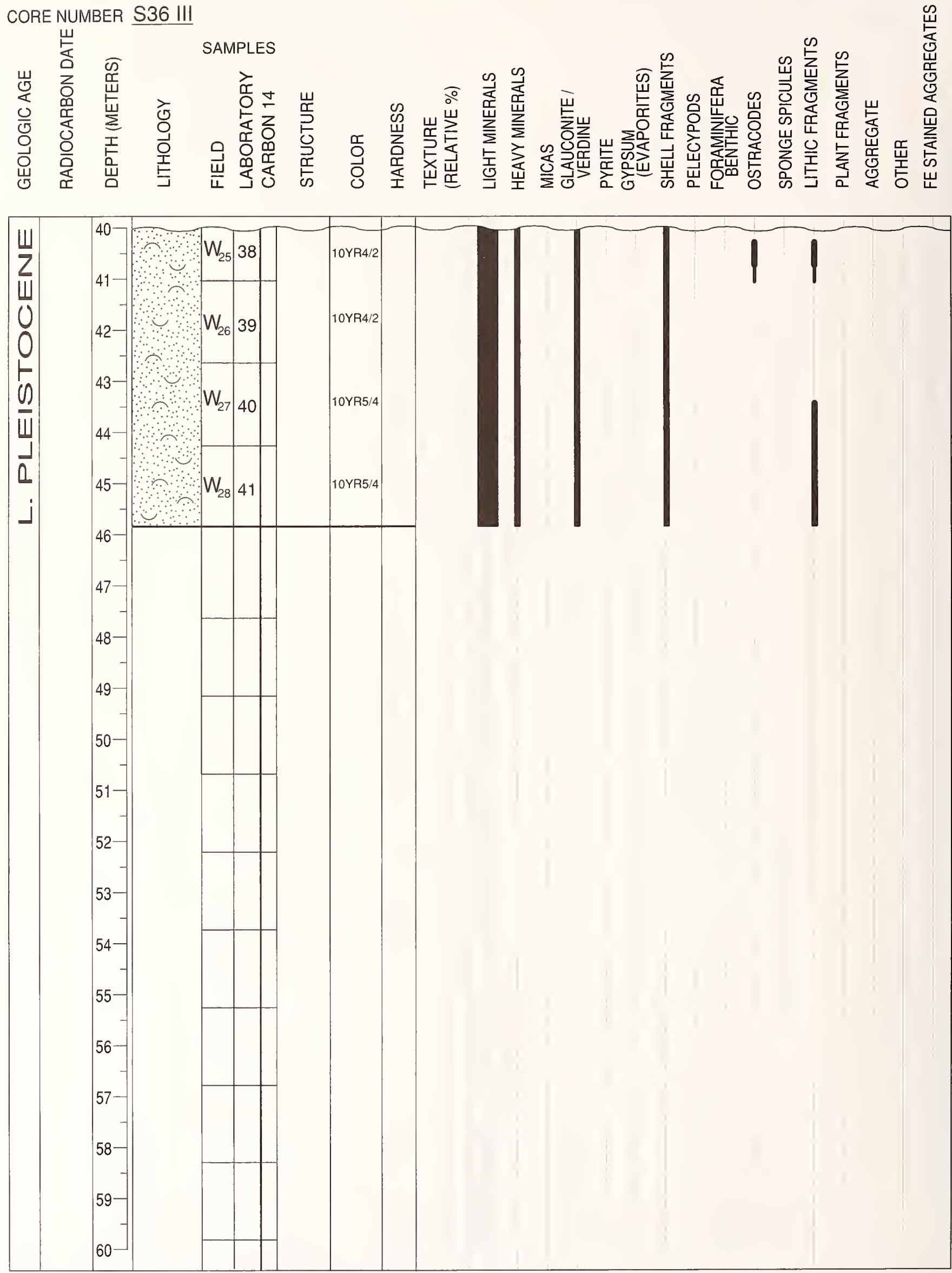
APPENDIX 1.-Continued.

CORE NUMBER S37 I

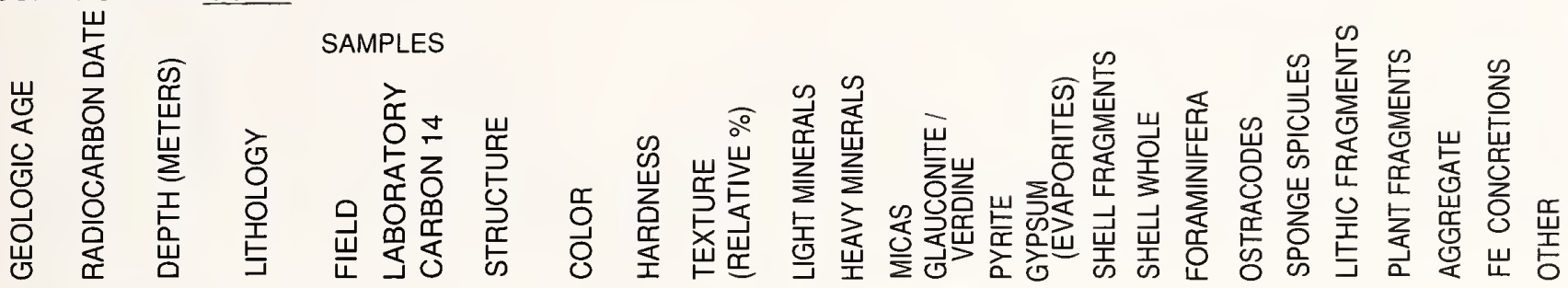

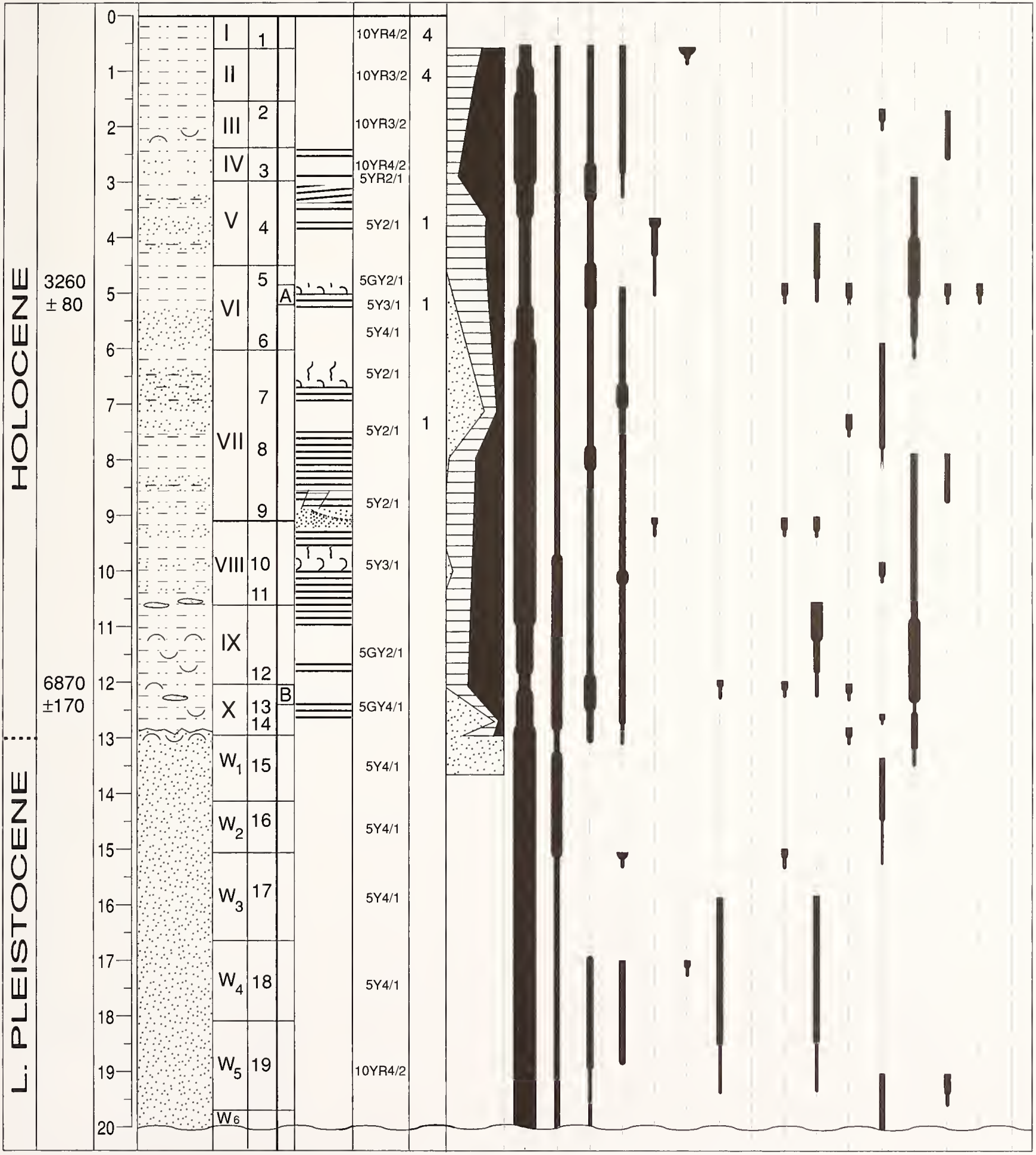


APPENDIX 1.-Continued.

CORE NUMBER S37 II

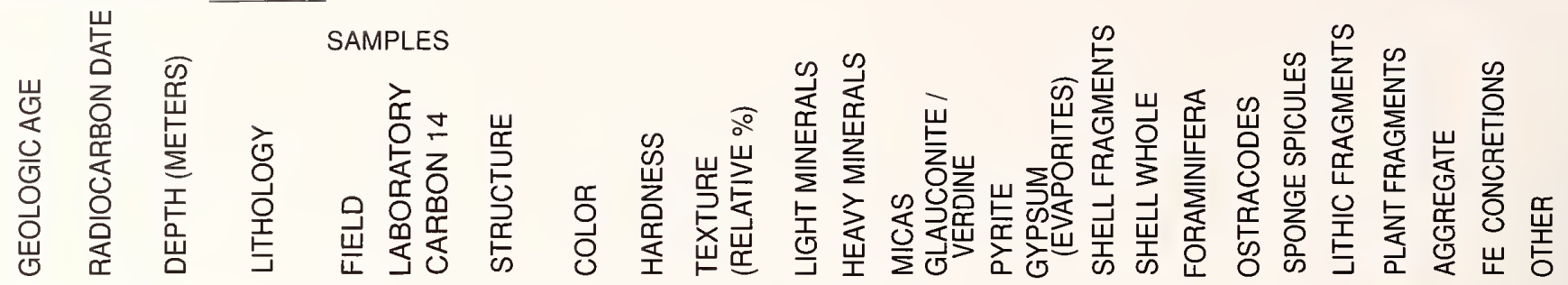

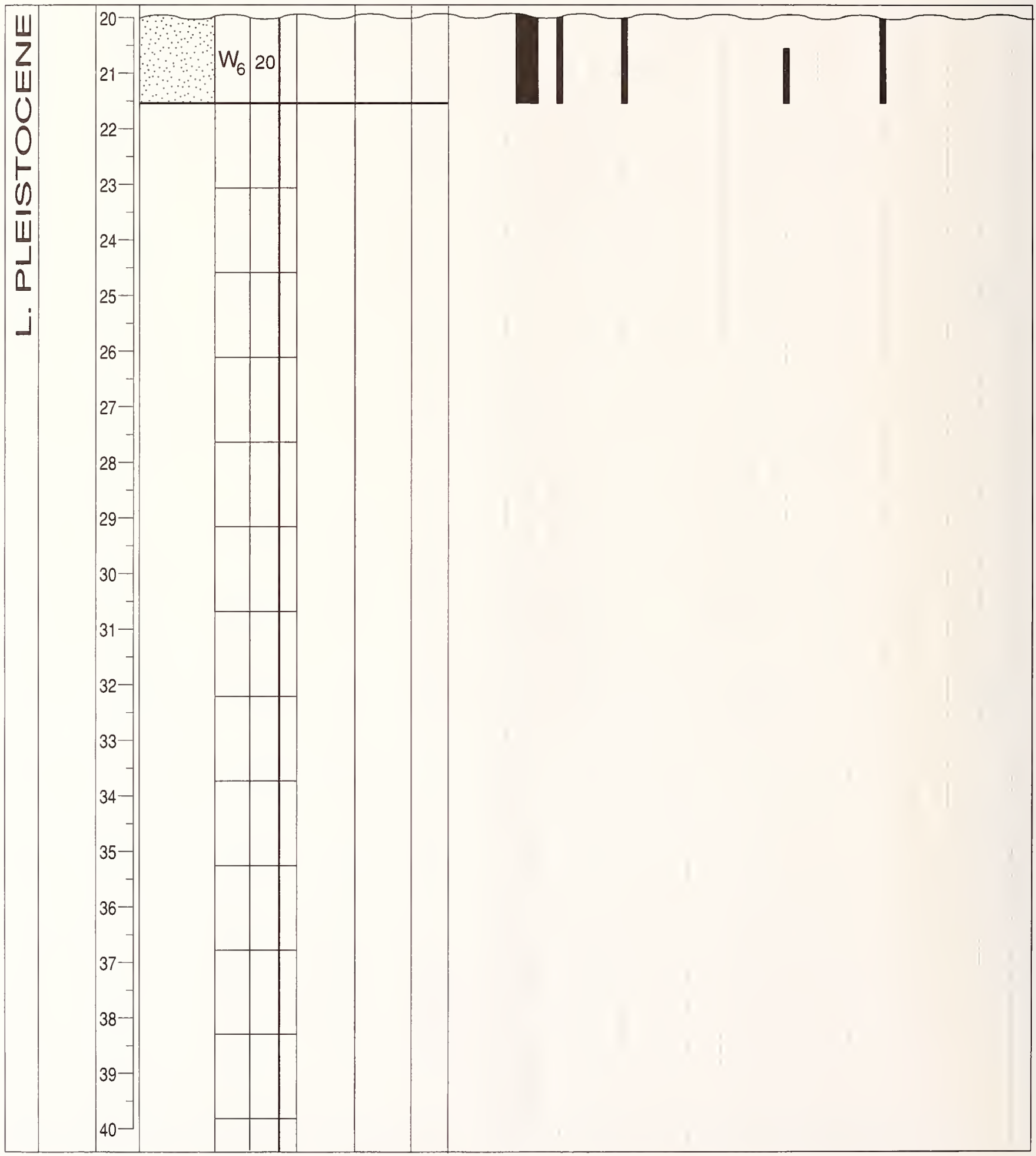


APPENDIX 1.-Continued.

CORE NUMBER S38 I
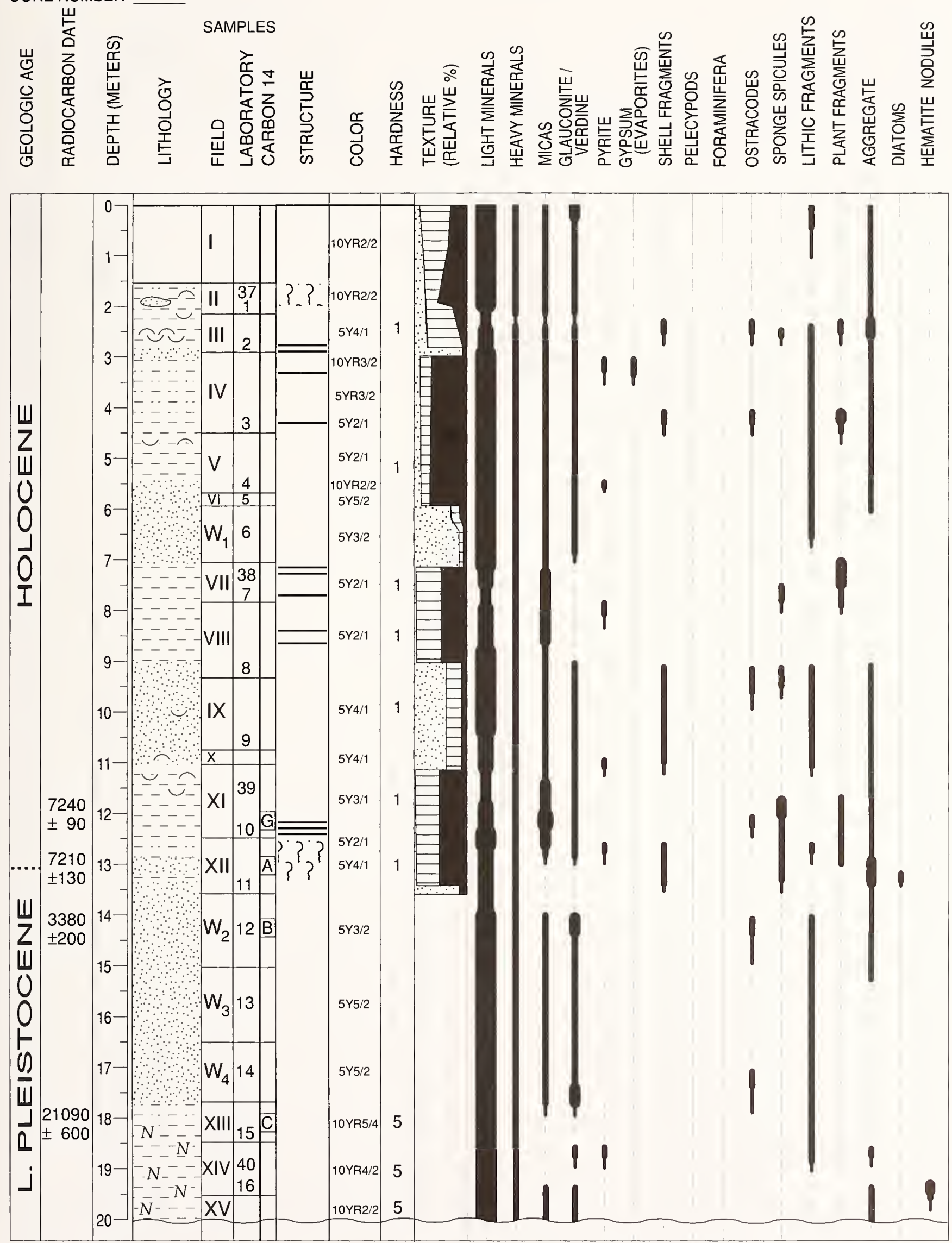
APPENDIX 1.-Continued.

CORE NUMBER S38 II

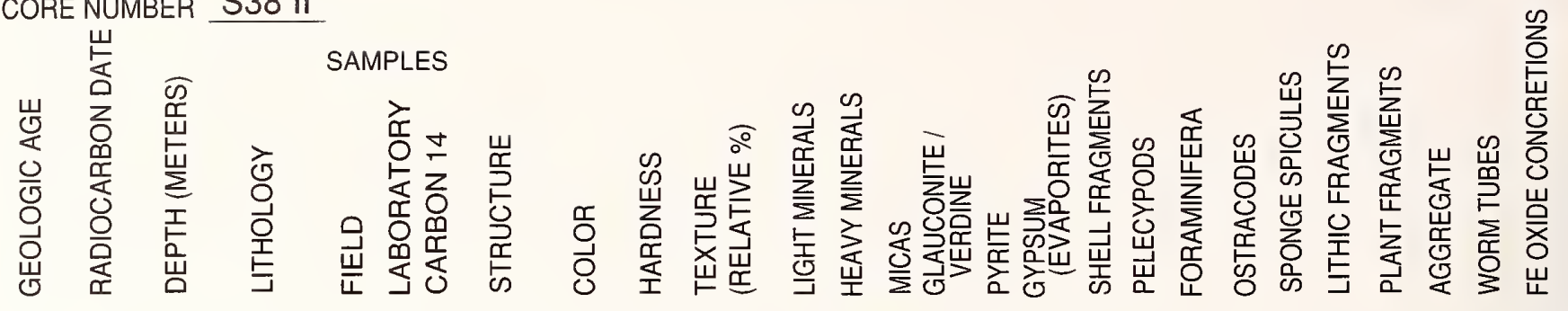

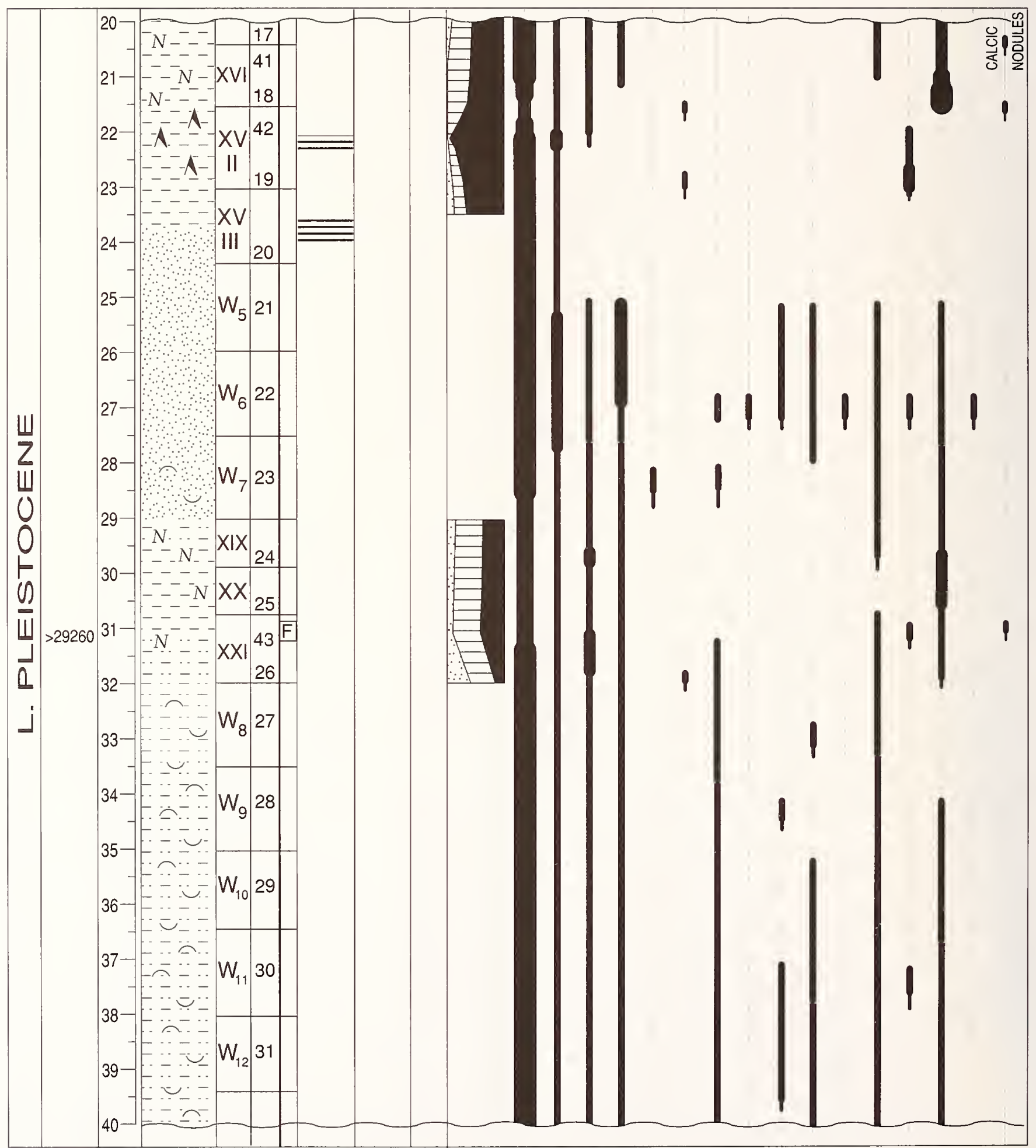


APPENDIX 1.-Continued.

\section{CORE NUMBER S38 III}

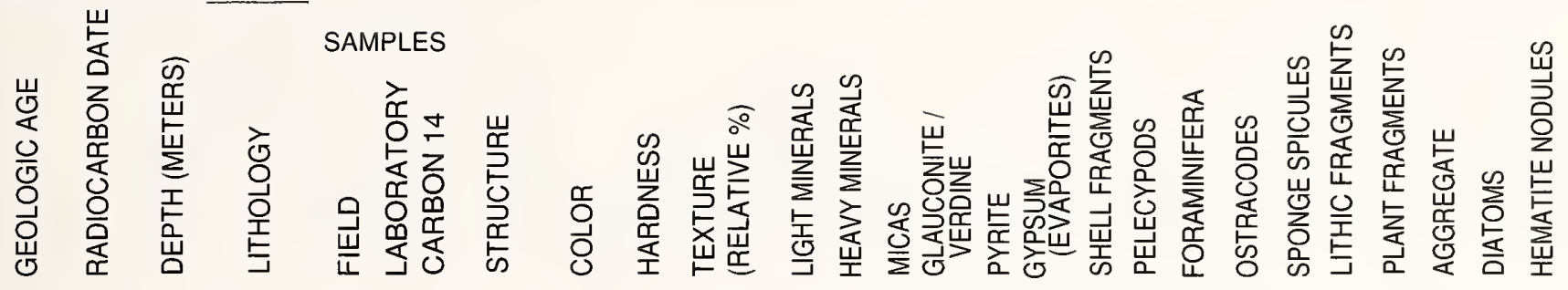

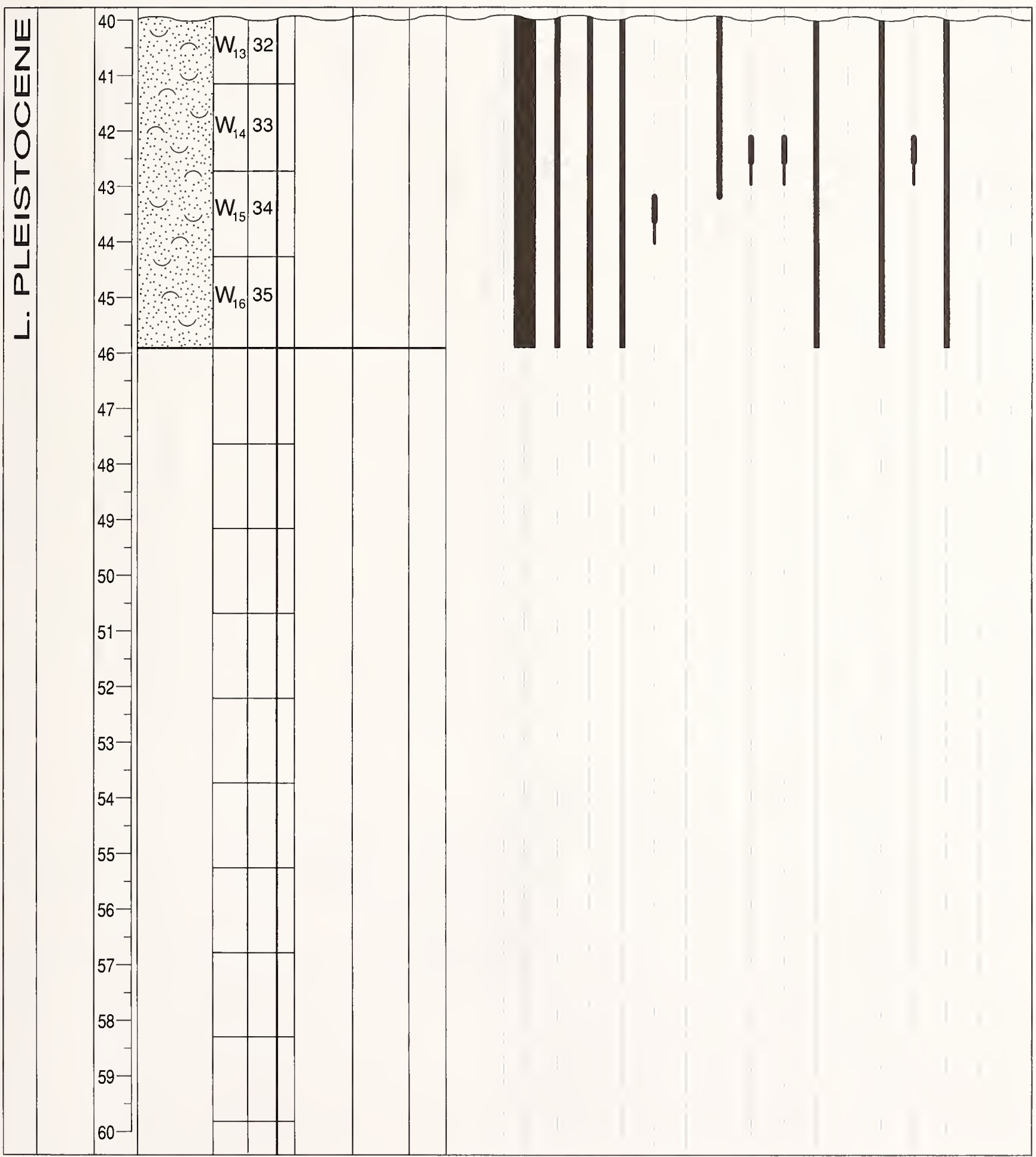


APPENDIX 1.-Continued.
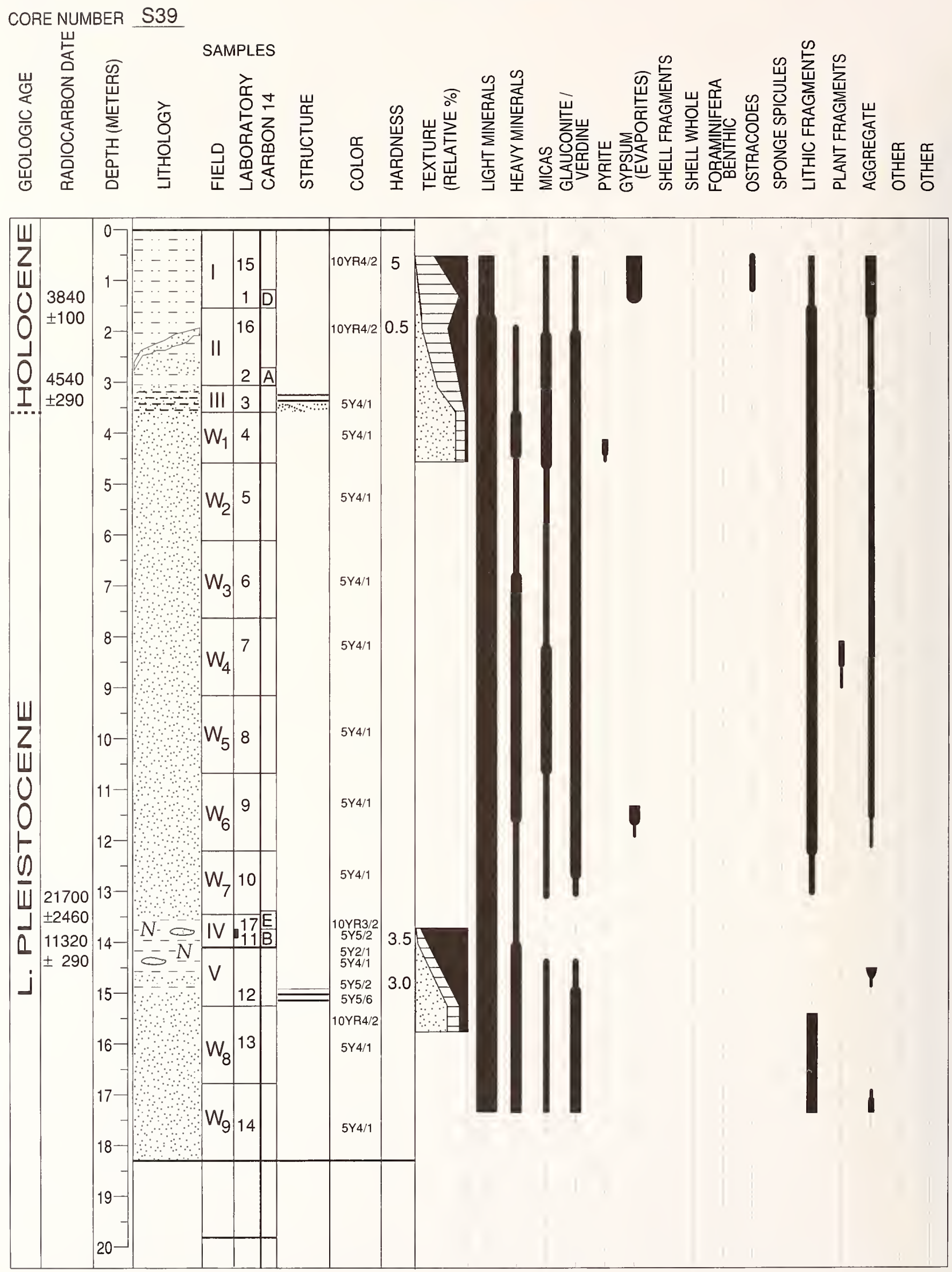
APPENDIX 1.-Continued.

CORE NUMBER S40I

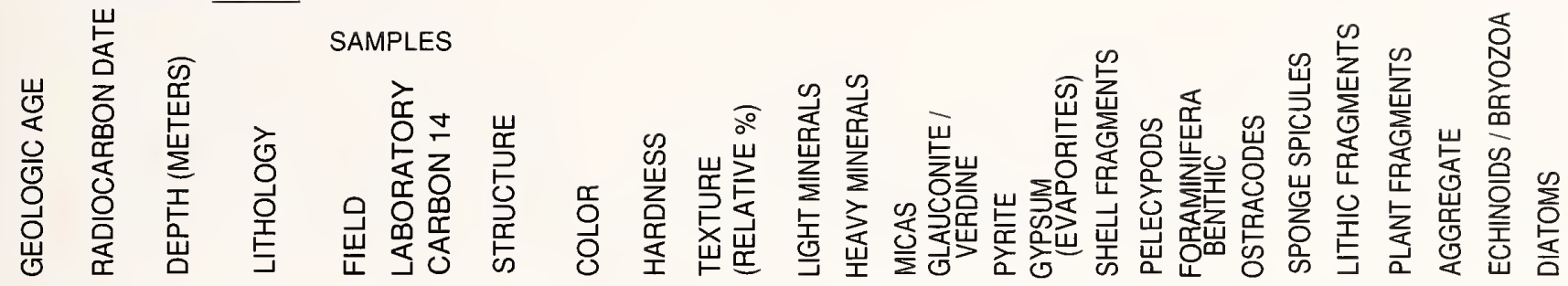

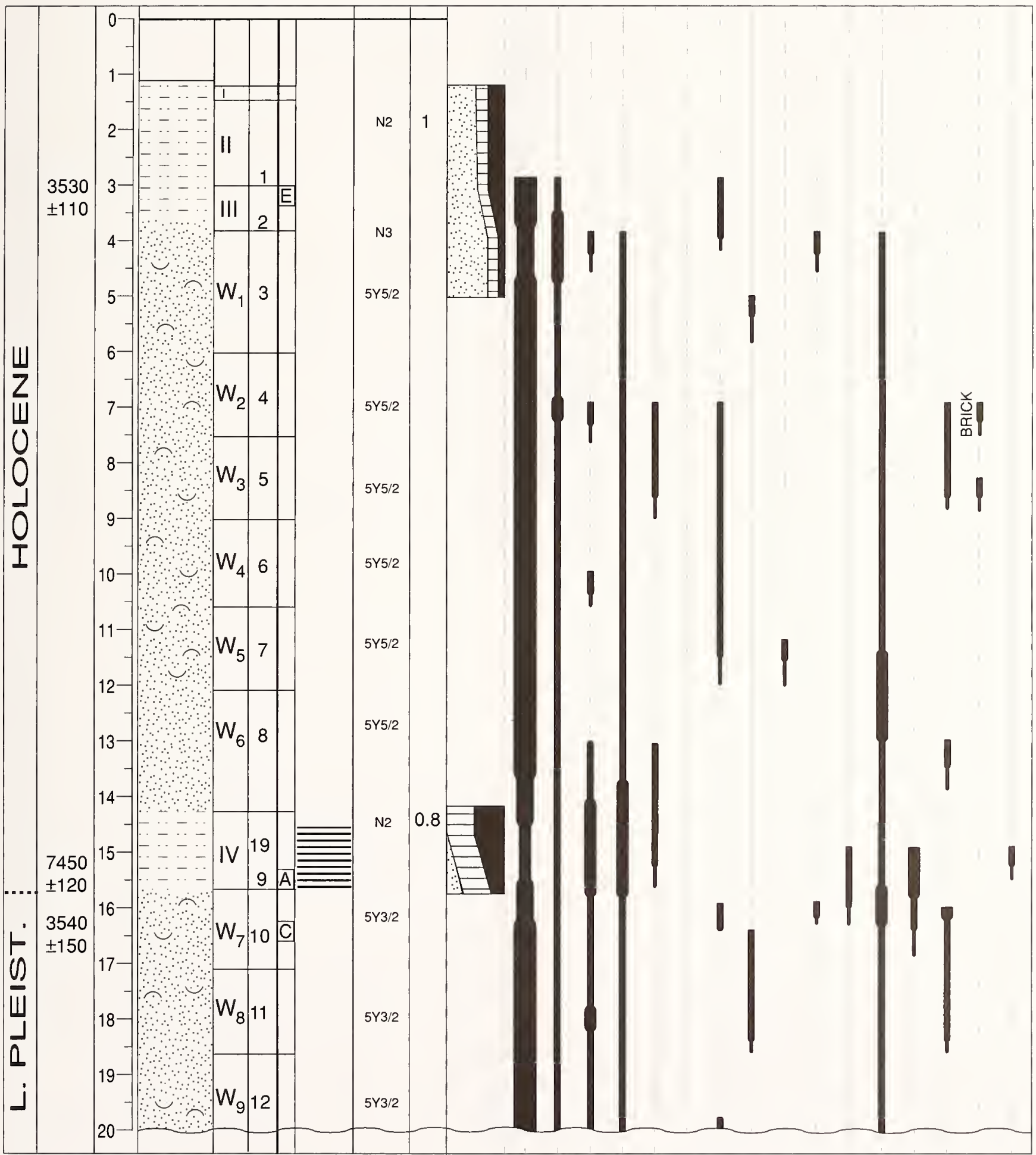


APPENDIX 1.-Continued.

CORE NUMBER S 40 II

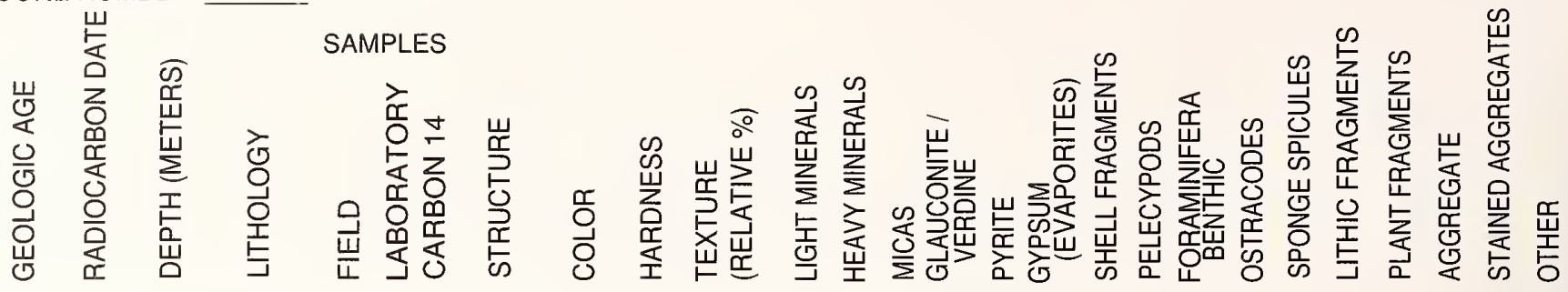

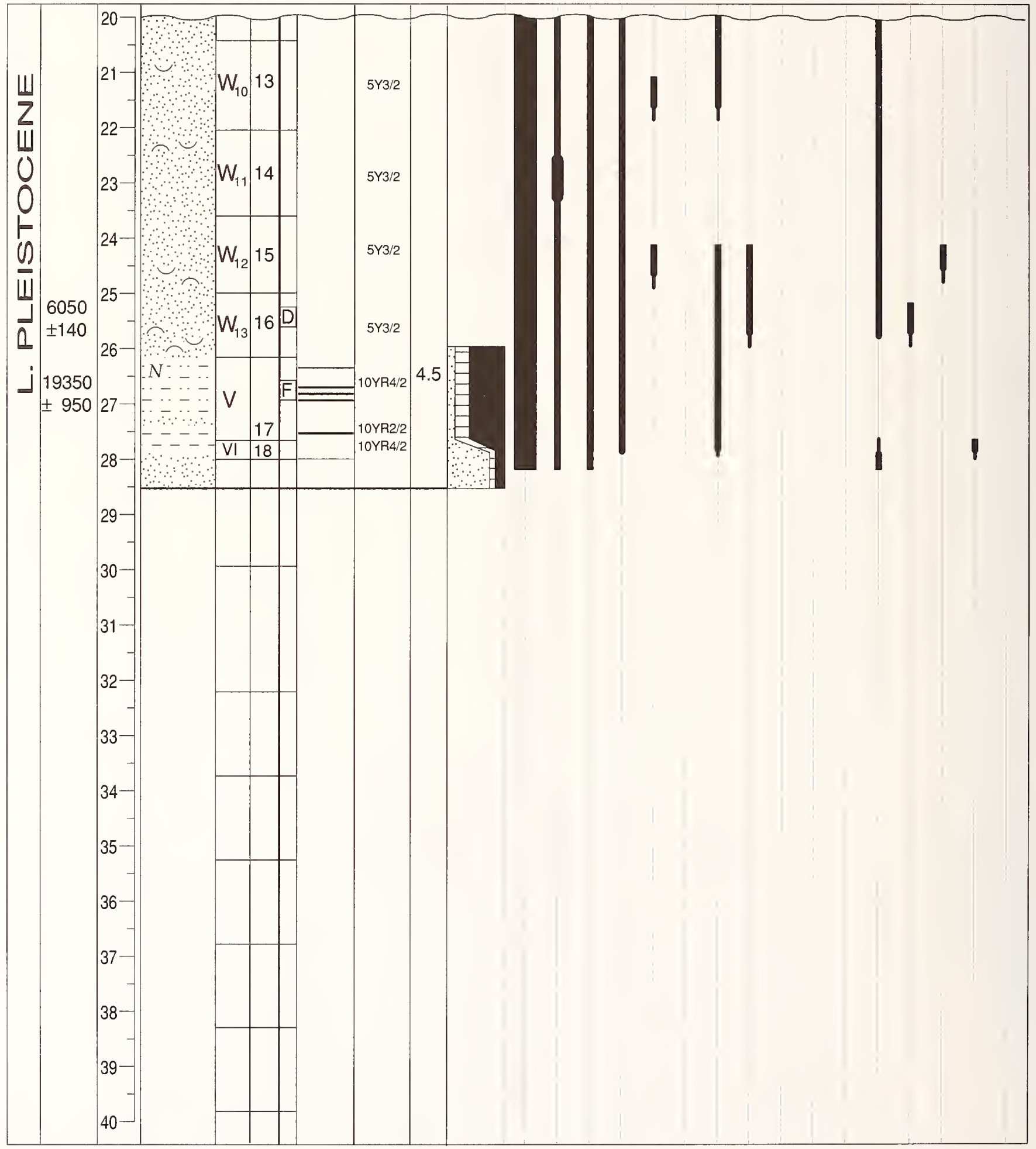


APPENDIX 1.-Continued.

CORE NUMBER S41I

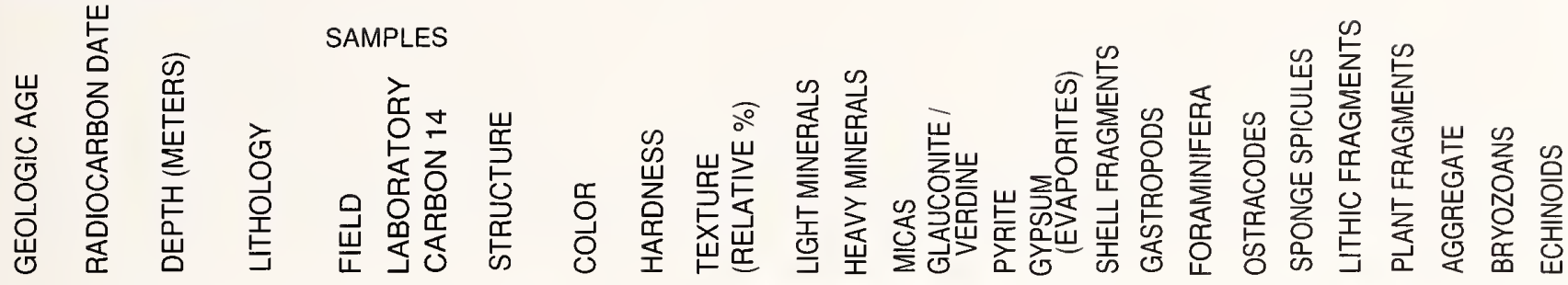

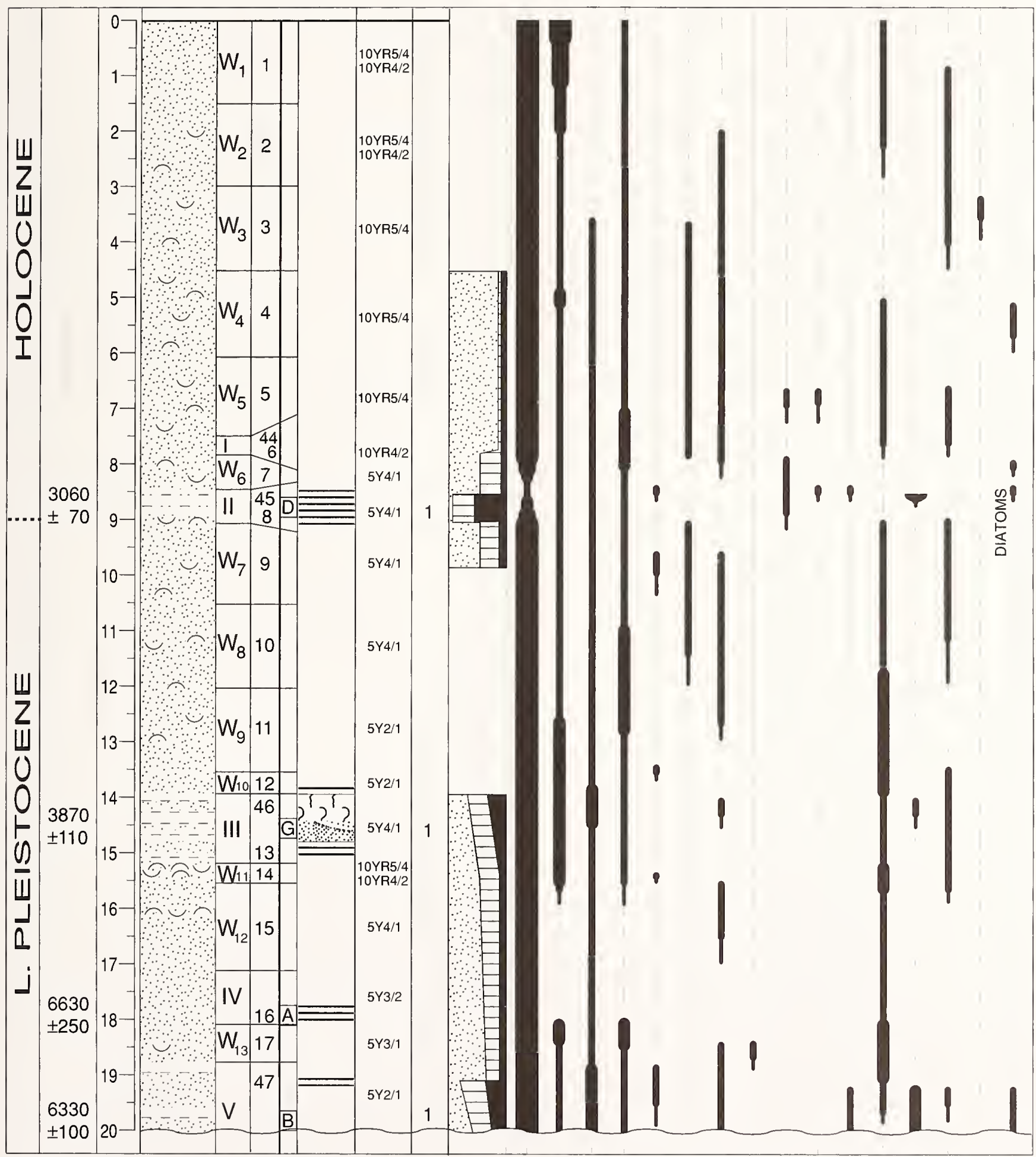


APPENDIX 1.-Continued.

CORE NUMBER S41II
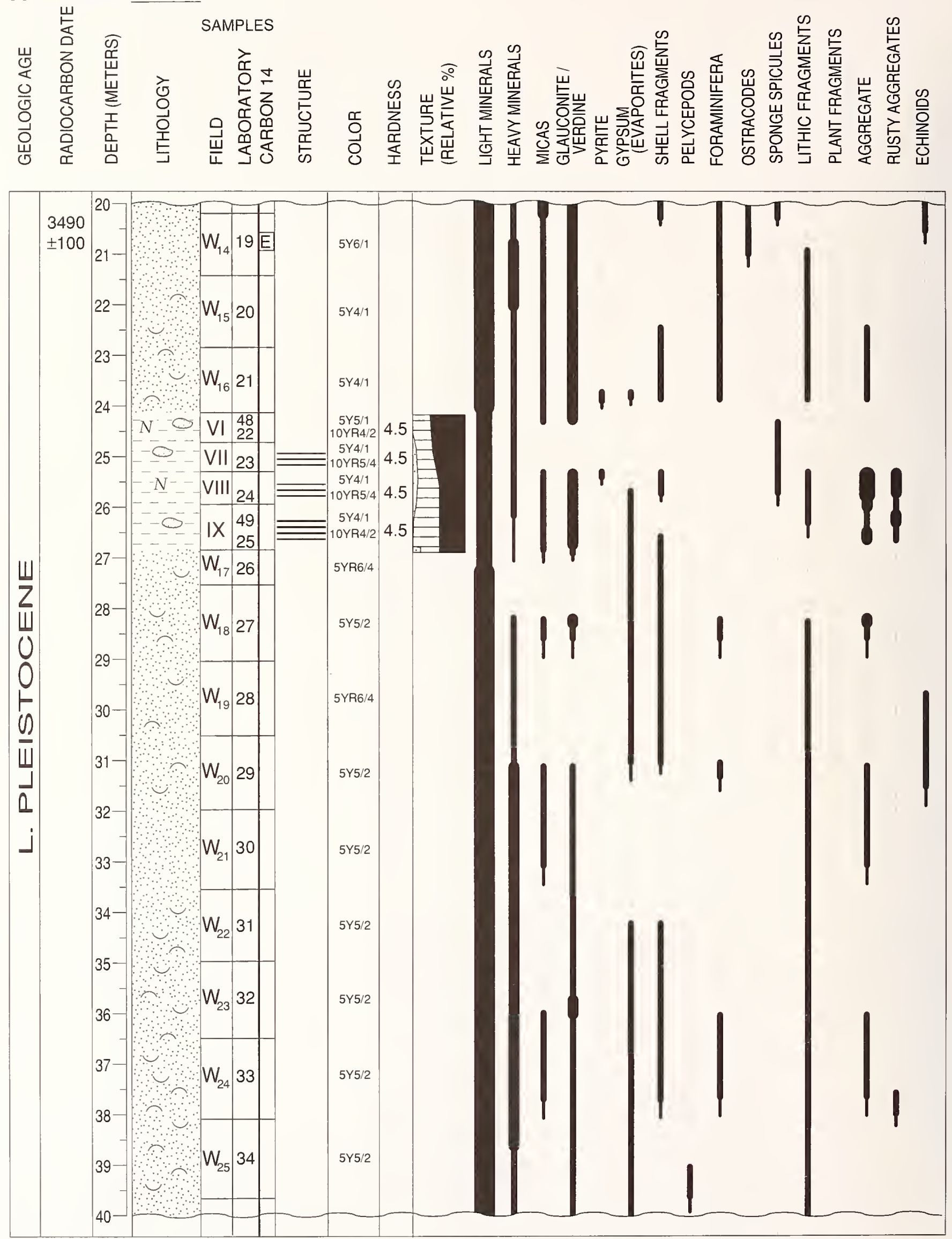
APPENDIX 1.-Continued.

CORE NUMBER S41 III

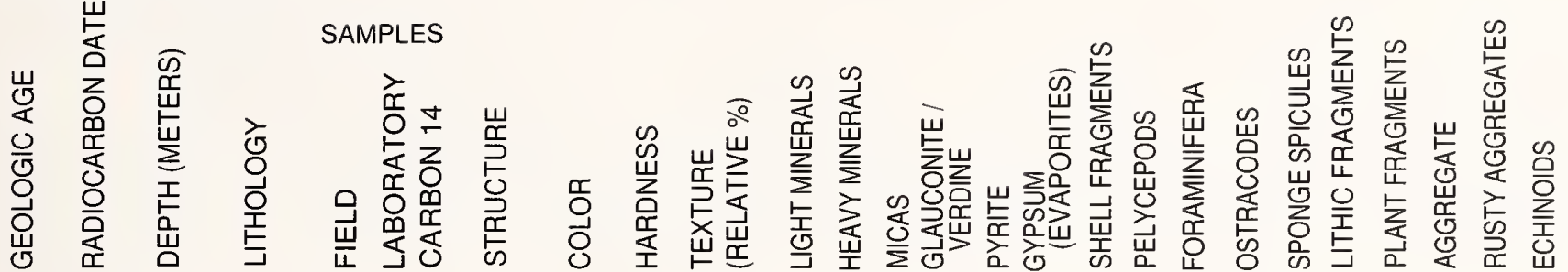

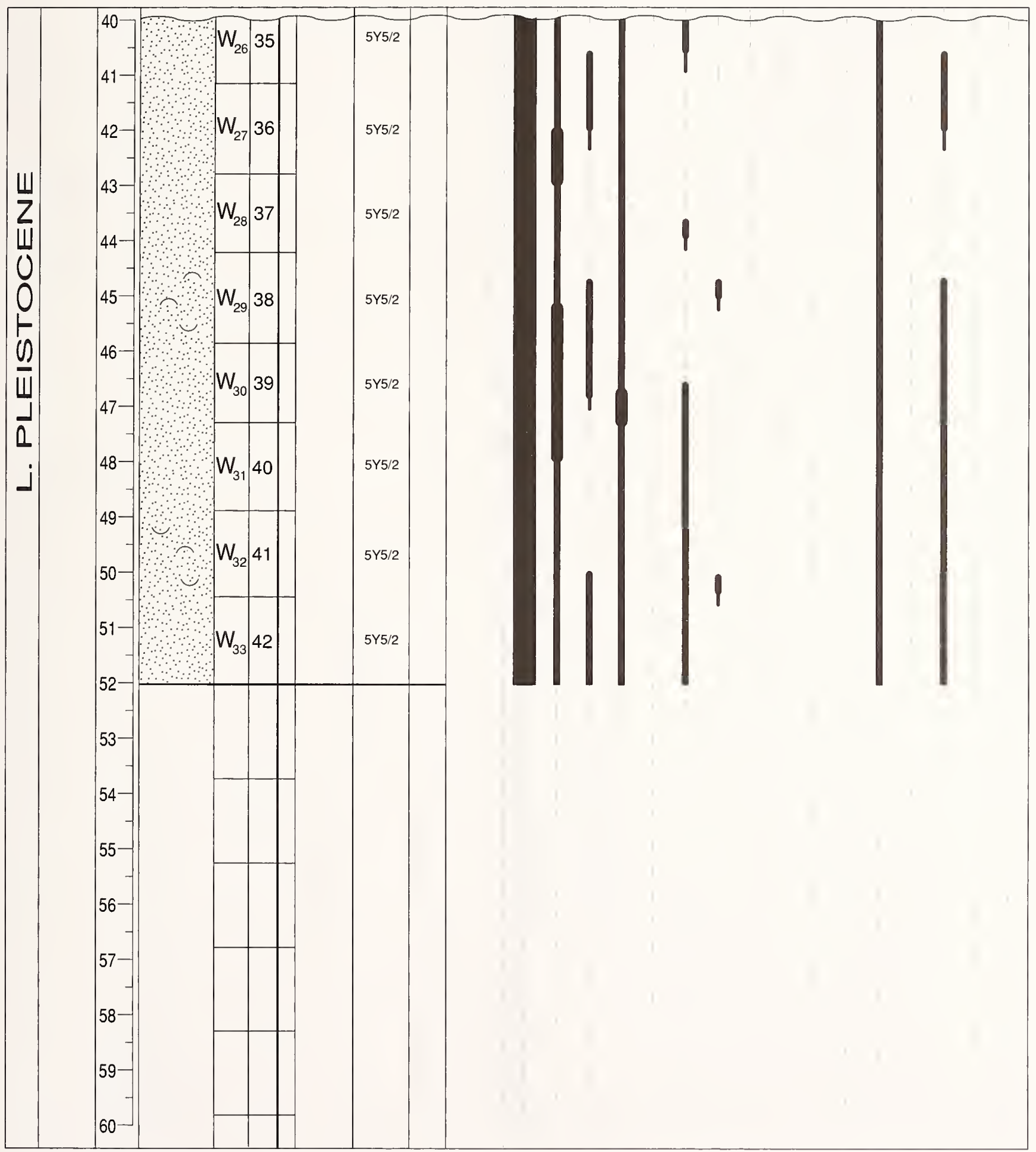


APPENDIX 1.-Continued.

CORE NUMBER S42I

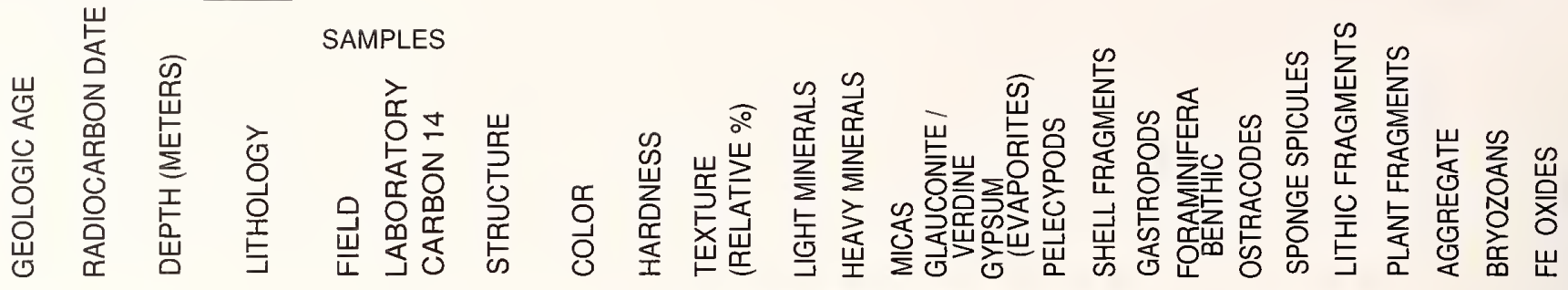

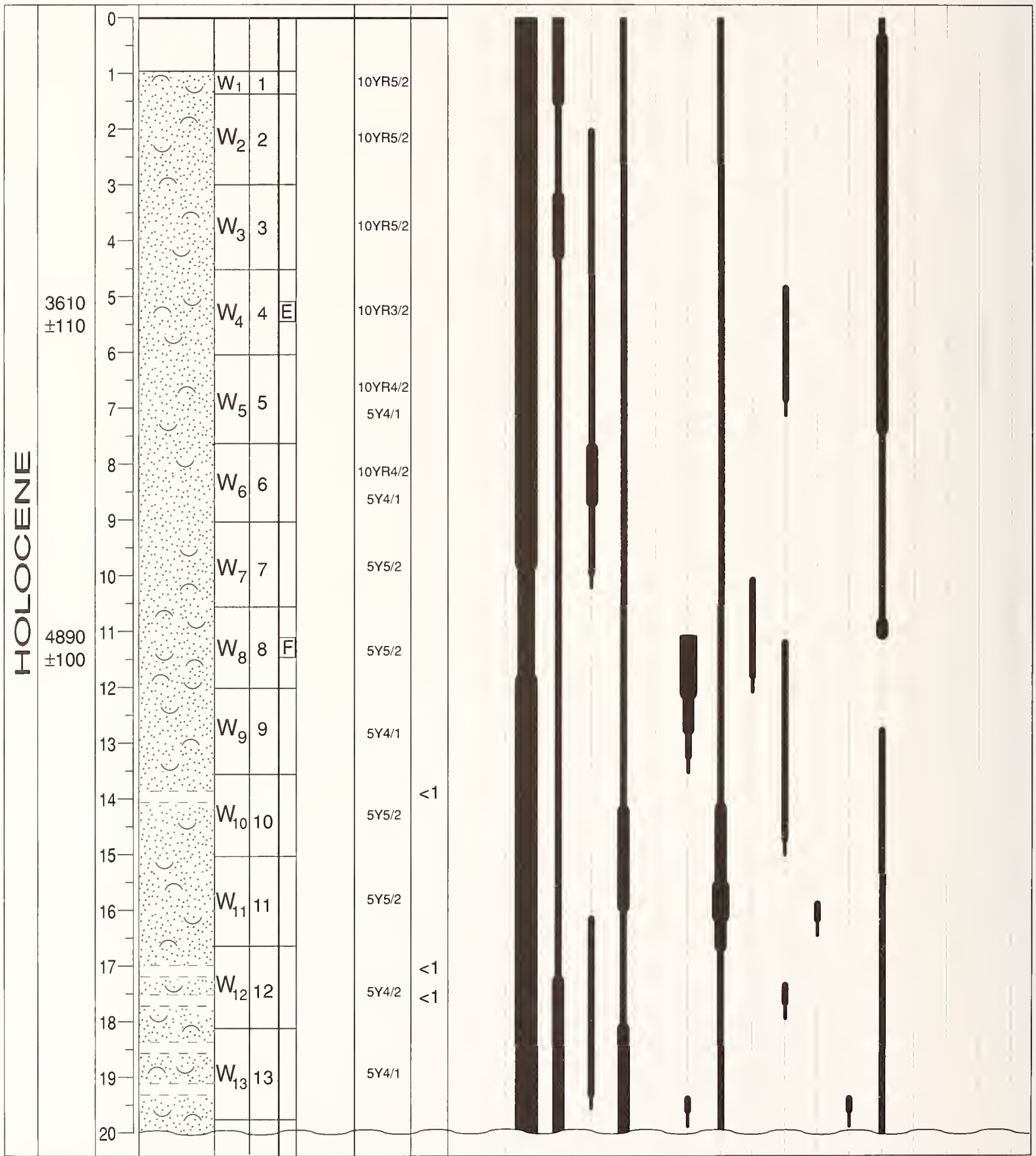


APPENDIX 1.-Continued

CORE NUMBER S42 II

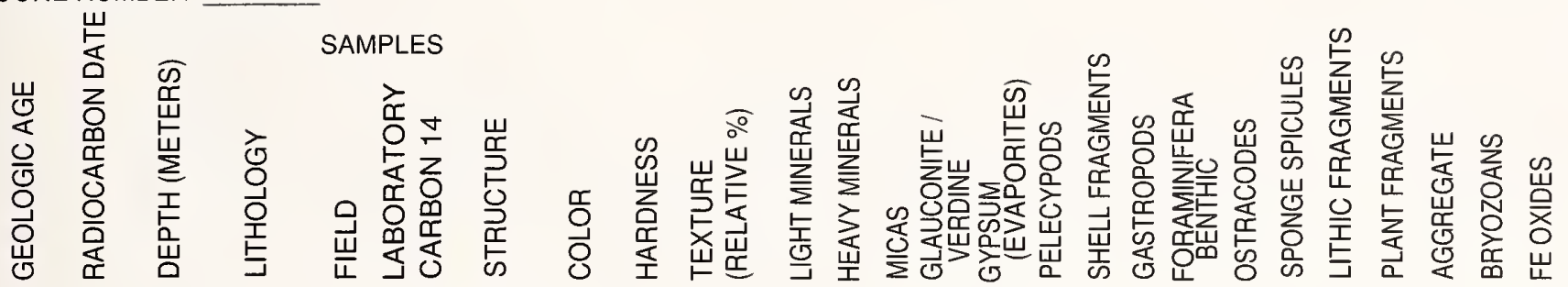

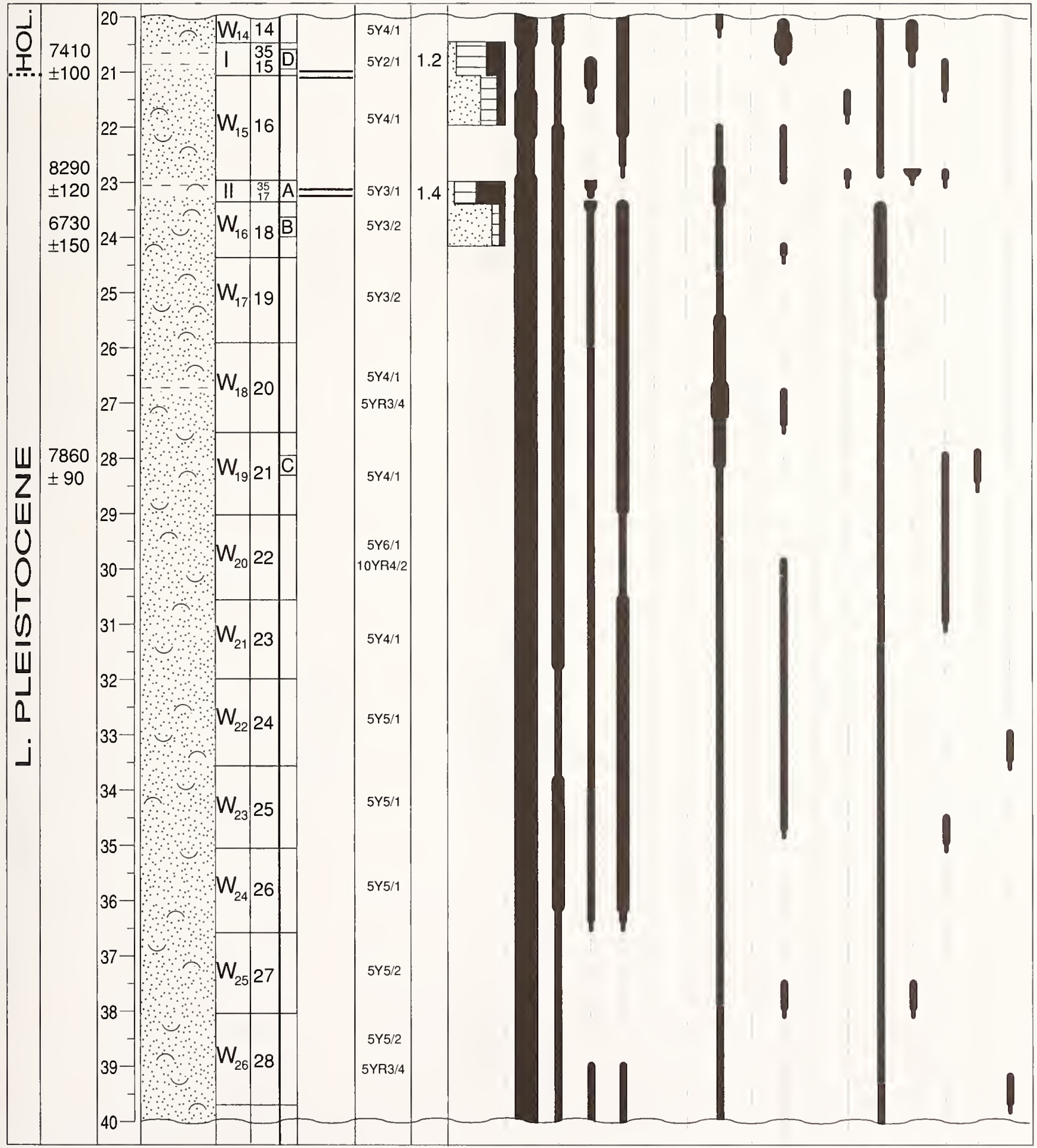


APPENDIX 1.-Continued.

CORE NUMBER S42 III

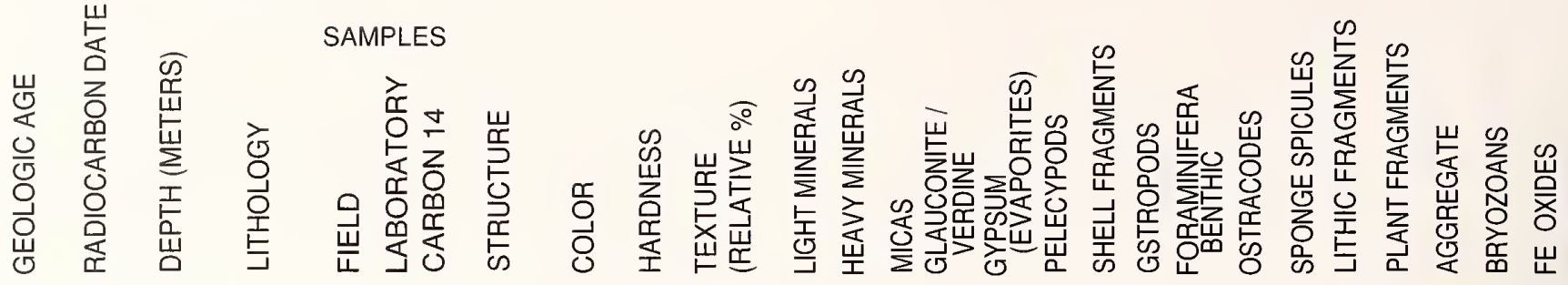

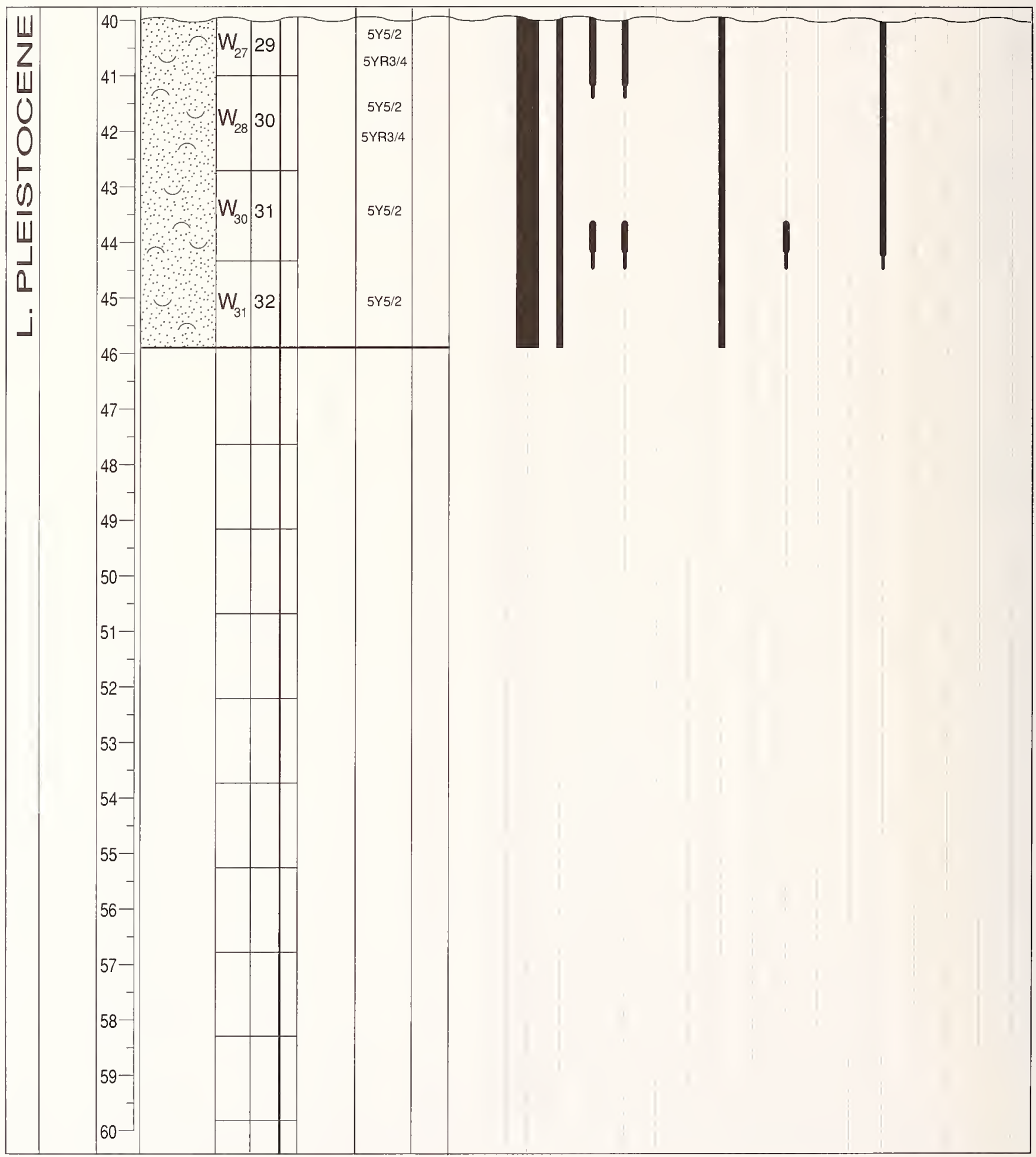


APPENDIX 1.-Continued.

CORE NUMBER S43I

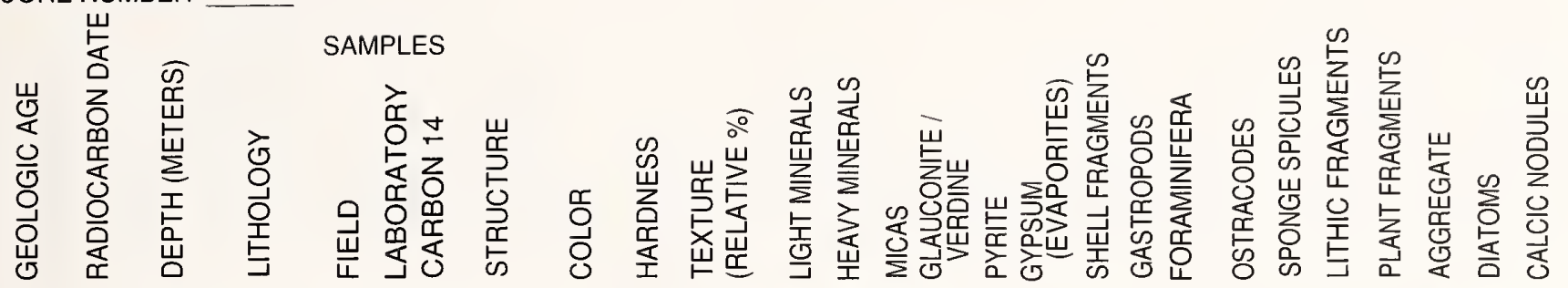

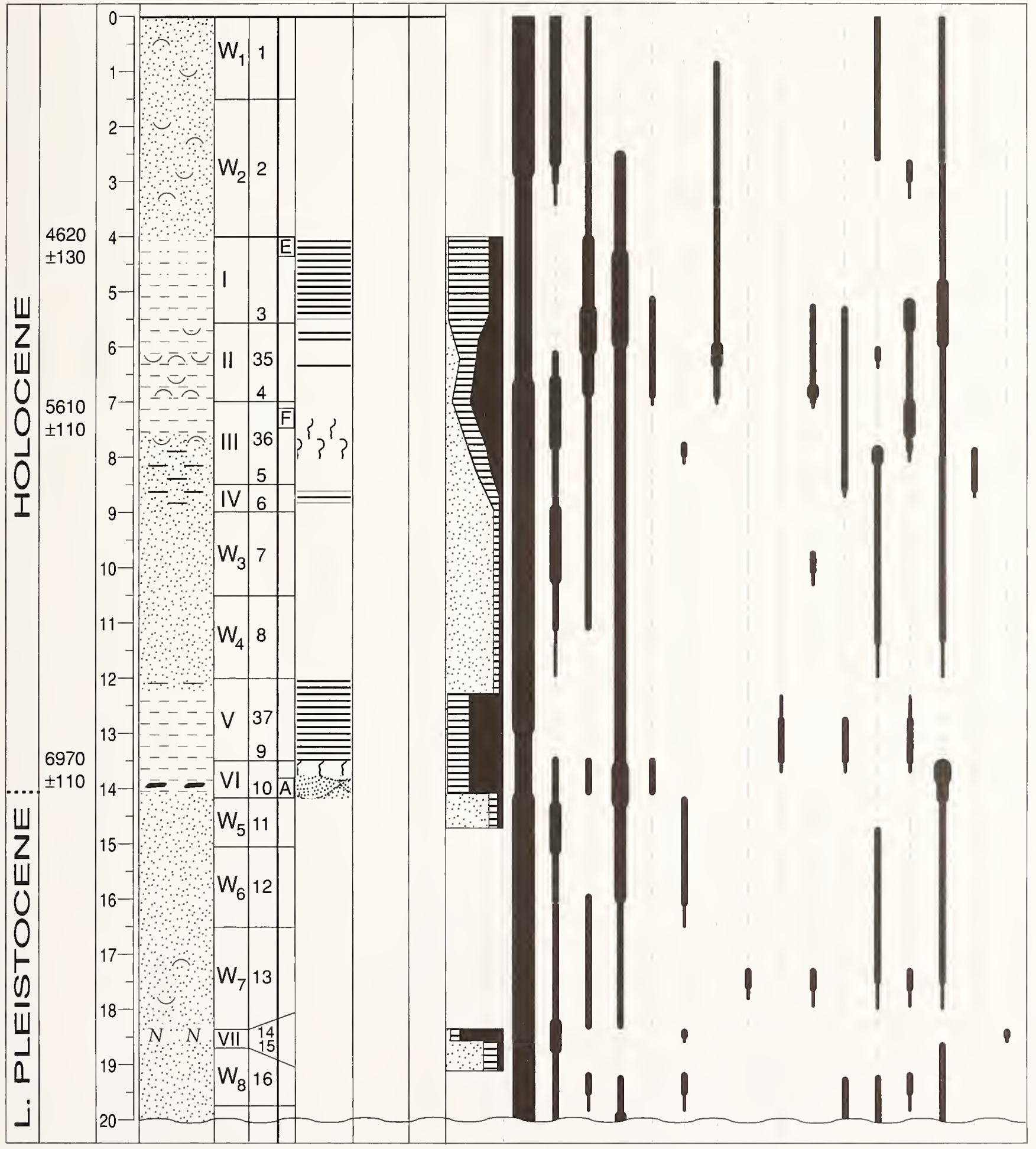


APPENDIX 1.-Continued.

CORE NUMBER S43 II

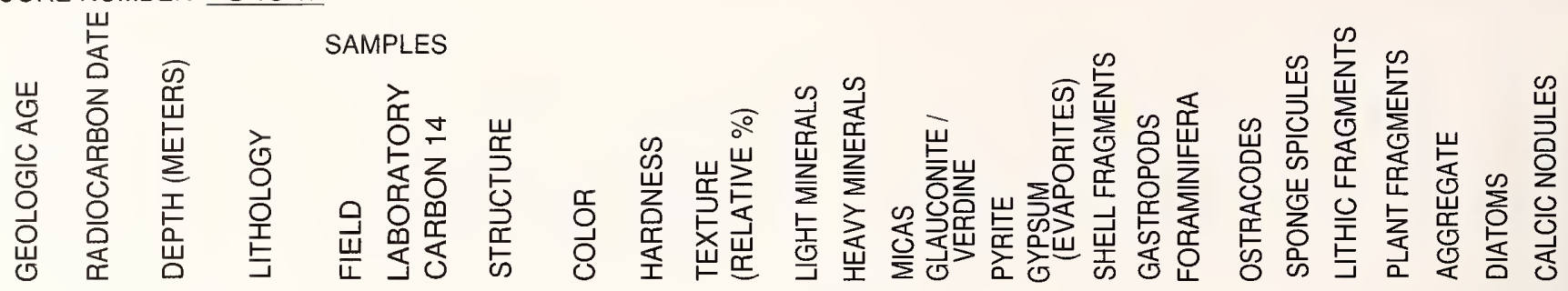

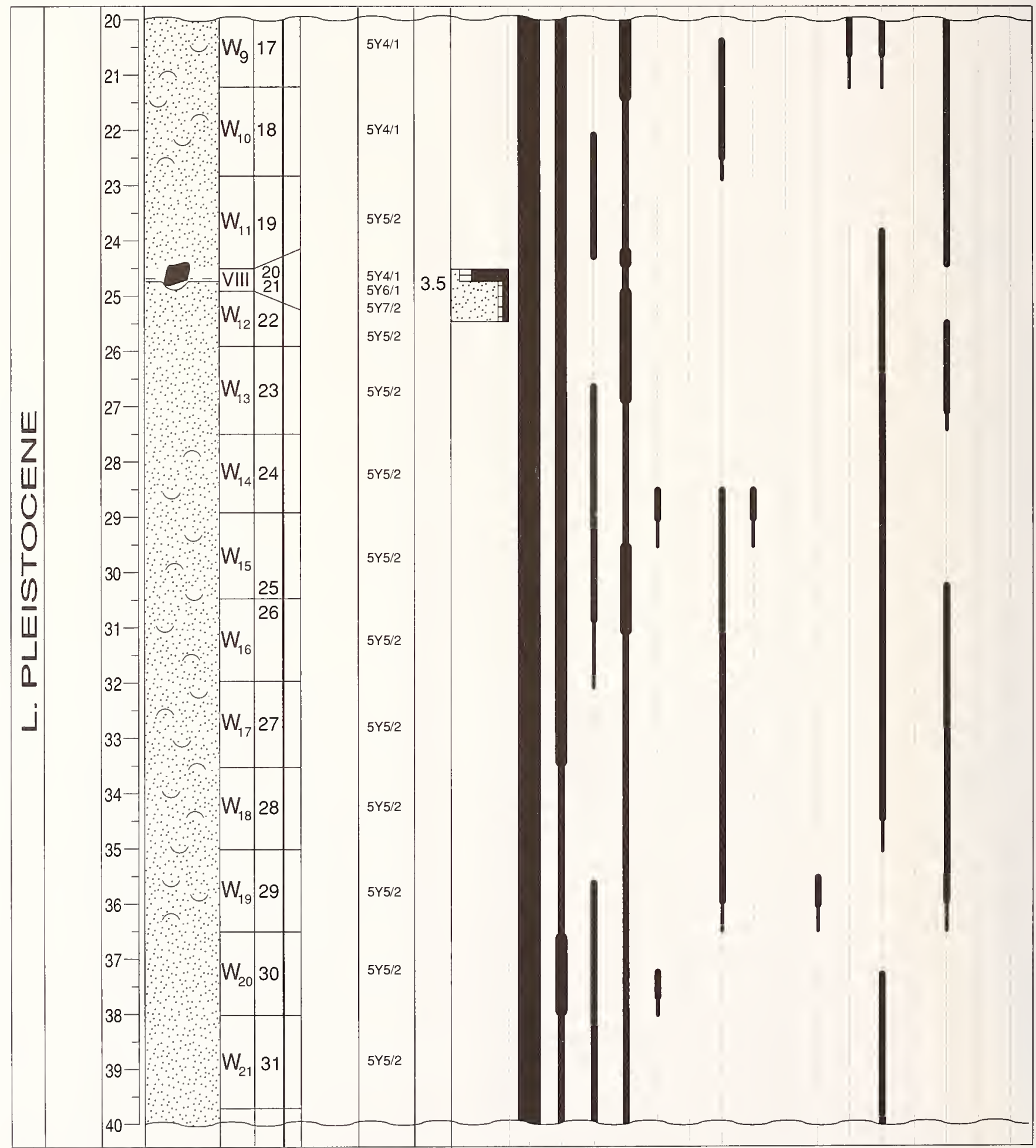


APPENDIX 1.-Continued.

CORE NUMBER S43 III

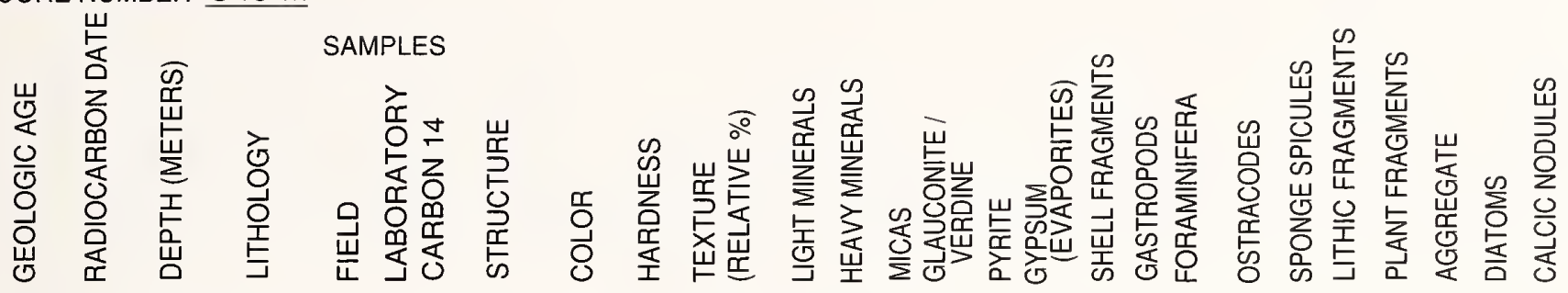

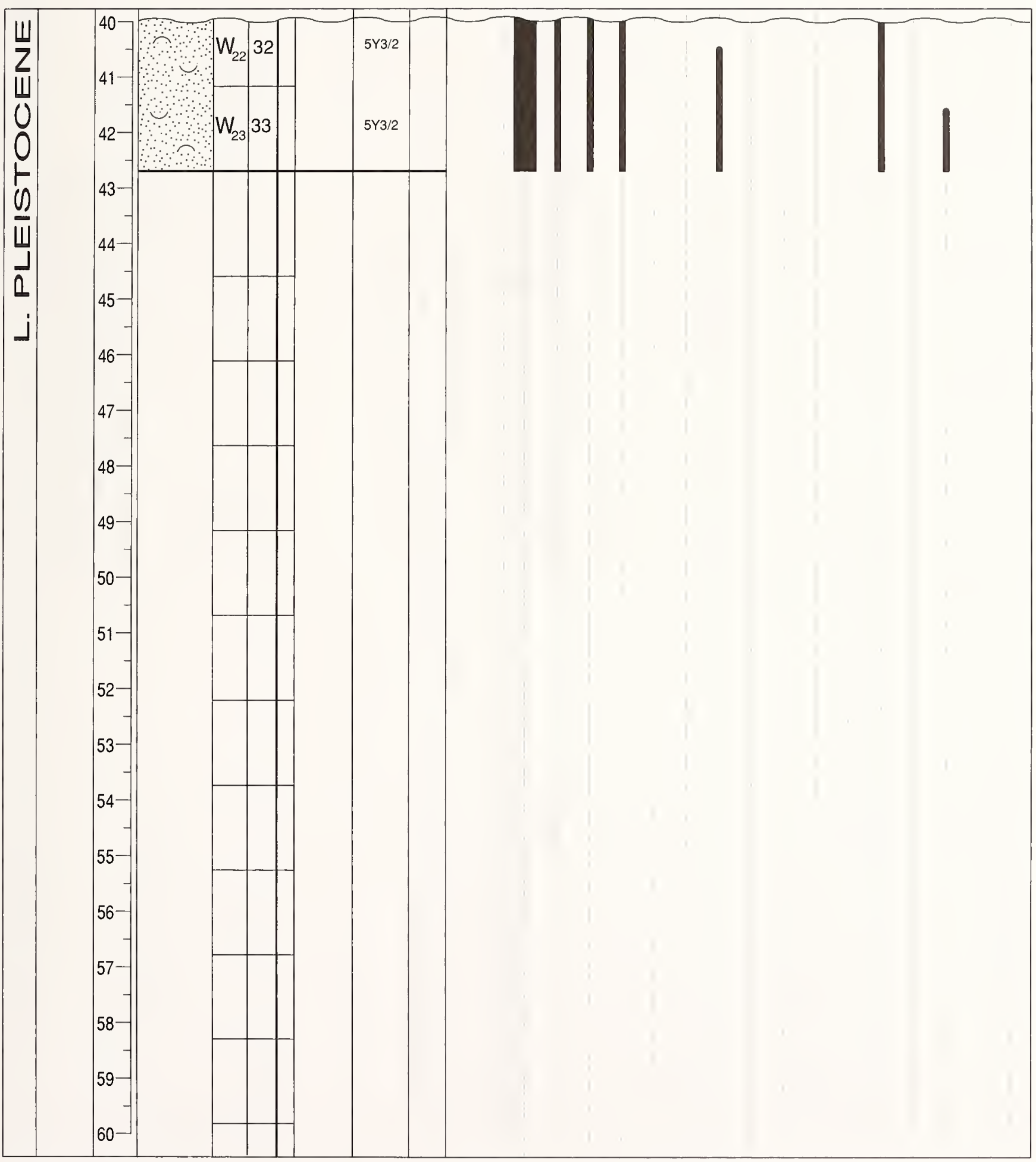


APPENDIX 1.-Continued.

CORE NUMBER S44I
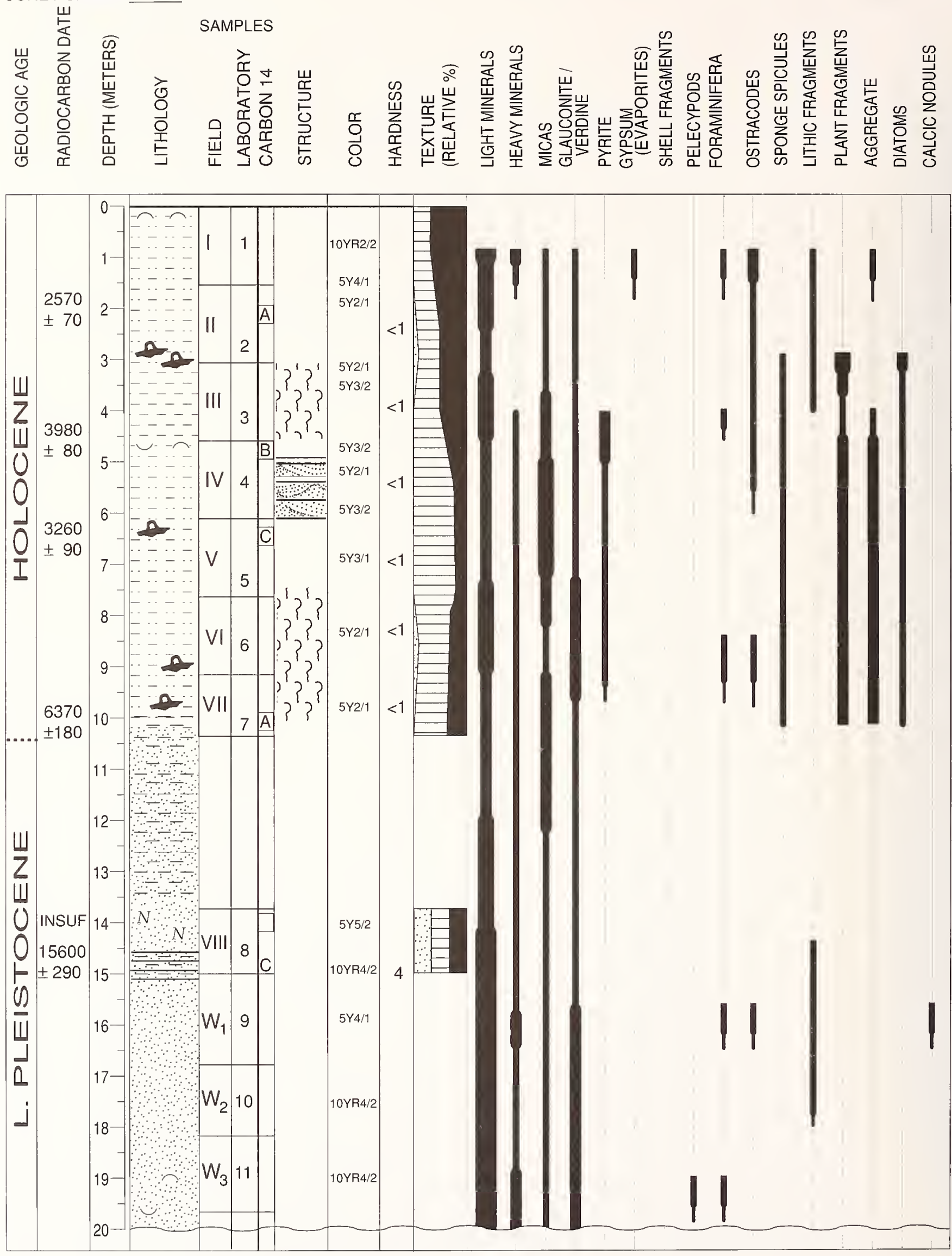
APPENDIX 1.-Continued.
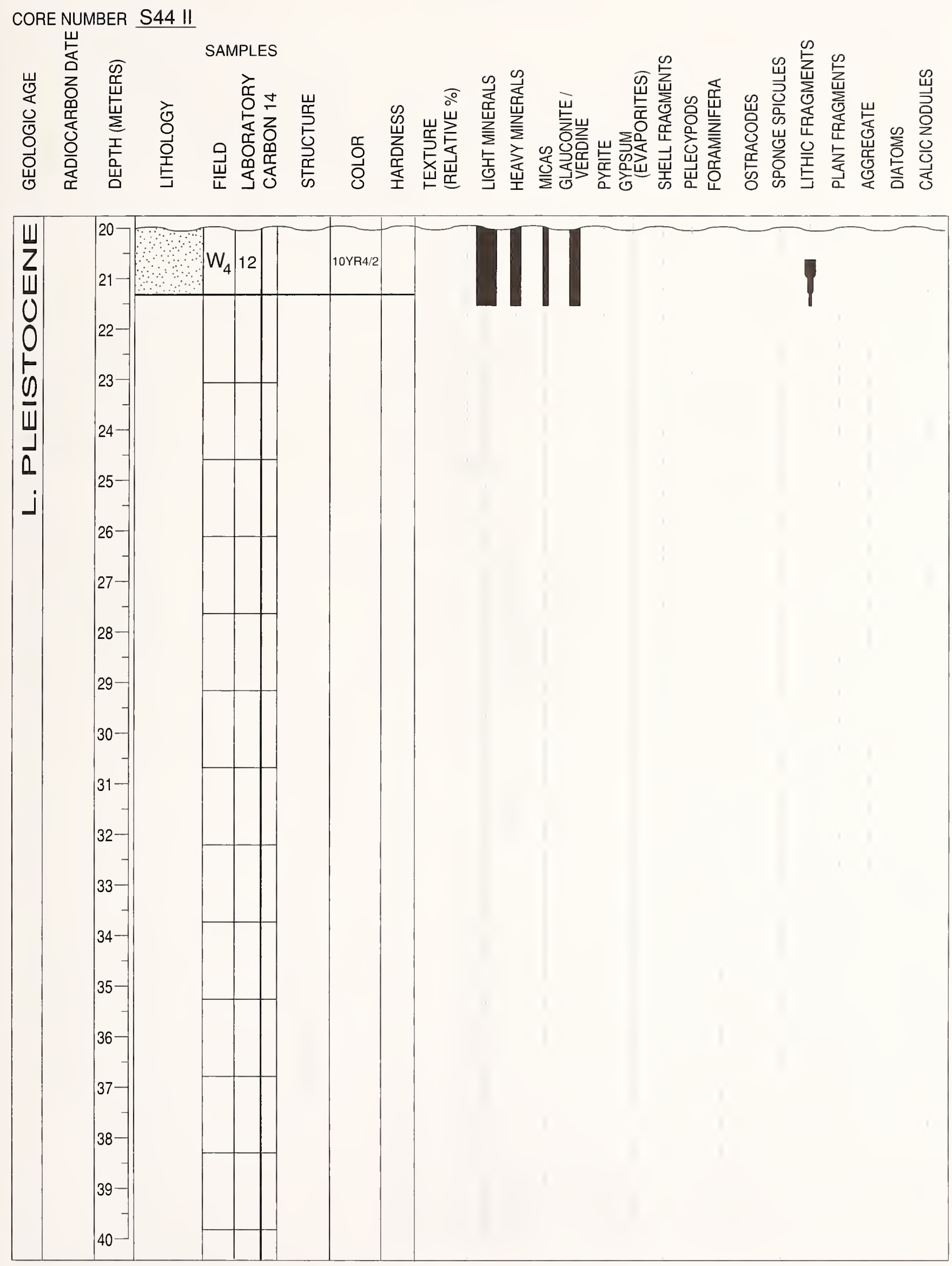
APPENDIX 1.-Continued.

CORE NUMBER S45I
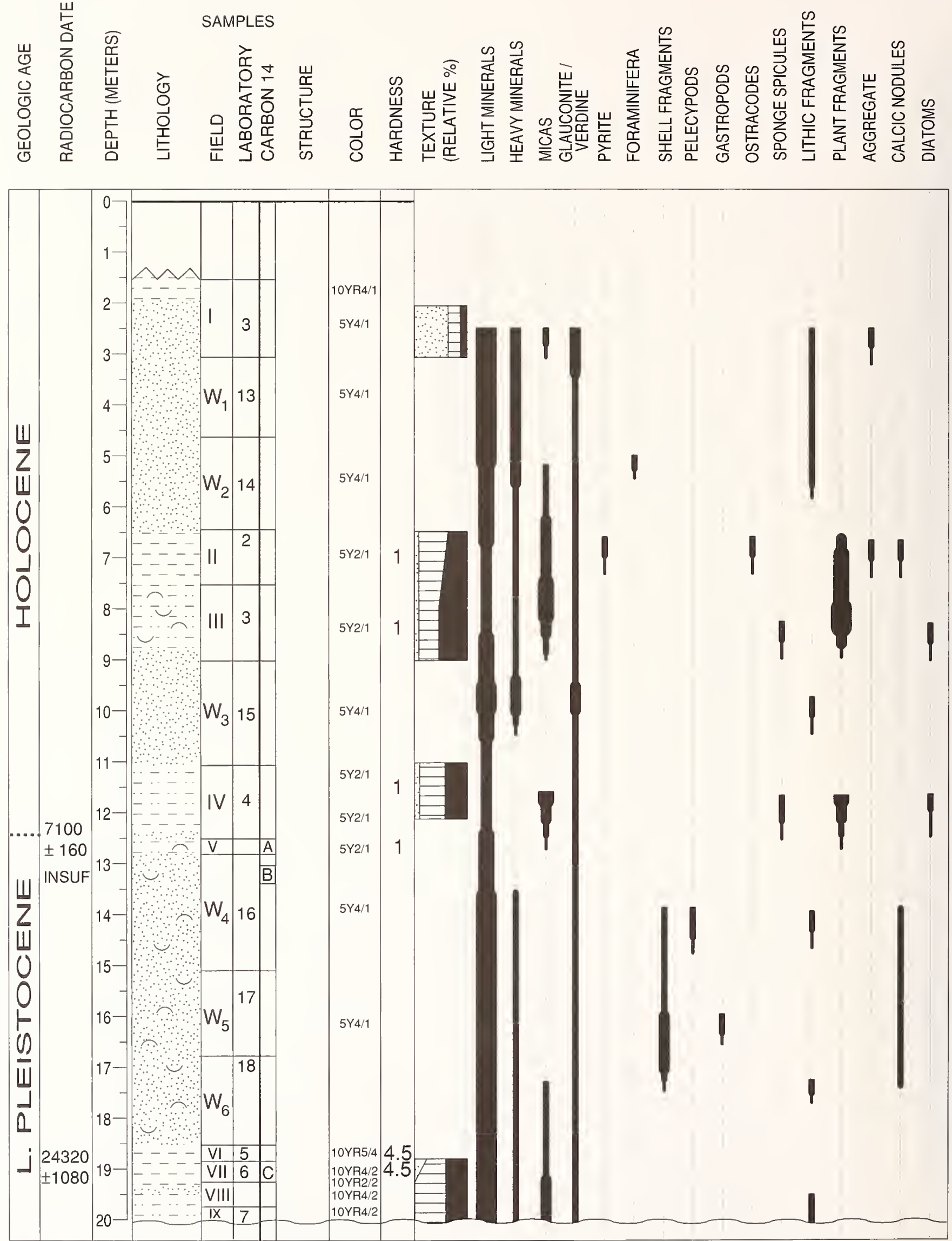
APPENDIX 1.-Continued.

CORE NUMBER S45 II

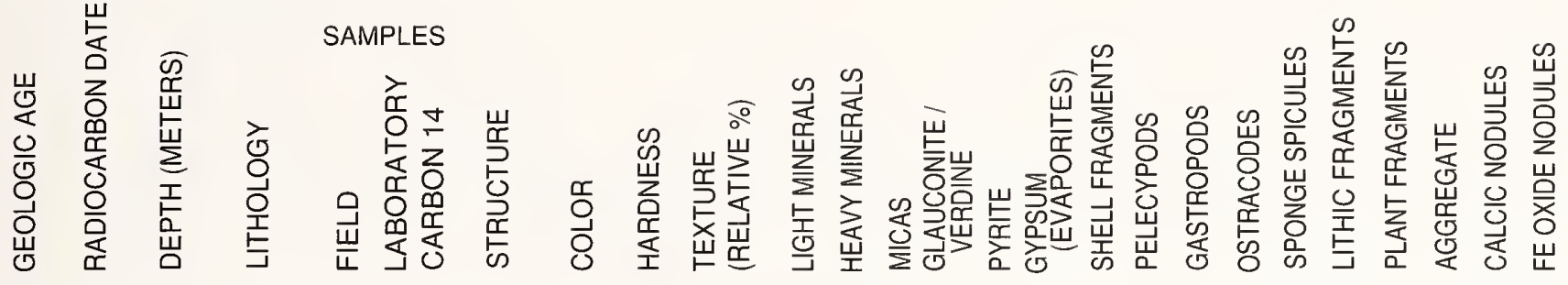

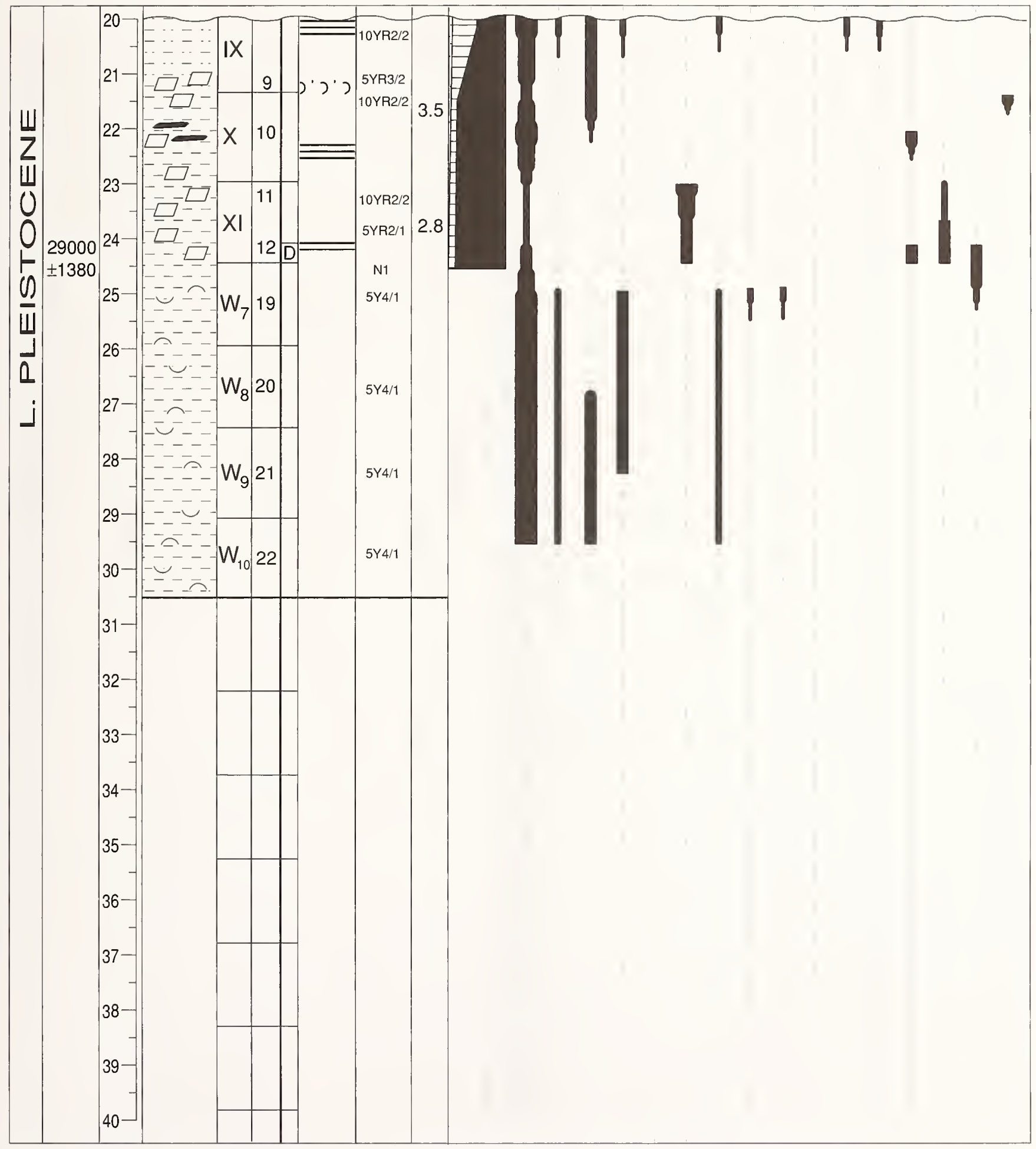


APPENDIX 1.-Continued.

CORE NUMBER S46 I
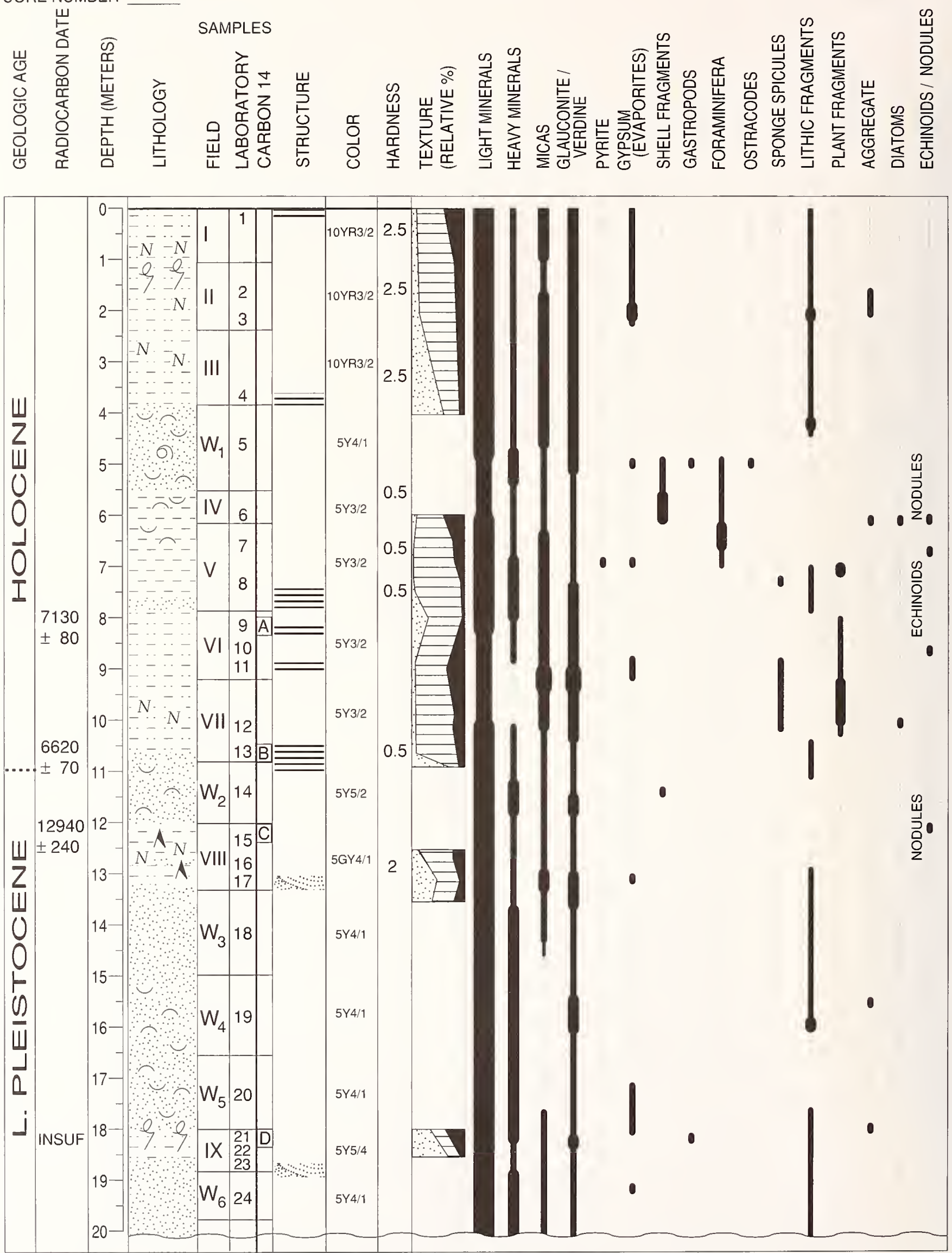
APPENDIX 1.-Continued.

\section{CORE NUMBER S46 II}

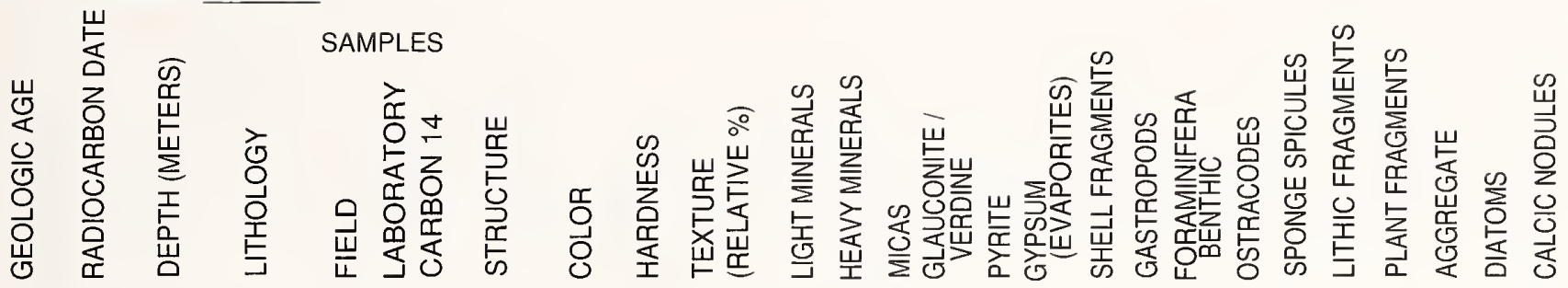

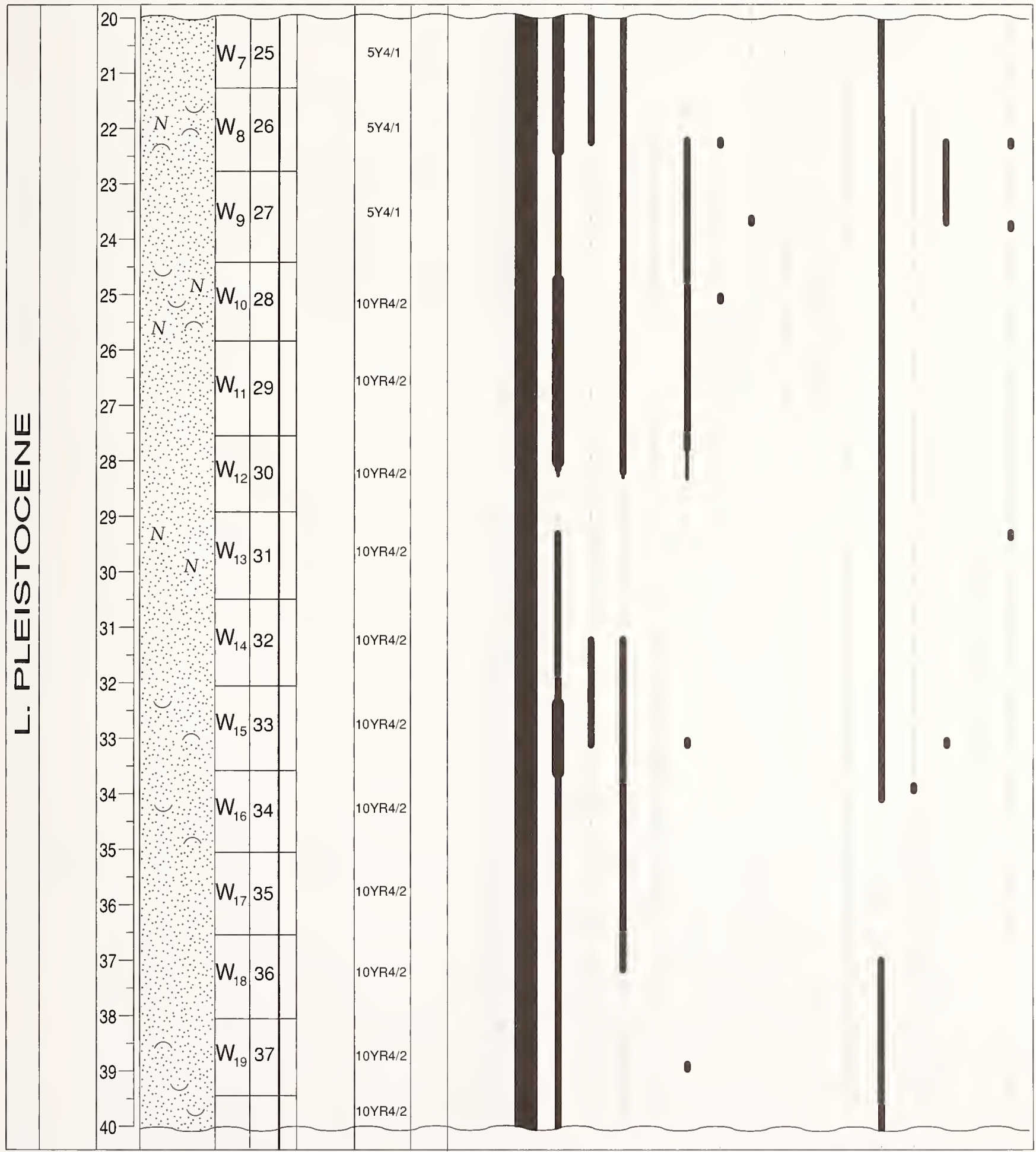


APPENDIX 1.-Continued.

\section{CORE NUMBER S46 III}
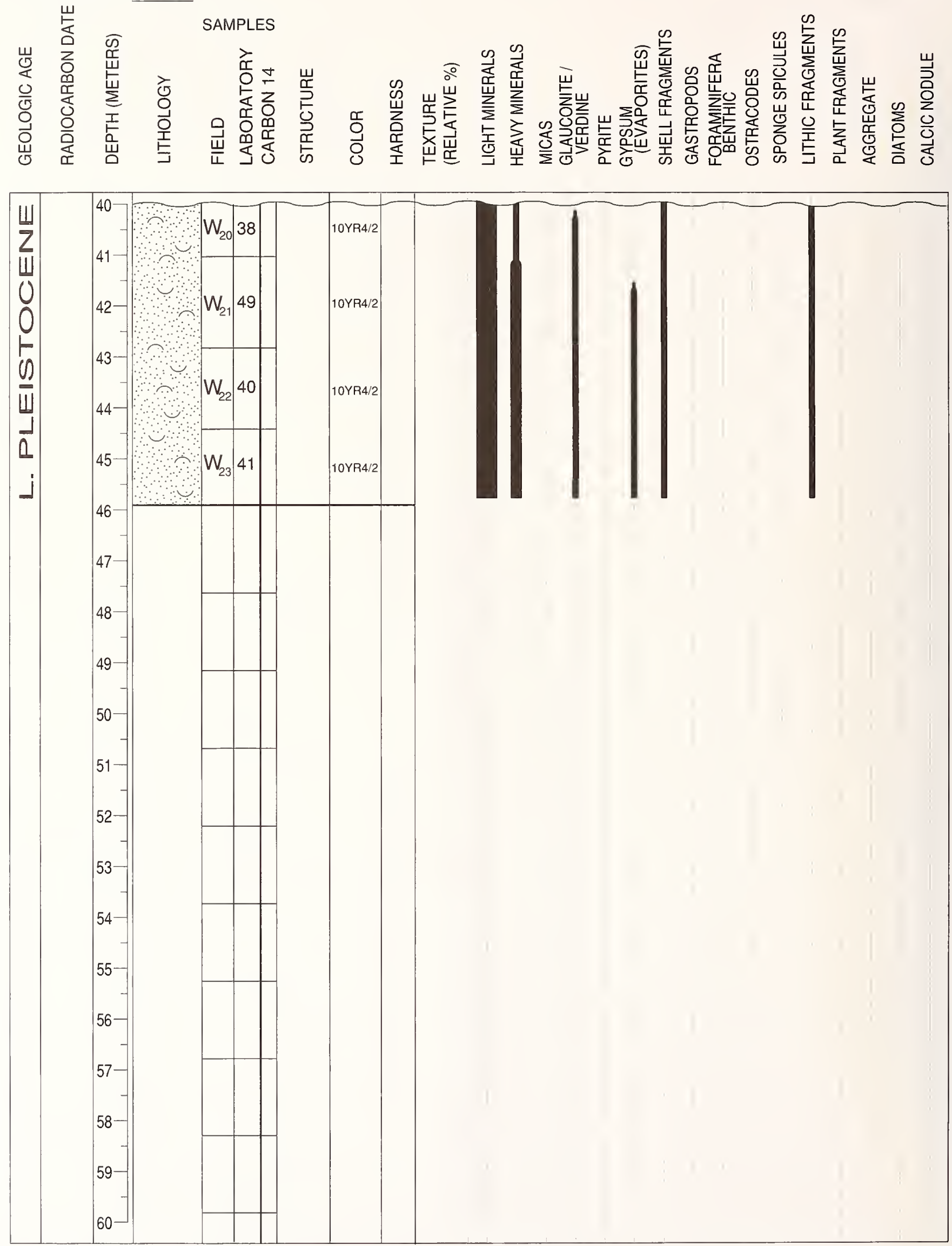
APPENDIX 1.-Continued.

CORE NUMBER S47 I
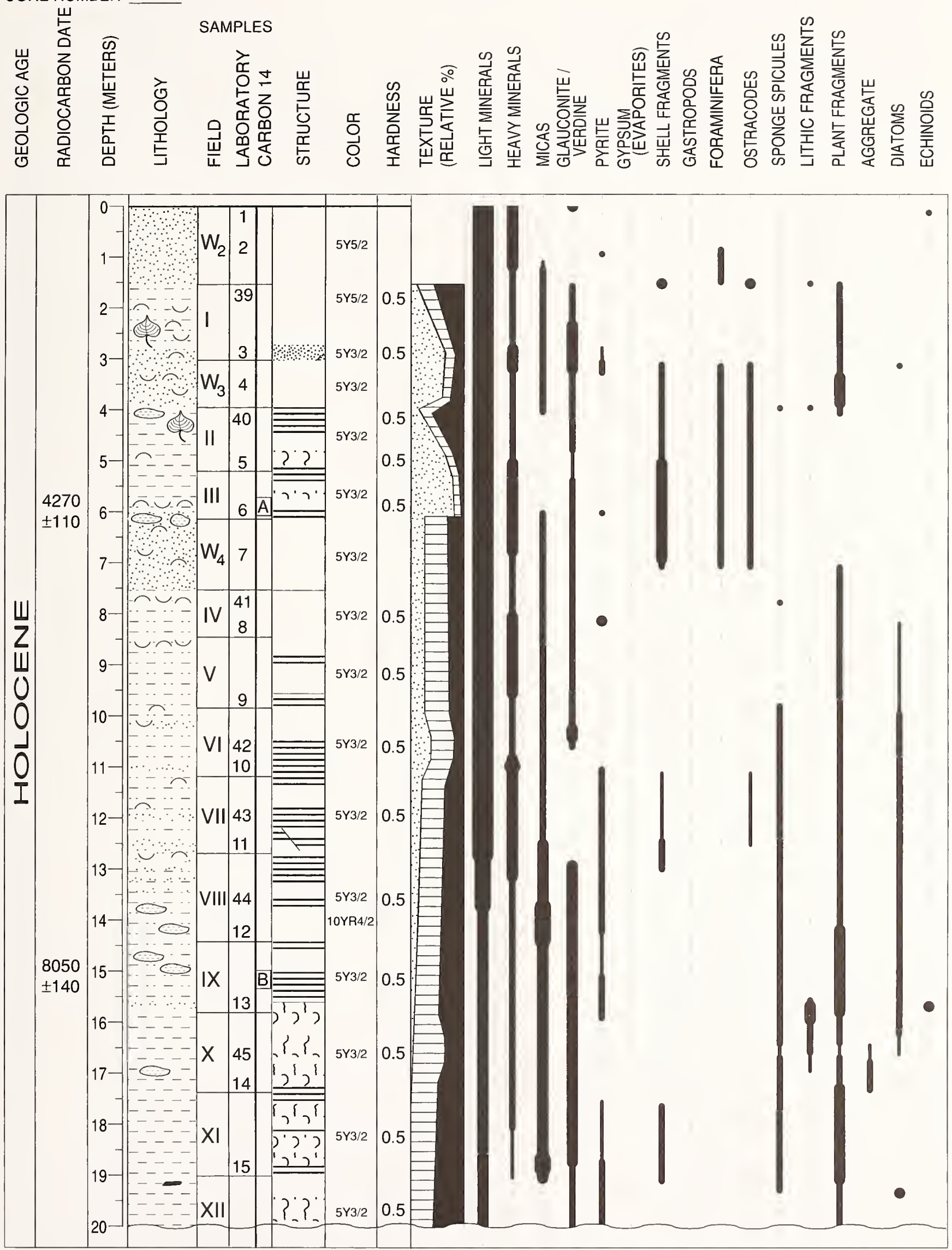
APPENDIX 1.-Continued.

CORE NUMBER S47 II
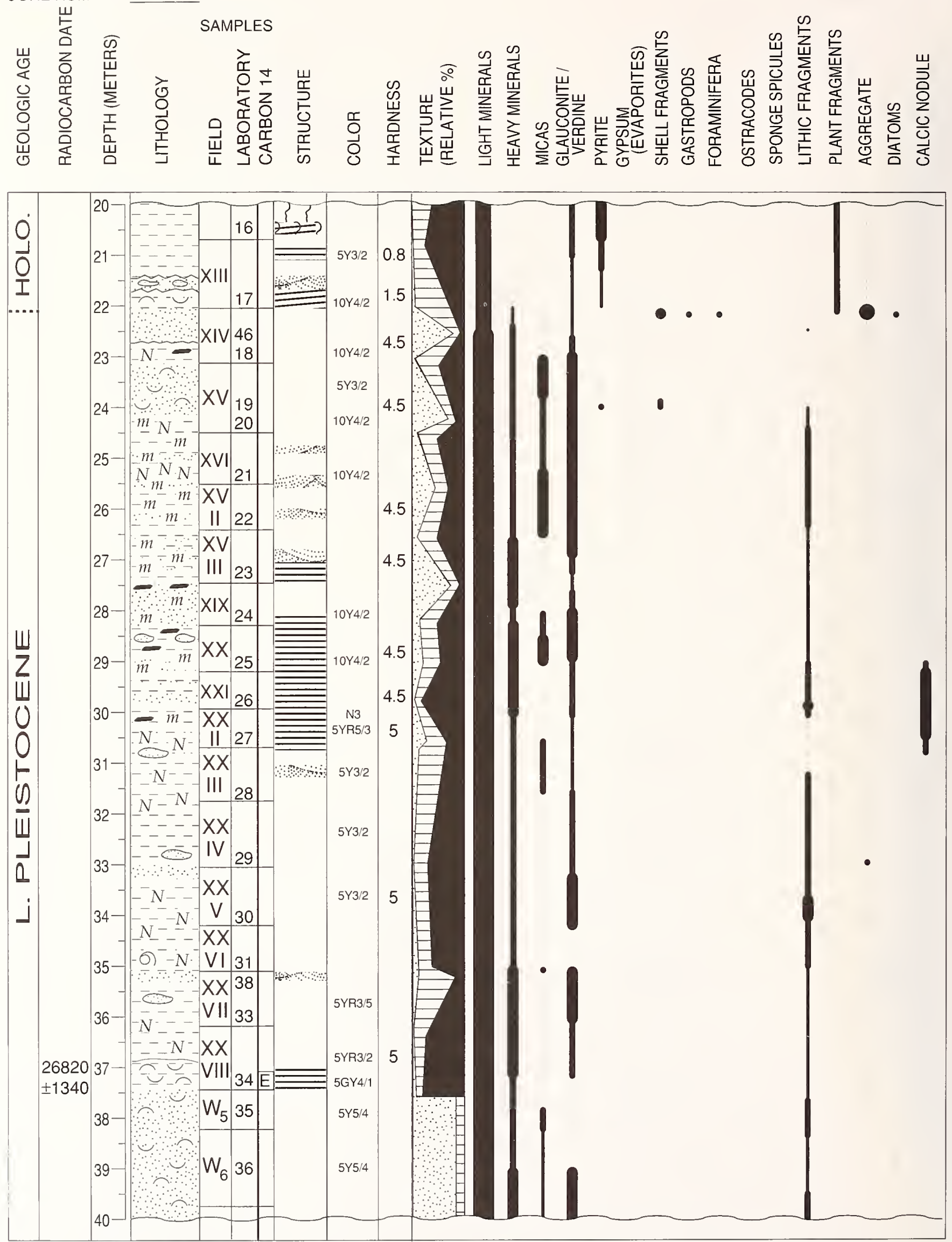
APPENDIX 1.-Continued.
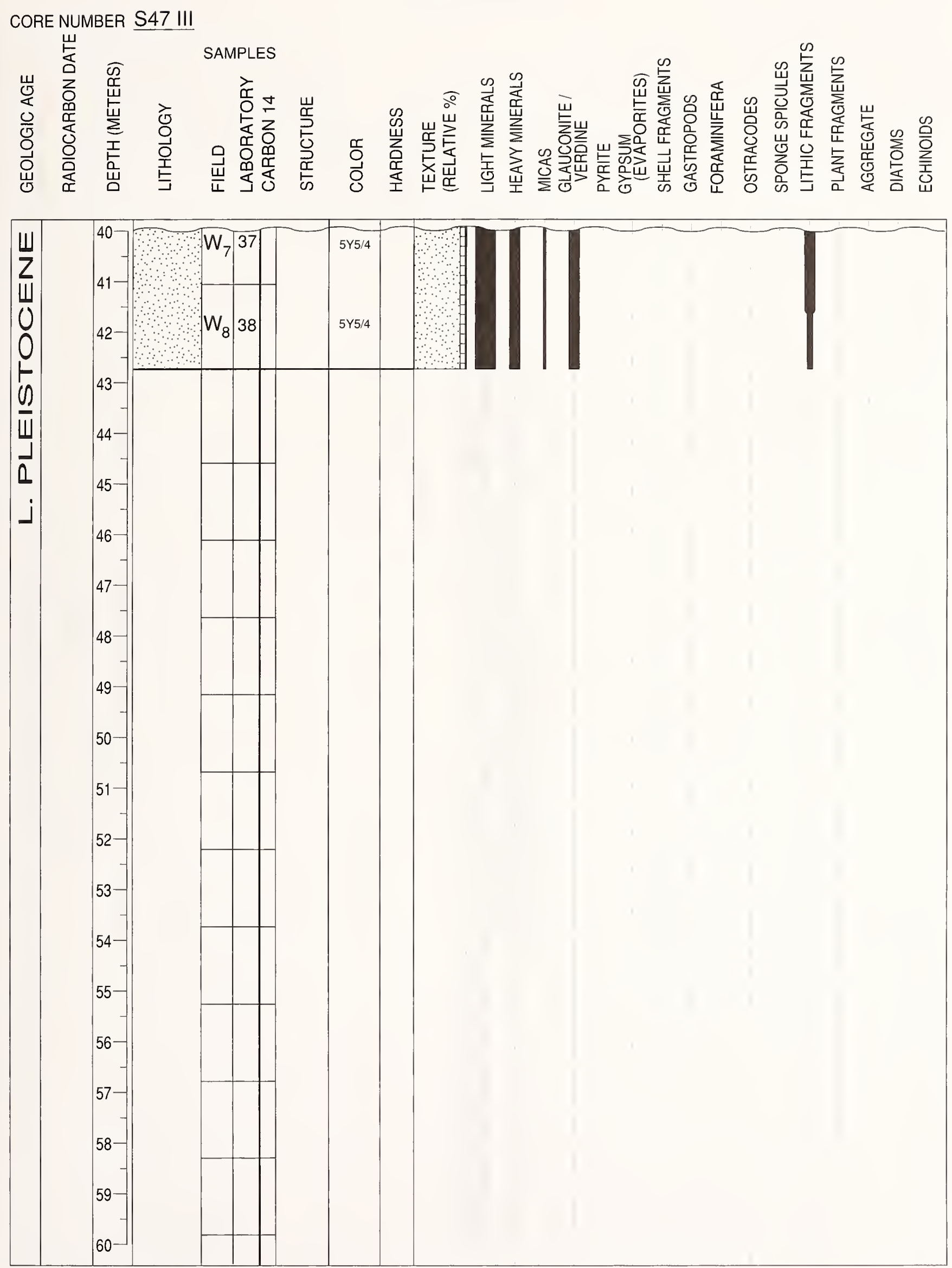
APPENDIX 1.-Continued.

CORE NUMBER S48I
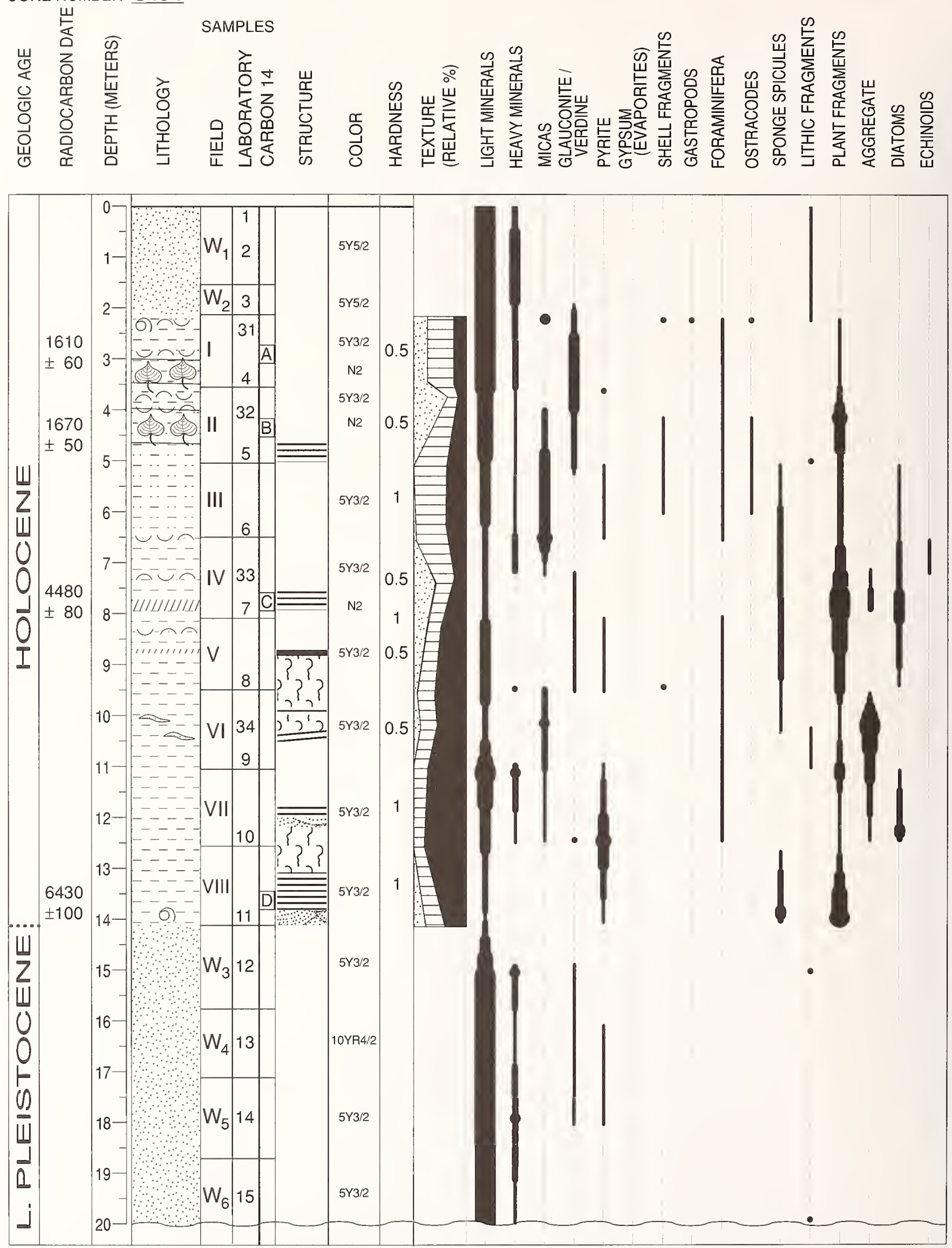
APPENDIX 1.-Continued.

CORE NUMBER S48 II

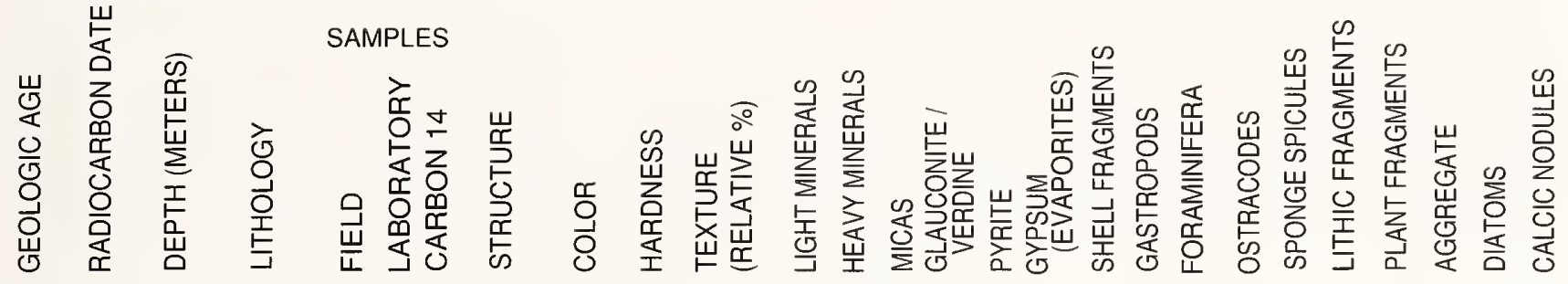

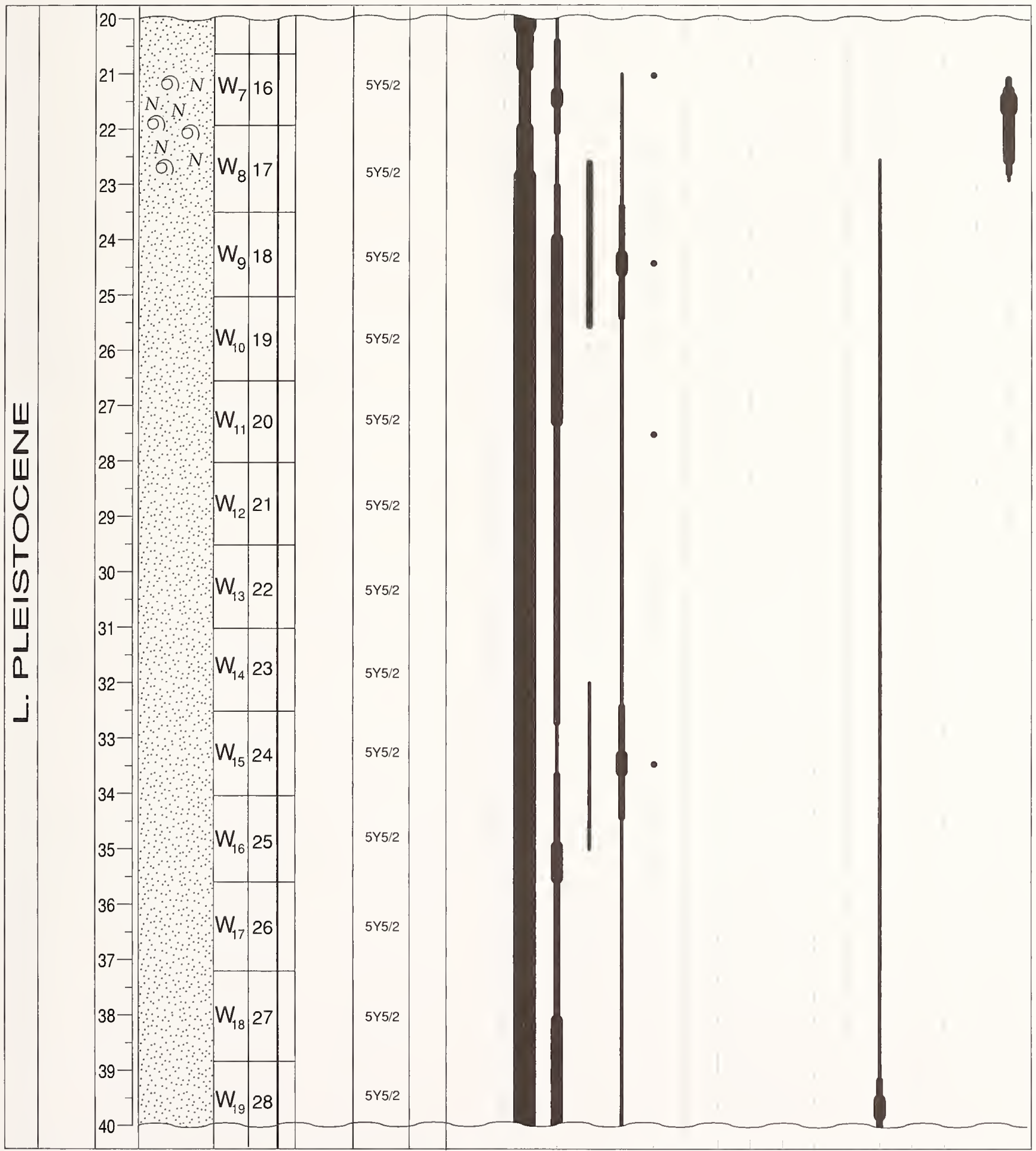


APPENDIX 1.-Continued.

CORE NUMBER S48 III

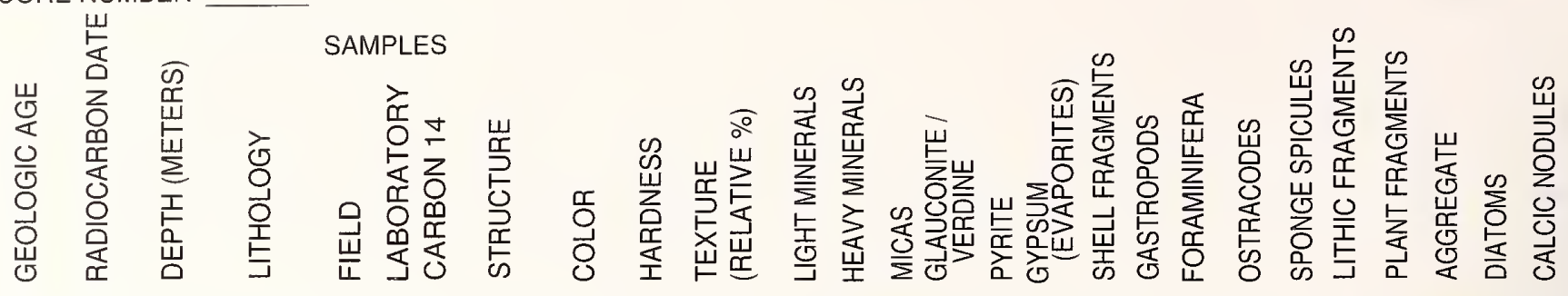

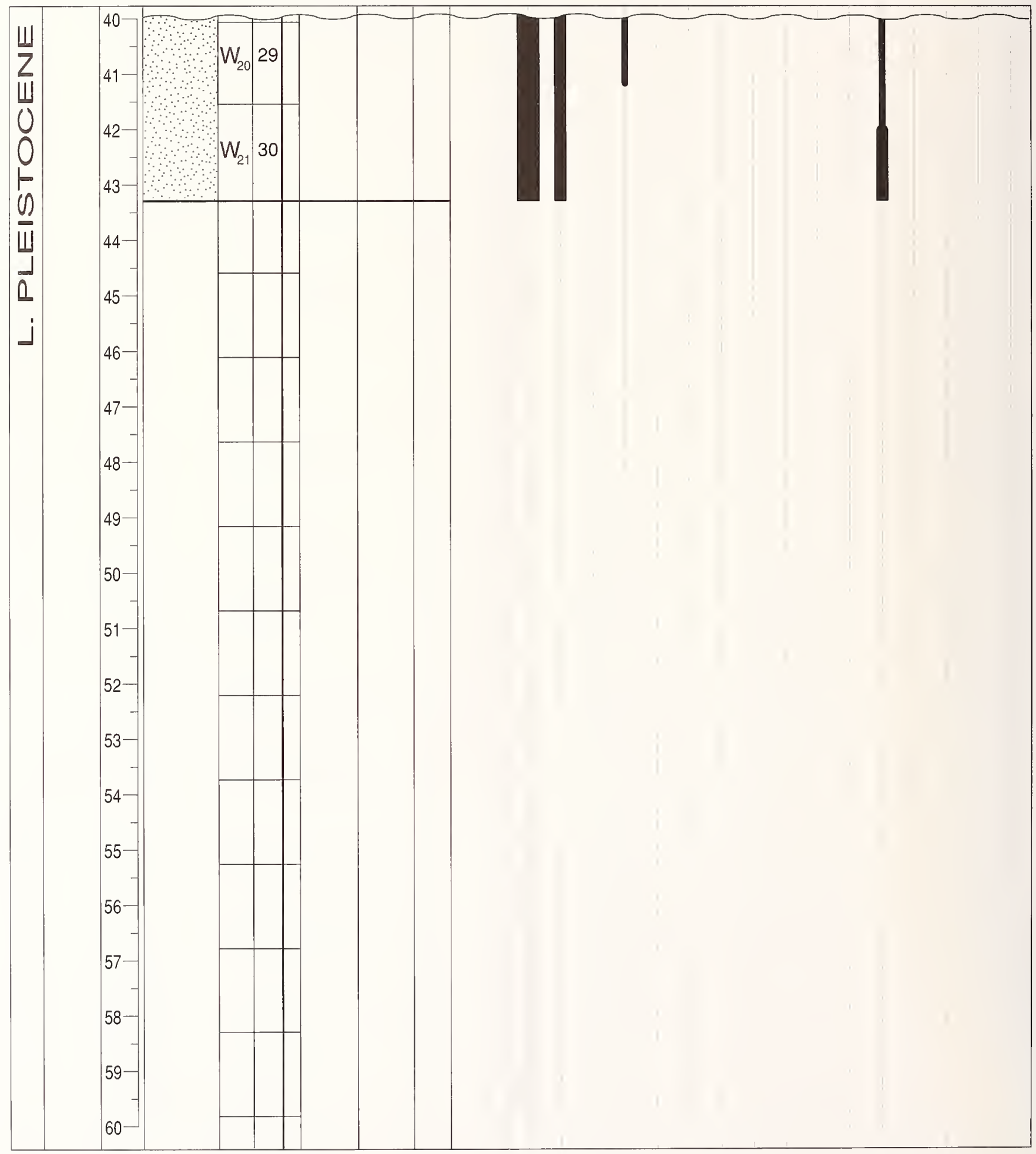


APPENDIX 1.-Continued.

CORE NUMBER S49I
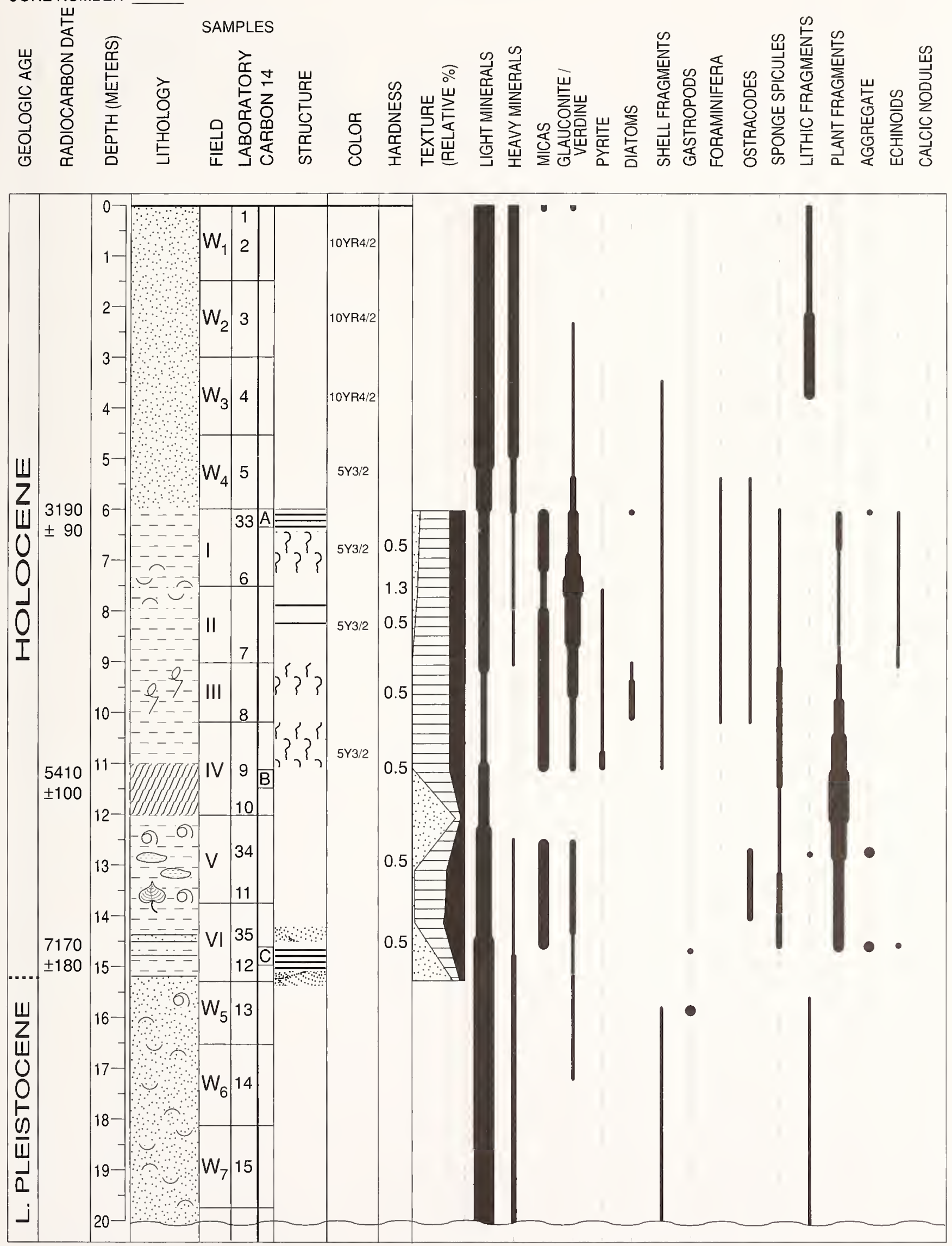
APPENDIX 1.-Continued.

CORE NUMBER S49 II

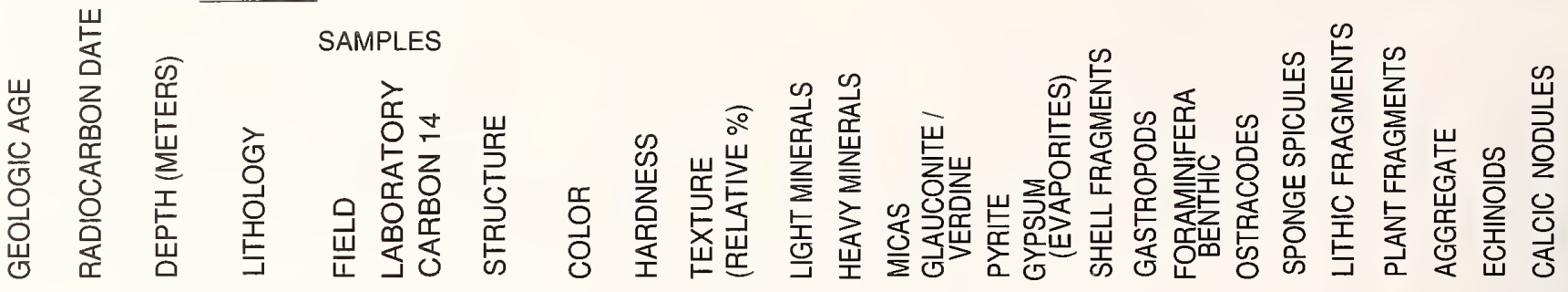

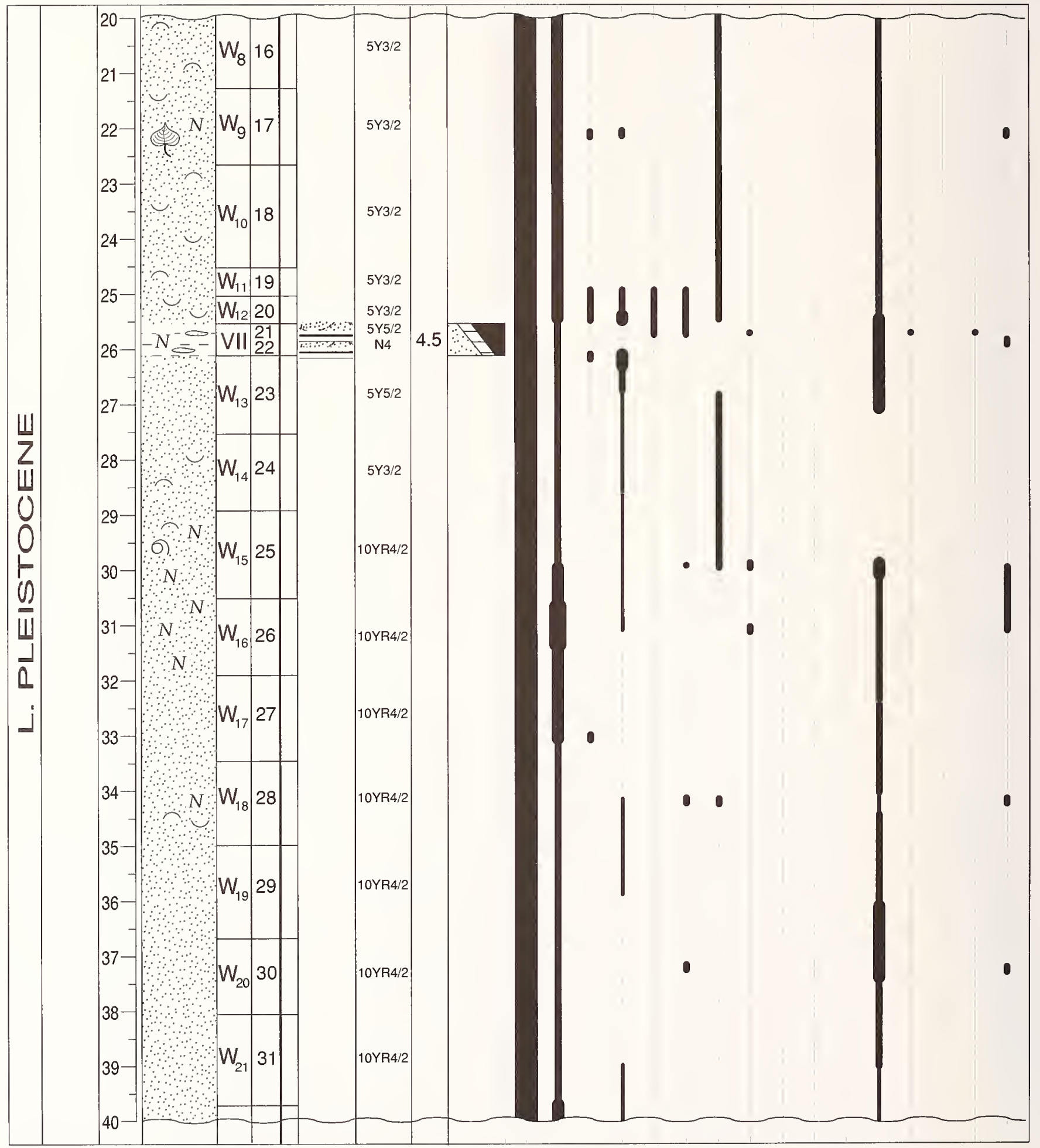


APPENDIX 1.-Continued.
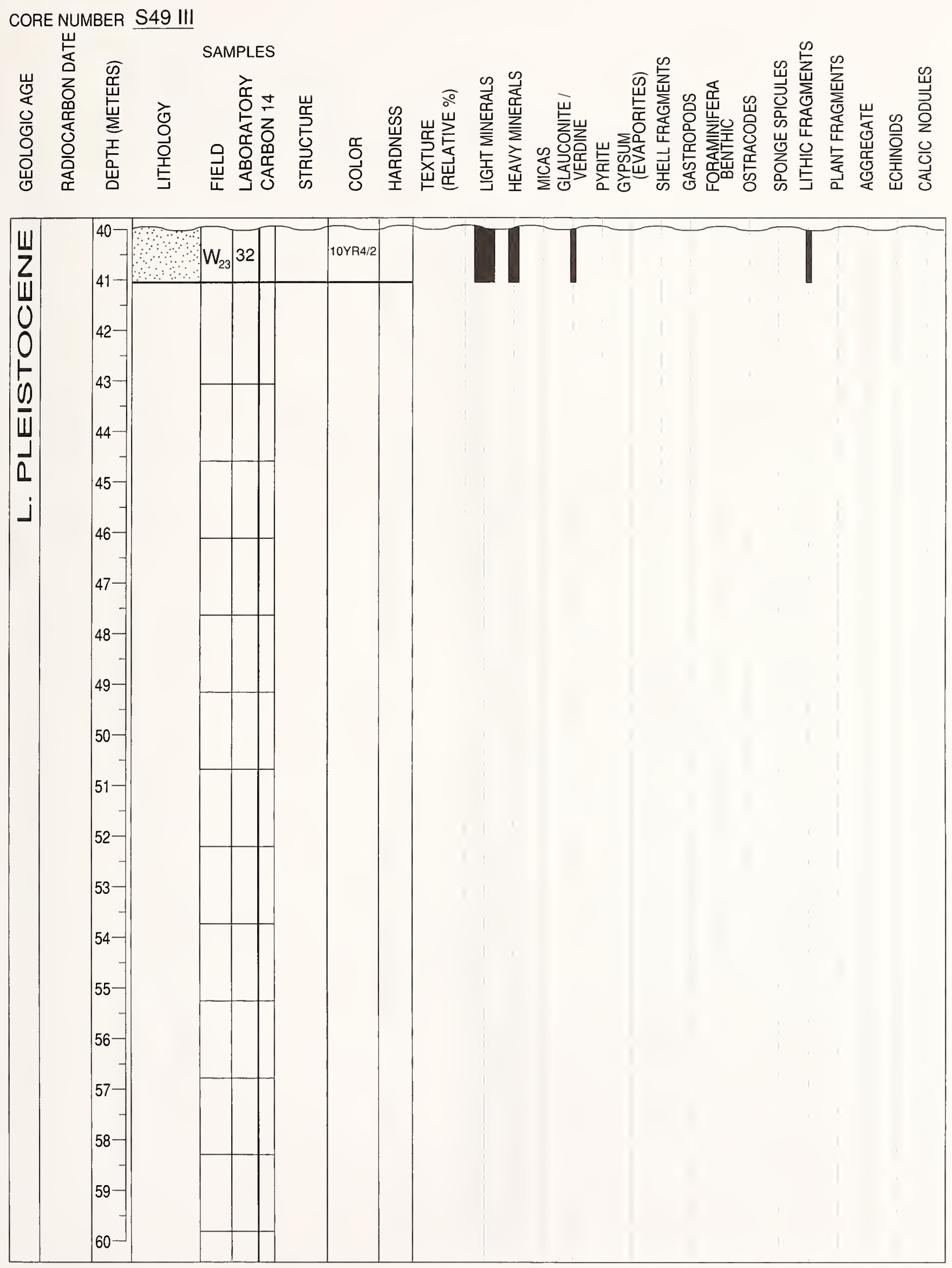
APPENDIX 1.-Continued.

CORE NUMBER S50 I

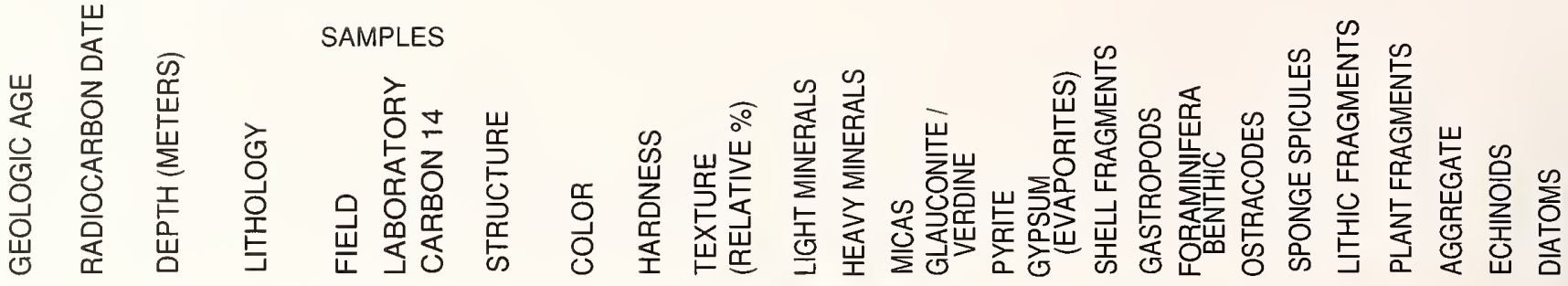

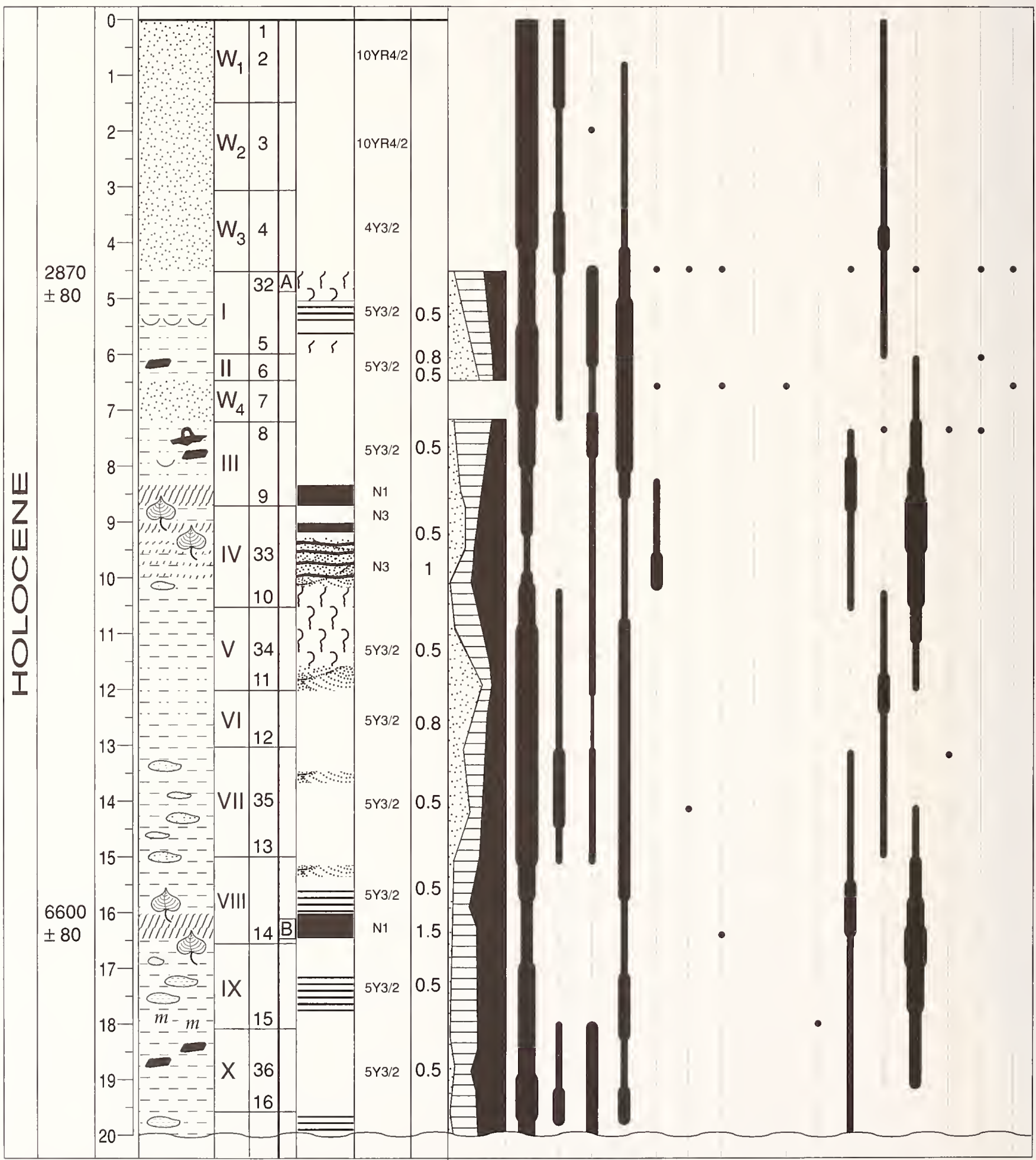


APPENDIX 1,-Continued.

CORE NUMBER S50 II
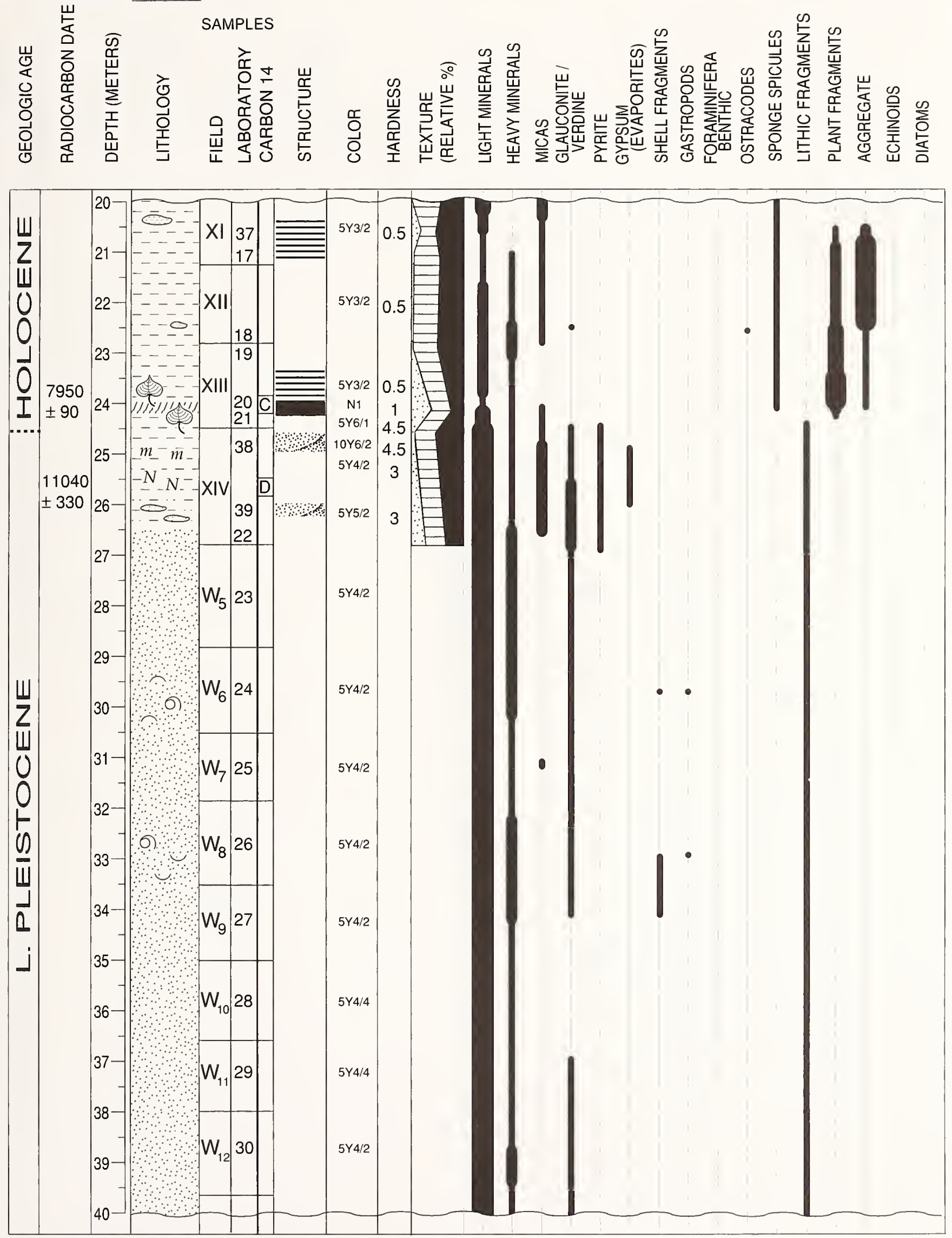
APPENDIX 1.-Continued.

CORE NUMBER S5O III

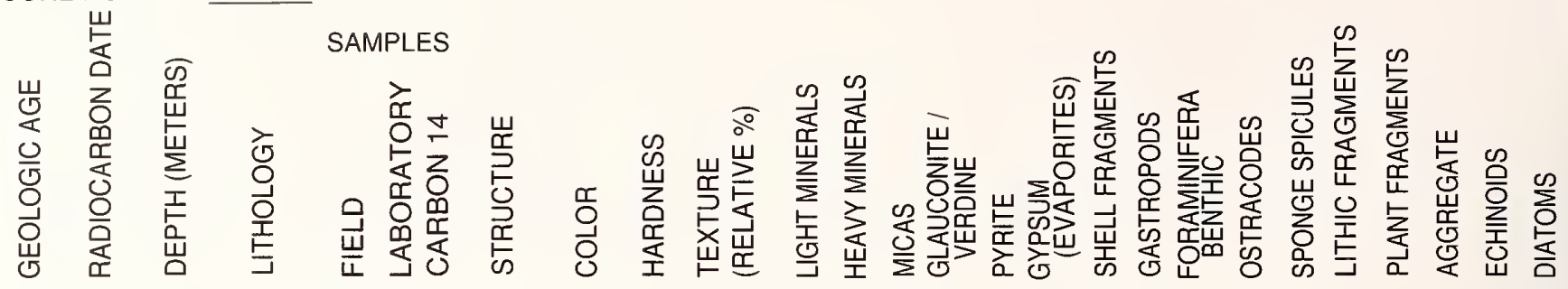

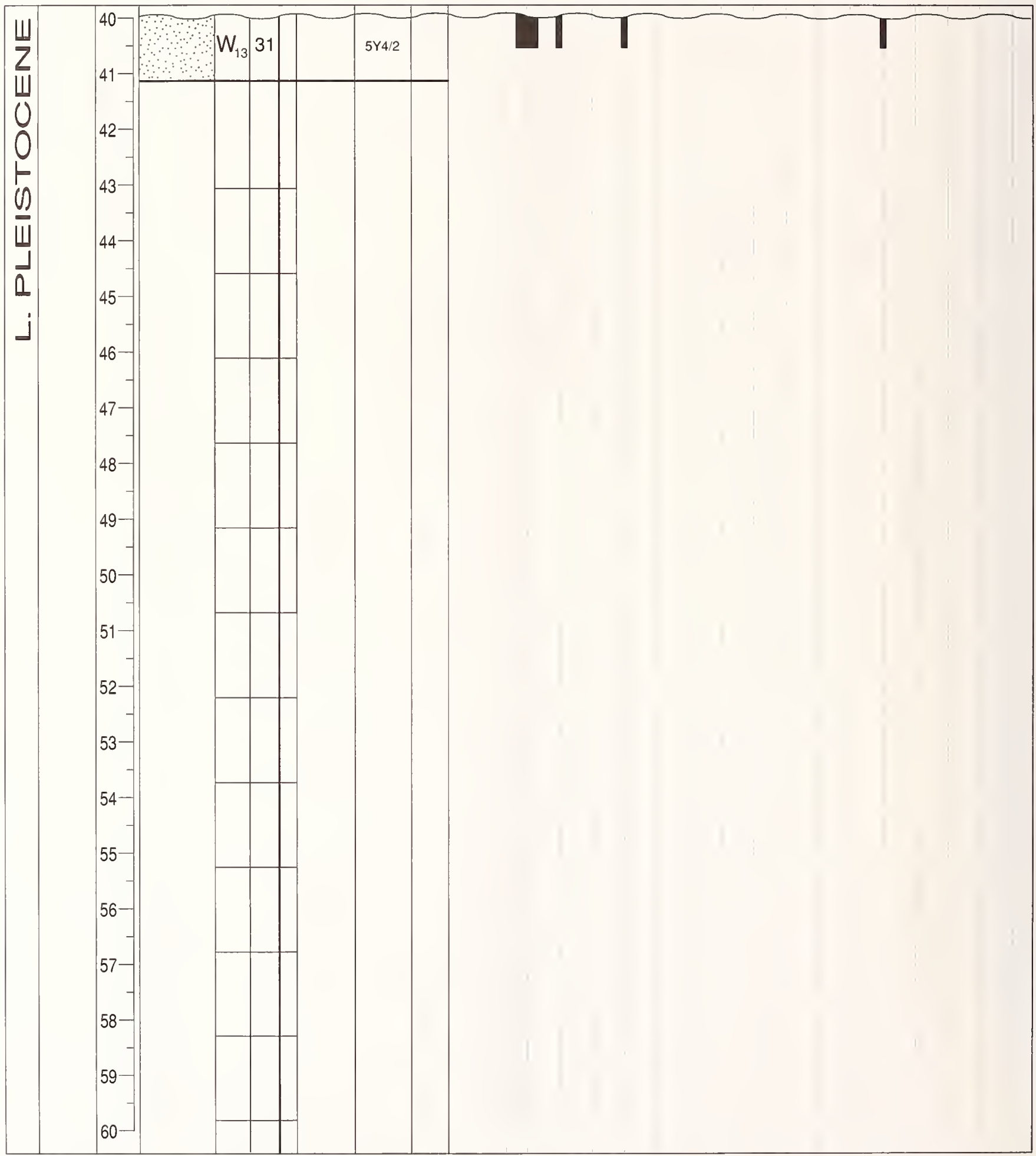


APPENDIX 1.-Continued.

CORE NUMBER S51I

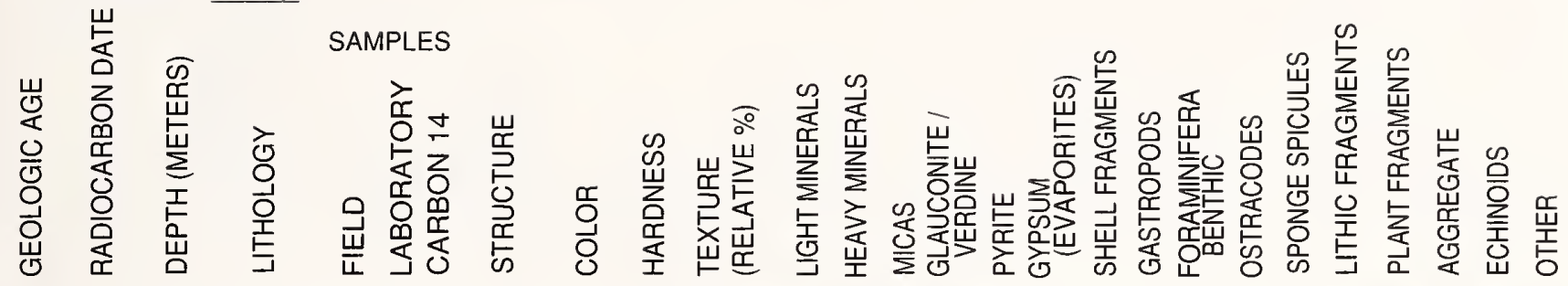

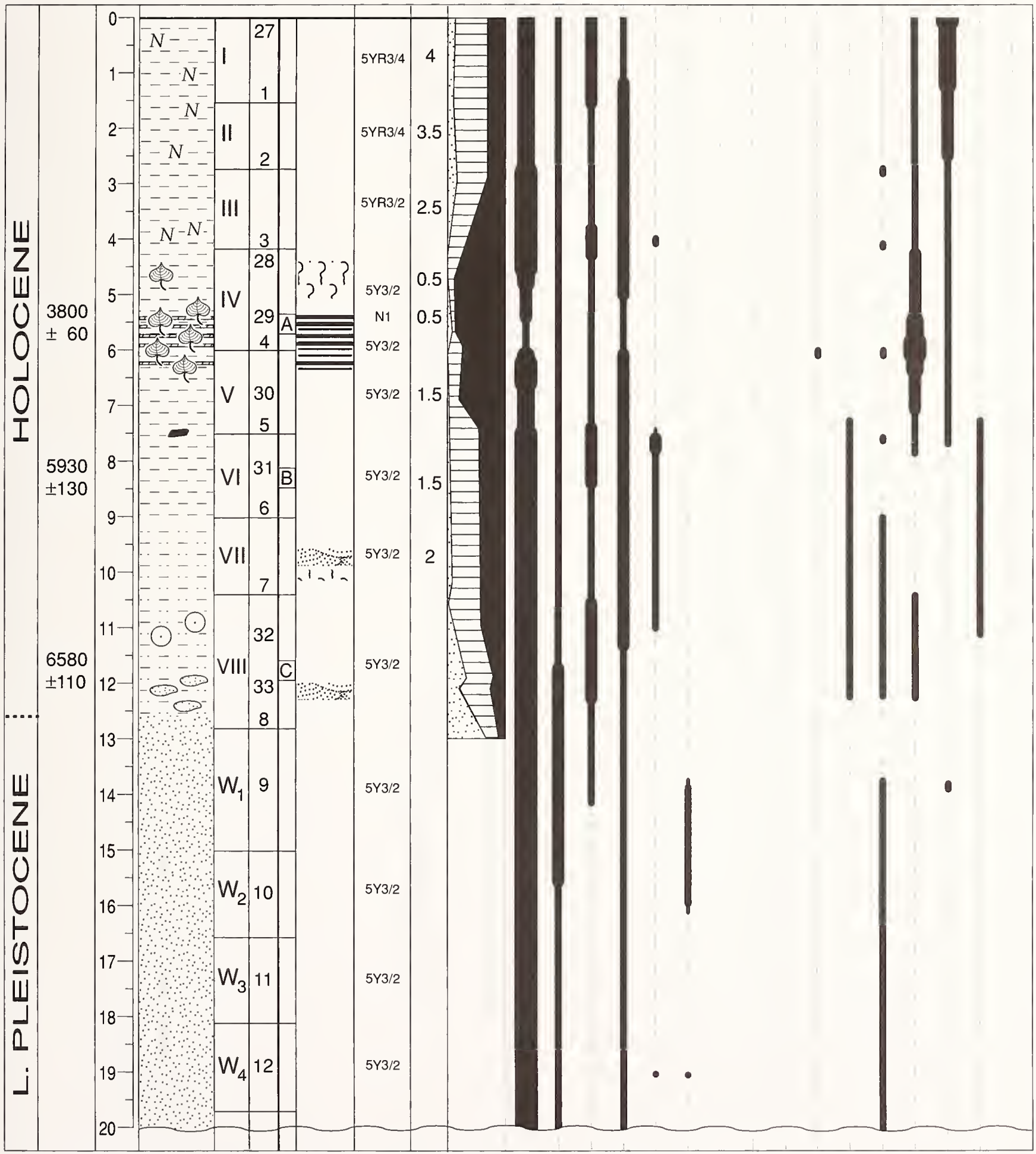


APPENDIX 1.-Continued.

CORE NUMBER S51 II

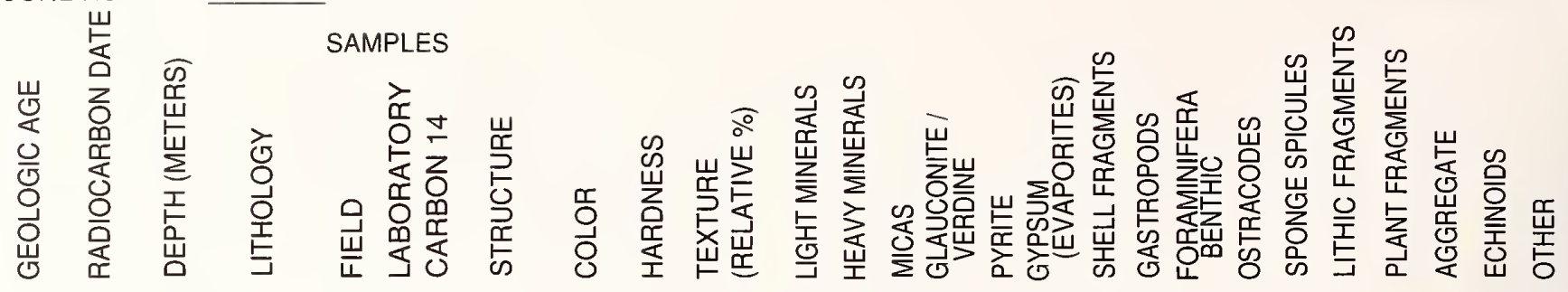

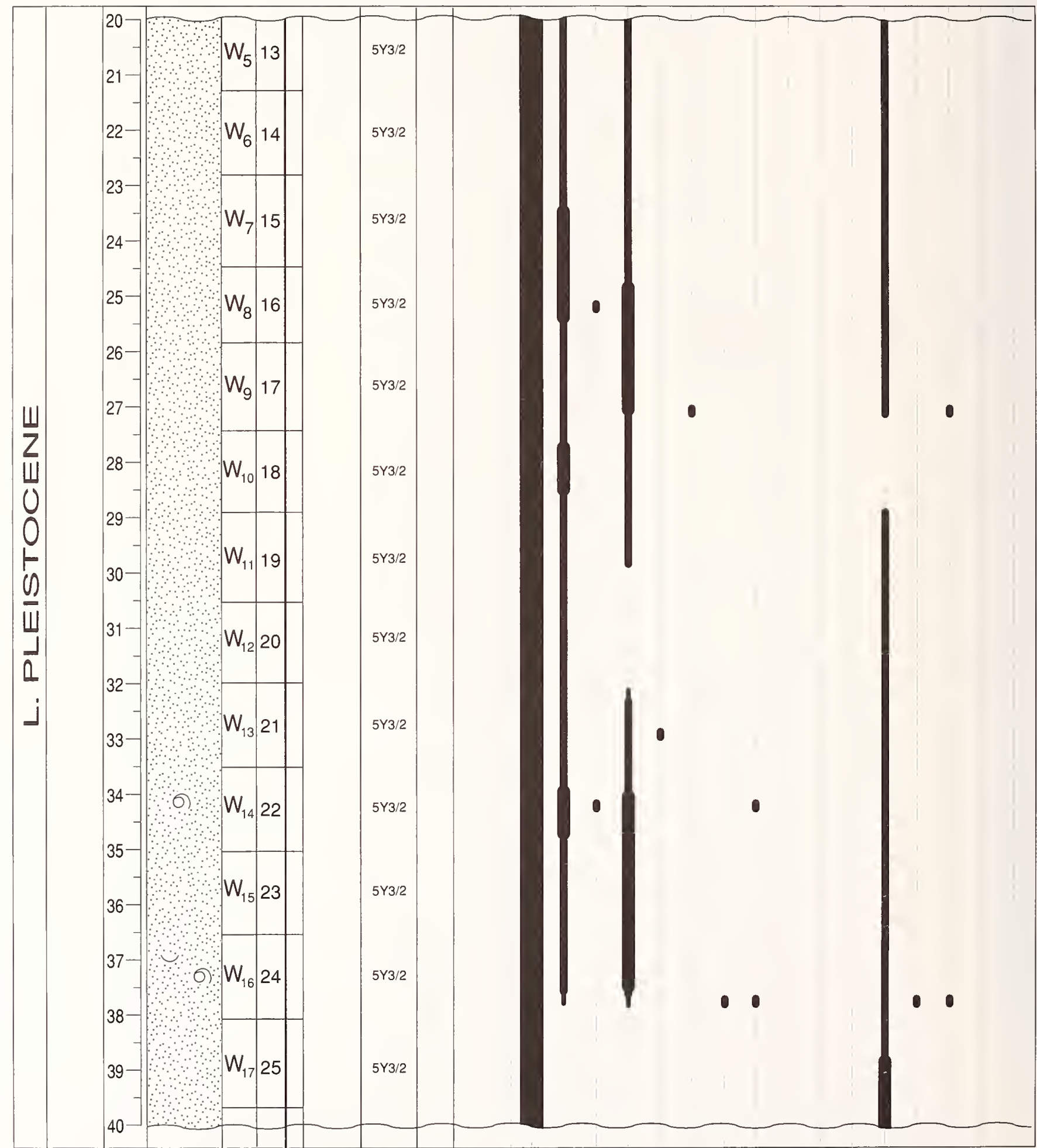


APPENDIX 1.-Continued.
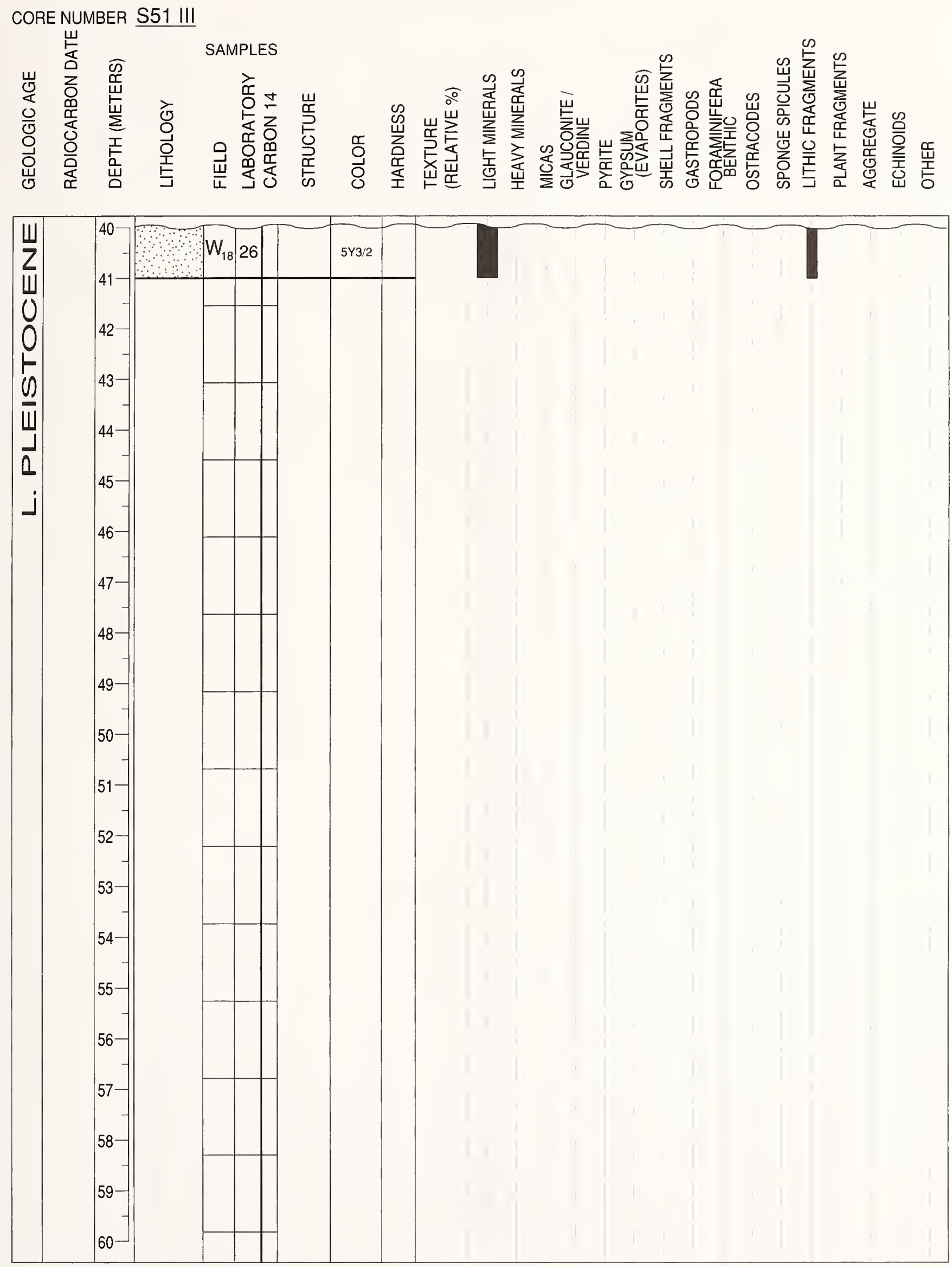
APPENDIX 1.-Continued.

CORE NUMBER S52 I

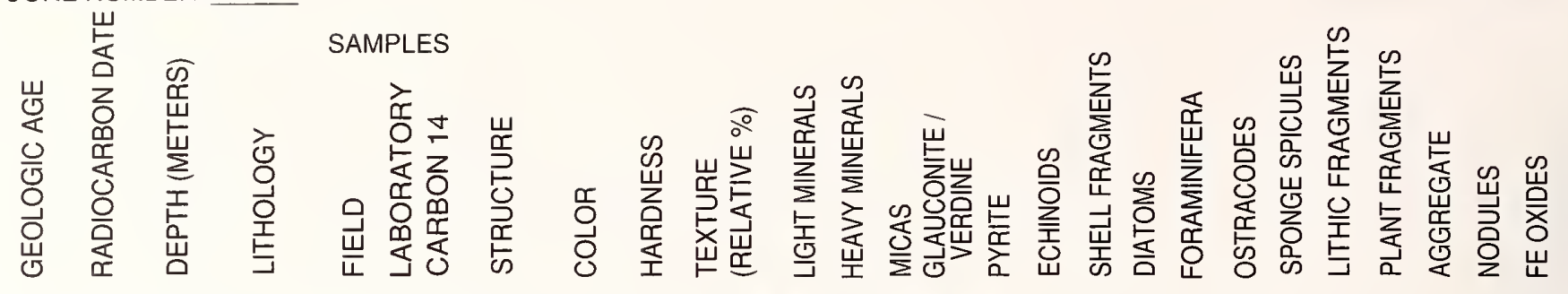

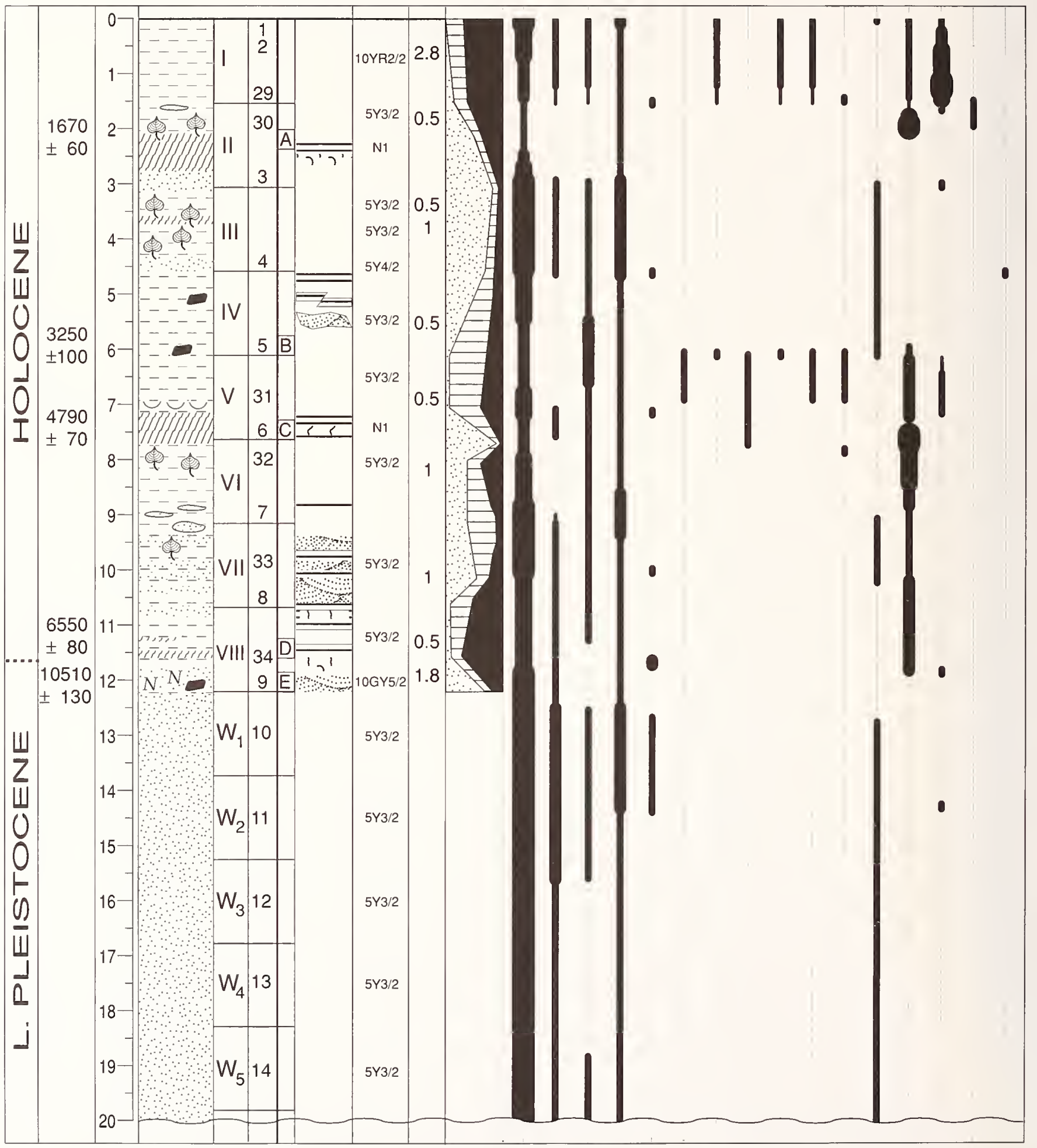


APPENDIX 1,-Continued.
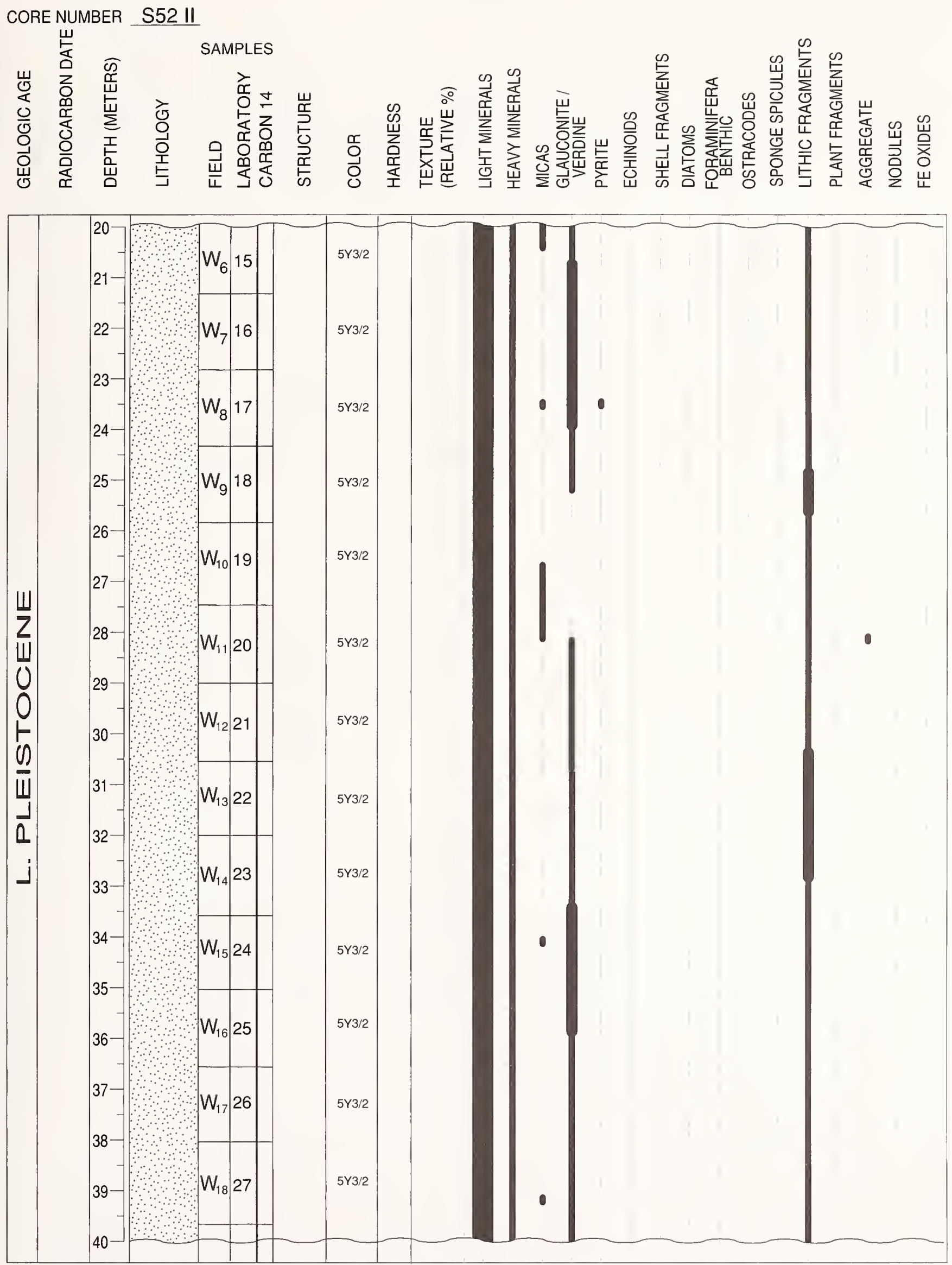
APPENDIX 1,-Continued.

CORE NUMBER S52 III

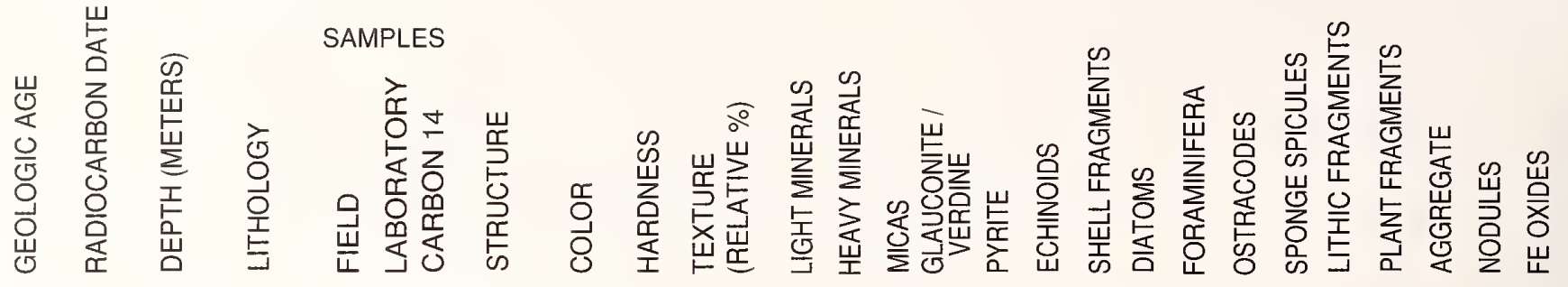

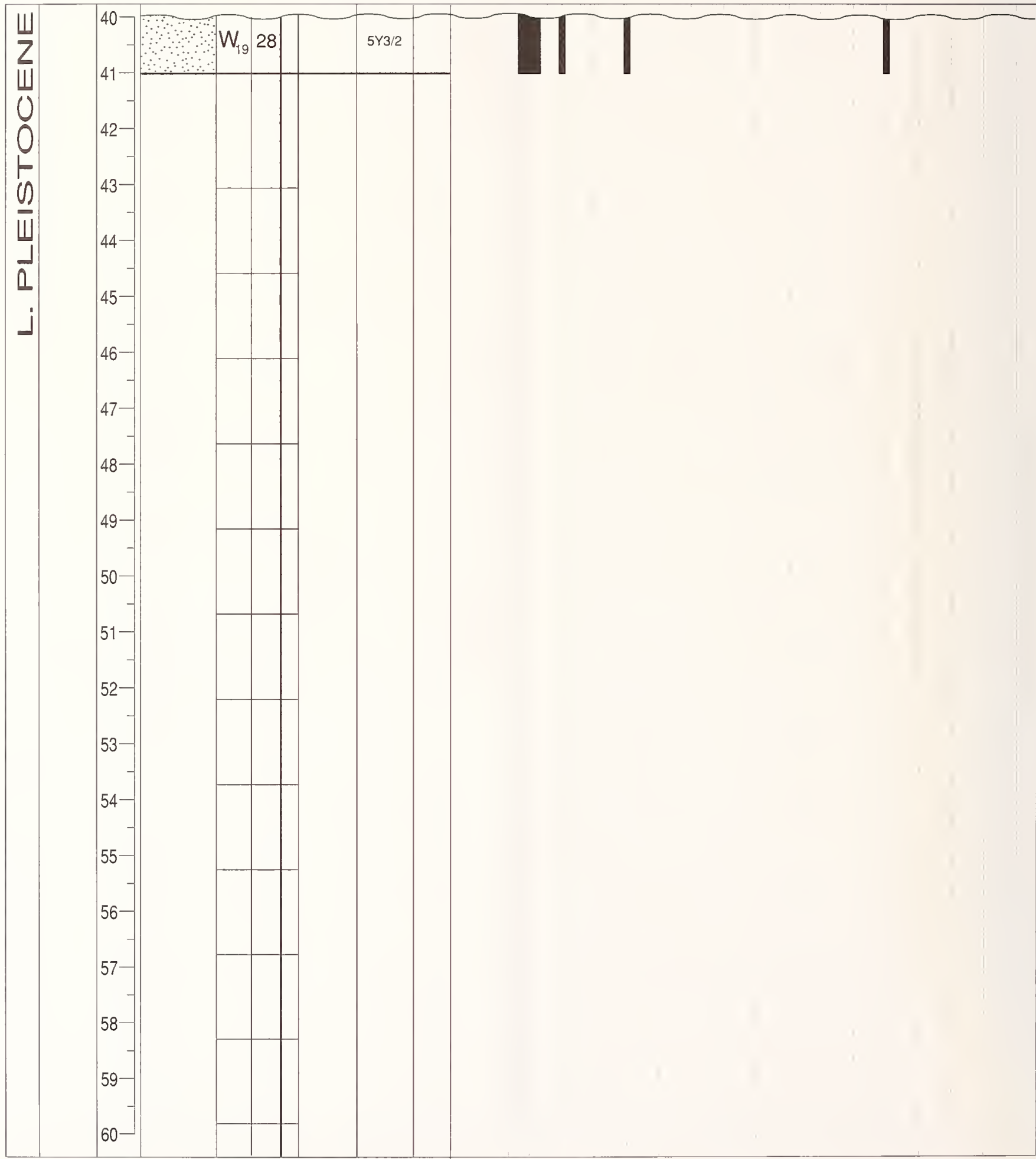


APPENDIX 1.-Continued.

CORE NUMBER S53 I

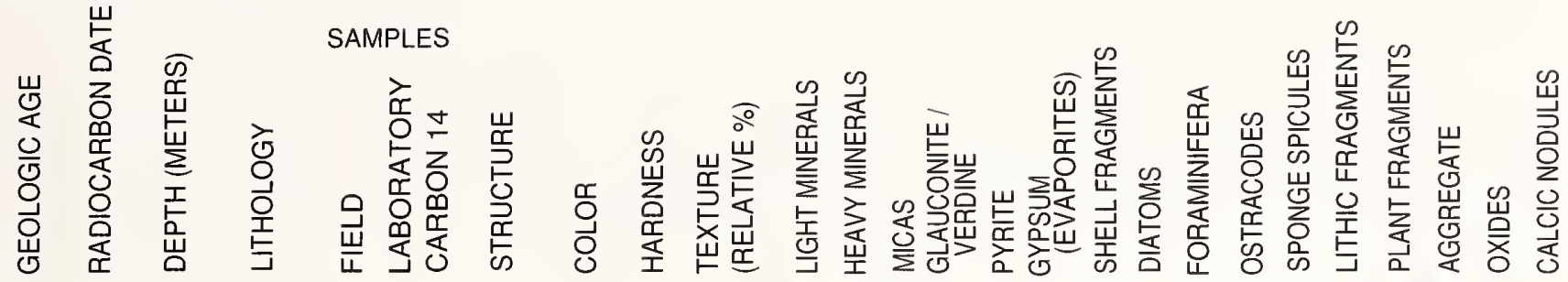

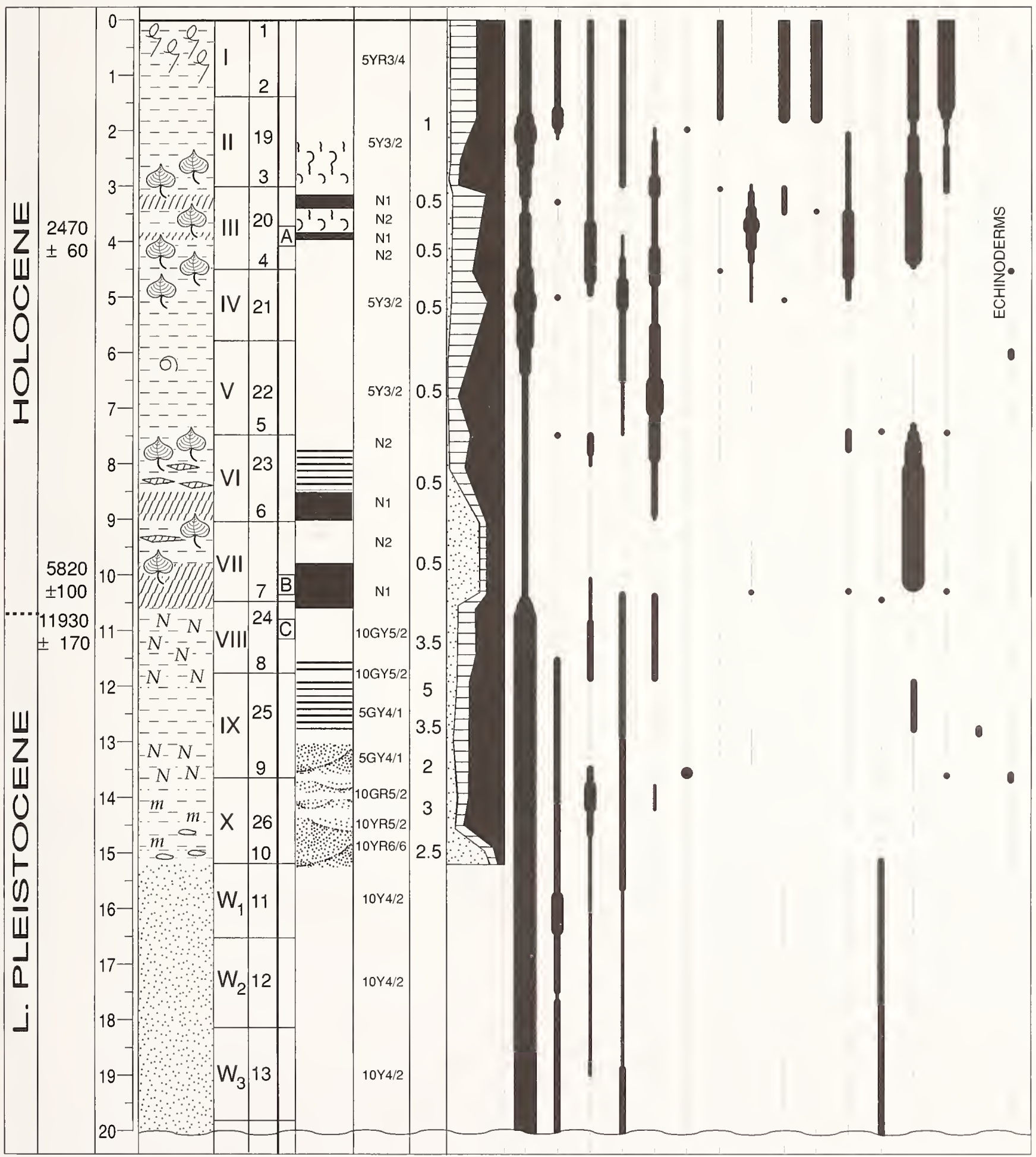


APPENDIX 1.-Continued.

CORE NUMBER S53 II

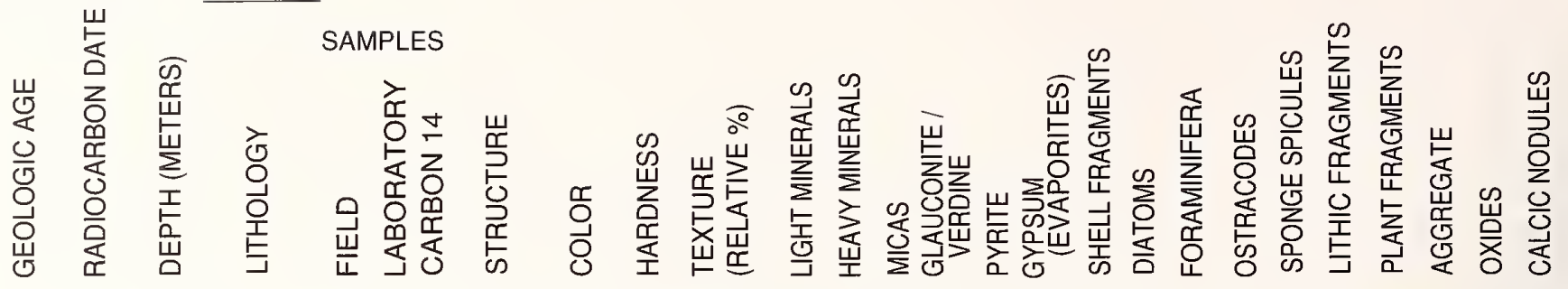

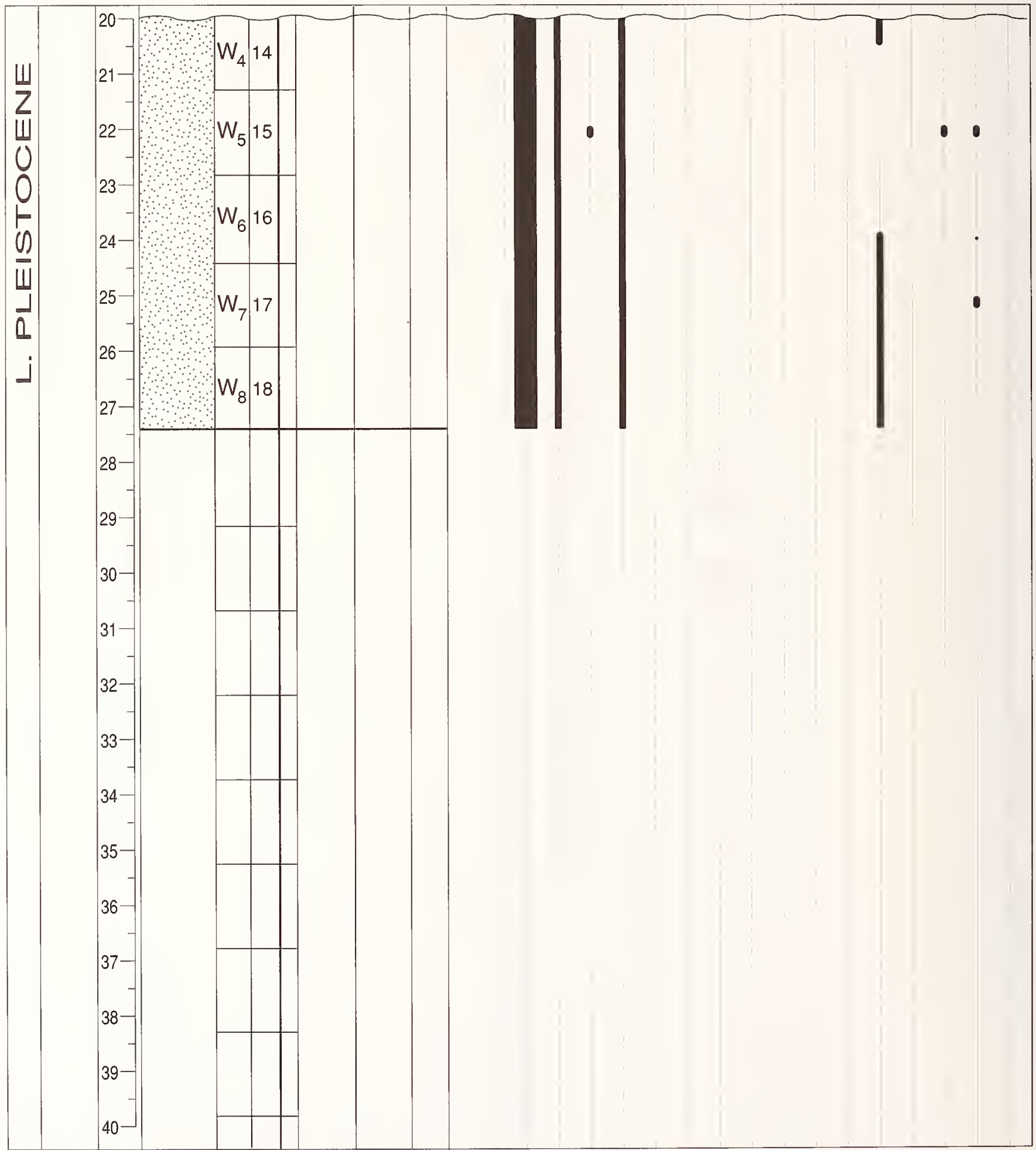


APPENDIX 1.-Continued.

CORE NUMBER $\$ 54$
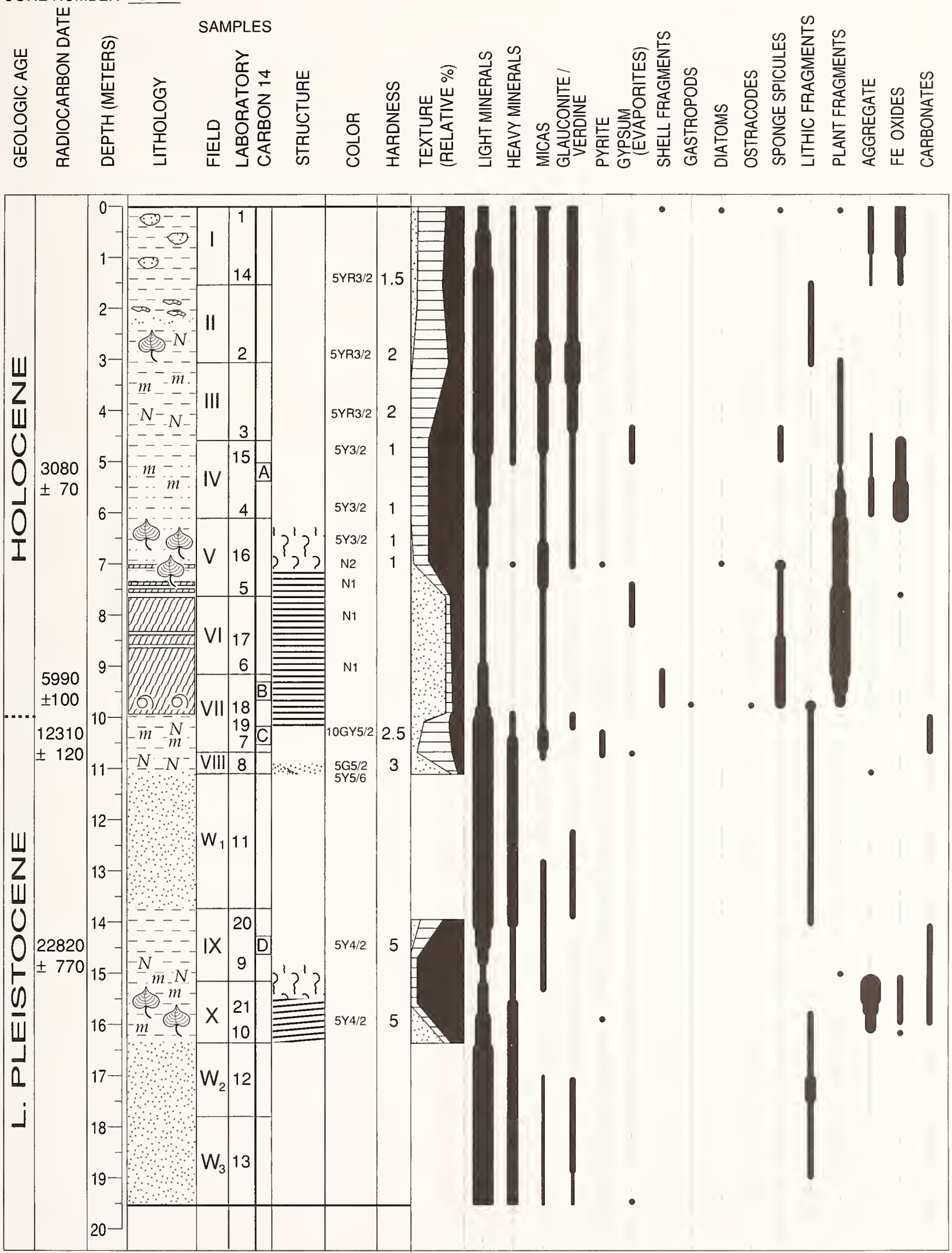
APPENDIX 1.-Continued.

CORE NUMBER \$55

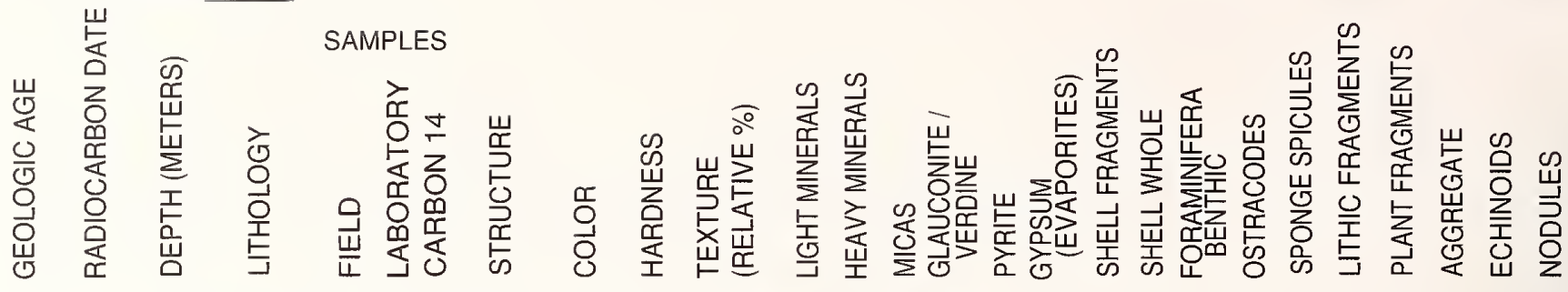

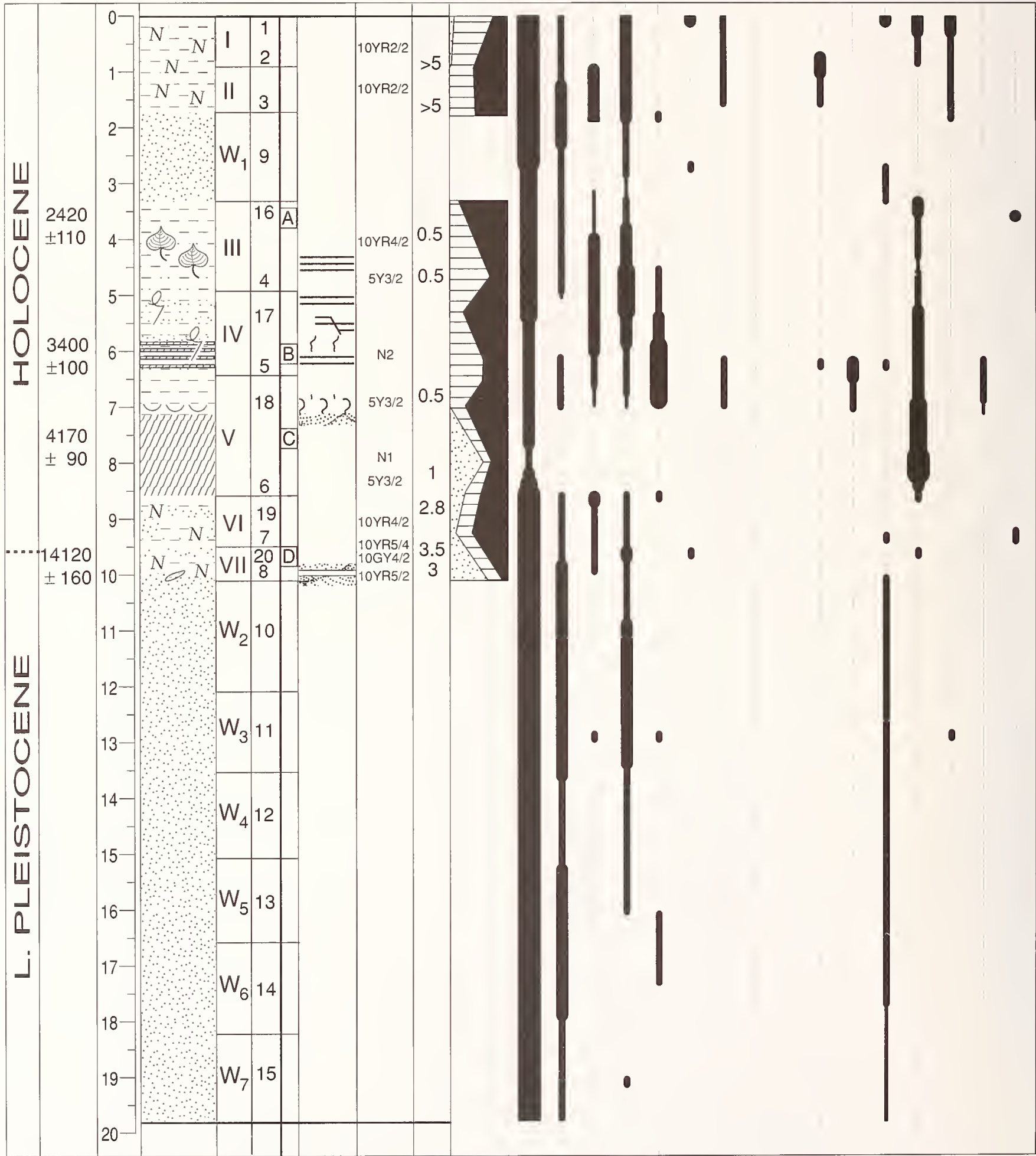


APPENDIX 1.-Continued.

CORE NUMBER S56

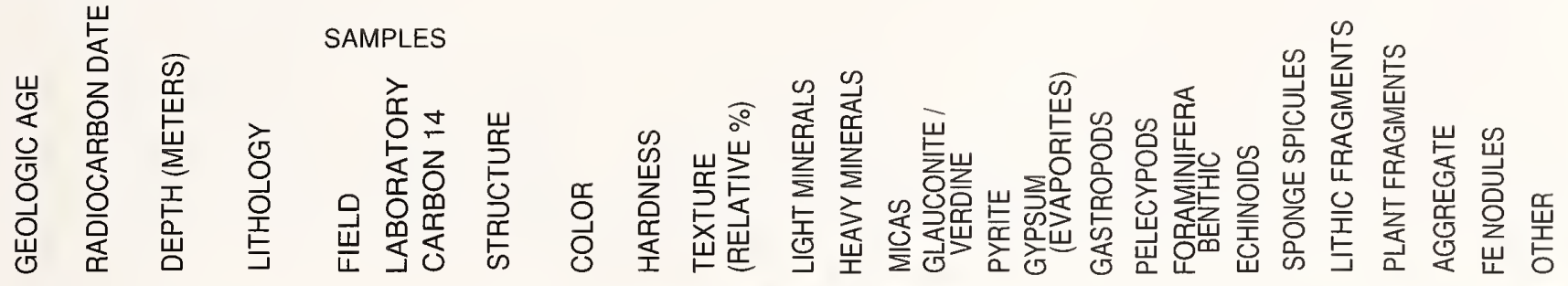

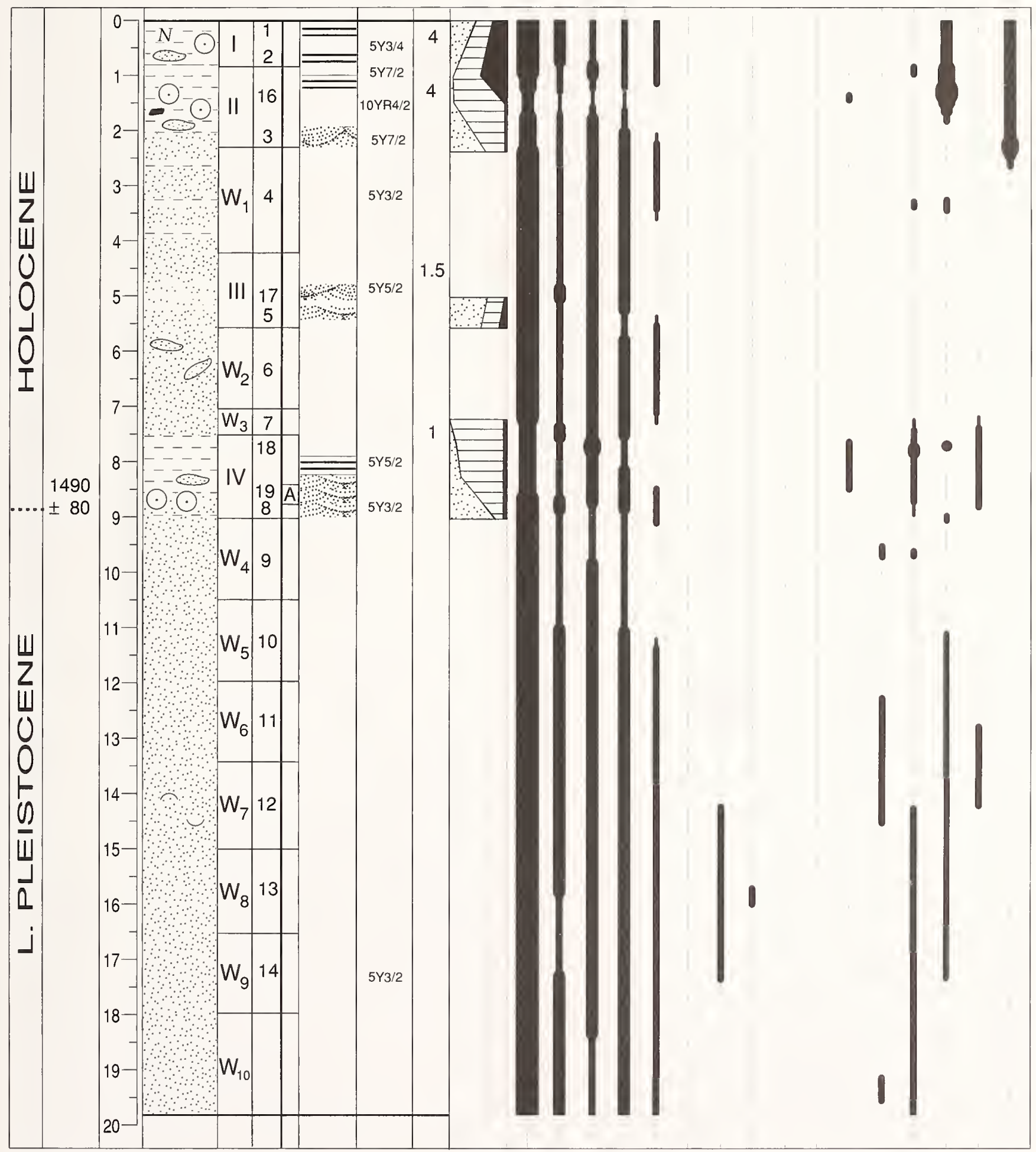


APPENDIX 1.-Continued.

CORE NUMBER S57

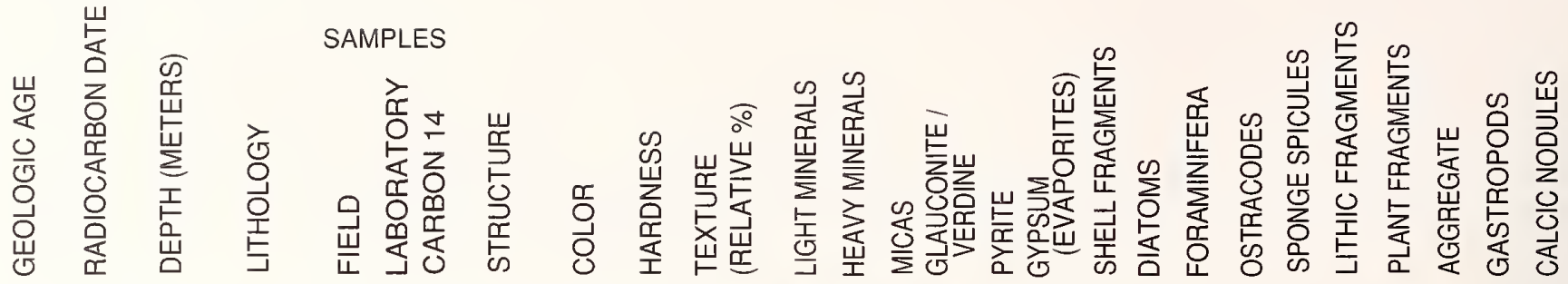

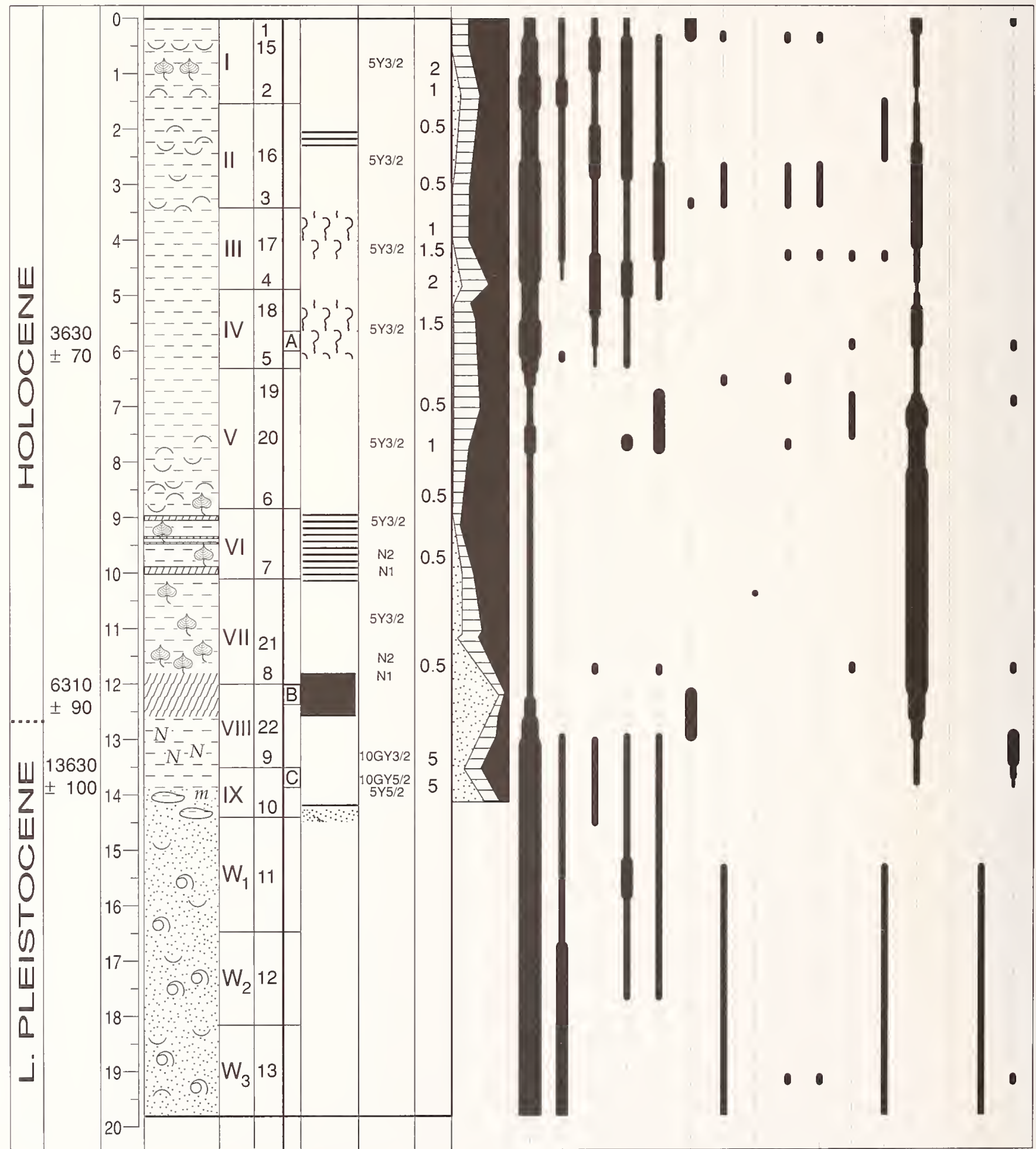


APPENDIX 1.-Continued.

CORE NUMBER S58 I

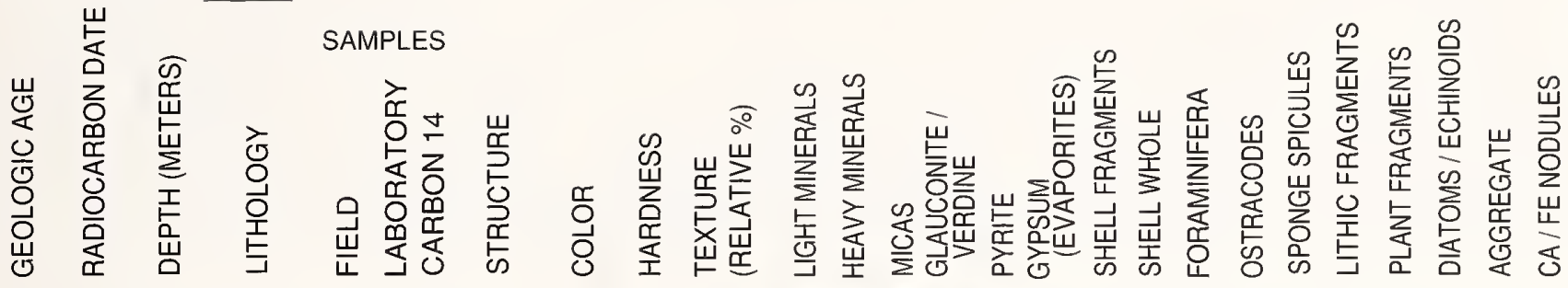

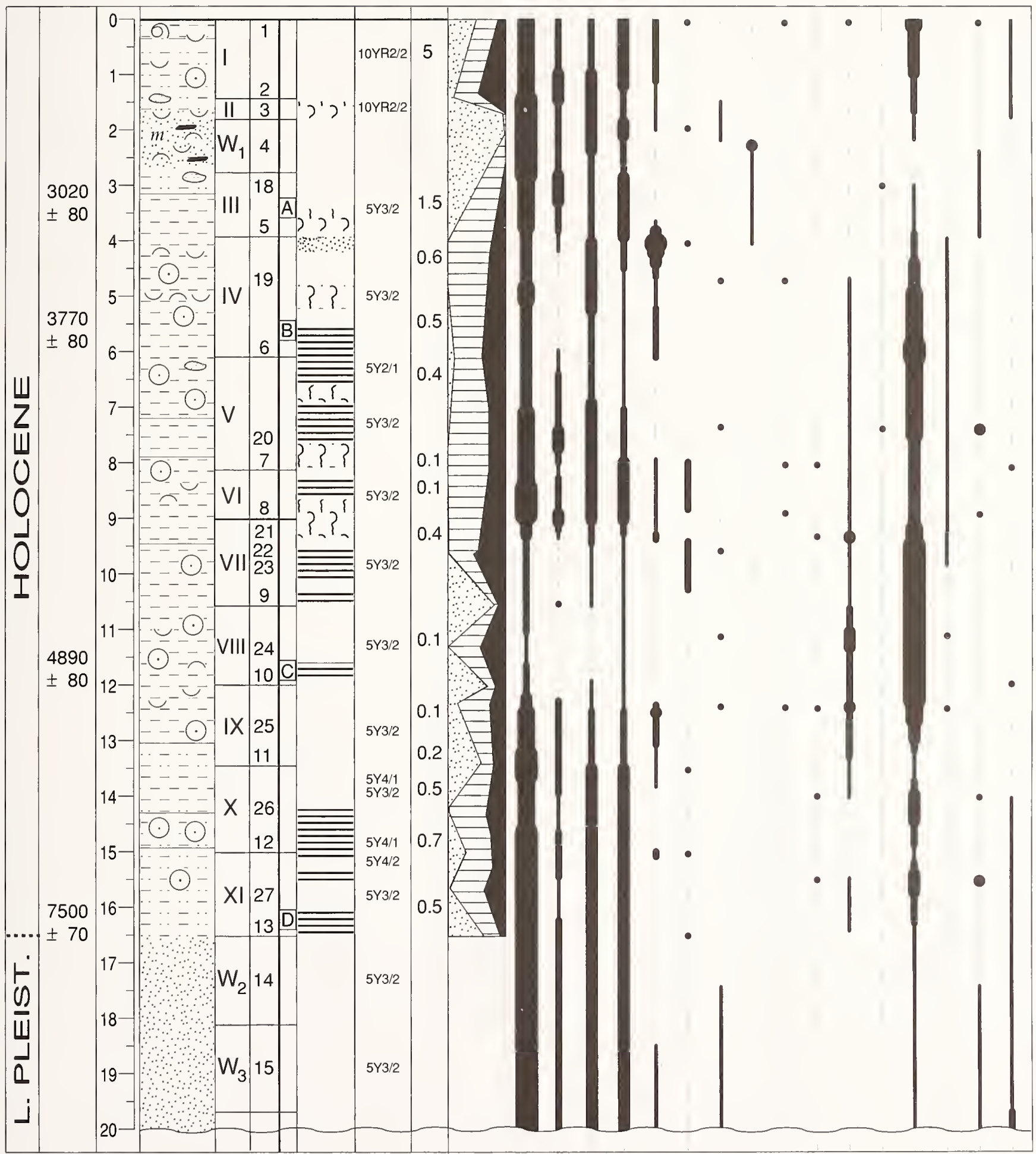


APPENDIX 1.-Continued.
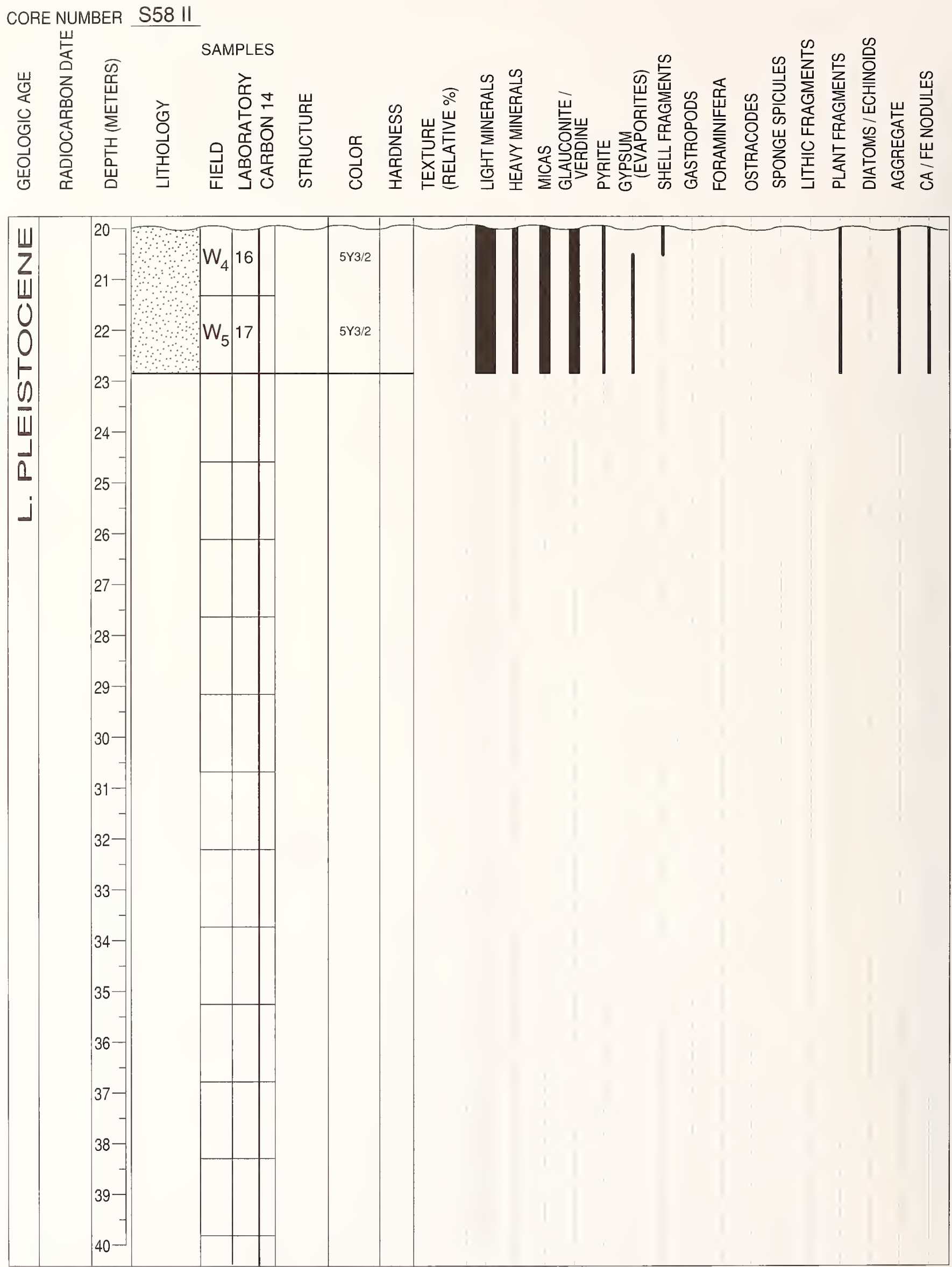
APPENDIX 1.-Continued.

CORE NUMBER S59I
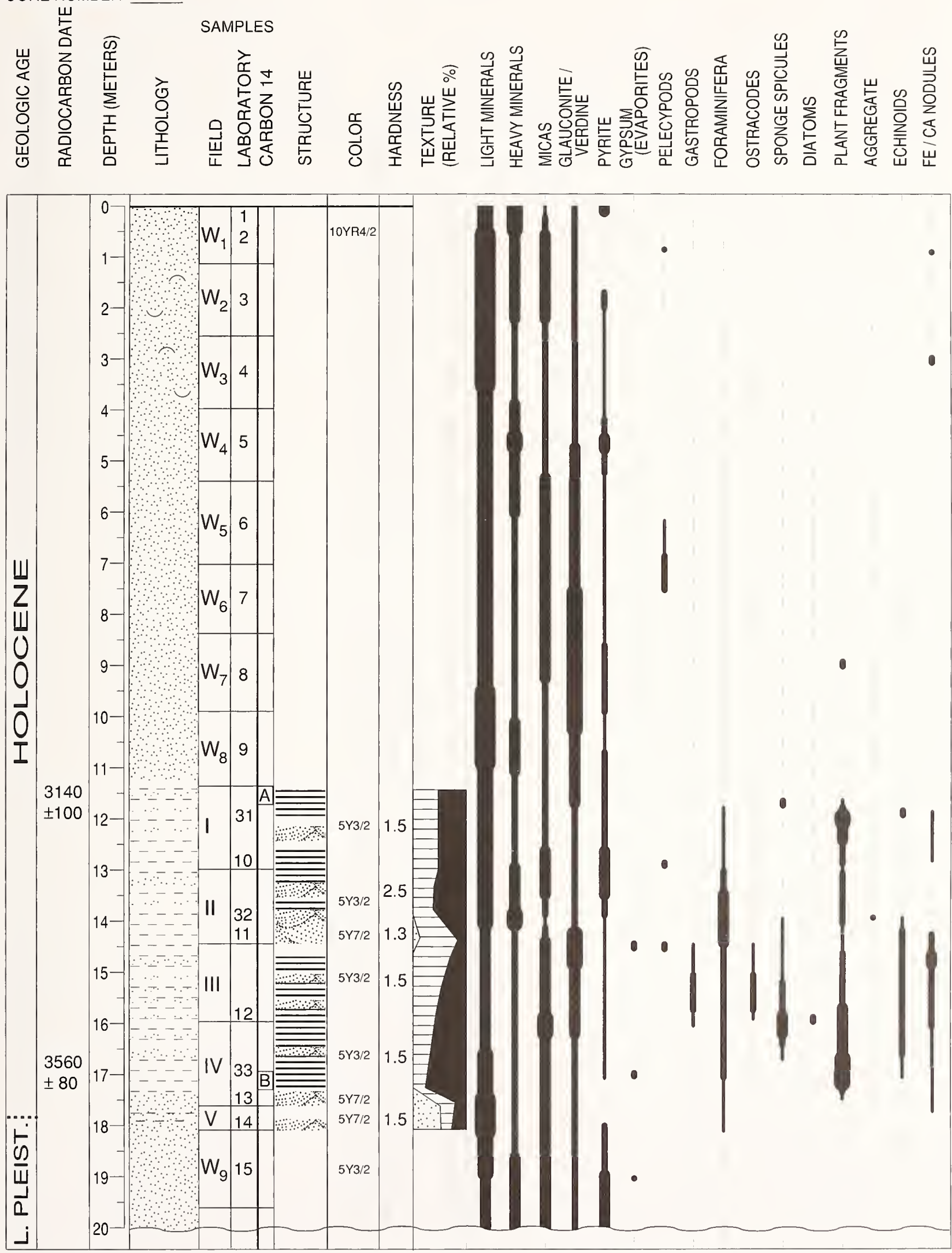
APPENDIX 1.-Continued.
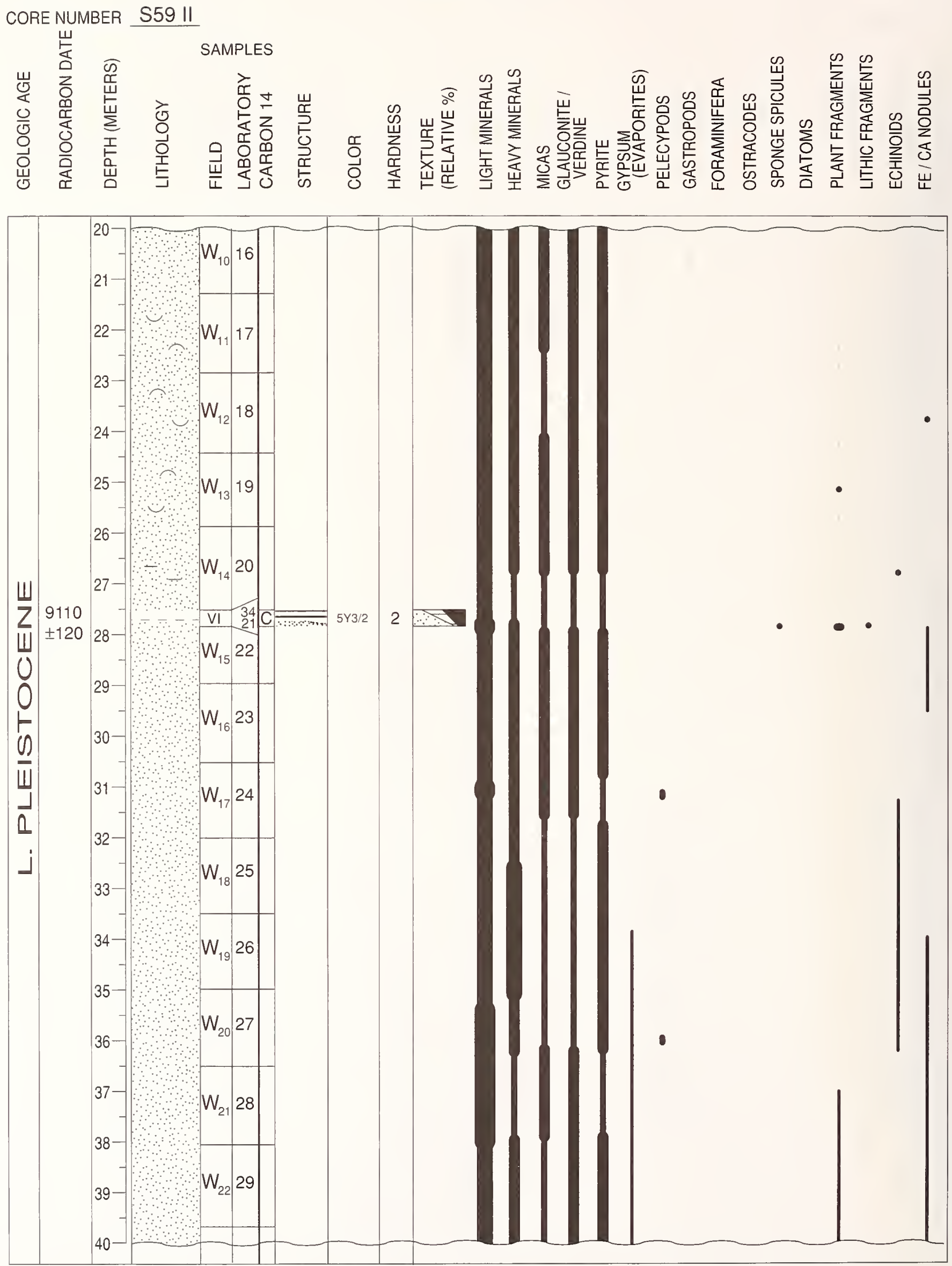
APPENDIX 1.-Continued.

CORE NUMBER S59 III

SAMPLES

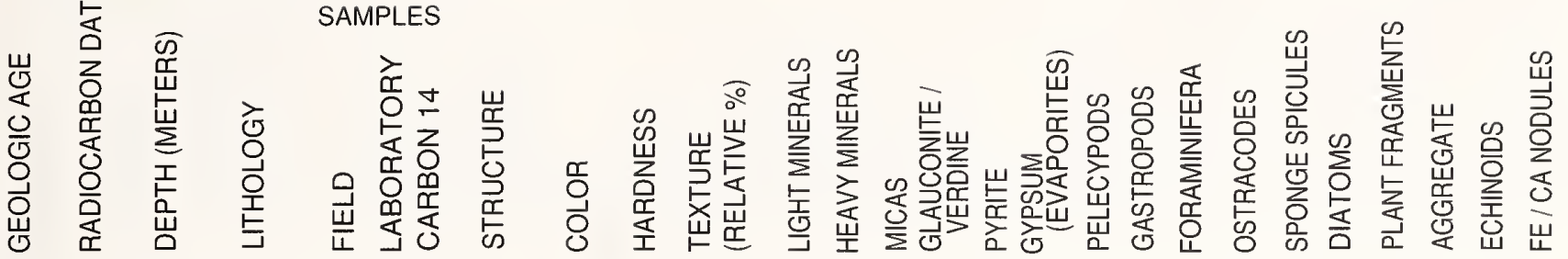

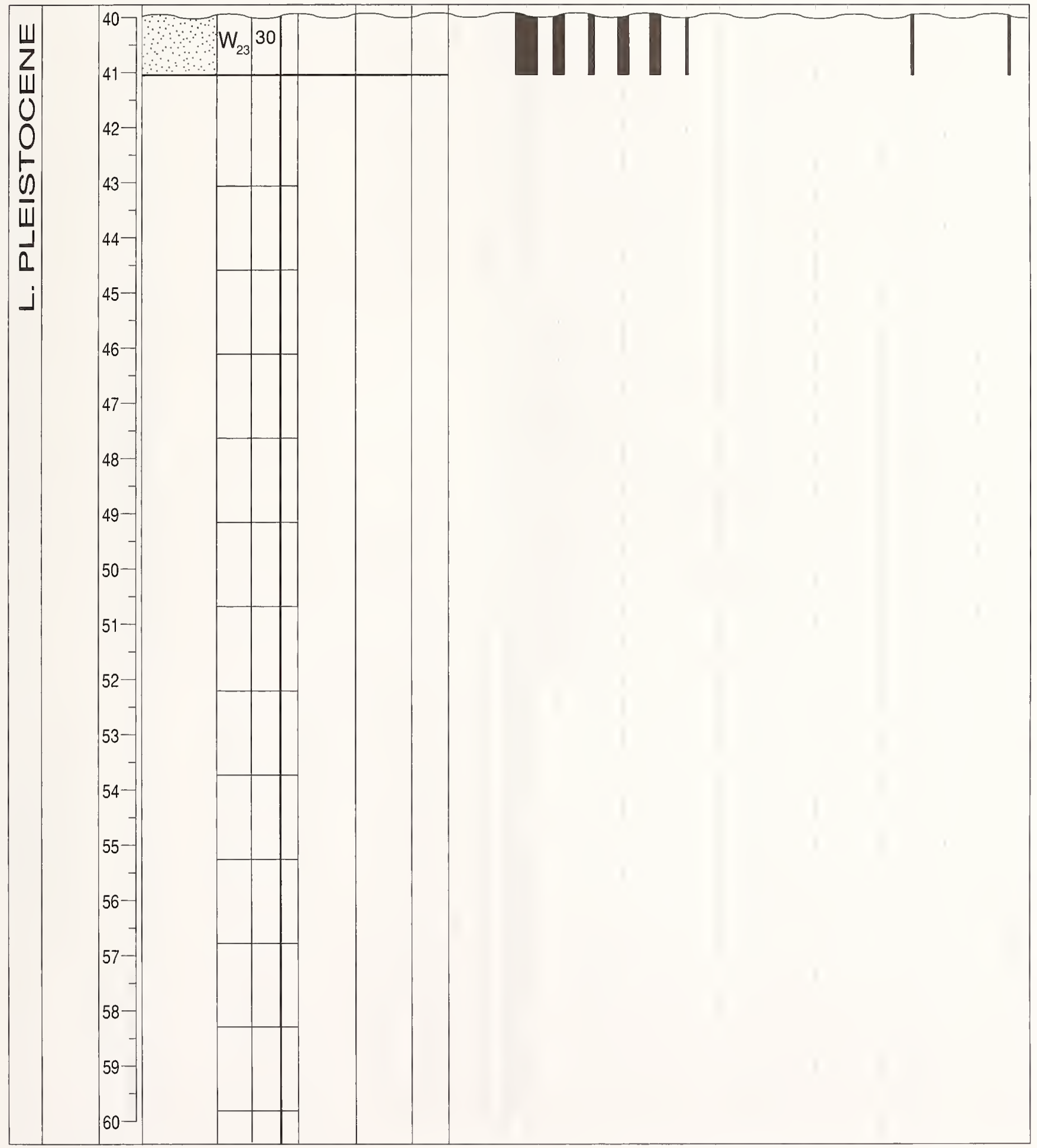


APPENDIX 1.-Continued.

CORE NUMBER S6OI
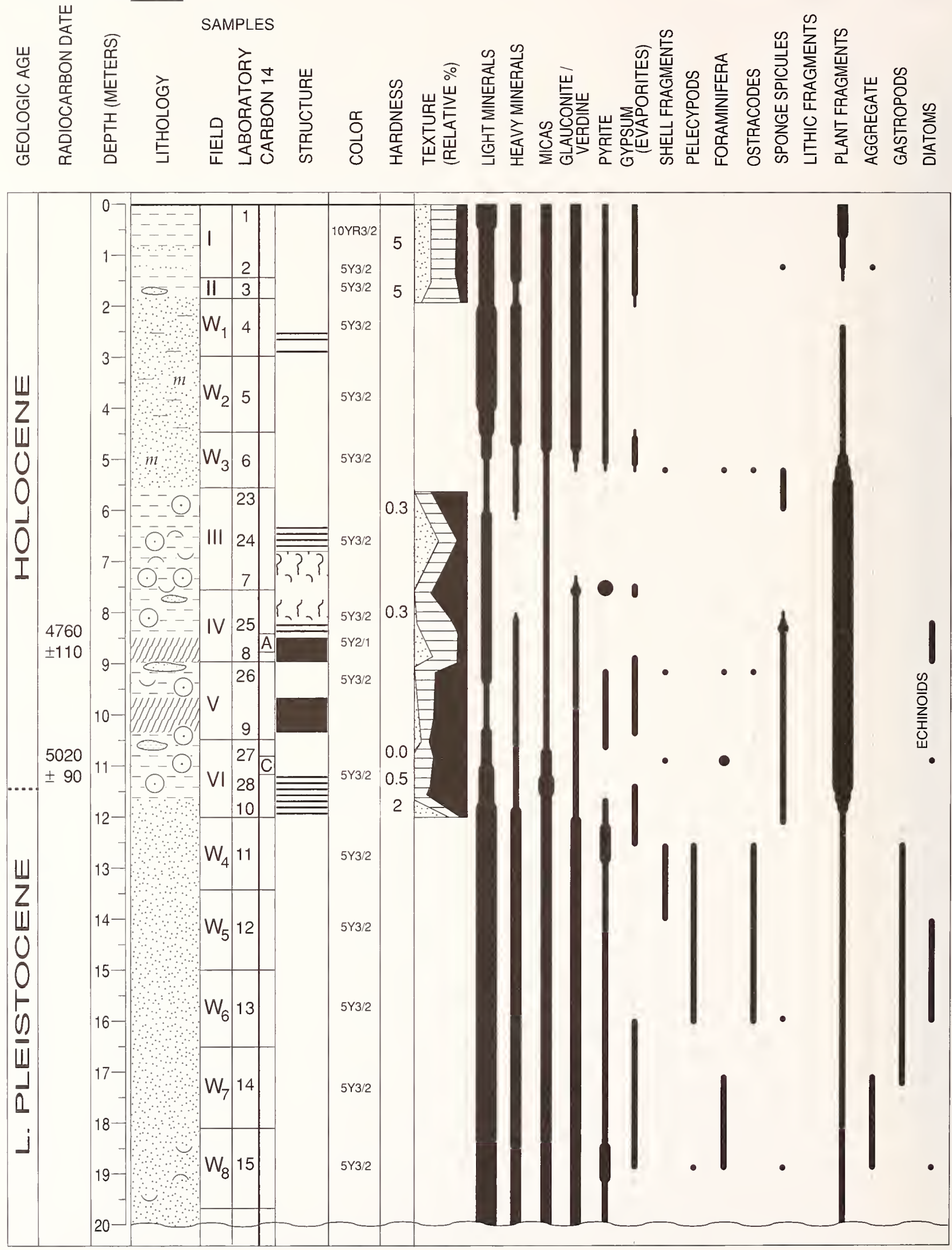
APPENDIX 1.-Continued.
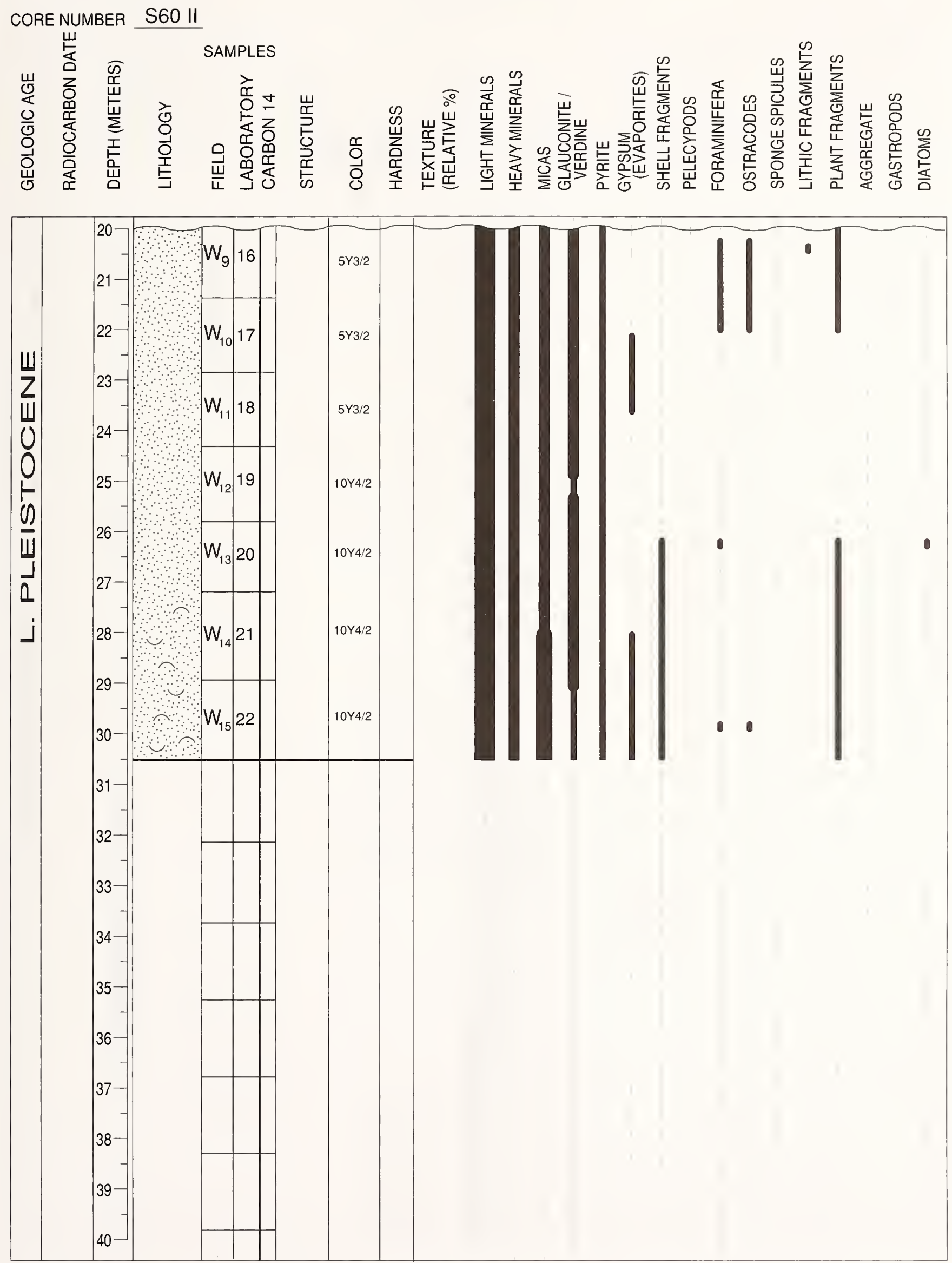
APPENDIX 1.-Continued.

CORE NUMBER S61I
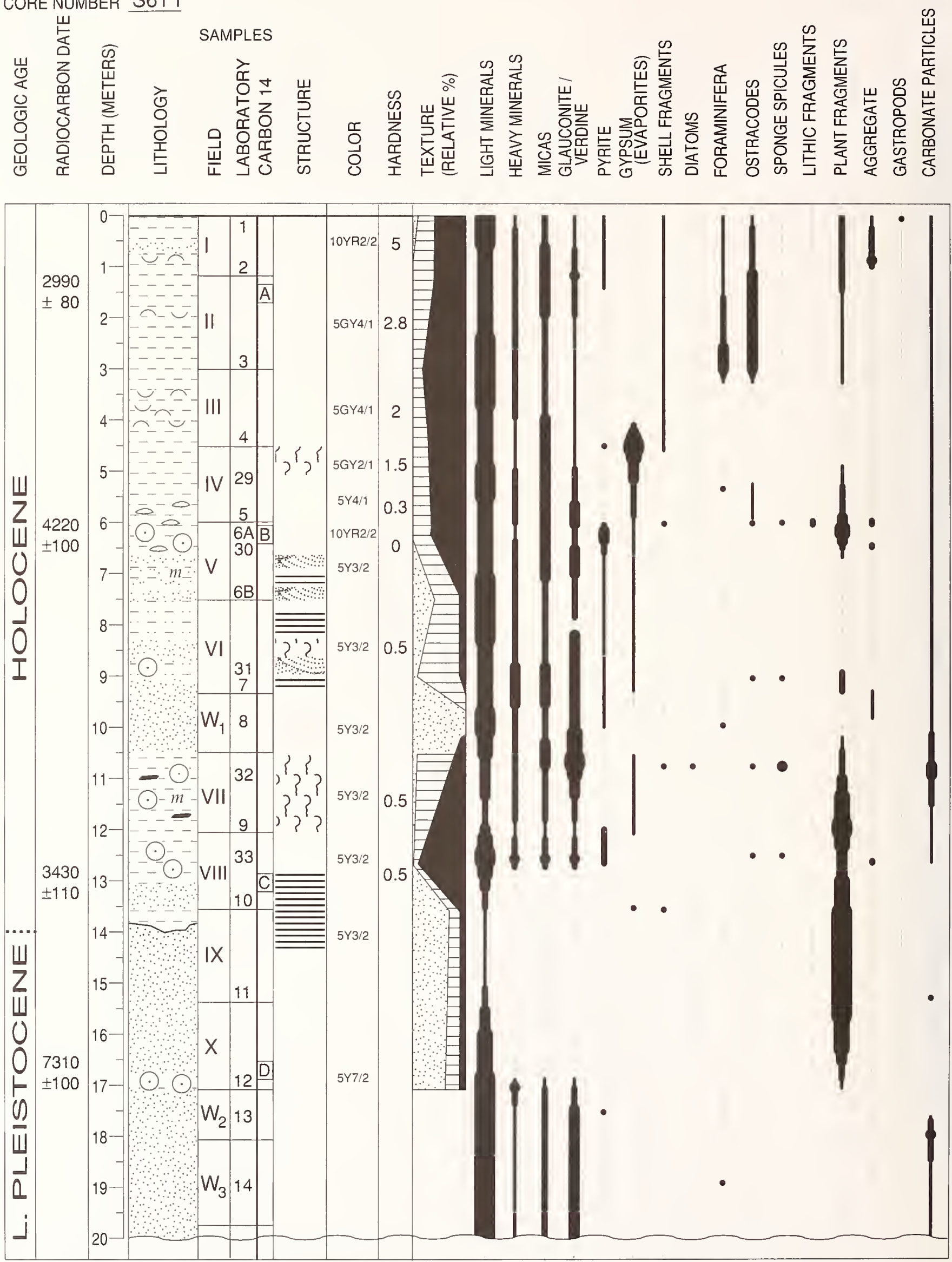
APPENDIX 1.-Continued.
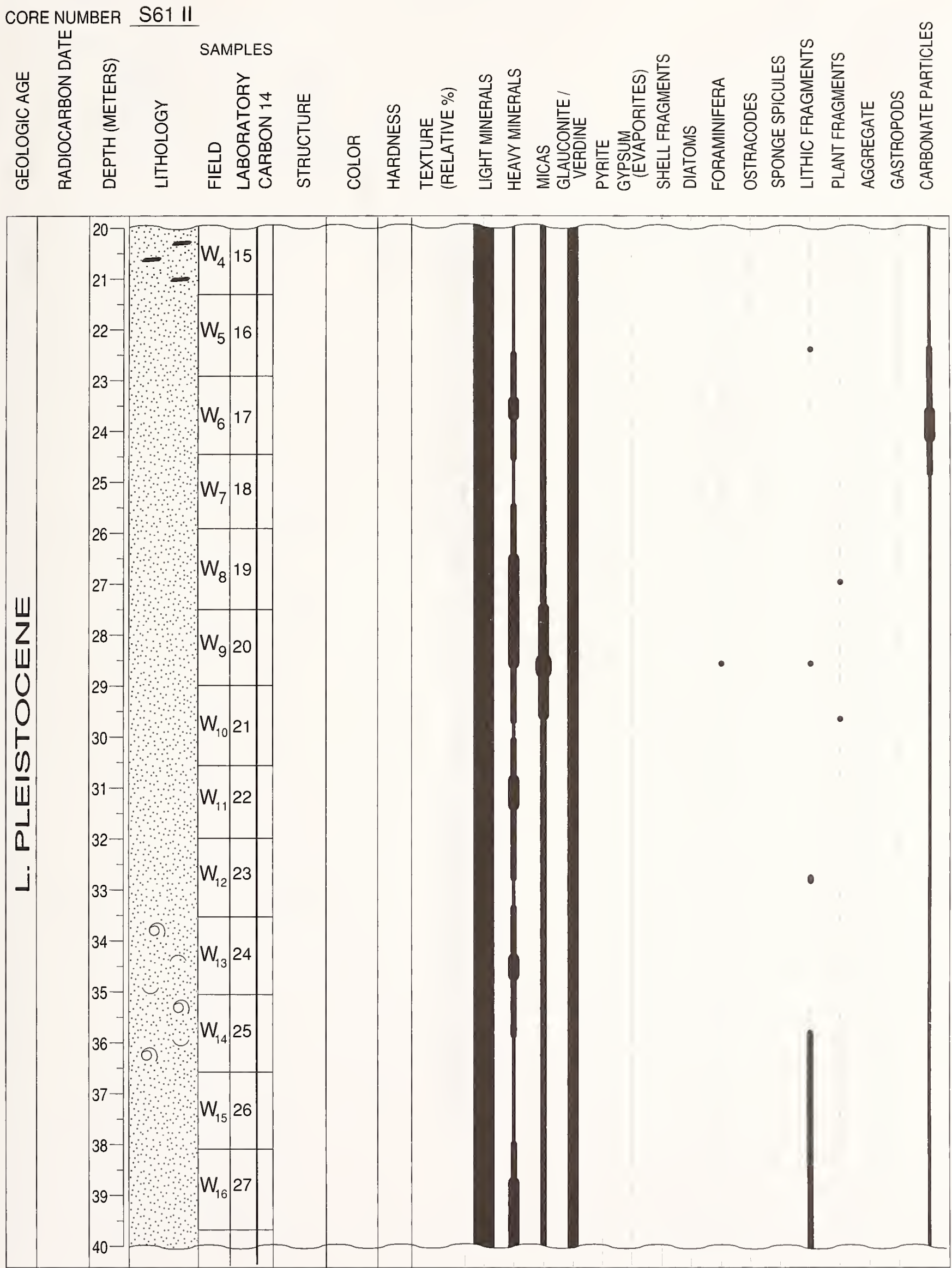
APPENDIX 1.-Continued.
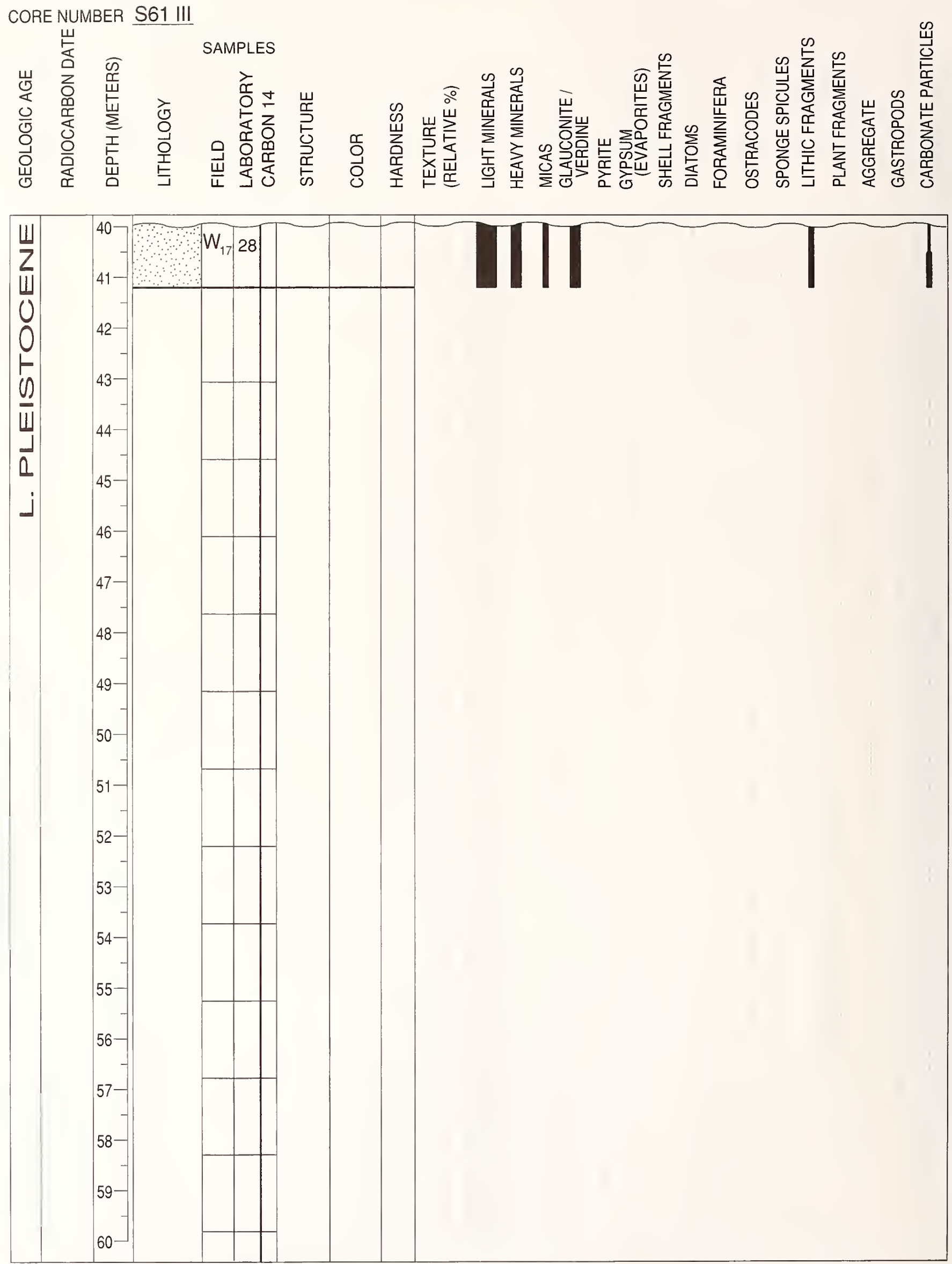
APPENDIX 1.-Continued.

CORE NUMBER S62I
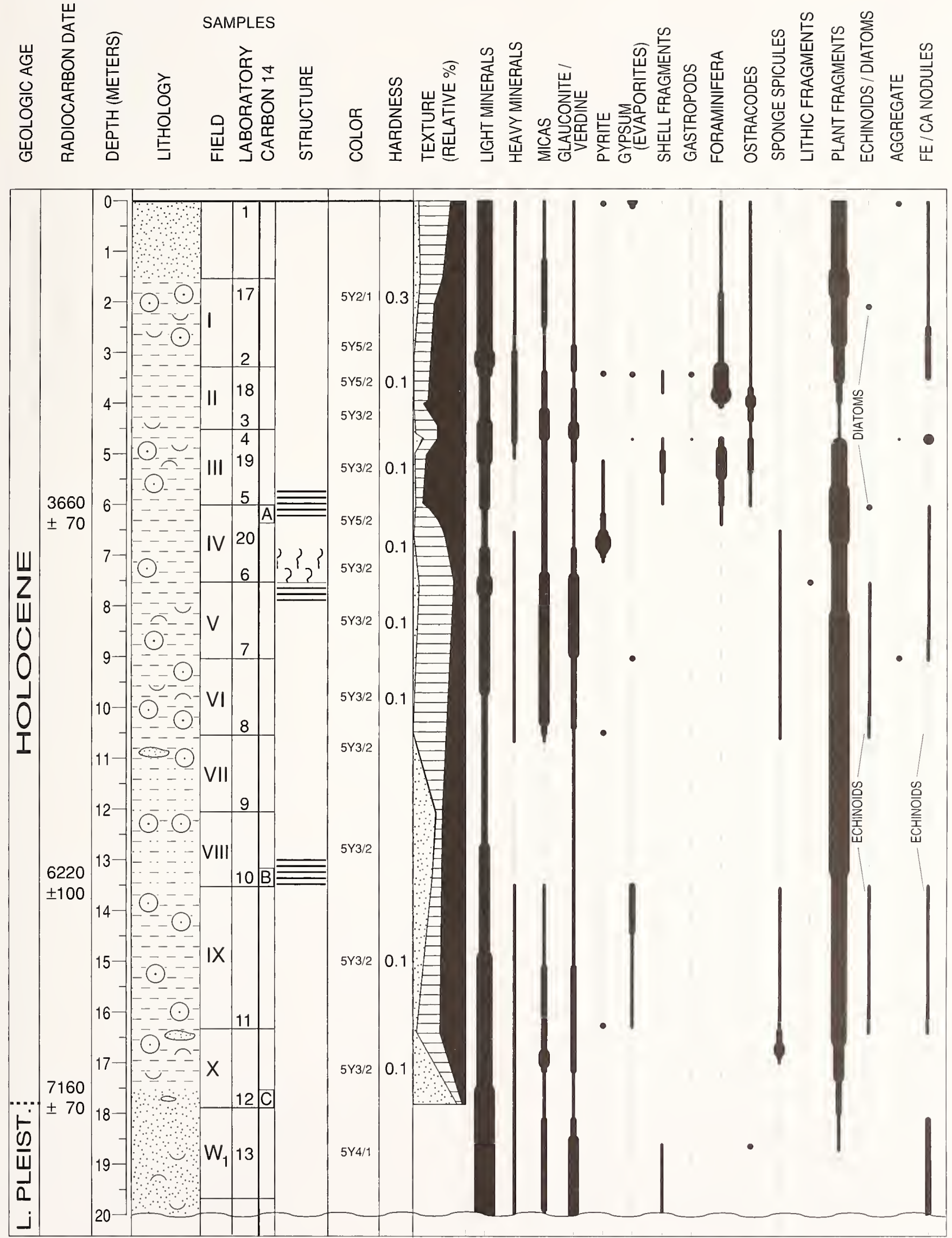
APPENDIX 1.-Continued.
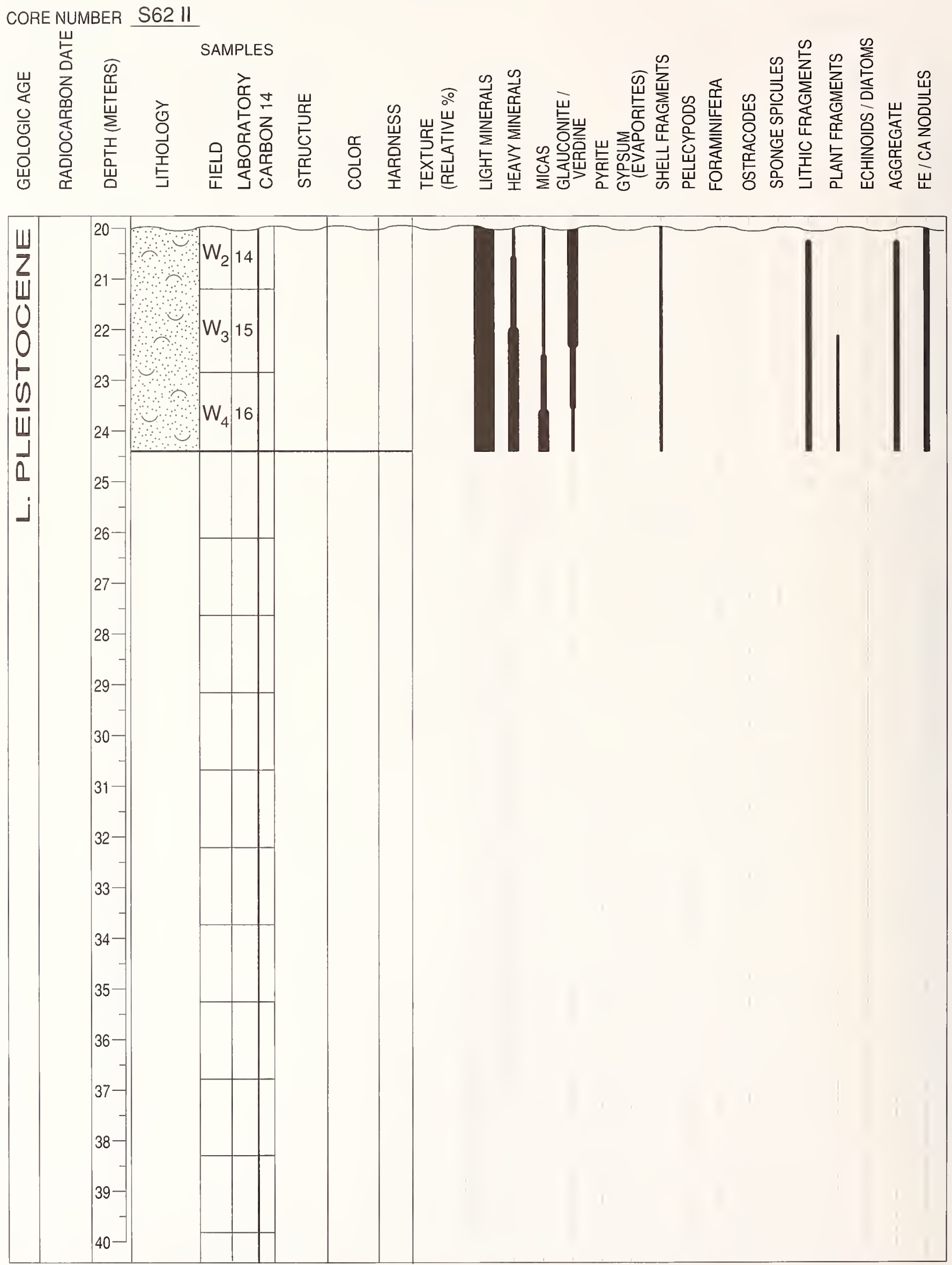
APPENDIX 1.-Continued.

CORE NUMBER S63 I

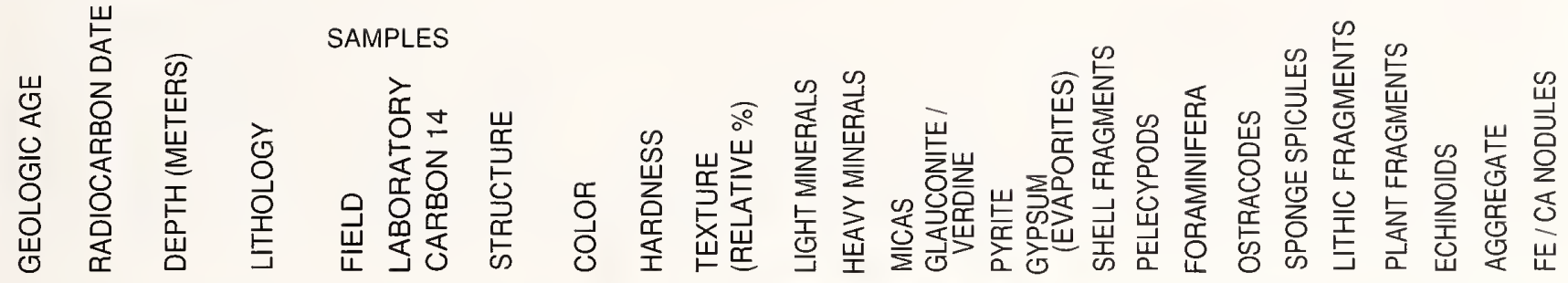

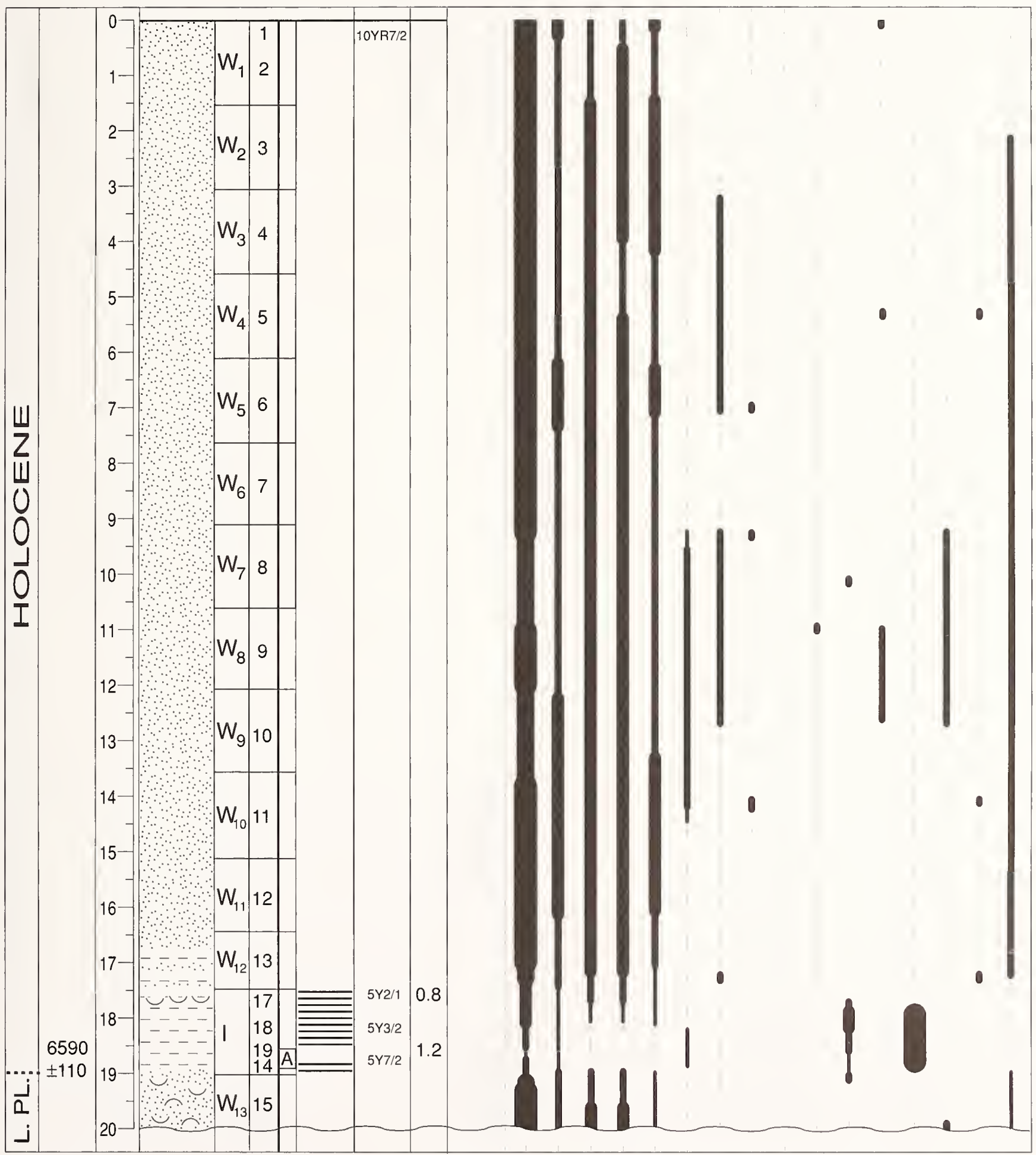


APPENDIX 1.-Continued.

CORE NUMBER S63 II

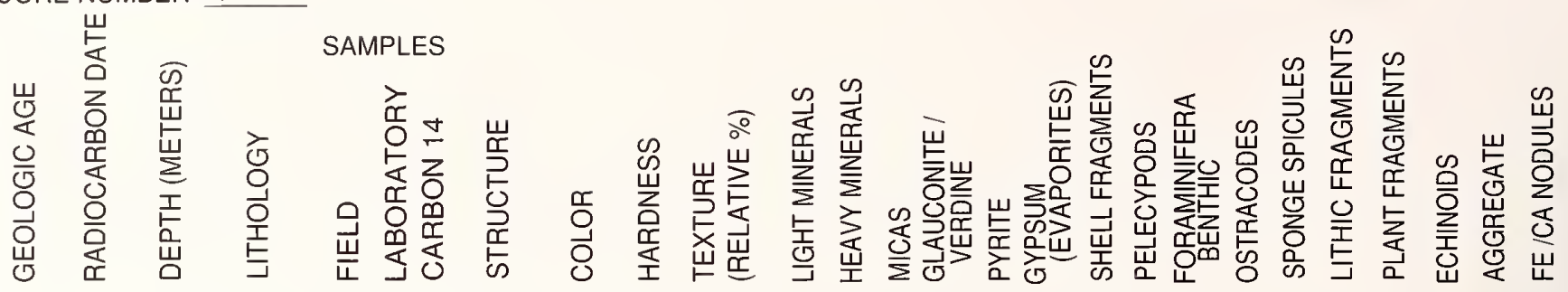

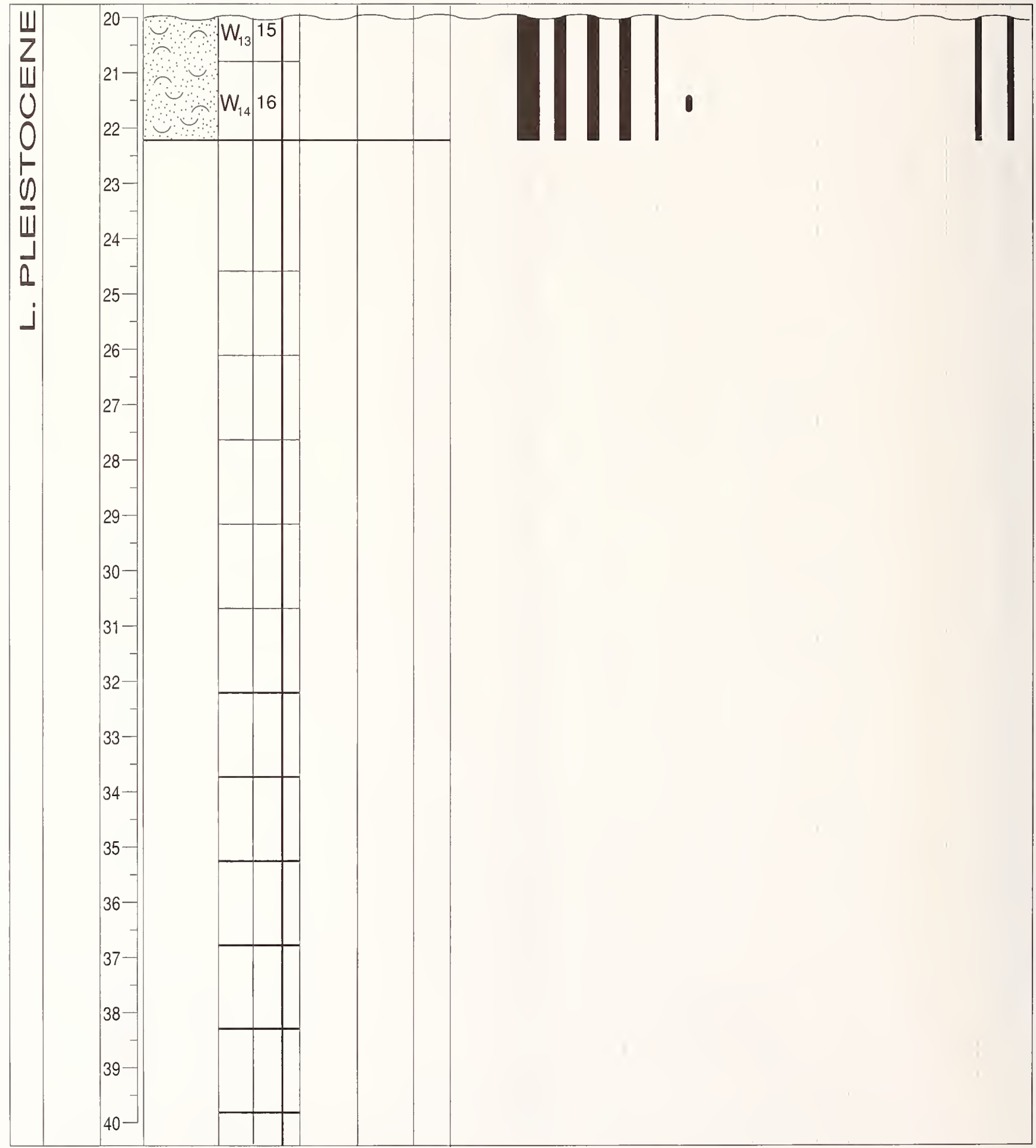


APPENDIX 1.-Continued.

\section{CORE NUMBER S64 I}
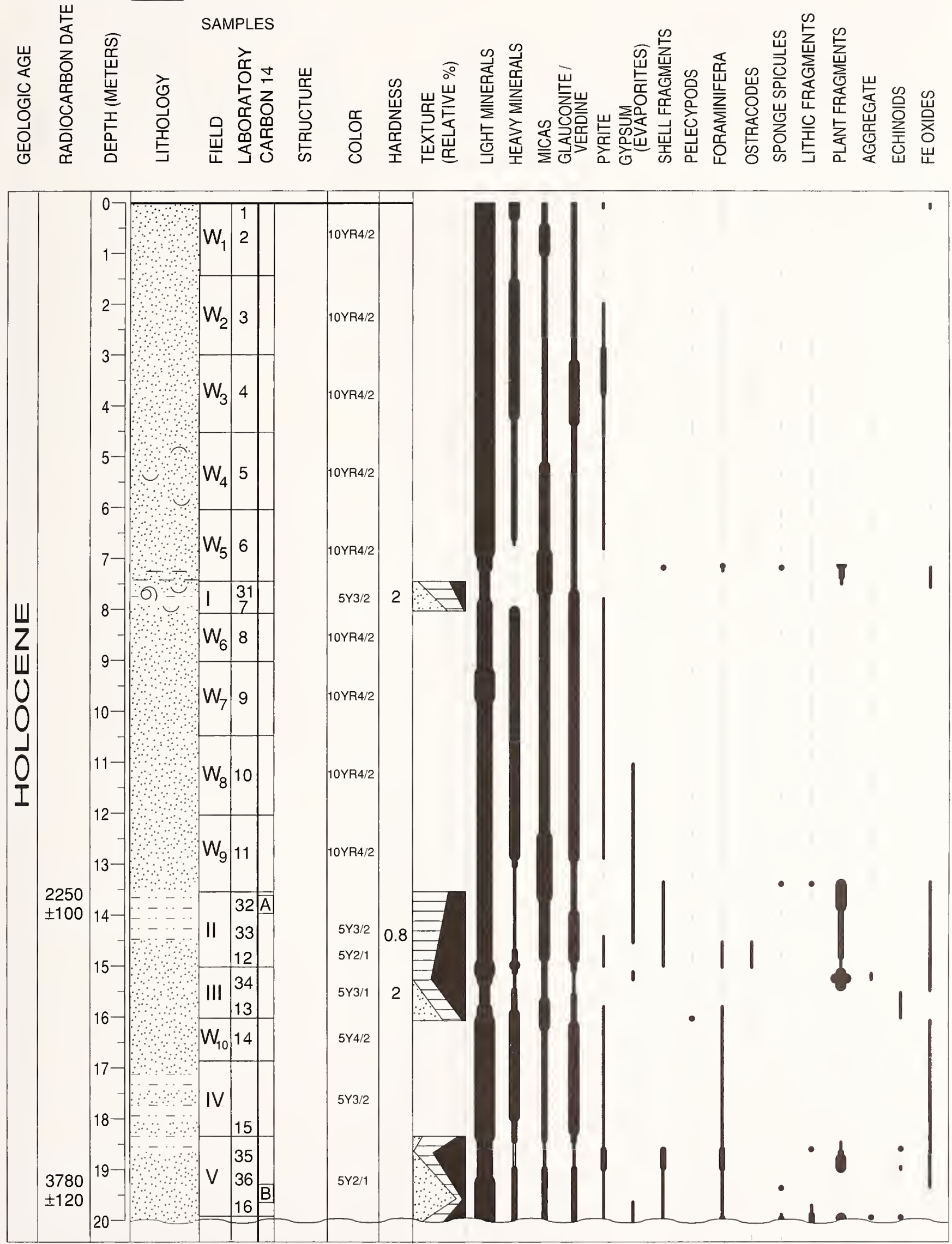
APPENDIX 1.-Continued.

CORE NUMBER S64 II

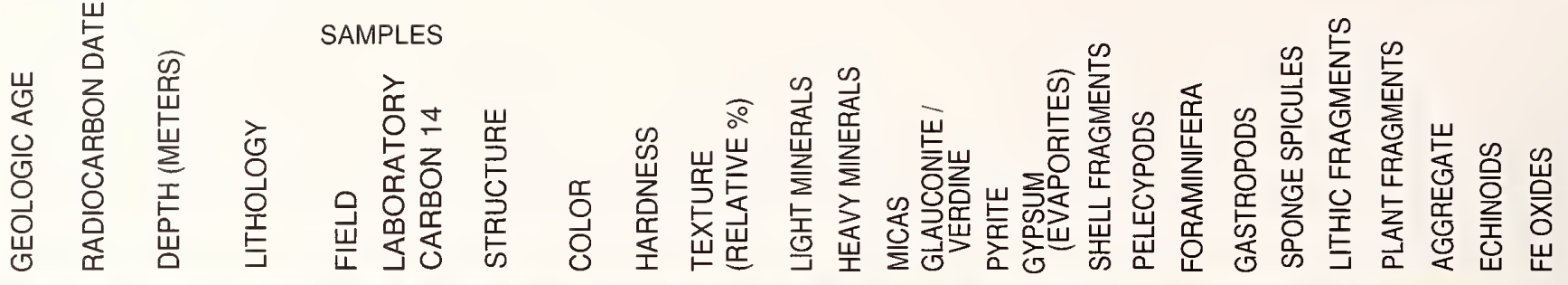

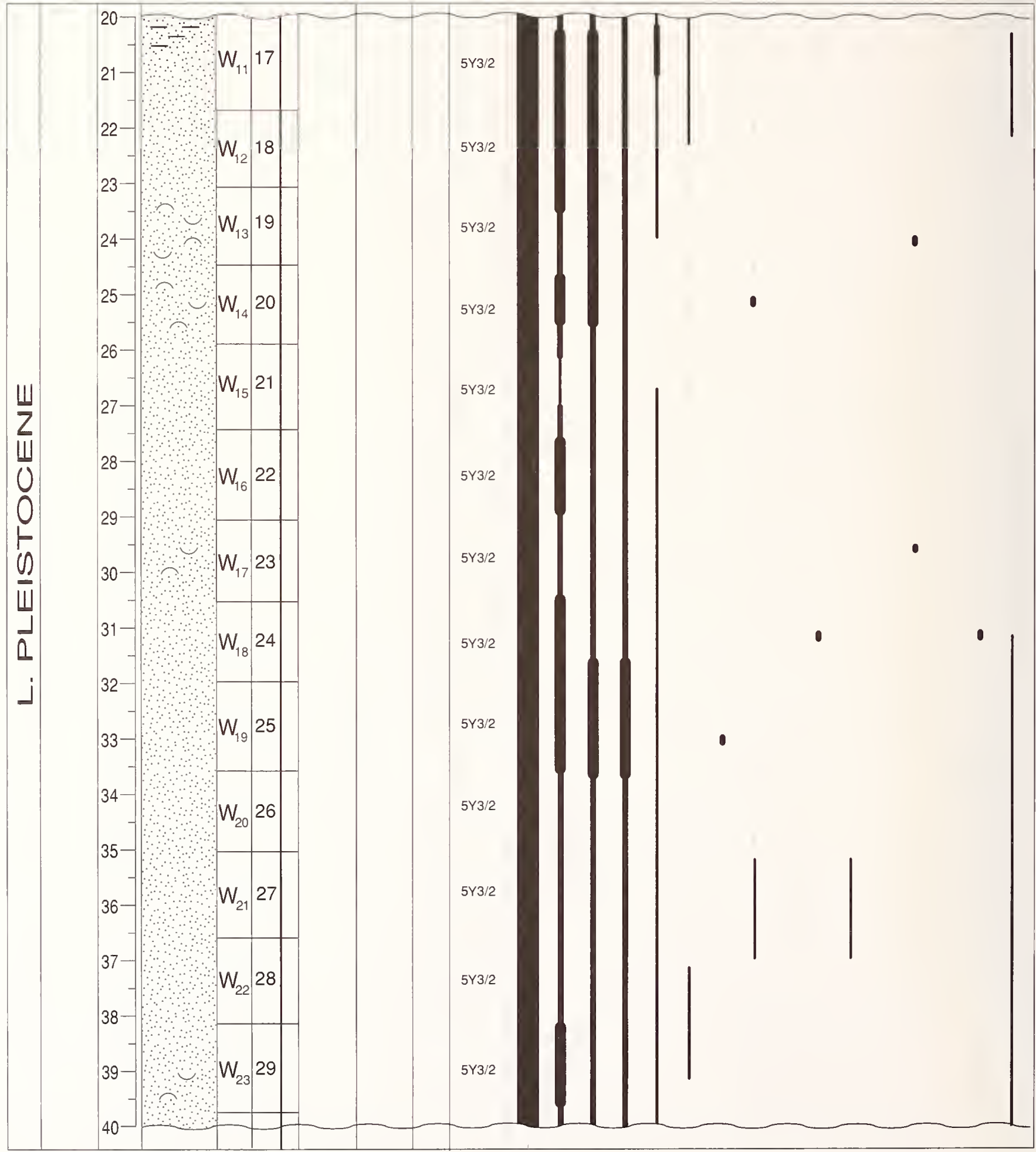


APPENDIX 1.-Continued.

CORE NUMBER S64 III

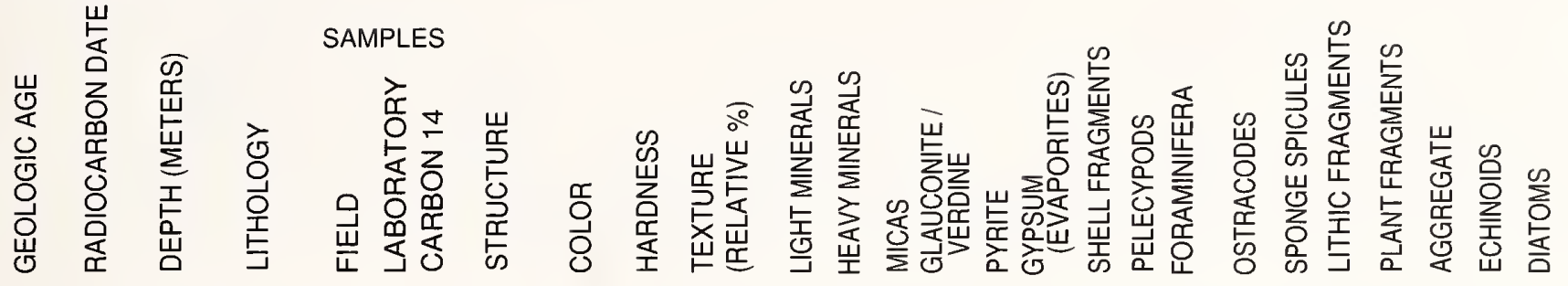

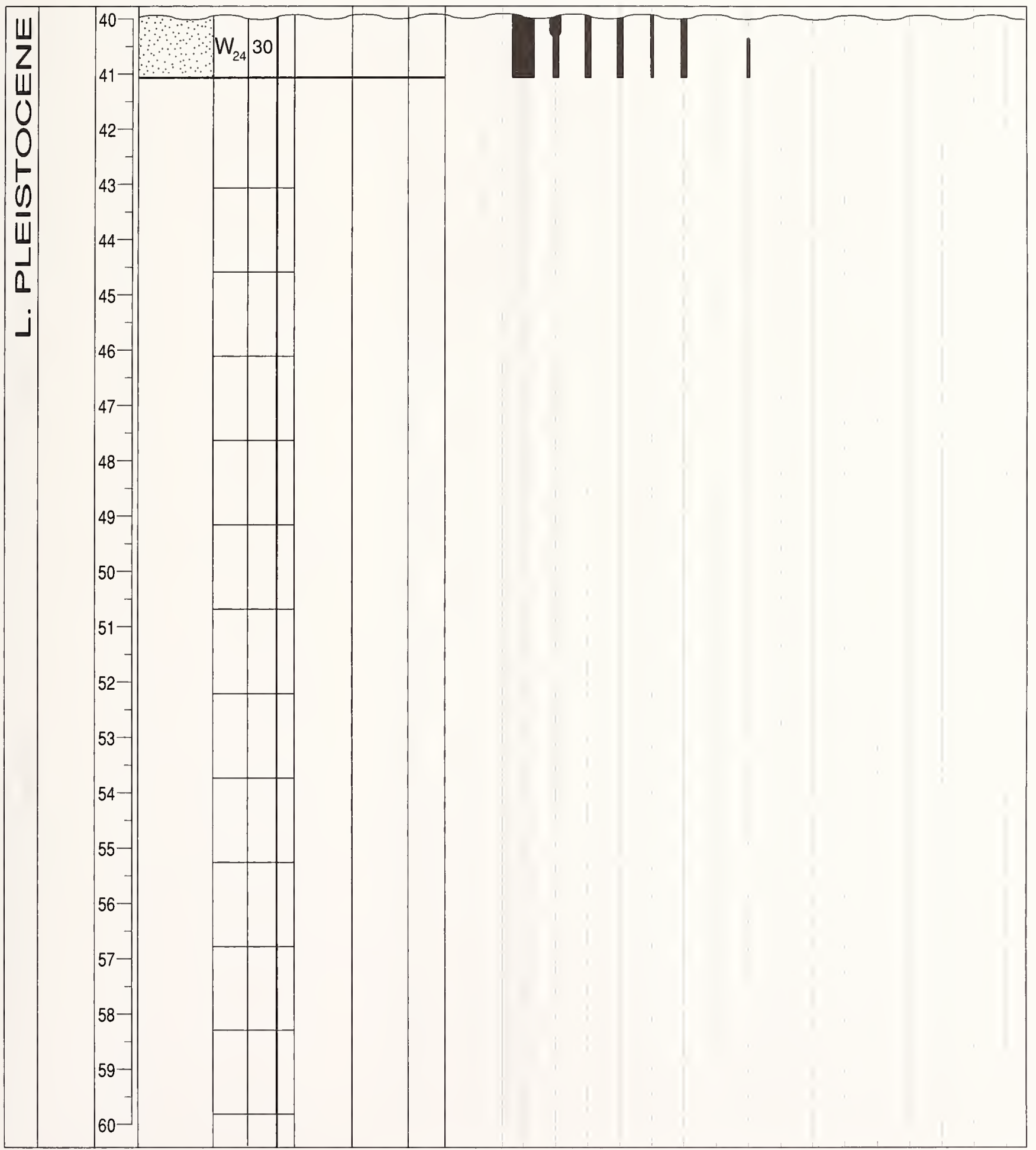


APPENDIX 1.-Continued.

CORE NUMBER S65I

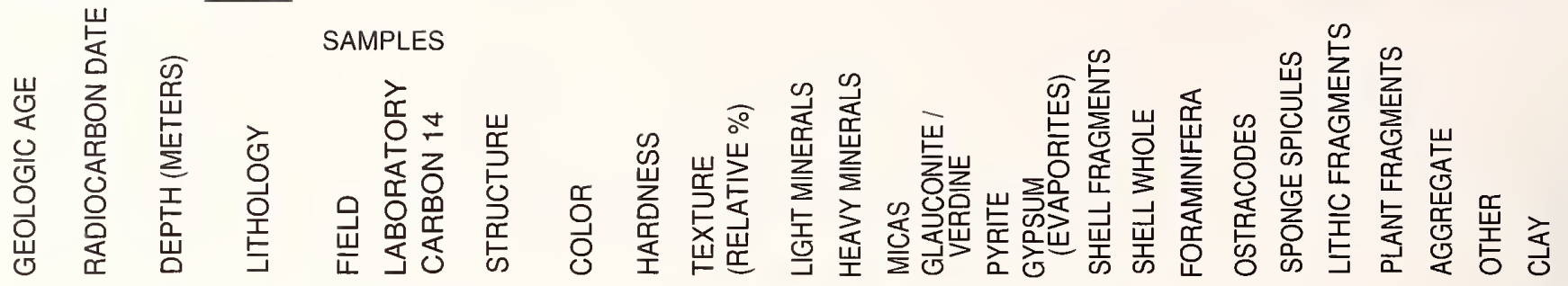

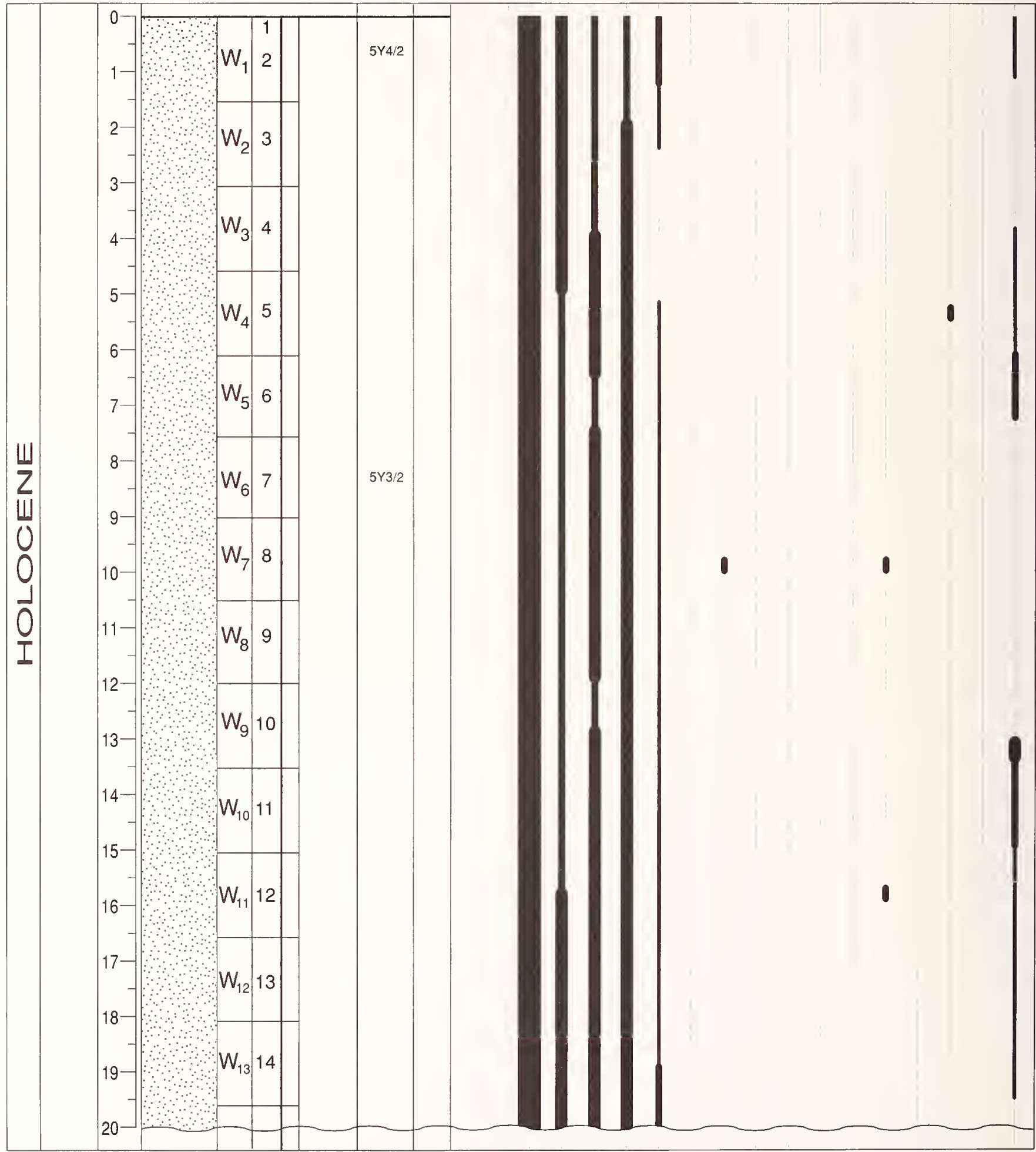


APPENDIX 1.-Continued.

CORE NUMBER S65 II
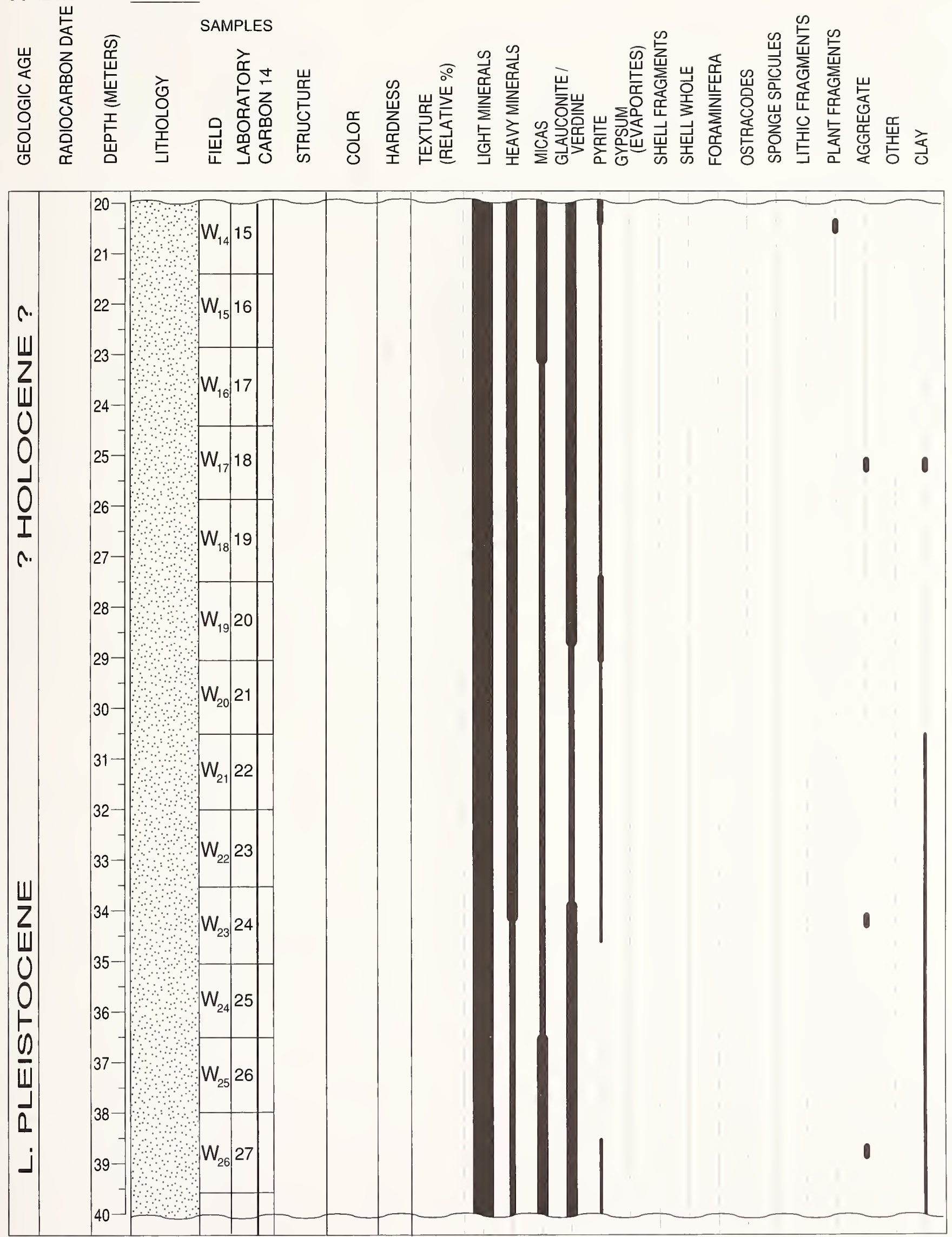
APPENDIX 1.-Continued.

CORE NUMBER S65 III

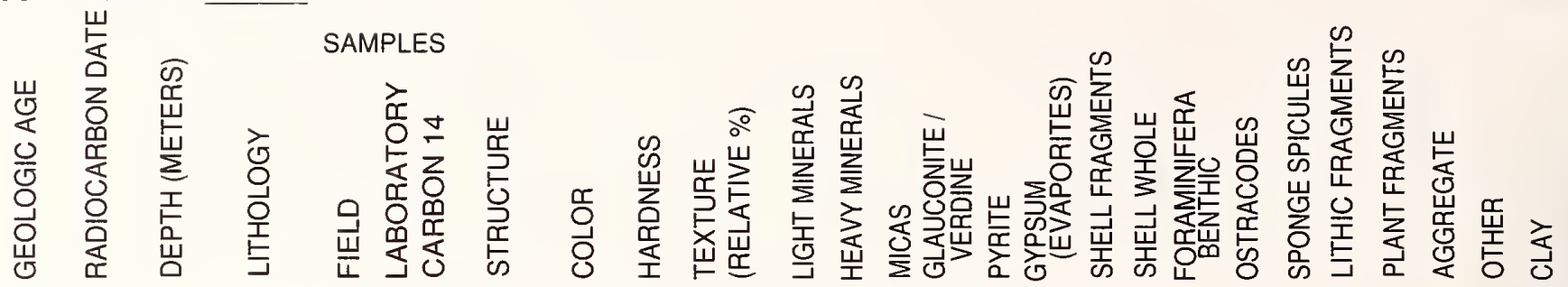

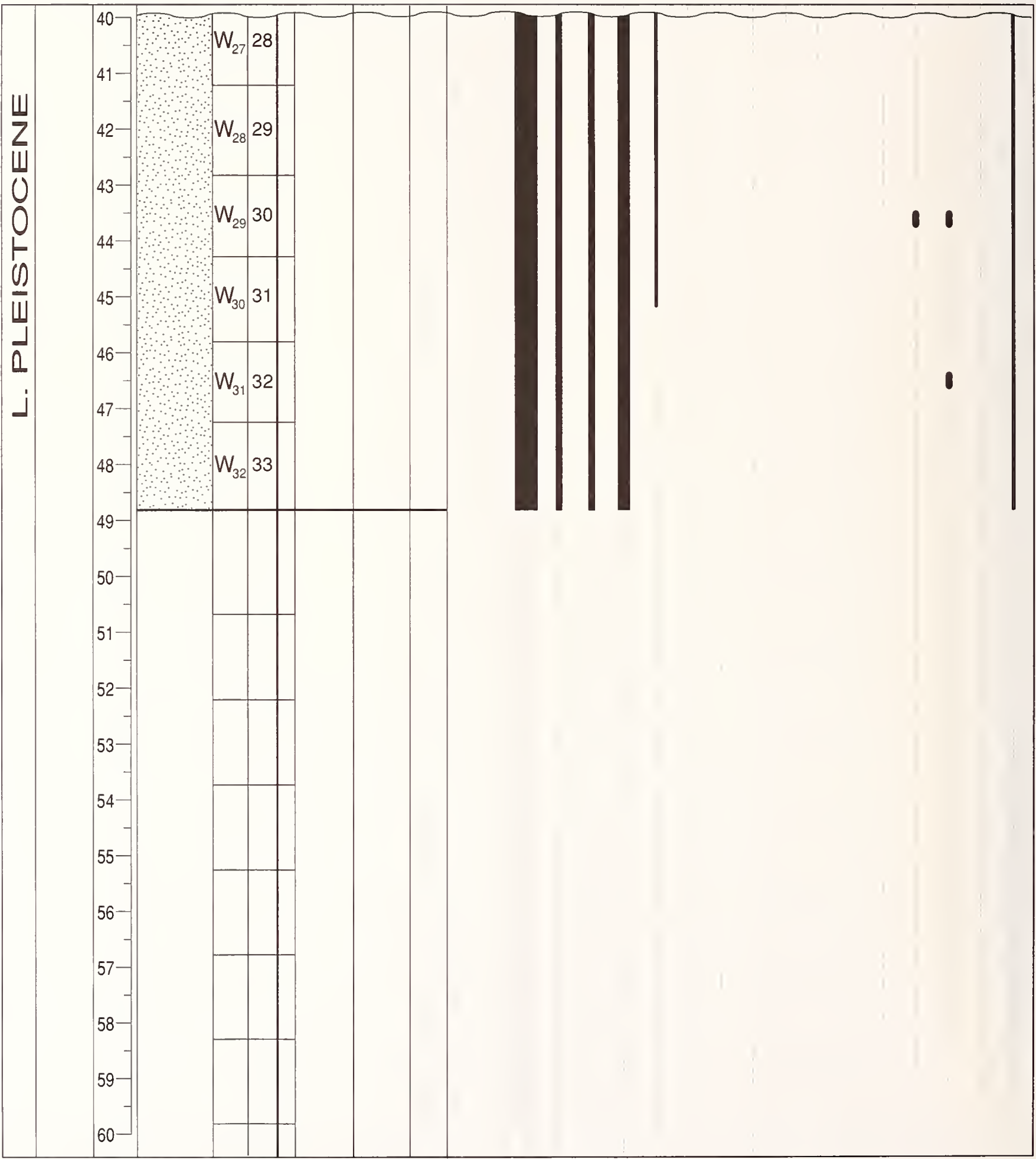


APPENDIX 1.-Continued.

CORE NUMBER S66
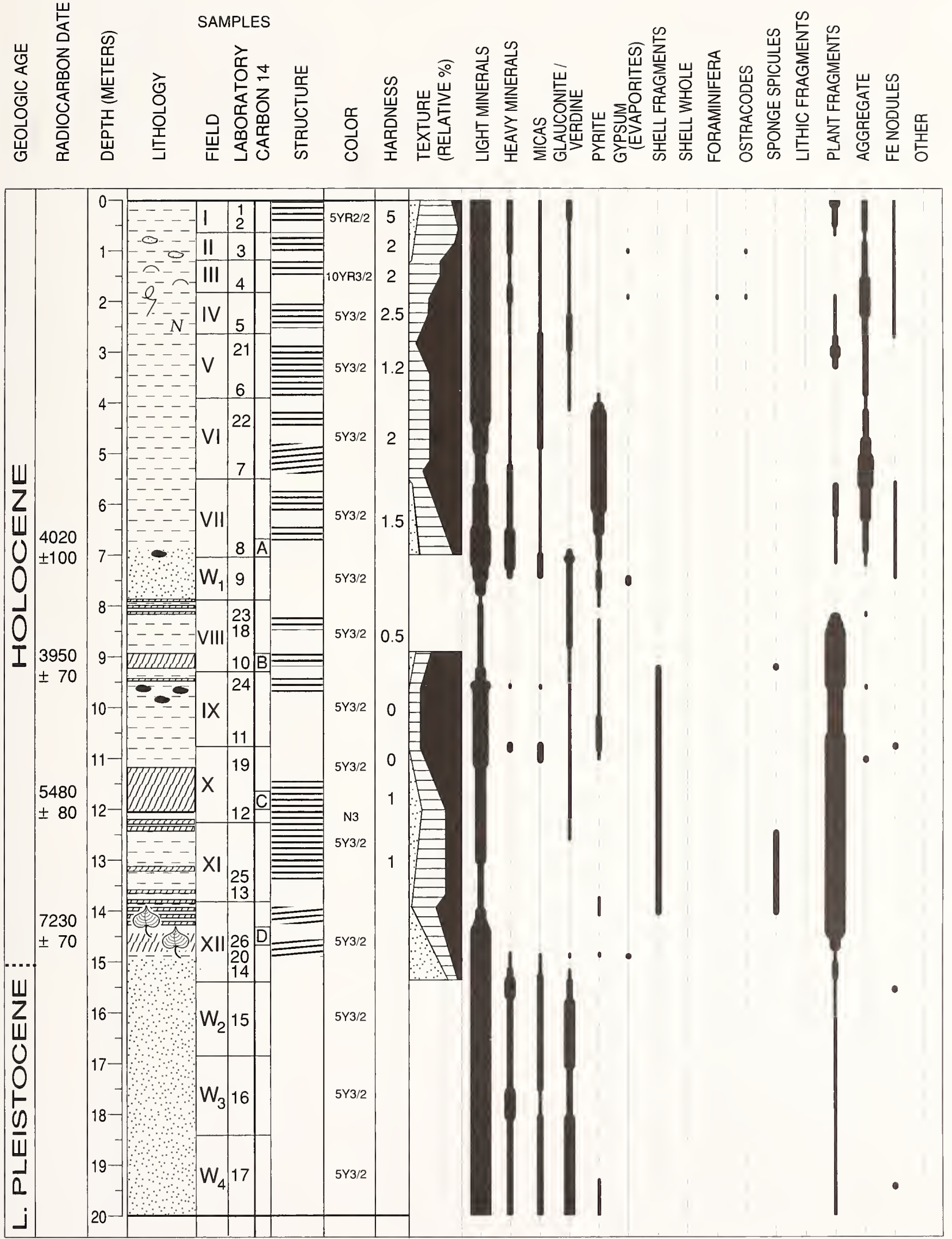
APPENDIX 1.-Continued.

CORE NUMBER S67

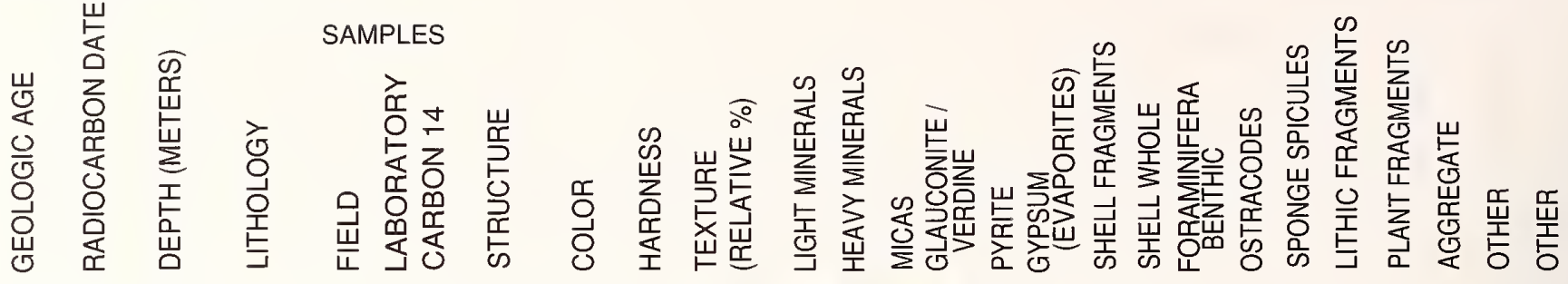

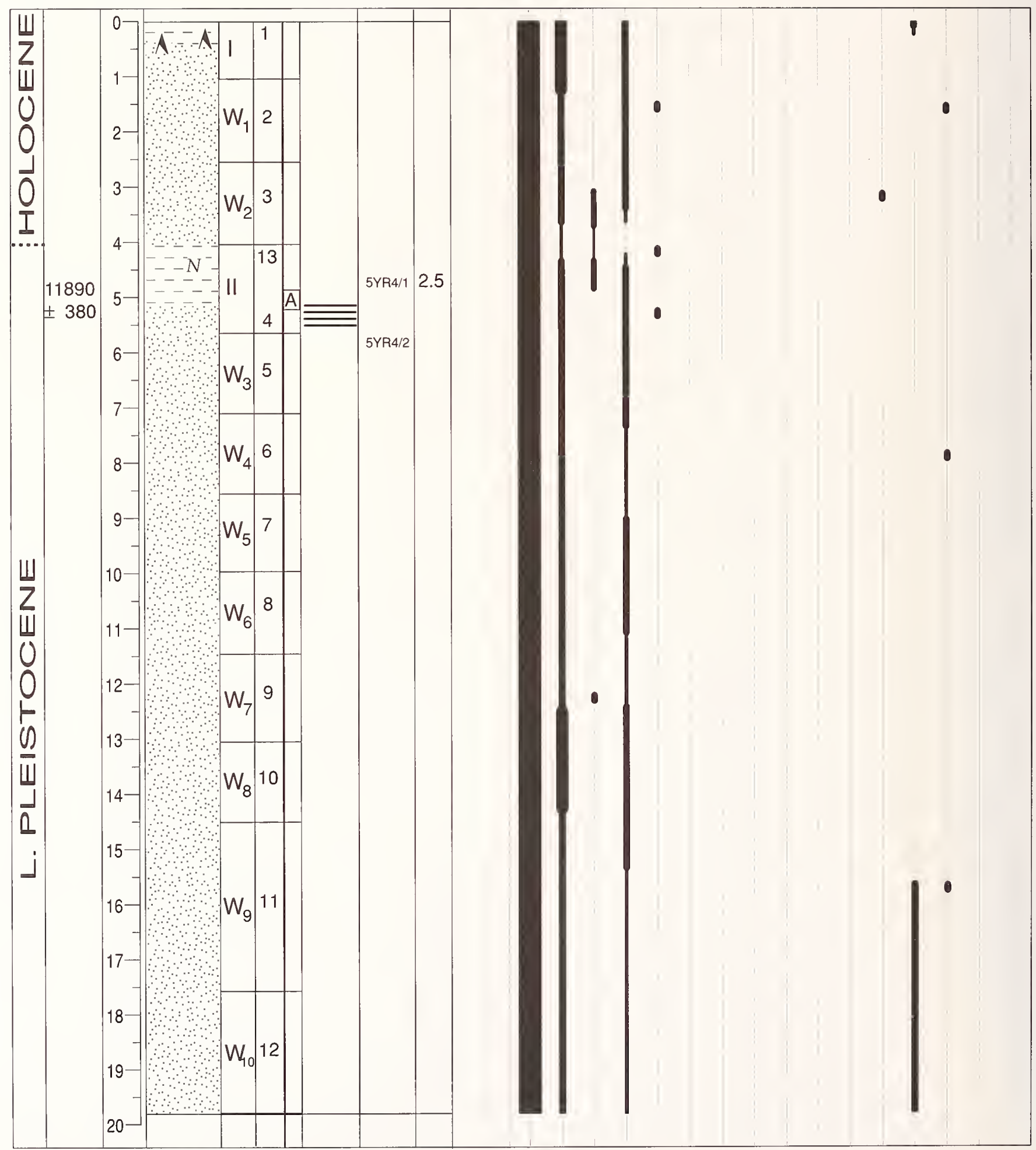


APPENDIX 1.-Continued.

CORE NUMBER S68I

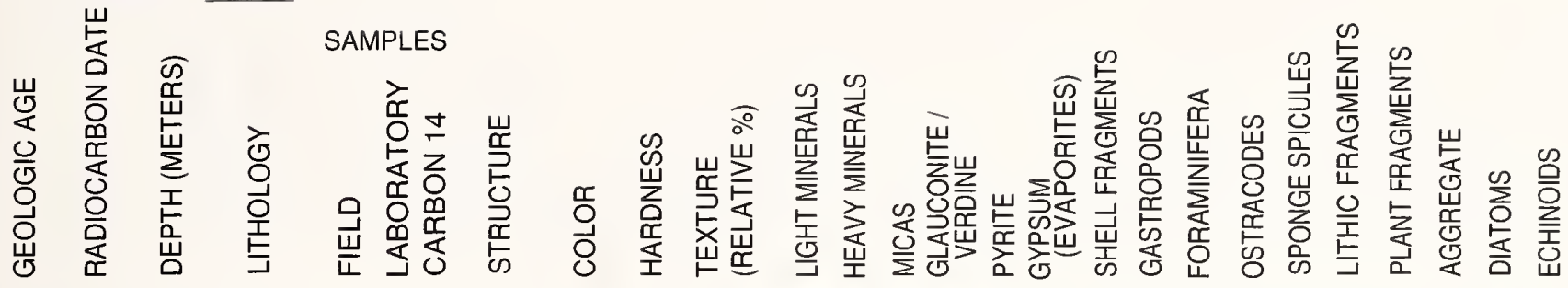

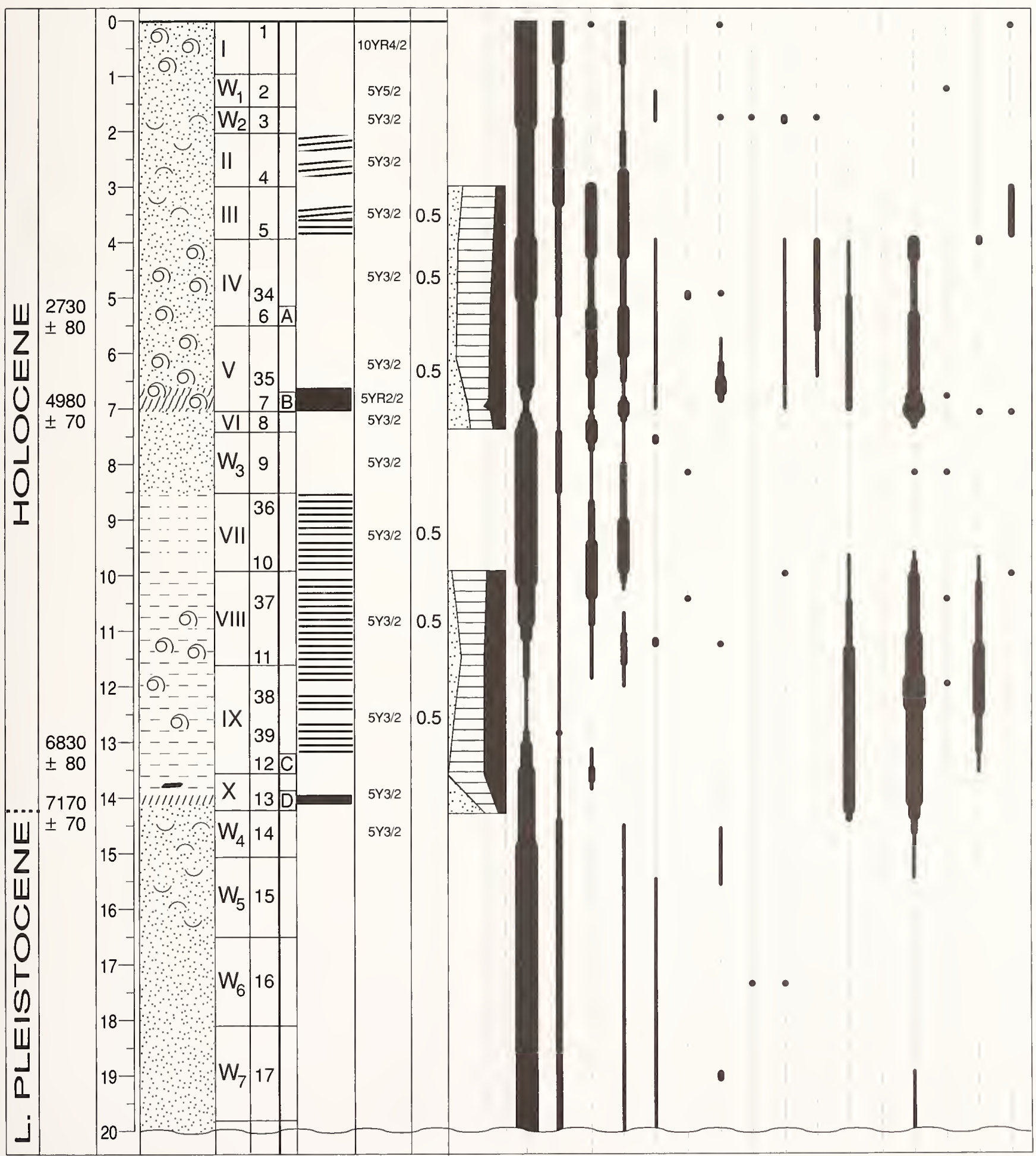


APPENDIX 1.-Continued.

CORE NUMBER S68 II

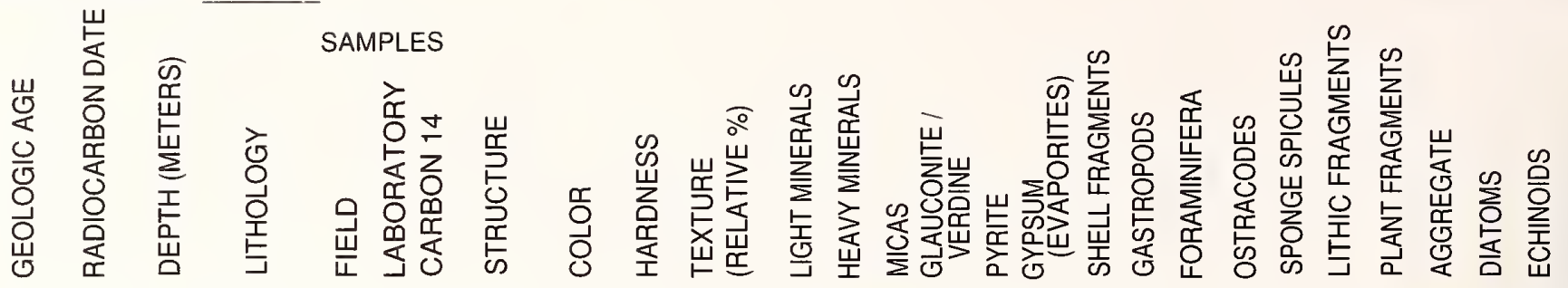

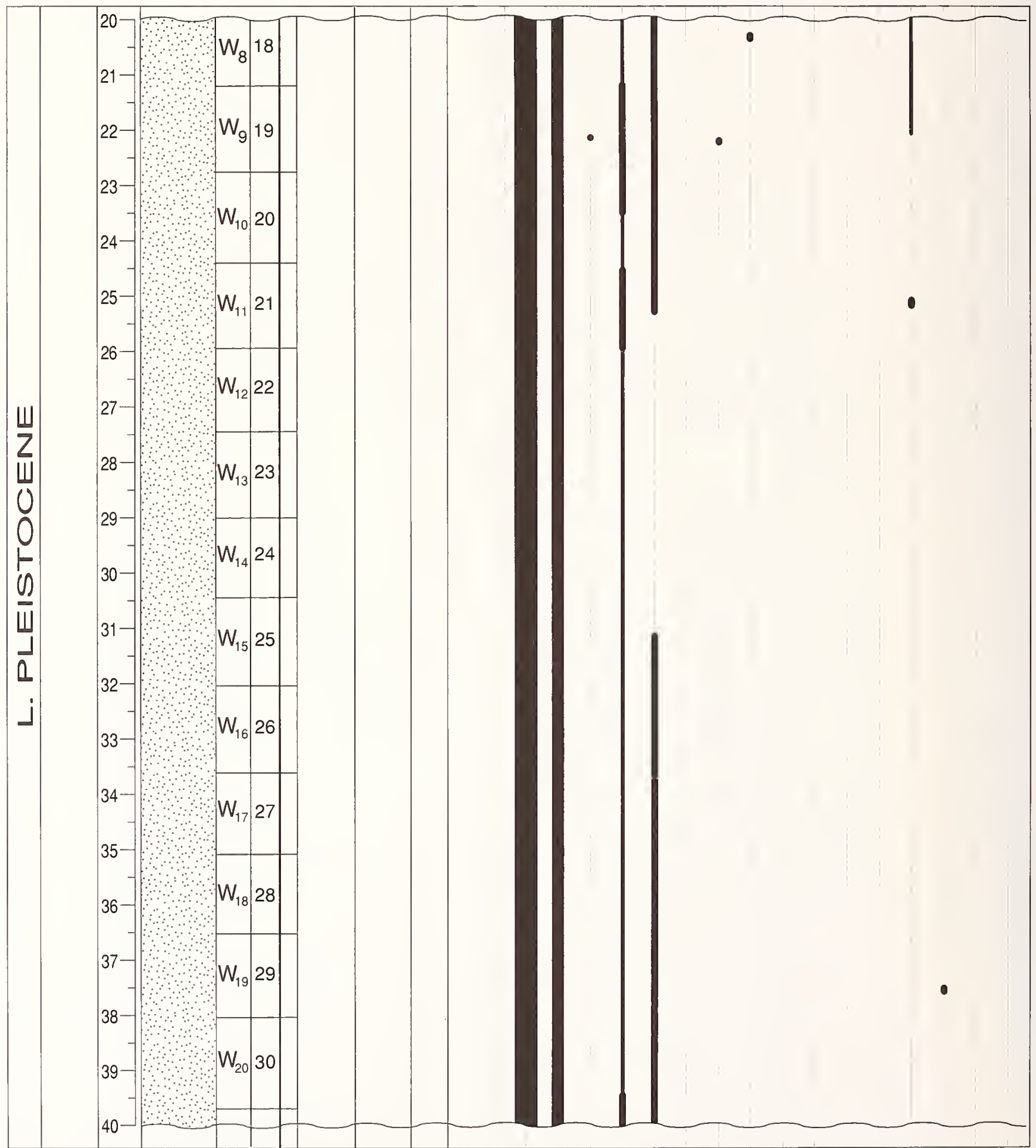


APPENDIX 1.-Continued.
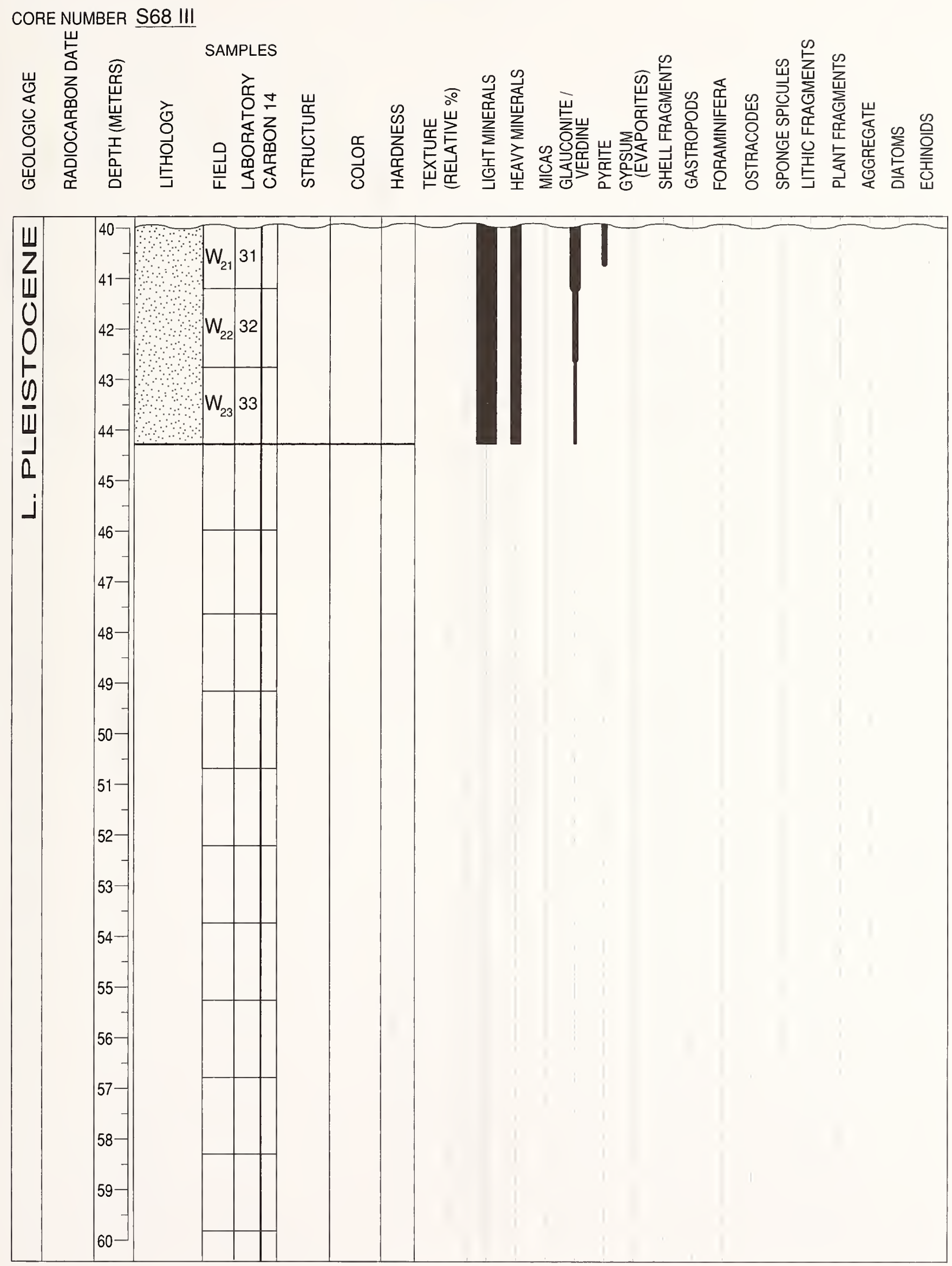
APPENDIX 1.-Continued.

CORE NUMBER S69

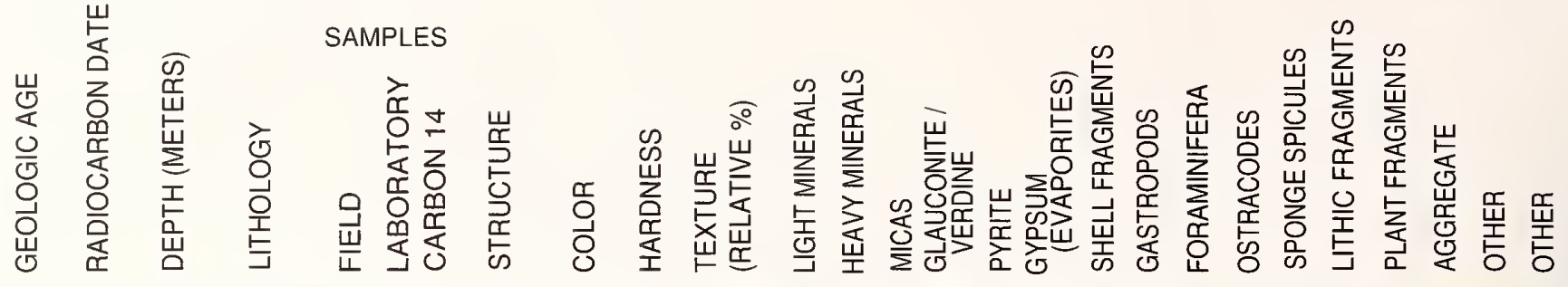

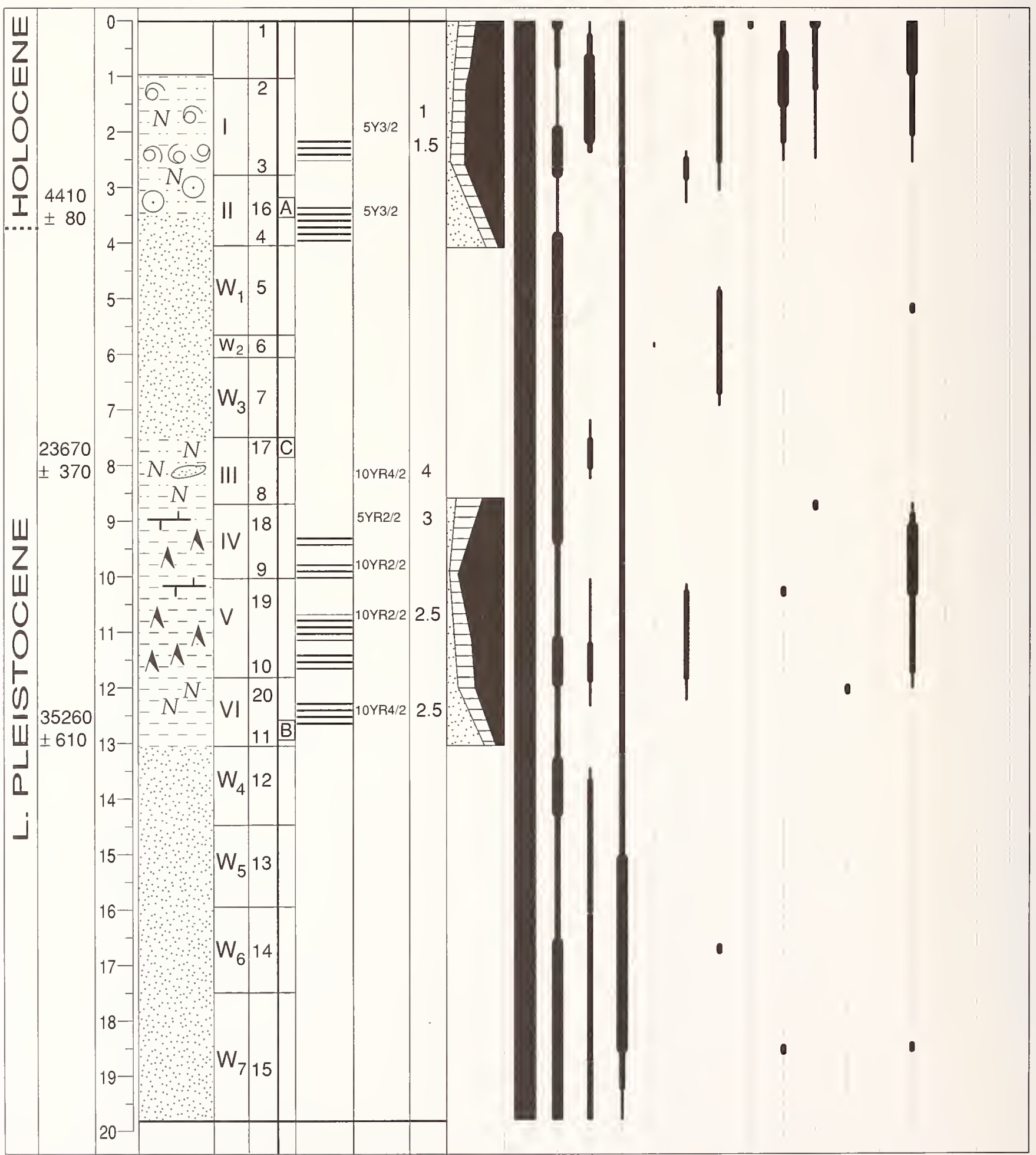


APPENDIX 1.-Continued.

CORE NUMBER S70

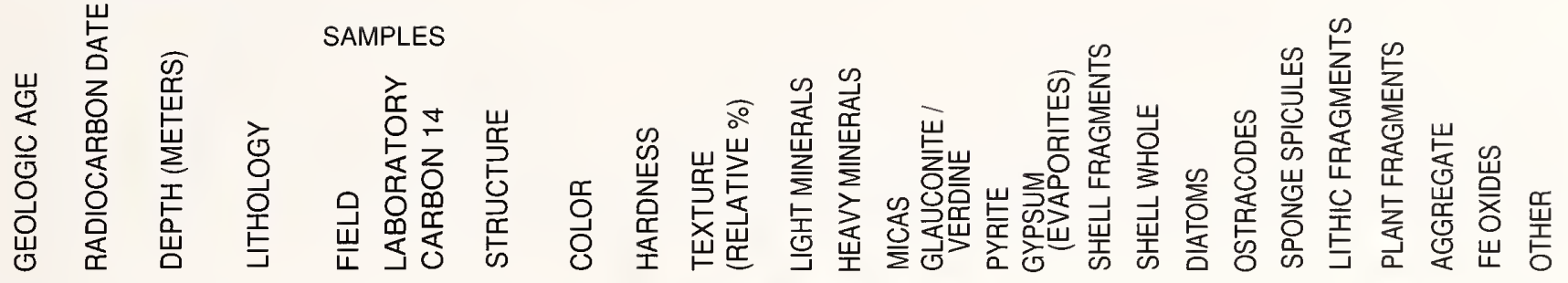

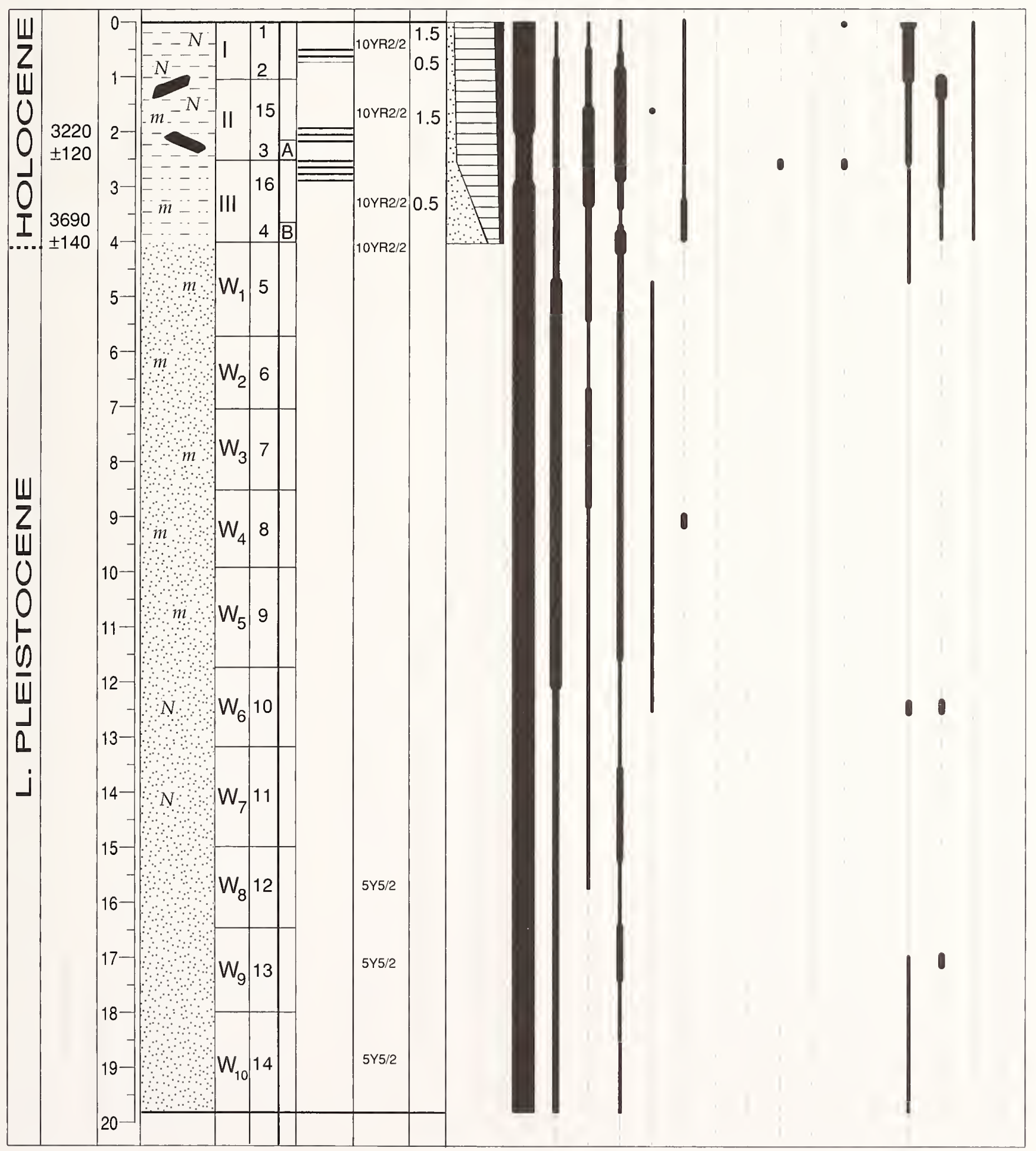


APPENDIX 1.-Continued.

CORE NUMBER S71I

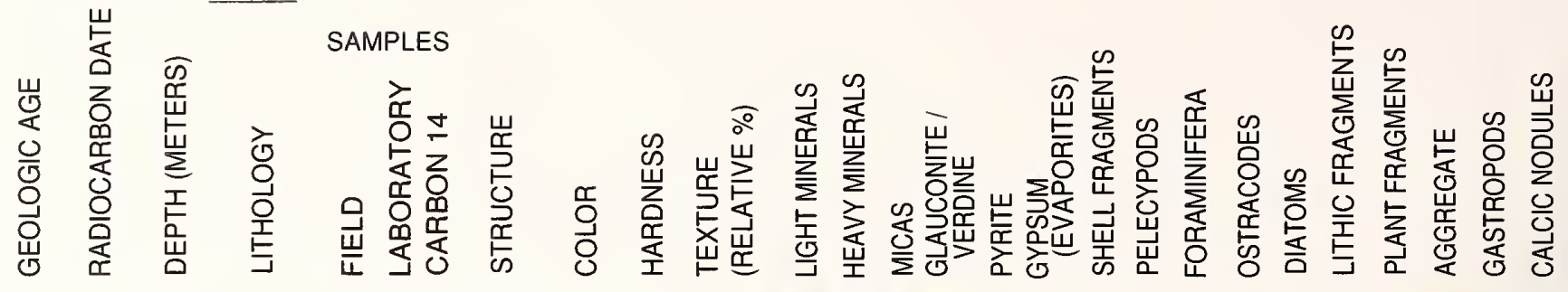

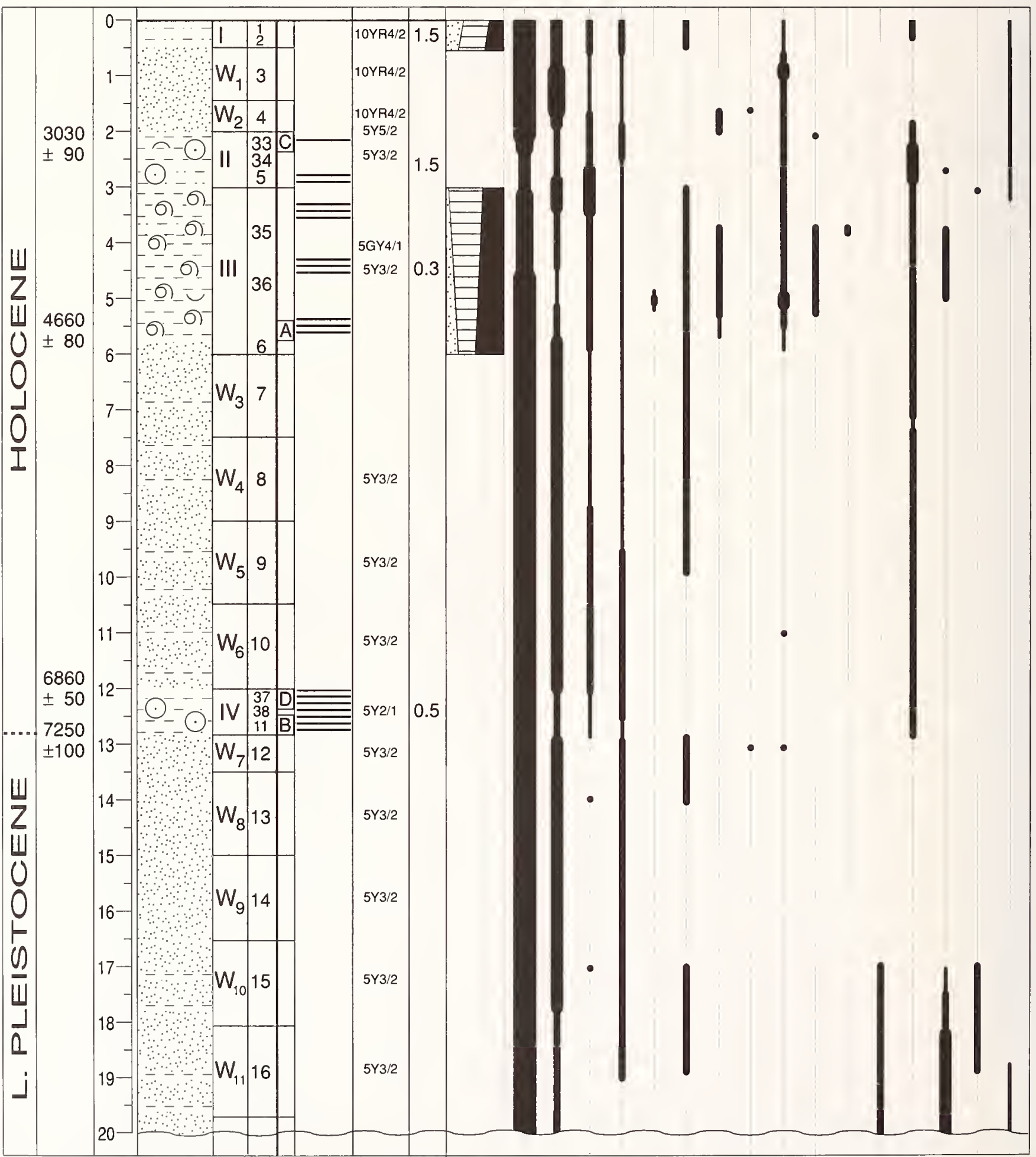


APPENDIX 1.-Continued.

CORE NUMBER S71 $\|$

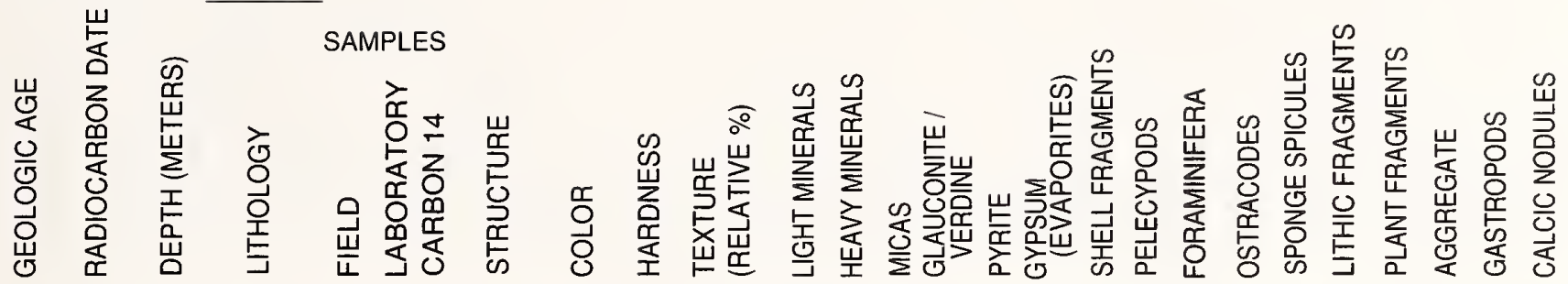

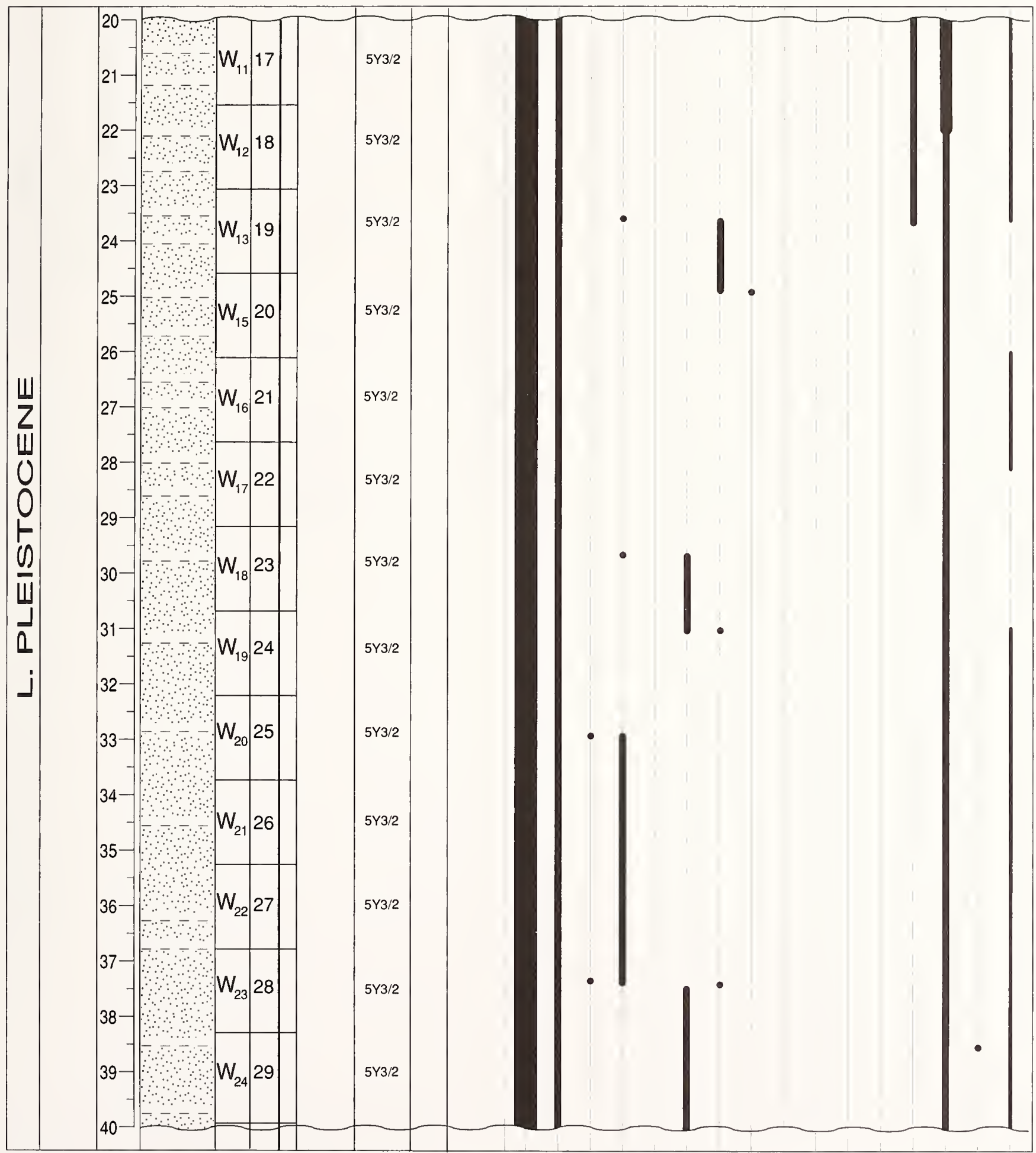


APPENDIX 1.-Continued.

CORE NUMBER S71 III

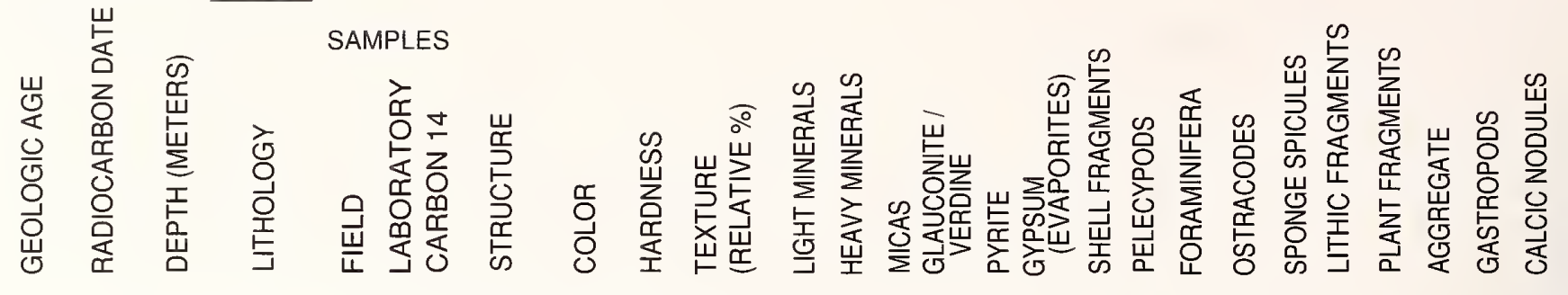

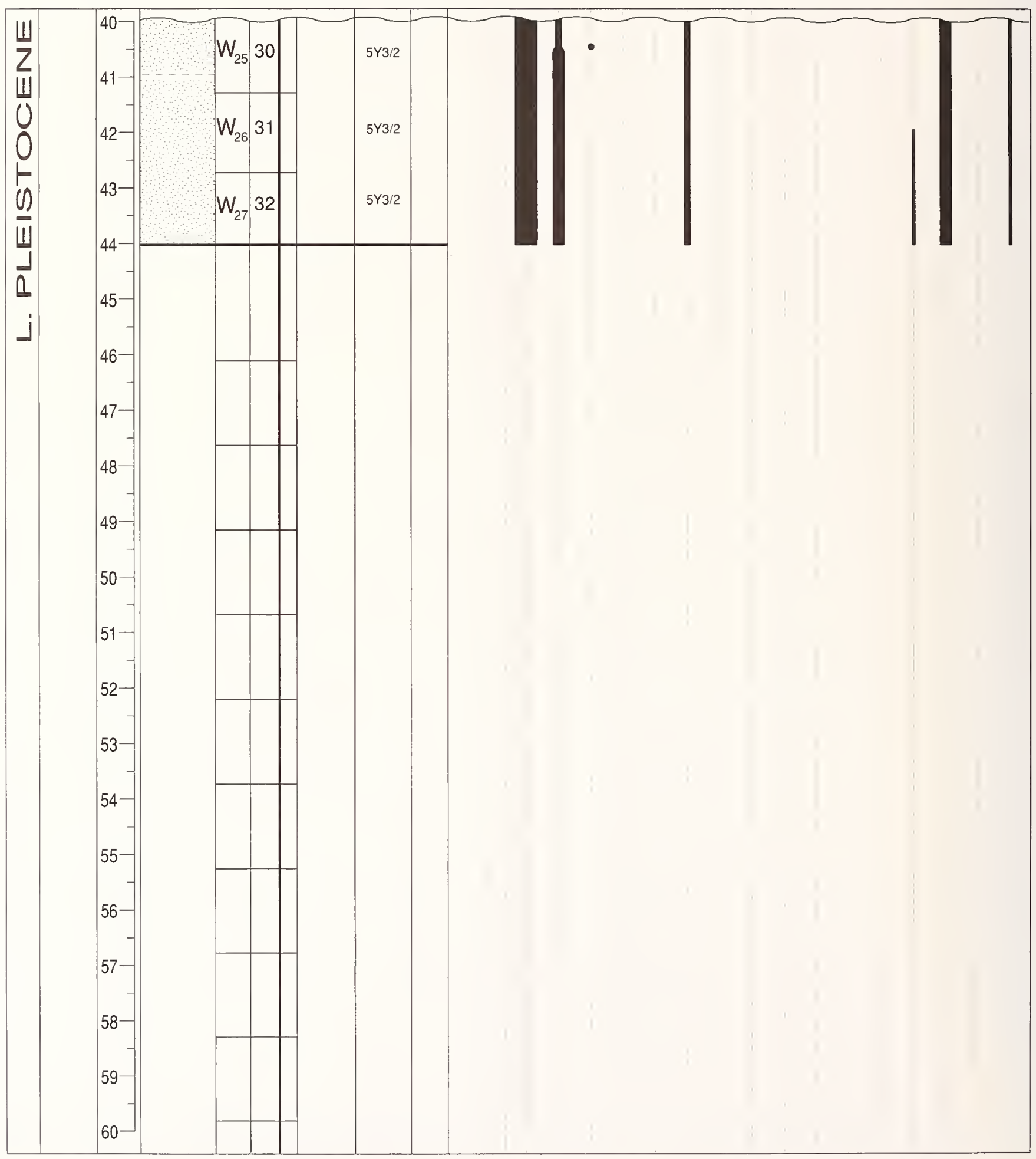


APPENDIX 1.-Continued.

CORE NUMBER $\quad$ S72
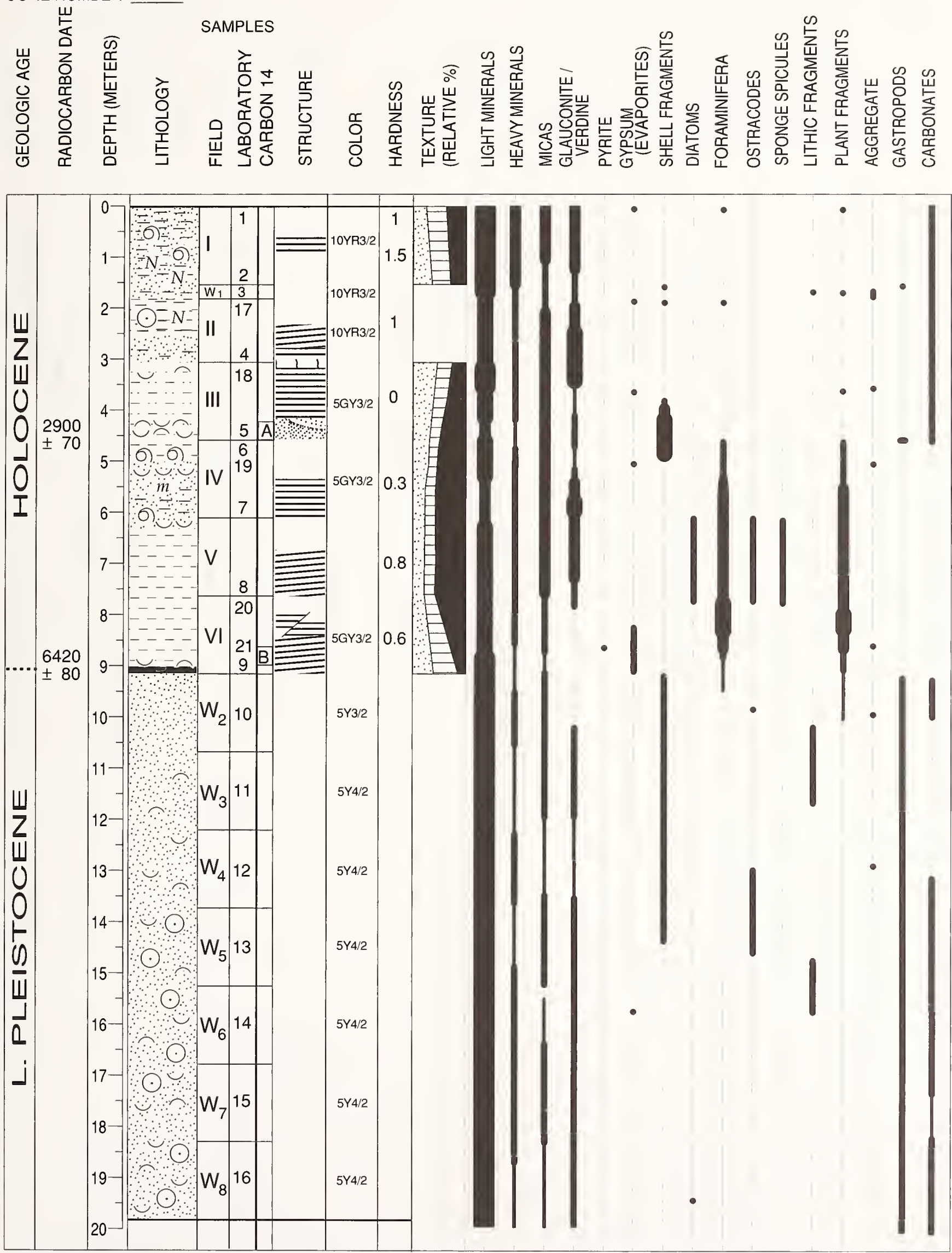
APPENDIX 1.-Continued.

CORE NUMBER S73 I
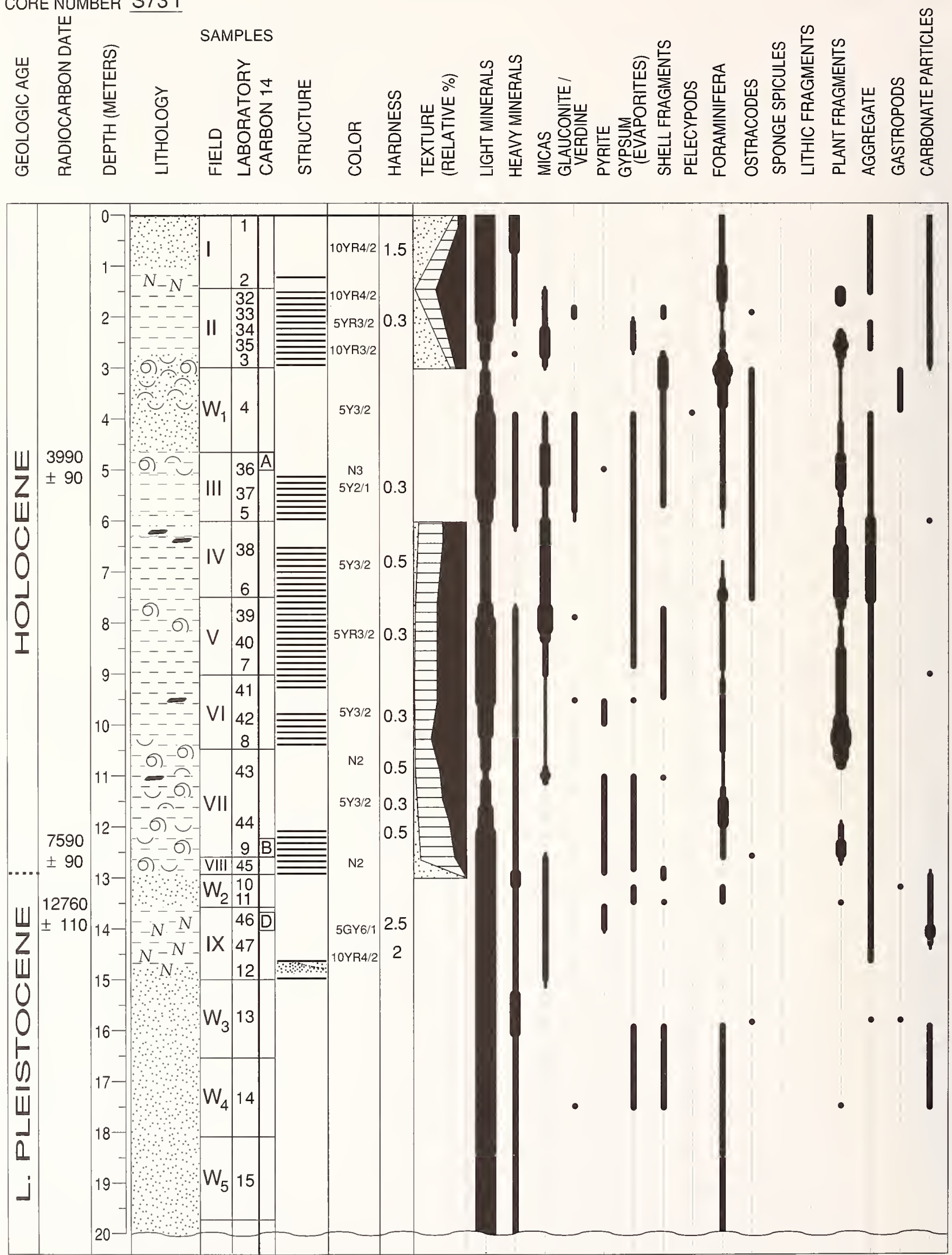
APPENDIX 1.-Continued.

CORE NUMBER S73 ॥

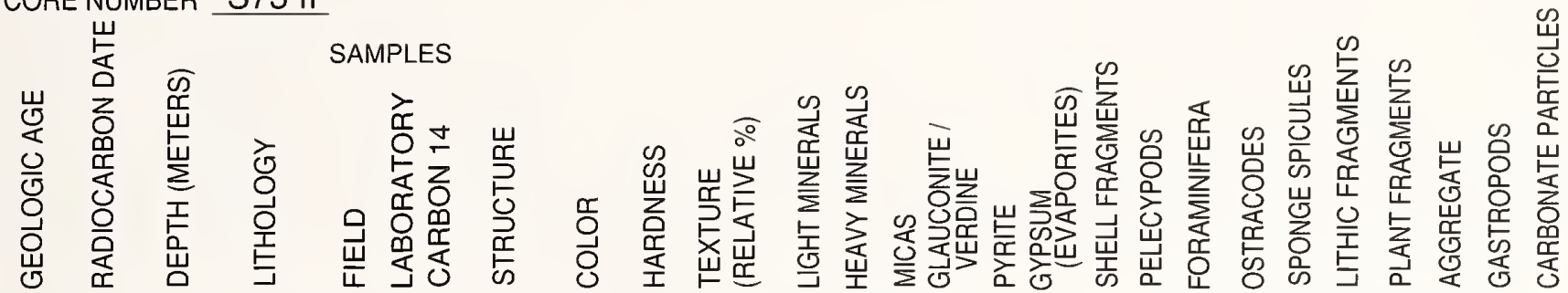

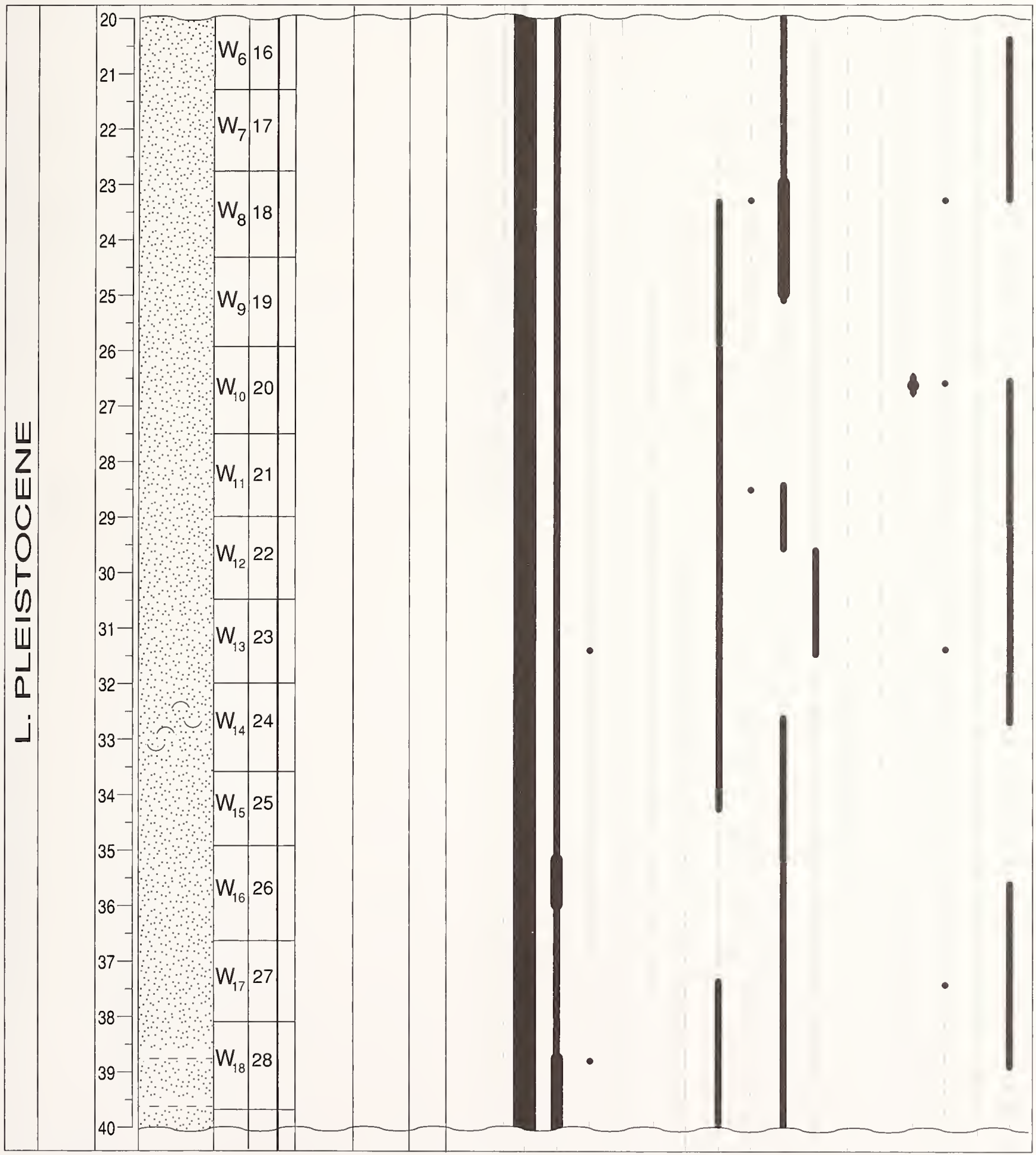


APPENDIX 1.-Continued.
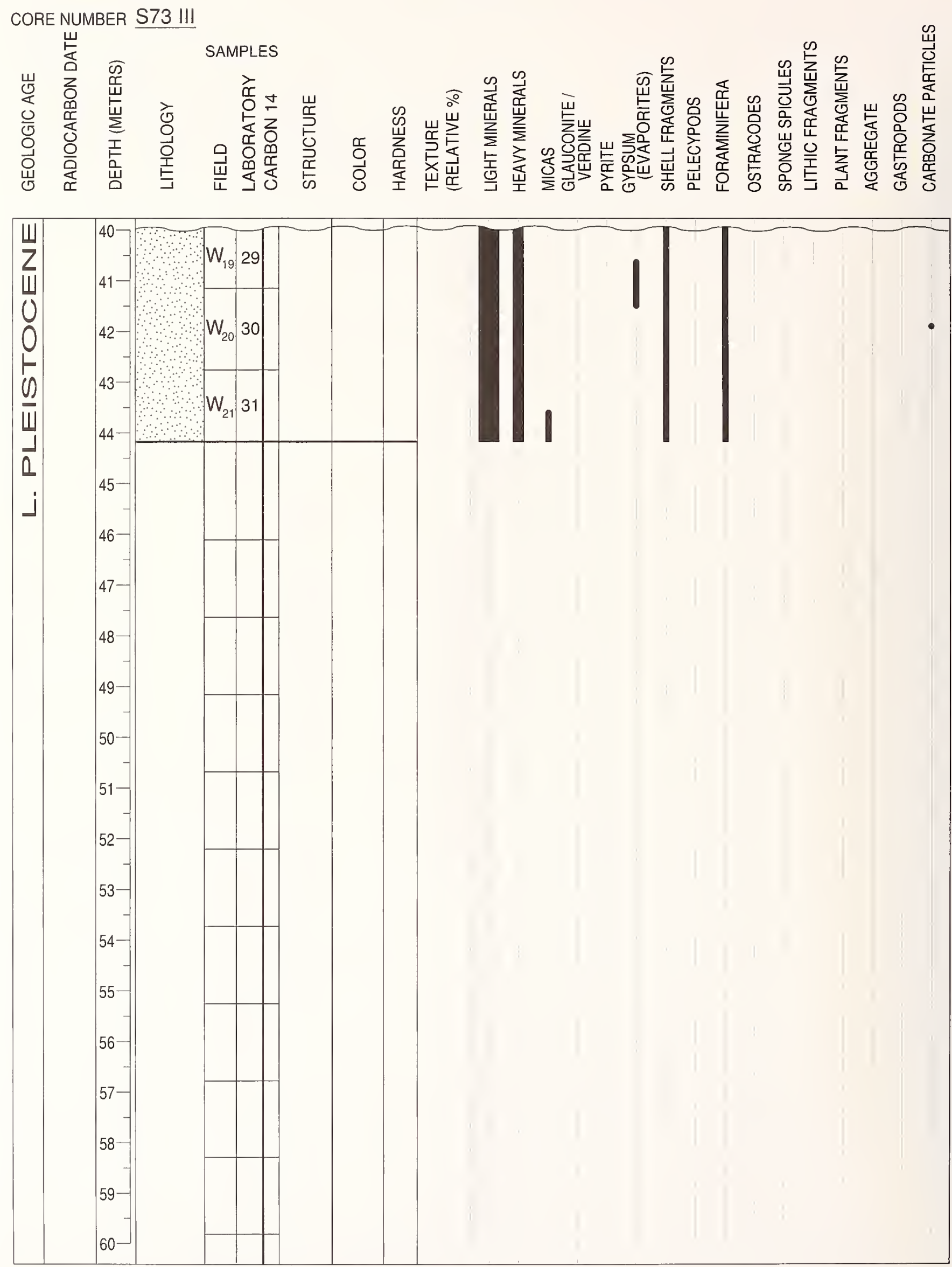
APPENDIX 1.-Continued.

CORE NUMBER $\$ 74$

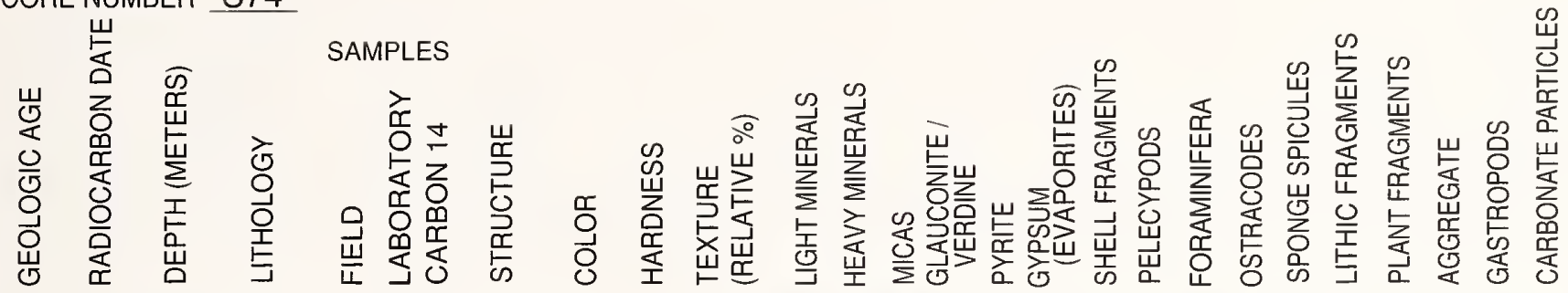

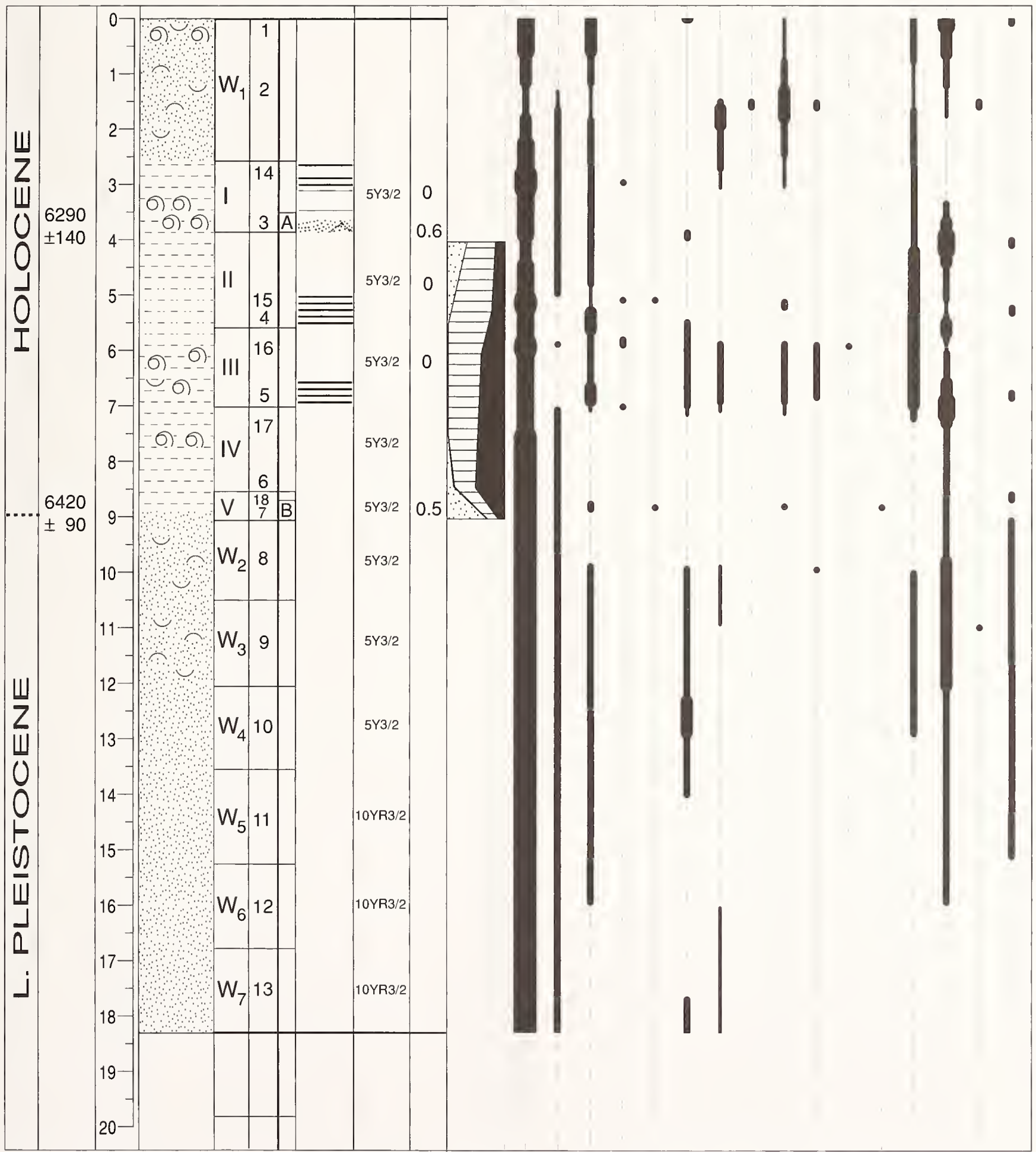


APPENDIX 1.-Continued.
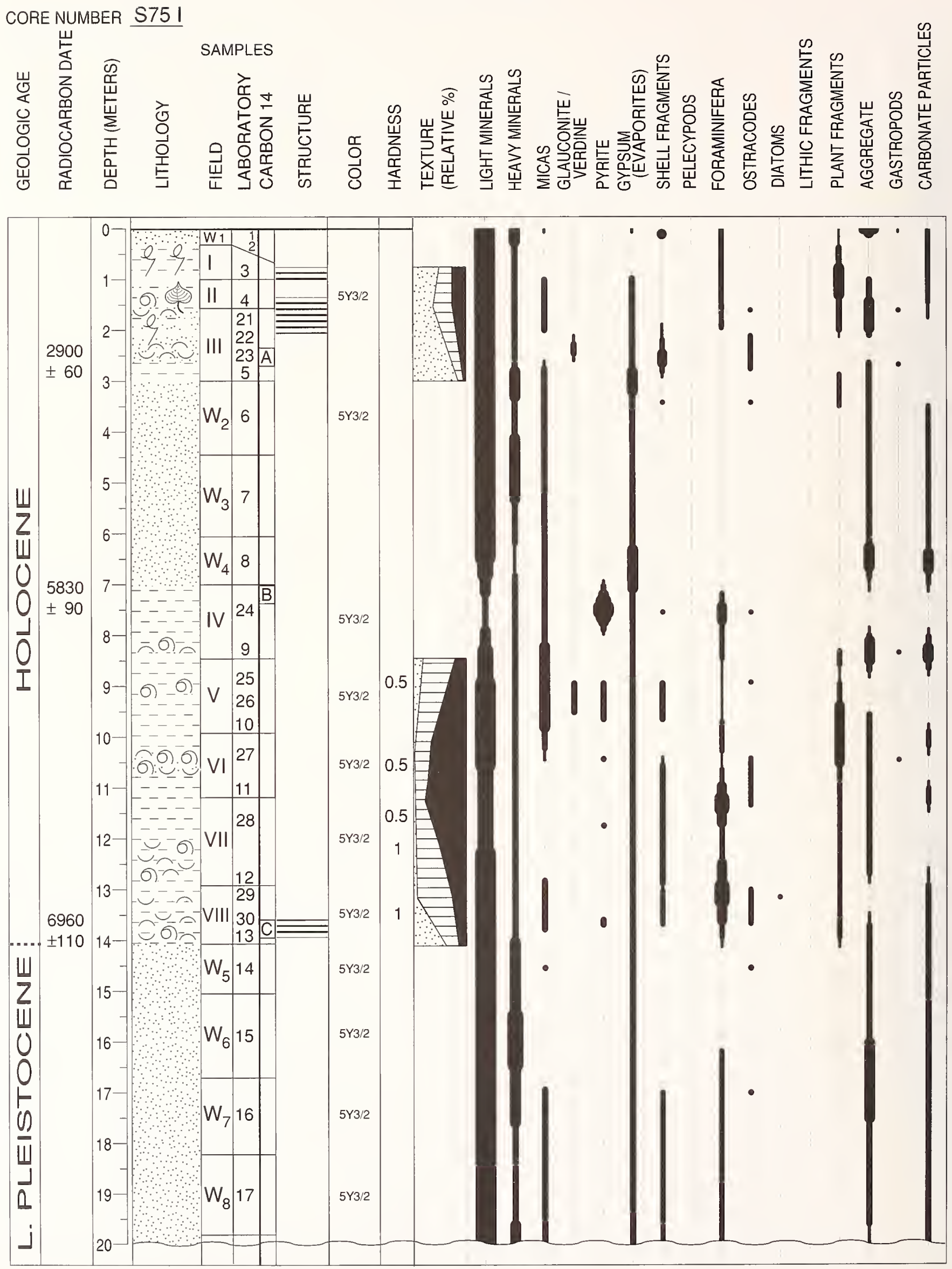
APPENDIX 1.-Continued.

CORE NUMBER S75 II

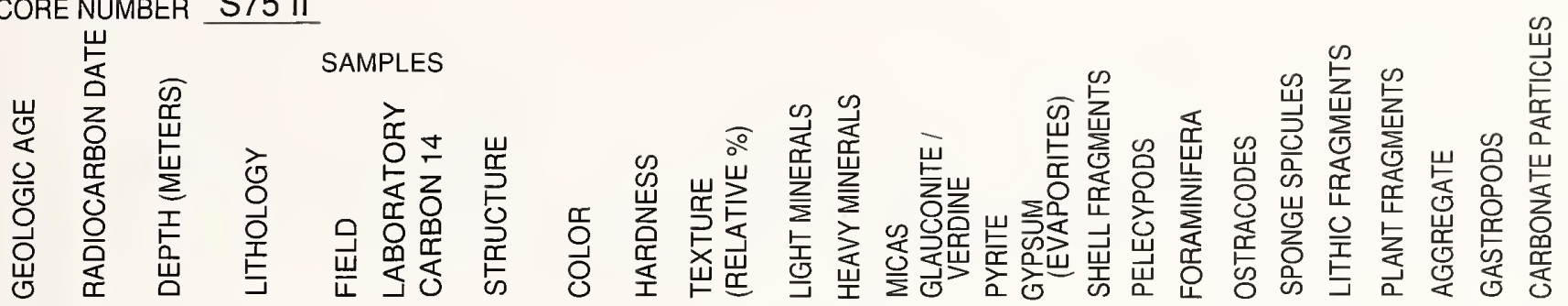

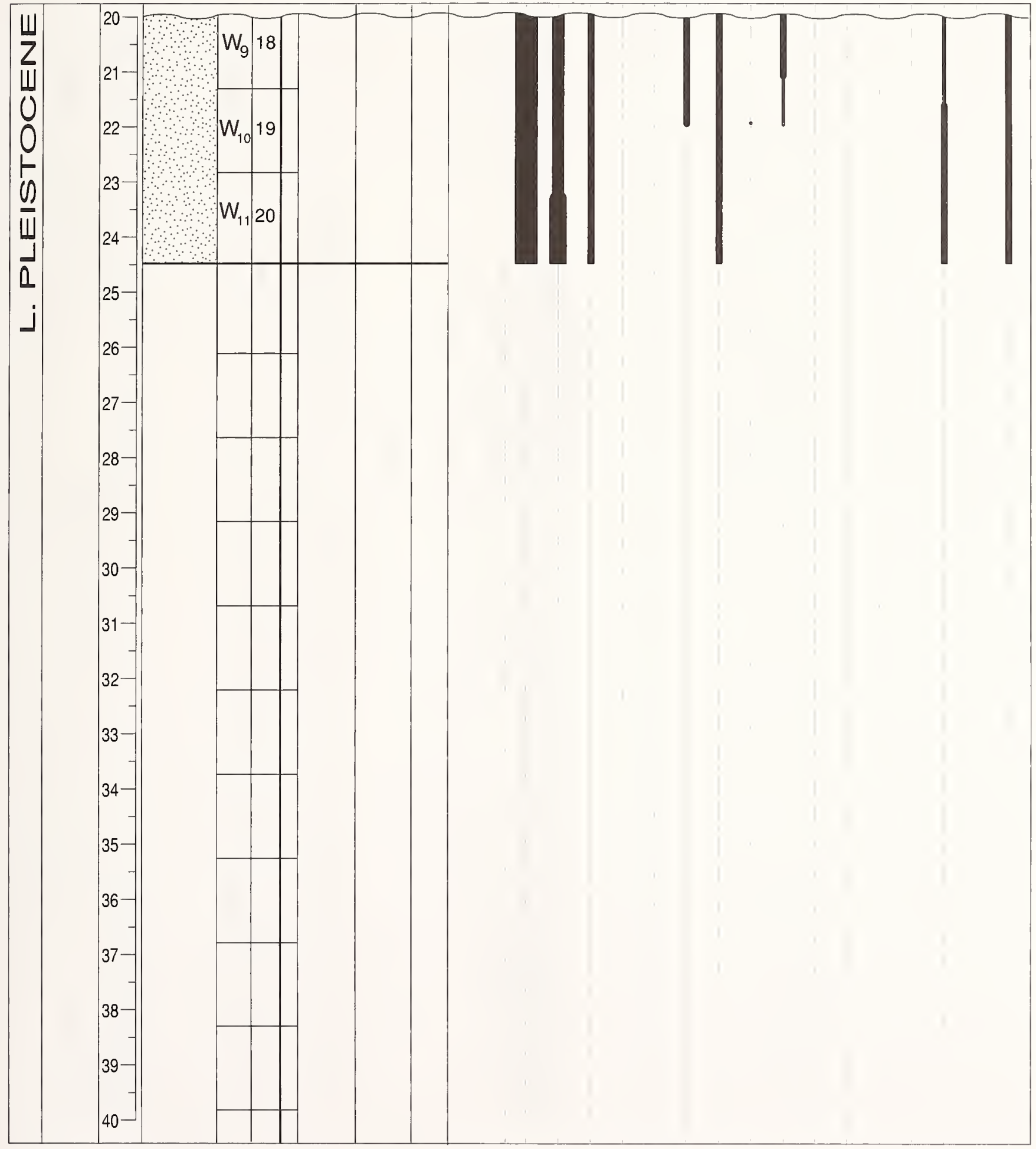


APPENDIX 1.-Continued.

CORE NUMBER S76
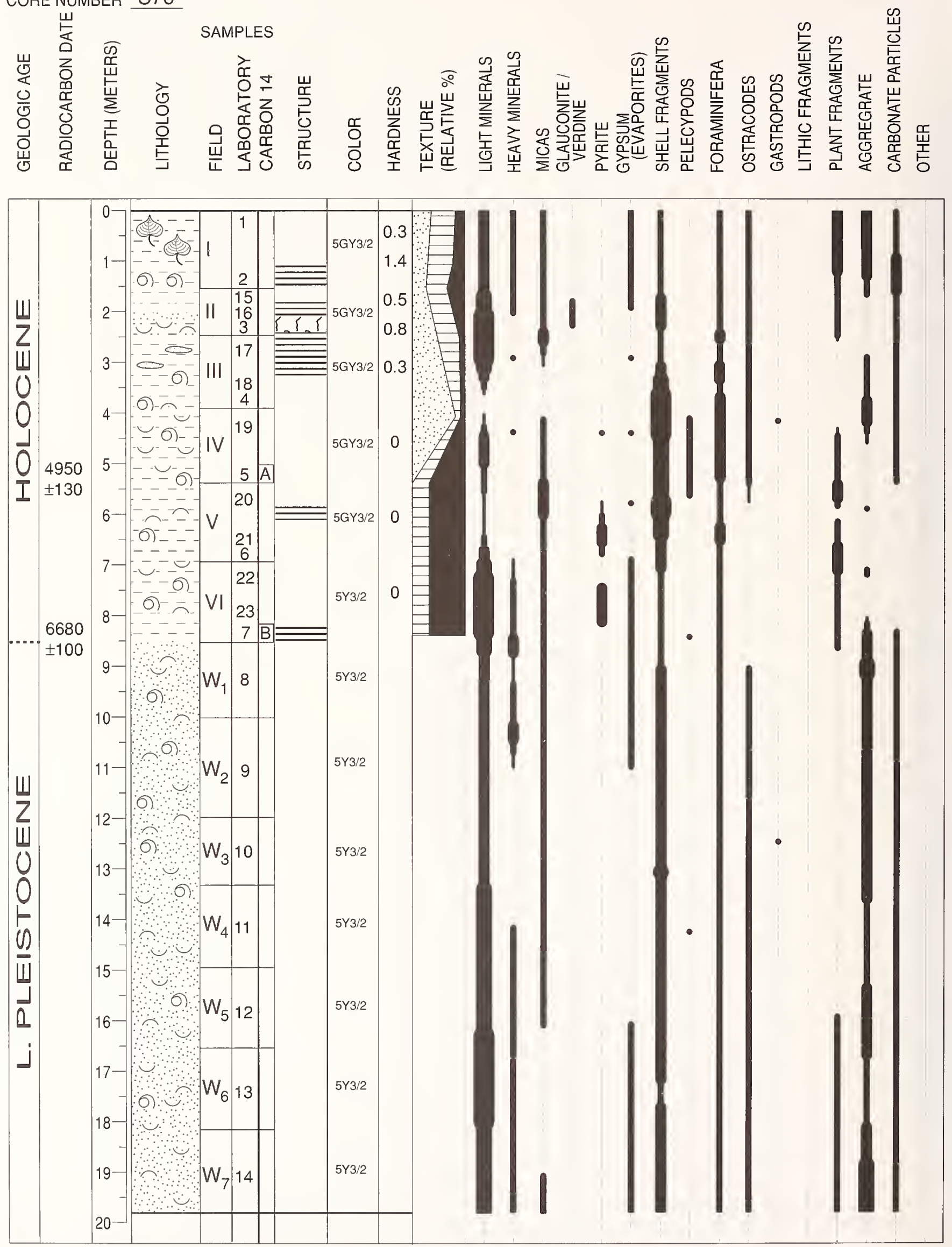
APPENDIX 1.-Continued.

CORE NUMBER S77।
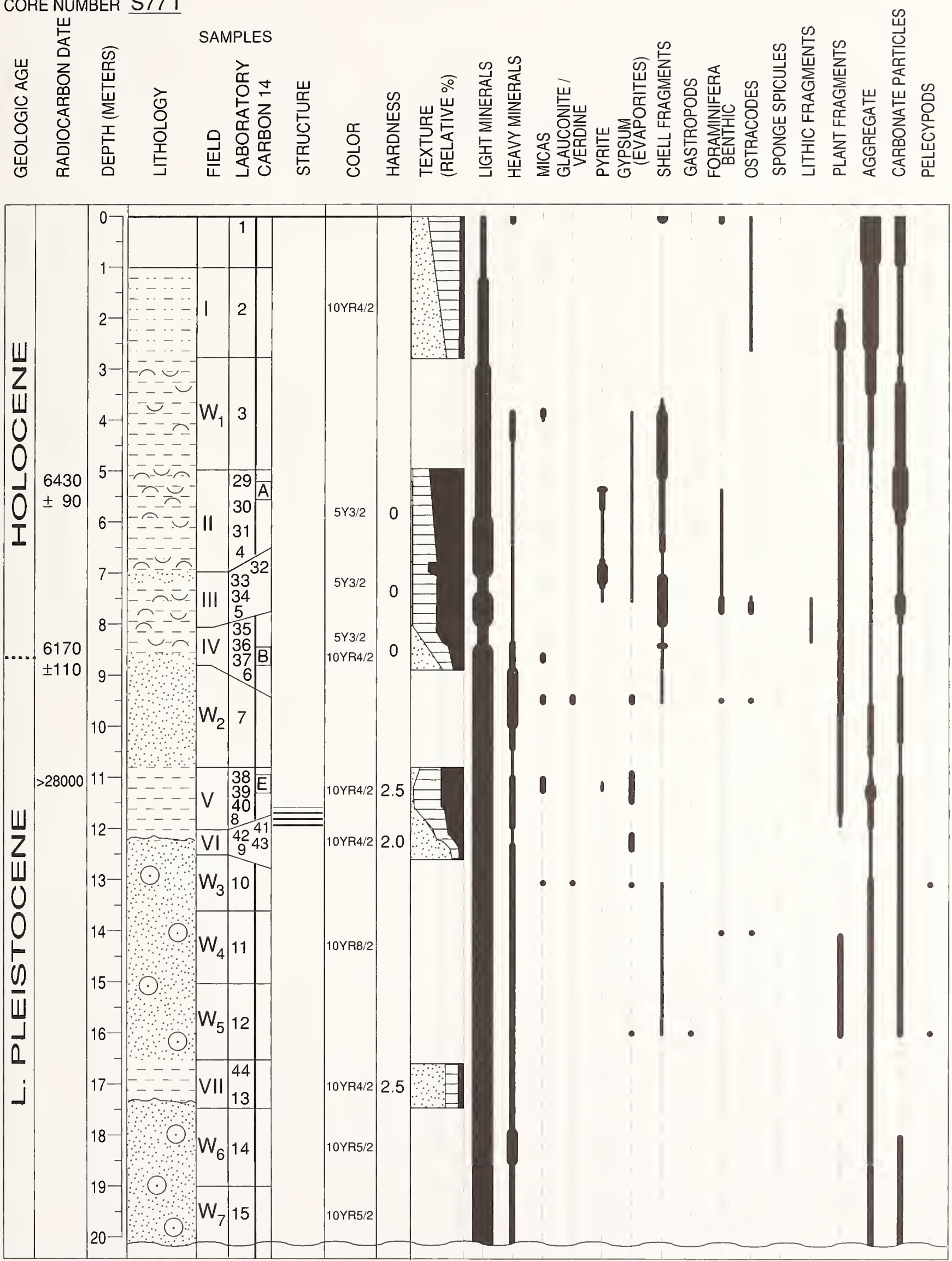
APPENDIX 1.-Continued.
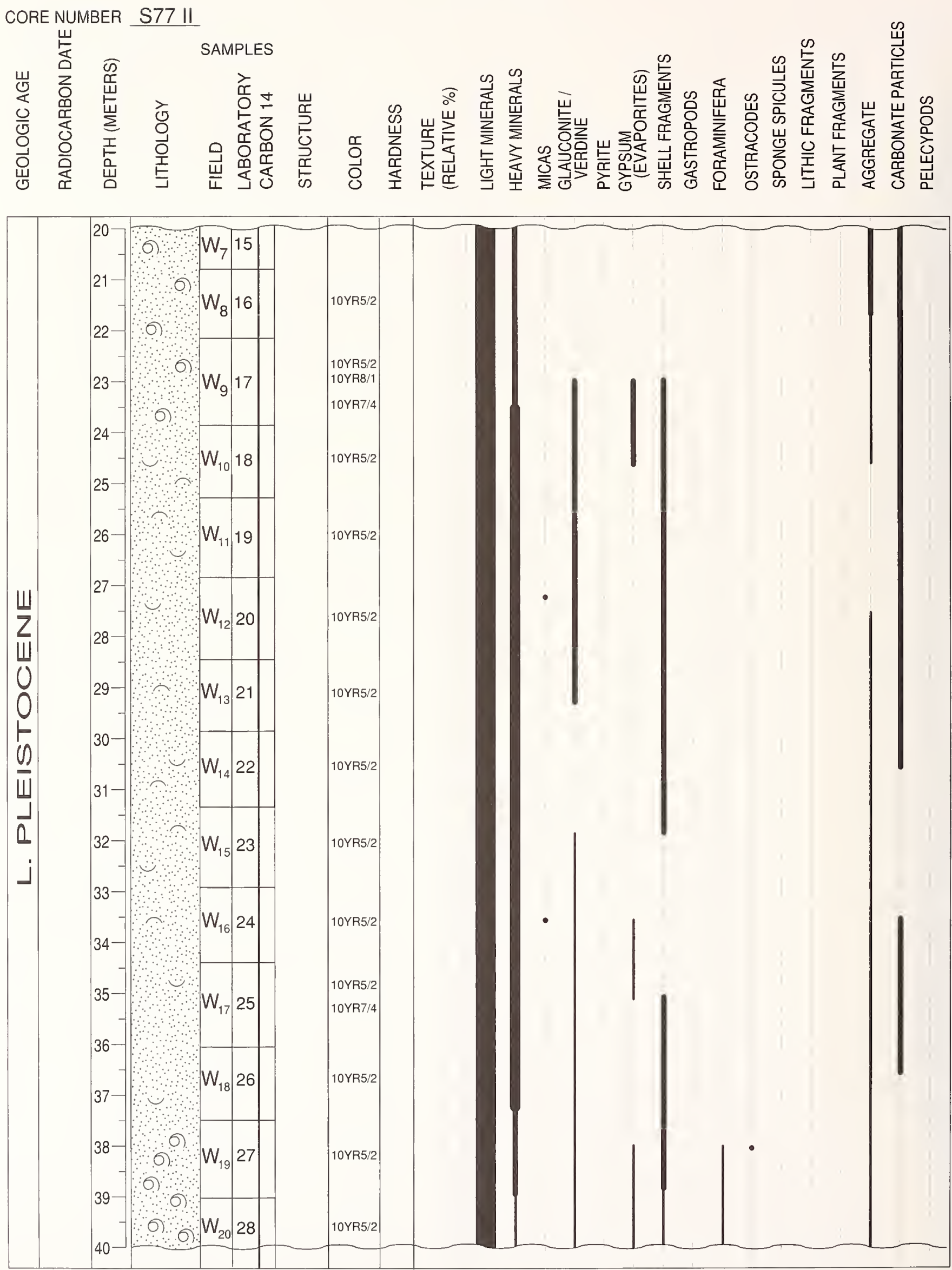
APPENDIX 1.-Continued.

\section{CORE NUMBER S77 III}

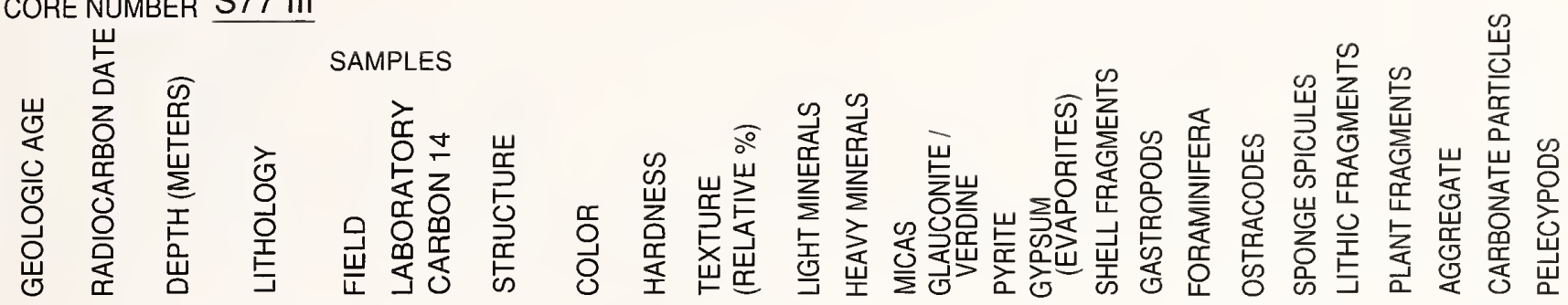

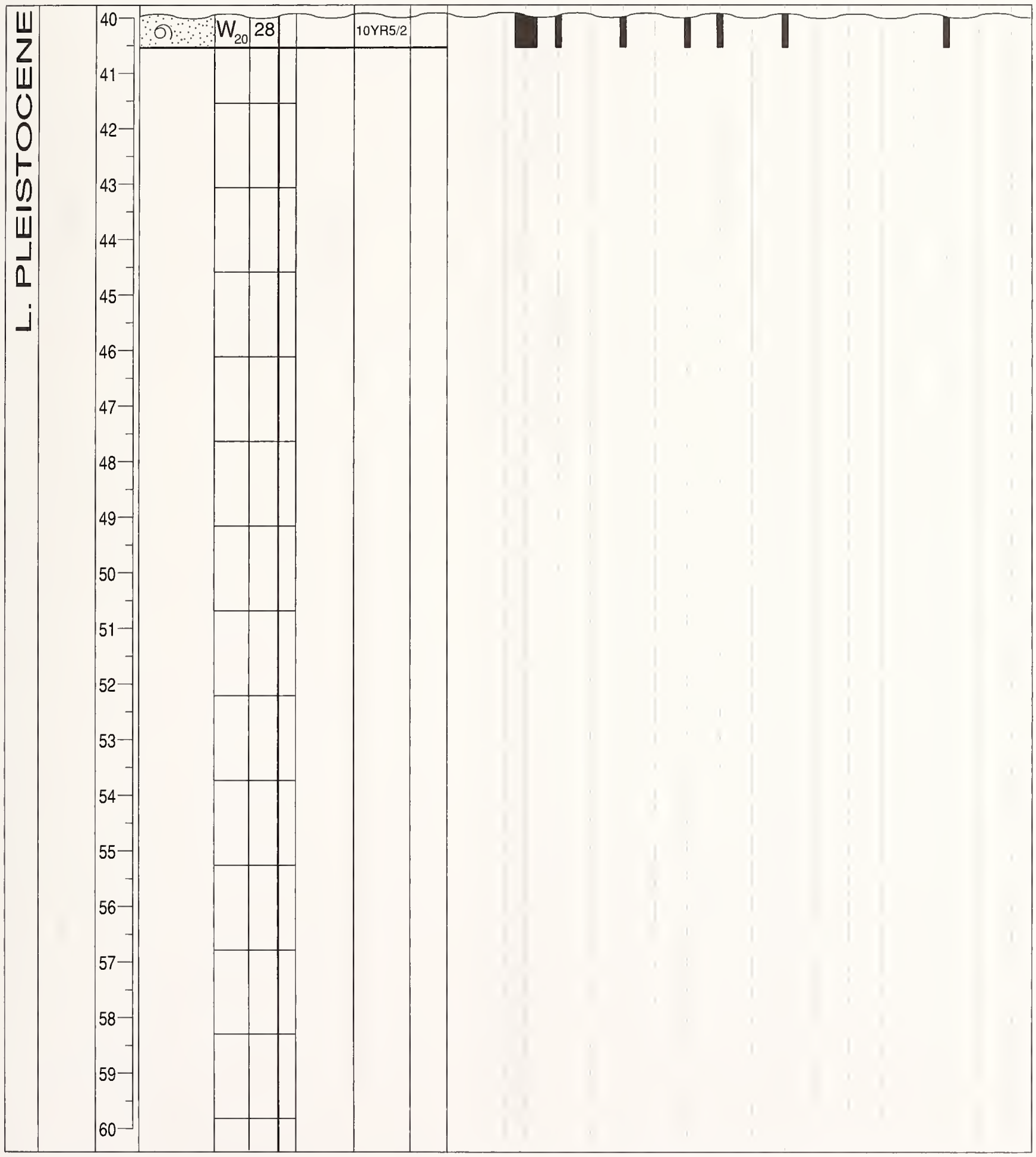


APPENDIX 1.-Continued.
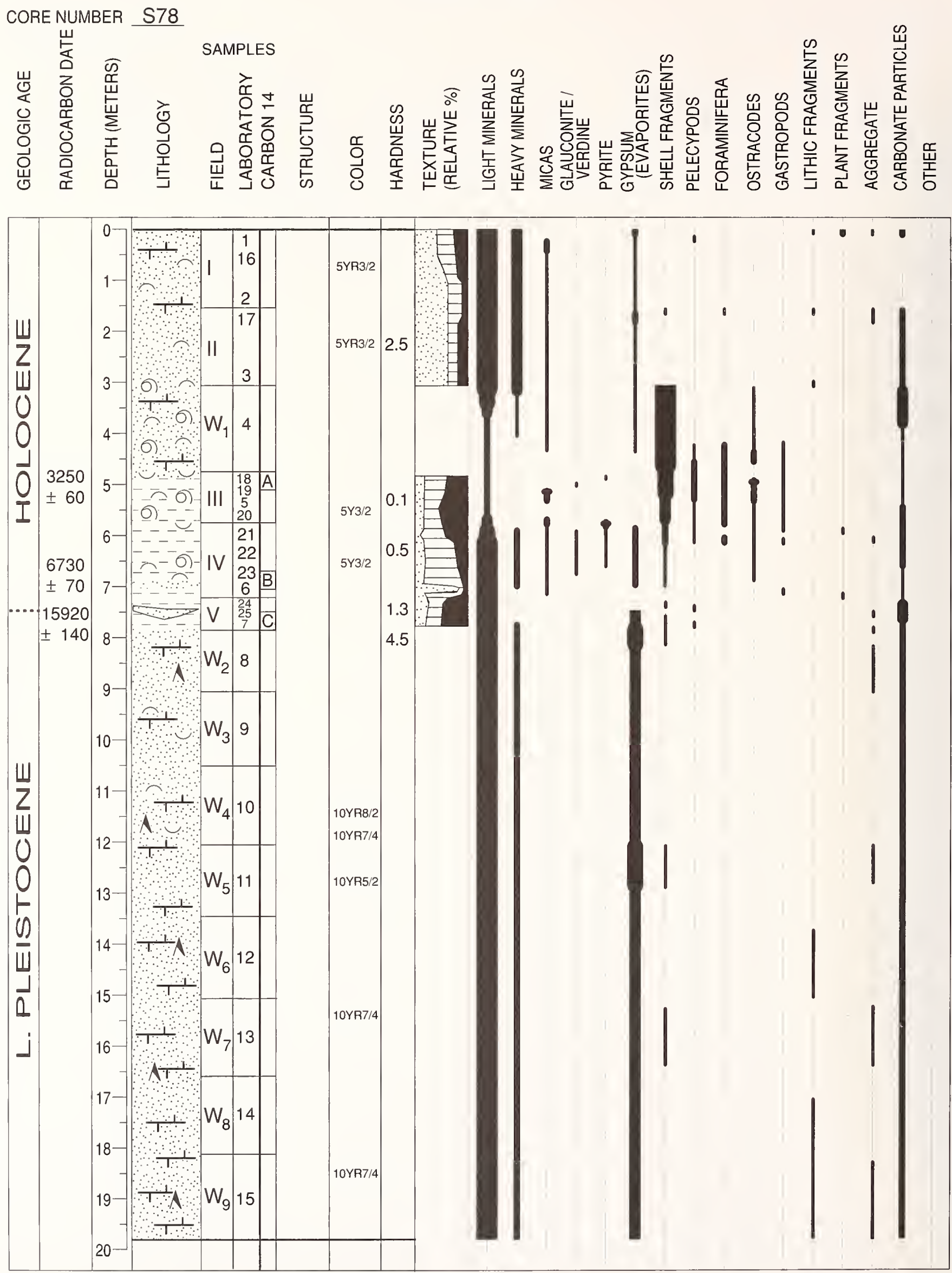
APPENDIX 1.-Continued.

CORE NUMBER S79 I

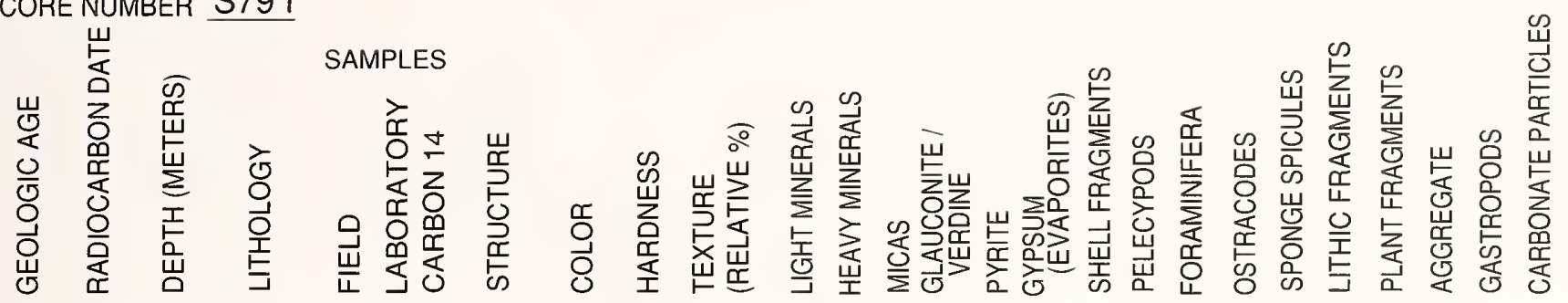

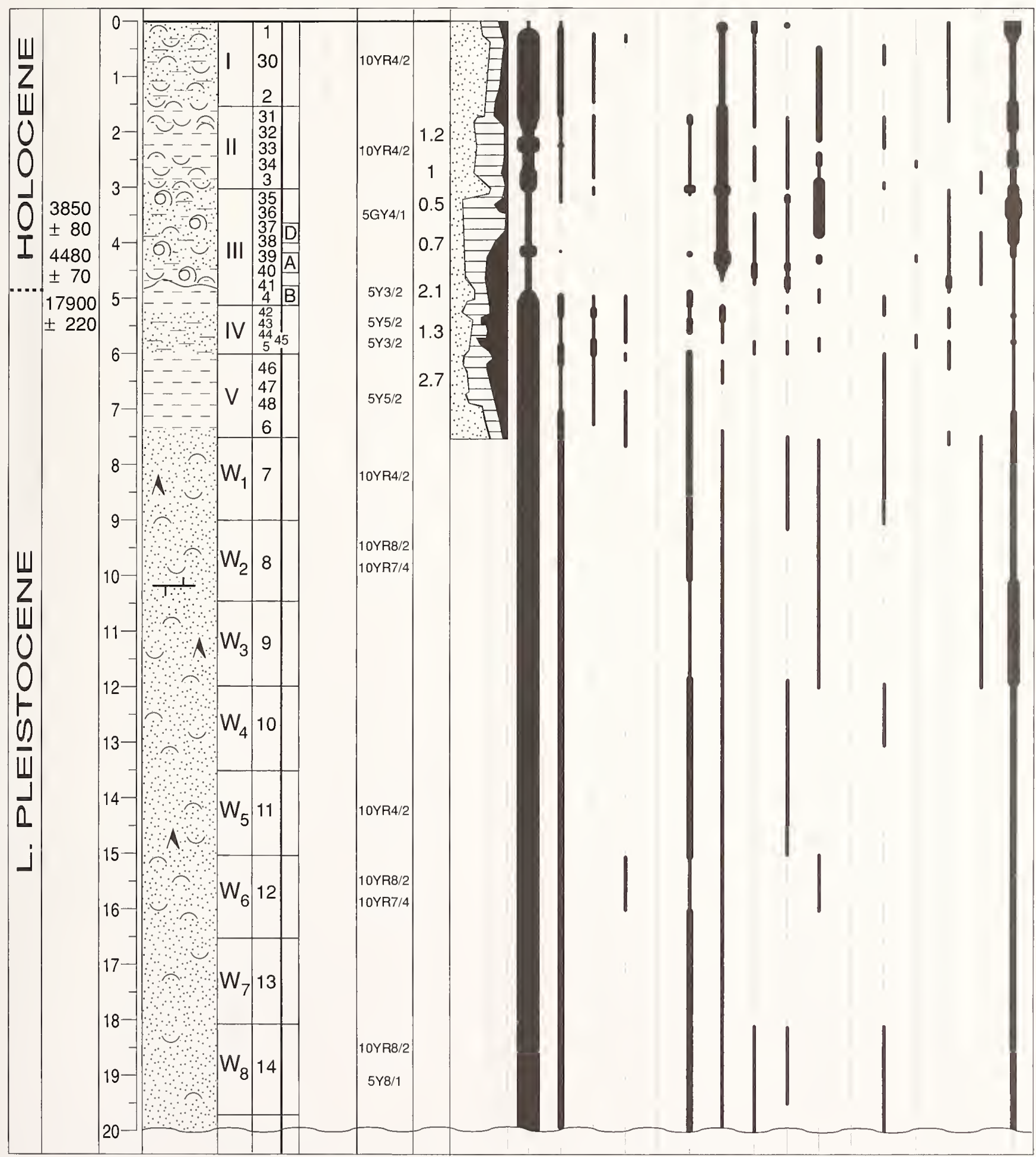


APPENDIX 1.-Continued.
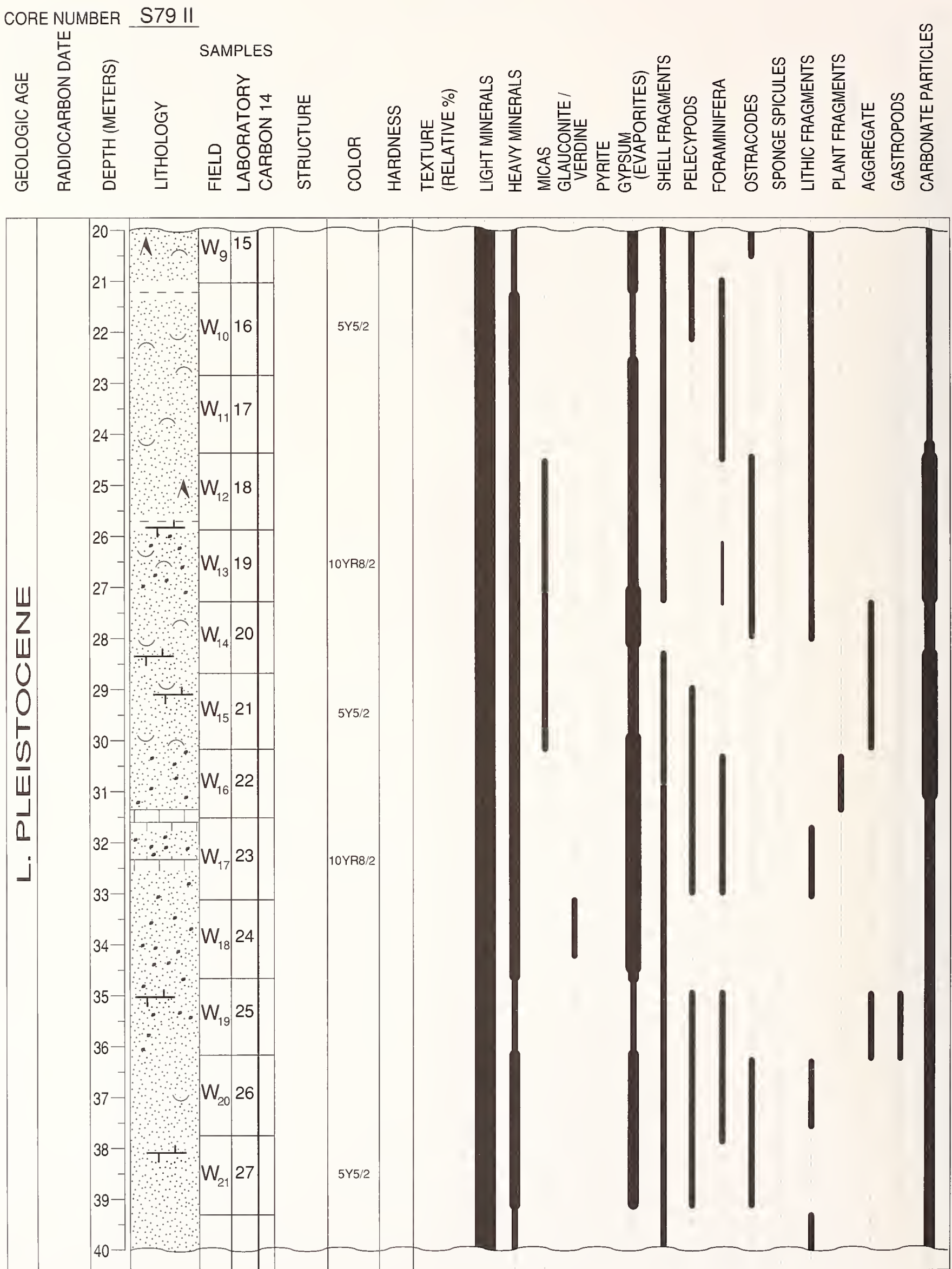
APPENDIX 1.-Continued.

CORE NUMBER S79 III

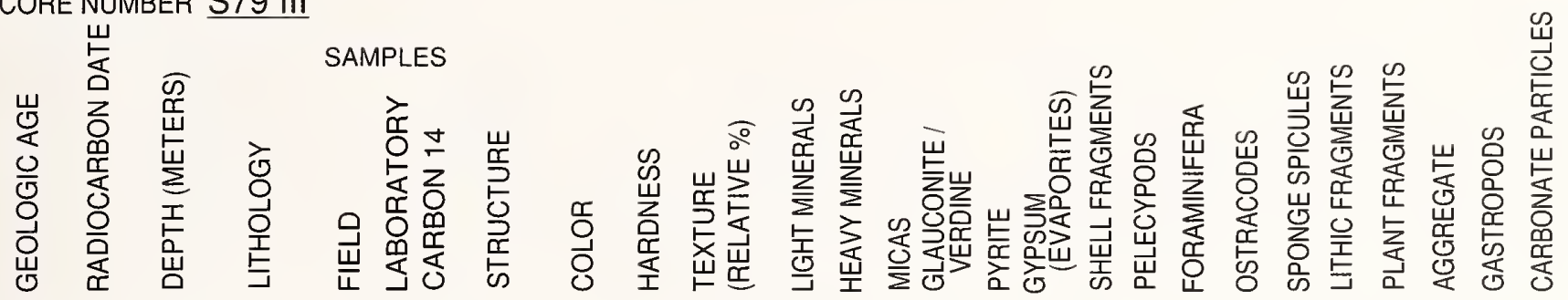

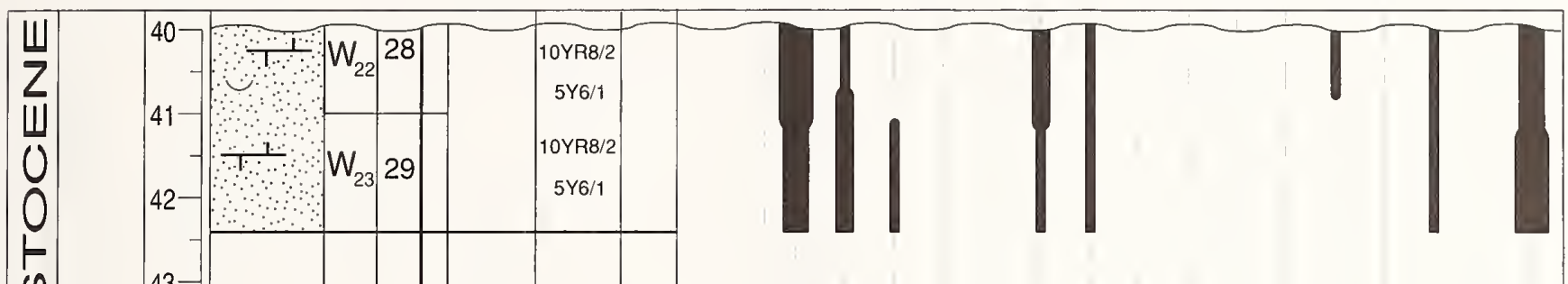


APPENDIX 1.-Continued.

CORE NUMBER S80 I
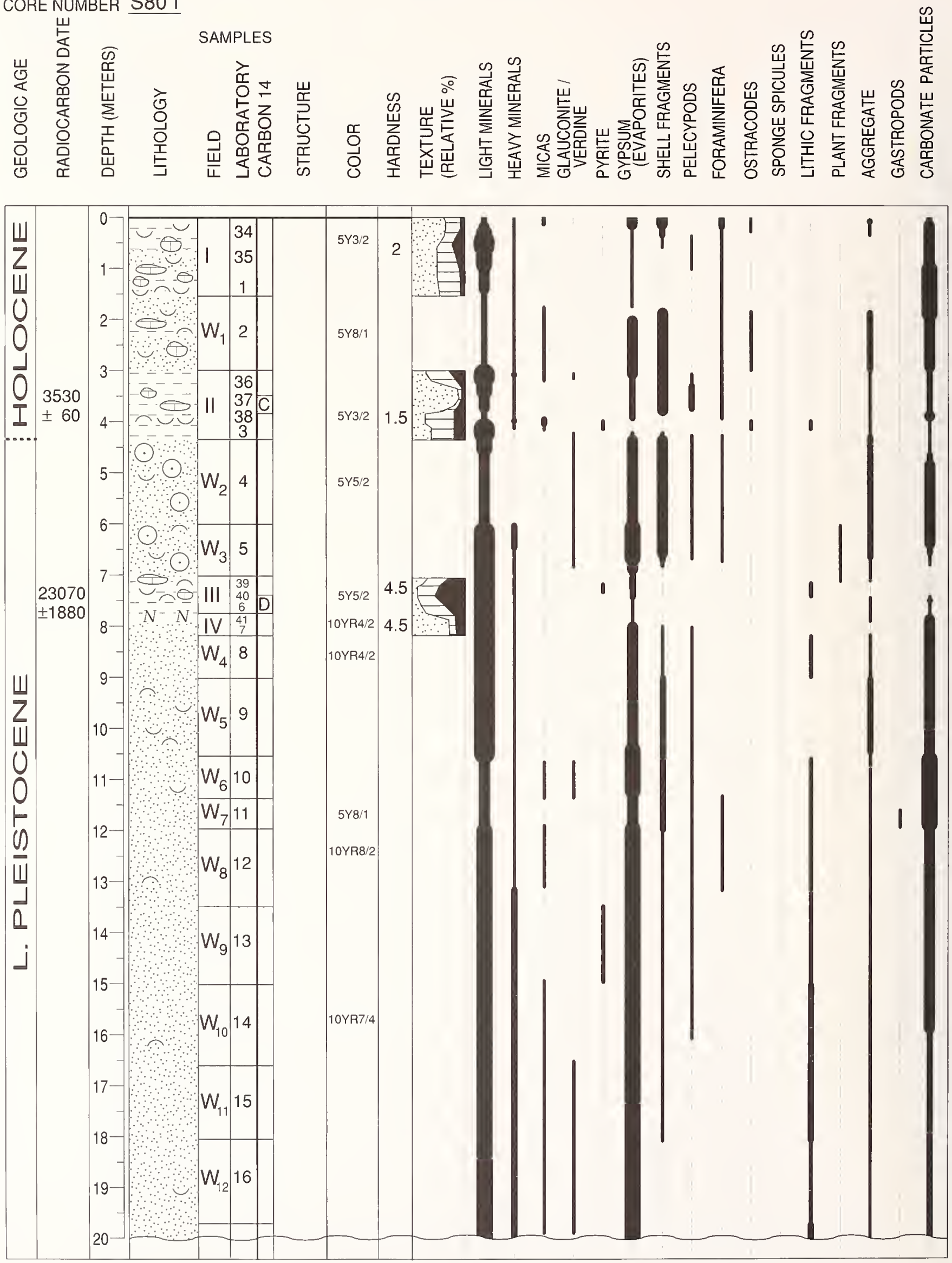
APPENDIX 1.-Continued.

CORE NUMBER S8O II

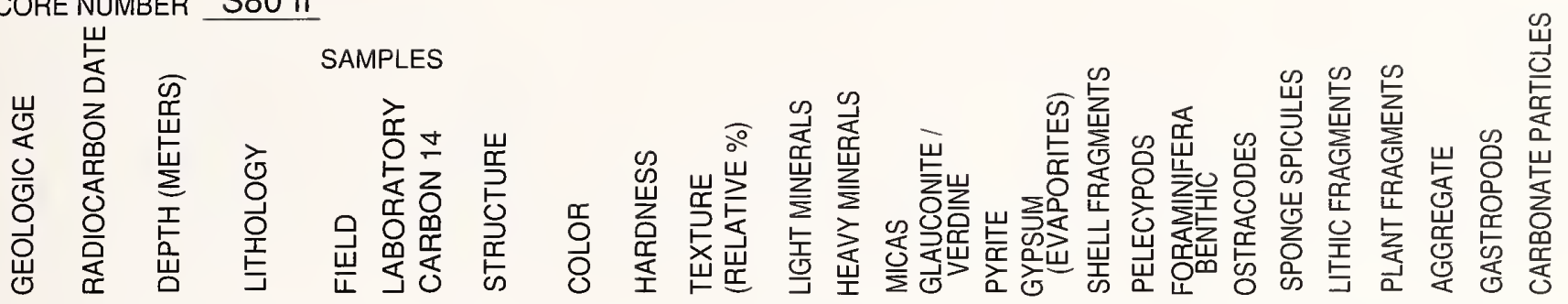

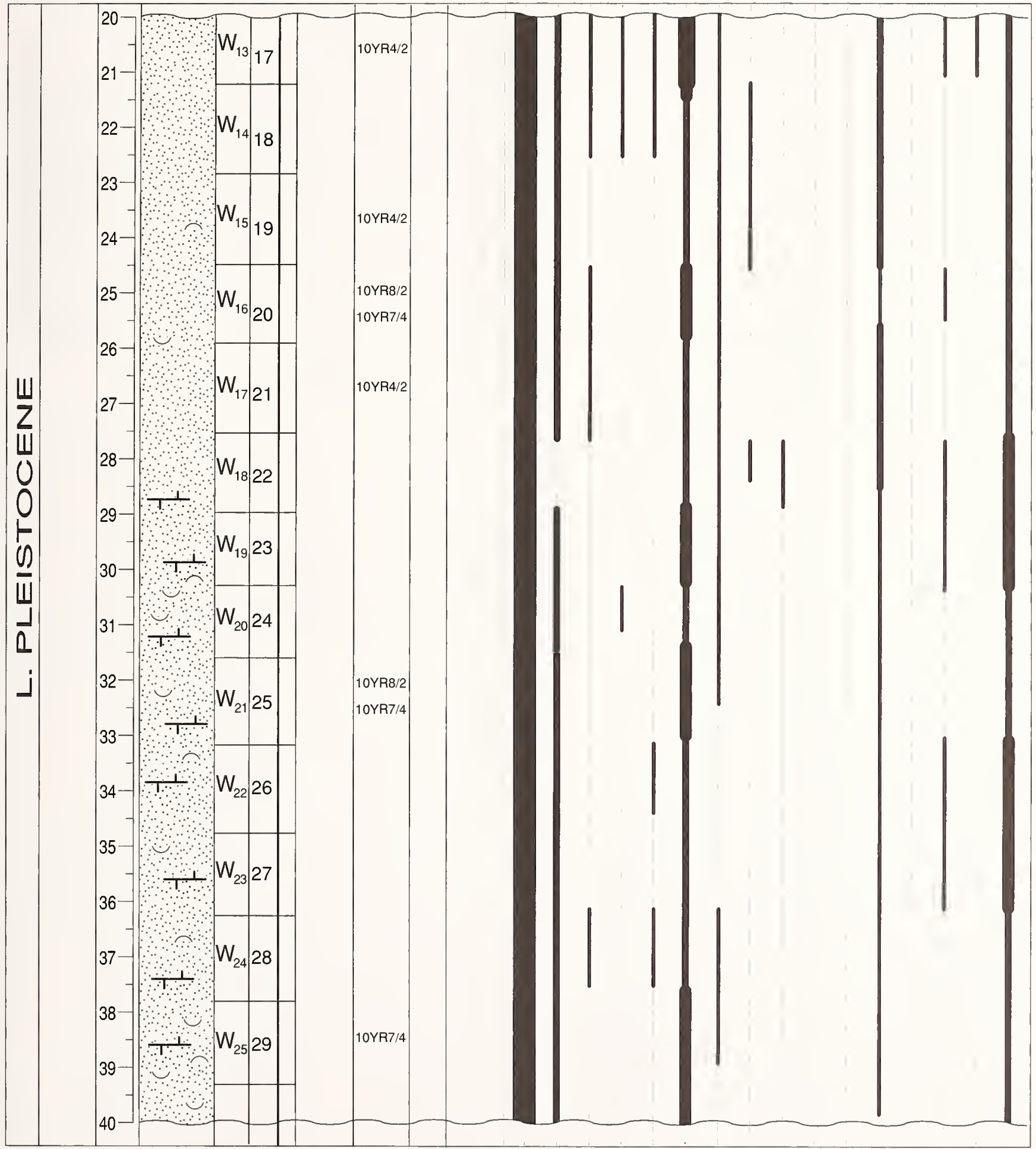


APPENDIX 1.-Continued.

CORE NUMBER S80 III

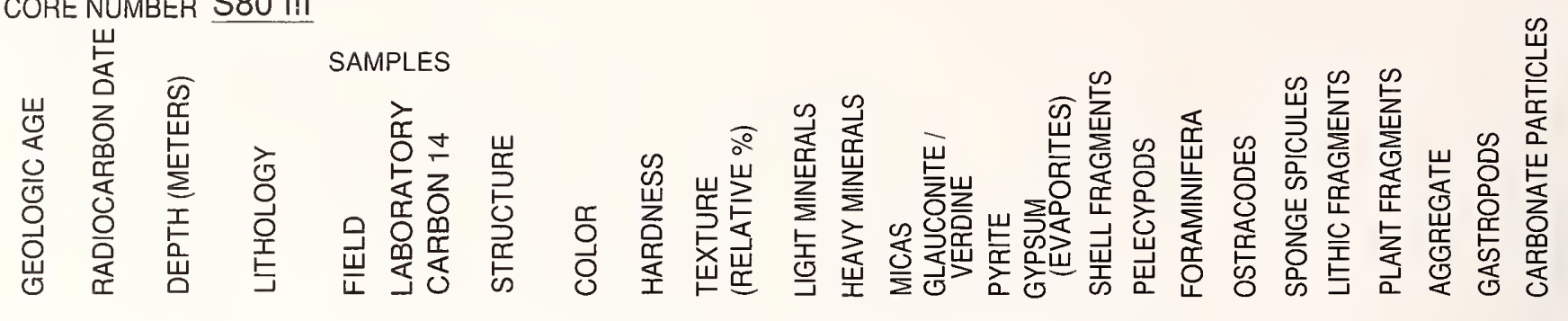

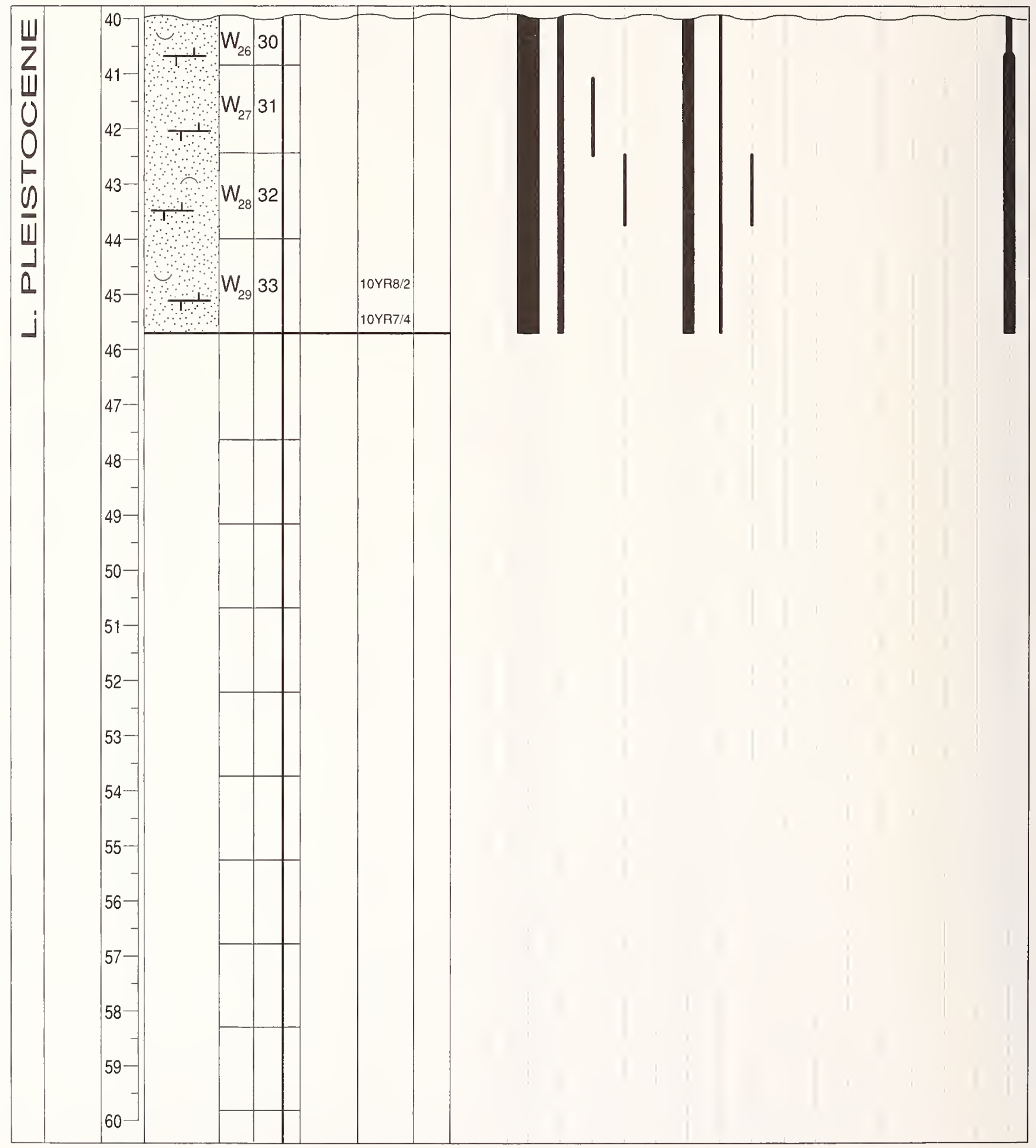


APPENDIX 1.-Continued.

CORE NUMBER S81 I

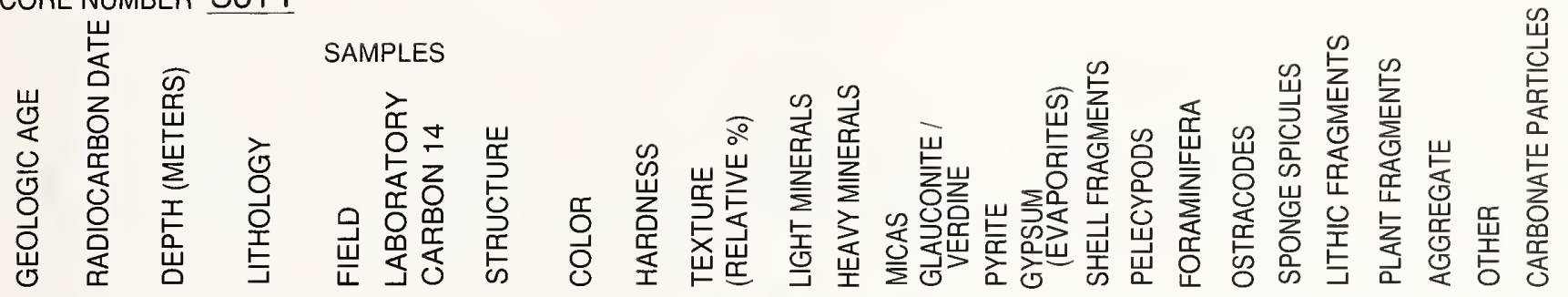

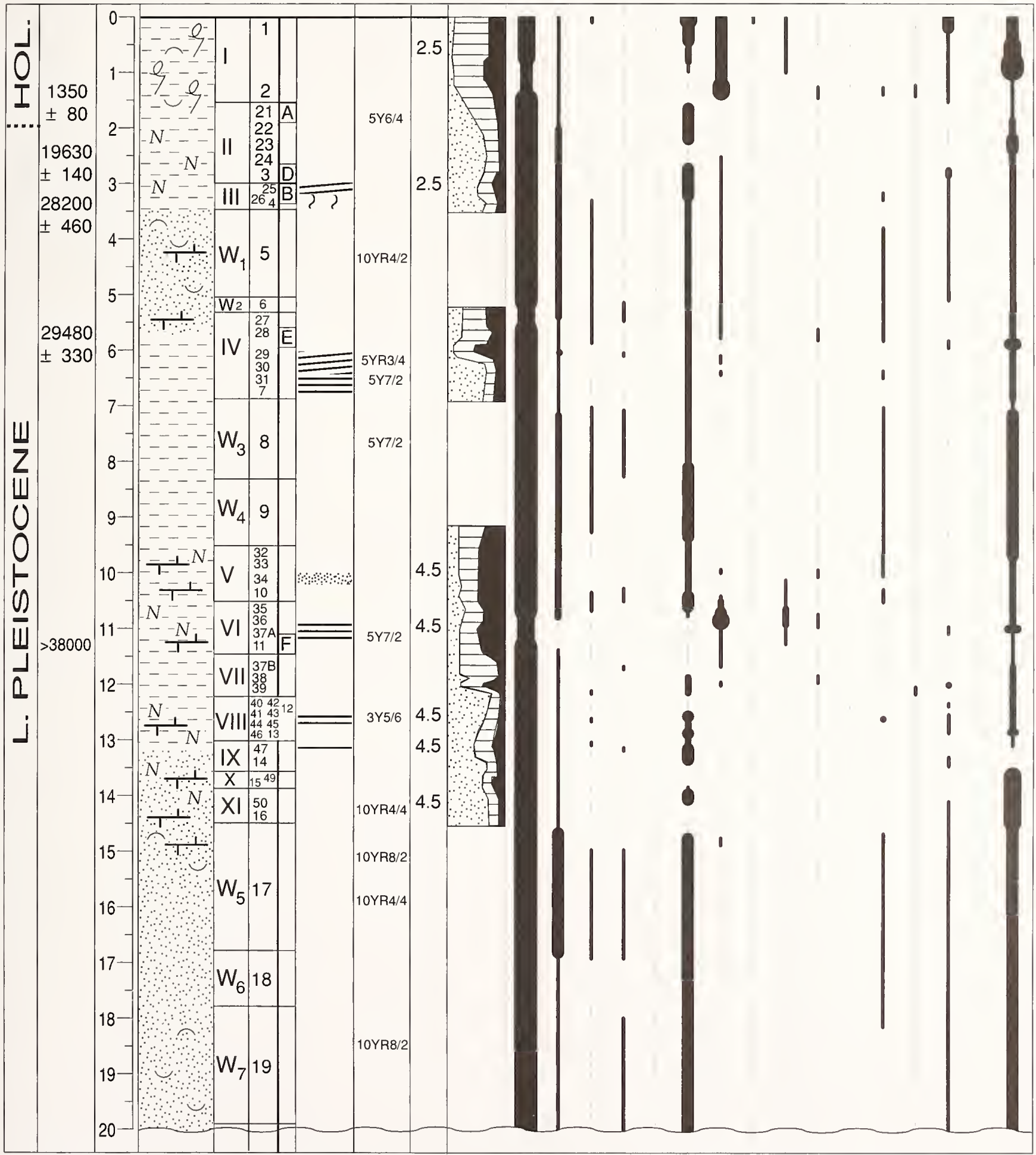


APPENDIX 1.-Continued.
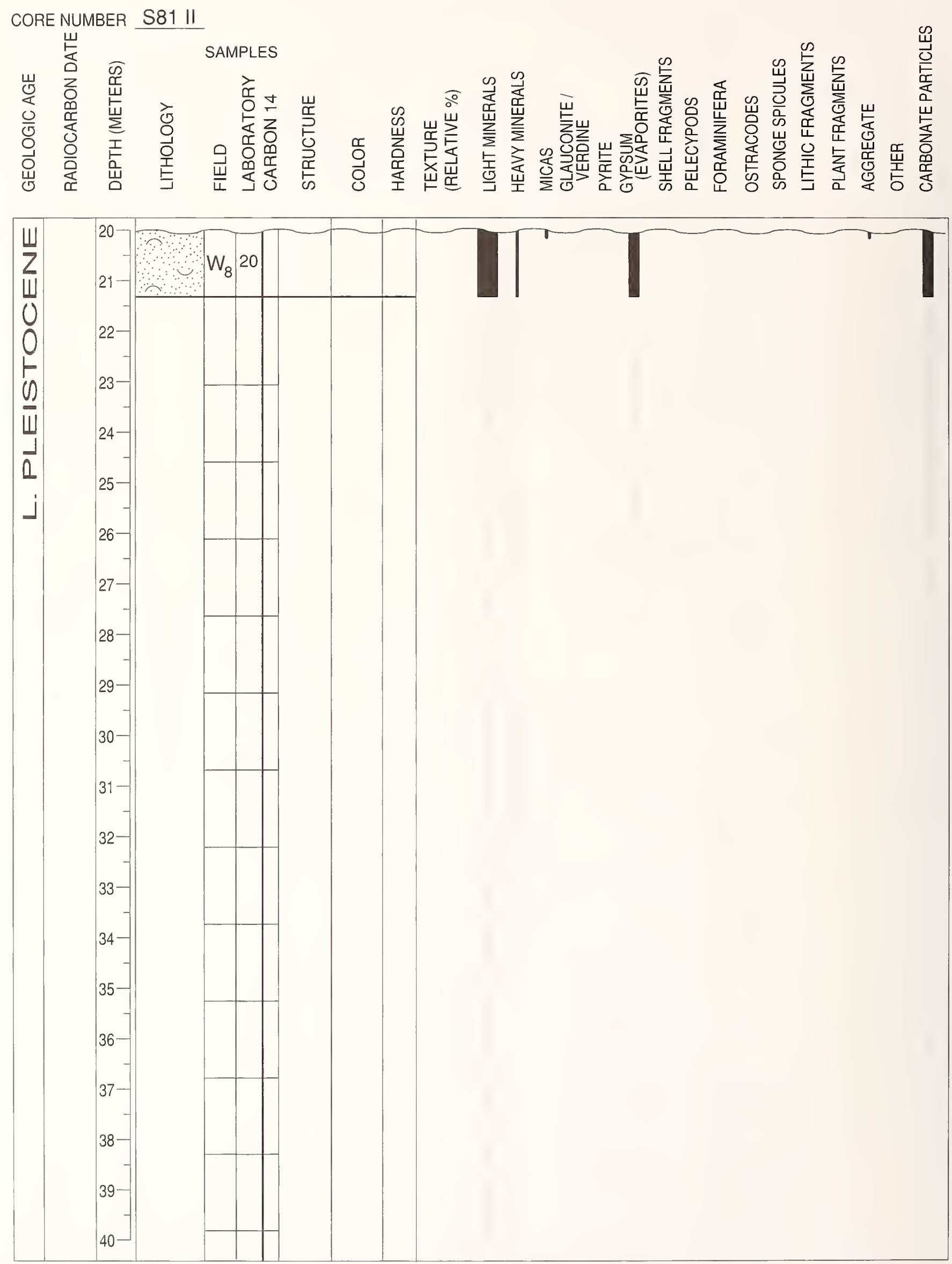
APPENDIX 1.-Continued.

CORE NUMBER S82I

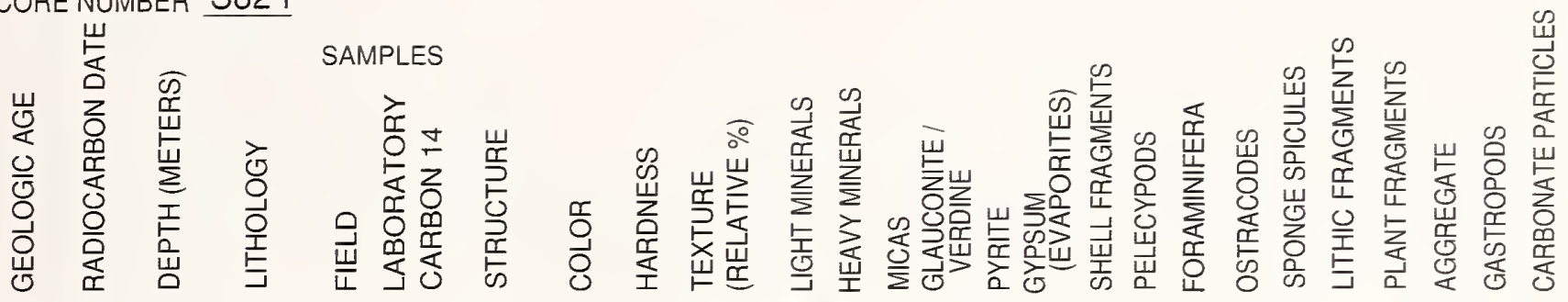

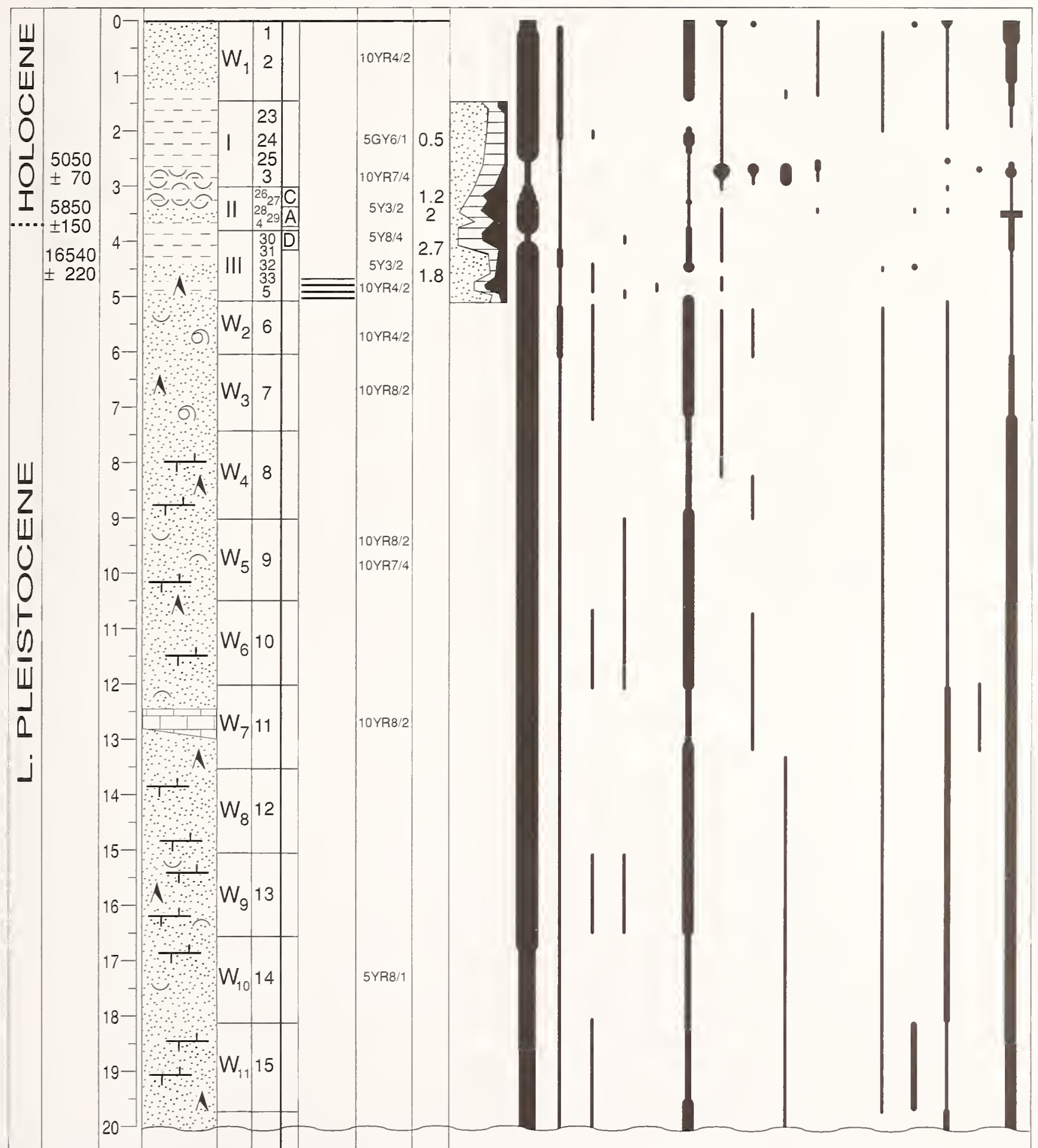


APPENDIX 1.-Continued.

CORE NUMBER S82 II

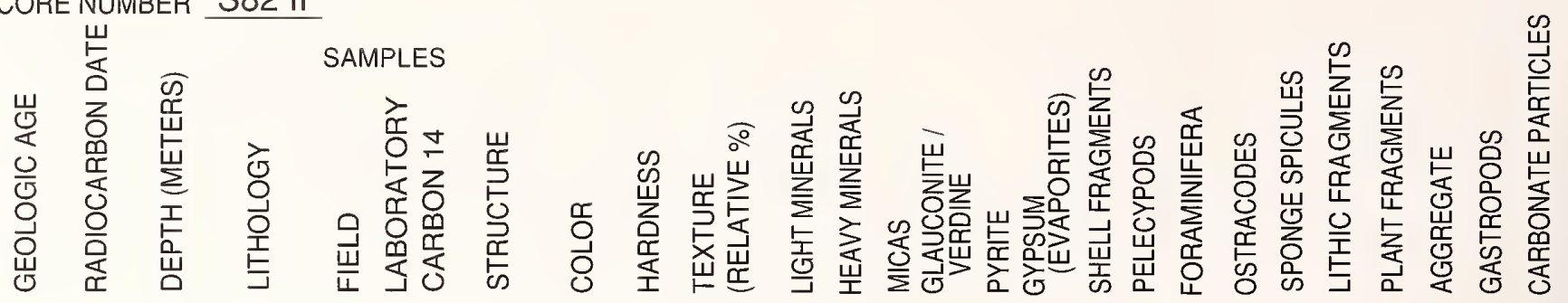

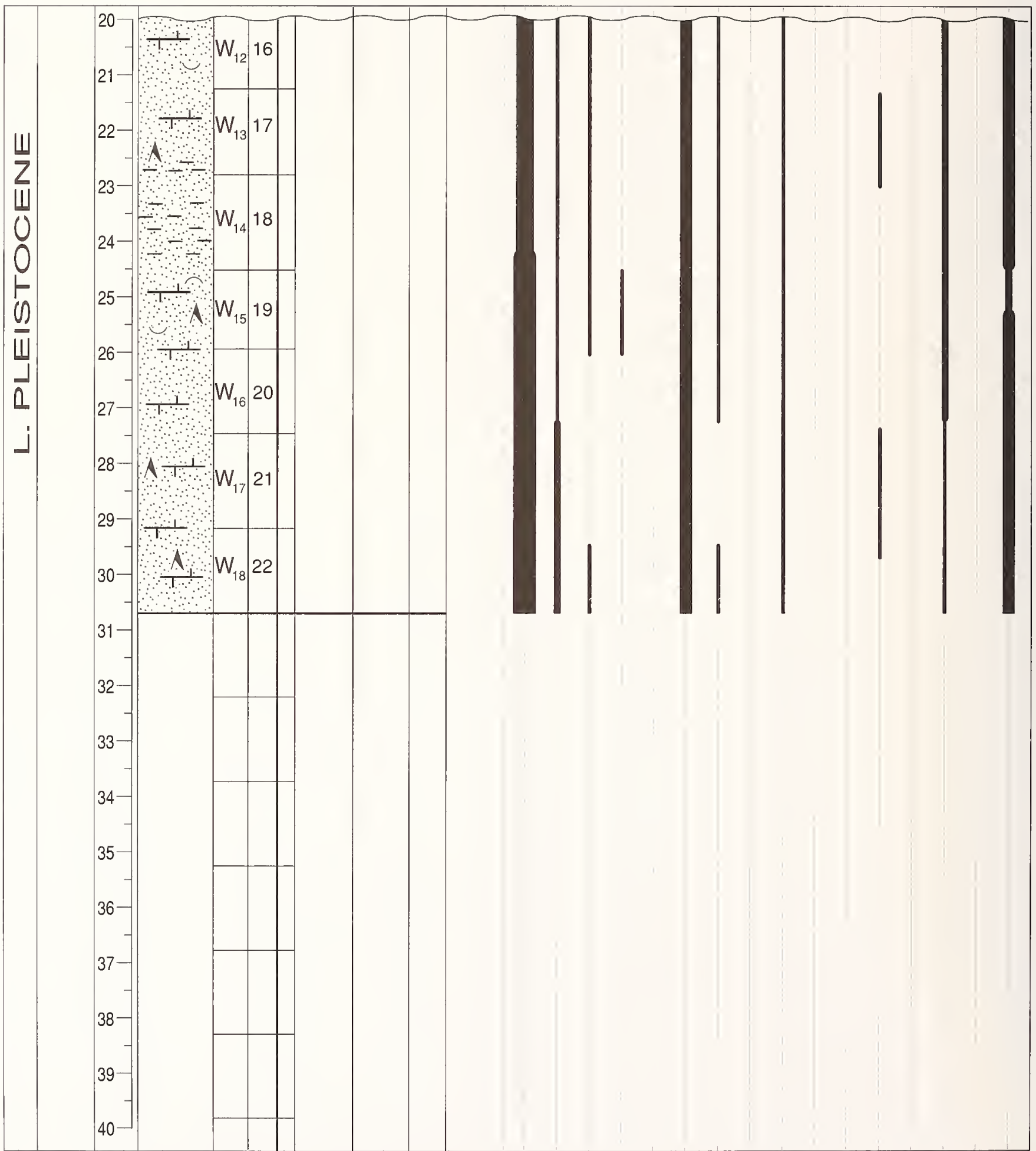


APPENDIX 1.-Continued.

CORE NUMBER S83I

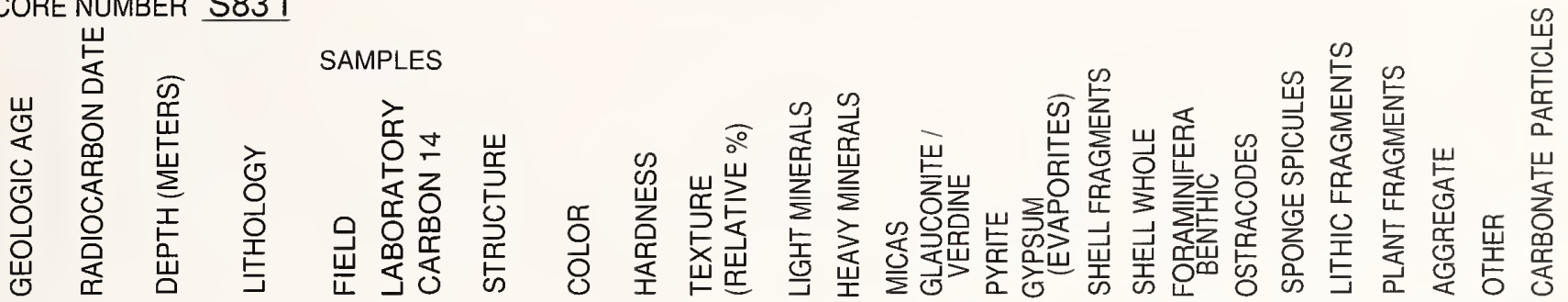

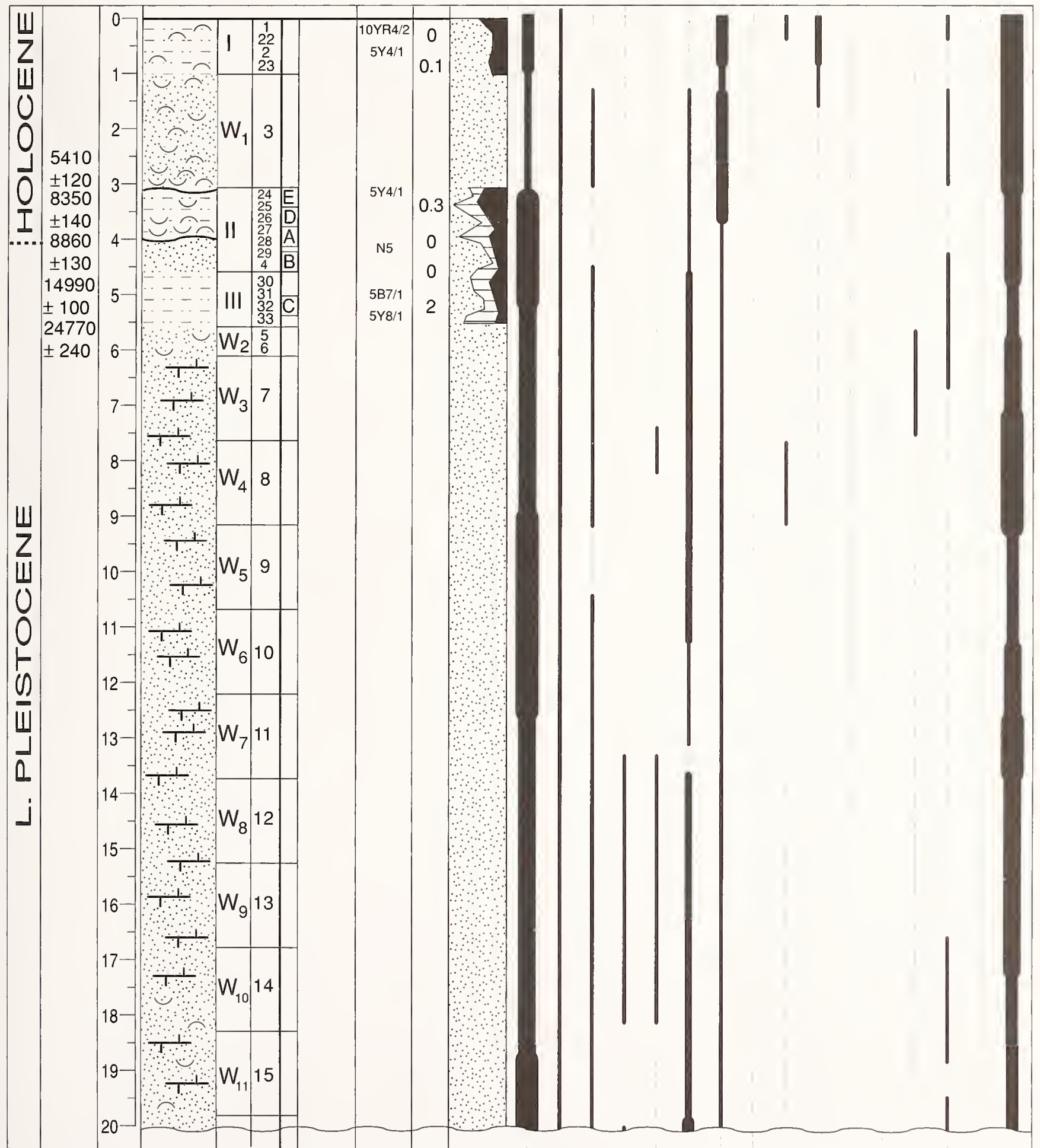


APPENDIX 1.-Continued.
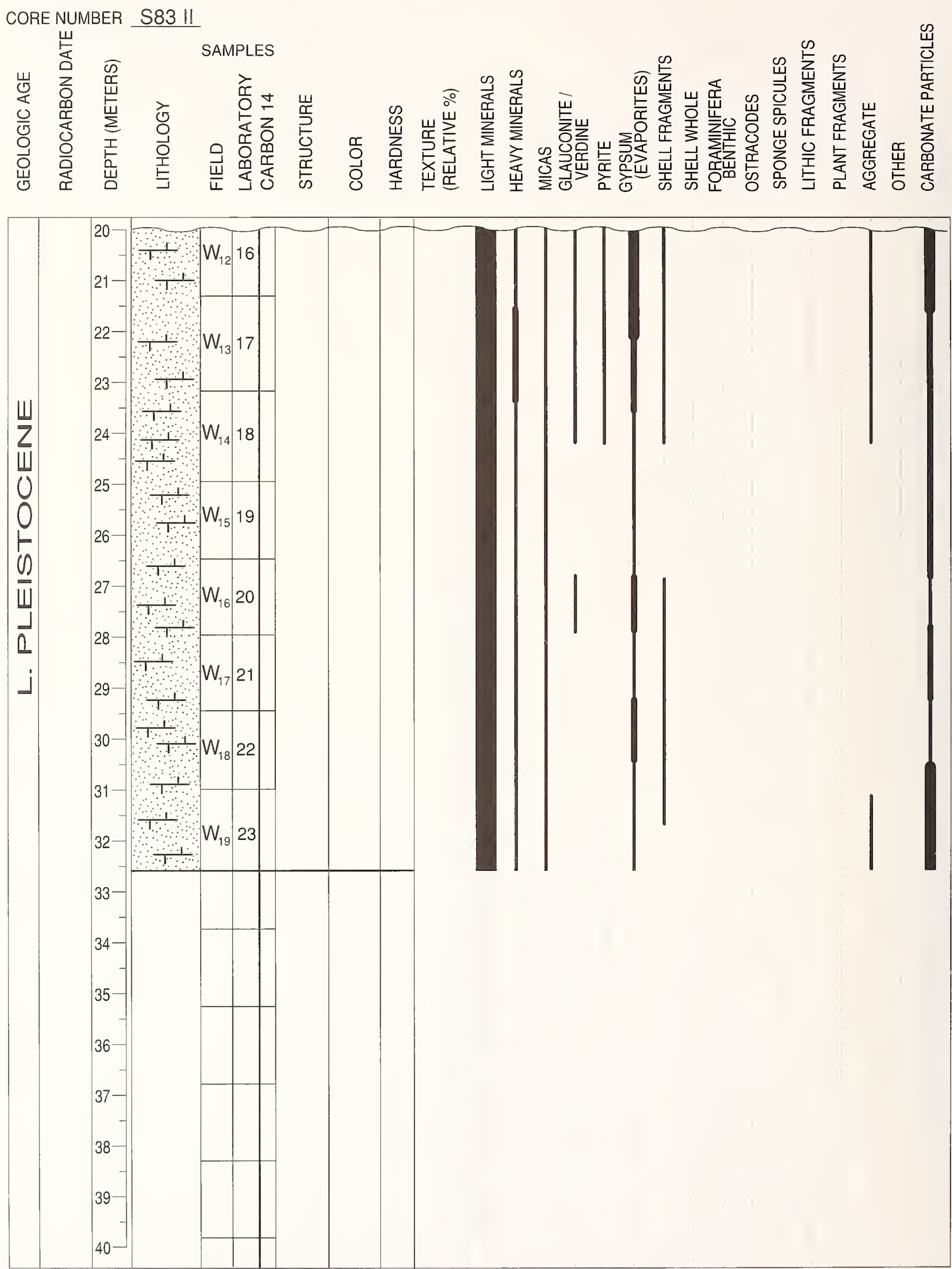
APPENDIX 1.-Continued.
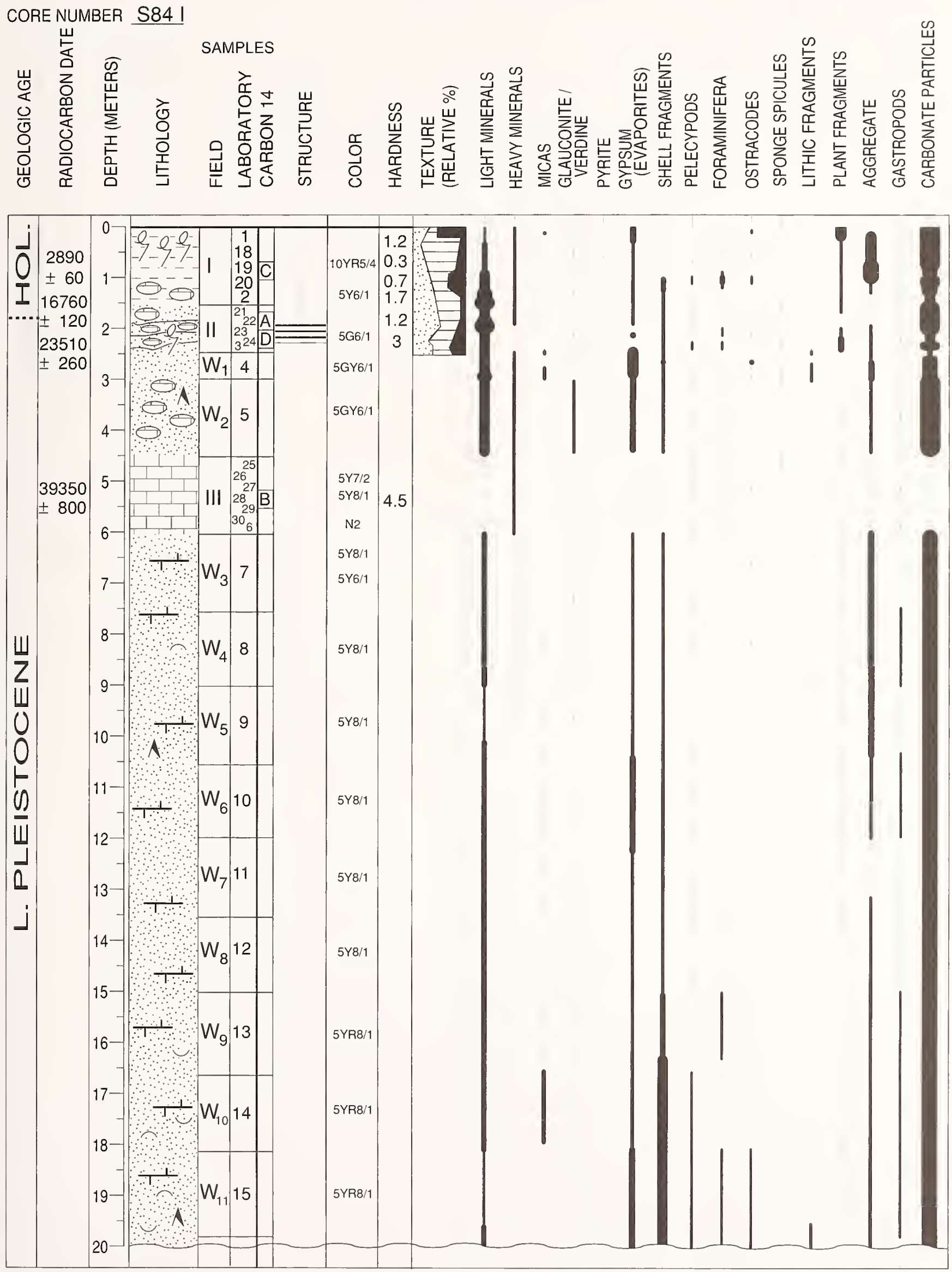
APPENDIX 1.-Continued.
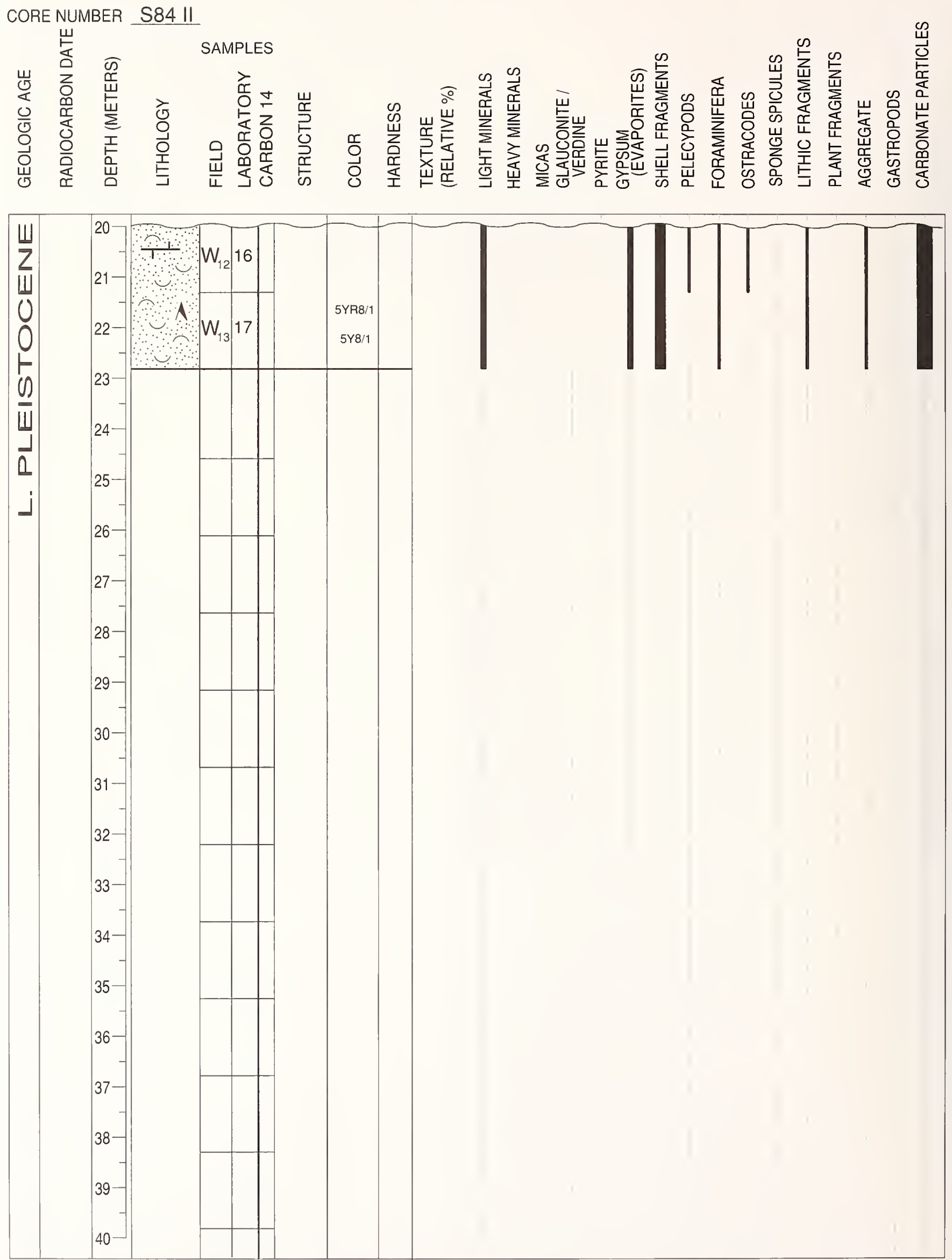
APPENDIX 1.-Continued.
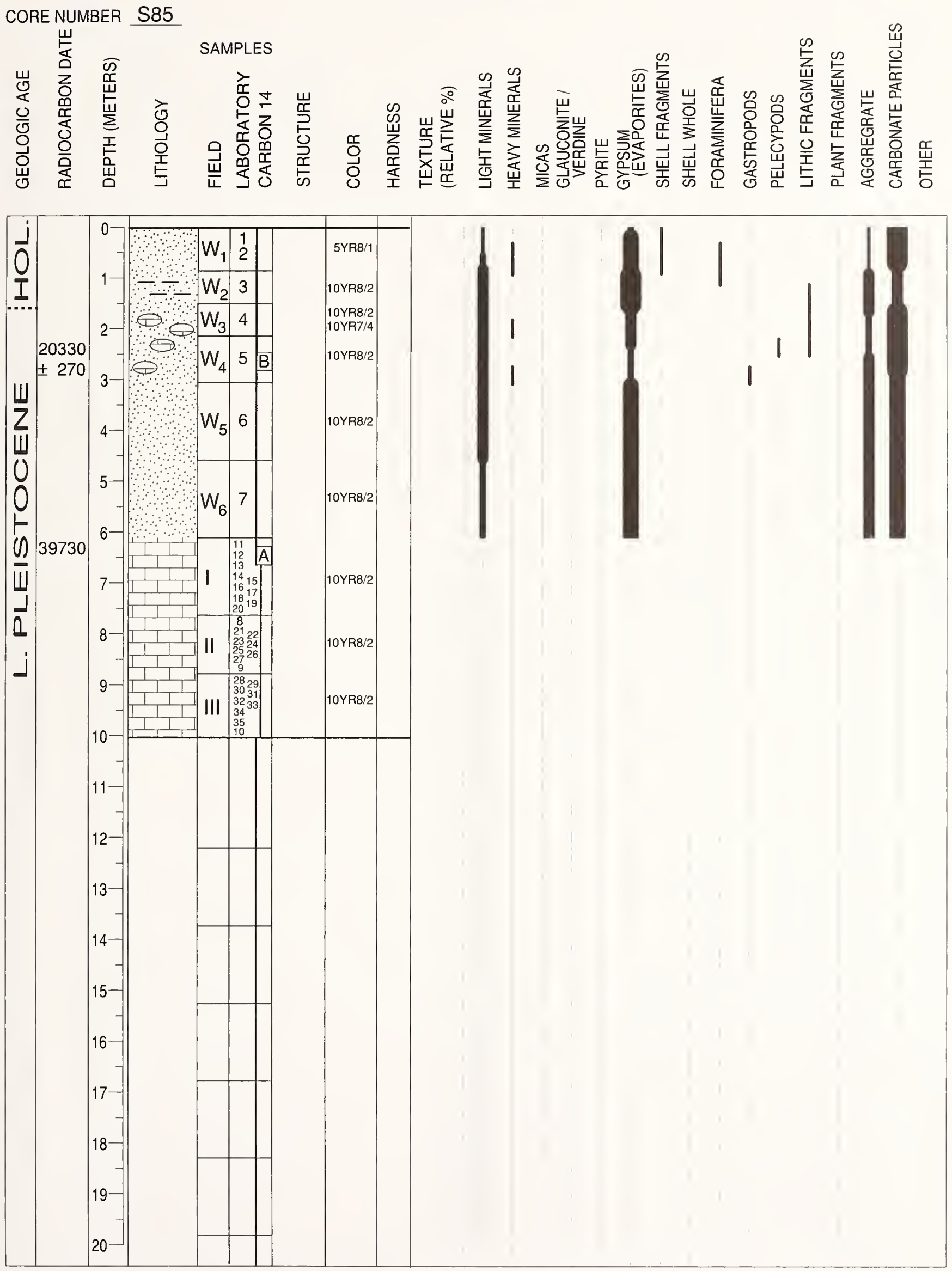
APPENDIX 1.-Continued.
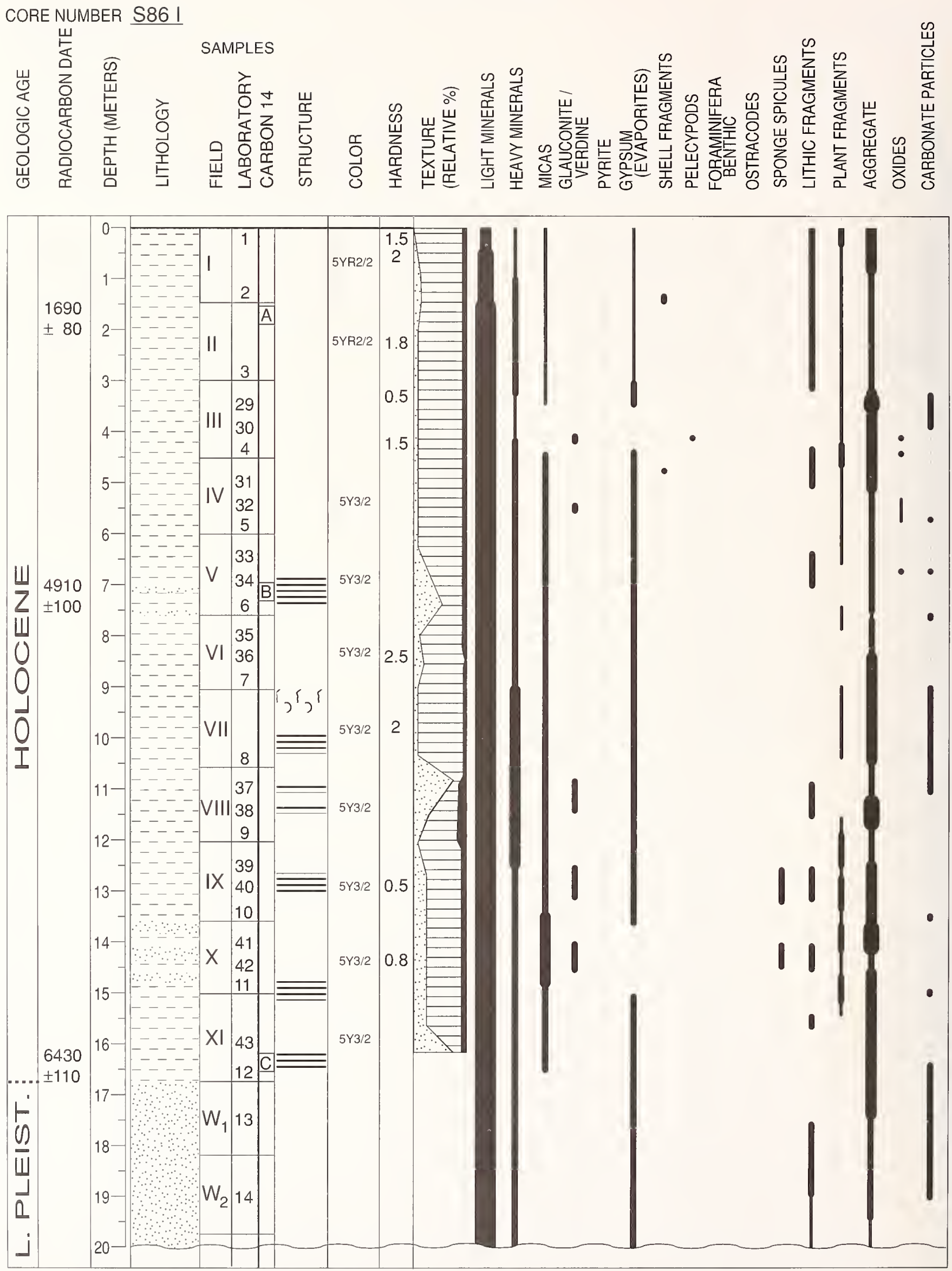
APPENDIX 1.-Continued.

CORE NUMBER S86 II

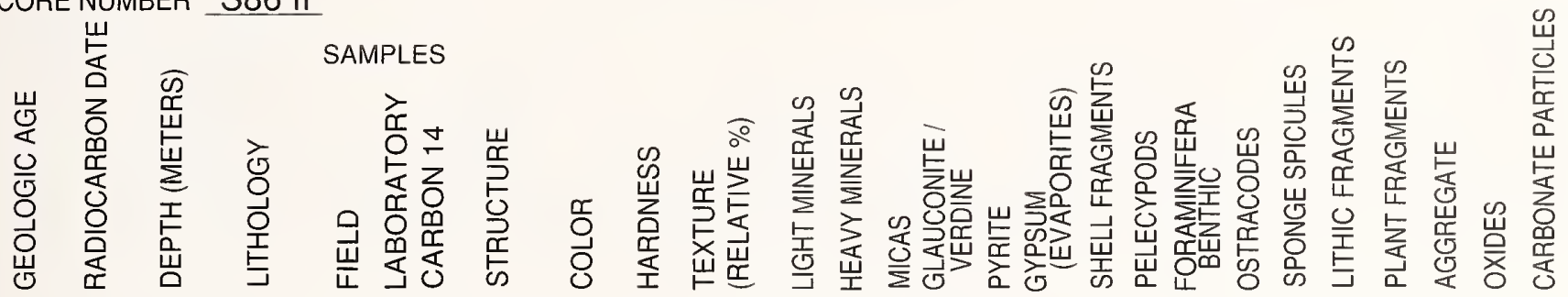

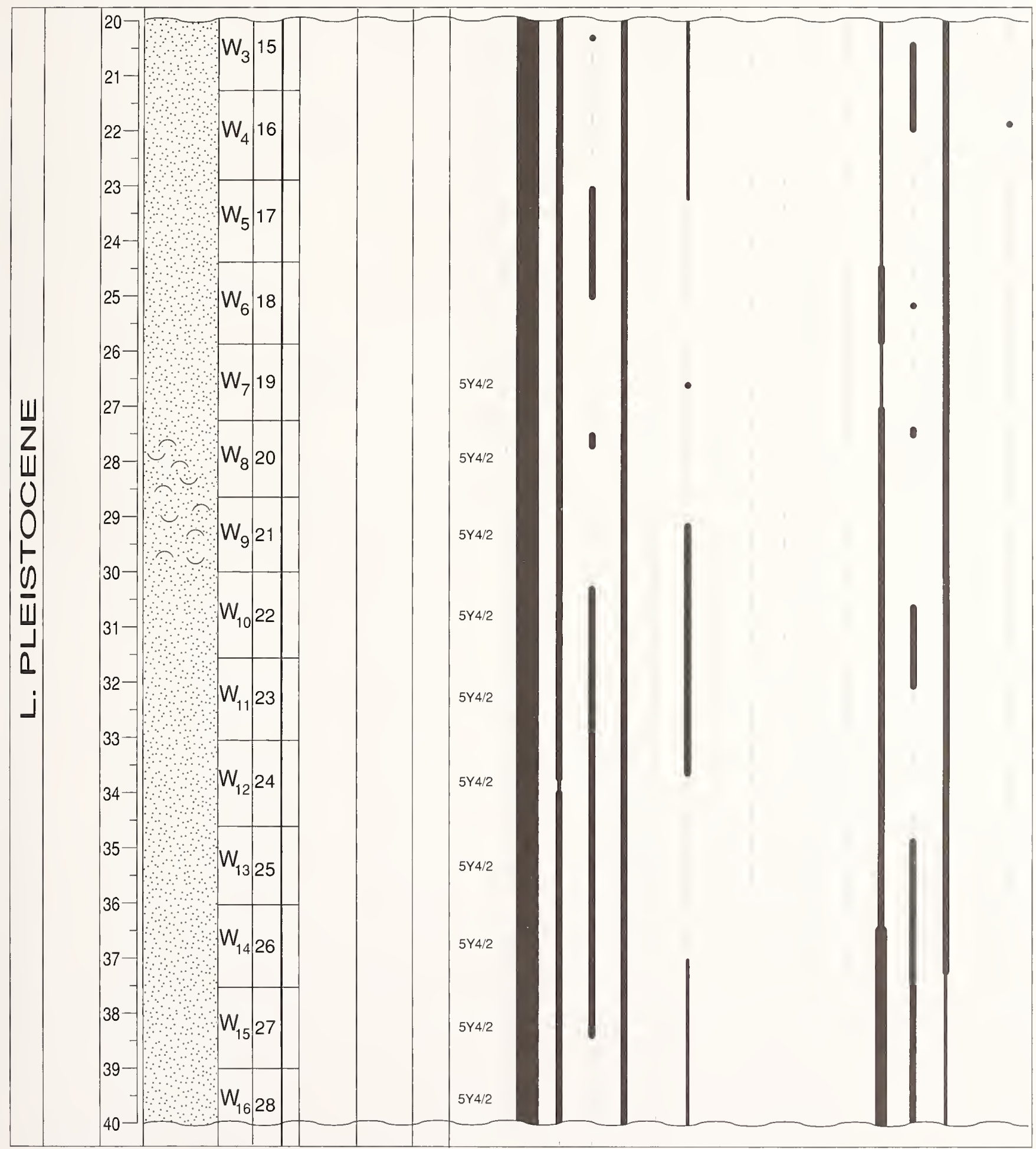


APPENDIX 1.-Continued.

\section{CORE NUMBER S86 III}
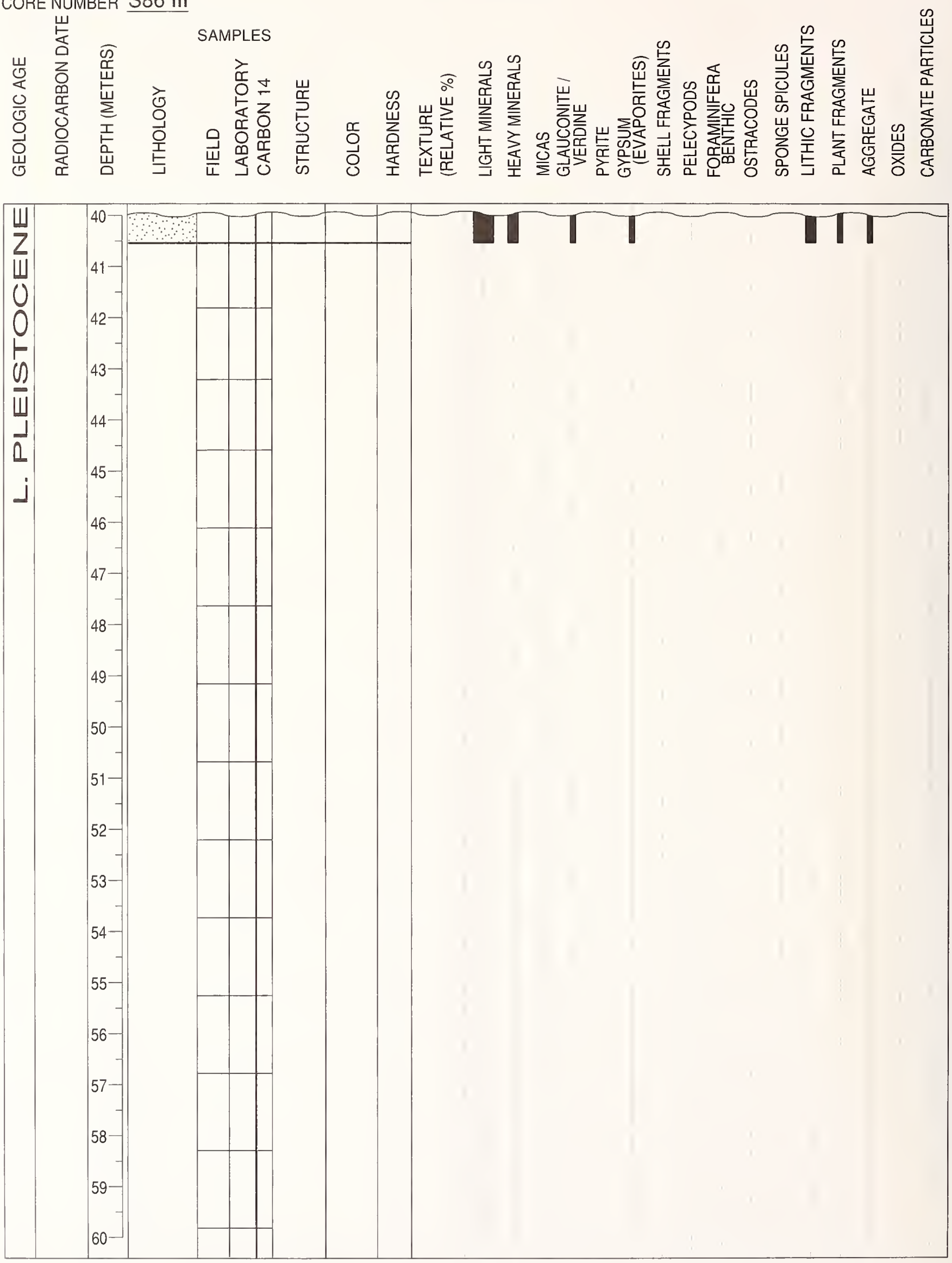
APPENDIX 1.-Continued.
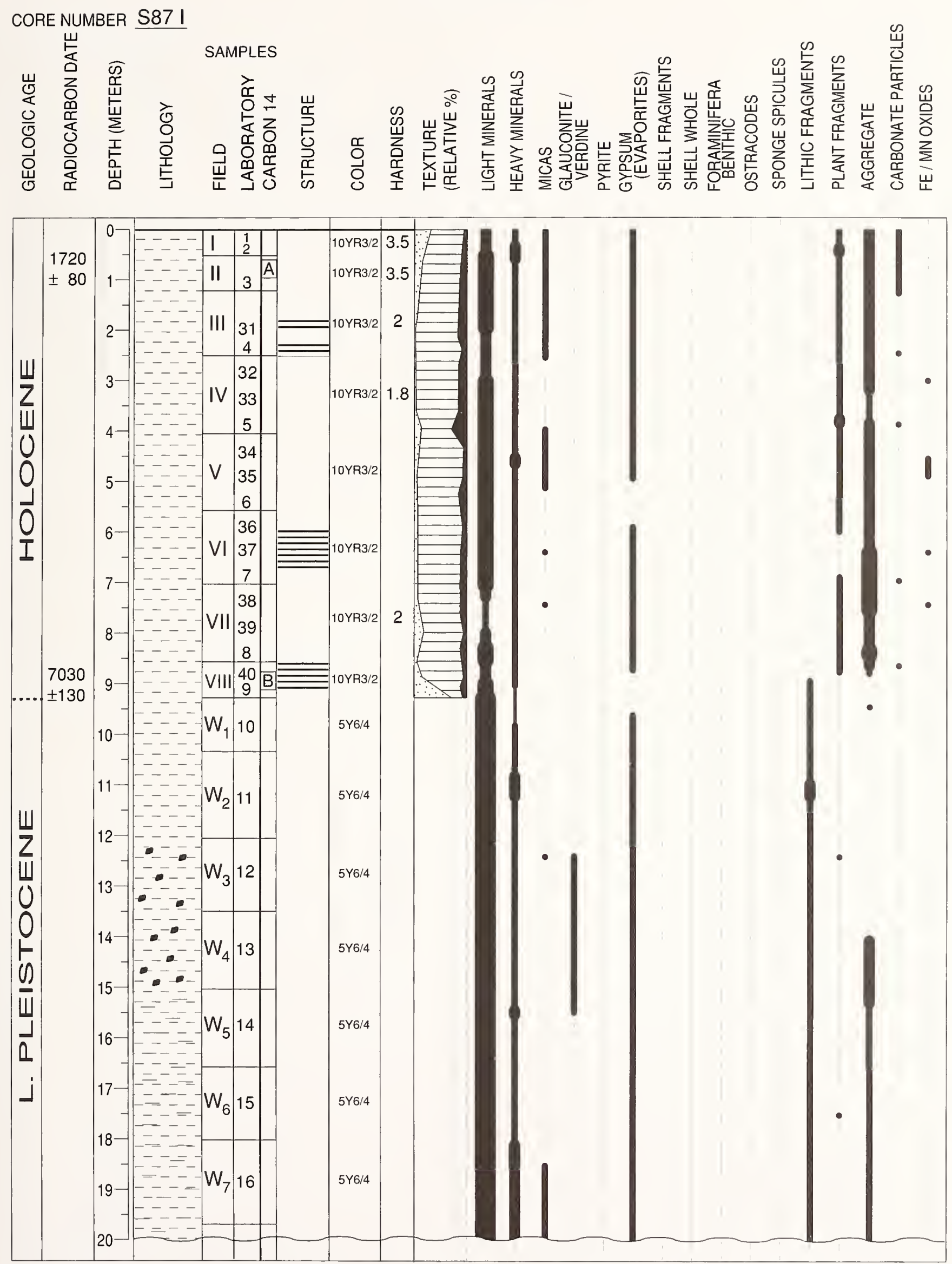
APPENDIX 1.-Continued.
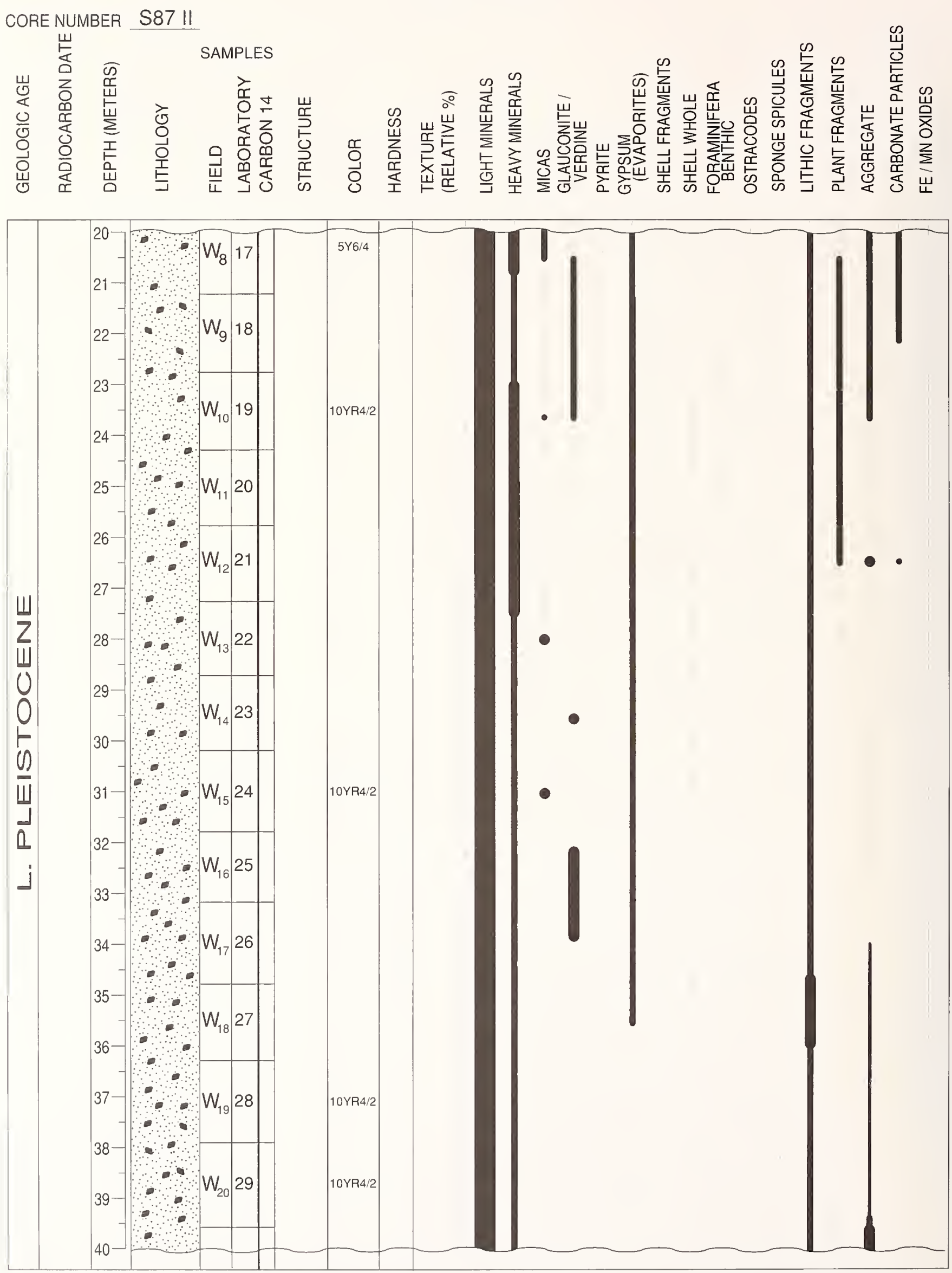
APPENDIX 1.-Continued.

CORE NUMBER S87 III

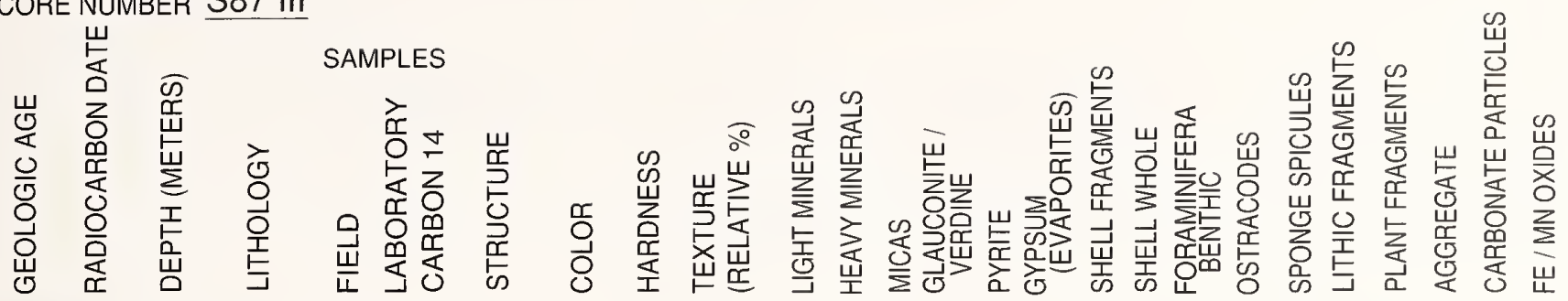

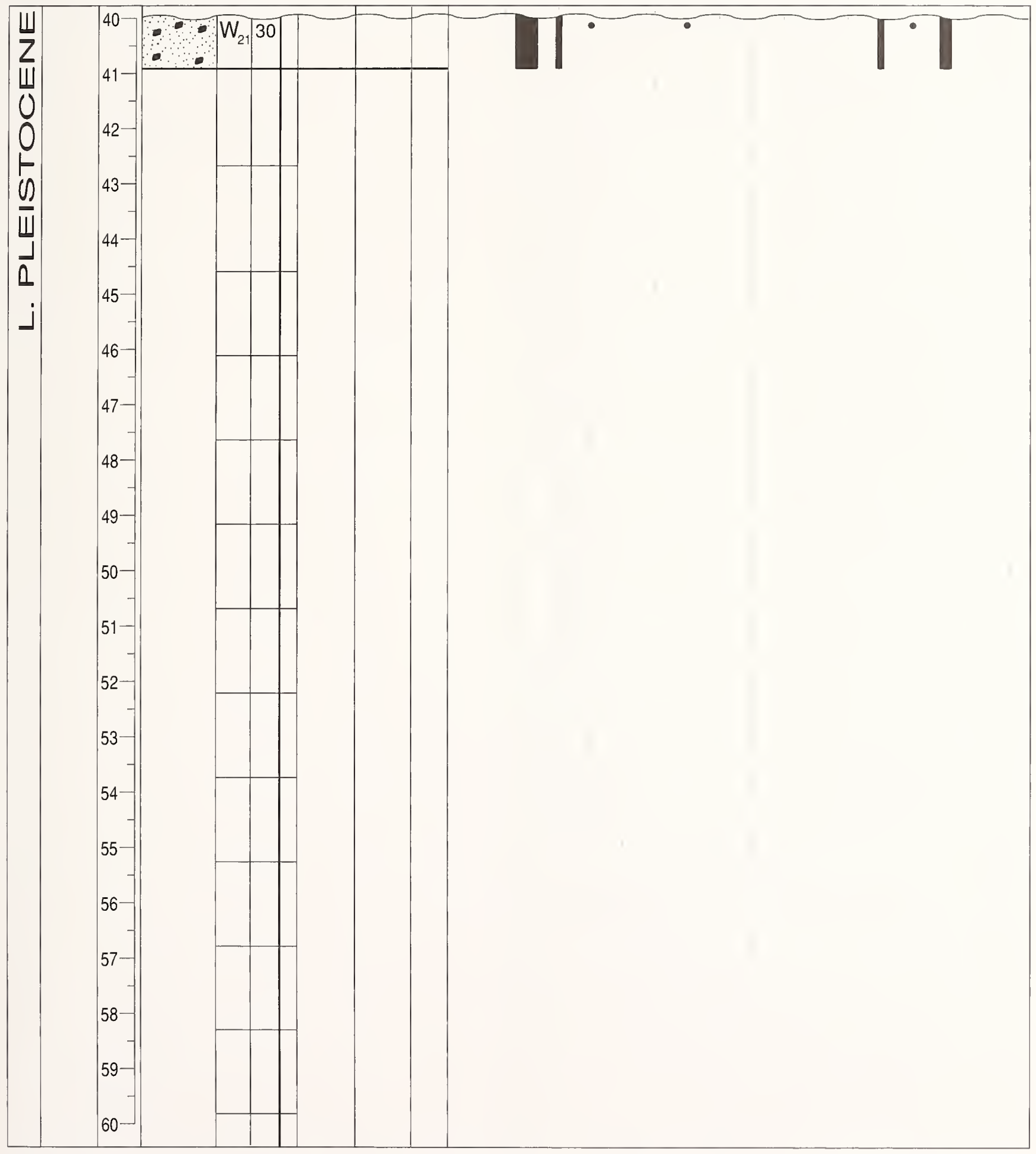




\section{Appendix 2: Core Sample Data Listings}

\section{Legend}

\section{TEXTURAL ANALysis}

\begin{tabular}{lr} 
SAND & \multicolumn{1}{c}{ Sand } \\
SILT & Silt \\
CLAY & Clay \\
& TOTAL $=100 \%$
\end{tabular}

\begin{tabular}{ll}
\multicolumn{2}{c}{ POINT COUNT ANALYSIS } \\
\cline { 2 - 2 } Abbreviation & Composition \\
LT & Light mineral \\
HVY & Heavy mineral \\
MICA & Mica \\
GLAU & Glauconite/Verdine \\
PYRT & Pyrite \\
EVAP & Evaporite \\
GYP & Gypsum \\
LITH & Lithic fragment \\
AGG & Aggregate \\
PLTM & Plant material \\
FORB & Foraminifera, Benthic \\
FORP & Foraminifera, Planktonic \\
GSHW & Gastropod shell, Whole \\
GSHF & Gastropod shell, Fragment \\
PSHW & Pelecypod shell, Whole \\
PSHF & Pelecypod shell, Fragment \\
SHLW & Shell, Whole \\
SHLF & Shell, Fragment \\
OSTR & Ostracode \\
SPNG & Sponge spicule \\
ECHIN & Echinoderm \\
BRYO & Bryozoa \\
WRMT & Worm tube \\
PTER & Pteropod \\
DIAT & Diatom \\
INSCT & Insect \\
RADIO & Radiolaria \\
OTH & Other \\
Fe OXIDE & Iron Oxide \\
WHITE & White calcareous carbonite \\
CARBT & Carbonate \\
& Too\% \\
& \\
&
\end{tabular}

CARBON-14
Radiocarbon date 
APPENDIX 2.-Continued.

产

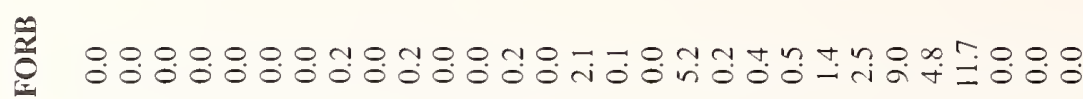

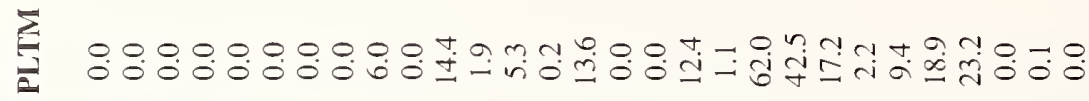

选

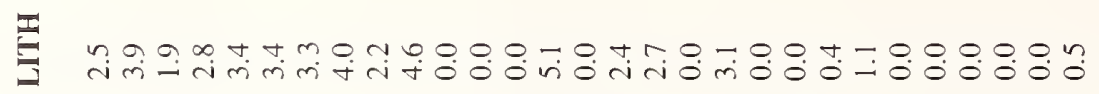

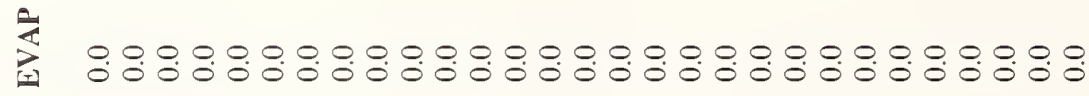

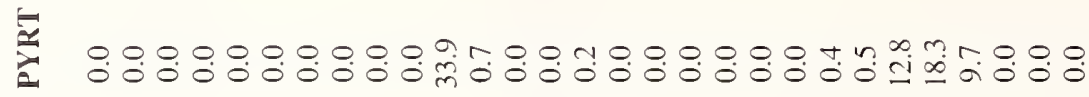

过

送

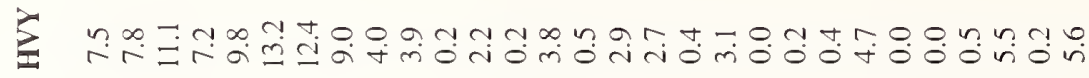

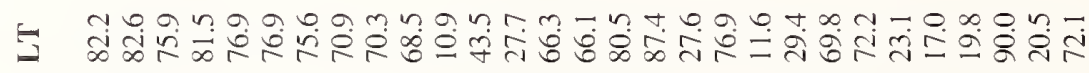

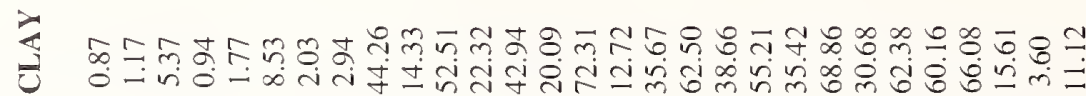

ติ

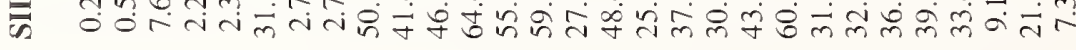

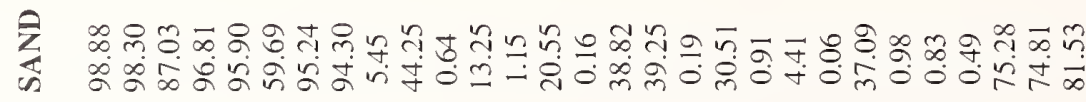

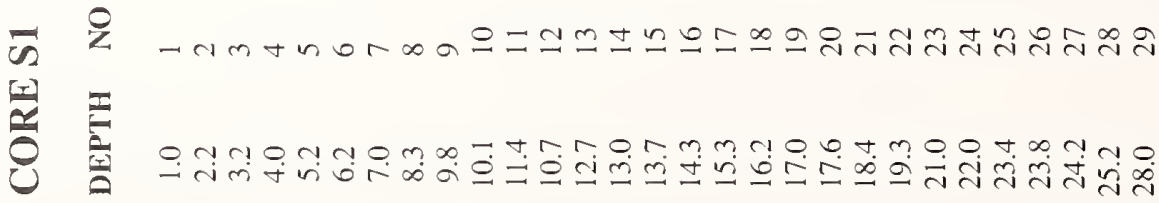


APPENDIX 2.-Continued.

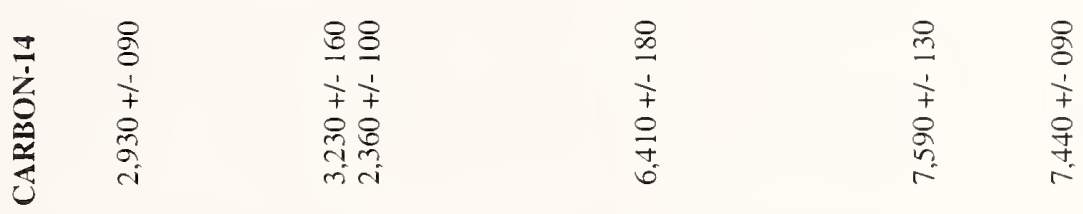

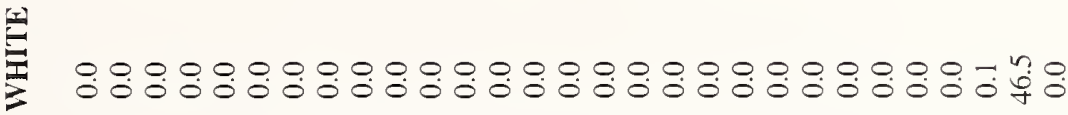

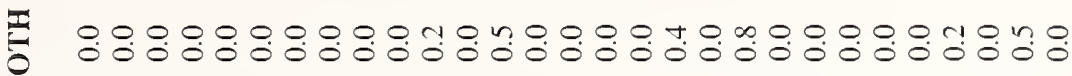

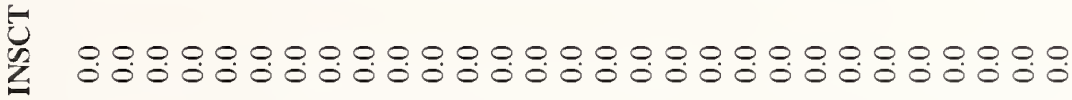

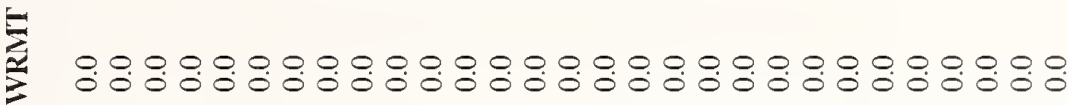

啳

玄

苟

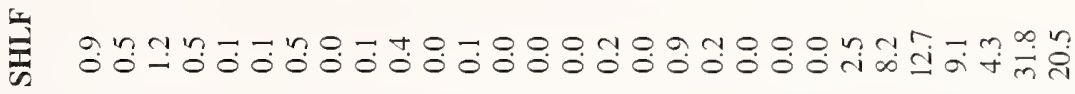

至

चี 
APPENDIX 2.-Continued.

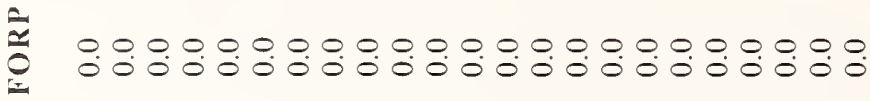

药

至

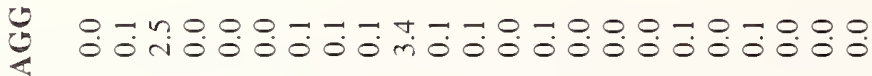

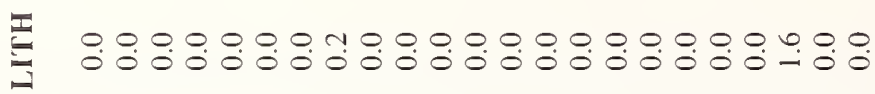

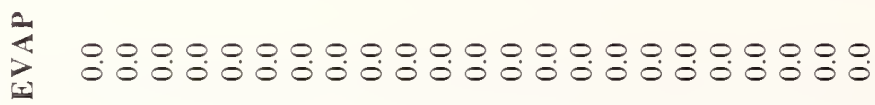

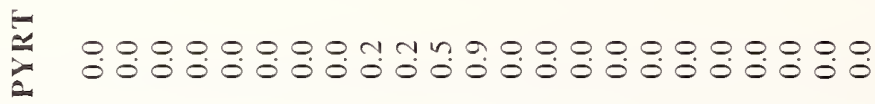

$\int_{0}$ o

还

¿

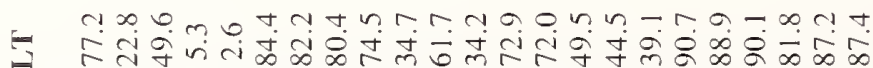

\

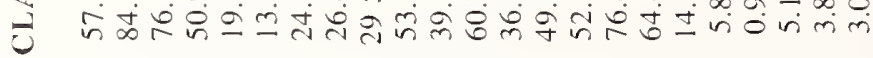

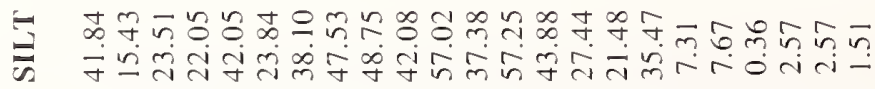

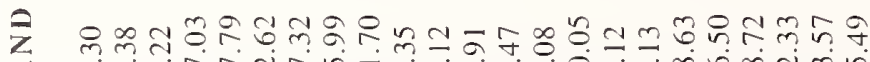

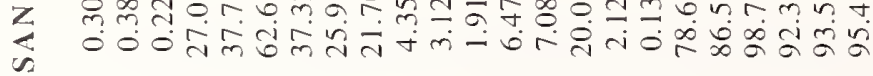

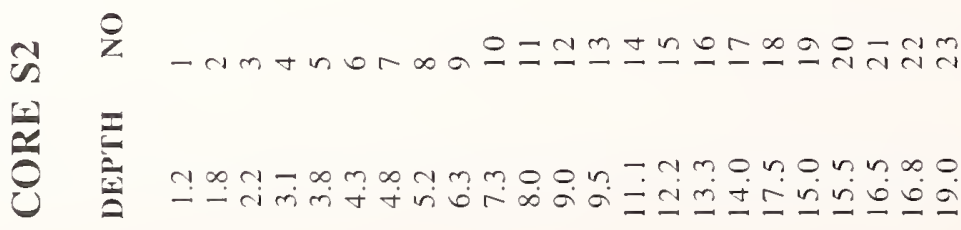


APPENDIX 2,-Continued.

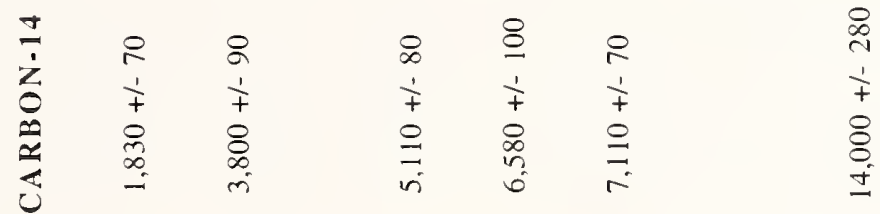

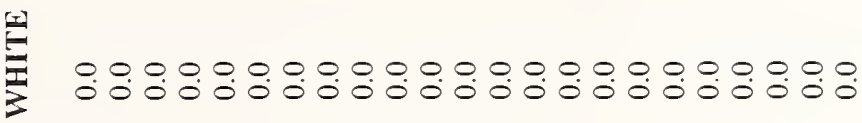

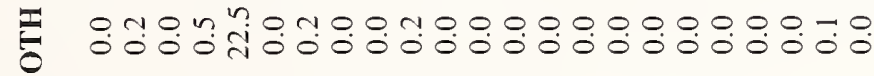

茎

i

茟

茪

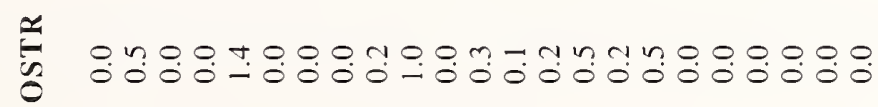

专

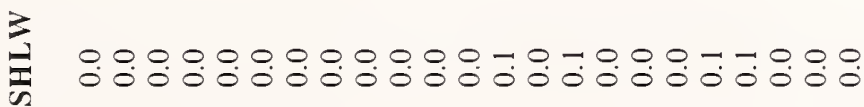

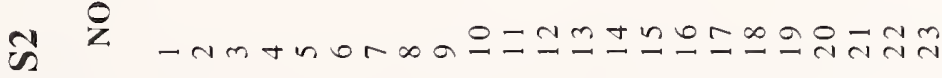

告 
APPENDIX 2.-Continued.

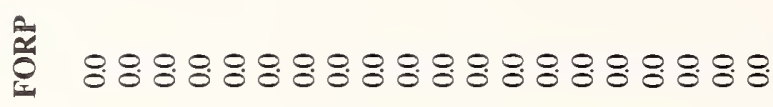

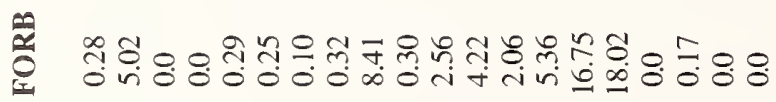

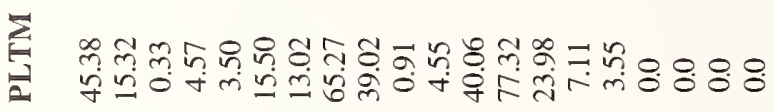

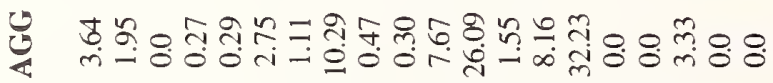

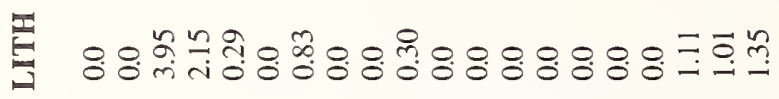

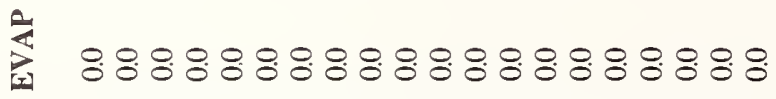

ส

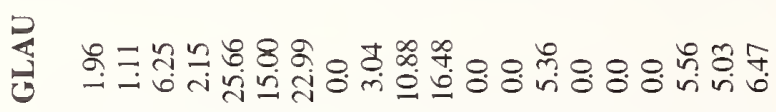

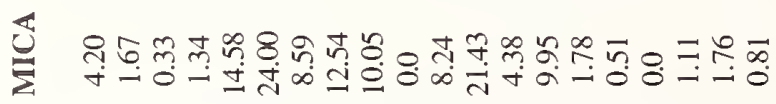

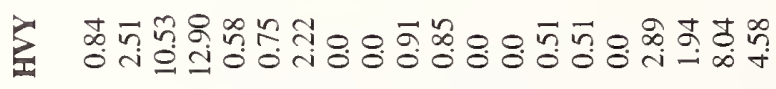

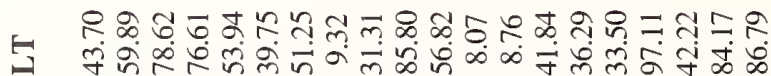

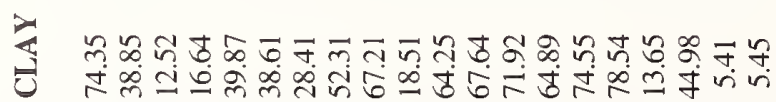

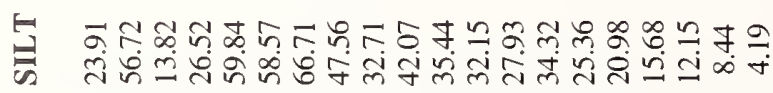

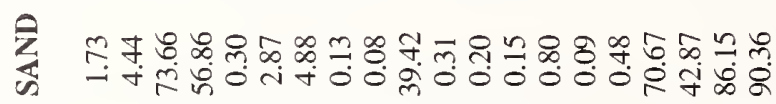

$\approx \sum$ ช

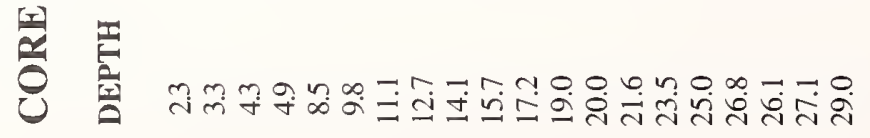


APPENDIX 2.-Continued.

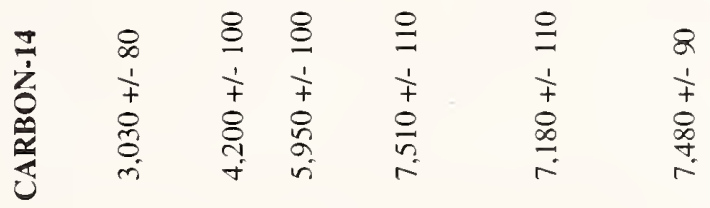

$8.8 \% 8.8 \% 8 \% 8.8 .8 .8 .8 \%$

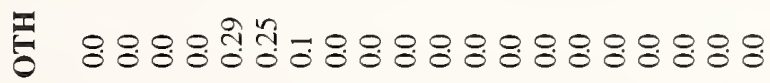

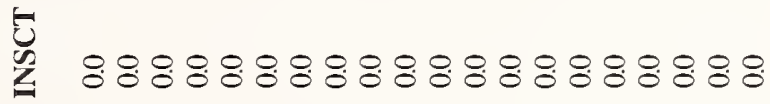

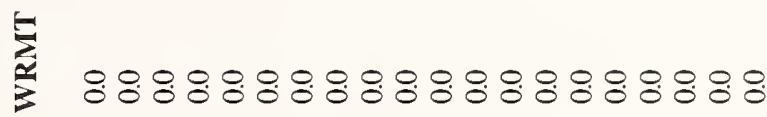

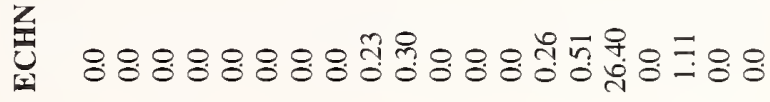

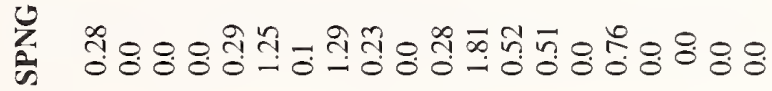

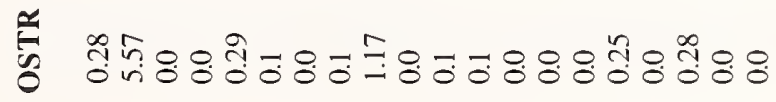

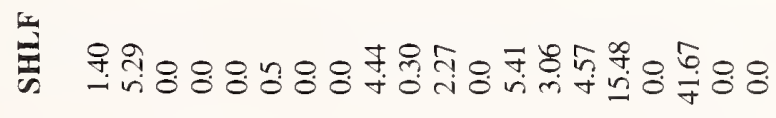

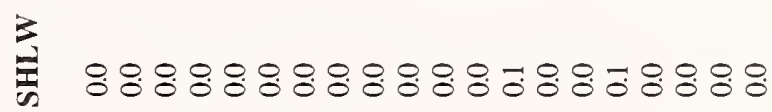

ข

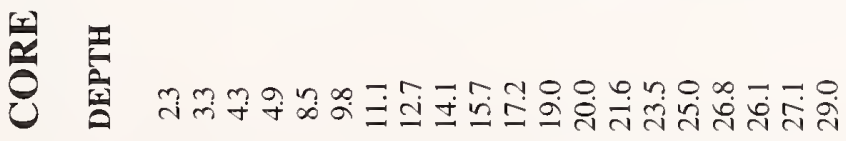


APPENDIX 2.-Continued.

$\frac{1}{0}$

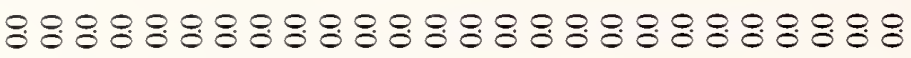

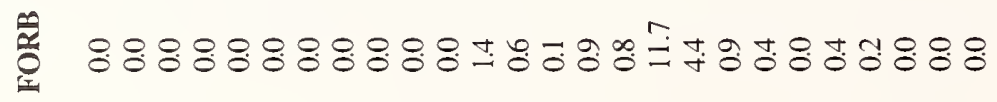

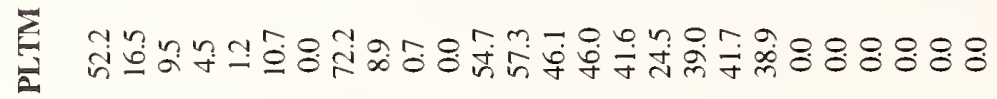

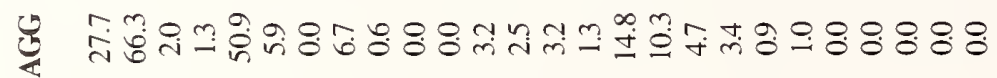

돈

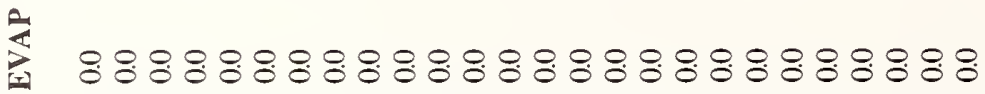

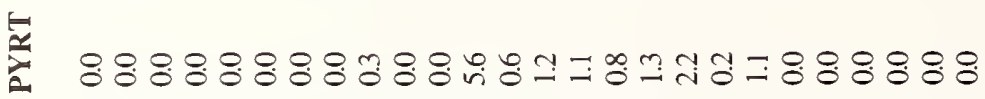

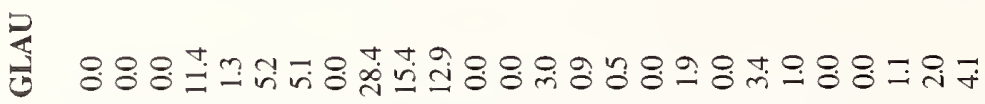

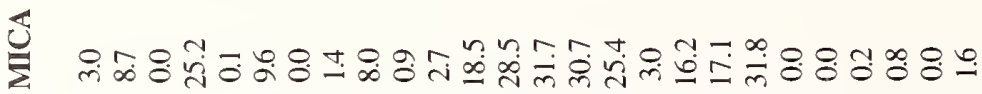

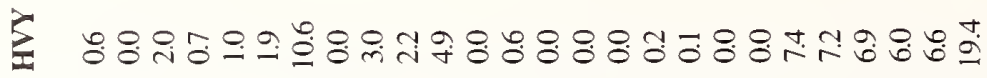

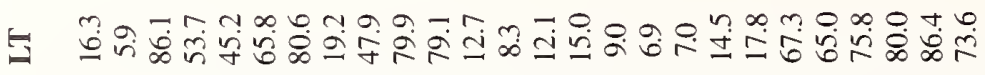

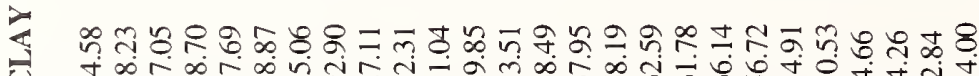

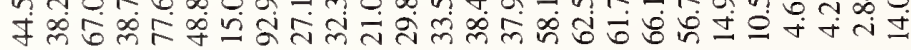

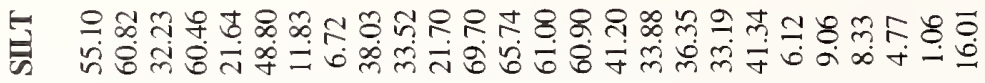

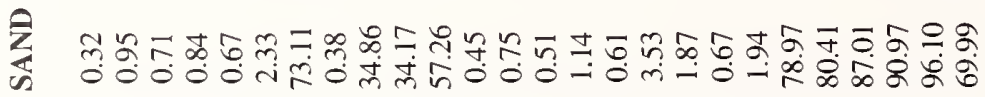

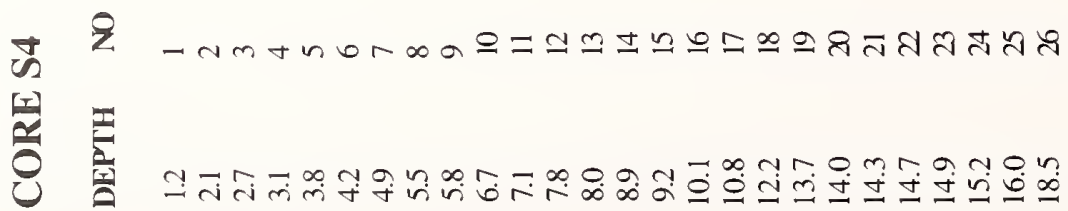


APPENDIX 2.-Continued.

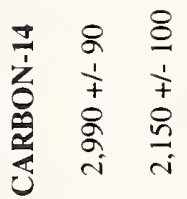

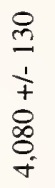

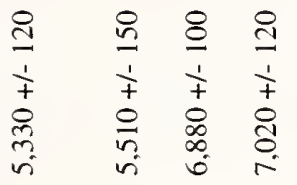

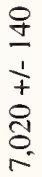

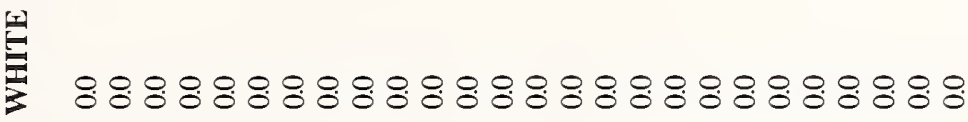

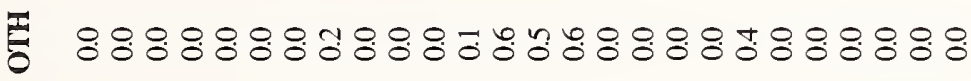

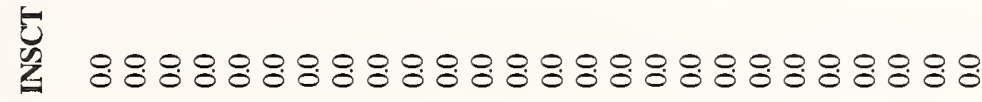

$\sum_{3}^{2}$

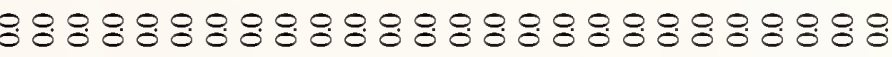

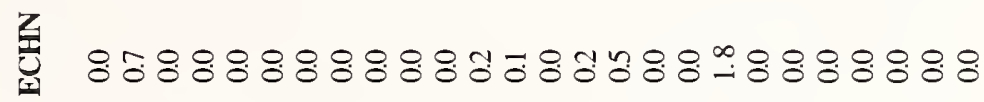

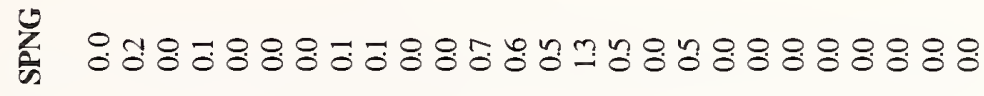

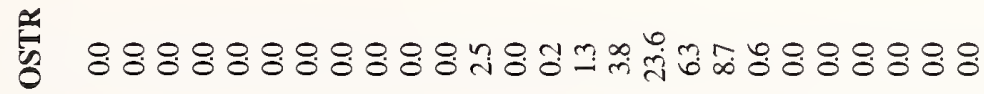

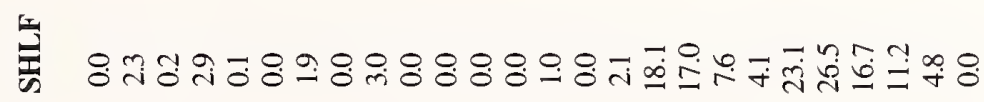

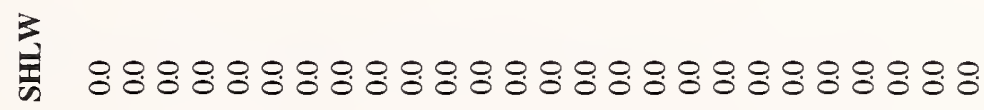

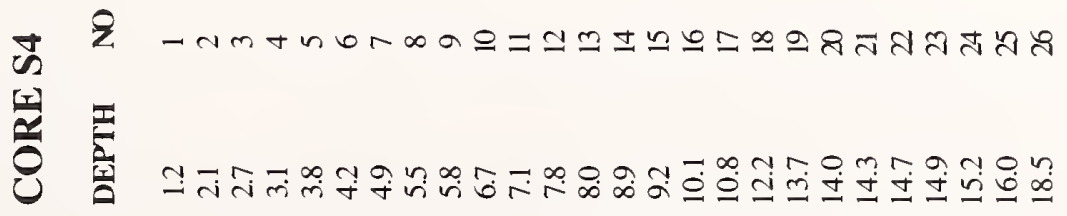


APPENDIX 2.-Continued.

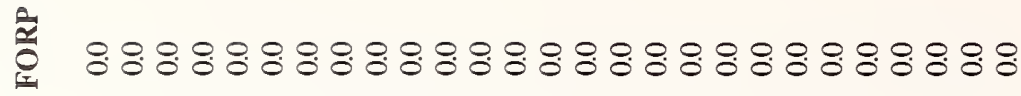

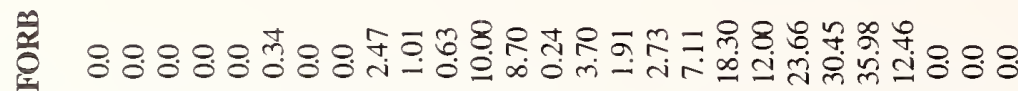

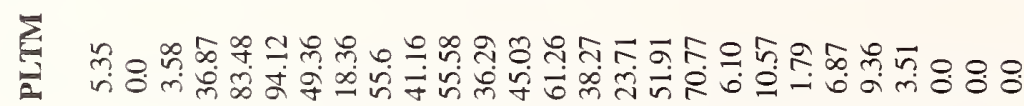

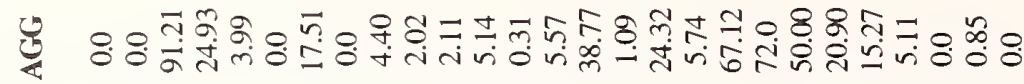

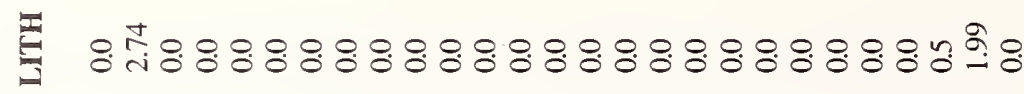

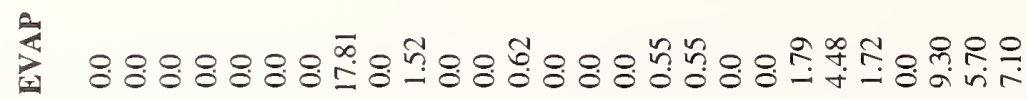

啇

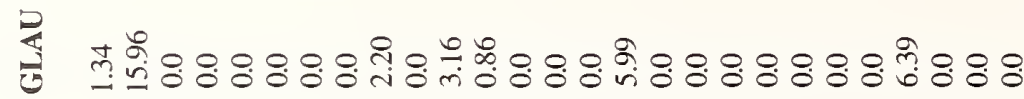

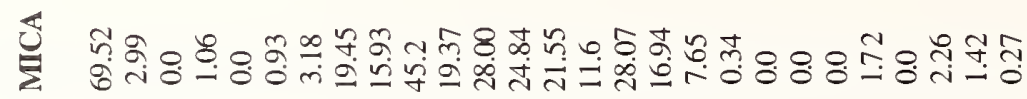

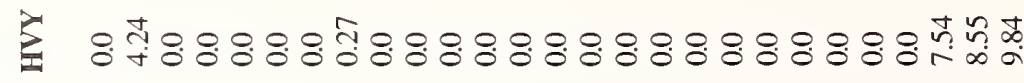

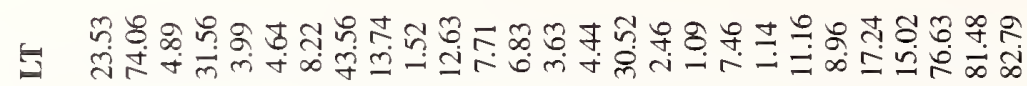

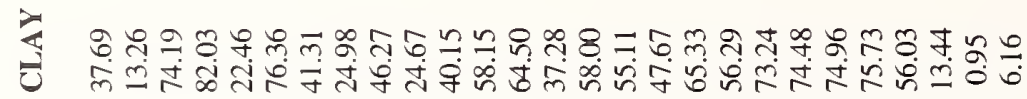

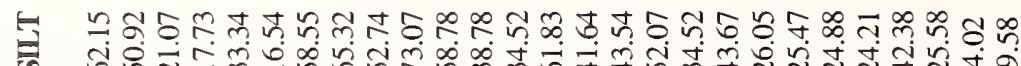

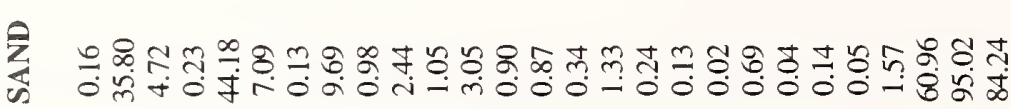

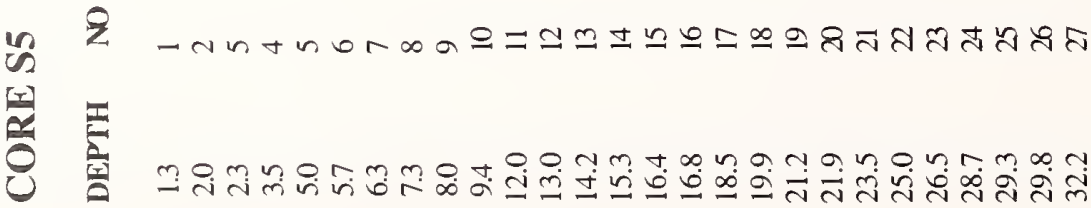


APPENDIX 2--Continued.

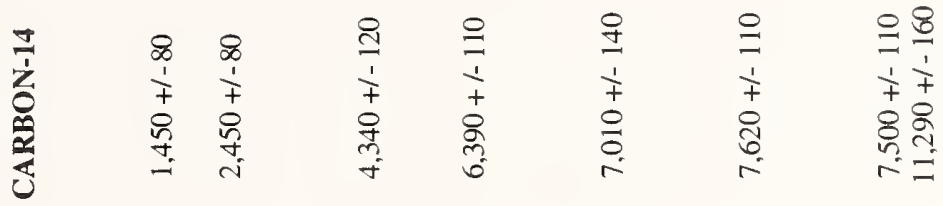

8.8 .8 .8 .8 .8 .8 .8 .8 .8 .8 .8 .8 .8 .8 .8

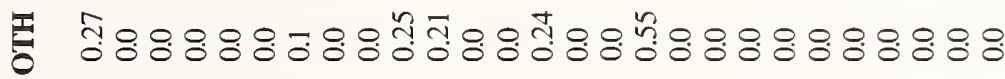

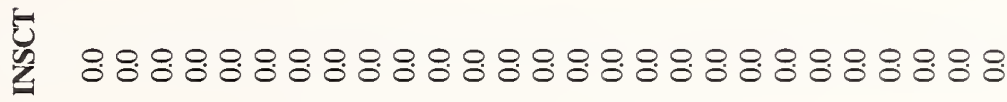

:

خ

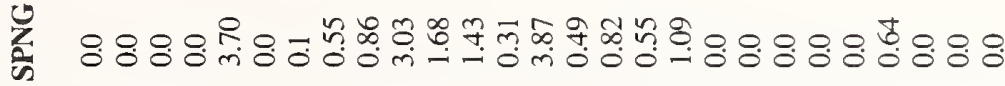

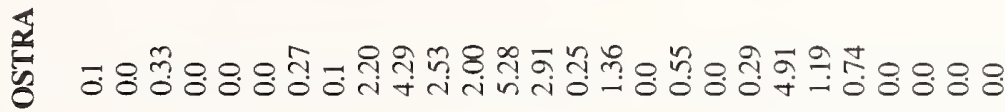

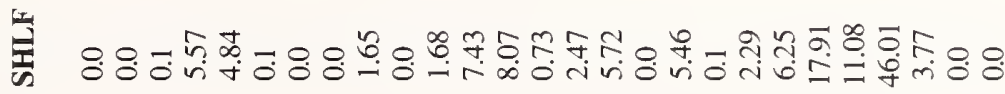

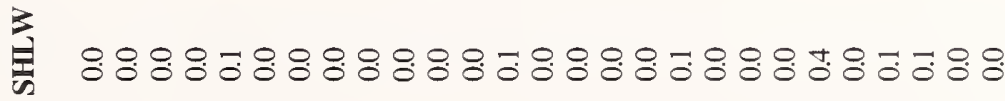

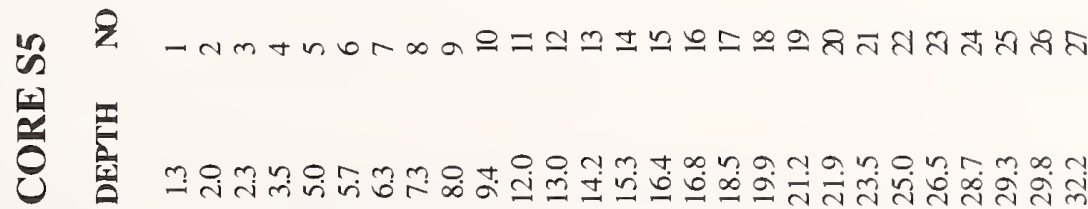


APPENDIX 2.-Continued.

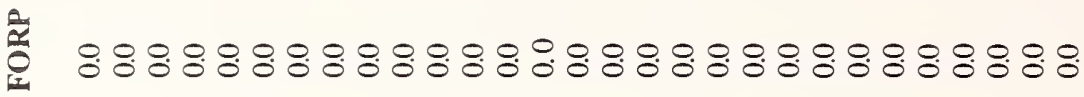

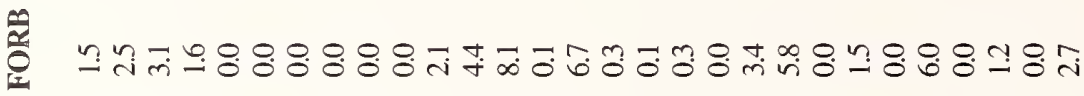

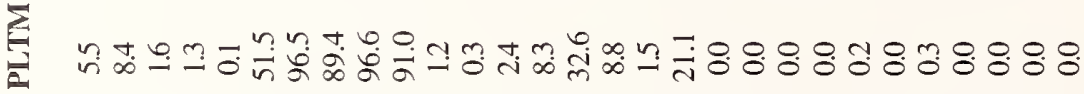

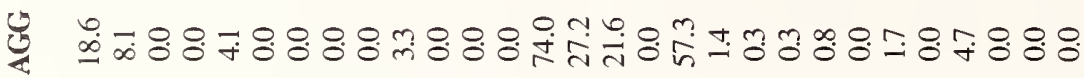

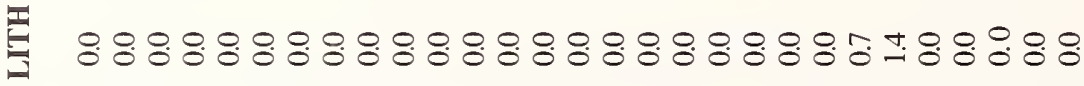

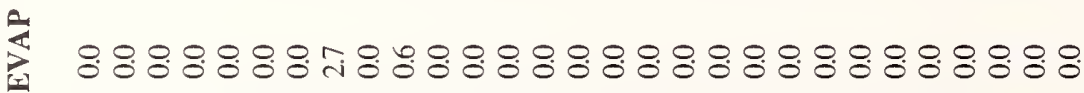

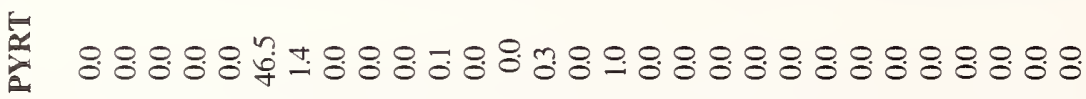

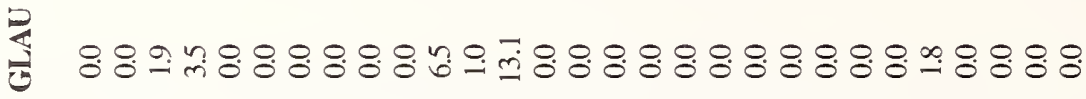
选

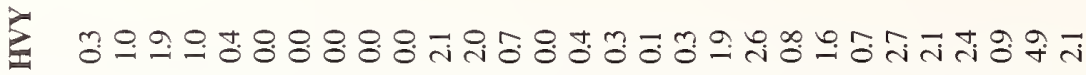

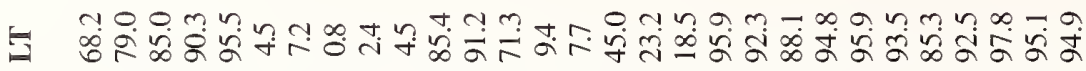

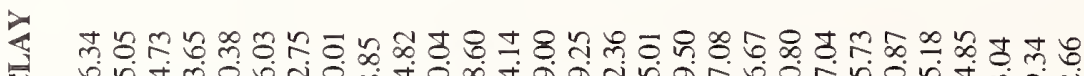

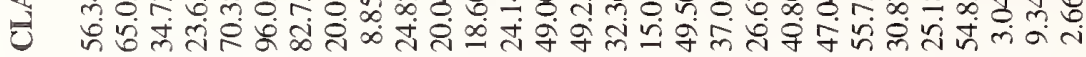

莫

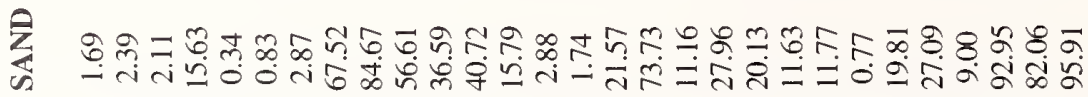

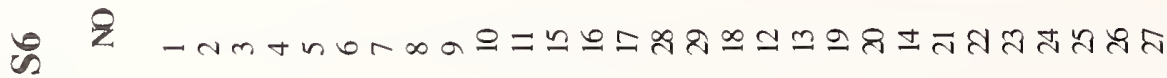


APPENDIX 2.-Continued.

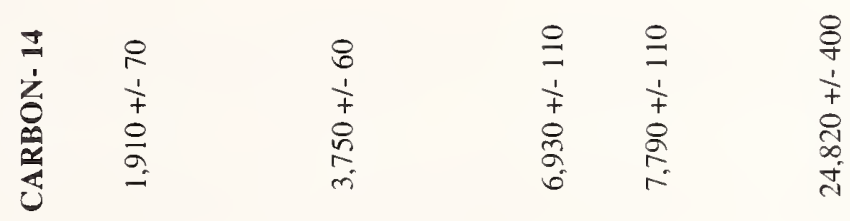

$8.8: 8.8 .8 .8 .8 .8 .8 .8 .8 .8 .8 .8 .8$

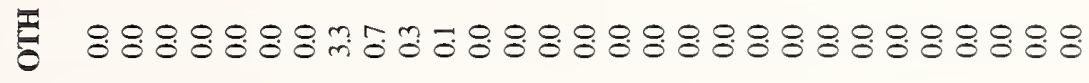

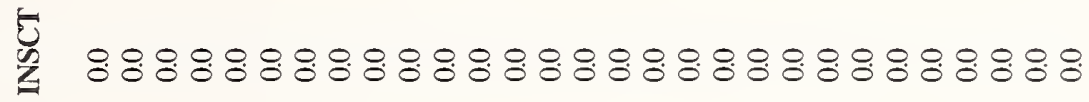

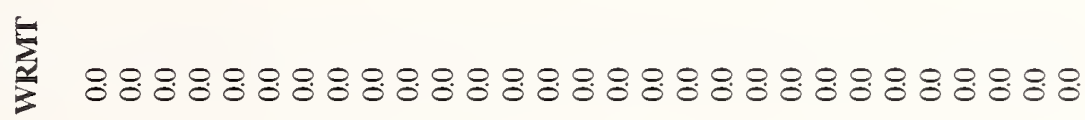

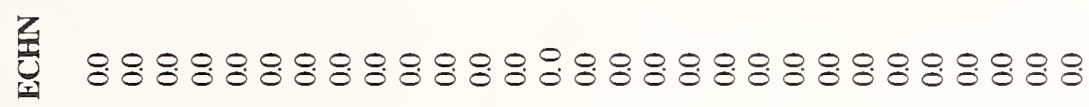

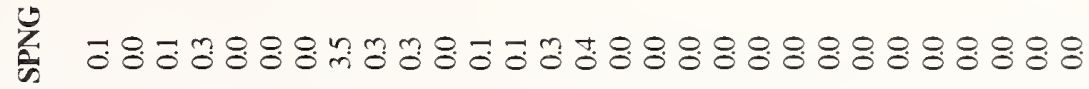

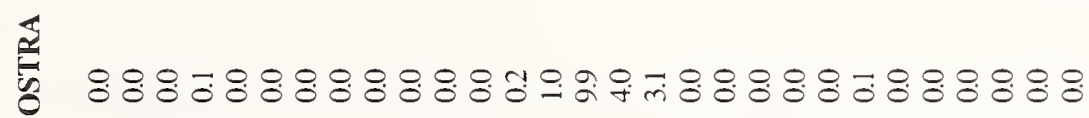

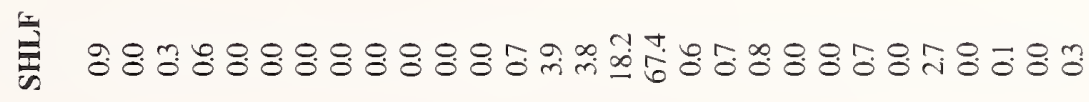

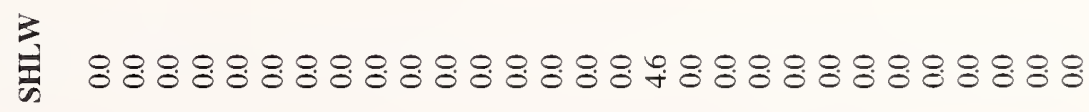

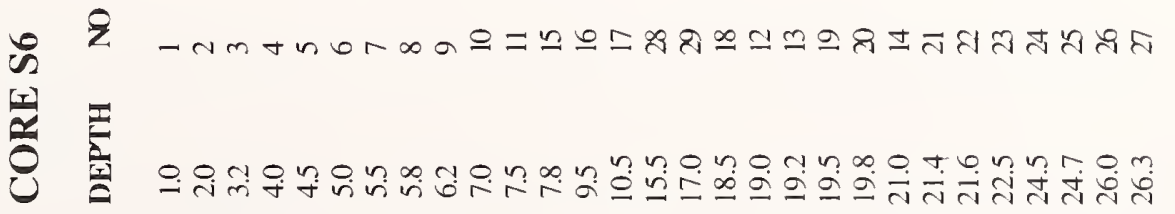


APPENDIX 2,-Continued.

$\stackrel{ }{2}$

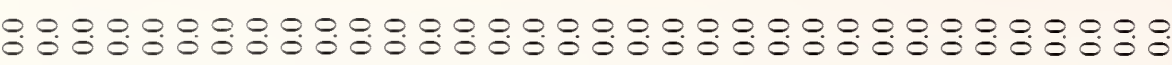

$\underset{1}{0}$

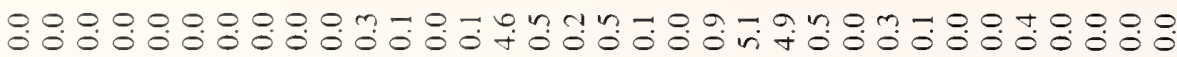

$\sum$

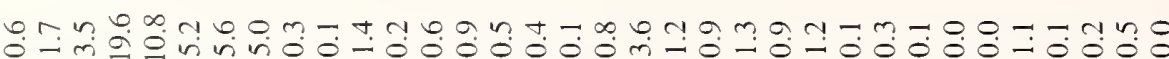

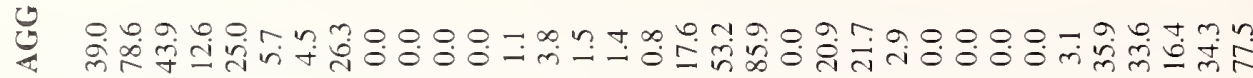

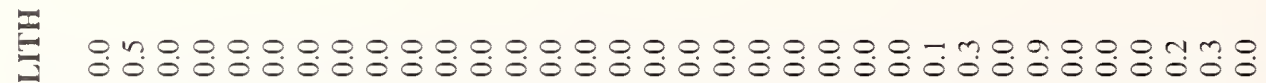
2 i

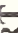

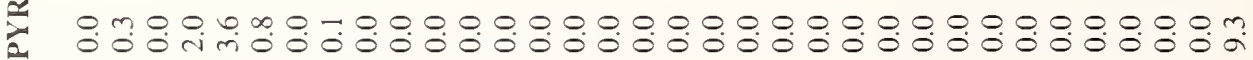

ए 五

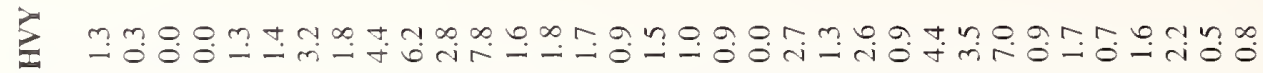

G

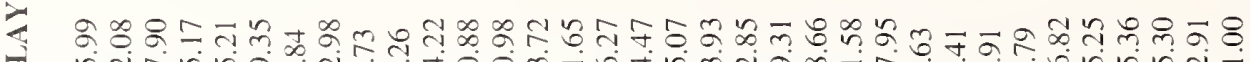

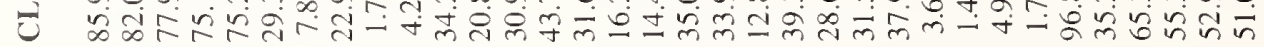

" ज

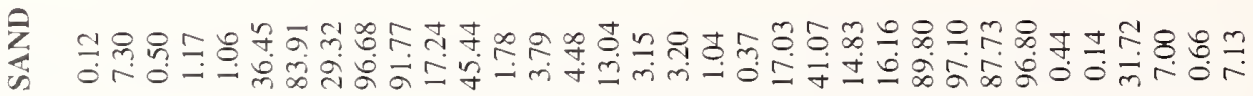

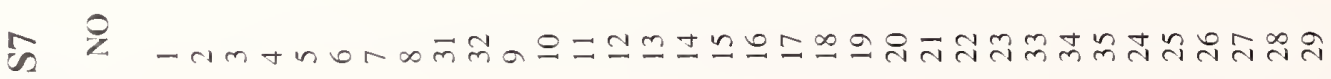


APPENDIX 2.-Continued.

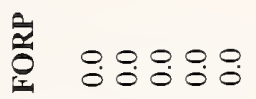

을 0.0980

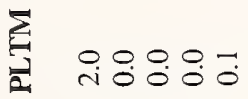

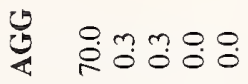

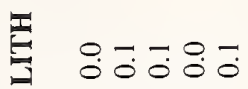

椊

荧 809800

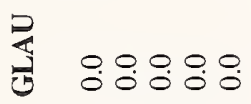

夰

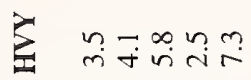

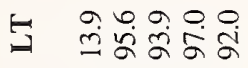

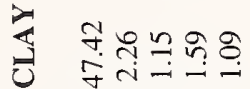

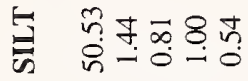

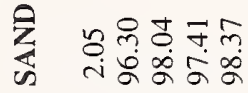

is ₹ 유에에

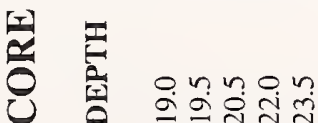




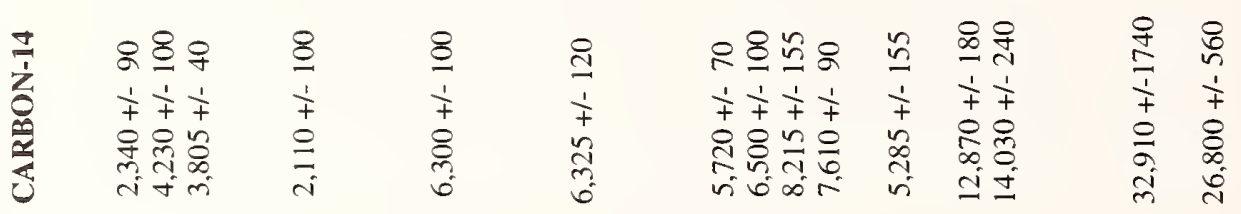

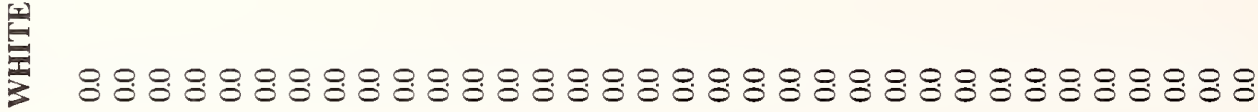

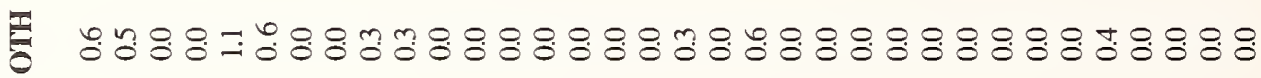

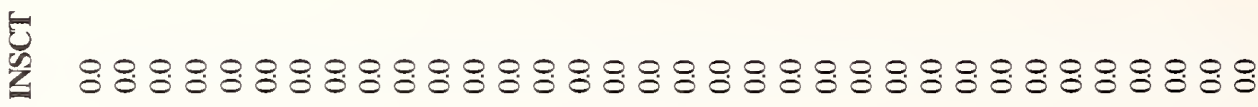

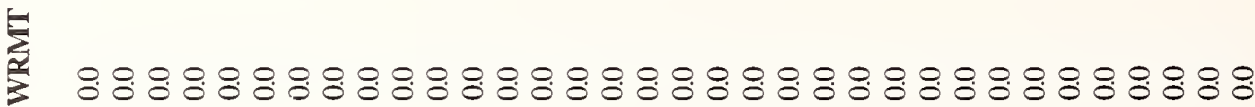

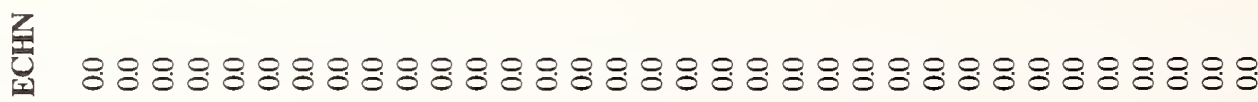

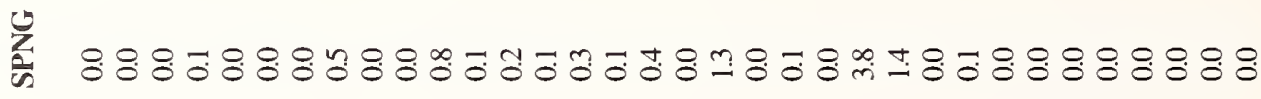

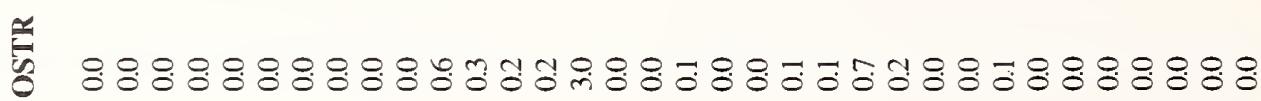

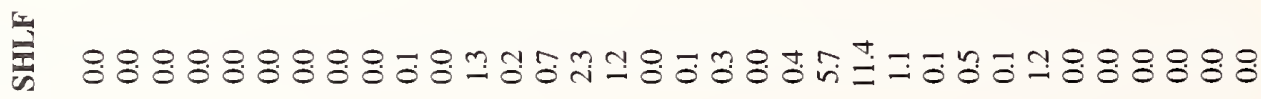

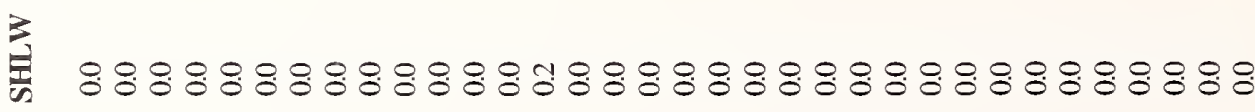

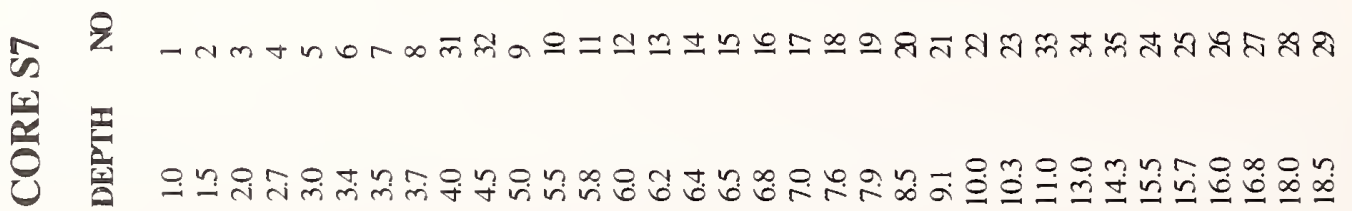


APPENDIX 2.-Continued.

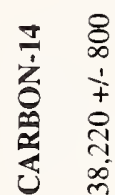

$8: 8: 8$

픙 $n 8888$

ㄴ.

88888

屏 $8888 \%$

言 $88: 8: 8$

蒾 88888

宸 88888

意 88888

is Z

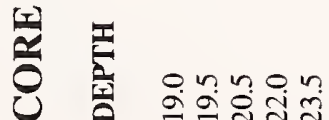


APPENDIX 2.--Continued.

产

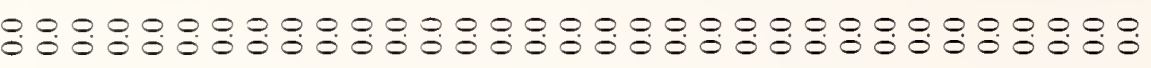

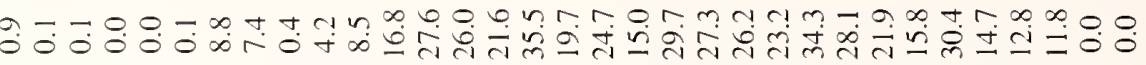

$\underset{\mathrm{E}}{\mathrm{E}}$

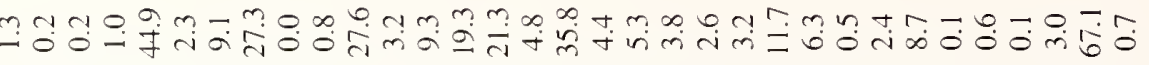

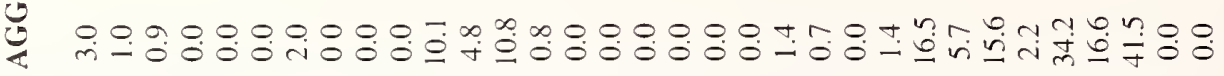

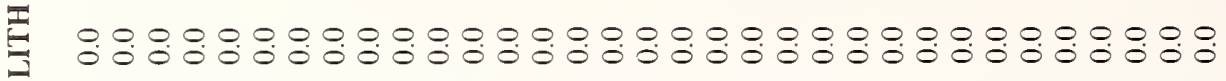

i

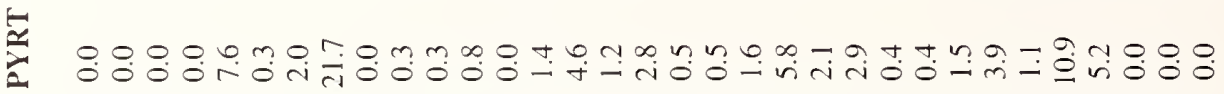

苞

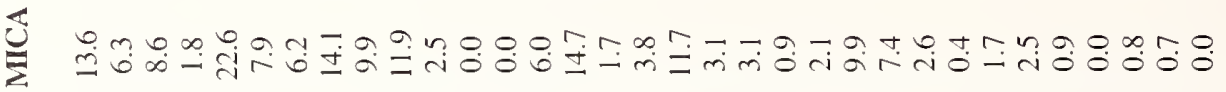

¿

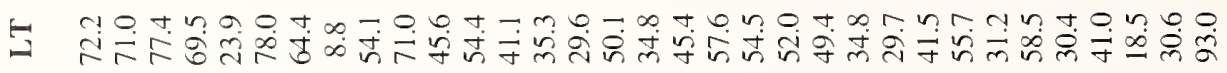

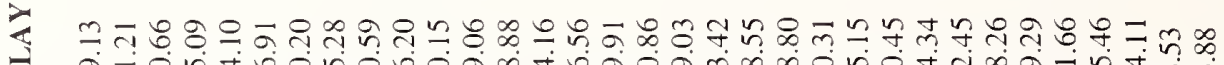

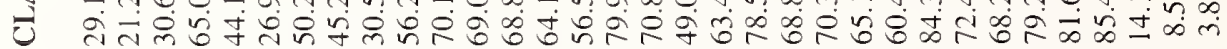

H

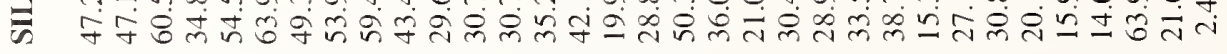

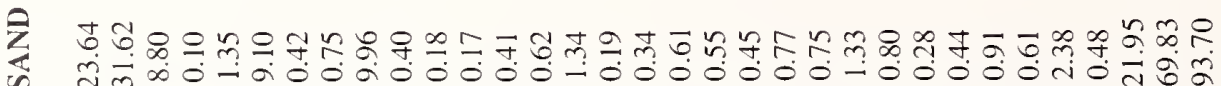

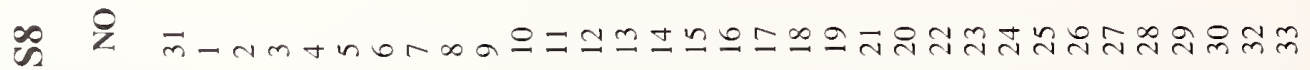

눙 혼

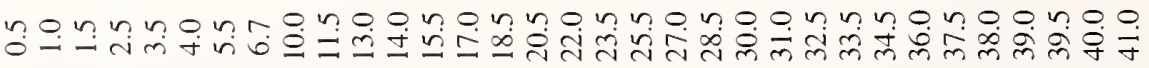


APPENDIX 2.-Continued.

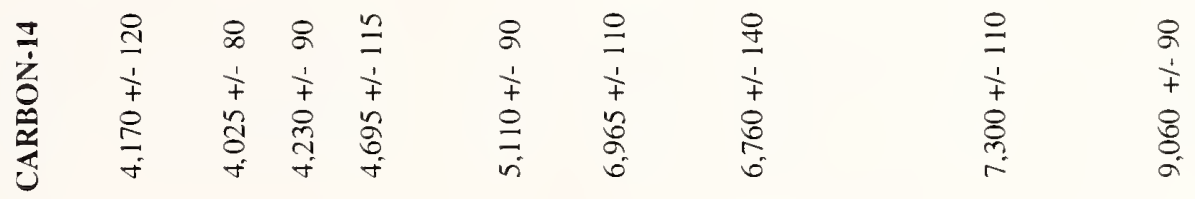

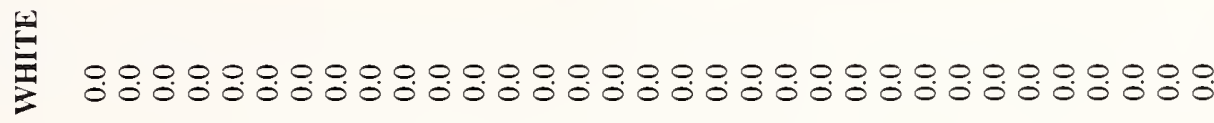

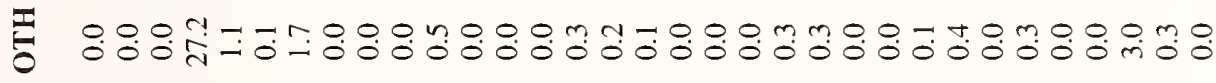

䒘

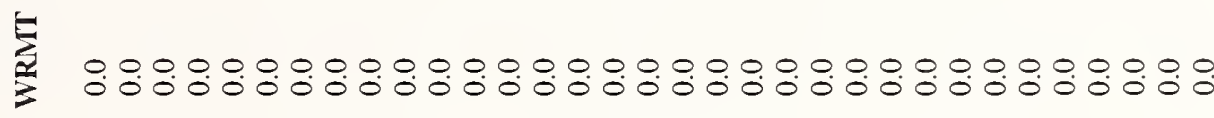

苜

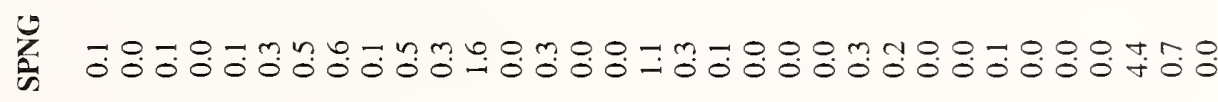

若

崖

药

艎

造 
APPENDIX 2.-Continued.

: $: 8: 8: 8: 8: 8: 8: 8: 8: 8: 8$

: $: 8: 8: 8: 8: 8: 8: 8: 8: 8:$

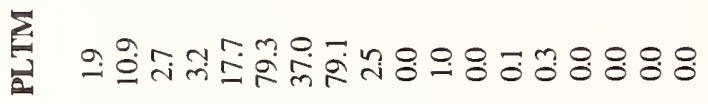

莓

T.

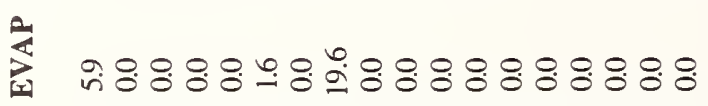

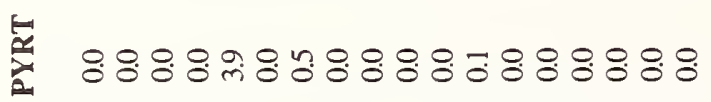

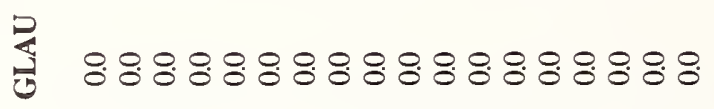

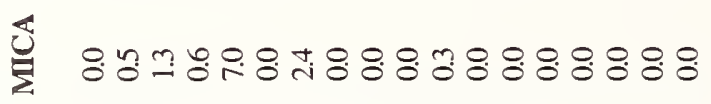

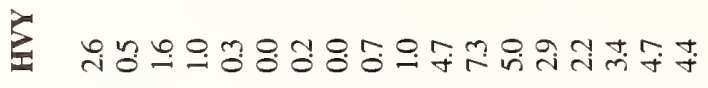

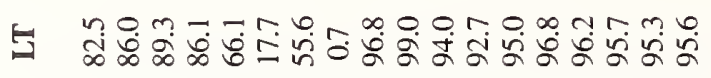

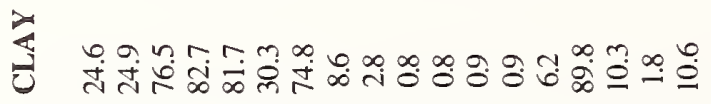

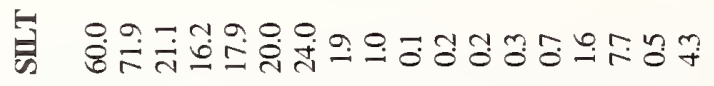

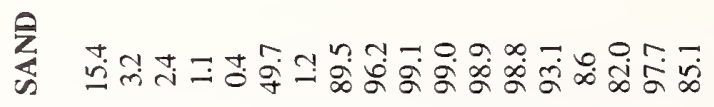

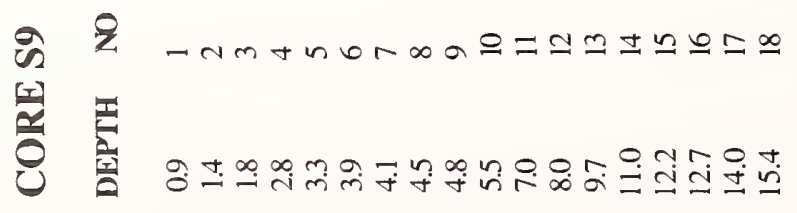


APPENDIX 2.-Continued.

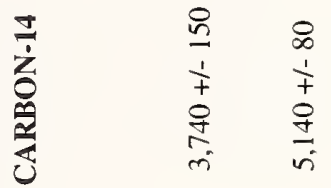

$8: 88: 88: 88: 8: 58.88$

등

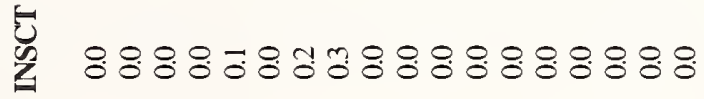

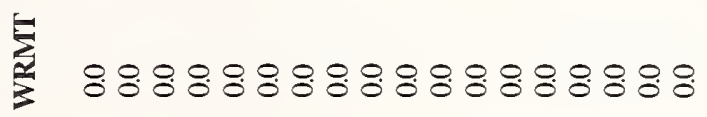

خ

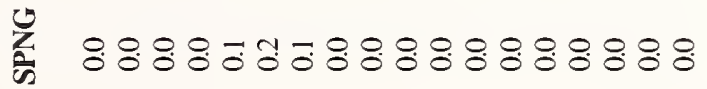

\%

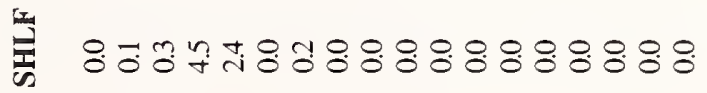

基 $8: 8: 88: 88: 88: 88: 88$

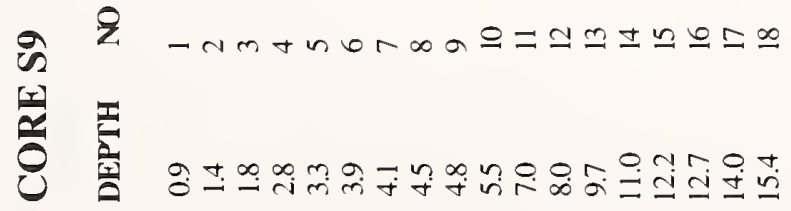


APPENDIX 2.-Continued.

产 $8: 8: 8: 8: 8: 8: 8: 8: 8$

管 8.88 .88 .88 .88 .88 .88

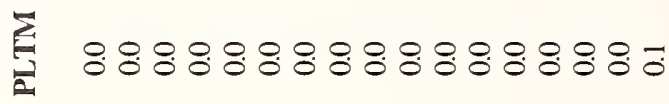

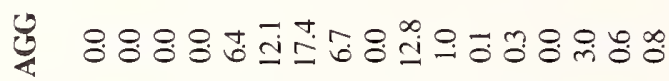

产

位 $8: 8: 8: 8: 8: 8: 8: 8: \circ$

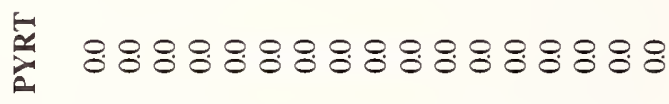

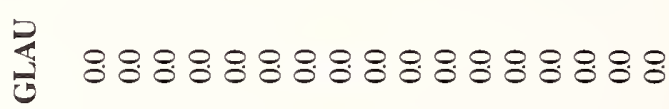

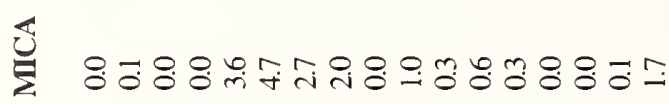

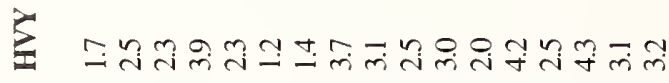

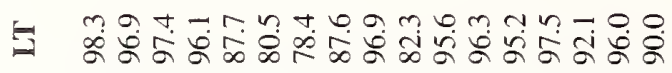

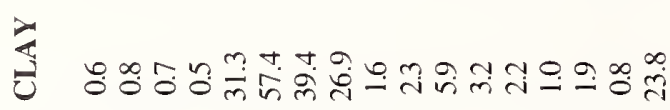

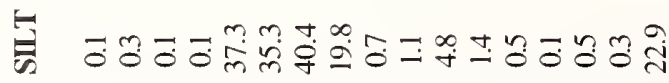

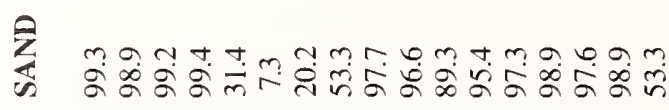

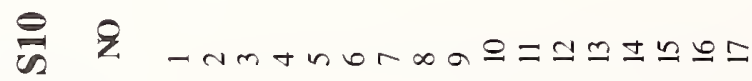

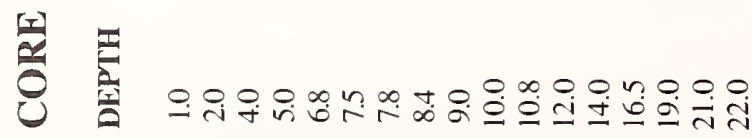


APPENDIX 2.-Continued.

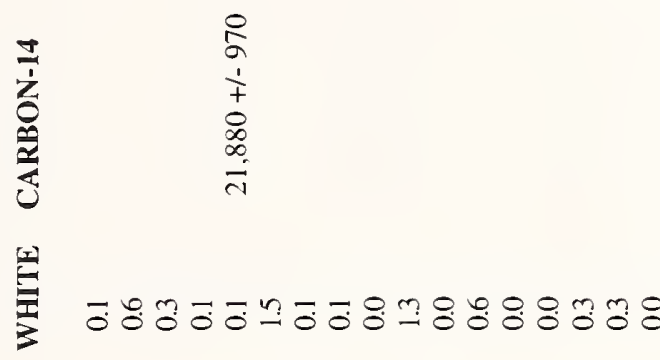

F 88888888888888888

范 8888888888888888.

$8: 88888888888888:$

慮 888888888888888888

茪 88888888888888888

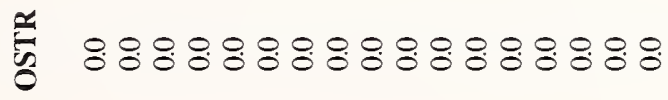

跑 88888888888888888

言 88888888888888888

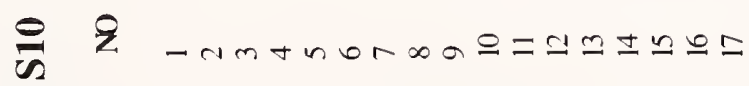

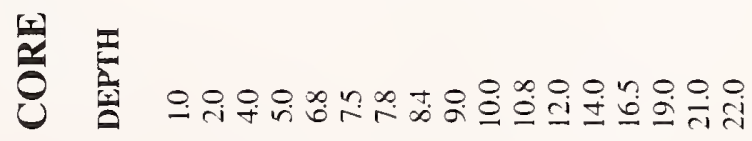


APPENDIX 2.-Continued.

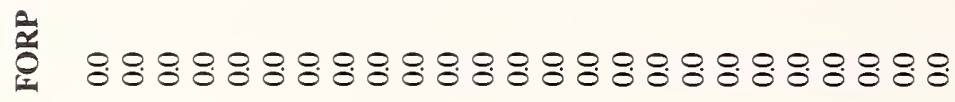

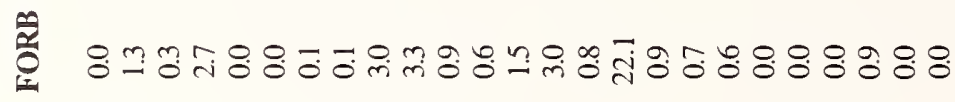

费

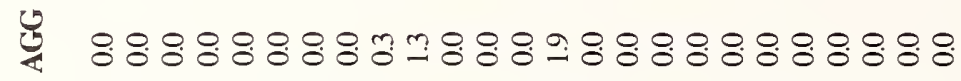

E

育

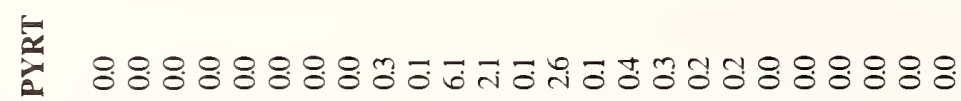

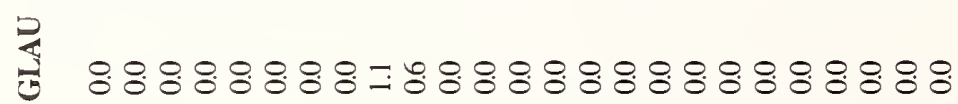

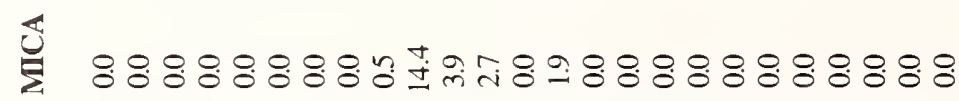

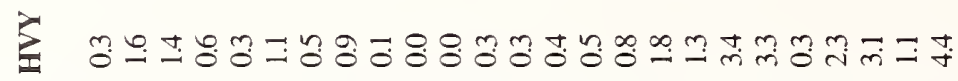

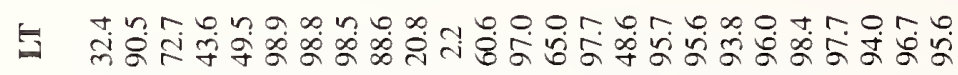

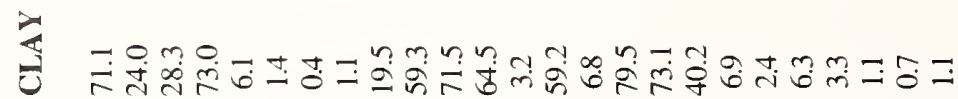

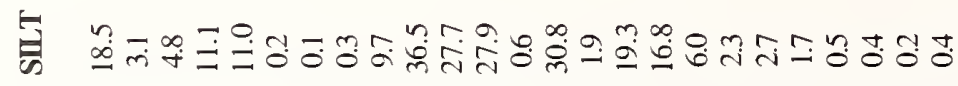

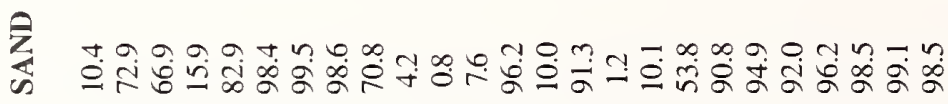

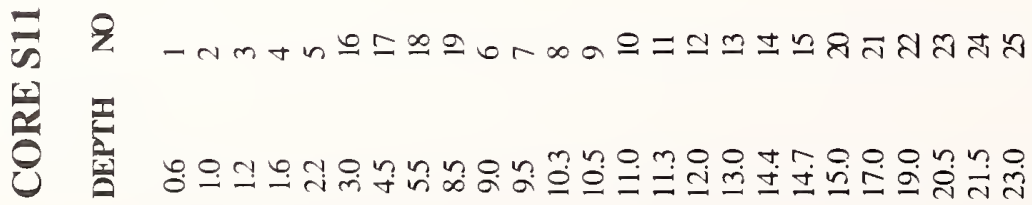


APPENDIX 2.-Continued.

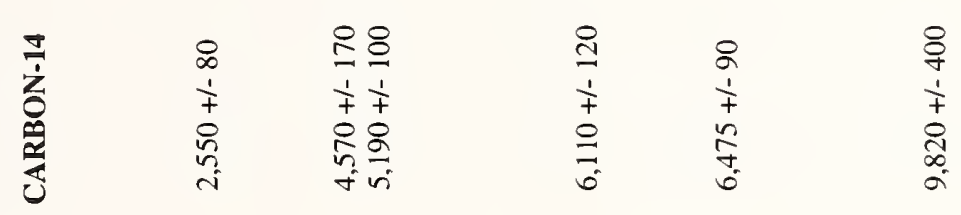

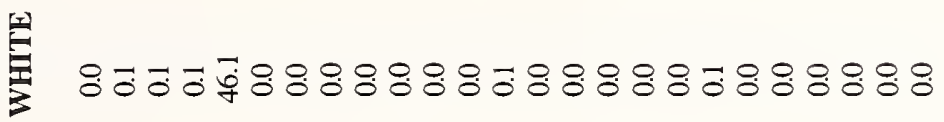

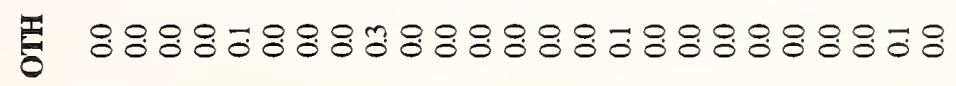

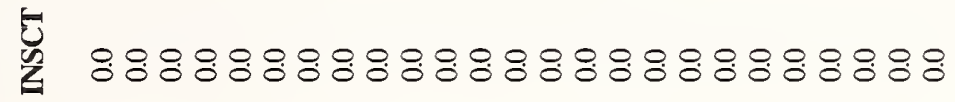

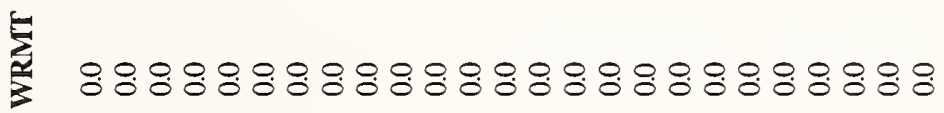

茎

芒

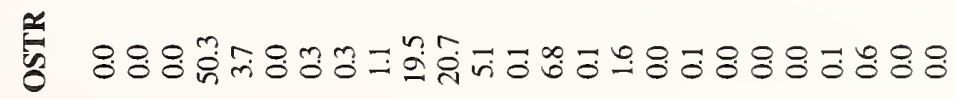

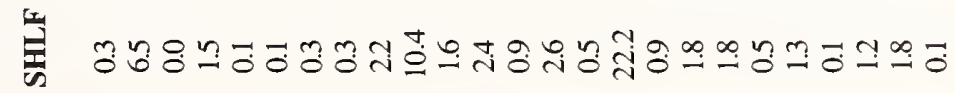

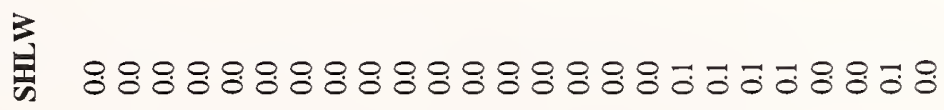

Еี そ -

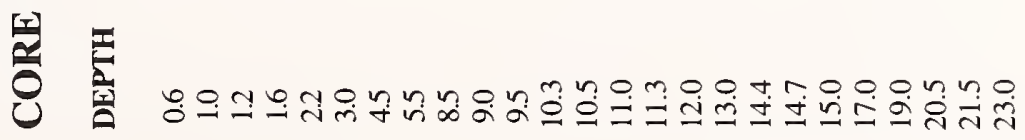


APPENDIX 2.-Continued.

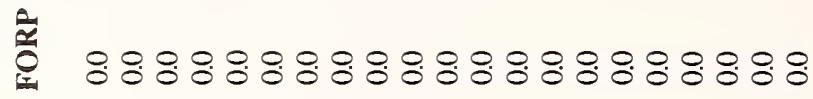

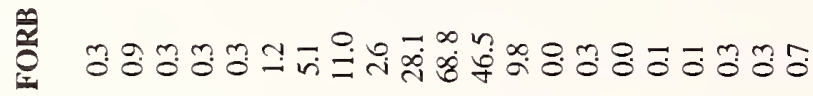

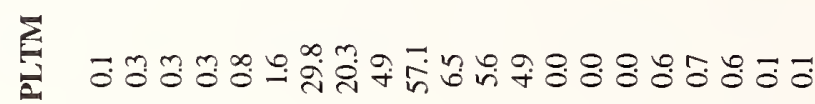

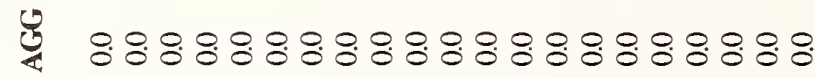

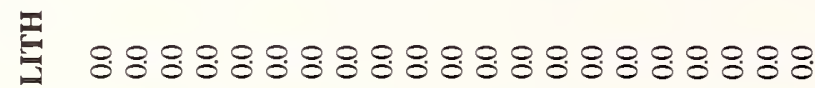

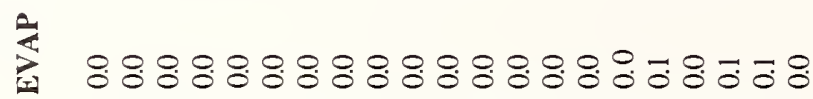

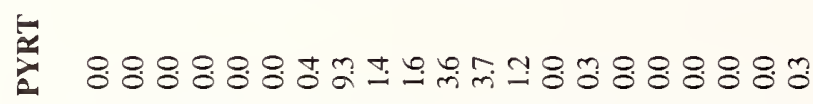

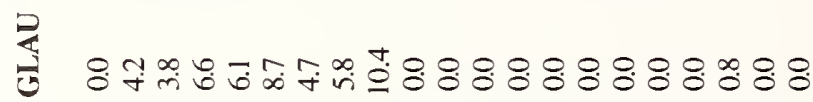

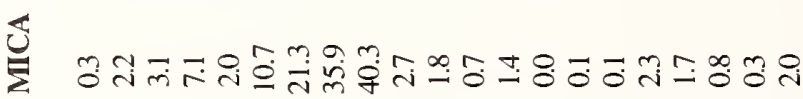

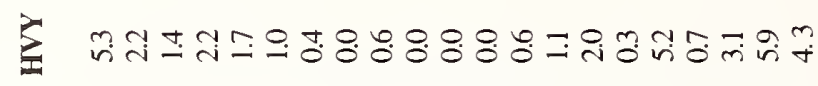

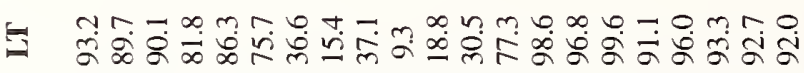

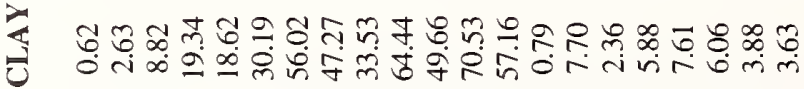

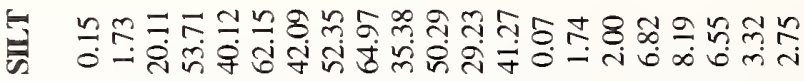

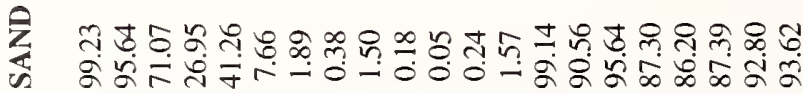

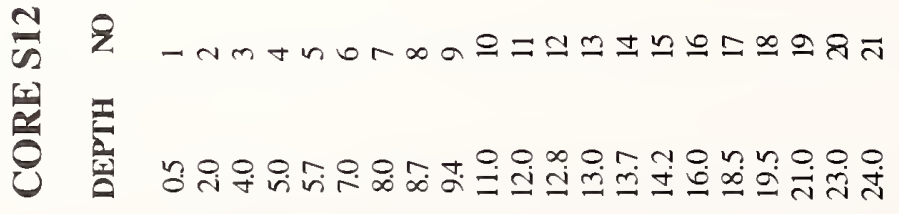


APPENDIX 2.-Continued.

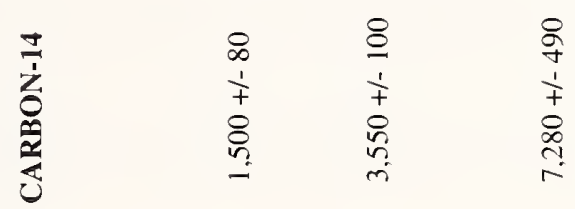

$8.88 .88 .88 .88 .88 .88 .88 .8 \%$

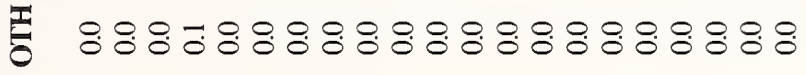

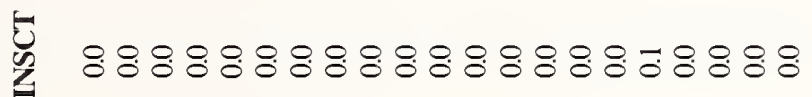

$8.8: 8.8: 8.8: 8: 8: 8: 8: 8: 8.8$

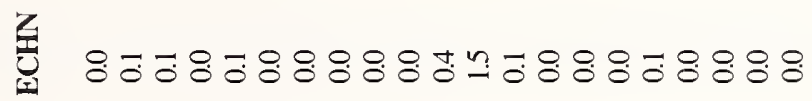

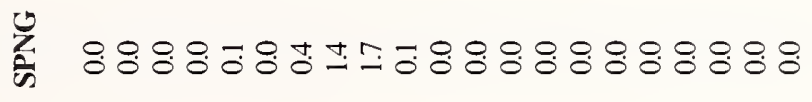

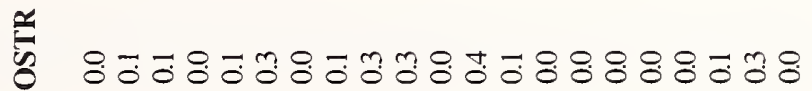

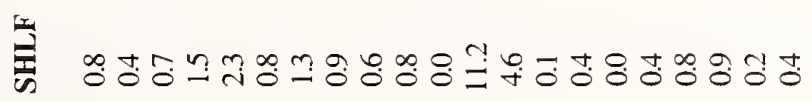

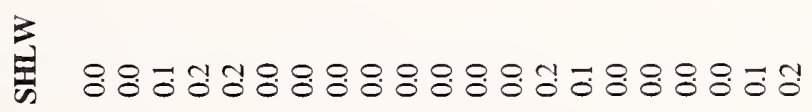

ปี Z -

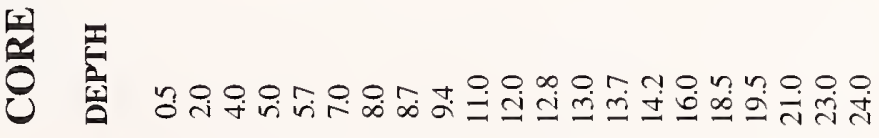


APPENDIX 2.-Continued.

옹

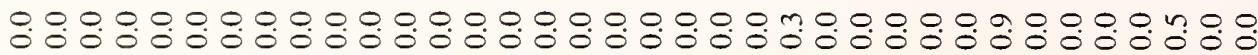

옹

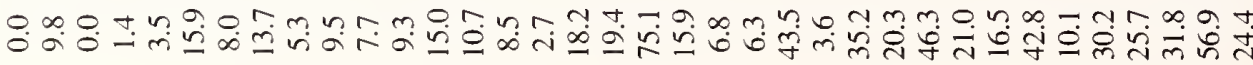

总

y

彗

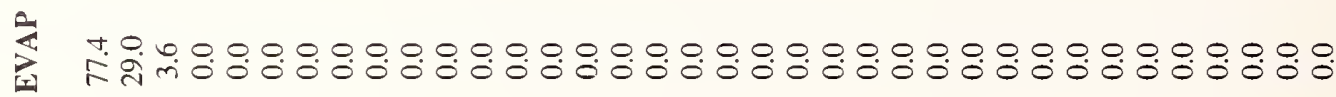

E

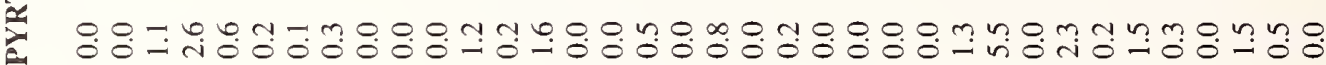

昰

岁

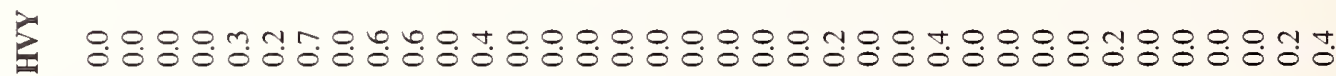

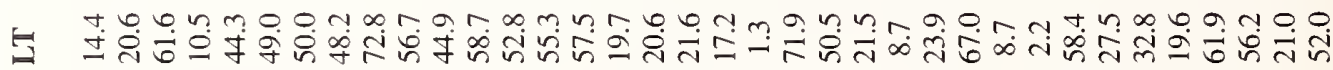

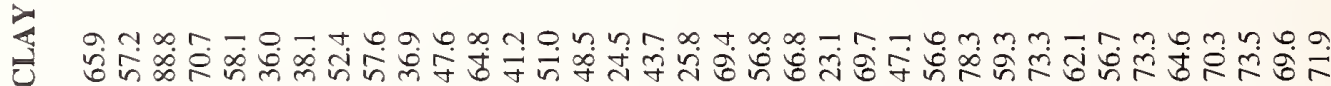

H

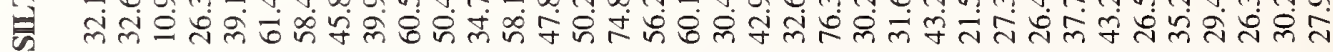

育

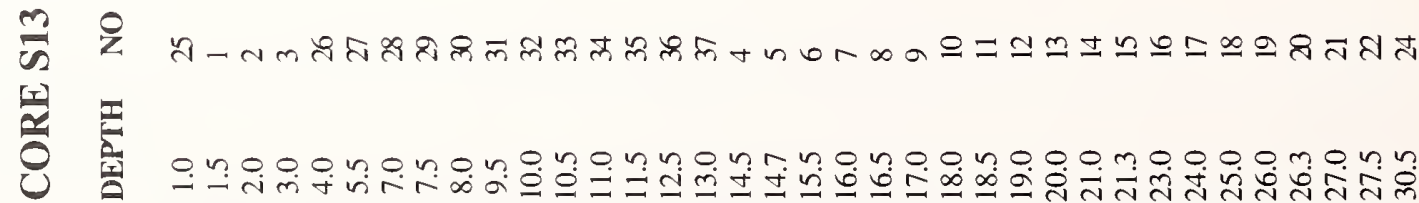


APPENDIX 2.-Continued.

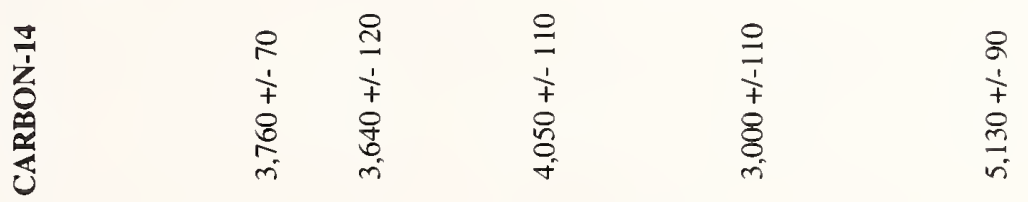

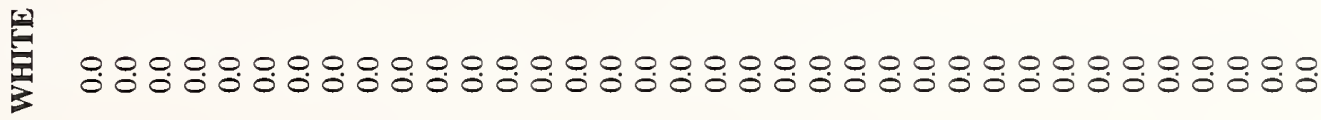

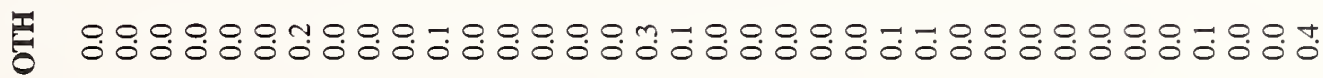

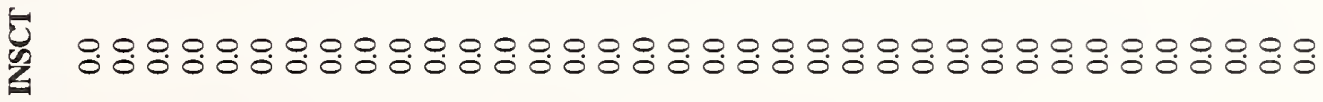

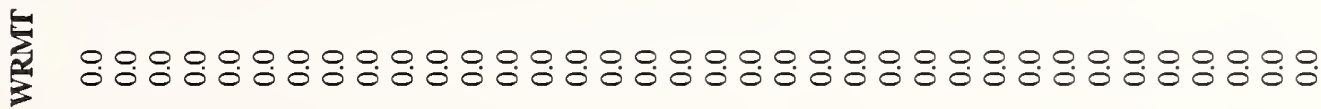

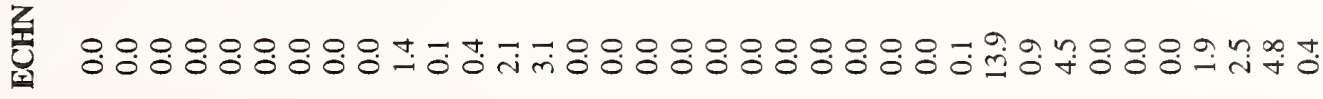

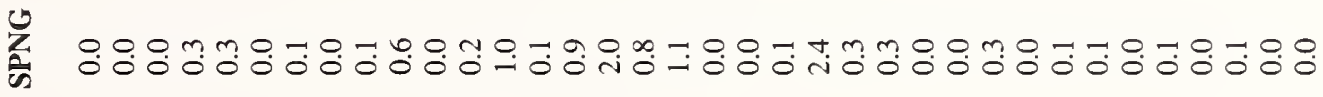

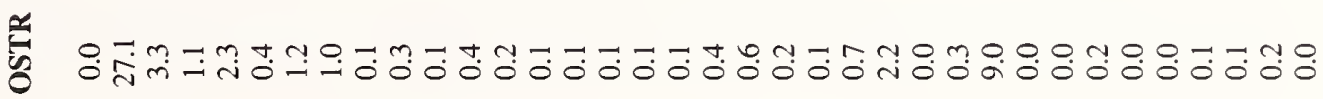

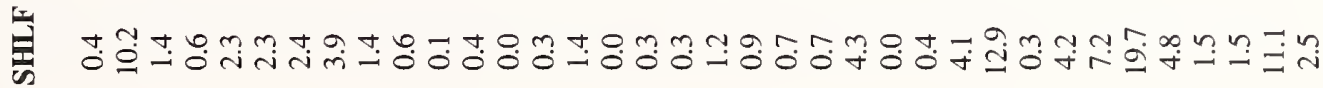

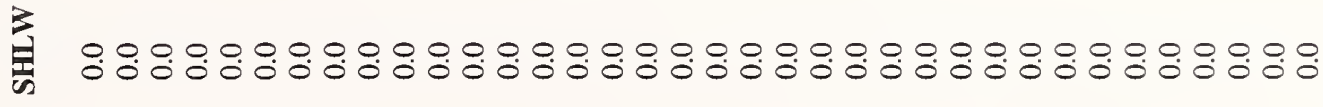

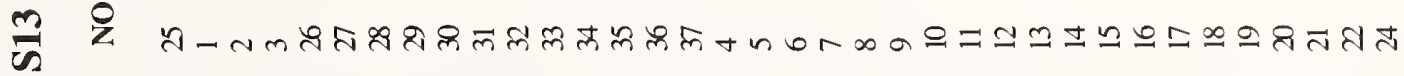

送

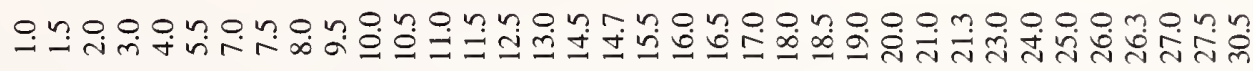


APPENDIX 2,-Continued.

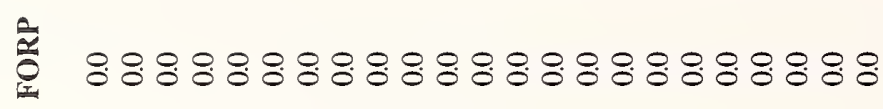

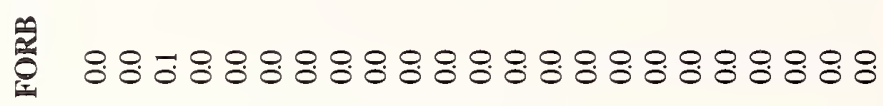

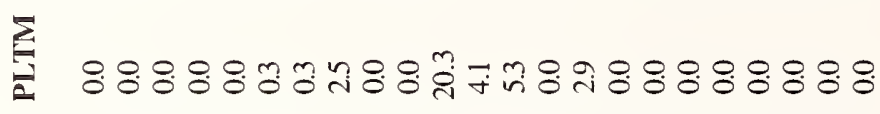

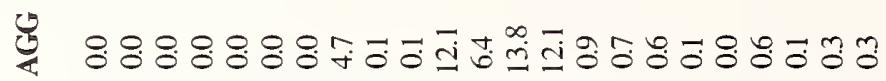

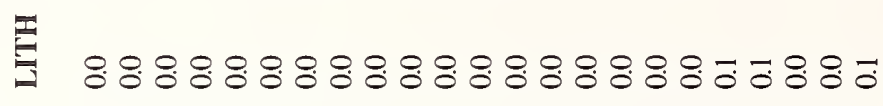

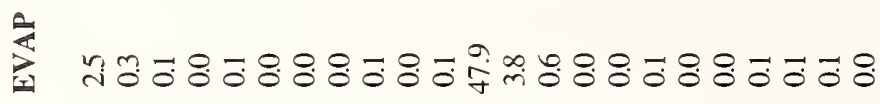

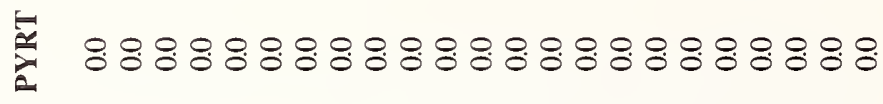

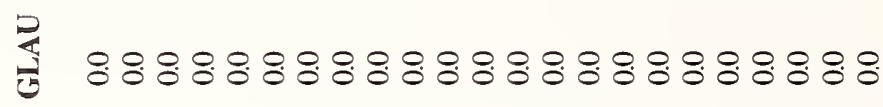

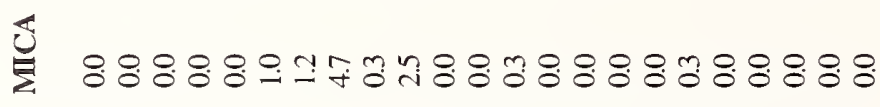

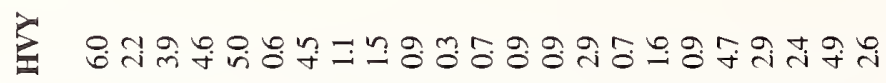

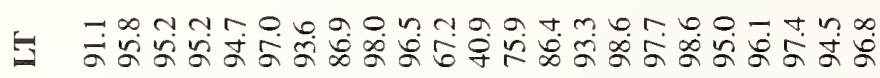

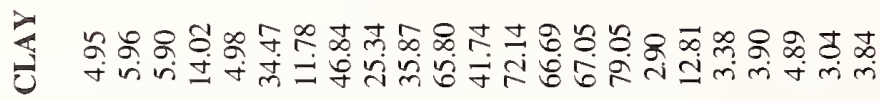

官

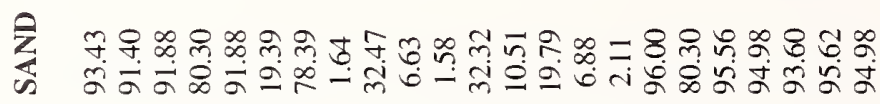

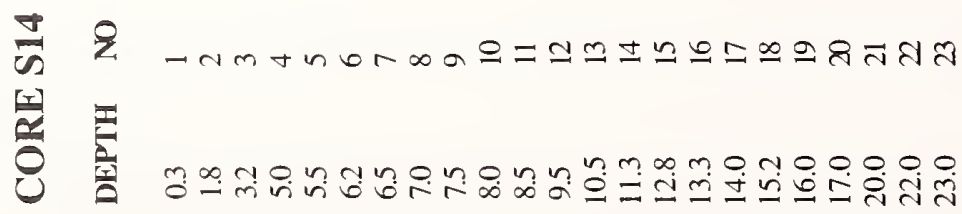


APPENDIX 2.-Continued.
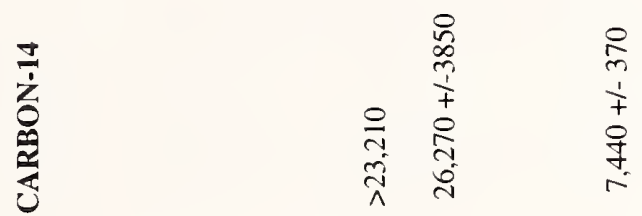

8.

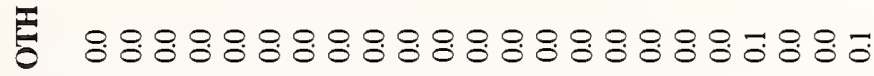

苞 $8: 8: 8: 8: 8: 8: 8: 8: 8: 8: 8: 8:$

$8: 8: 8: 8: 8: 8: 8: 8: 8: 8: 8: 8$

触 $888888: 88: 888: 88: 888888$

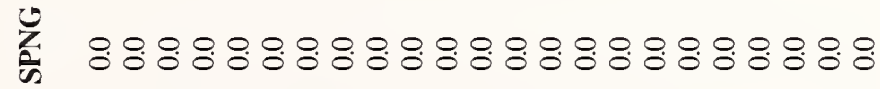

苞 $: 8: 8: 8: 8: 8: 8: 8: 8:: 8: 8: 8: 8$

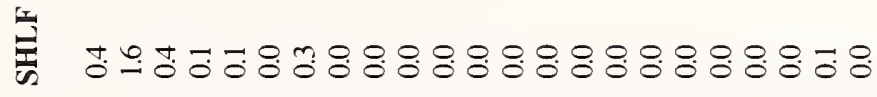

产 $8: 8: 8: 8: 8: 8: 8: 8: 8: 5: 8: 8$

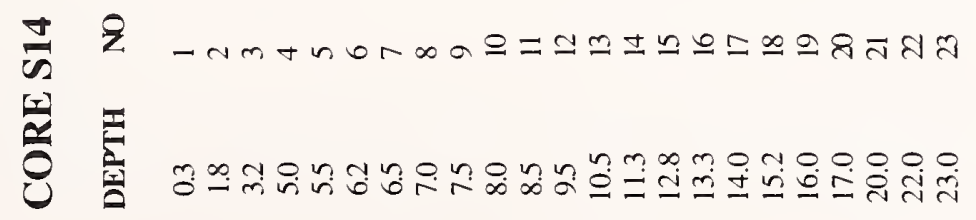


APPENDIX 2.-Continued.

ํㅡㅇ

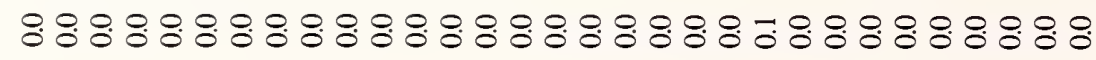

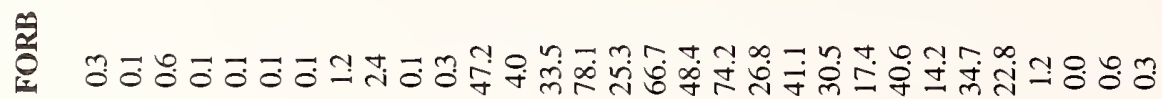

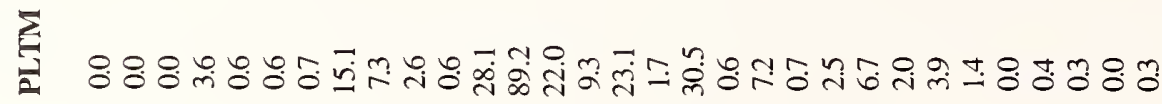

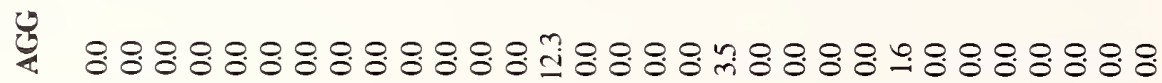

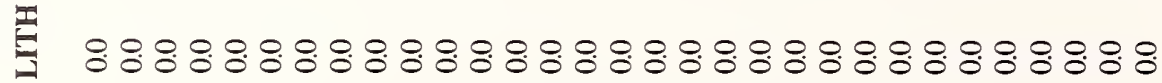

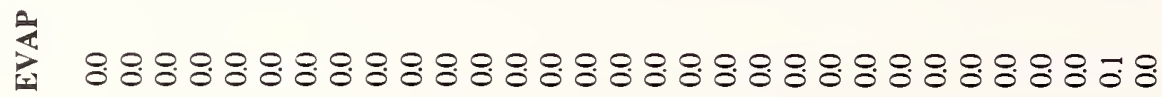

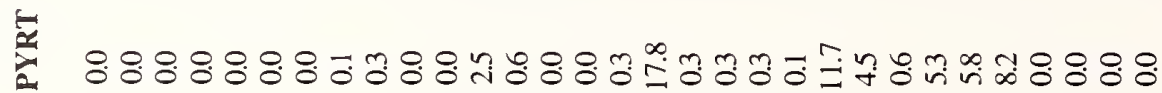

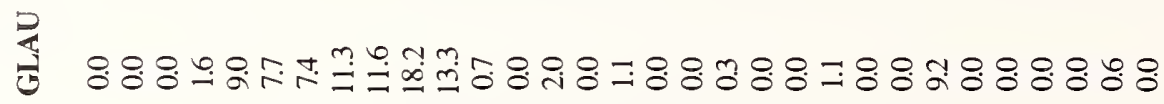

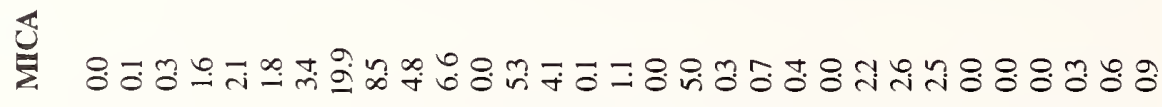

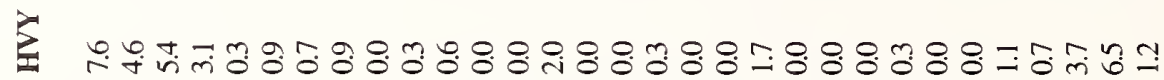

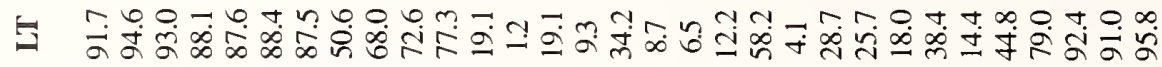

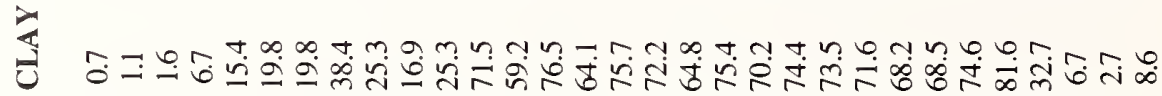

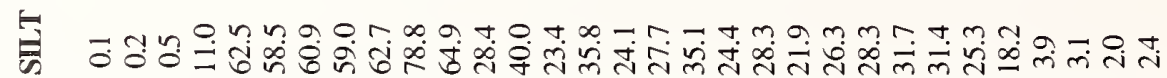

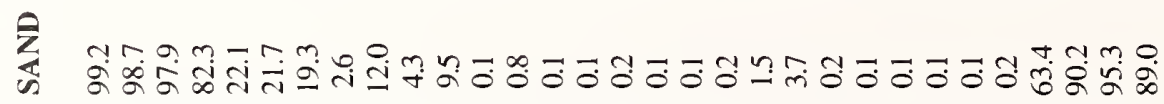

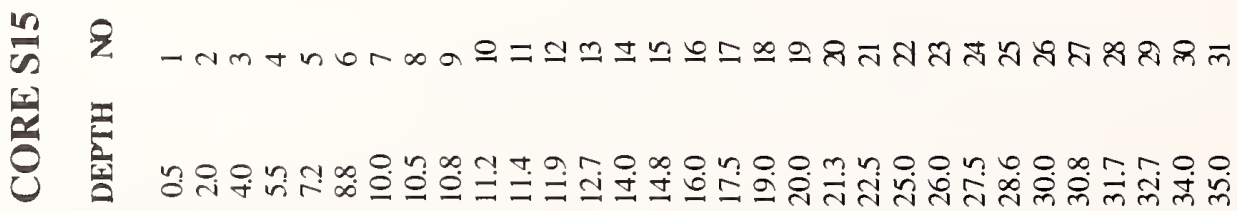


APPENDIX 2.-Continued.

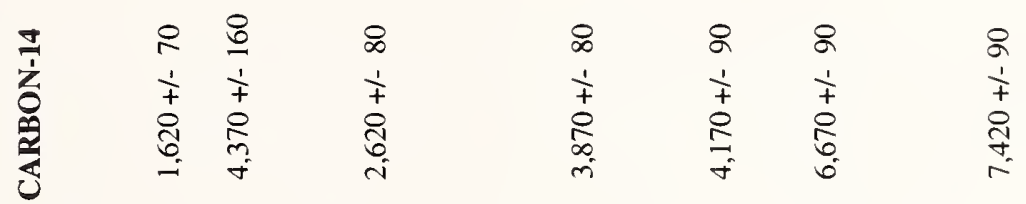

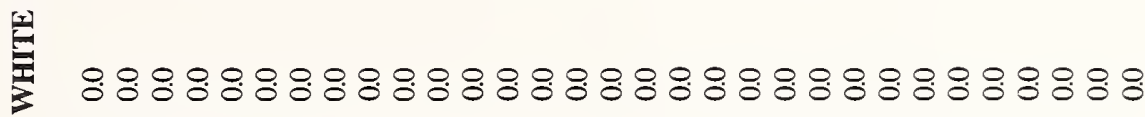

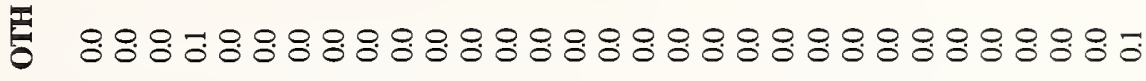

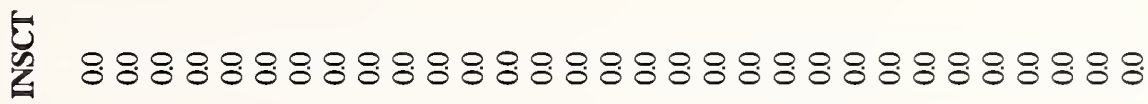

$8: 8: 8: 8: 8: 8: 8: 8: 8: 8: 8: 8: 8: 8: 8: 8$

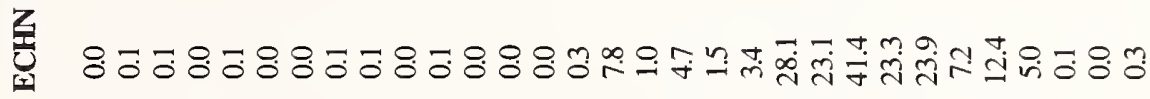

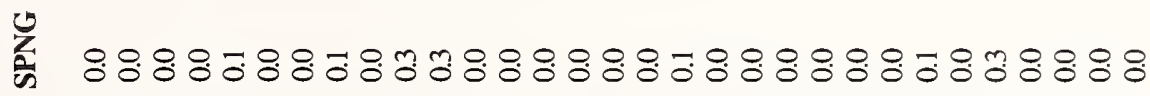

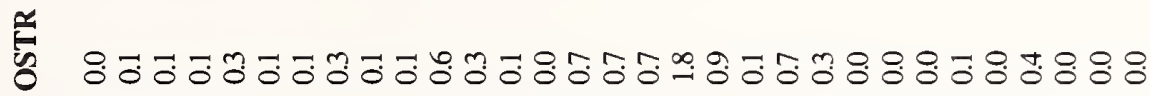

罢

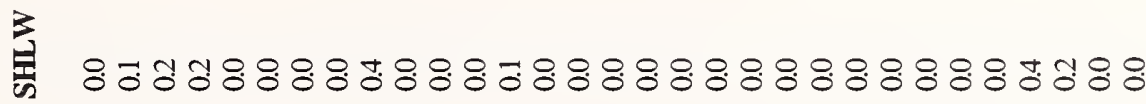

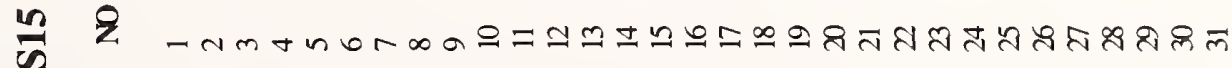

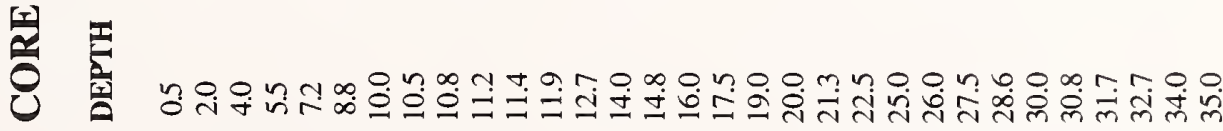


APPENDIX 2.-Continued.

응

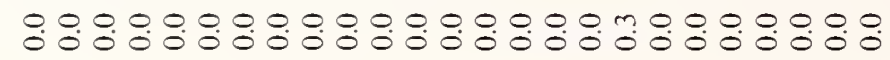

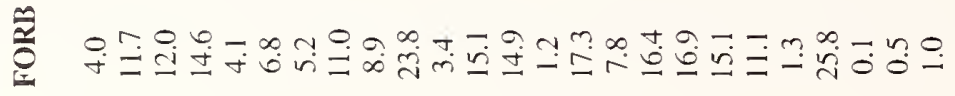

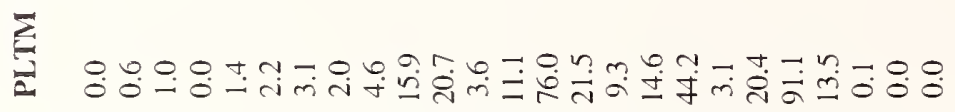

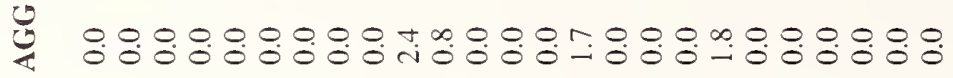

I

L

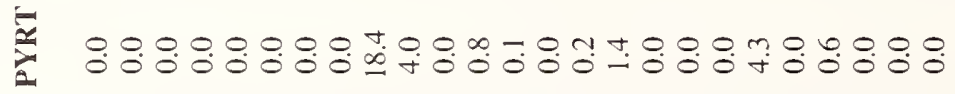

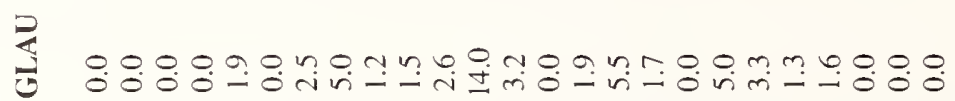

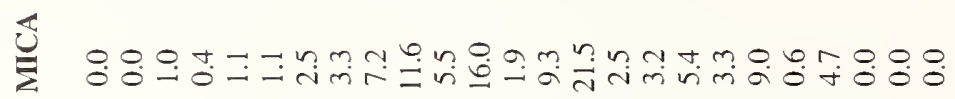

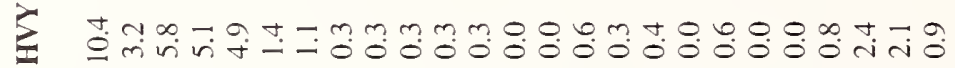

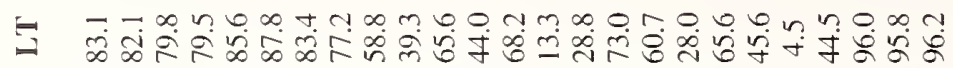

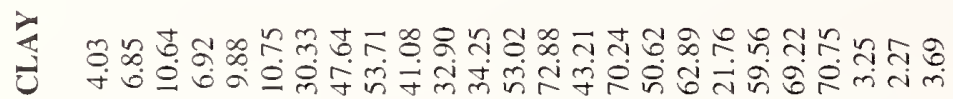

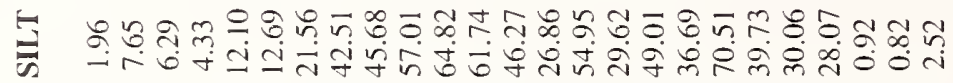

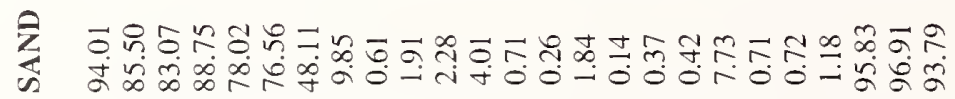

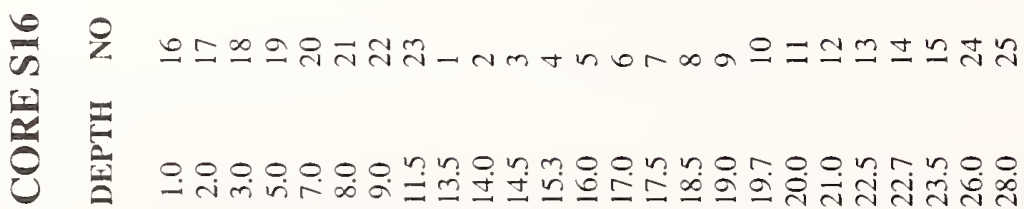


APPENDIX 2.-Continued.

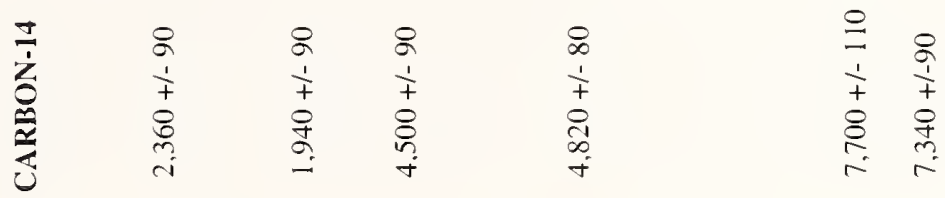

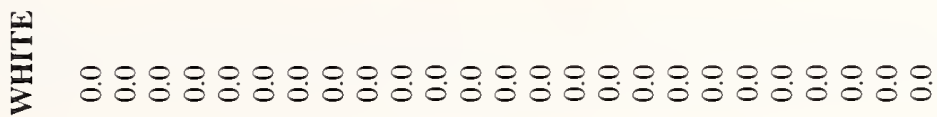

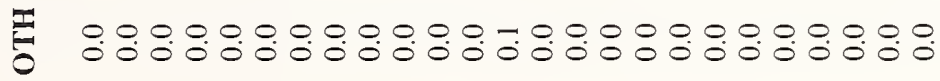

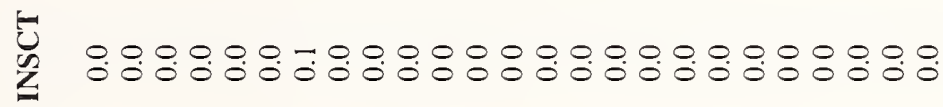

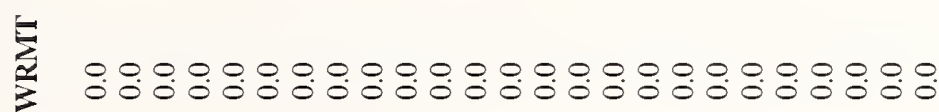

岁

䓪

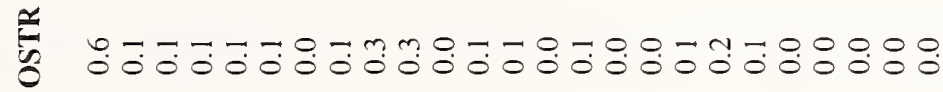

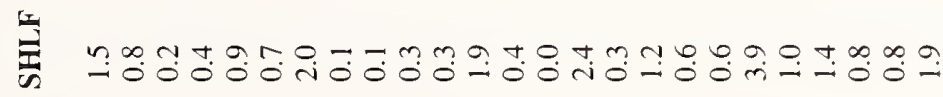

考

르

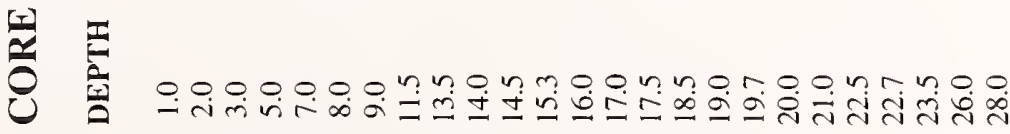


APPENDIX 2.-Continued.

产

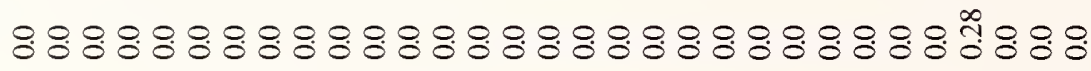

鱼

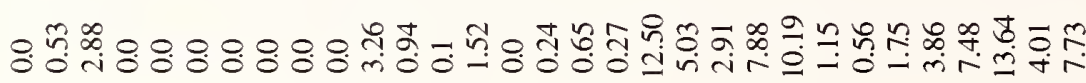

悬

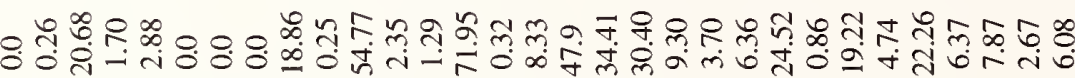

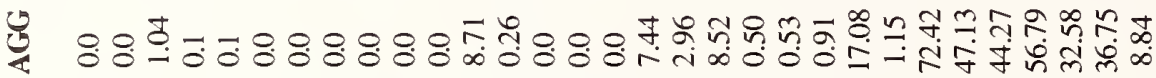

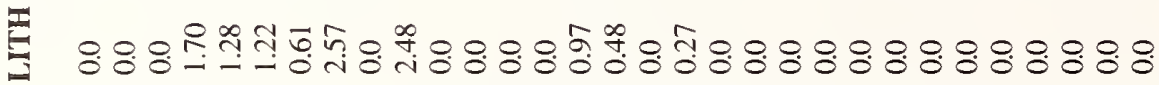

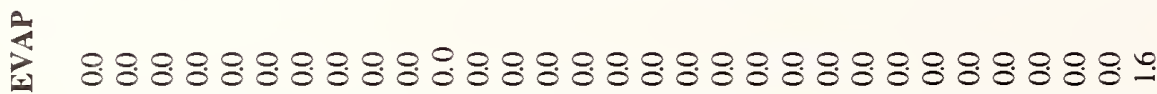

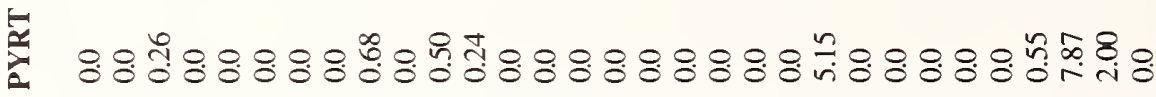

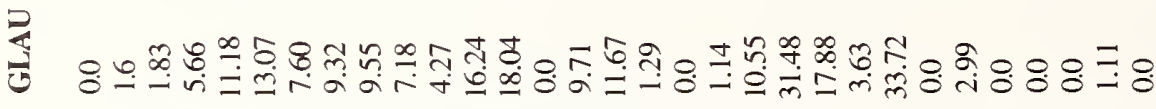
选

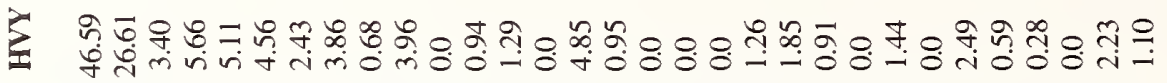

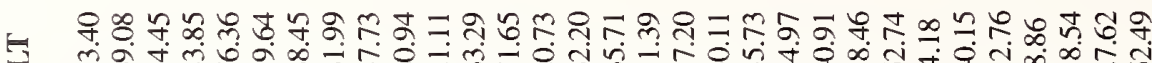

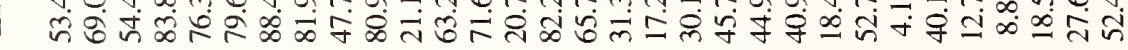

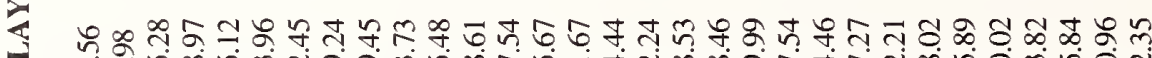

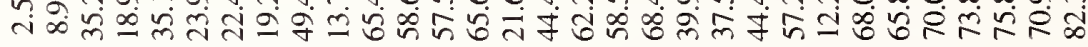

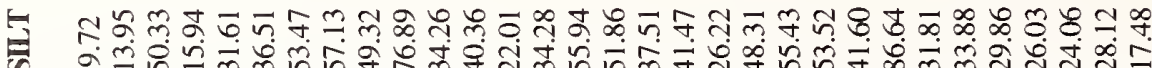

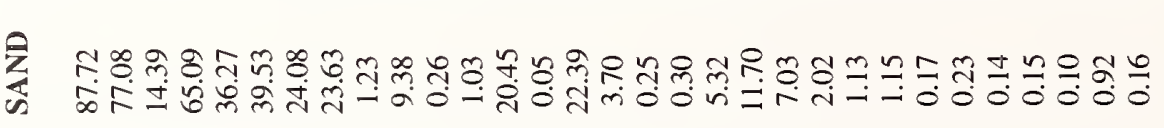

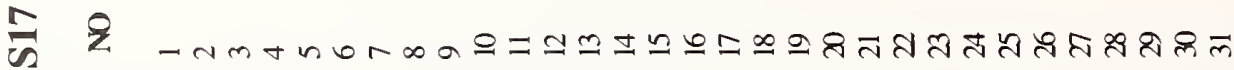

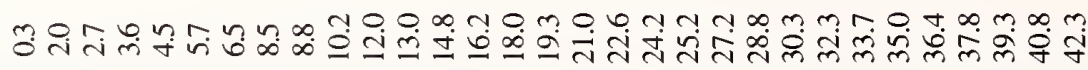


APPENDIX 2.-Continued.

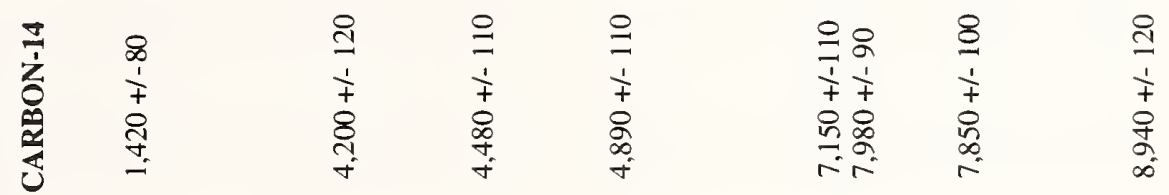

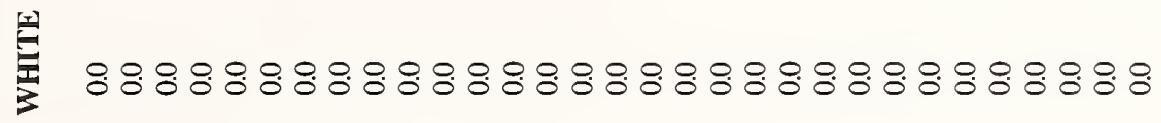

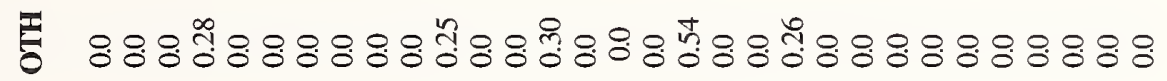

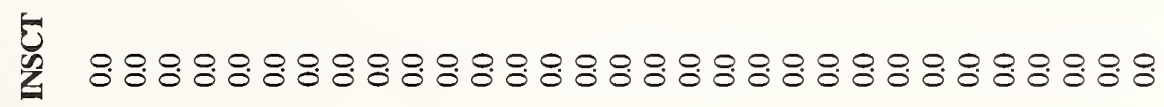

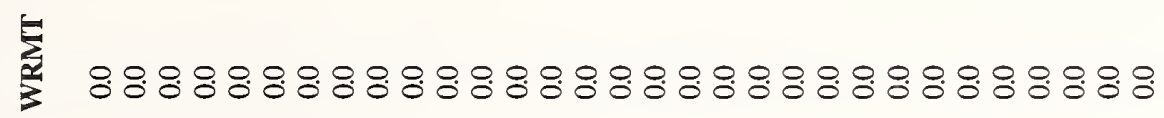

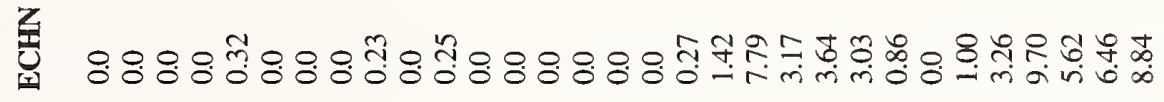

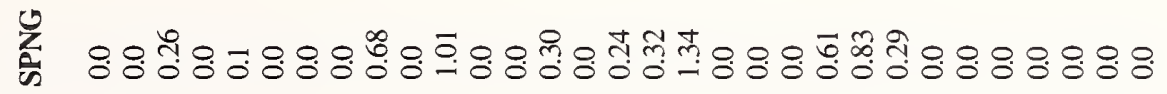

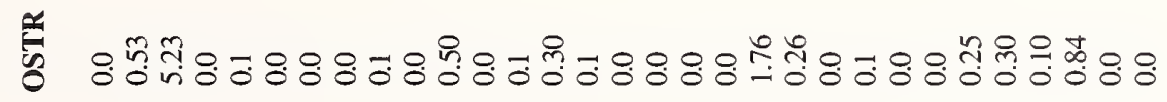

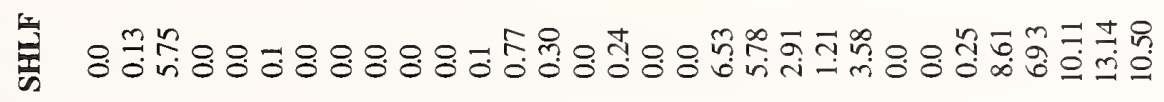

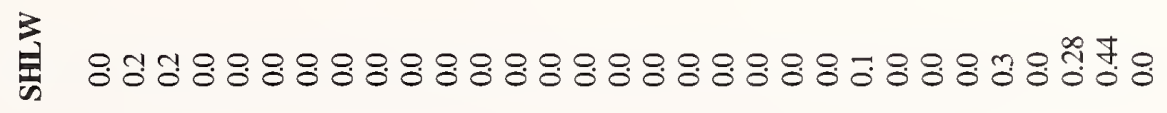

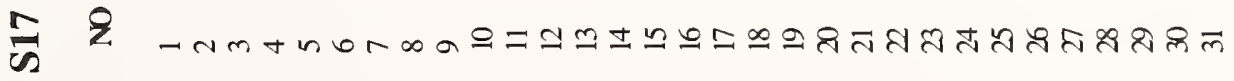

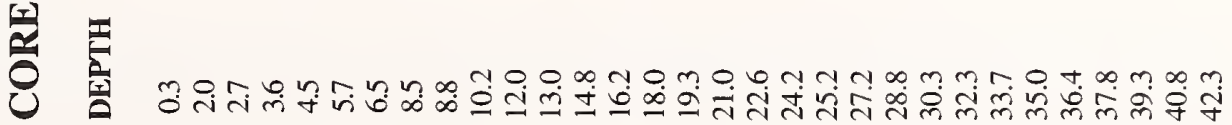


APPENDIX 2.-Continued.

일

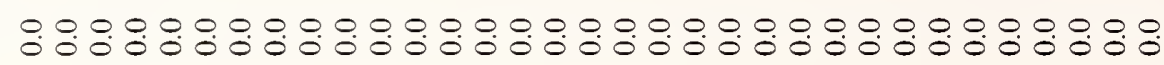

$\frac{1}{2}$

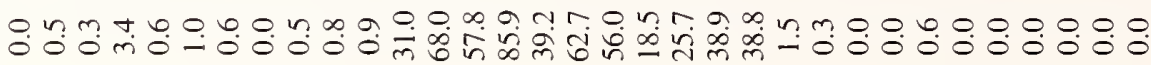

至

y

罪

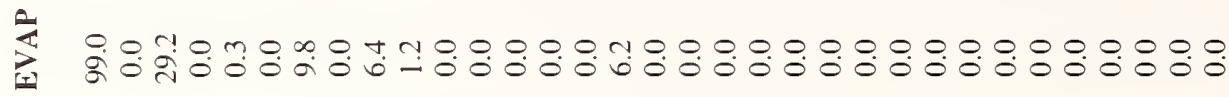

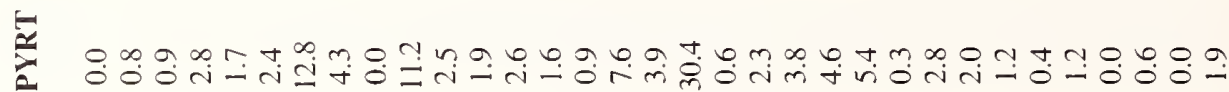

寻

岁

至

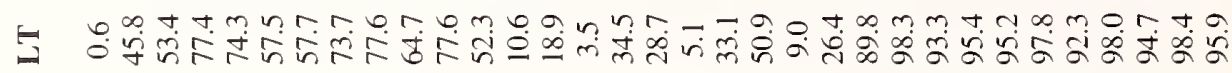

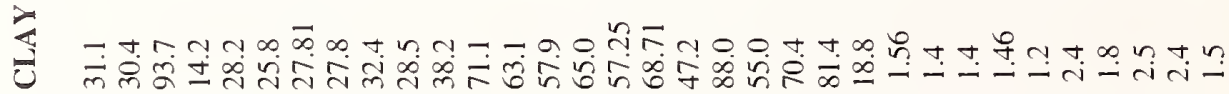

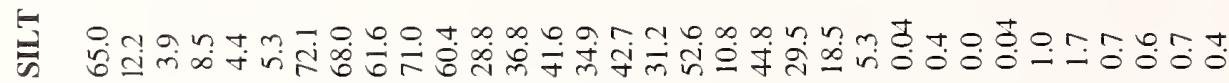

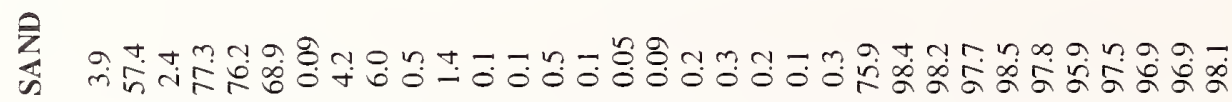

舟

है

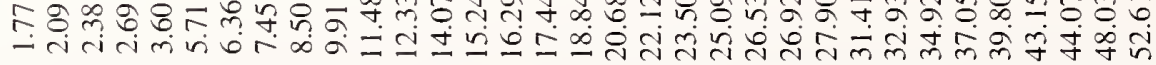


APPENDIX 2.-Continued.

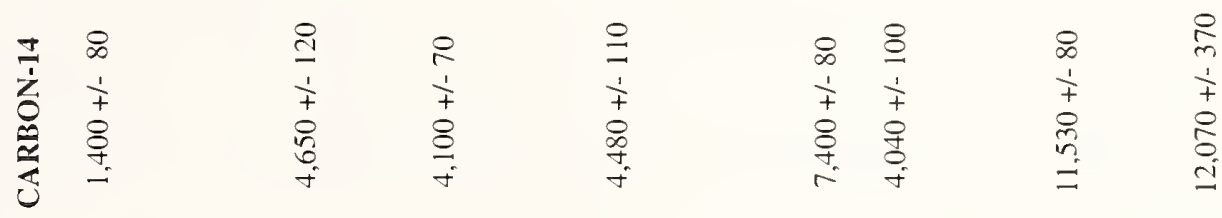

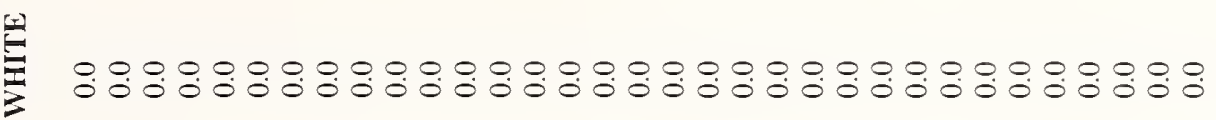

ప

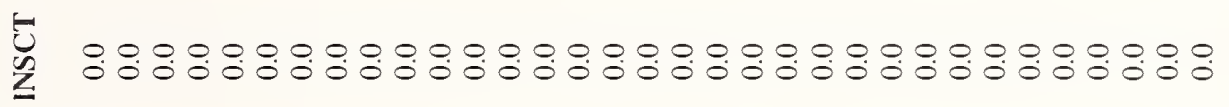

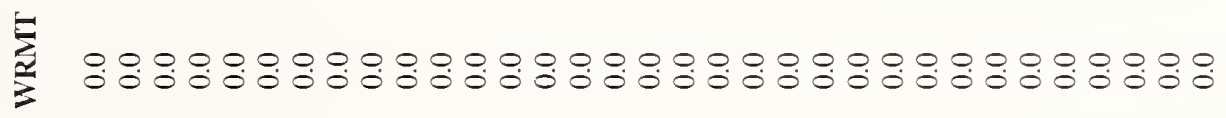

党

位

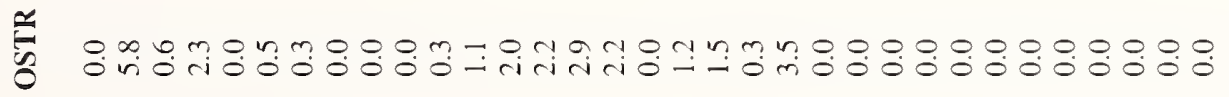

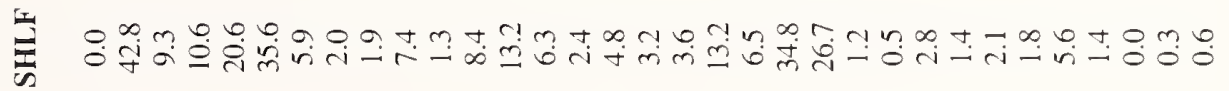

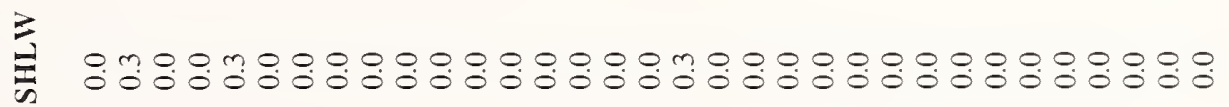

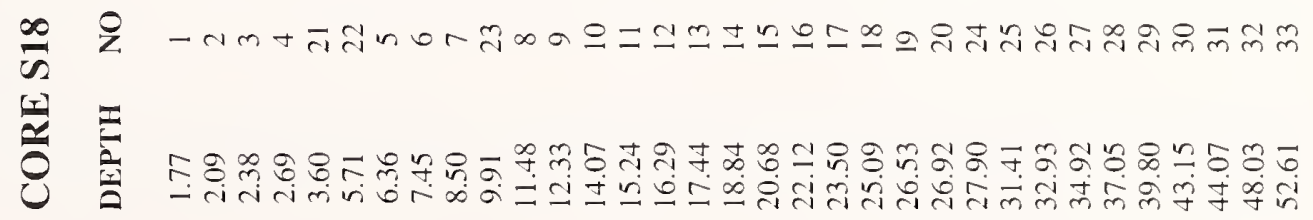


APPENDIX 2.-Continued.

茫 $8: 8: 8: 8: 8: 8: 8: 8: 8$

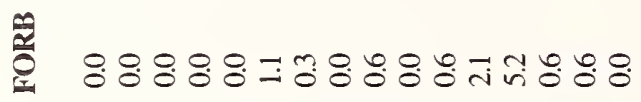

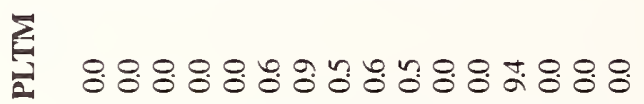

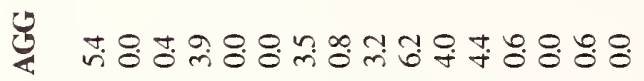

志

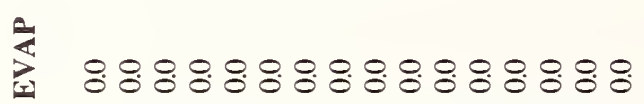

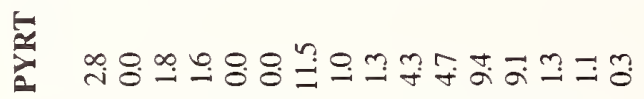

趟 $8: 8: 8: 8: 8: 8: 8: 8: 8$

选

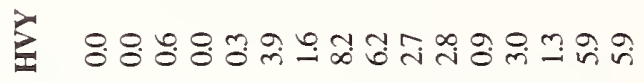

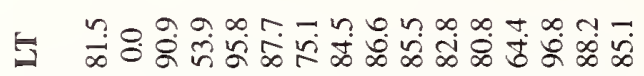

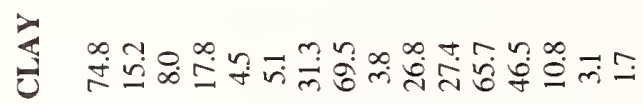

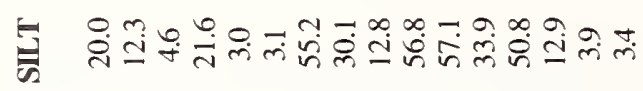

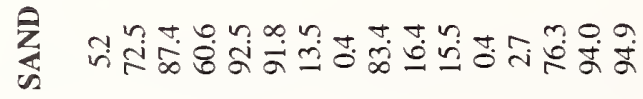

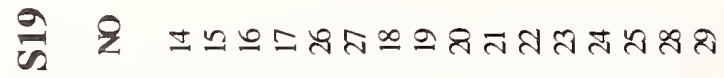

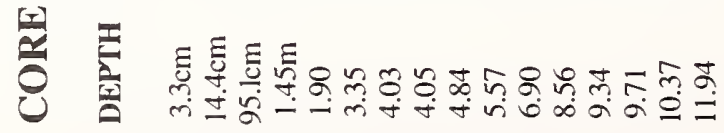


APPENDIX 2.-Continued.

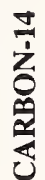

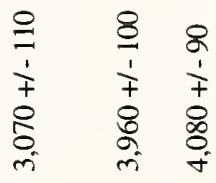

$8: 88: 88: 888: 88: 8$

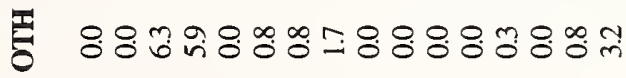

苟 $8: 8: 8: 8: 8: 8: 8: 8: \circ$

$88: 88: 88: 88: 88: 8$

党 $8: 88: 8: 8: 8: 88: 8:$

离 $8: 8: 8: 8: 8: 8: 8: 8: 8$

\%

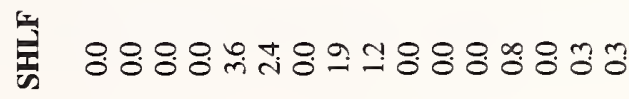

息 $8: 88: 8: 8: 8: 88: 88$

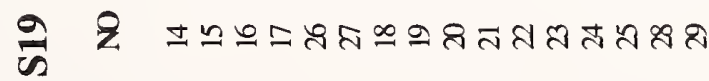

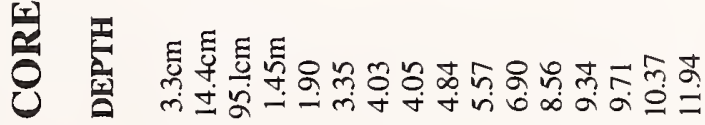


APPENDIX 2.-Continued.

0

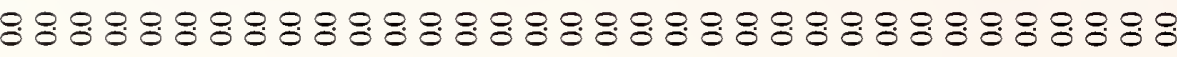

旁

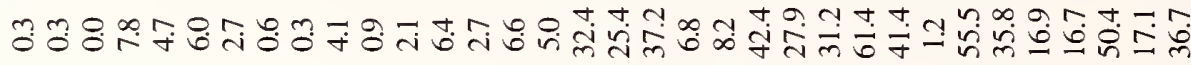

$\sum_{1}$

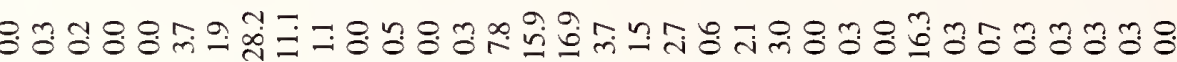

象

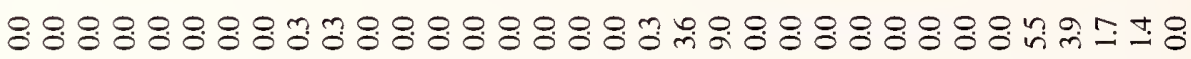

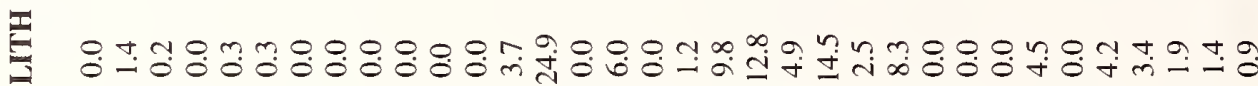

2

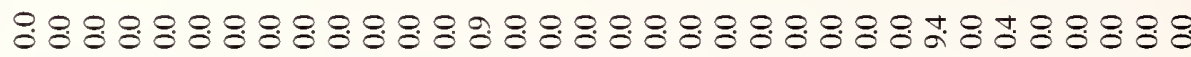

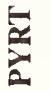

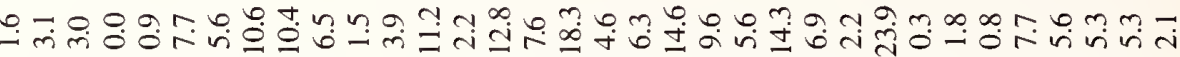

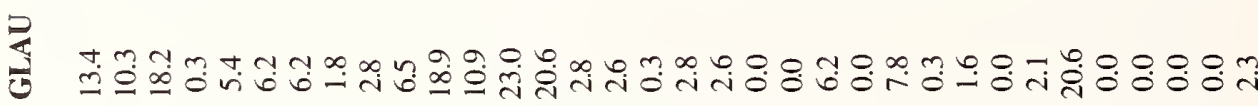

¿

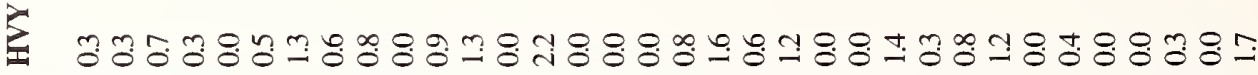

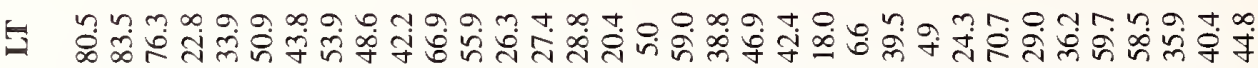

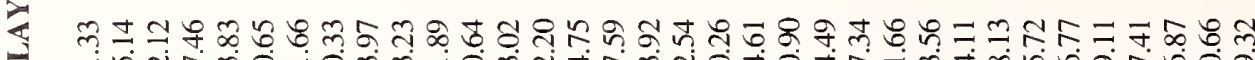

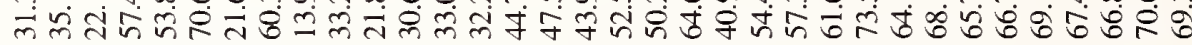

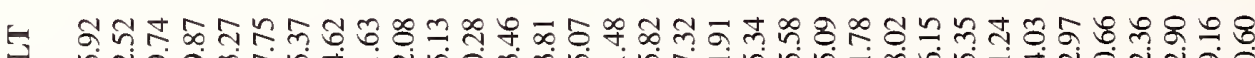

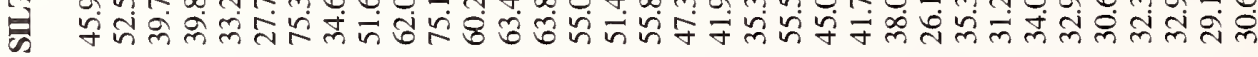

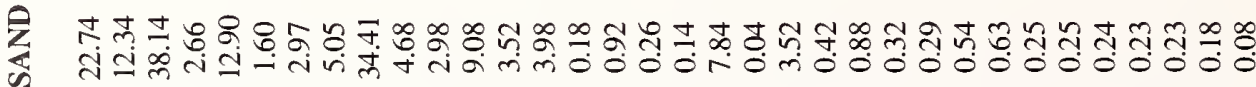

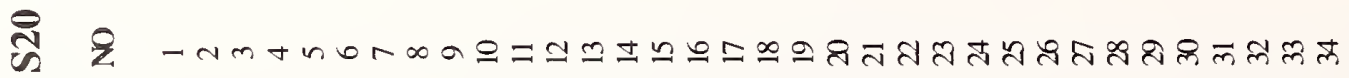

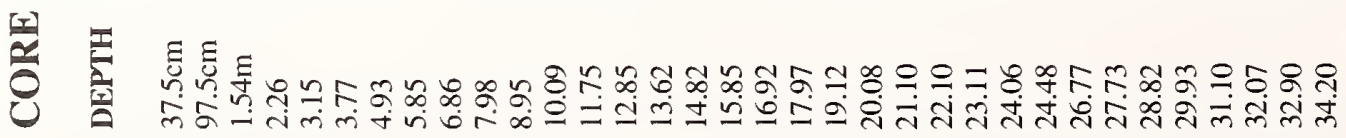


APPENDIX 2.-Continued.

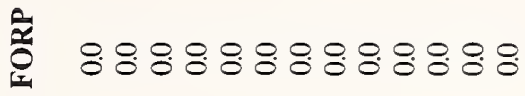

突

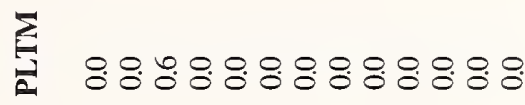

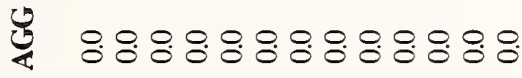

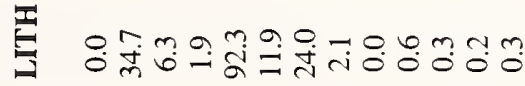

i $8.88 .888 .88 .8 \%$

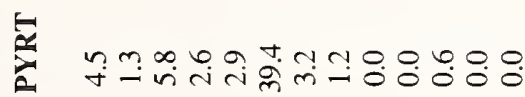

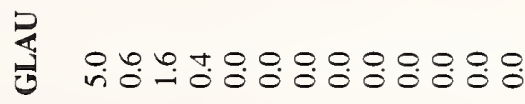

岁

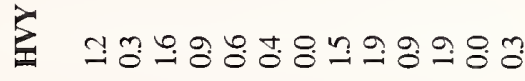

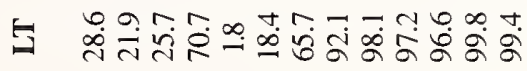

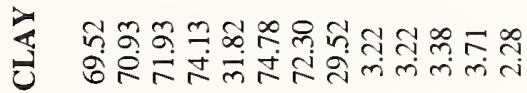

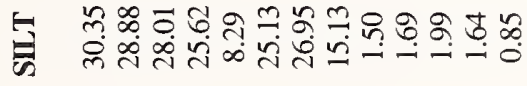

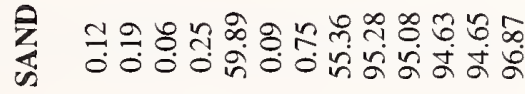

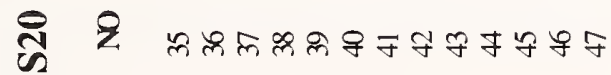

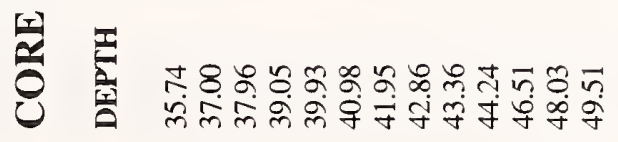


APPENDIX 2.-Continued.

$\frac{ \pm}{2}$

$\stackrel{8}{-}$
$\frac{1}{+}$
0
$\infty$
$\infty$
ci

$\stackrel{8}{\frac{1}{+}}$
$\frac{8}{8}$

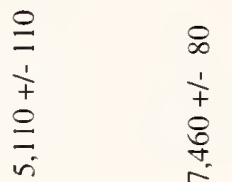

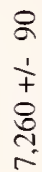

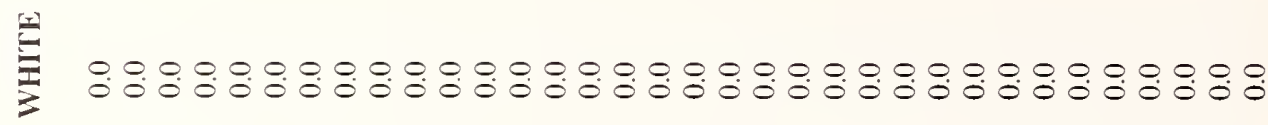

I

艺

产

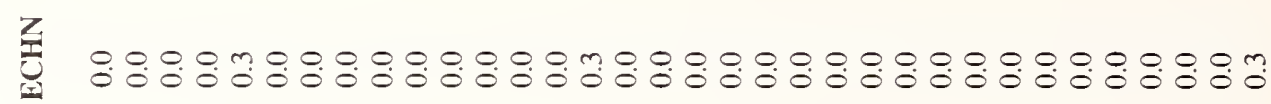

青

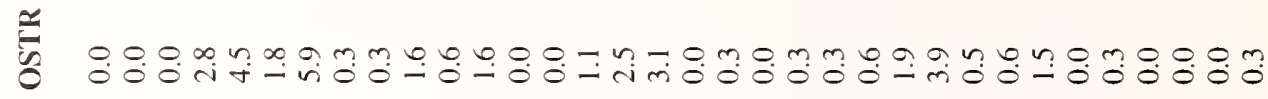

灵

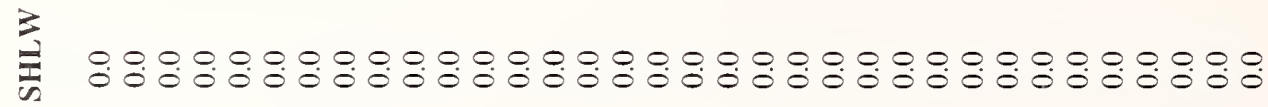

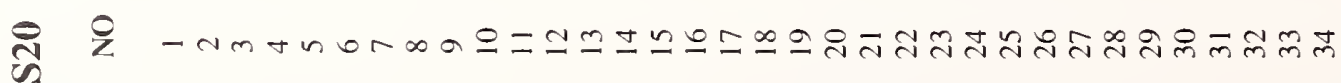

농

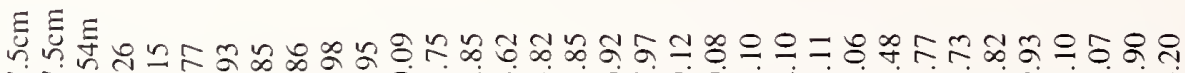

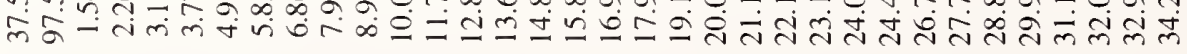


APPENDIX 2.-Continued.

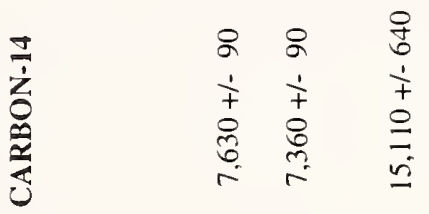

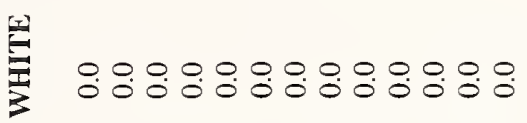

荡

㫐

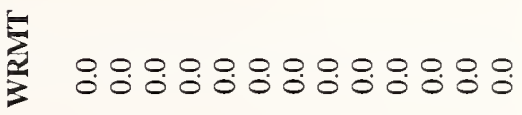

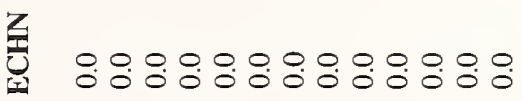

壳

\%

的

严

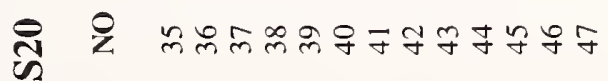

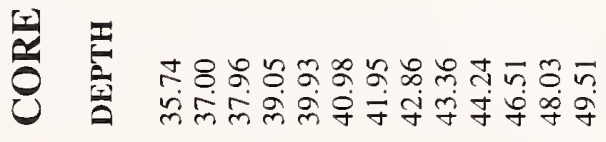


APPENDIX 2.-Continued.

응

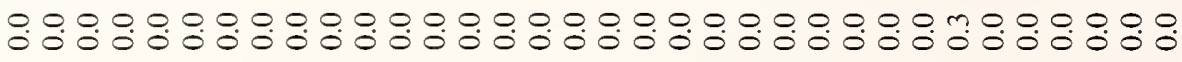

อํำ

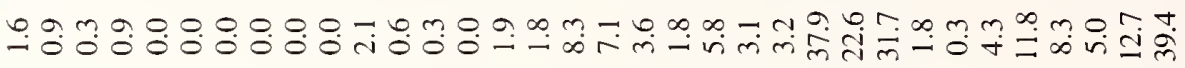

$E$

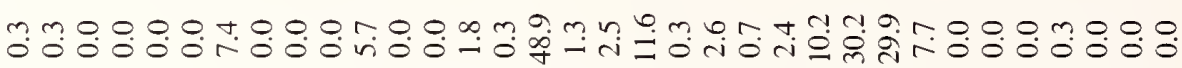

$\bigcup_{\zeta}^{\infty}$

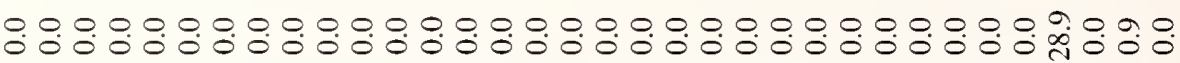

를

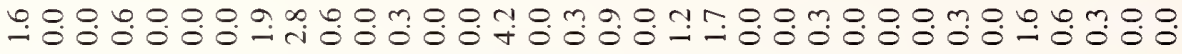

$\sum_{i=1}^{2}$

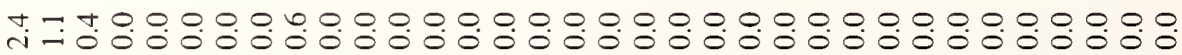

2ี

m品

$\frac{2}{3}$

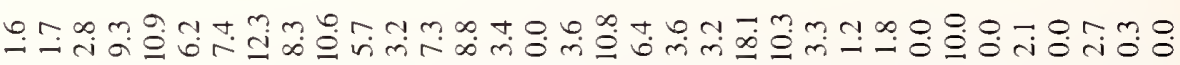

$\Xi$ m킁ํㅇำ

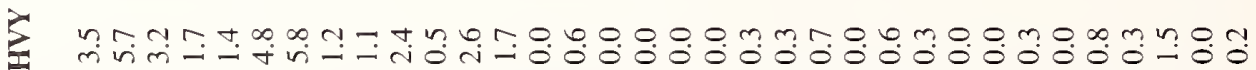

ت

\

J

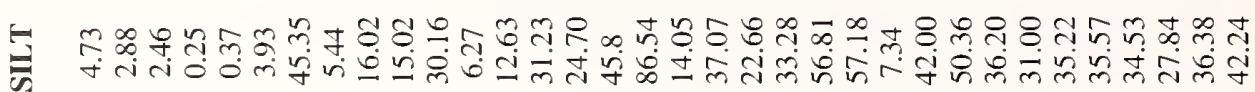

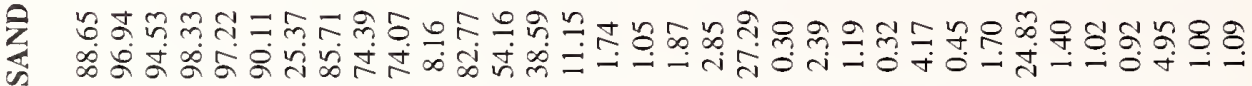

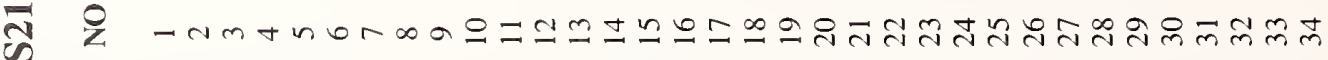

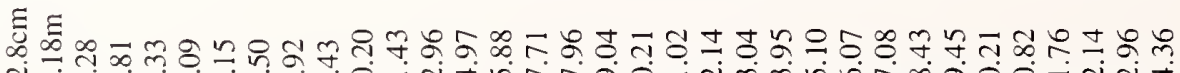
لj 
APPENDIX 2.-Continued.

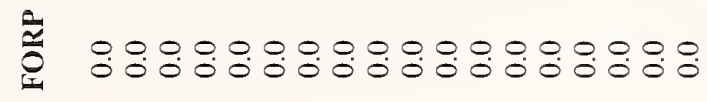

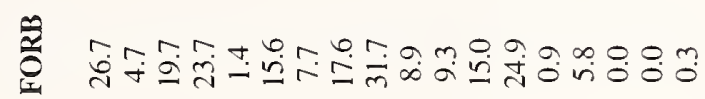

育

년

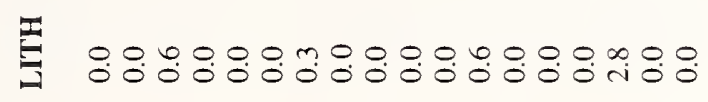

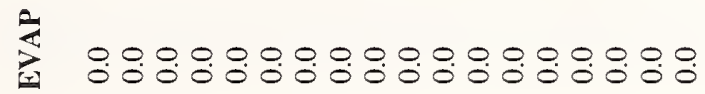

弚

过

¿

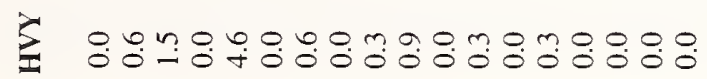

J

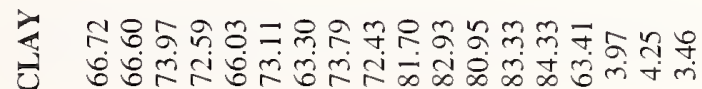

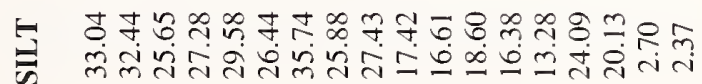

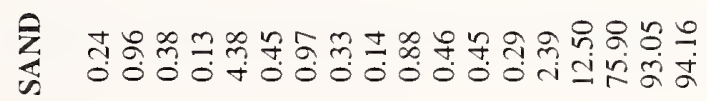

तु

重

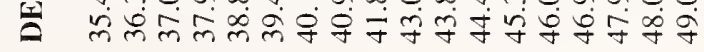


APPENDIX 2.-Continued.

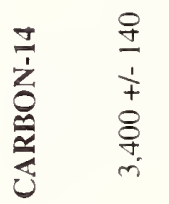

$\begin{array}{ll}8 & 8 \\ \dot{1} & \frac{1}{+} \\ + & \frac{1}{+} \\ 0 & \infty \\ n & \infty \\ m & m\end{array}$

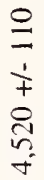

$\stackrel{\infty}{1}$
$\frac{1}{+}$
$\stackrel{\infty}{\infty}$
$\stackrel{\sim}{i}$

닐

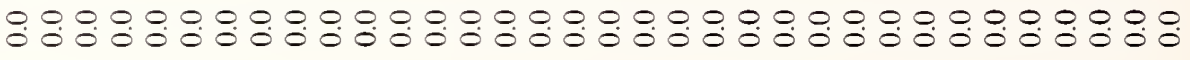

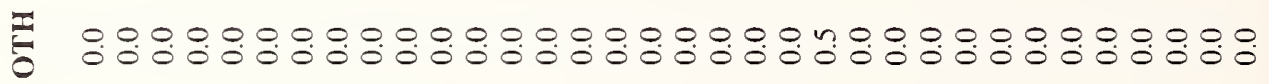

䒺

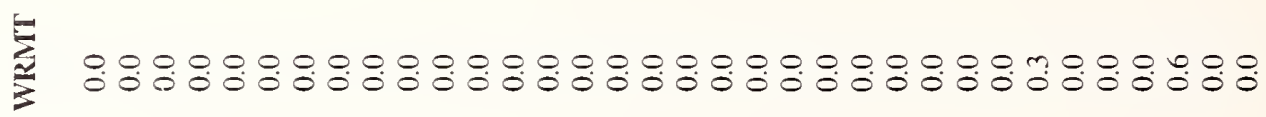

传

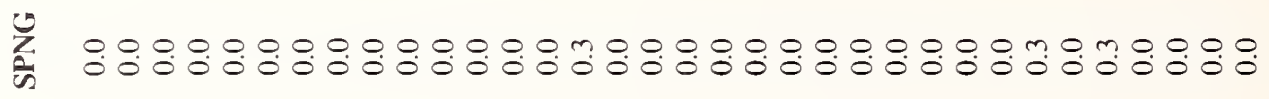

\%

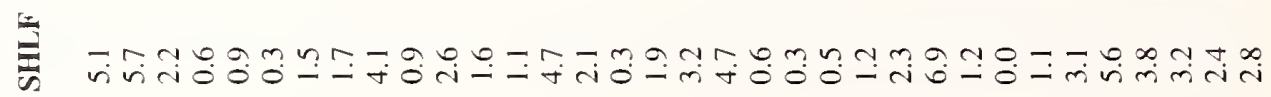

䓛

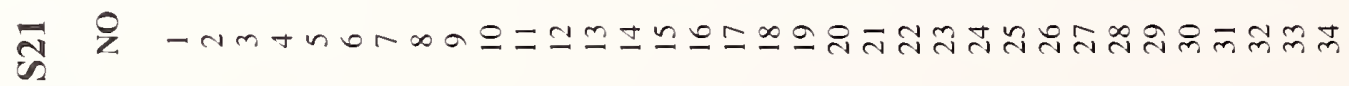

我 तु 
APPENDIX 2.-Continued.

E

荡

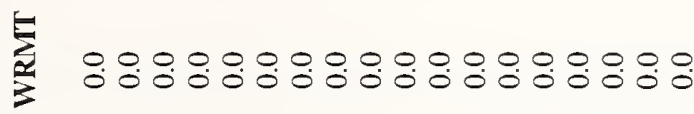

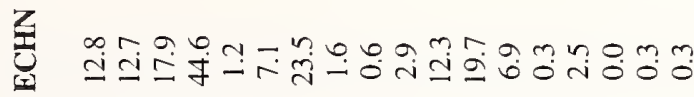

嵴

ஜ

岁

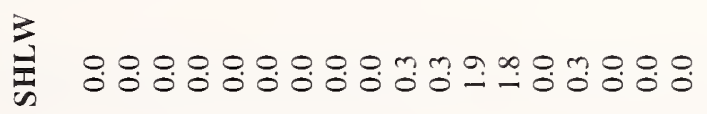

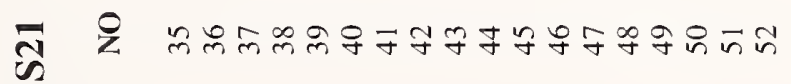

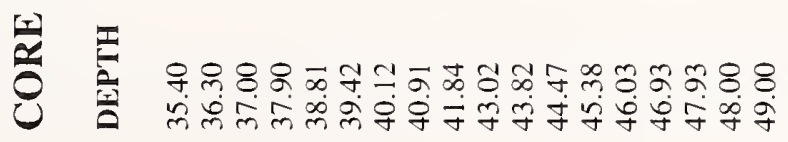


APPENDIX 2.-Continued.

芏

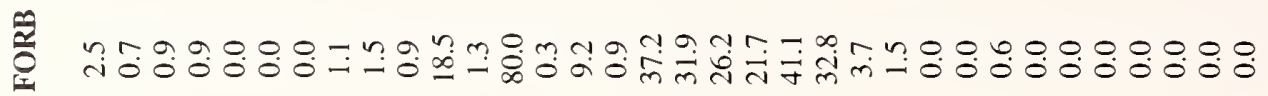

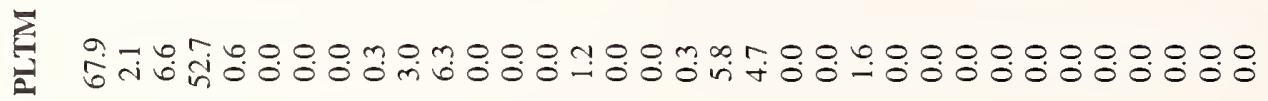

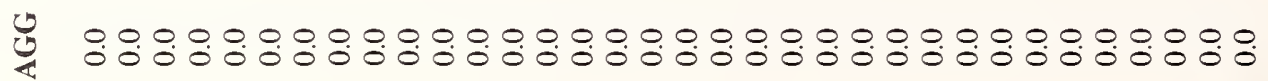

雷

i

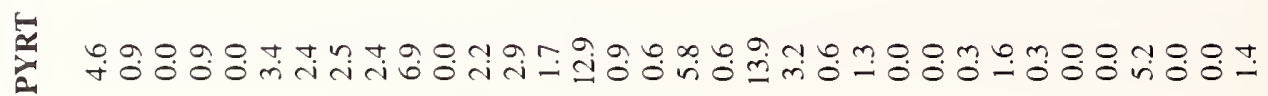

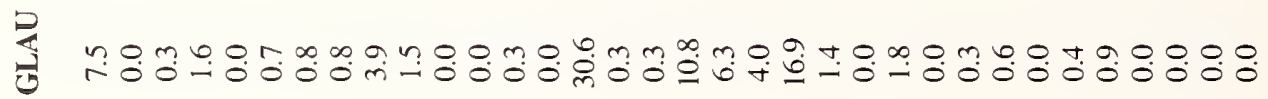

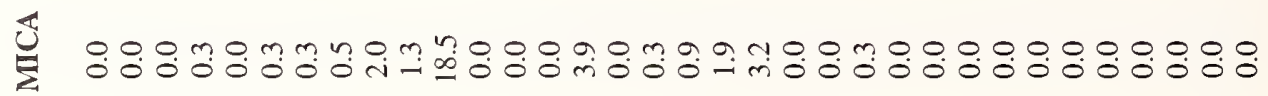

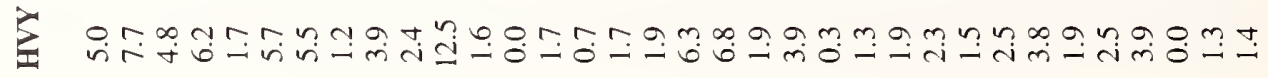

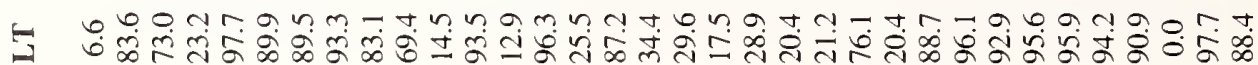

公

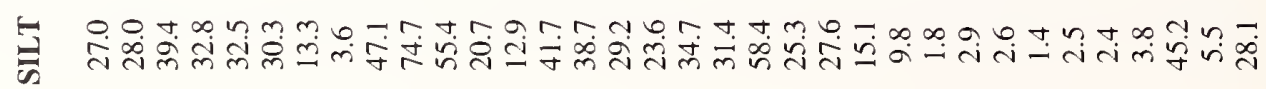

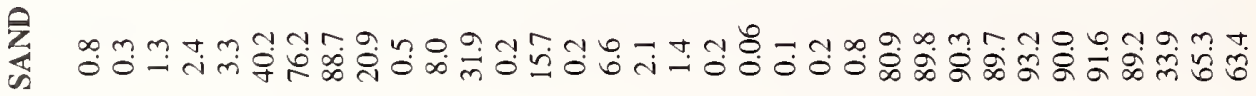

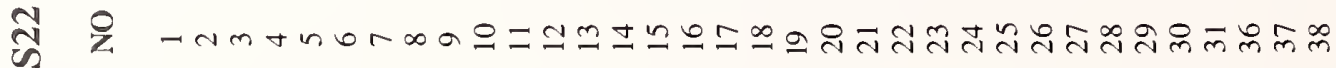

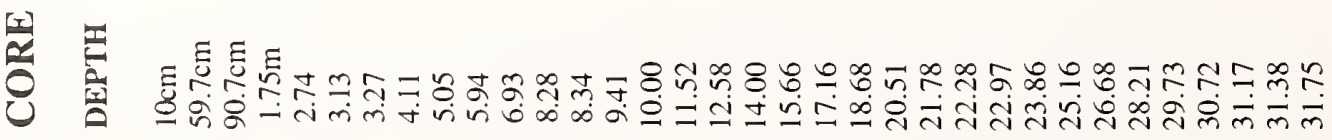


홍 $\stackrel{0}{0} \stackrel{0}{0} \stackrel{0}{0} \stackrel{0}{0} \stackrel{0}{0}:$

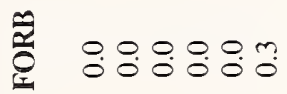

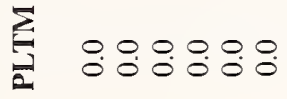

U 운용요용

声

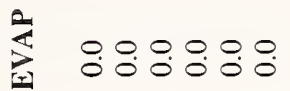

荧

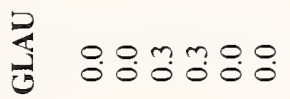

岁

¿ 9.

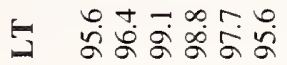

¿

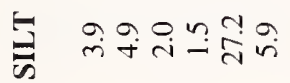

㐘

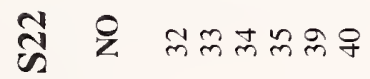

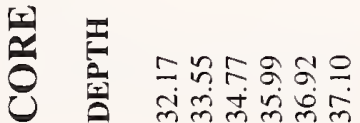


APPENDIX 2.-Continued.

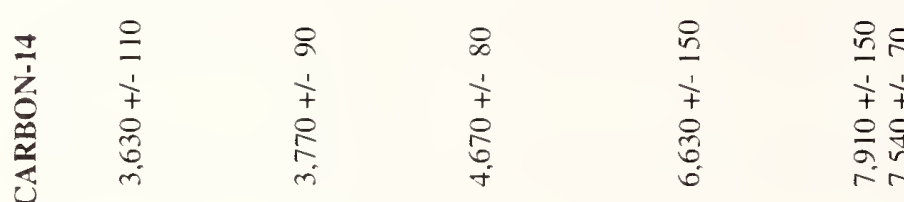

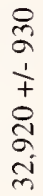

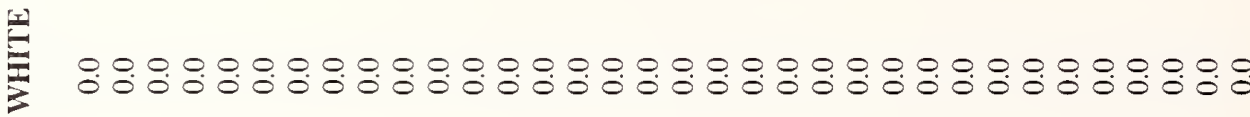

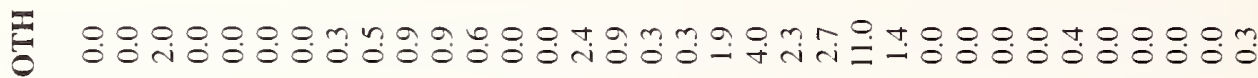

艺

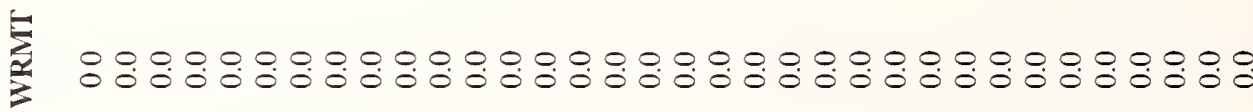

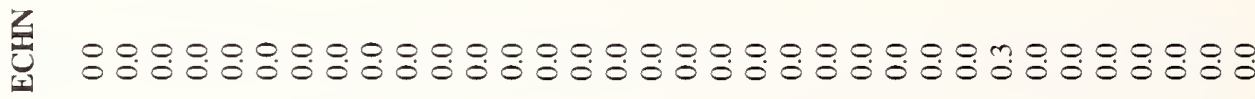

侯

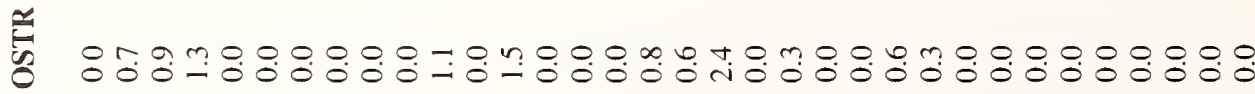

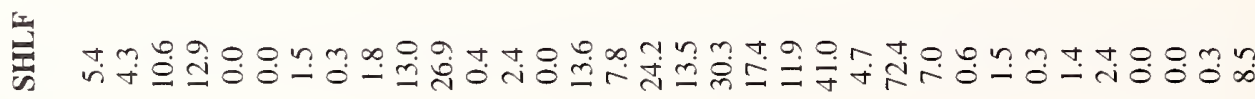

연

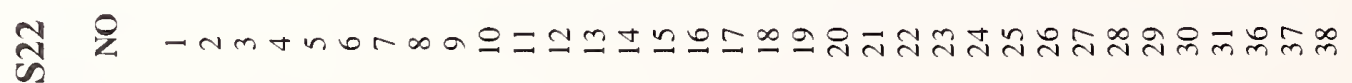

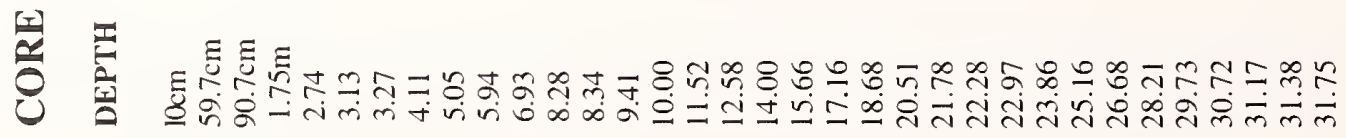


APPENDIX 2.-Continued.

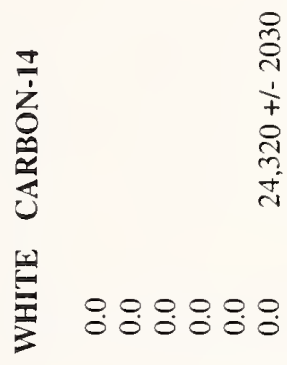

융용요

艺 0.00000

: $8: 80:$

坣 $80980 \%$

位

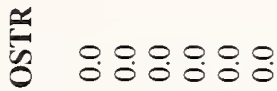

崖

莫 0.0080

กี

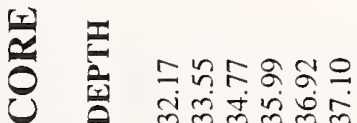


APPENDIX 2.-Continued.

部 88888888

옹 $8.8 .8: 8: 8$

鼠 $8 \% 8 \% 8: 8 \%$

난 용용ㅇㅇ용

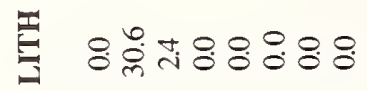

$\sum_{1}^{4} 888888$

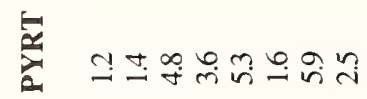

畓 $8 \% 88 \% 8 \% 8$

岂 $8 \% 8 \% 8 \%: \%$

ㅇํㅇ공ㅇํㅇํํ

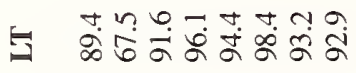

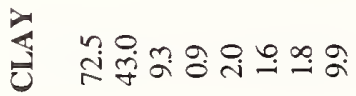

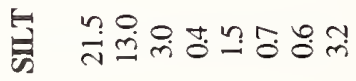

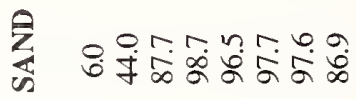

กี $Z$ กm+nor

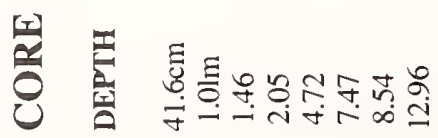


APPENDIX 2.-Continued.

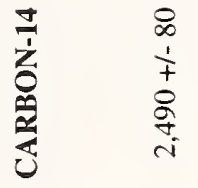

帘 $8.8 \% 8.8 \%$

I $8: 8: 8: 8: 88$

艺 $8.8 \% 8 \% 8 \%$

$8.808 .8 \%$

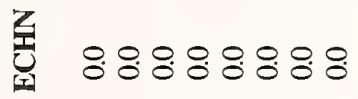

फ

\% $8: 8: 8: 8:$

荘

悬 80.808080

तु Z -nm+nor

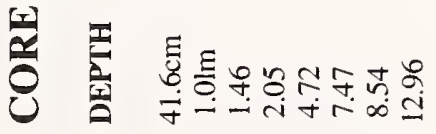


商

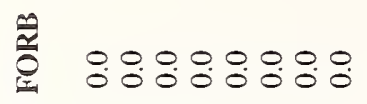

突 $8009080 \%$

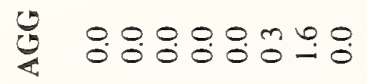

: $8: 08: 80: 0$

$\sum_{1}^{2} 8080: 8:$

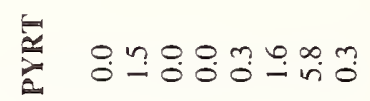

过 0.808080

远 $: 00: 0: 0: 0$

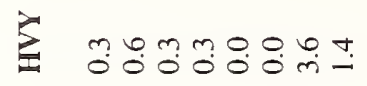

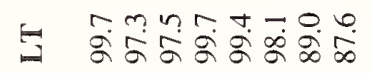

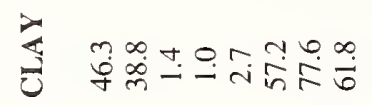

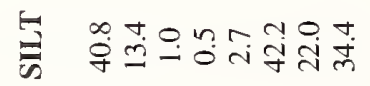

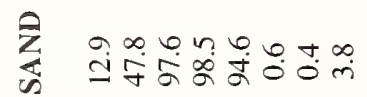

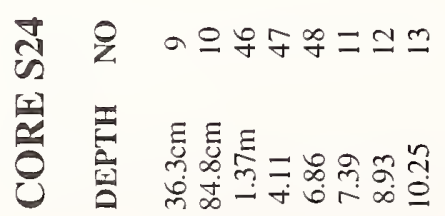


APPENDIX 2.-Continued.

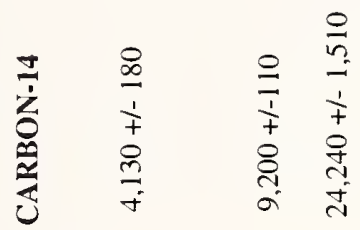

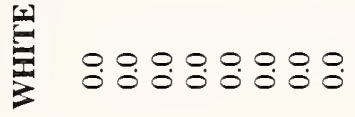

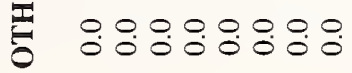

艺

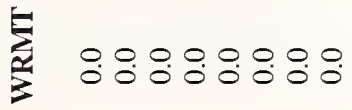

党

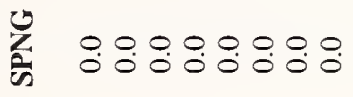

융

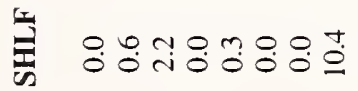

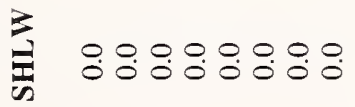

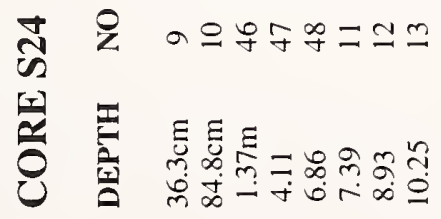


APPENDIX 2.-Continued.

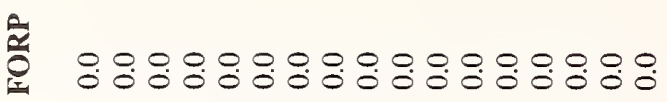

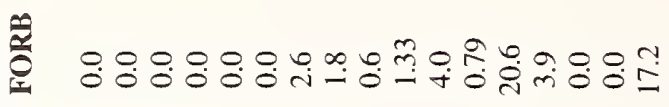

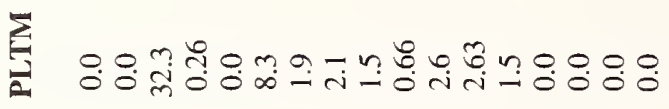

选

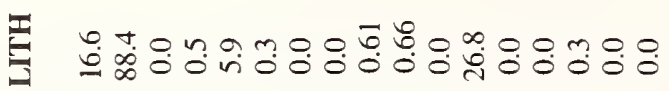

i

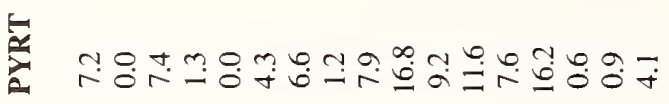

岁

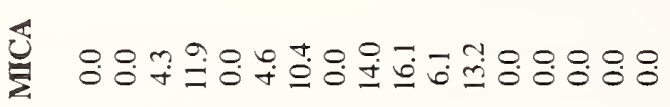

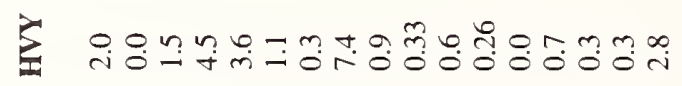

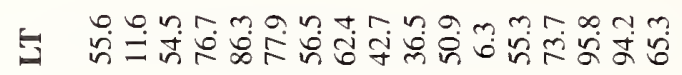

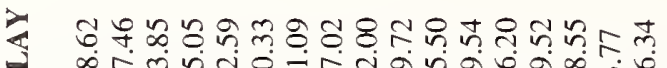

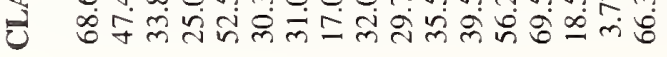

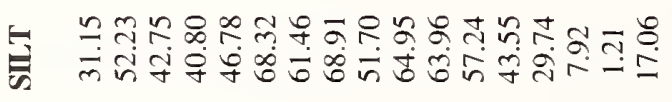

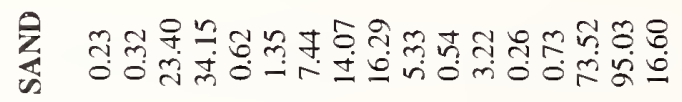

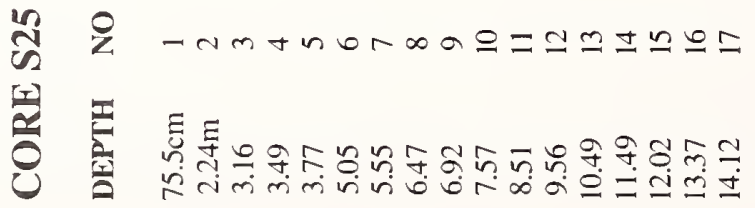


APPENDIX 2.-Continued.

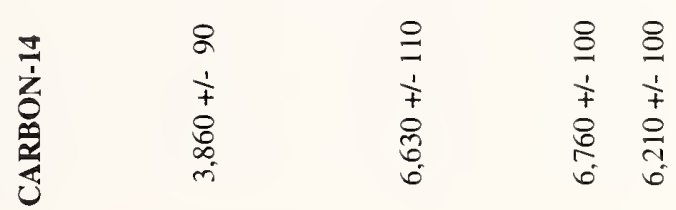

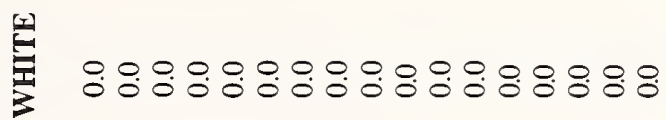

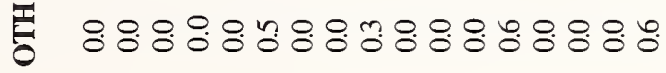

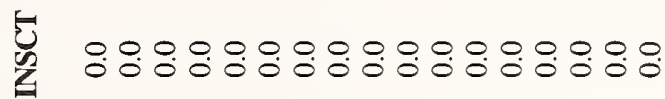

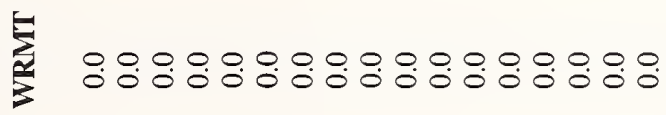

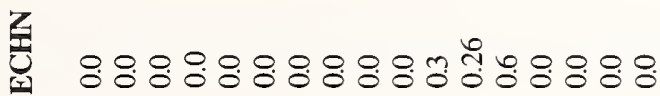

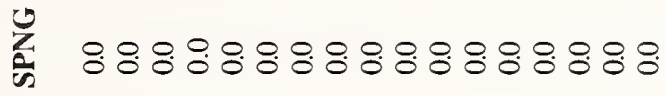

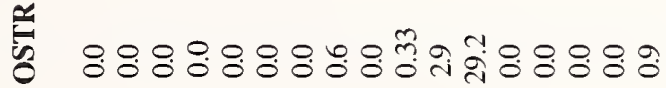

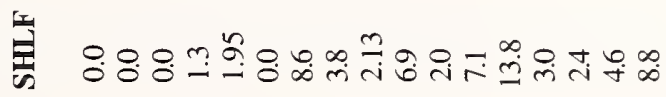

意 $808: 080: 80: 80808 \%$

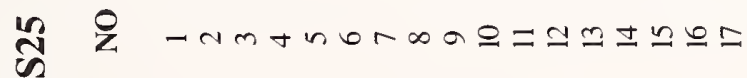

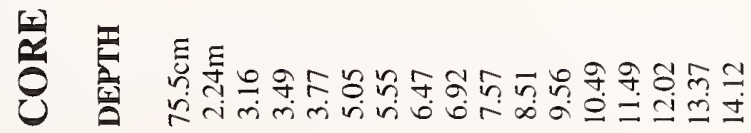


APPENDIX 2.-Continued.

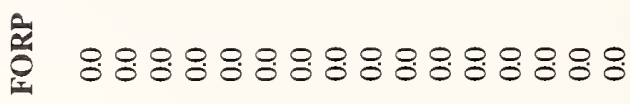

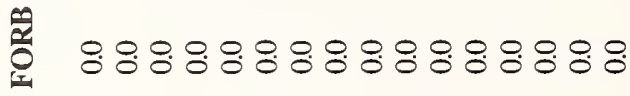

育 $8.88: 88: 8 \% .8 \% 8: 88$

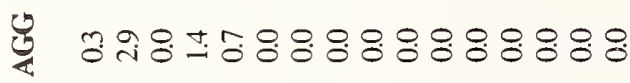

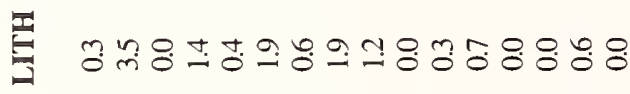

$\sum_{\text {l }}^{\circ} 8: 8 \%: 8: 8: 8: 8: 8$

氙

总

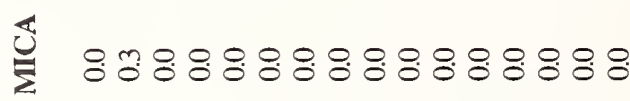

乡

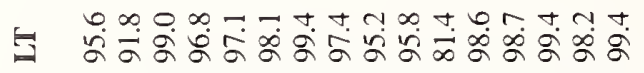

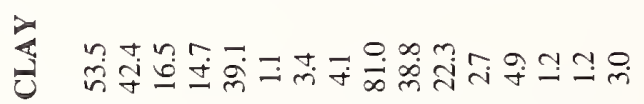

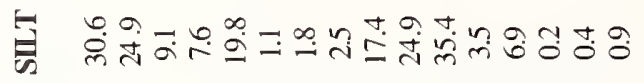

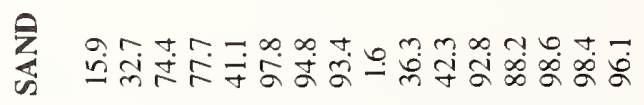

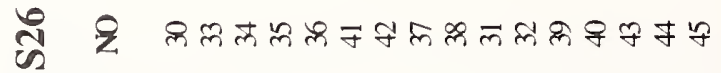

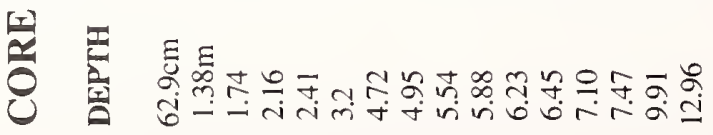


APPENDIX 2.-Continued.

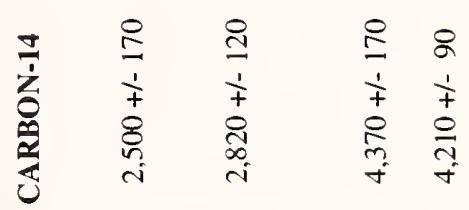

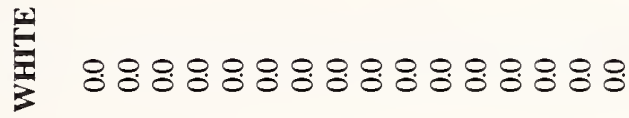

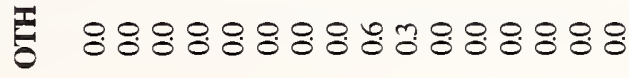

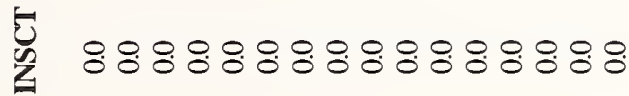

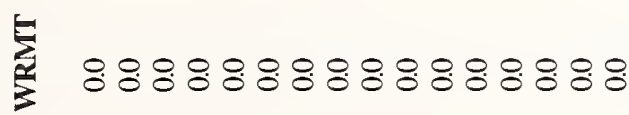

㽦 $88 \% 8 \% 88.8 \% 8 \% 8 \% 8 \%$

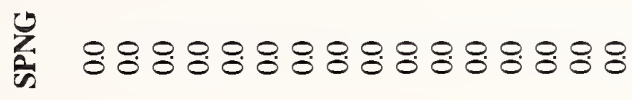

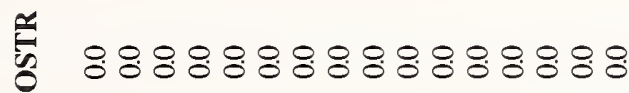

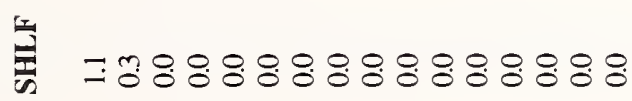

基 8888.8888 .888888

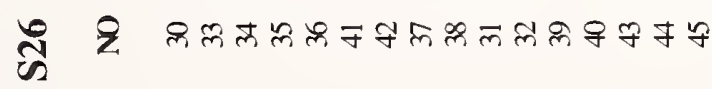

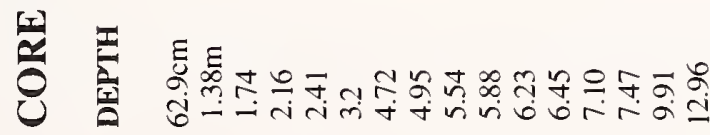


APPENDIX 2.-Continued.

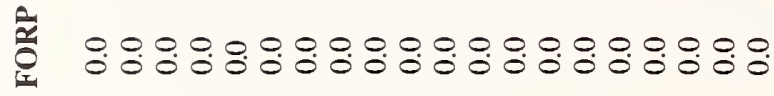

衰

产

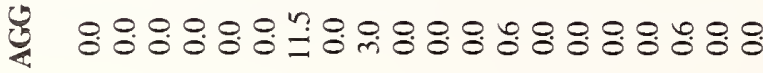

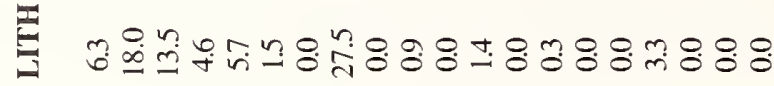

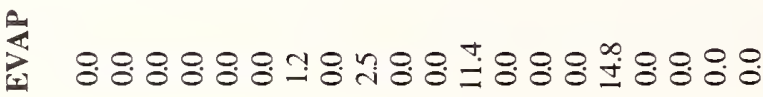

衰

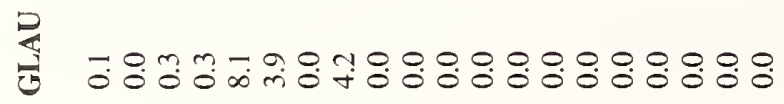

喜

完

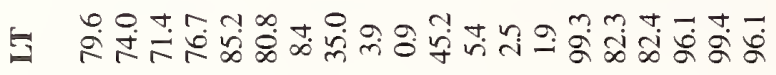

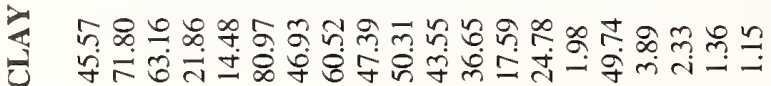

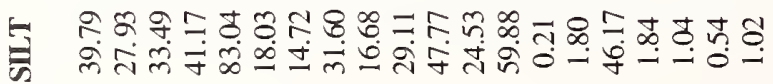

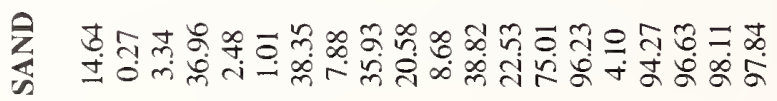

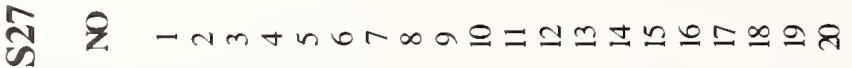

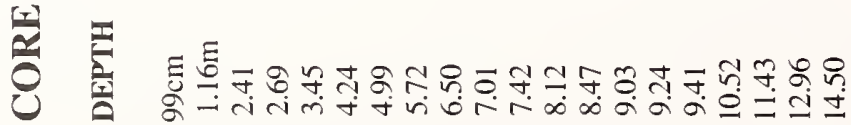


APPENDIX 2.-Continued.

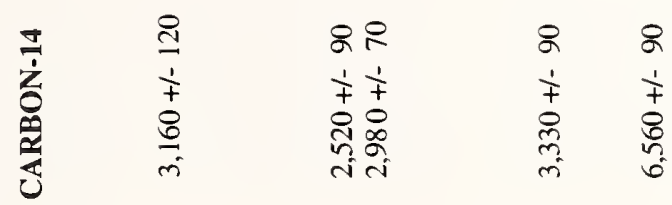

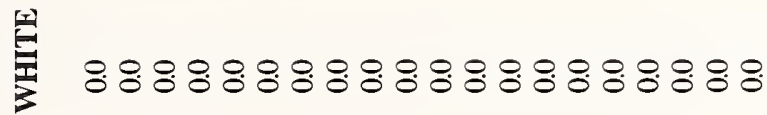

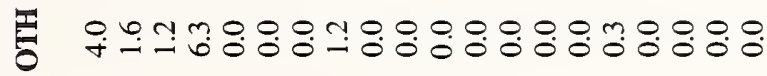

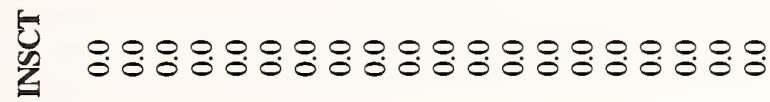

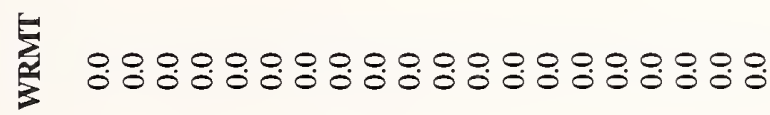

虚

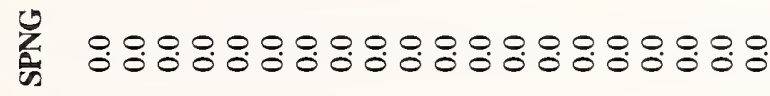

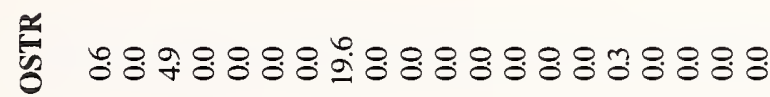

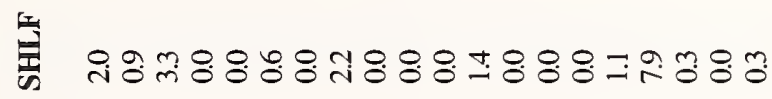

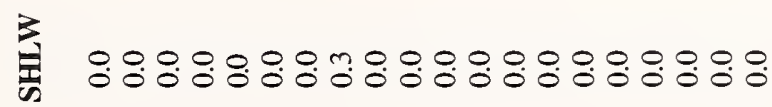

กู Z -

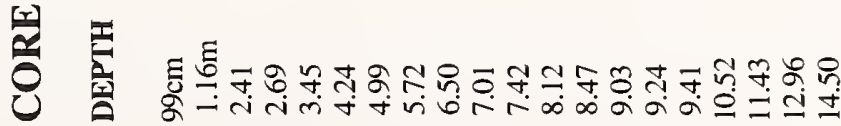


APPENDIX 2.-Continued.

里

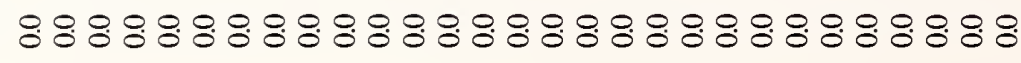

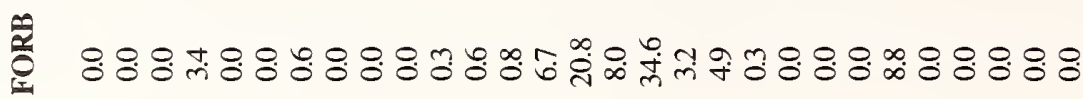

氙

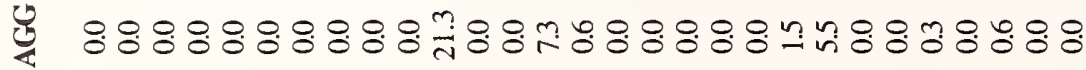

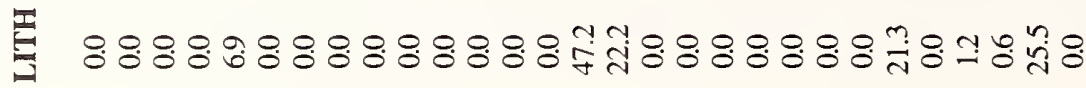

i $8: 8 \infty: 8: 8: 8: 8: 8: 8: 8: 8: 8: 8: 88.8$

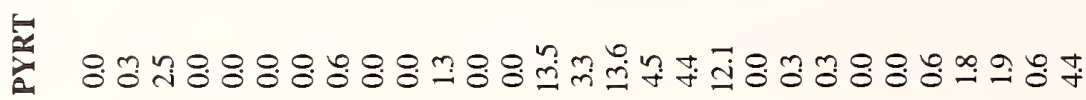

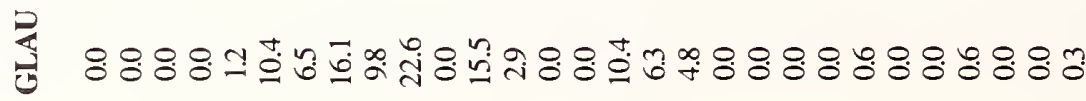

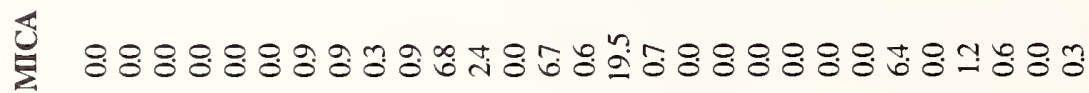

4⿻ำ

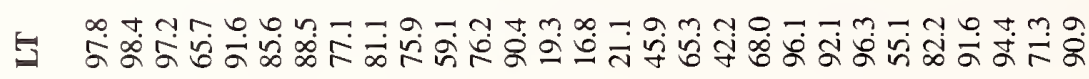

离

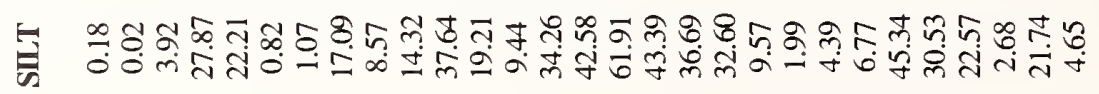

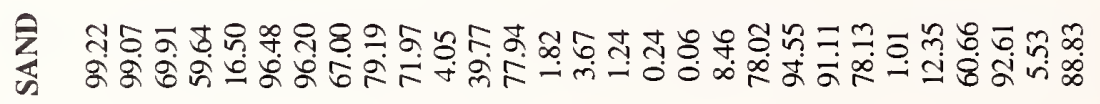

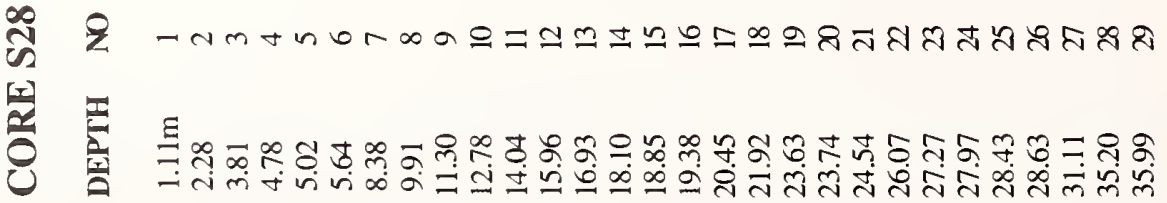


APPENDIX 2.-Continued.

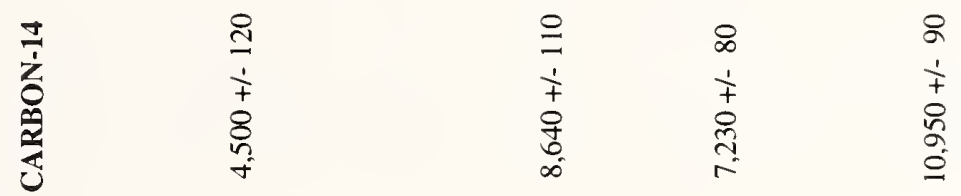

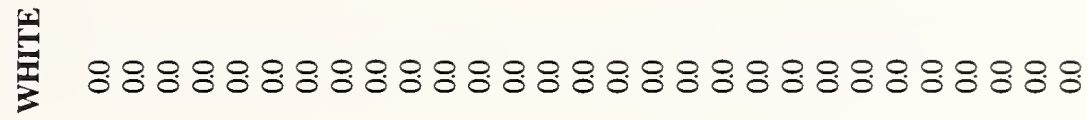

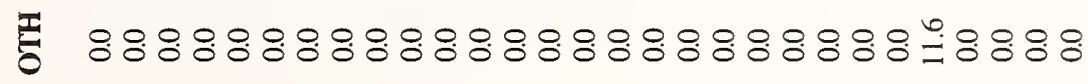

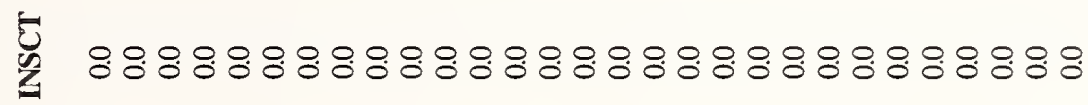

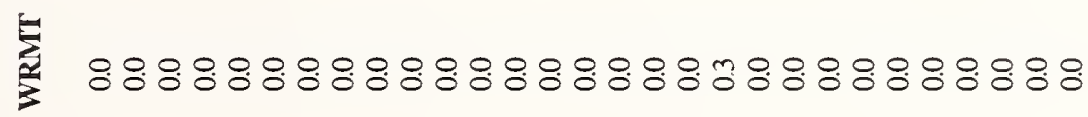

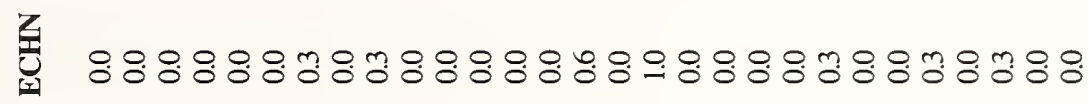

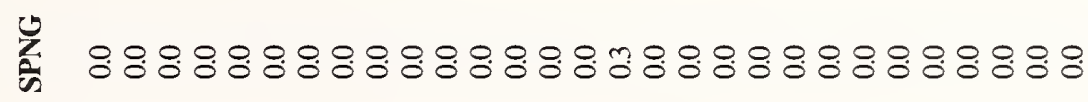

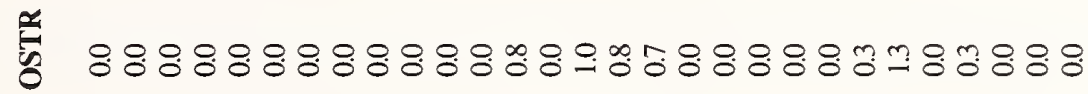

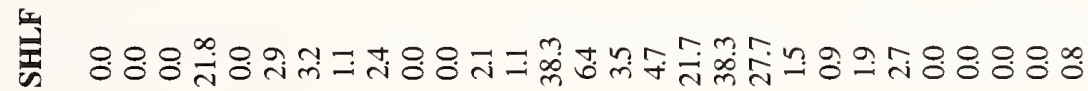

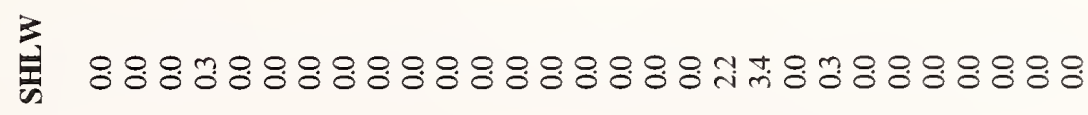

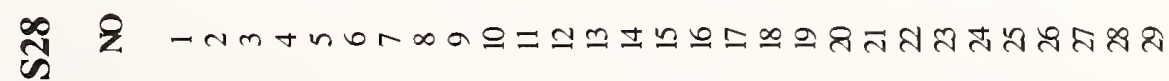

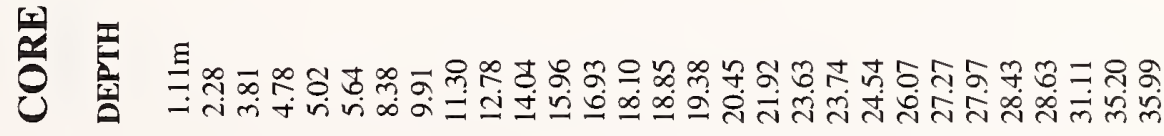


APPENDIX 2.-Continued.

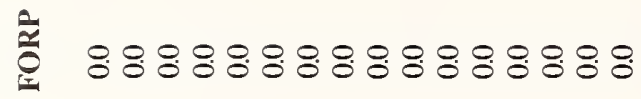

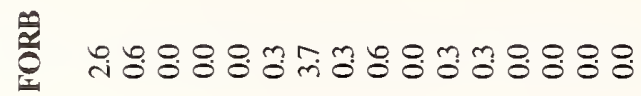

产

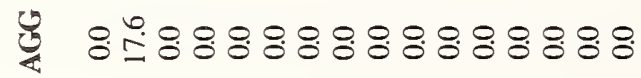

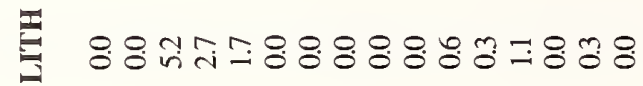

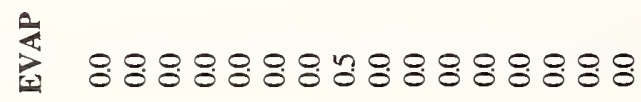

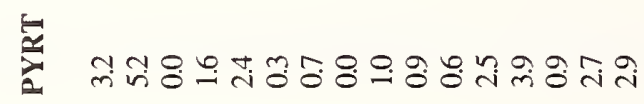

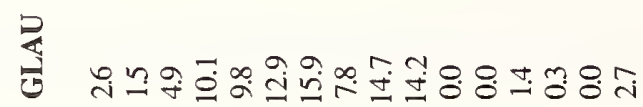

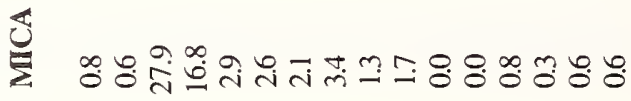

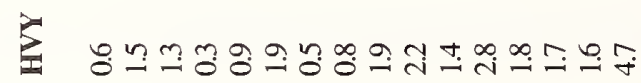

L

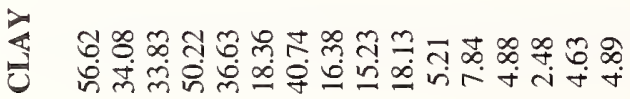

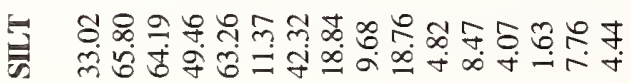

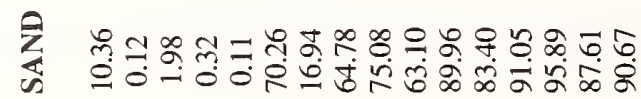

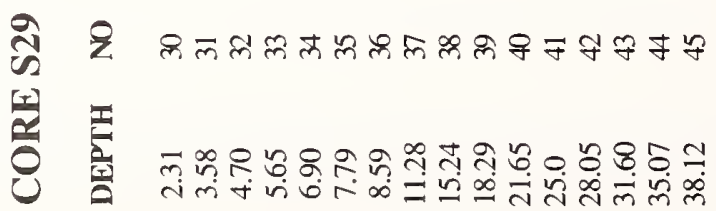


APPENDIX 2.-Continued.

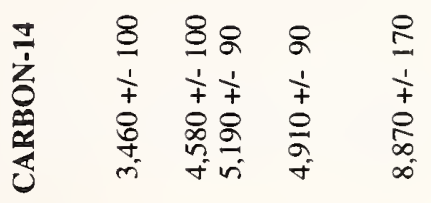

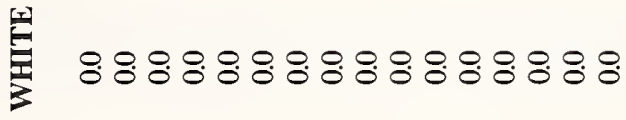

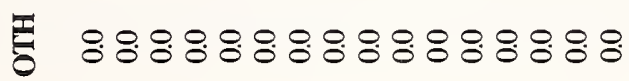

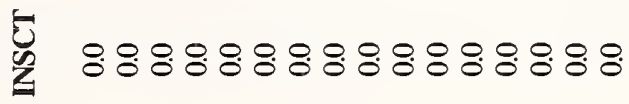

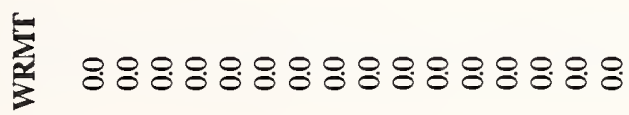

省 $8.8 \% 8.8 \% 8.8 \% 8.8 \%$

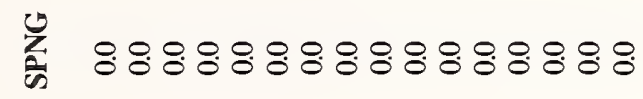

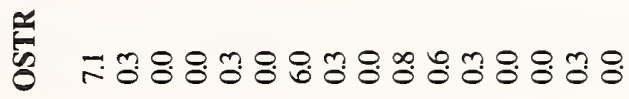

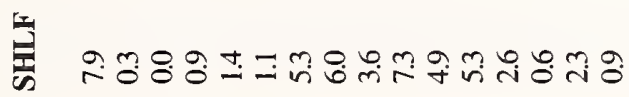

窧

त్

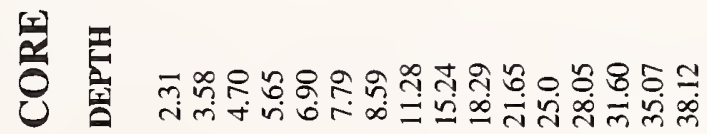


APPENDIX 2.-Continued.

产

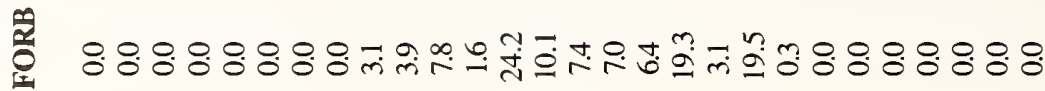

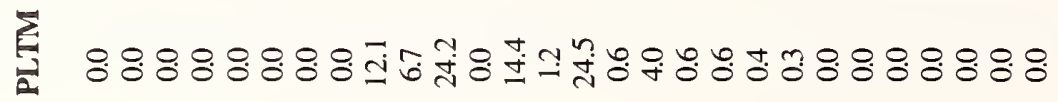

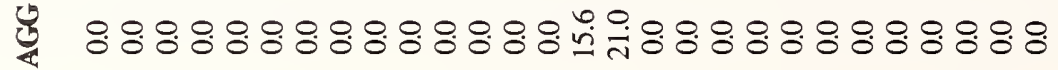

置

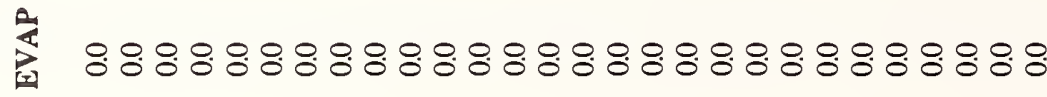

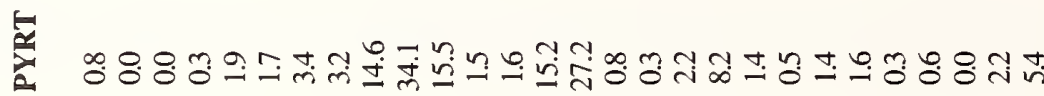

崫

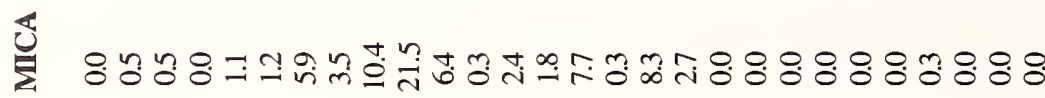

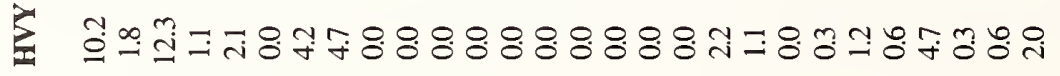

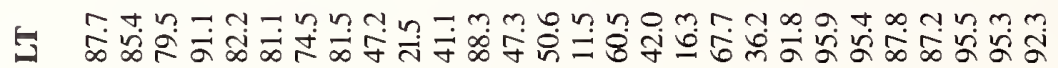

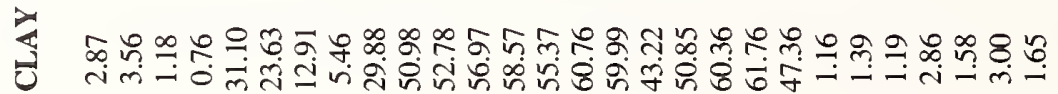

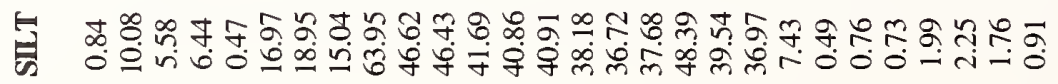

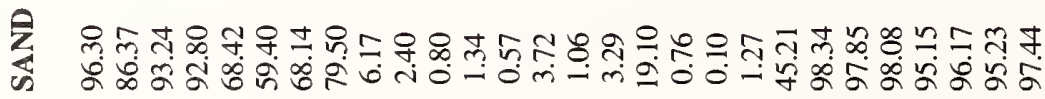

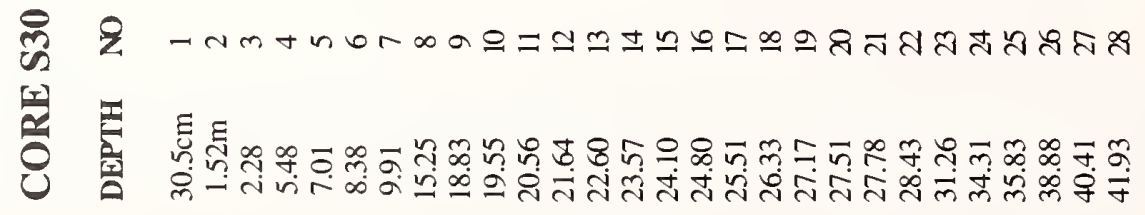


APPENDIX 2.-Continued.

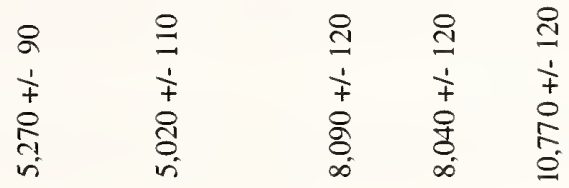

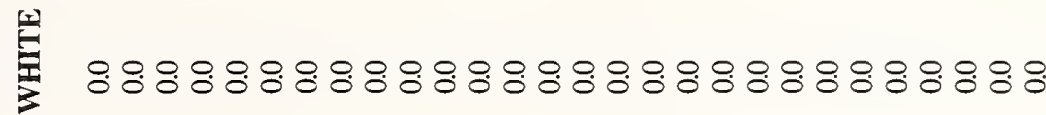

E $: 8: 8: 8: 8: 8: 3: 8: 8: 8: 8: 8: 9: 8: 8$

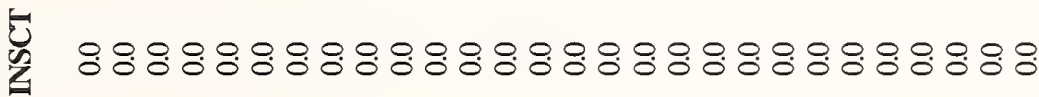

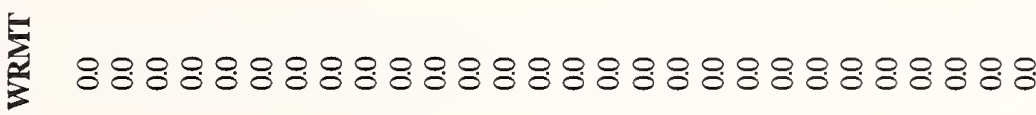

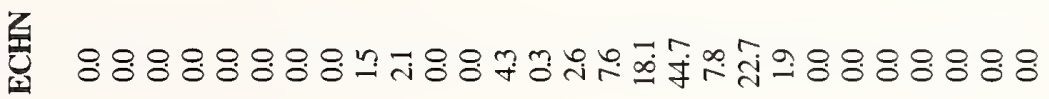

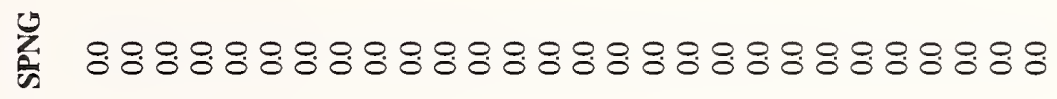

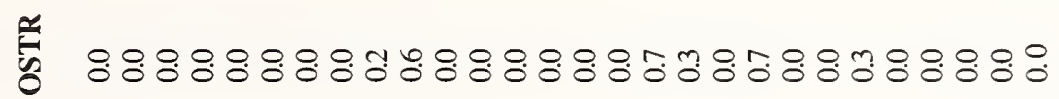

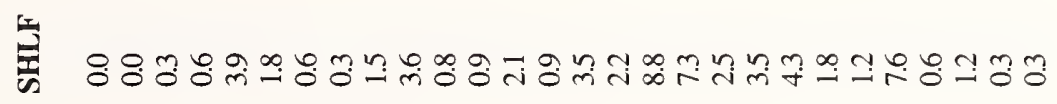

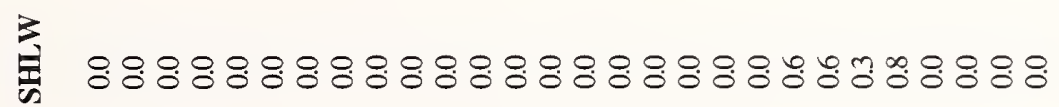

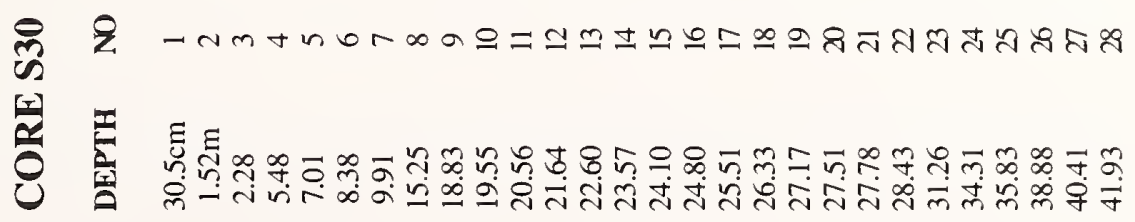


APPENDIX 2.-Continued.

을

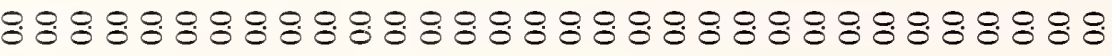

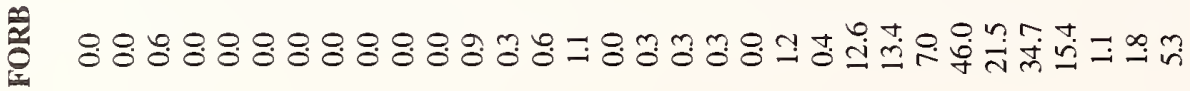

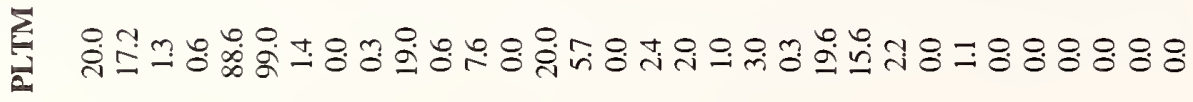

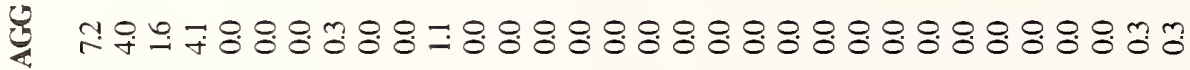

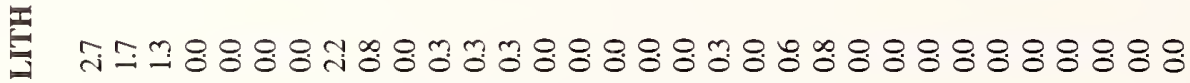

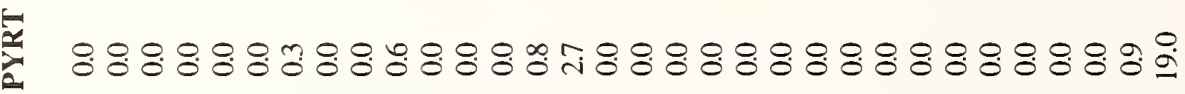

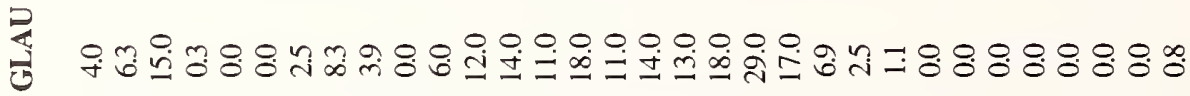

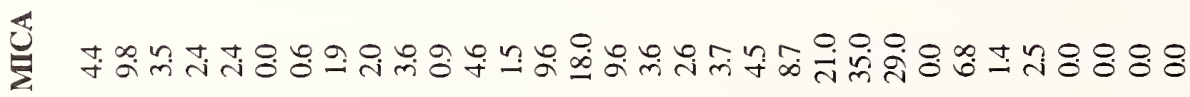

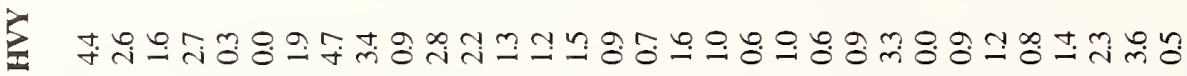

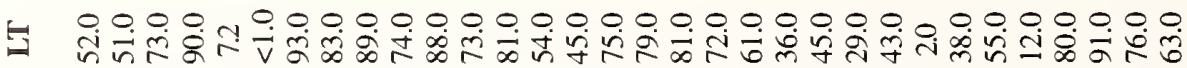

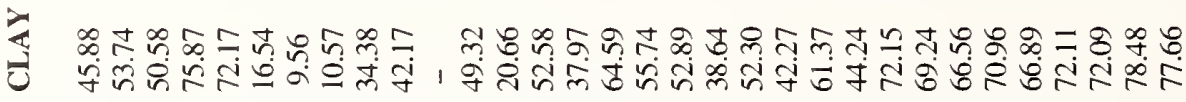

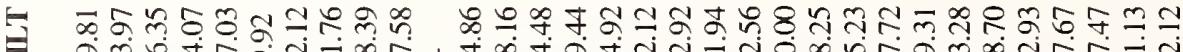

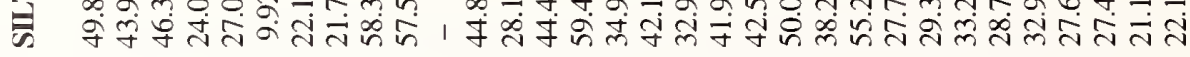

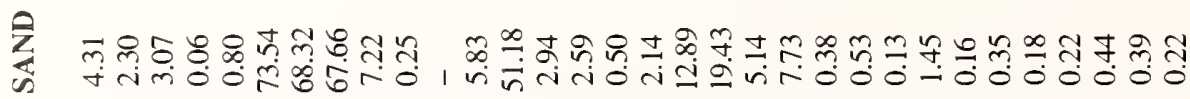

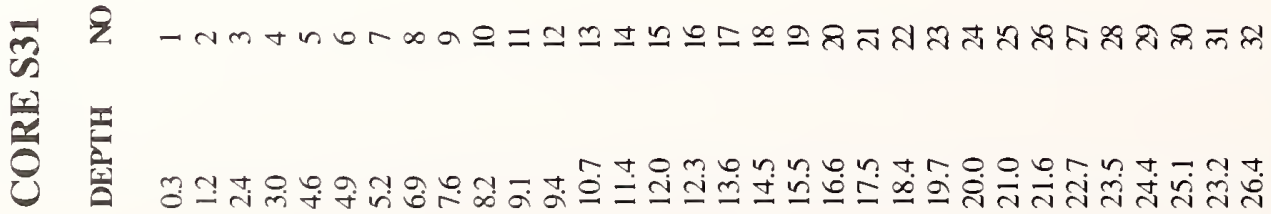


APPENDIX 2.-Continued.

突 $88: 8: 8$

苛

育 $808: 88$

帒 $8: 8: 8: 8$

$8: 8: 88$

$8: 88.8$

车 888888

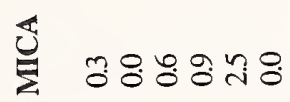

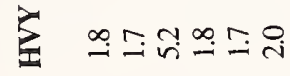

5

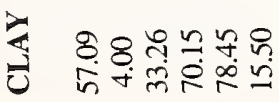

菌

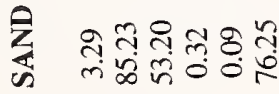

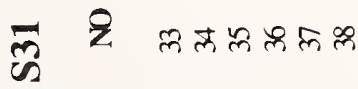

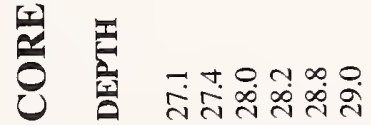


APPENDIX 2.-Continued.

Z

$$
\begin{aligned}
& 2 \\
& 1 \\
& +1 \\
& 0 \\
& 0 \\
& 0 \\
& m
\end{aligned}
$$

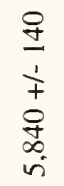

$\begin{array}{ll}\varrho & q \\ \frac{9}{1} & \frac{9}{1} \\ +1 & + \\ 0 & 0 \\ 0 & 0 \\ 0 & 0\end{array}$

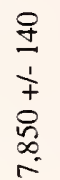

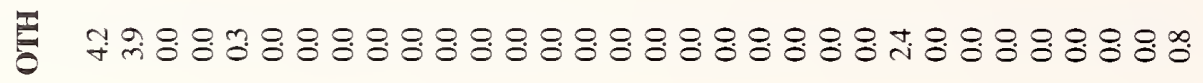

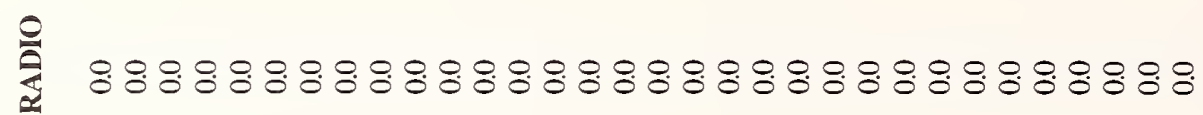

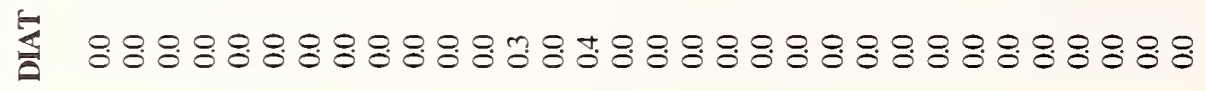

: $8: 8: 8: 8: 8: 8: 8: 8: 8: 8: 8: 8: 8: 8: 8: 8:$

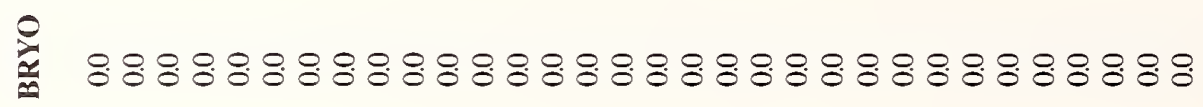

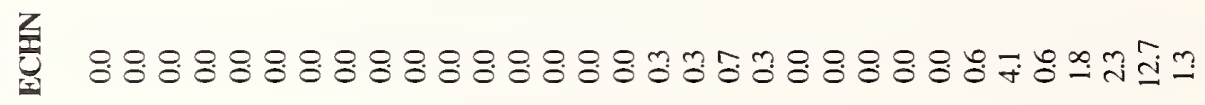

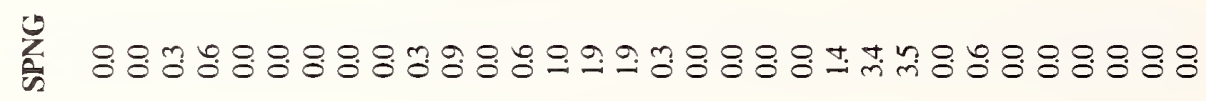

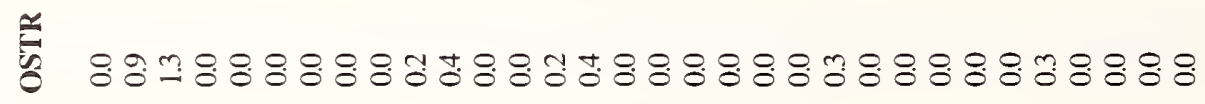

舅

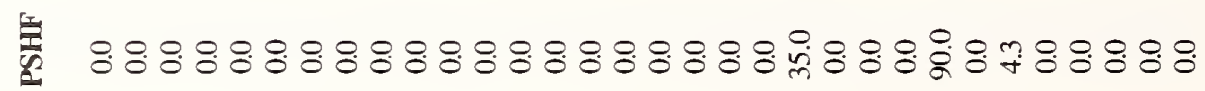

遂

悲 $8: 8: 8: 8: 8: 8: 8: 8: 8: 8: 8: 8: 8: 8: 8: 8: 0$

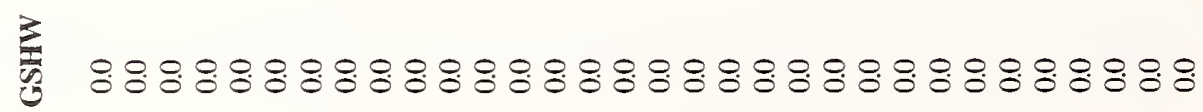

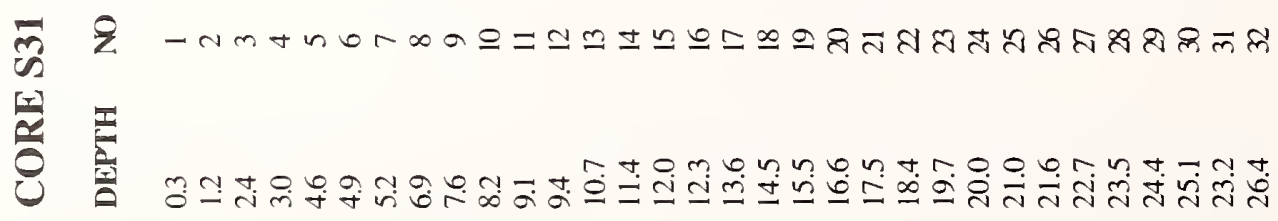


APPENDIX 2.-Continued.

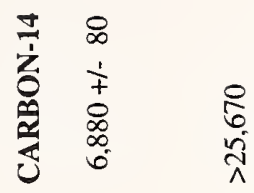

퐁 웅ㅇํㅇำำำ

은 $88.88 \%$

起 $8.808 \%$

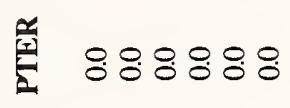

읍 8.88 .80

宓 $8.888:$

究 3.8 .88 .8

\%

온 8088808

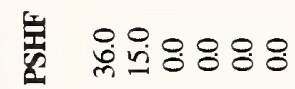

28.88:

웅용ㅇㅇㅇ

운 융용

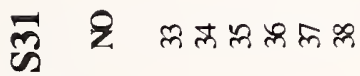

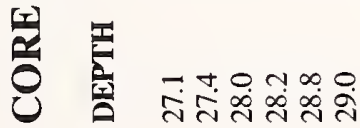


APPENDIX 2.-Continued.

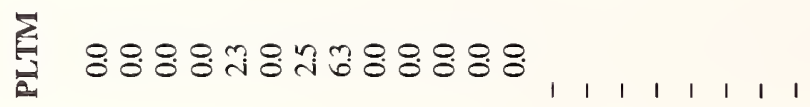

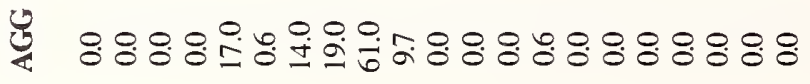

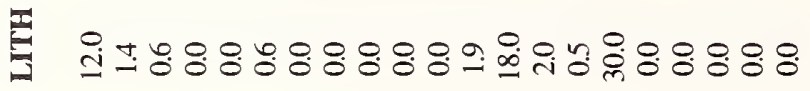

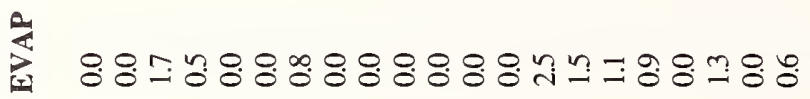

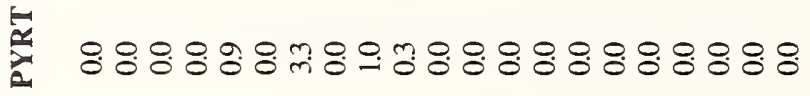

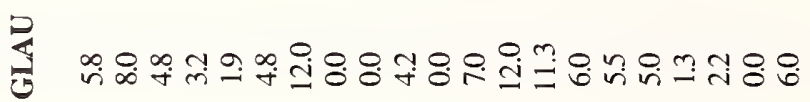

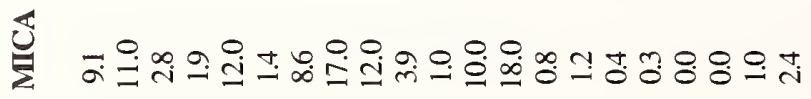

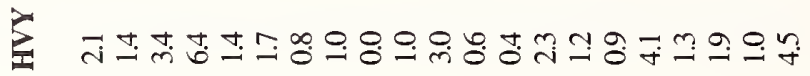

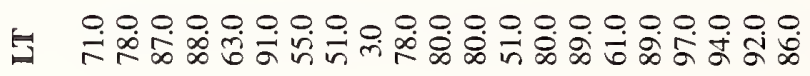

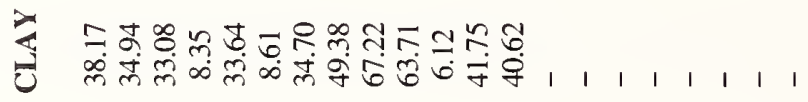

복

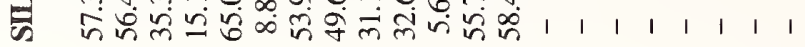

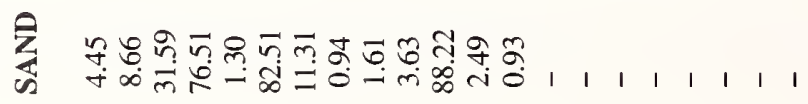

ข้ Z -

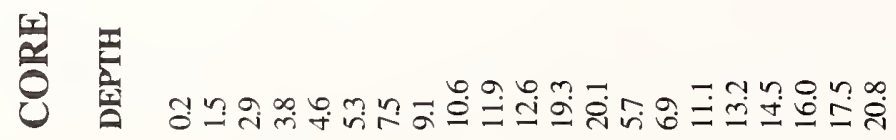


APPENDIX 2.-Continued.

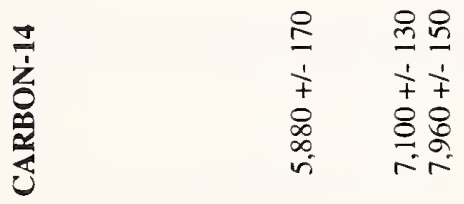

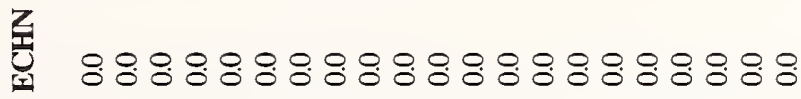

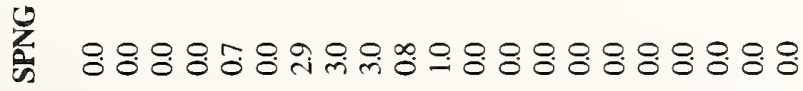

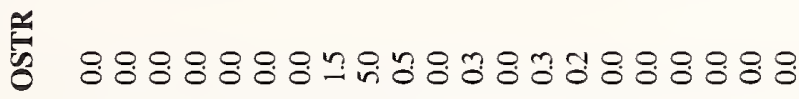

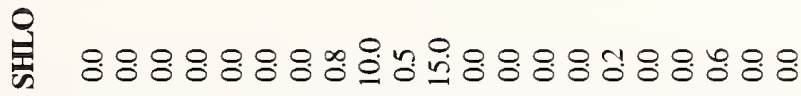

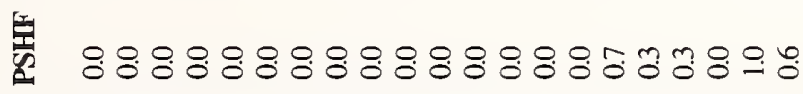

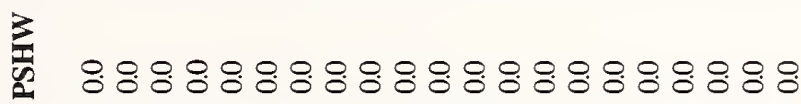

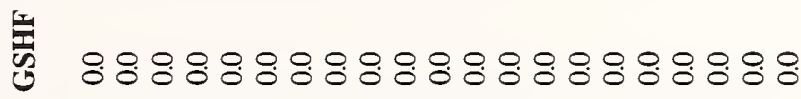

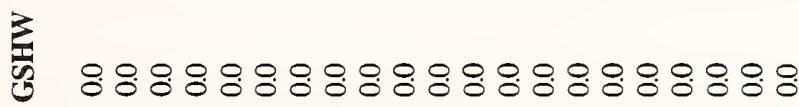

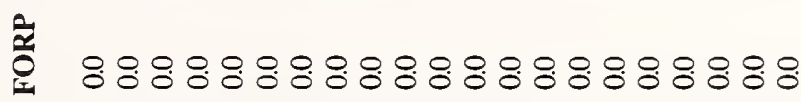

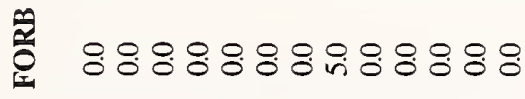

กี่ \& -

잉 동

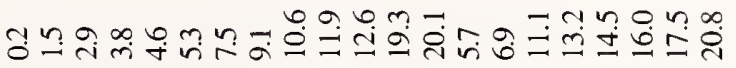


APPENDIX 2.-Continued.

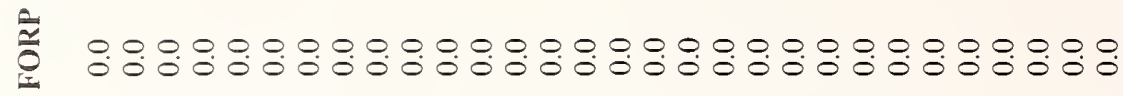

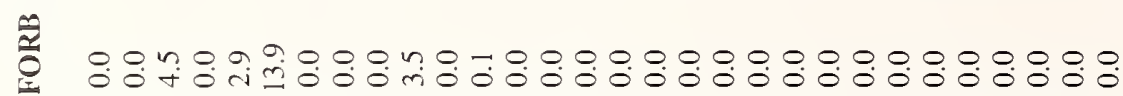

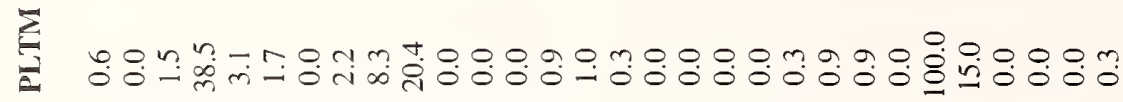

选

帠

这

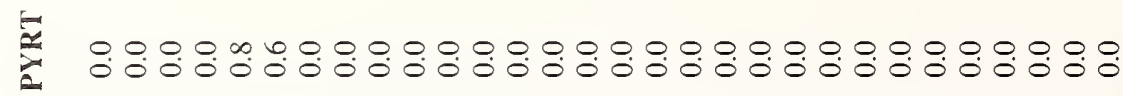

屬

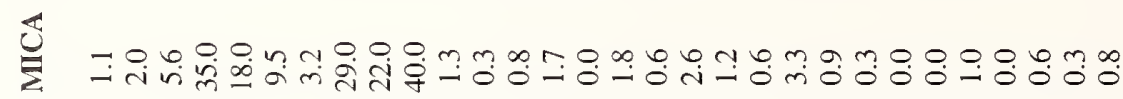

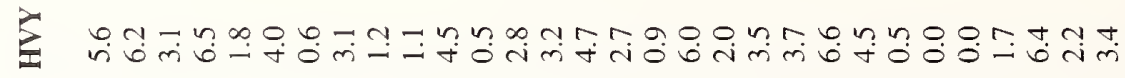

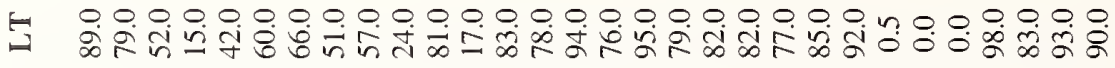

ᄂ

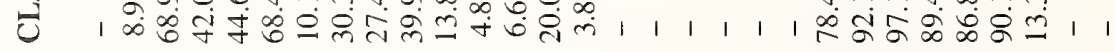

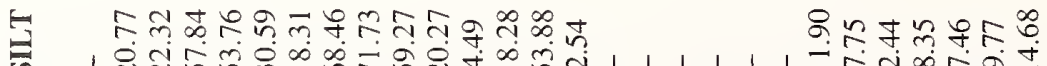

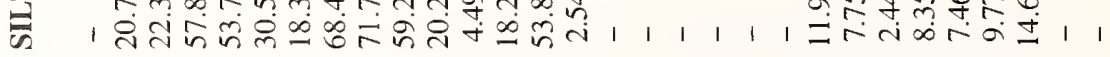

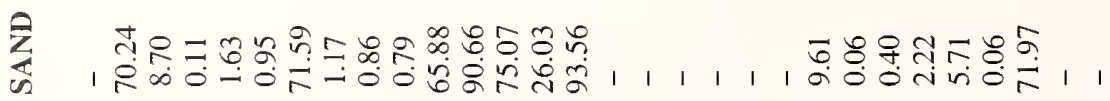

m Z

曾 
APPENDIX 2.-Continued.

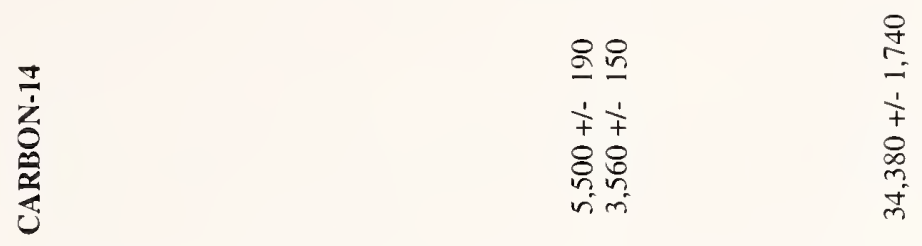

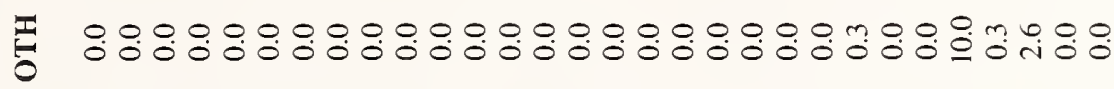

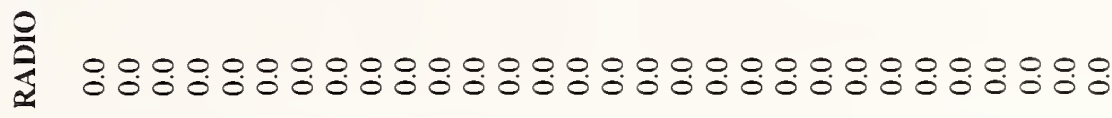

th

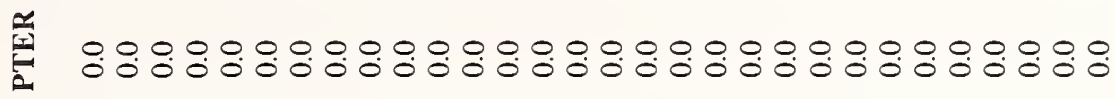

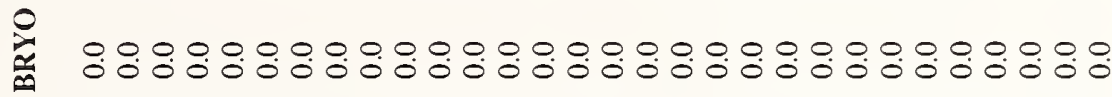
Z

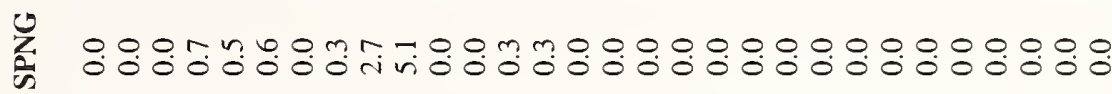

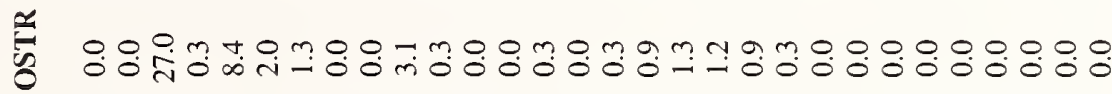
敬 는

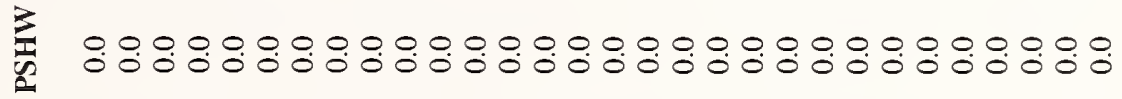

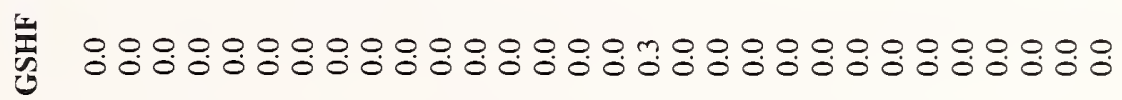

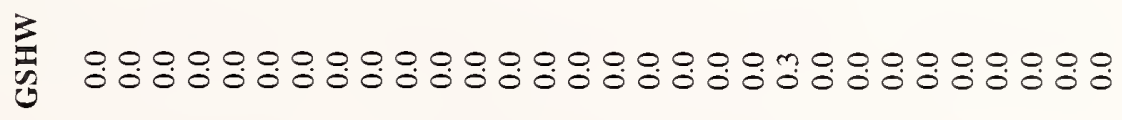

Øู่ 됭 
APPENDIX 2.-Continued.

空

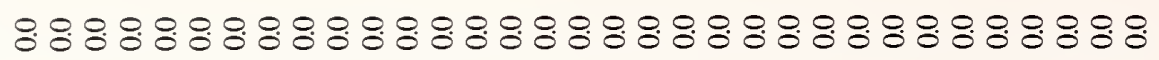

范

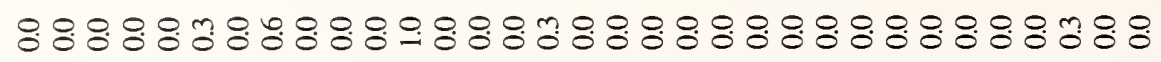

空

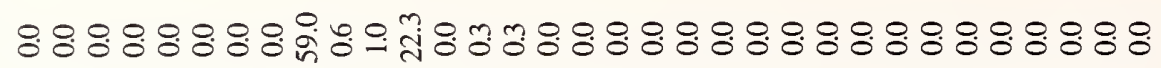

过

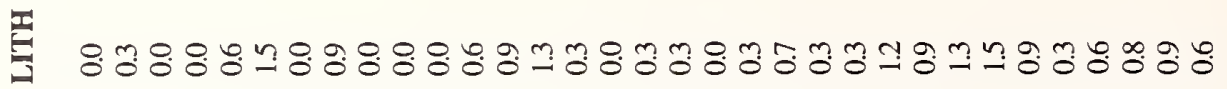

选

:

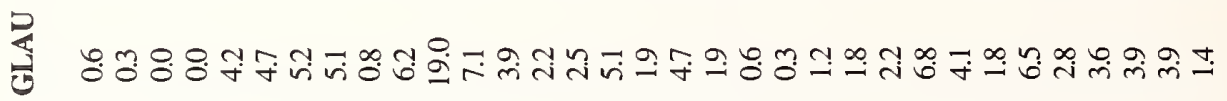

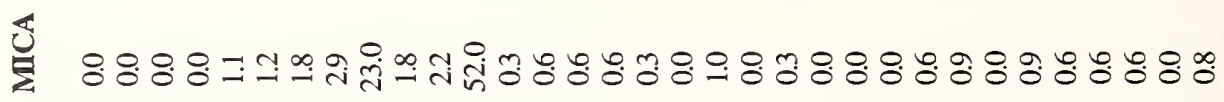

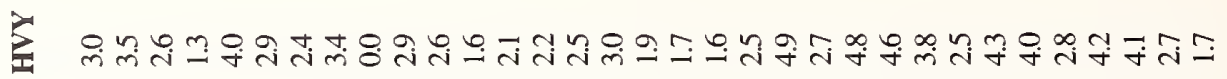

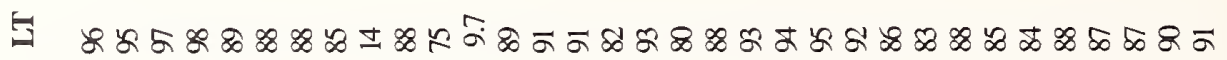

过

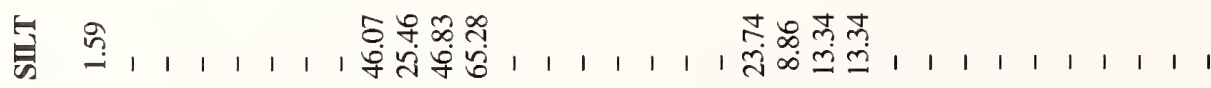

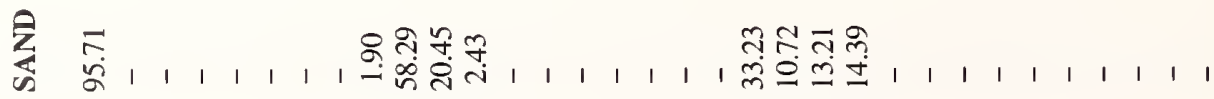

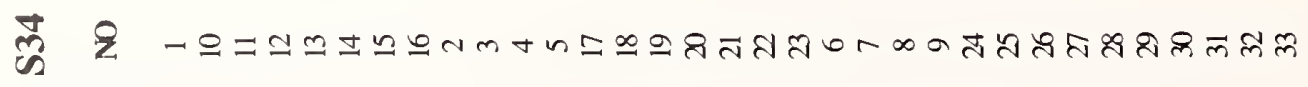

동

nom

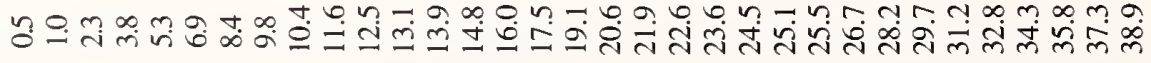


APPENDIX 2.-Continued.
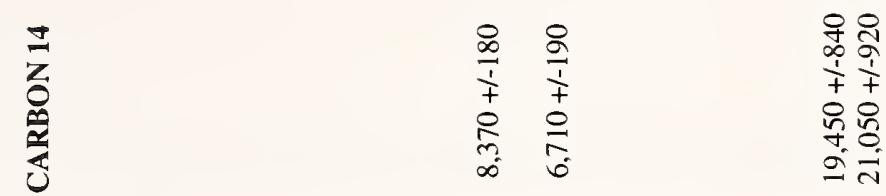

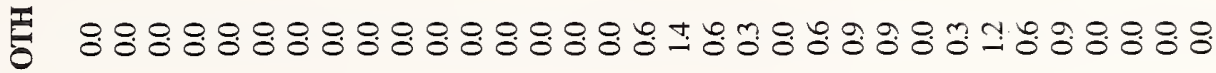

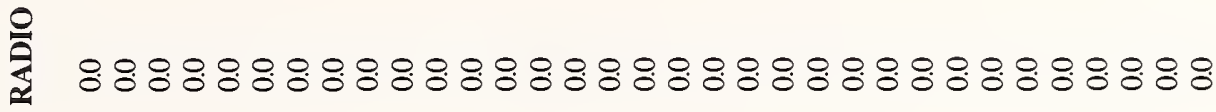
L $8: 8: 8: 8: 80: 8: 8: 8: 8: 8: 8: 8: 8: 8: 8: 8: 8$ L $8: 8: 8: 8: 8: 8: 8: 8: 8: 8: 8: 8: 8: 8: 8: 8: 8: 8: 8$

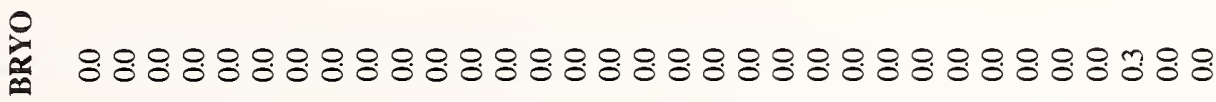

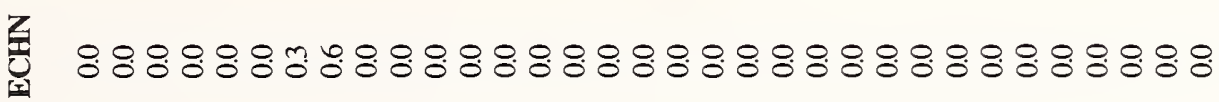

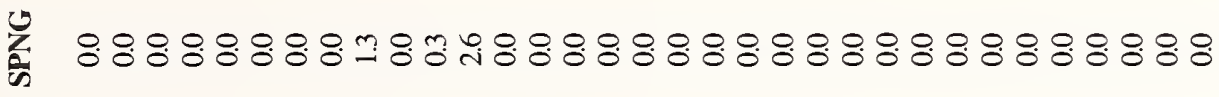

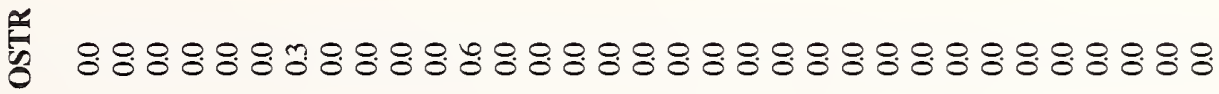

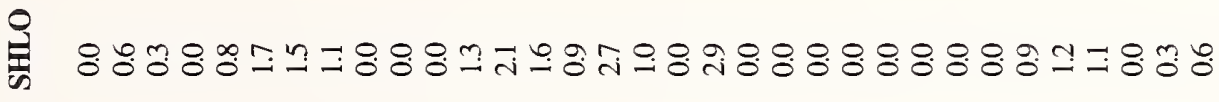

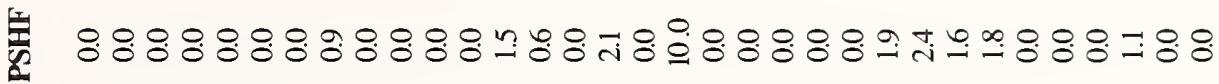

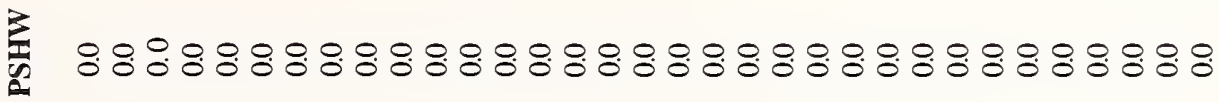

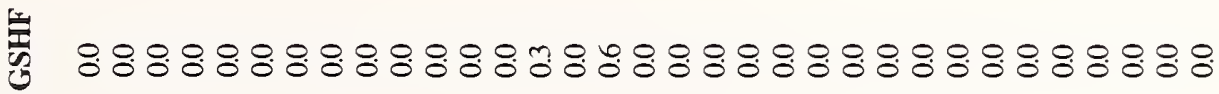
革 
APPENDIX 2.-Continued.

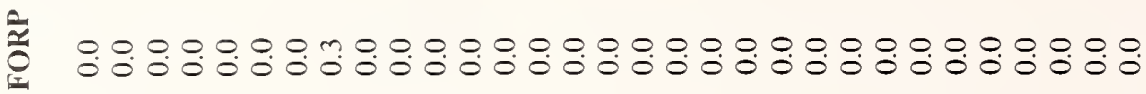

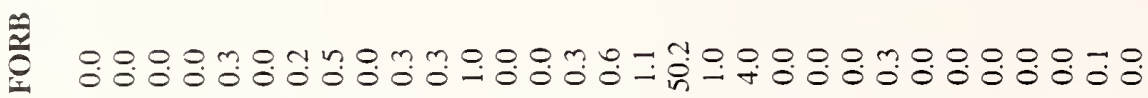

莫

过

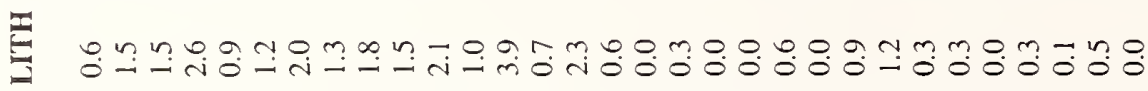

in

:

㐘

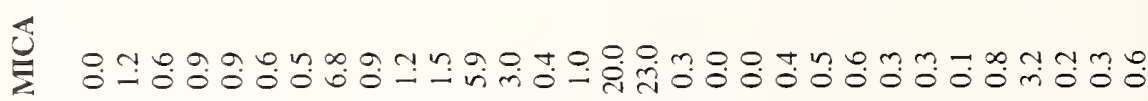

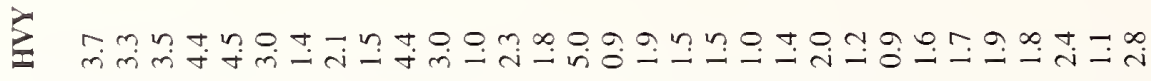

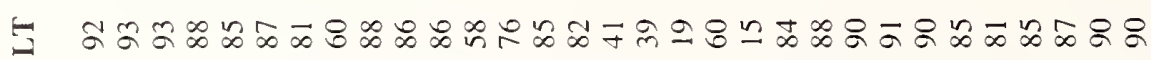

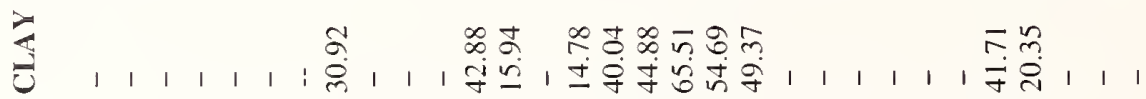

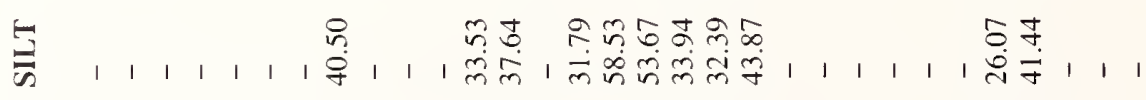

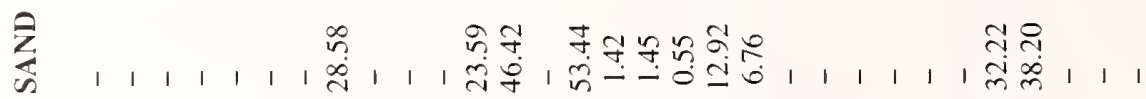

n?

至 
APPENDIX 2.-Continued.

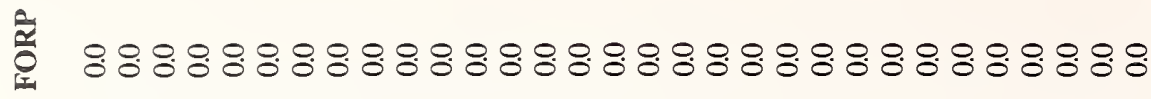

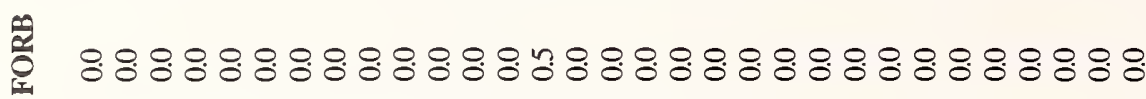

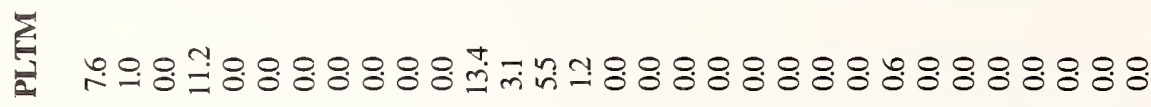

烍

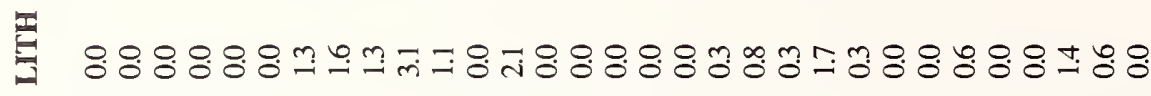

屈

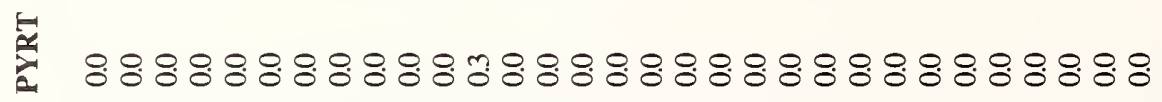

䓛

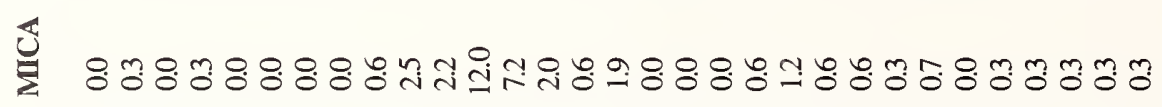

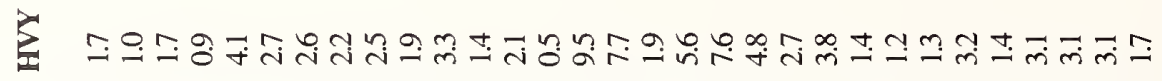

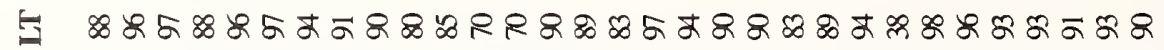

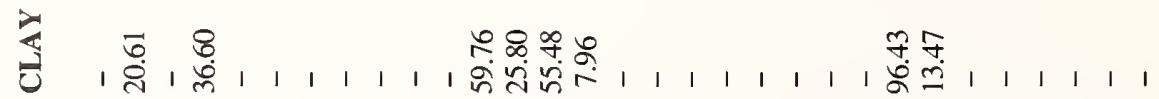

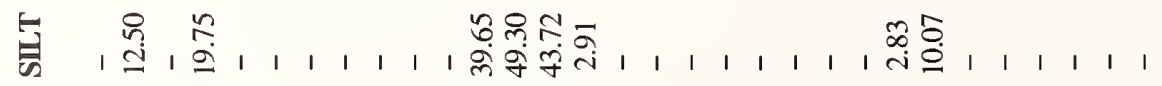

宓 1 量

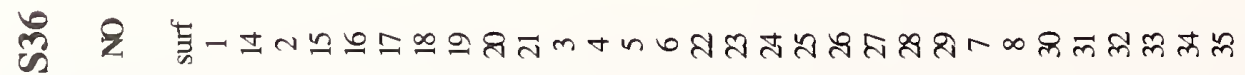

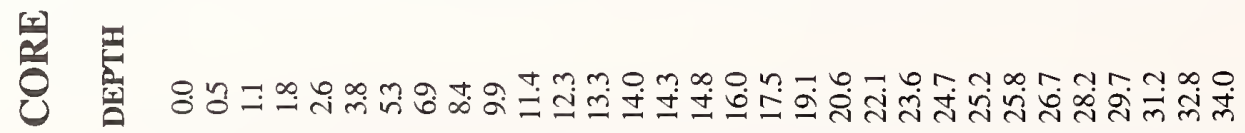


APPENDIX 2.-Continued.

突 $888: 88: 88: 8$

茓 $: 8: 8: 8: 8: 8:: 8$

골 88080008888888

帒 $8: 8 \%$ :

폭 888:888389.

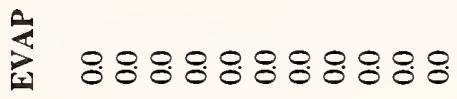

ะ $888: 8888888$

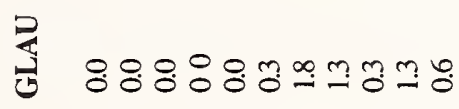

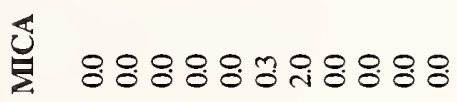

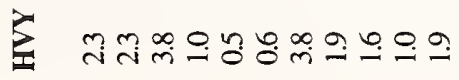

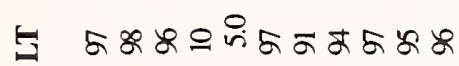

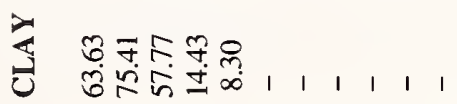

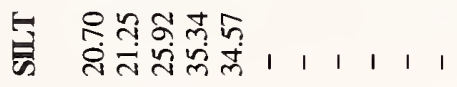

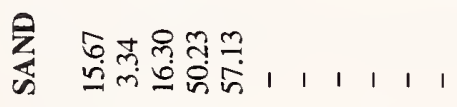

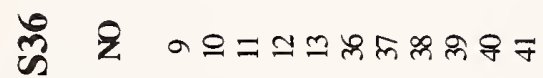

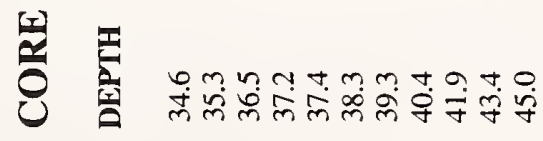


APPENDIX 2.-Continued.

$\frac{ \pm}{2}$

$\stackrel{0}{1}$
1
+
0
0
1

8
0
$\frac{1}{+}$
0
i
i

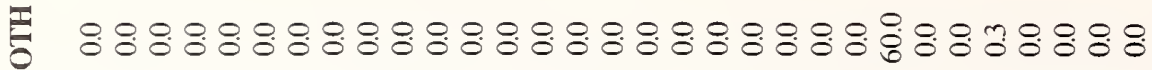

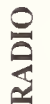

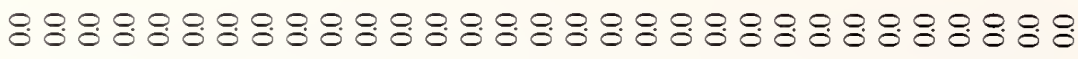

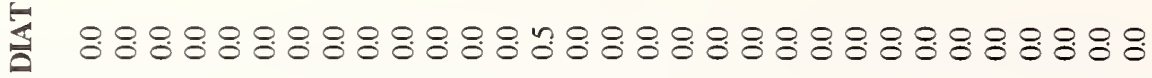

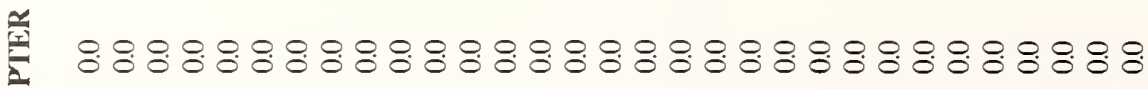

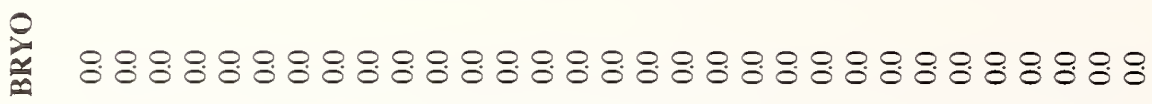

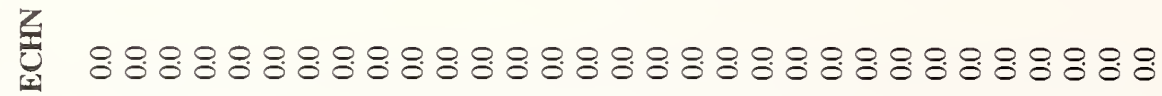

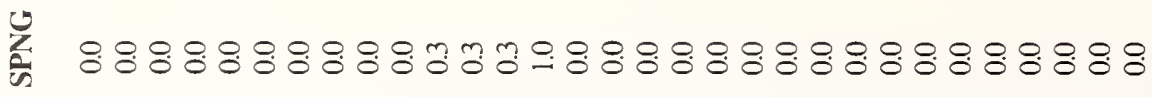

产

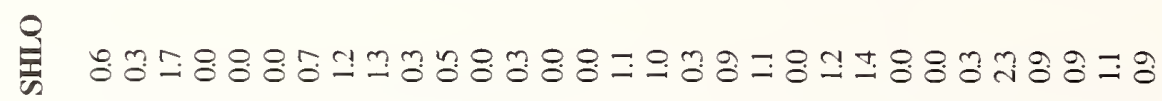

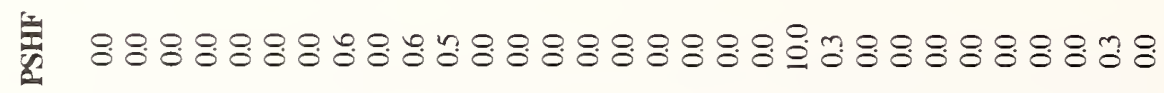

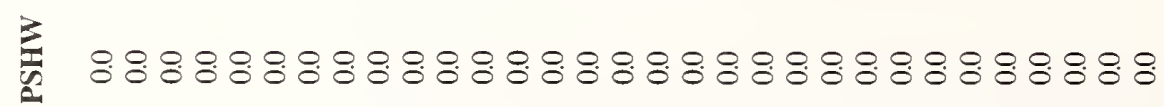

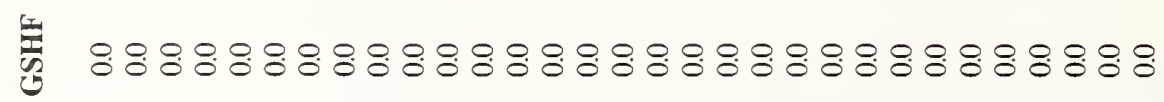

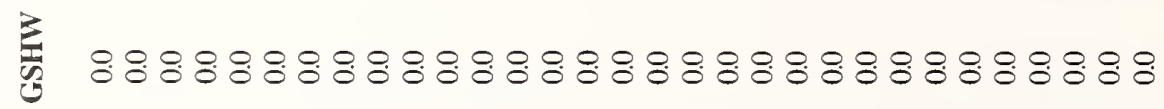

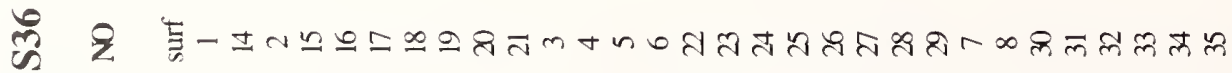

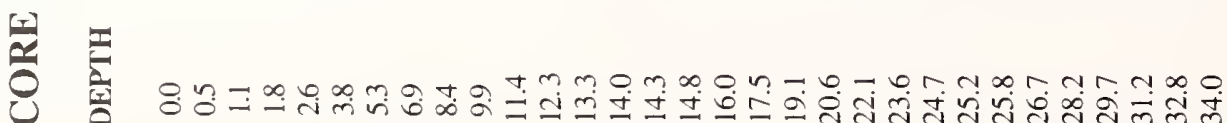


APPENDIX 2.-Continued.

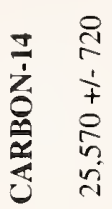

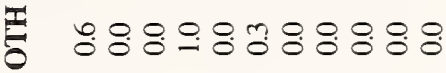

童 88888888888

产 $8888: 888: 88: 8$

尊 888888888888

产 $8: 8: 88: 88: 8:$

㟤 $888: 88888: 88$

粍 888888888888

善 88898887888

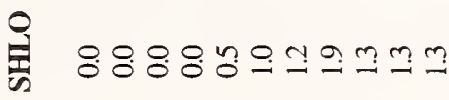

爵 88888888888

屡 88888888888

衰 $88888: 888: 8$

屡 $8: 88: 8: 8: 8: 8$

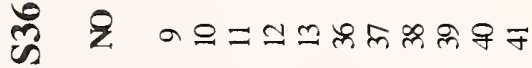

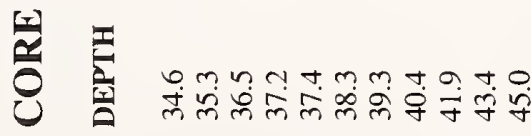


APPENDIX 2.-Continued.

중

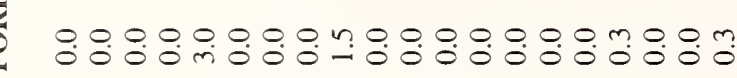

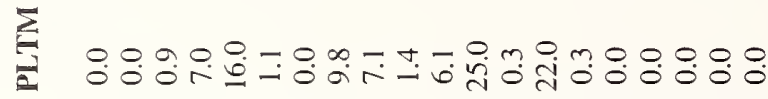

选

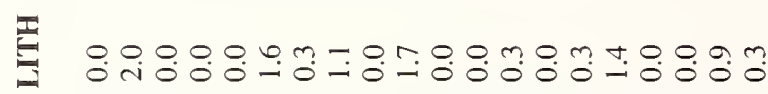

通

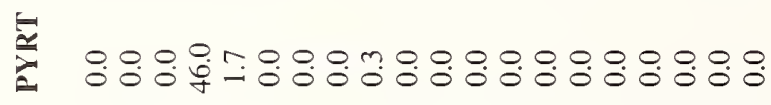

造

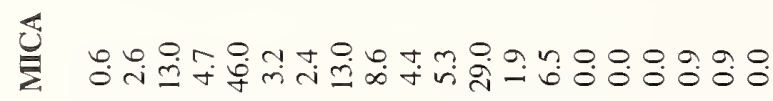

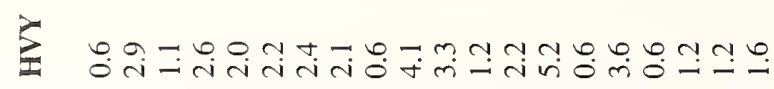

క

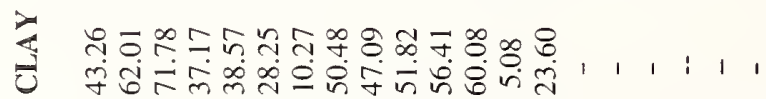

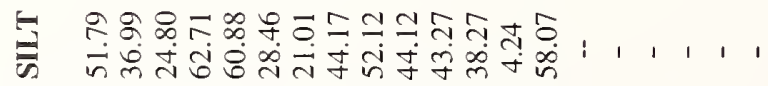

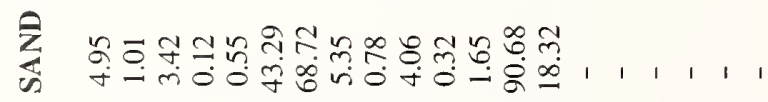

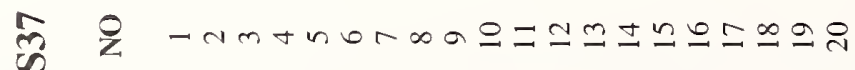

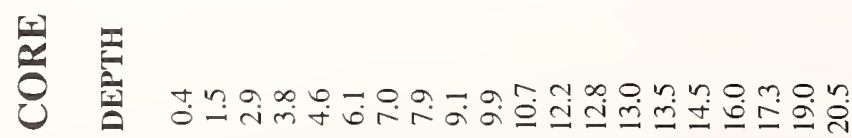


APPENDIX 2.-Continued.

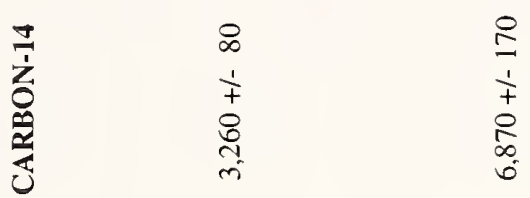

至

荒

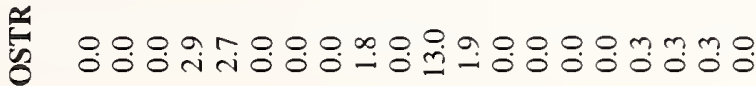

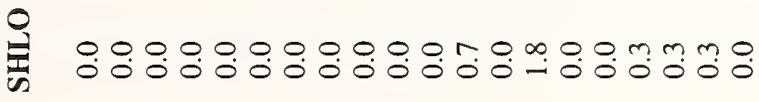

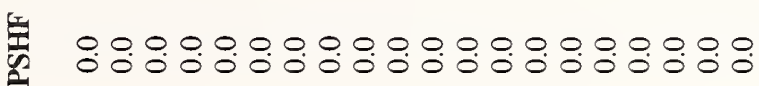

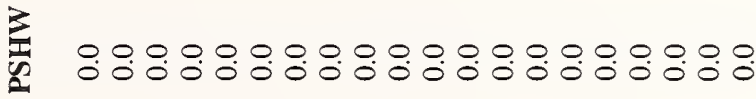

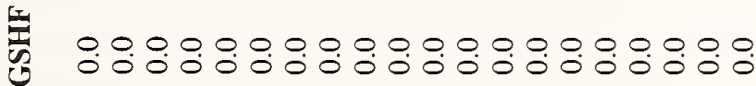

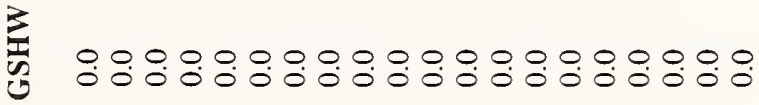

产

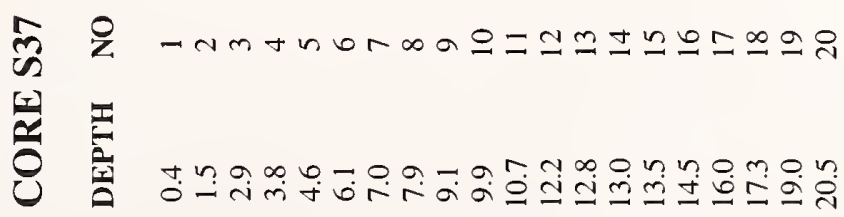


APPENDIX 2.-Continued.

홍

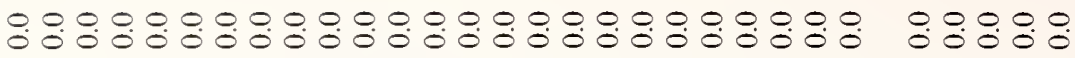

華

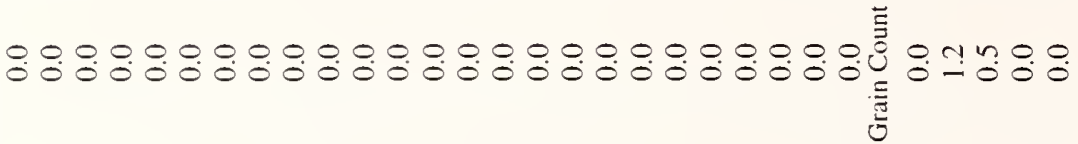

j

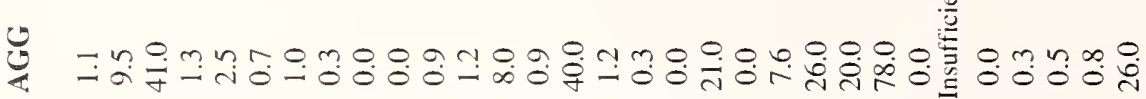

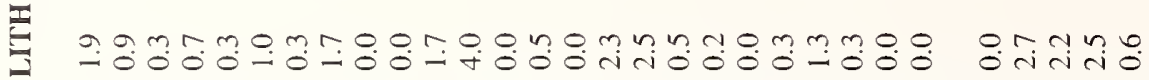

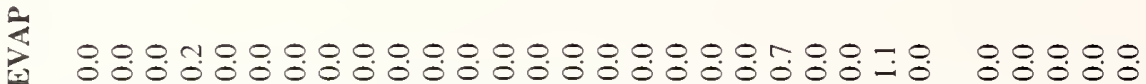

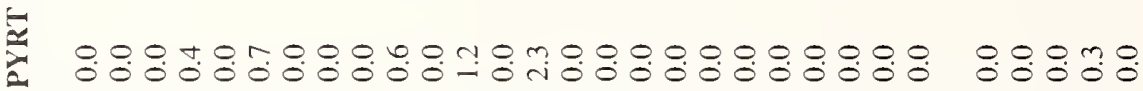

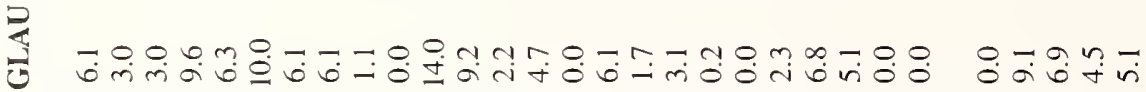

送

至

J

ङ

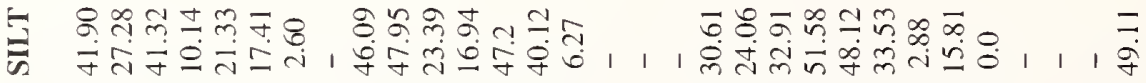

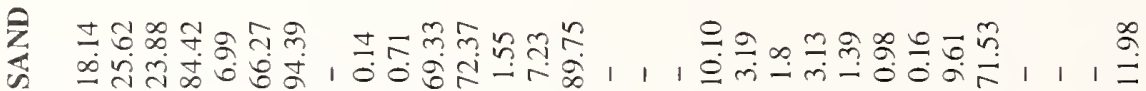

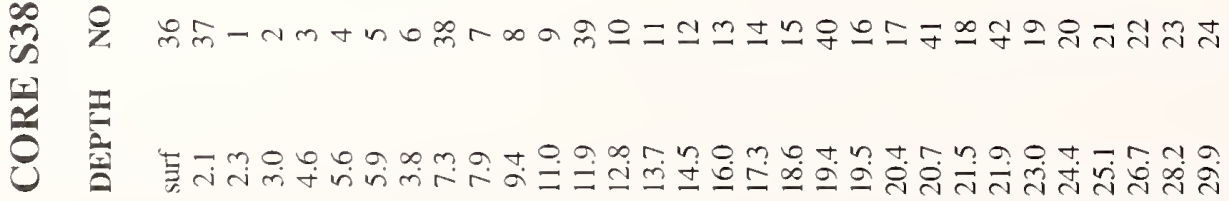




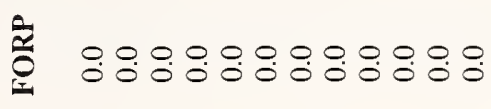

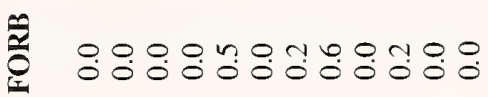

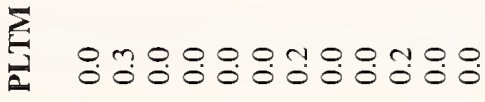

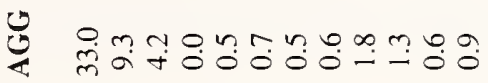

ప

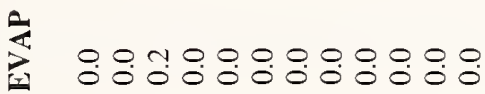

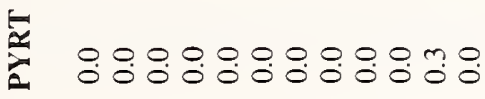

J

J

¿

= $\quad \begin{aligned} & 0 \\ & \text { అ }\end{aligned}$

¿ ำ

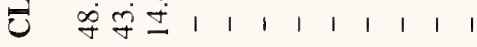

光

宓

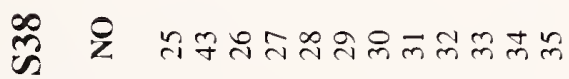

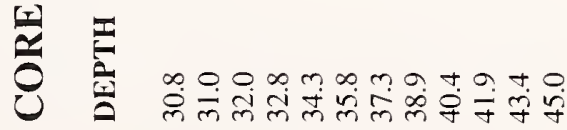


APPENDIX 2.-Continued.

$\frac{\square}{\frac{z}{2}}$

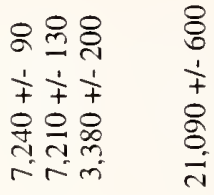

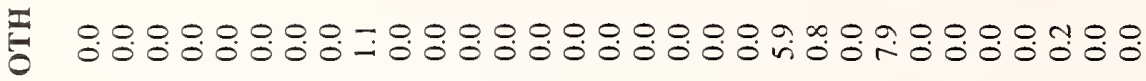

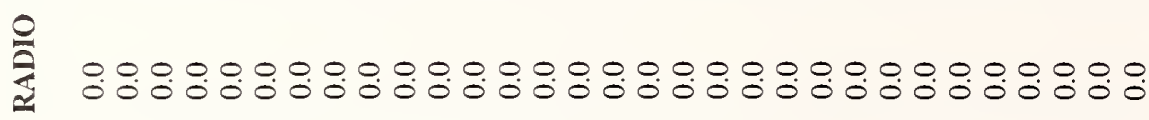

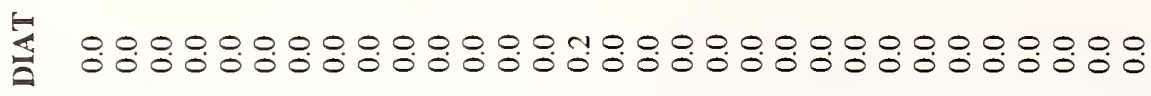

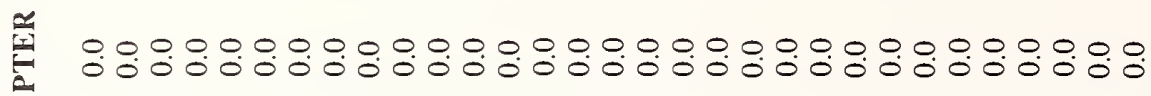

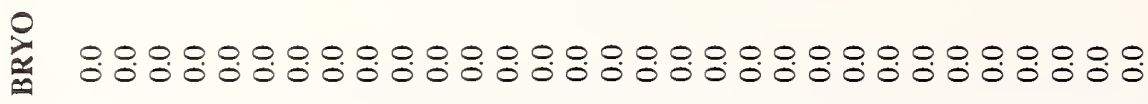

Z

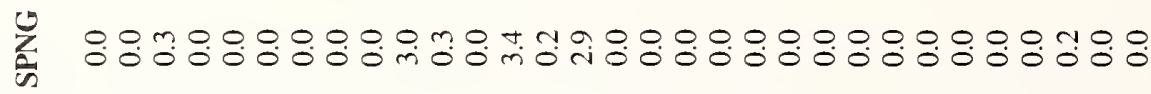

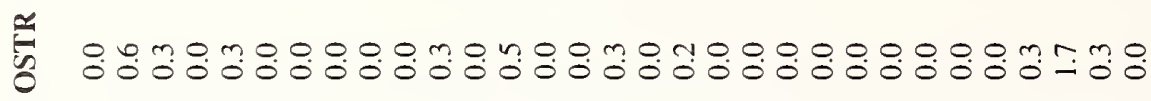

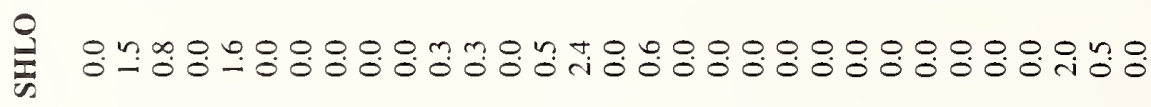

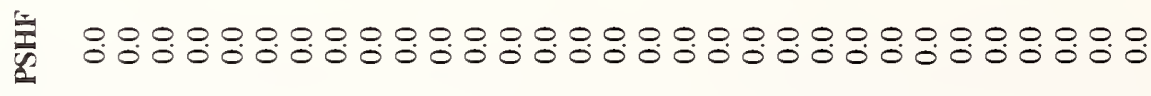

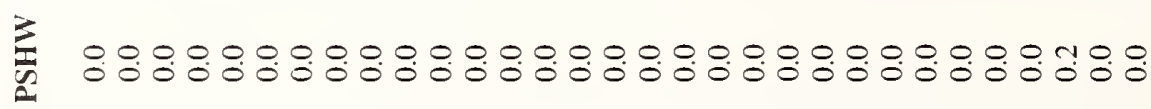

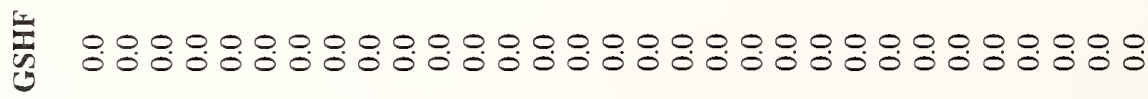

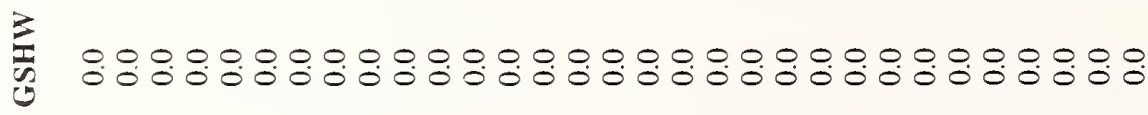

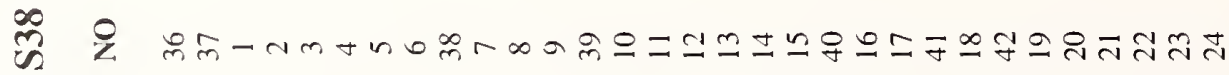

彭 


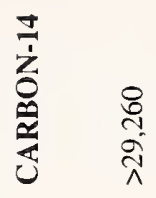

웅영용용요

鹿

这

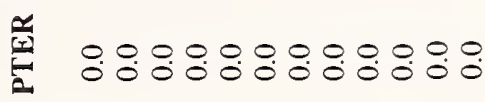

읍

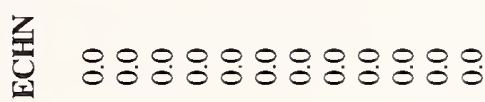

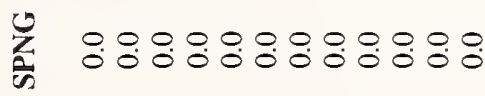

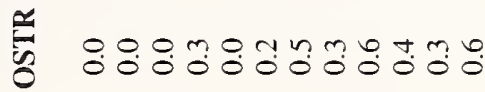

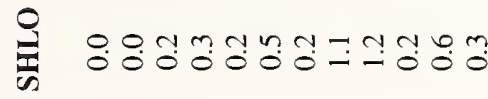

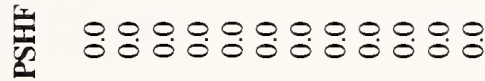

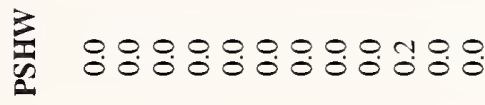

연

: $0: 0: 0: 0: 0: 0: 0$

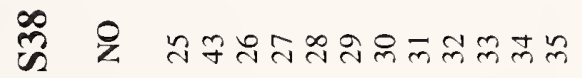

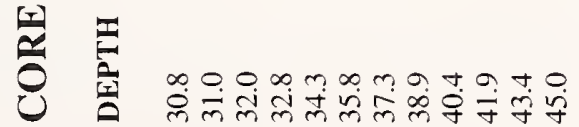


APPENDIX 2.-Continued.

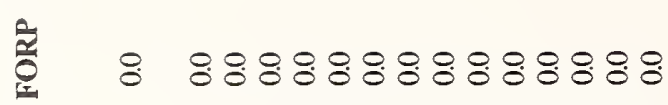

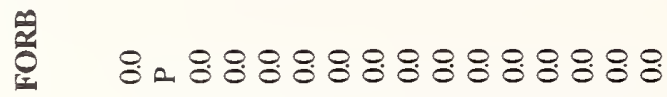

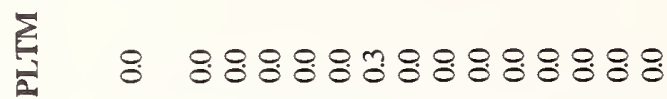

乐

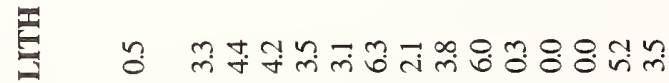

度

: $883888: 88: 88: 8$

窉

送

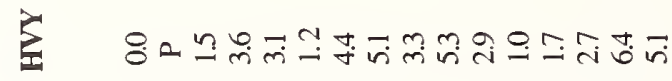

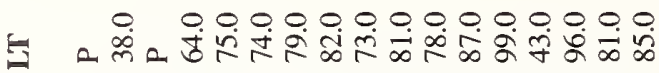

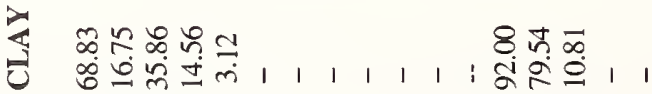

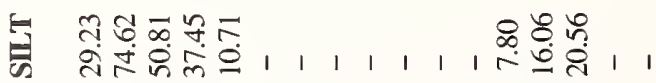

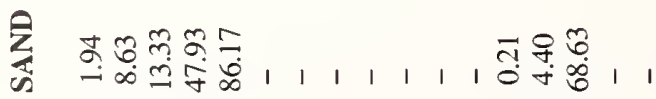

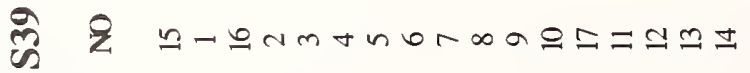

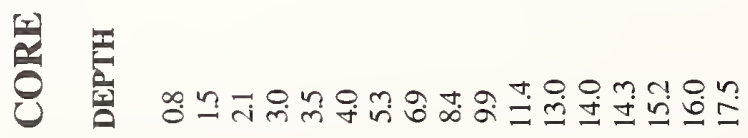


APPENDIX 2.-Continued.

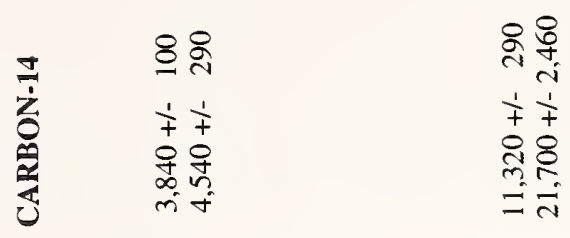

7. $88888: 88: 888: 88$

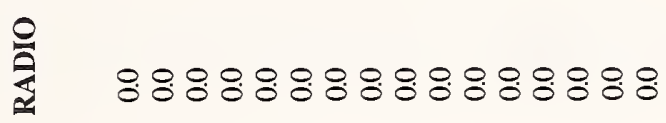

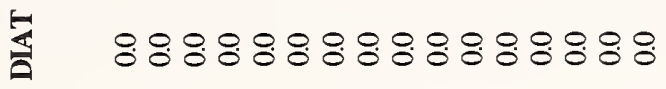

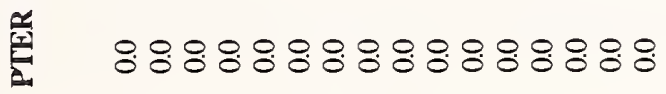

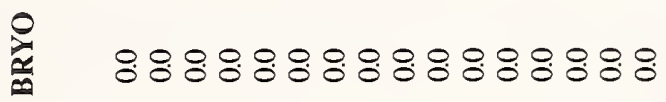

望 8888888888888888

玄 $8: 8: 8: 8: 8: 8: 8: 8: 8$

n

基 8.888 .88 .88 .8888 .8

: $8: 8: 8: 8: 8: 8: 8: 8: 8: 8$

88888888888888888

妍 $8: 8: 8: 8: 8: 8: 8: 8: 8:$

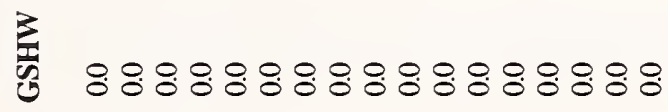

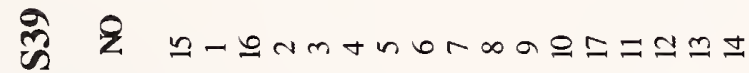

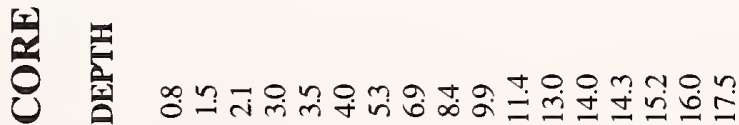


APPENDIX 2.-Continued.

융

尊 $8.88 .88 \% 8 \% 88.8888 .88$

运

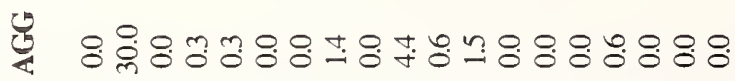

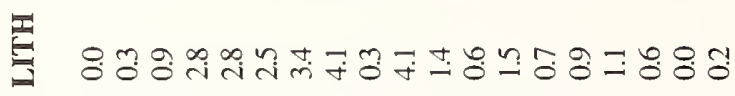

i

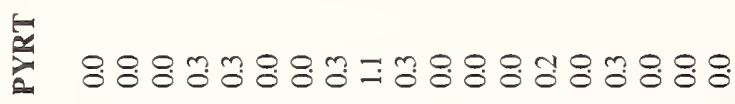

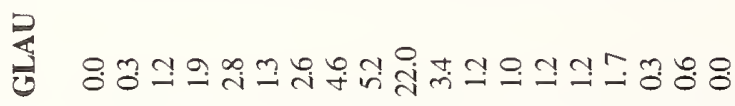

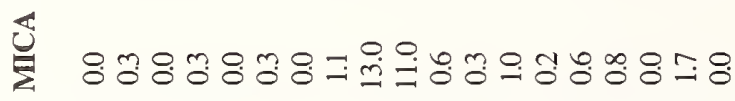

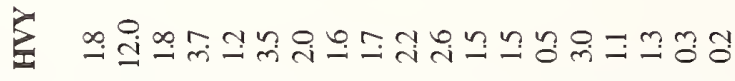

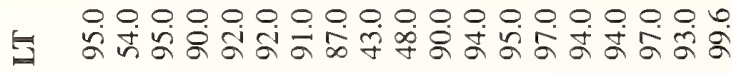

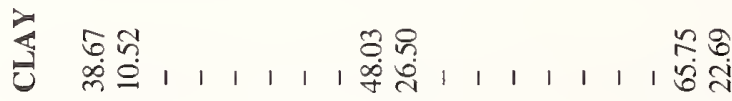

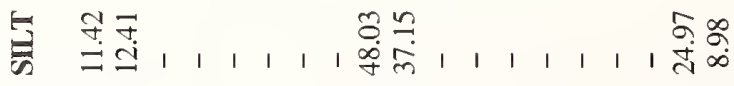

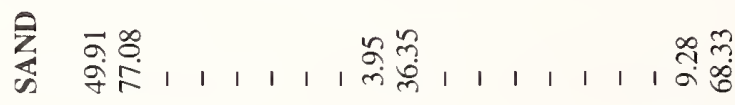

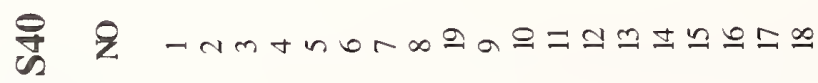

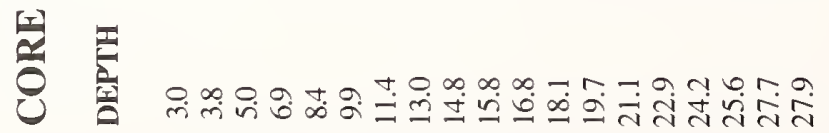


APPENDIX 2.-Continued.

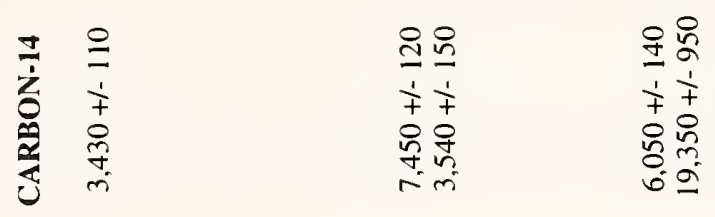

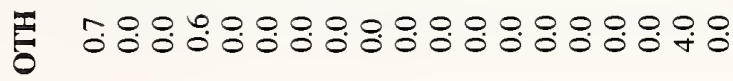

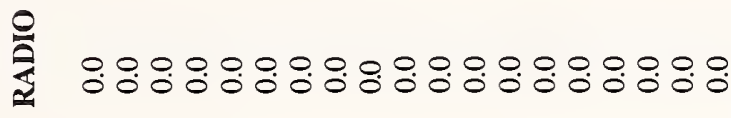

光

솔

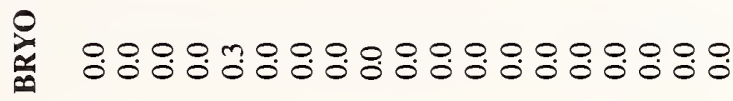

量

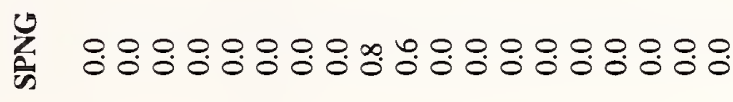

ร

曷

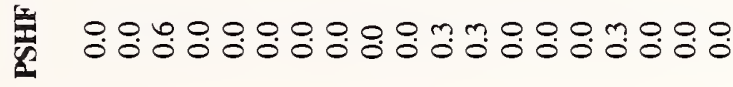

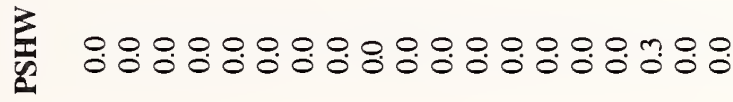

䆥

远

导 そ -

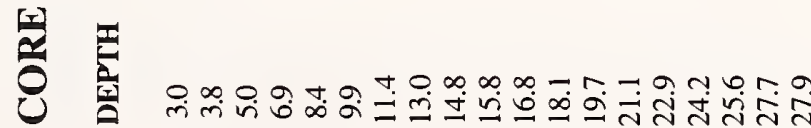


APPENDIX 2.-Continued.

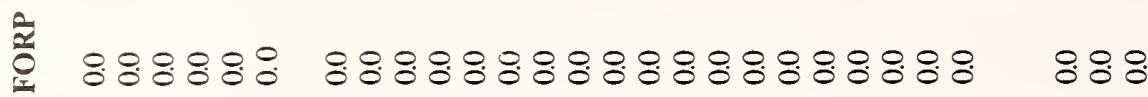

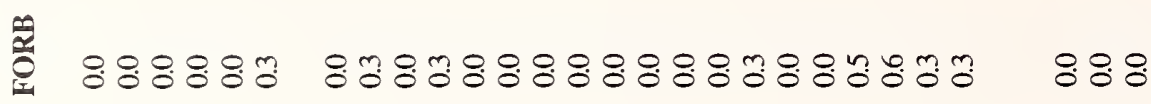

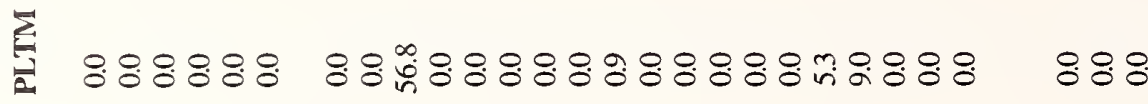

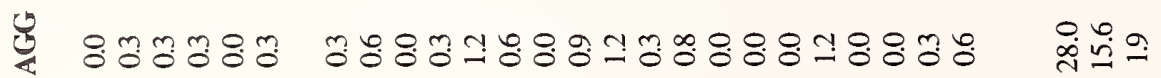

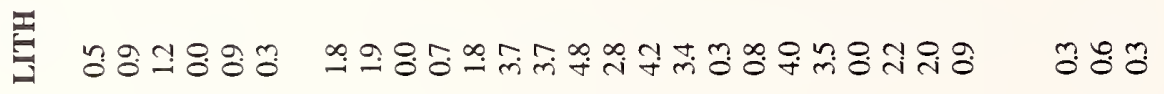

谣

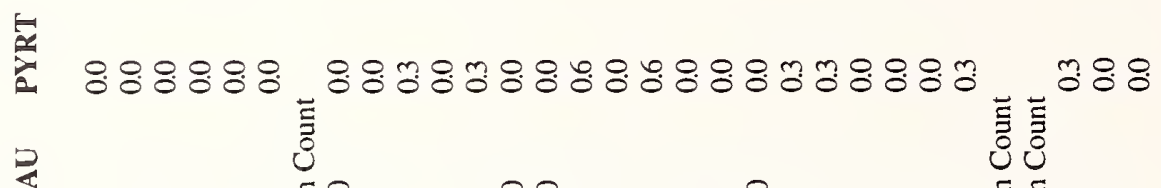

嵌 =

岁

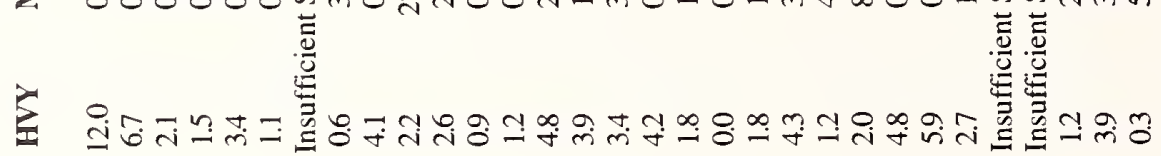

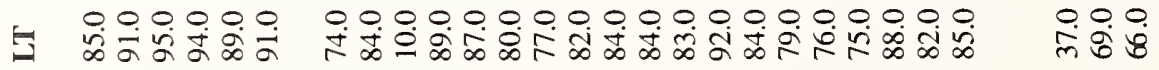

乡

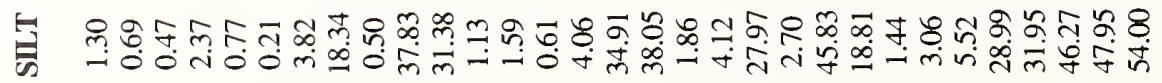

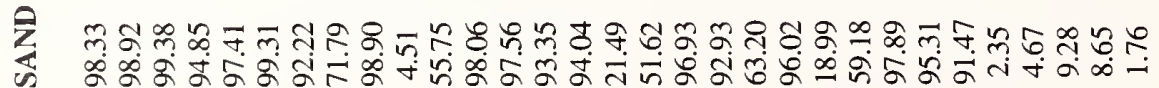

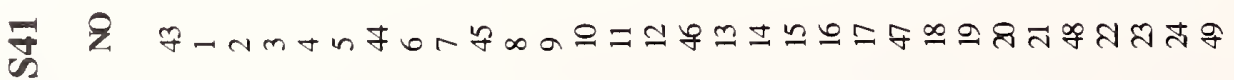

造

岁品 
APPENDIX 2.-Continued.

产 $8: 8: 8: 8: 8: 8: 8: 8: 8:$

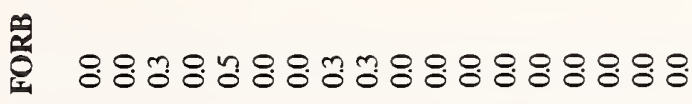

I $8: 8: 8: 8: 8: 8: 8: 8: 8:$

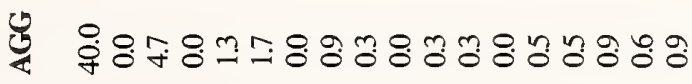

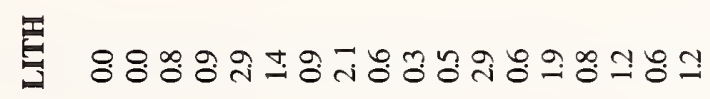

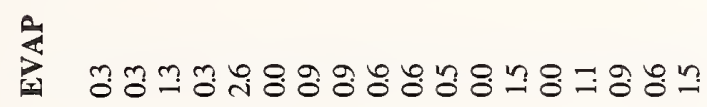

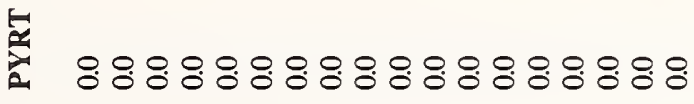

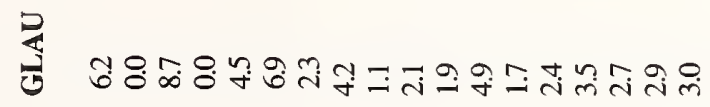

氙

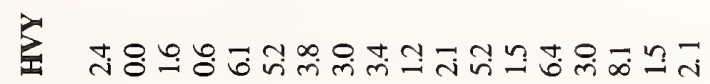

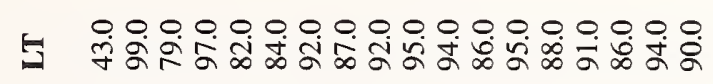

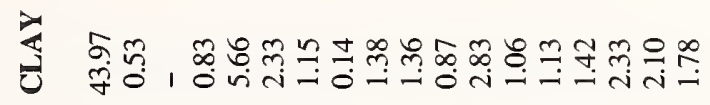

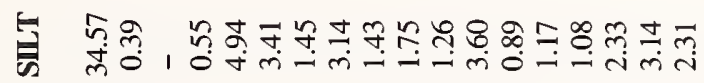

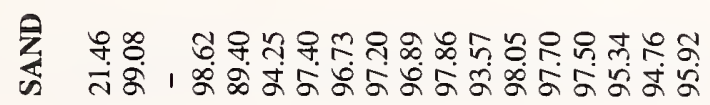

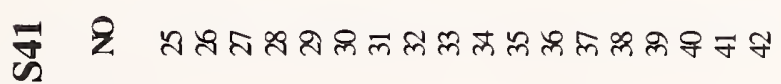

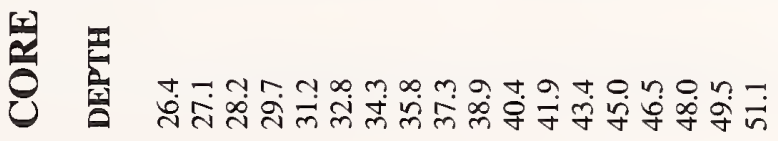


APPENDIX 2.-Continued.

突

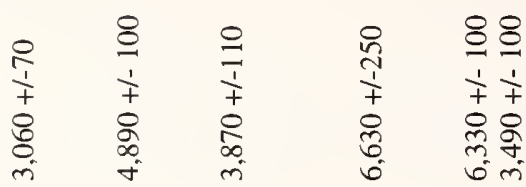

깅

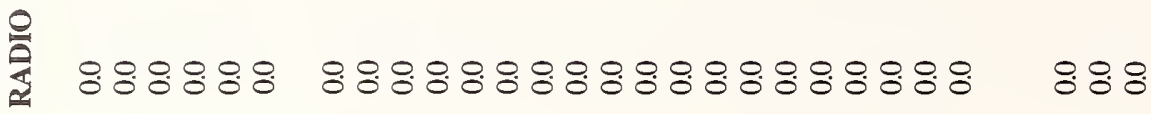

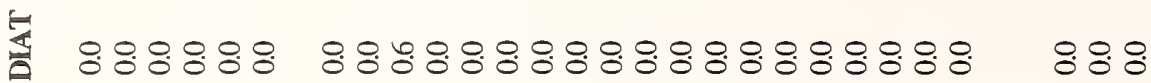

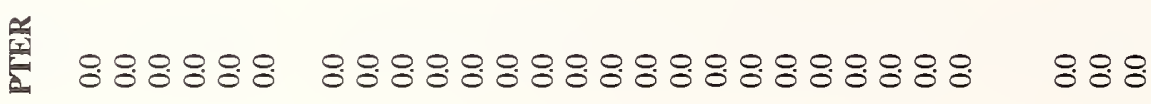

:

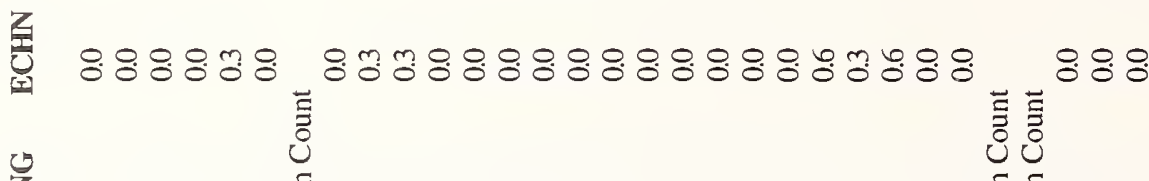

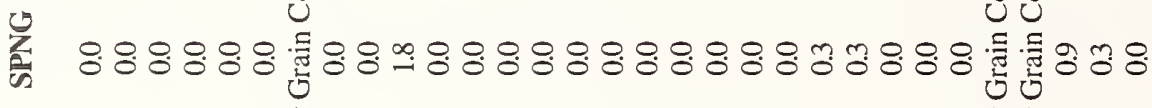

总

产

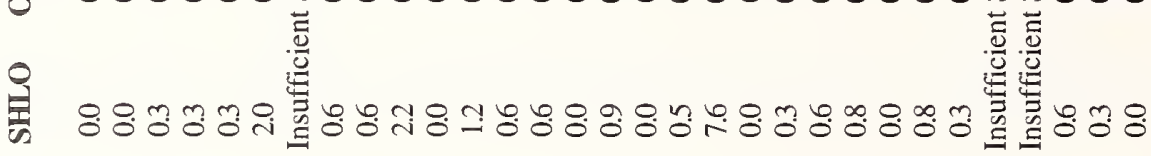

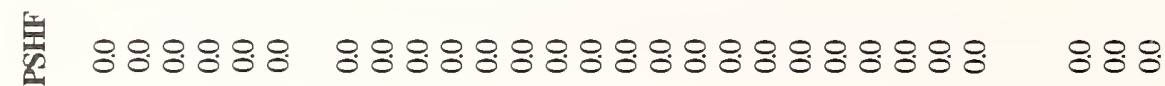

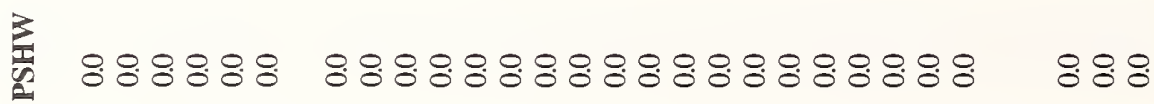

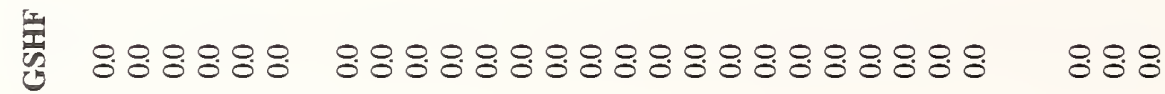

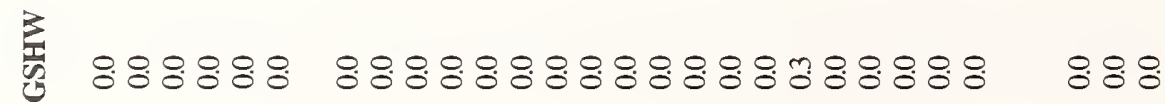

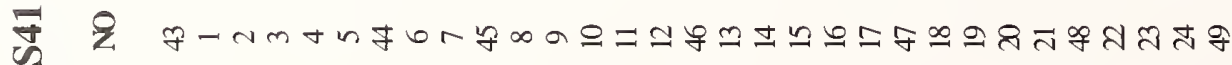

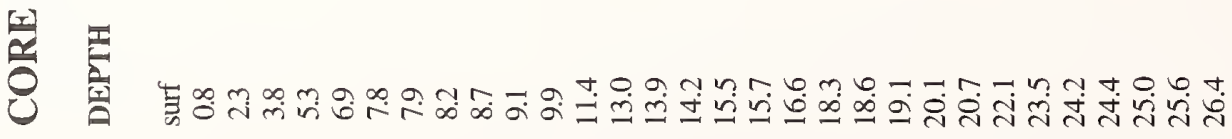


APPENDIX 2.-Continued.

竞

팡

产 $8: 8: 8: 8: 8 \%: 8: 8: 8 \%:$

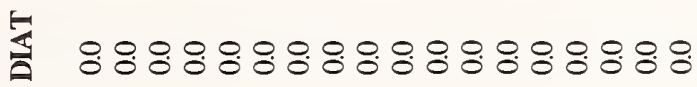

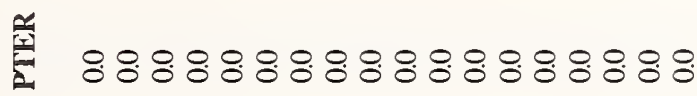

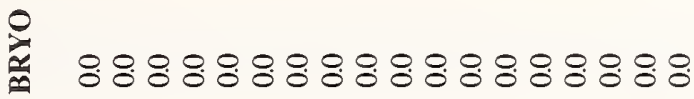

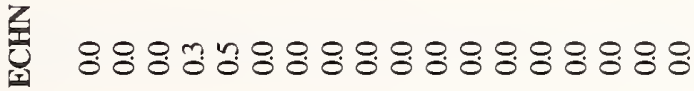

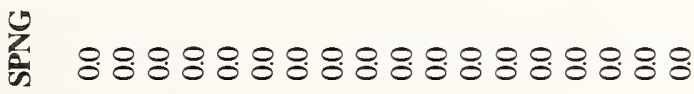

\%

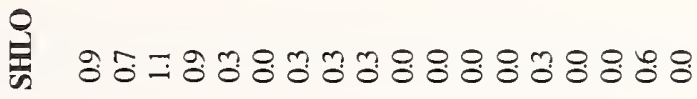

L

윤

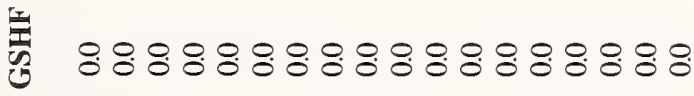

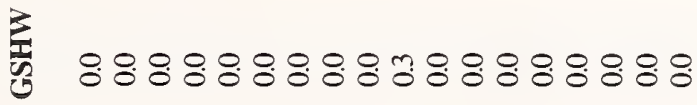

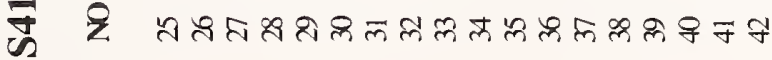

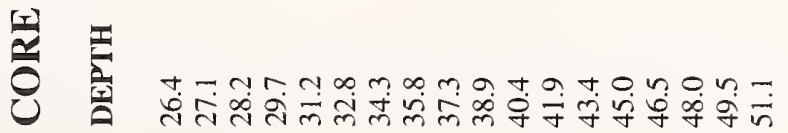


APPENDIX 2.-Continued.

총

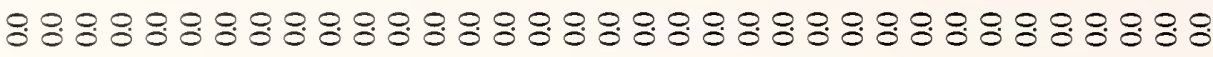

鱼

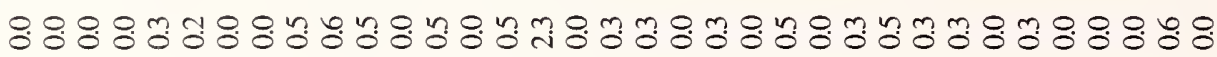

$\sum_{i=}$

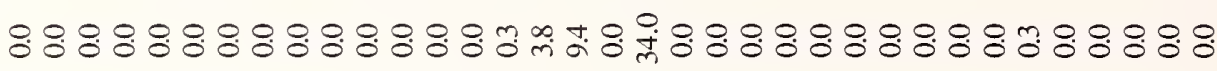

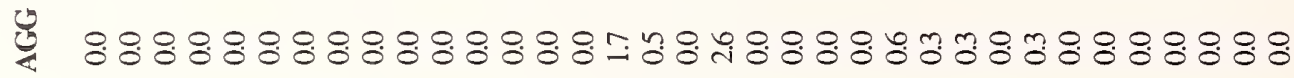

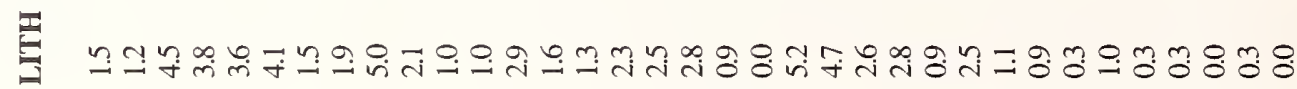

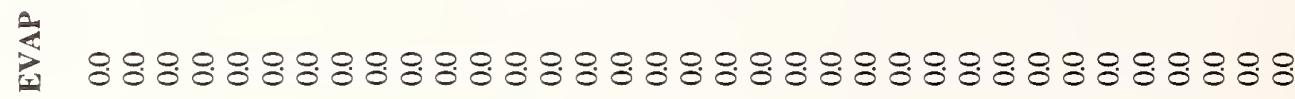

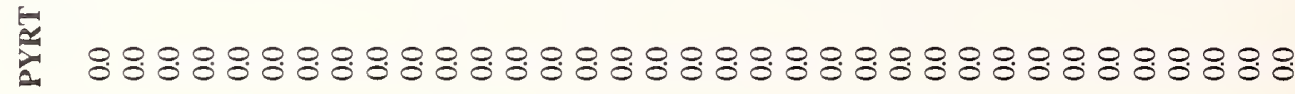

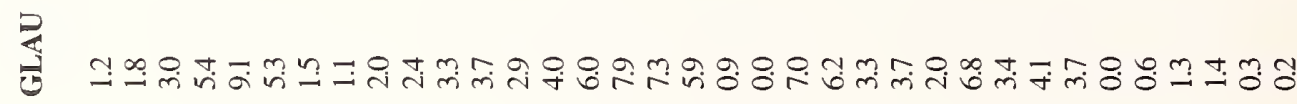

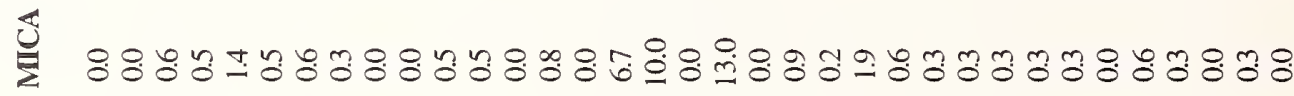

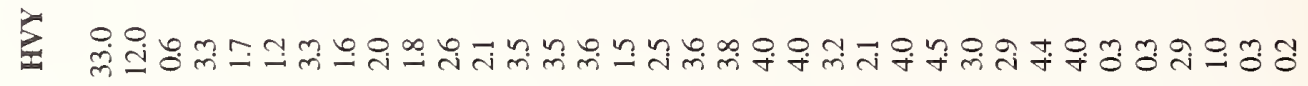

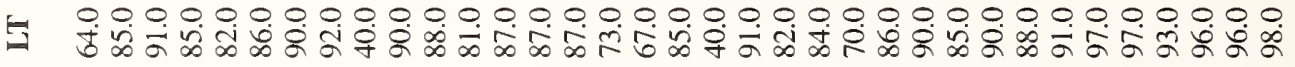

趟

岛

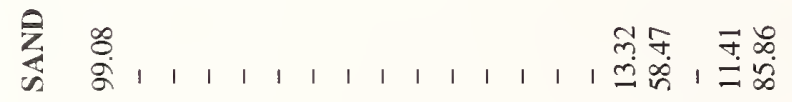

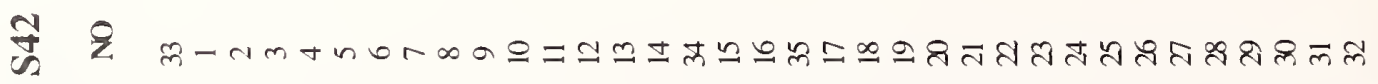

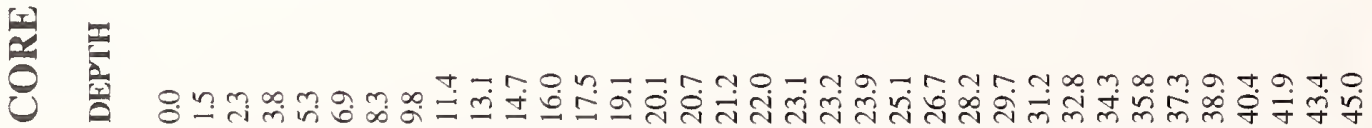


APPENDIX 2,-Continued.

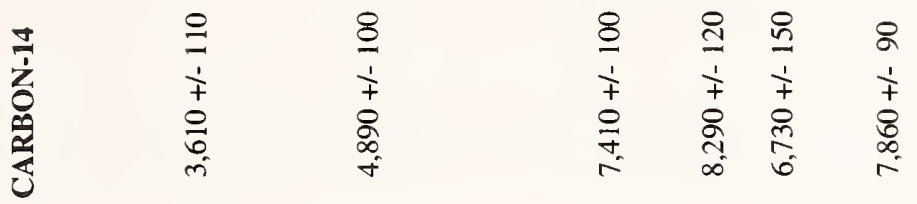

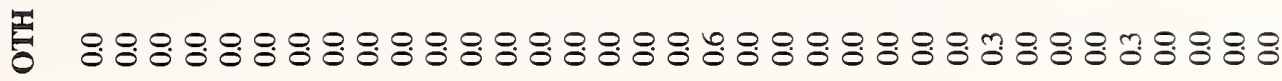

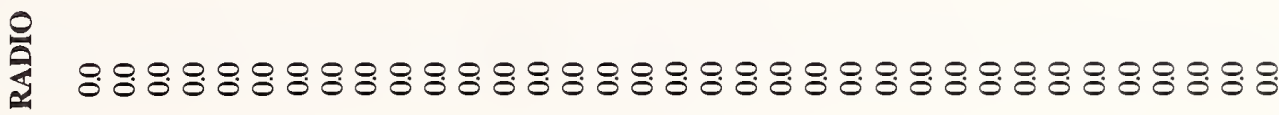

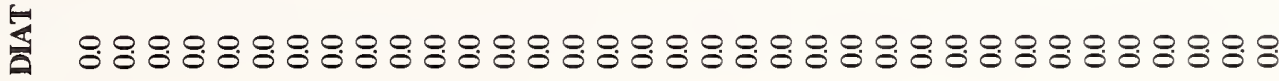
L :

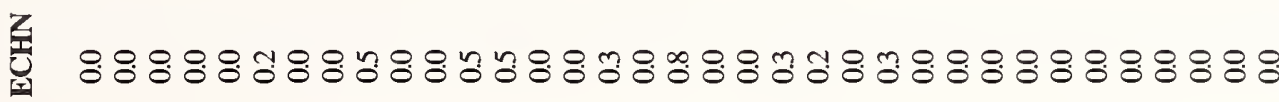

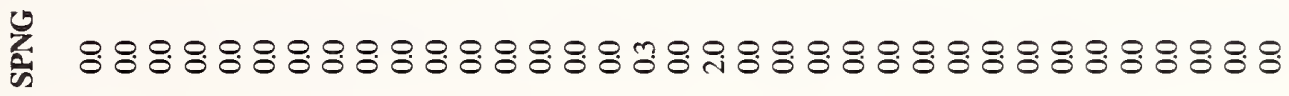

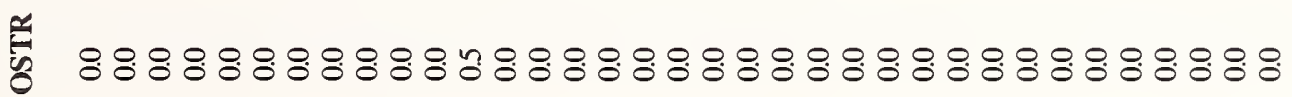

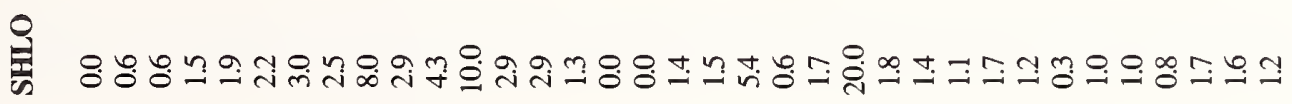
跑 逜

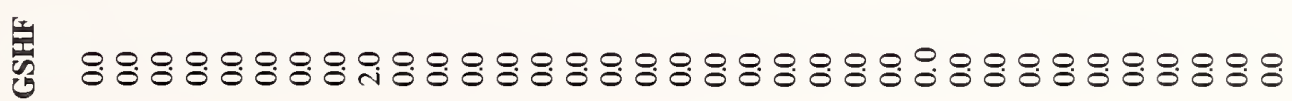

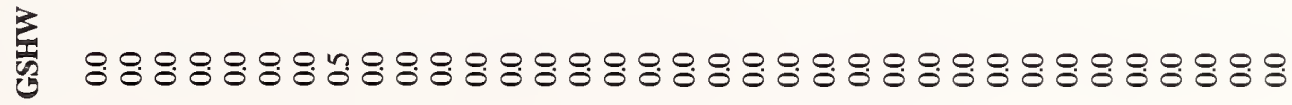

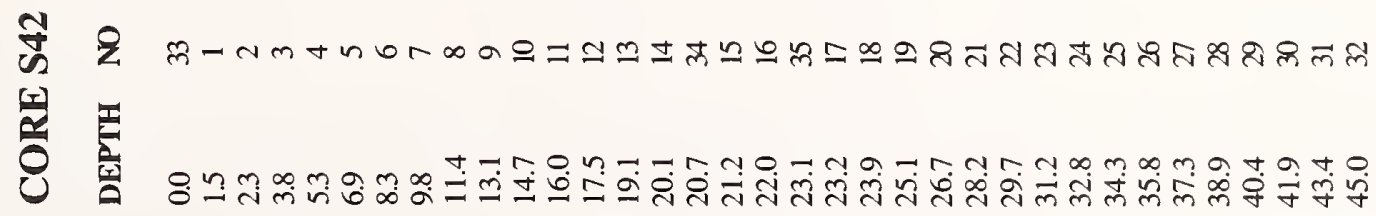


APPENDIX 2.-Continued.

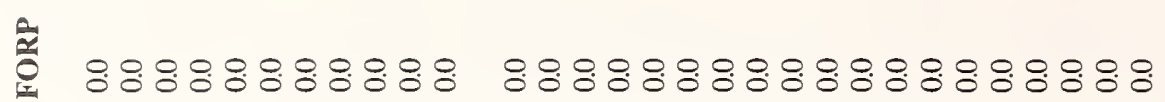

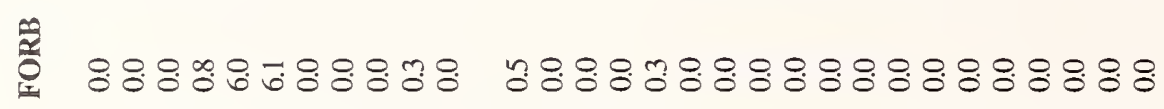

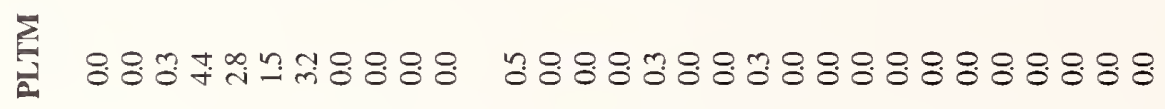

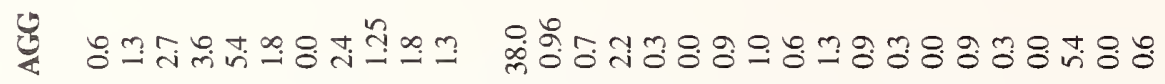

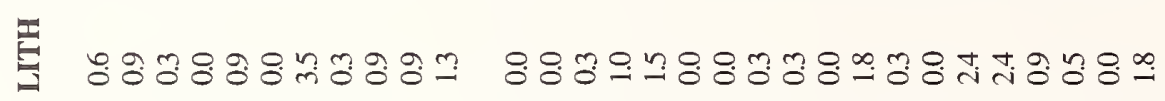

选

䒴

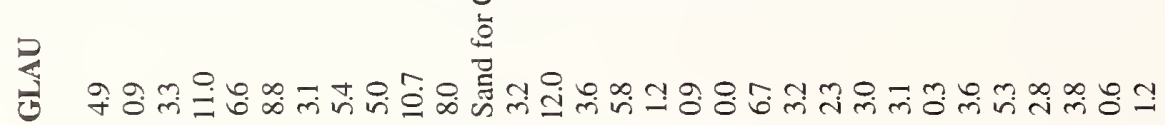

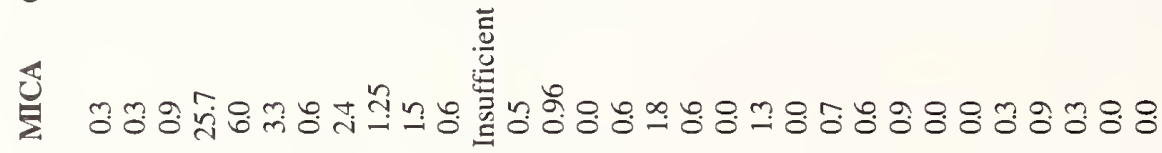

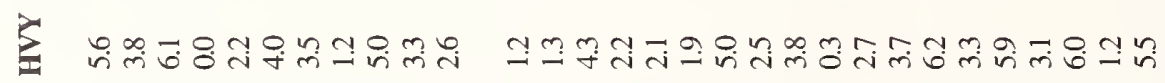

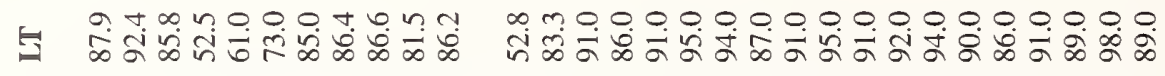

过

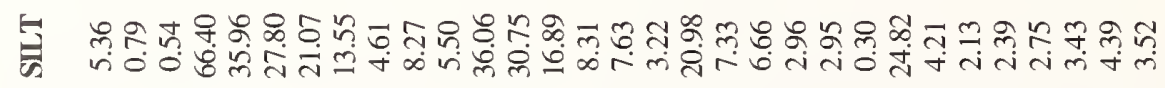

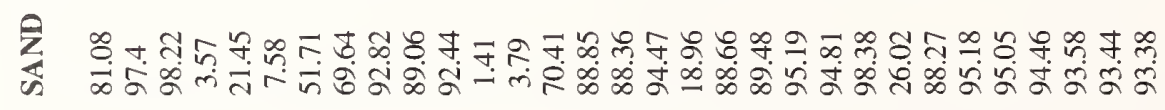

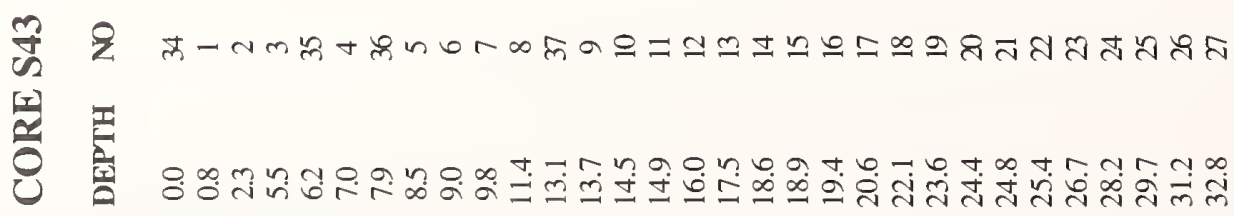


APPENDIX 2.-Continued.

$8: 88: 8$

紫 888888

8.88 .8

承 $308888 \%$

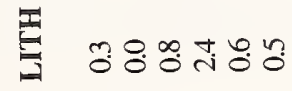

竞 888888

883888

葛

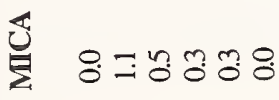

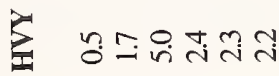

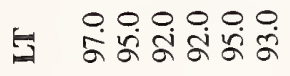

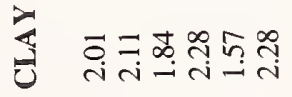

产

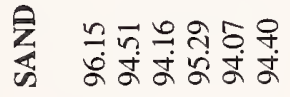

मैं वृत्रलमल

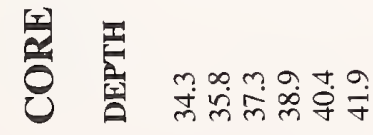


APPENDIX 2.-Continued.

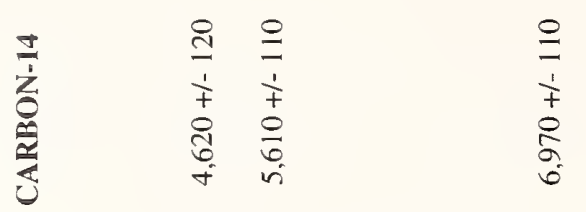

荫

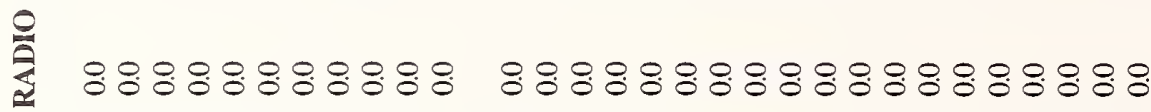

崩

논

욜

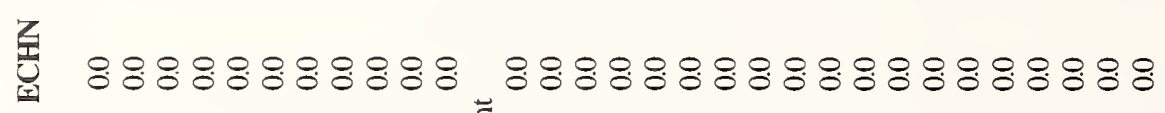
妄

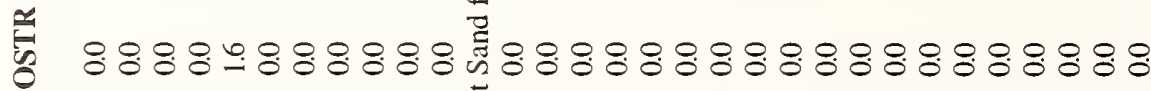
茬

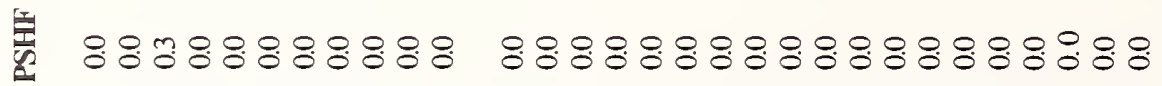

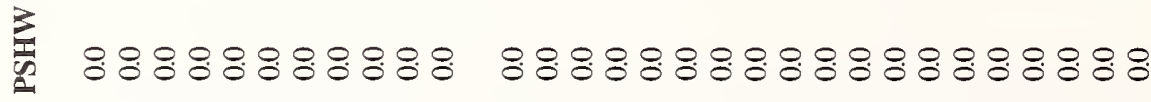

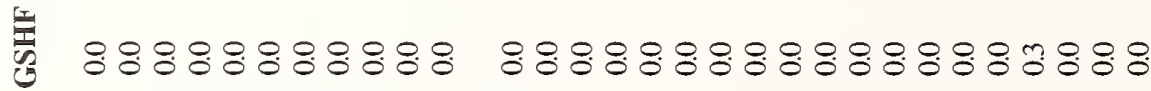
인

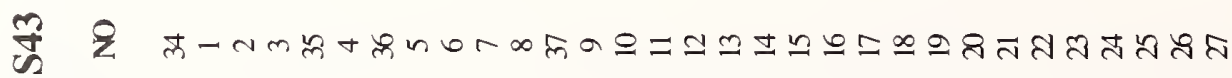


APPENDIX 2.-Continued.

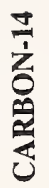

픙 8.808 .8

象 $8.8 \% 8.8$

崩 8.80 .80

돌 8.8088

욤요용

닌 8.8080.

筧 $8.88 .8 \%$

\%

웅 $2.808 \%$

崖 $8.88 .8 \%$

8.88 .8

䍃 $8.80 .8 ㅇ$

蒚 8.88 .808

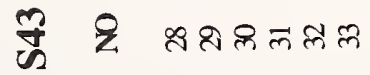

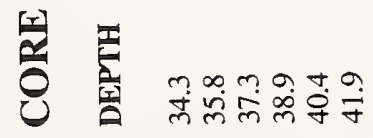


APPENDIX 2.-Continued.

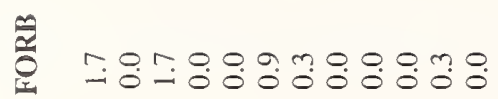

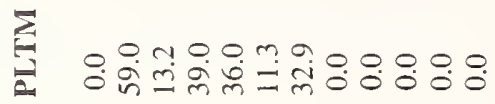

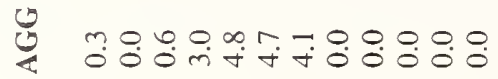

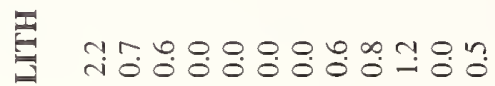

造

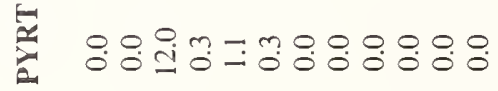

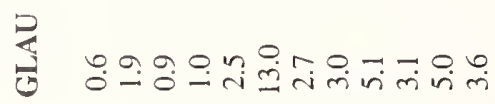

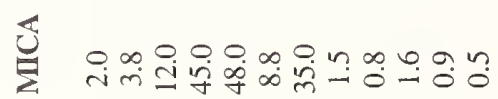

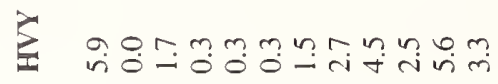

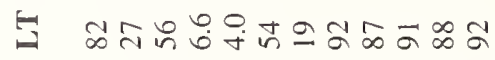

द ำ

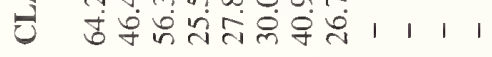

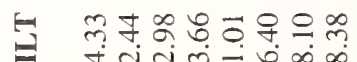

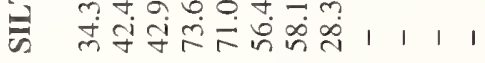

表 $\begin{aligned} & m \\ & \text { m }\end{aligned}$

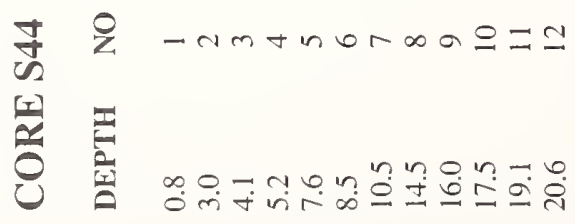


APPENDIX 2.--Continued.

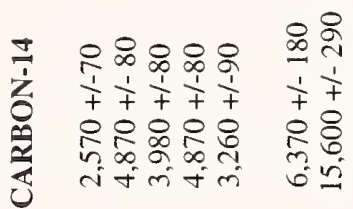

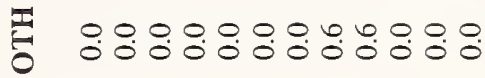

产 $8: 88: 88: 88: 8: 8$

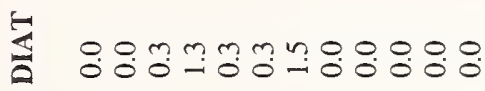

善 $8: 88: 88: 8: 8: 8:$

产 $88: 88: 88: 8: 8:$

着 $8: 88: 8: 8: 8: 8: 8:$

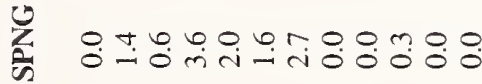

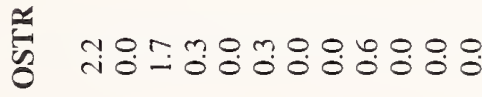

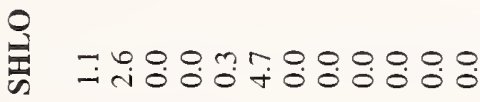

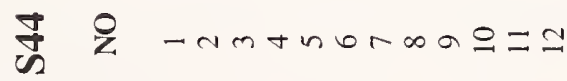

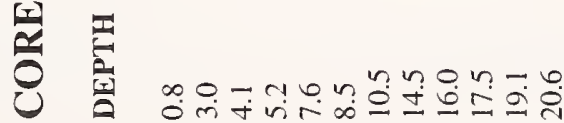


APPENDIX 2.-Continued.

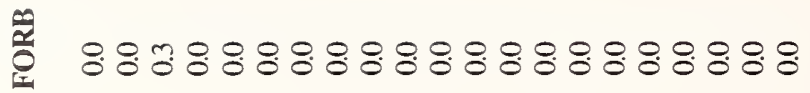

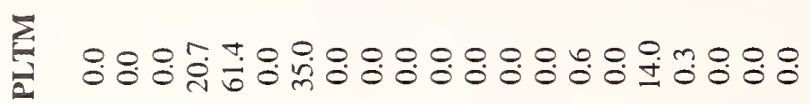

促

署

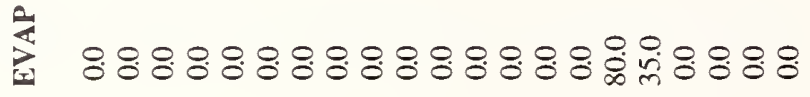

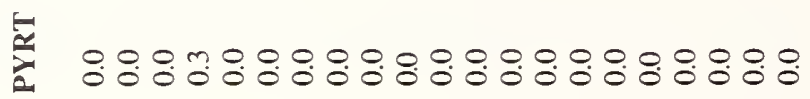

否

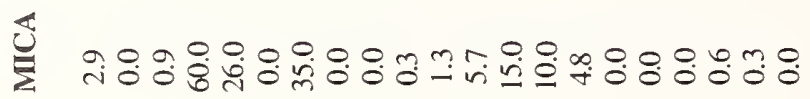

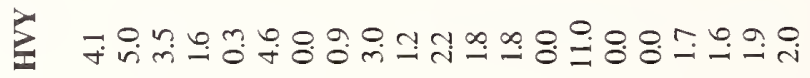

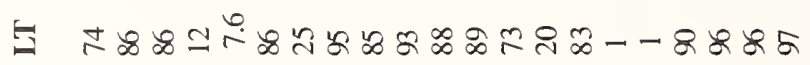

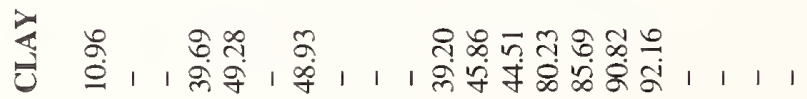

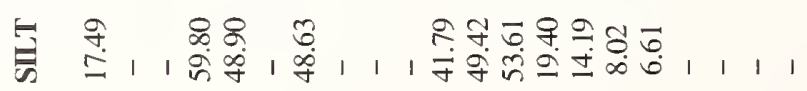

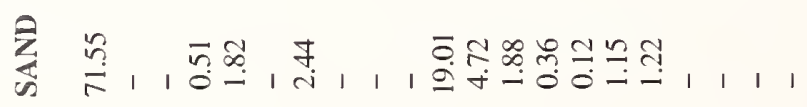

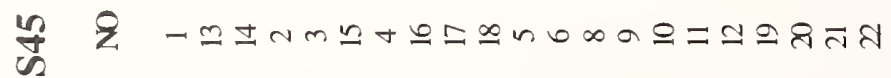

罗 
APPENDIX 2.-Continued.

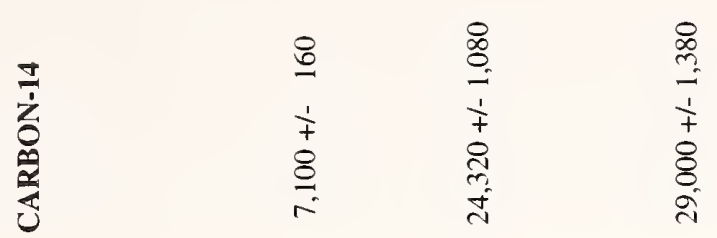

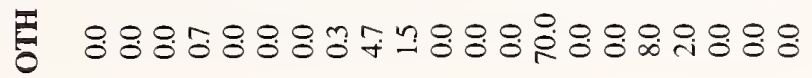

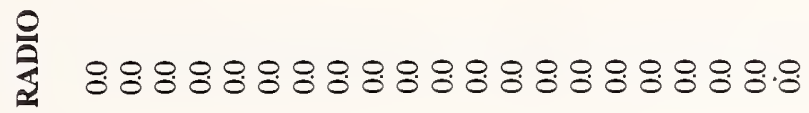

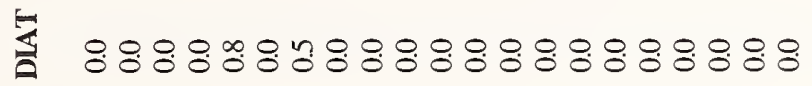

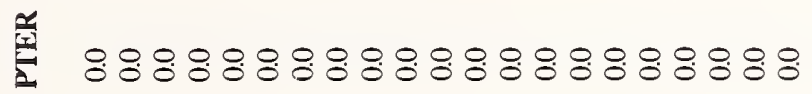

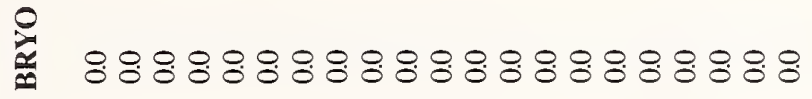

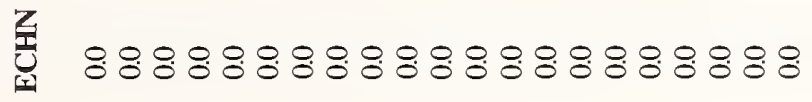

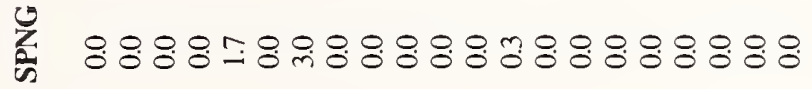

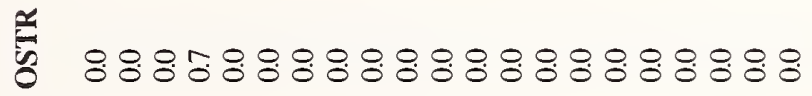

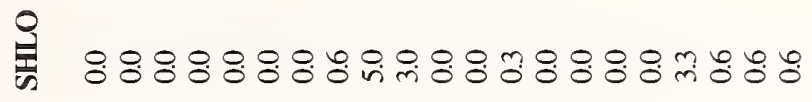

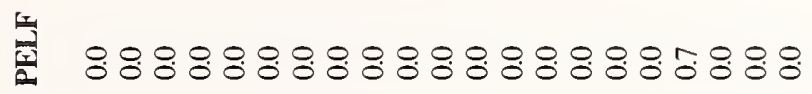

군

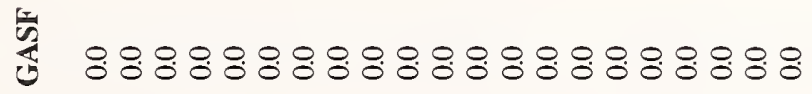

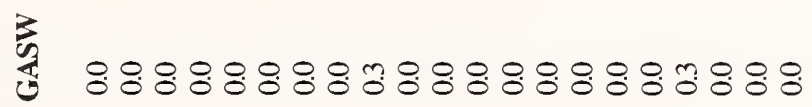

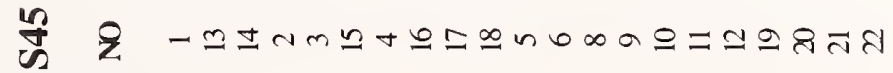

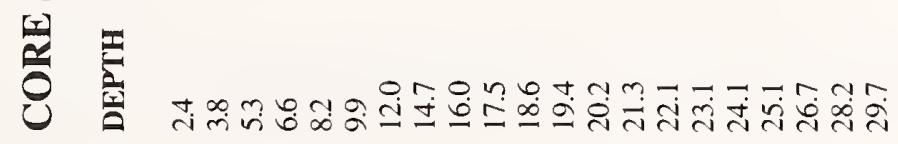


APPENDIX 2.-Continued.

\%

至

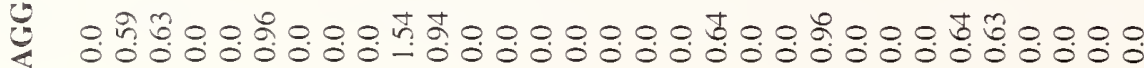

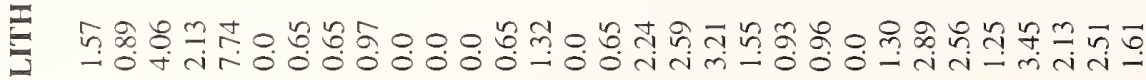

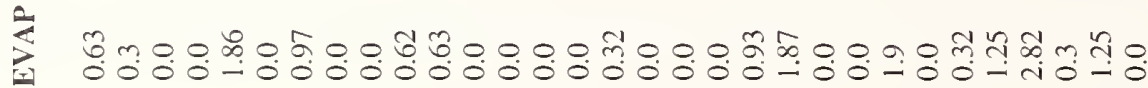

을

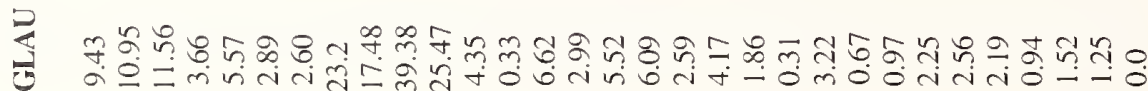

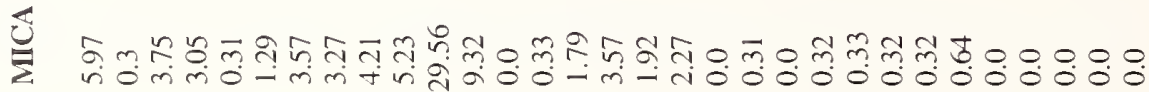

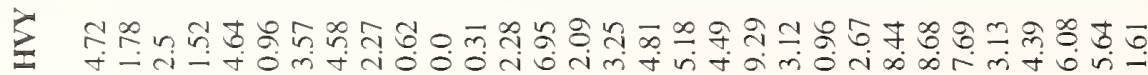

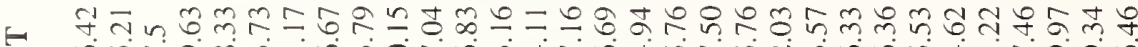

= एक

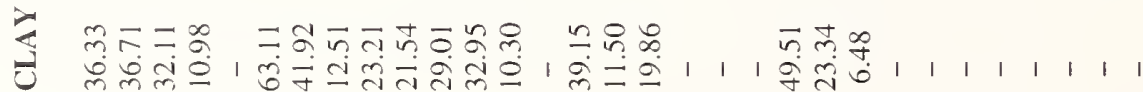

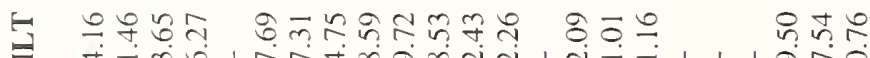

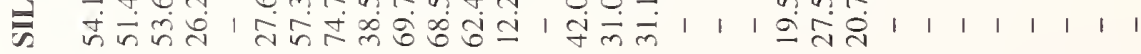

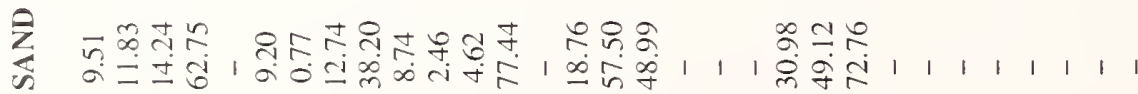

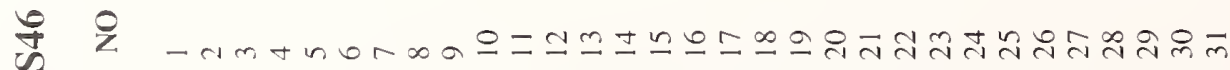

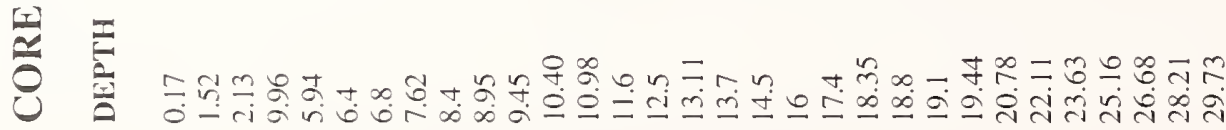


APPENDIX 2.-Continued.

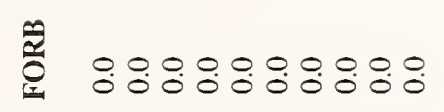

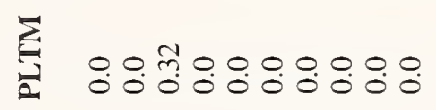

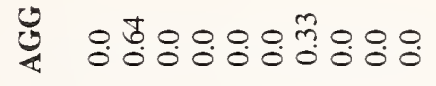

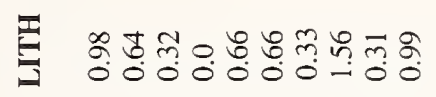

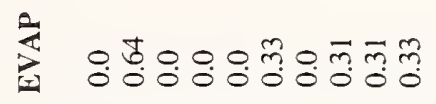

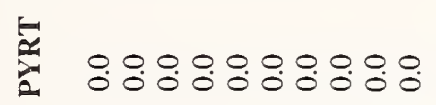

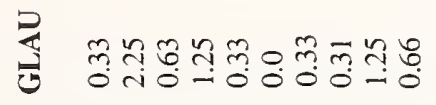

造

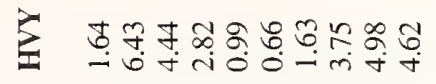

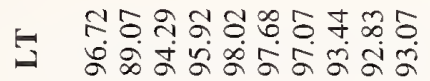

U

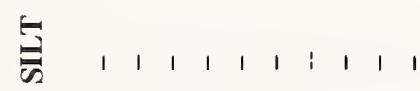

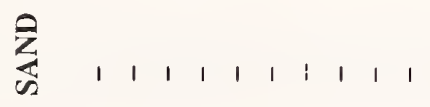

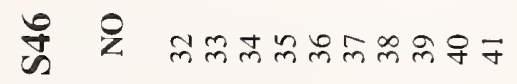

彭 
APPENDIX 2.-Continued.

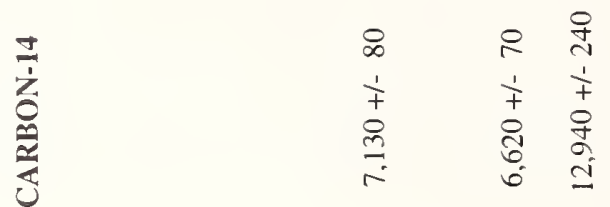

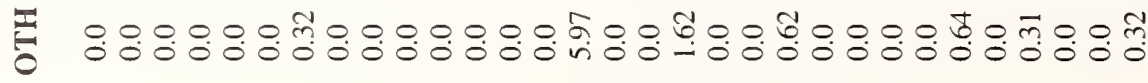

Е

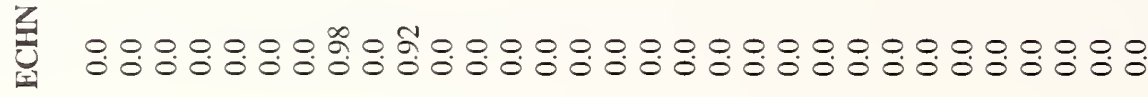

茩

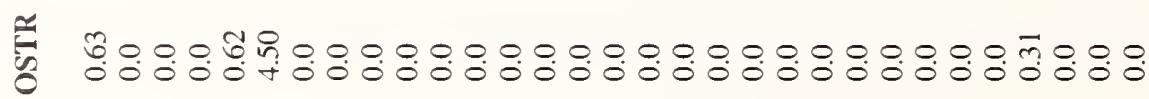

尝

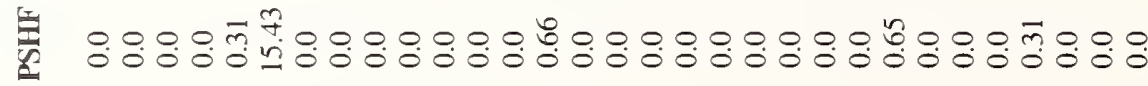

至

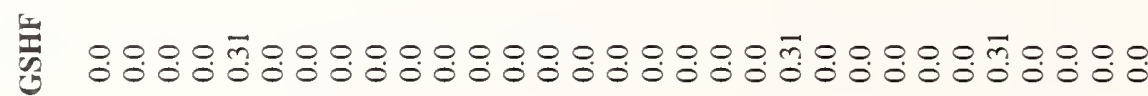

ป⿻ำ

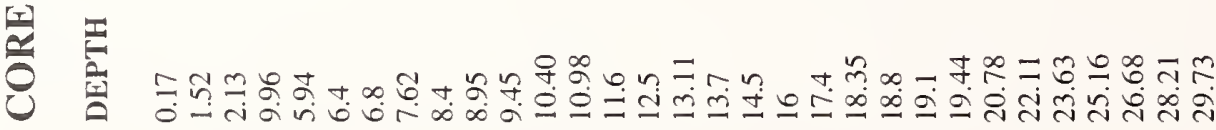


APPENDIX 2.-Continued.

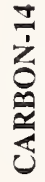

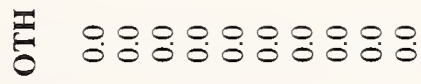

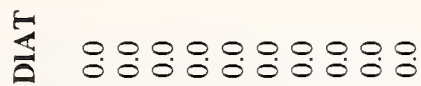

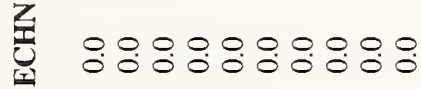

烍

采 영영영영용

㥒

爱

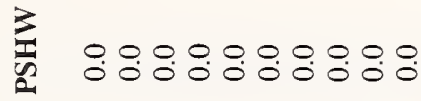

言 $8: 8: 8: 8: 8: 8:$

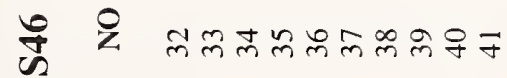

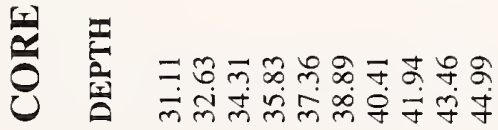


APPENDIX 2.-Continued.

$\frac{0}{2}$

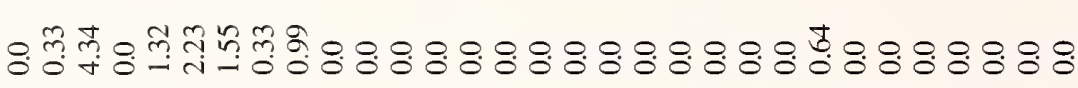

远

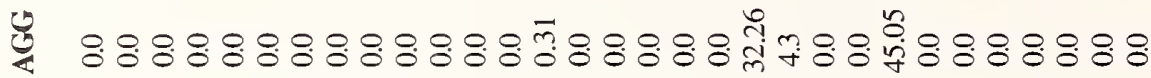

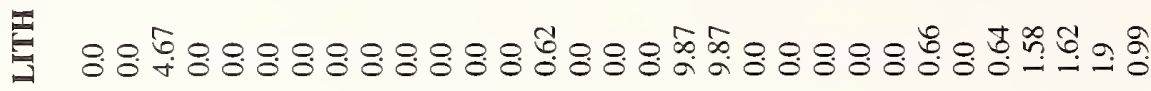

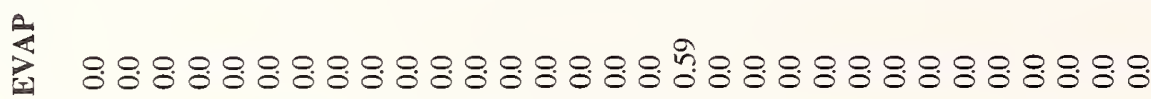

\&

秀

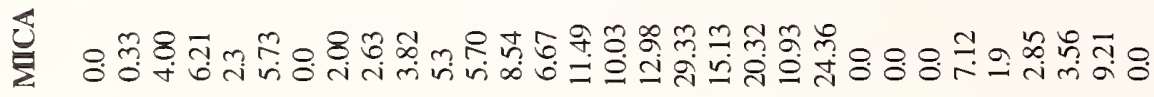

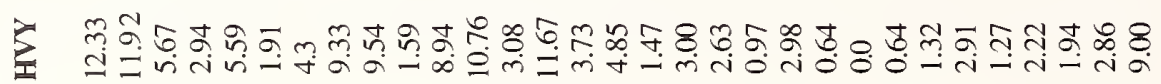

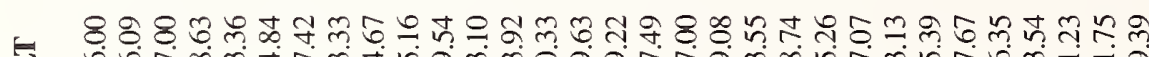

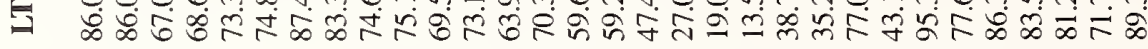

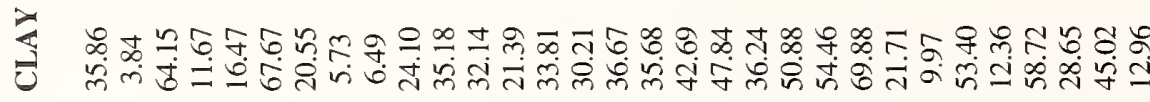

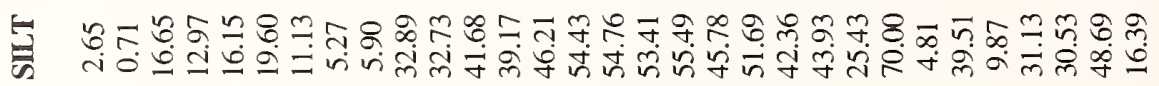

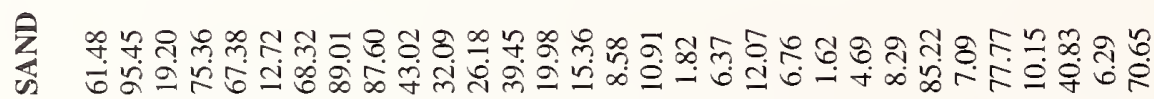

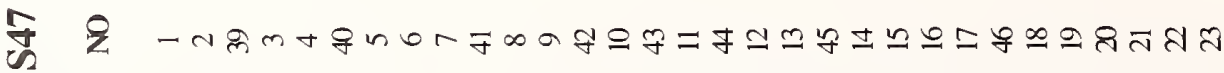

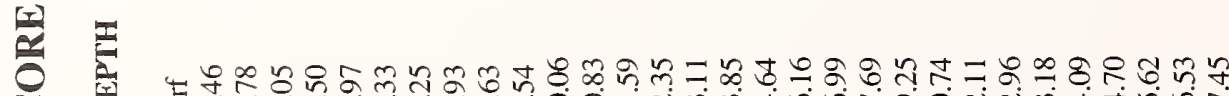

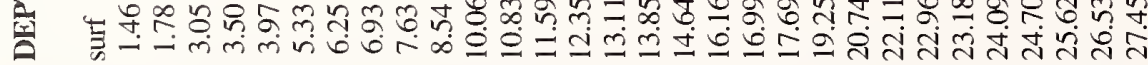


APPENDIX 2.-Continued.

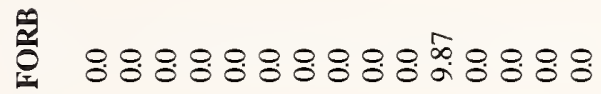

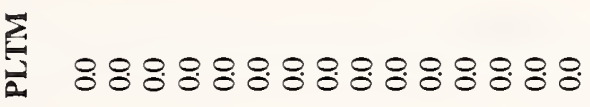

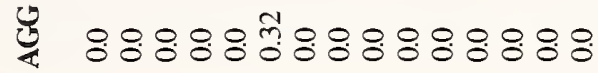

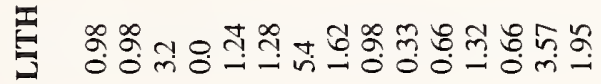

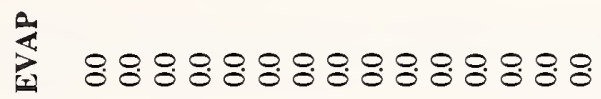

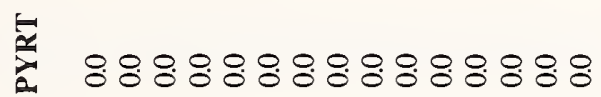

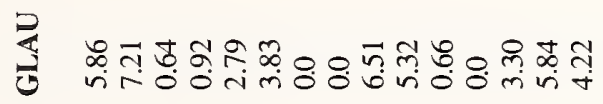

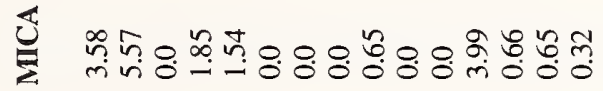

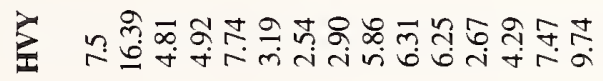

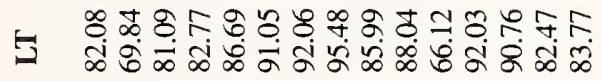

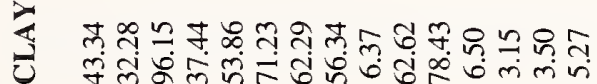

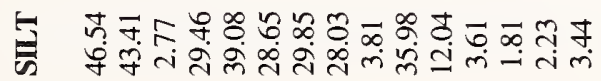

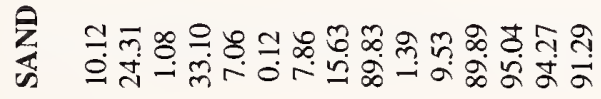

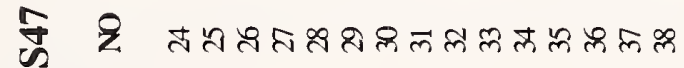

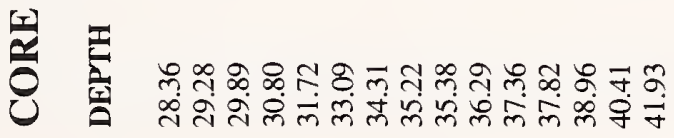


APPENDIX 2.-Continued.

竞

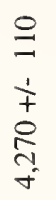

:

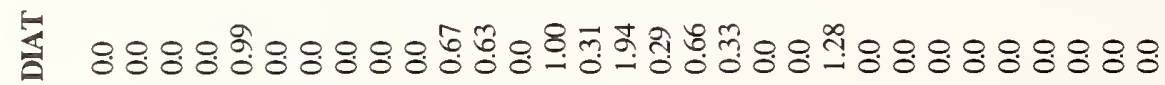

夏

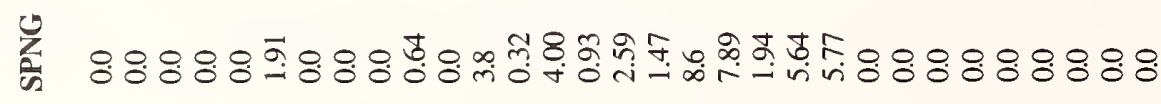

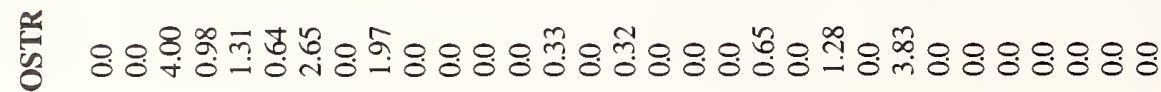

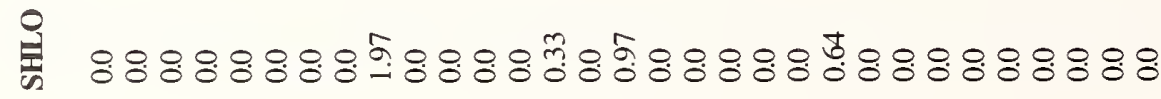

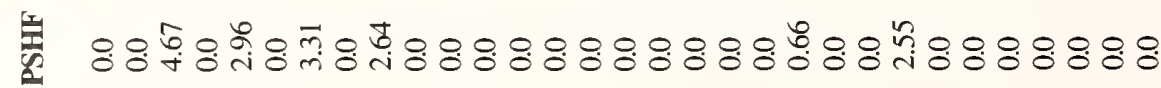

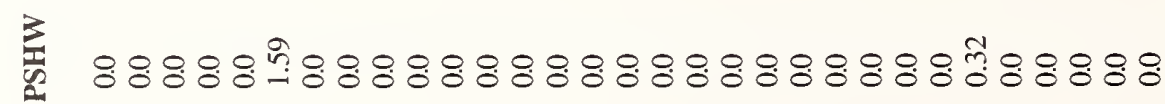

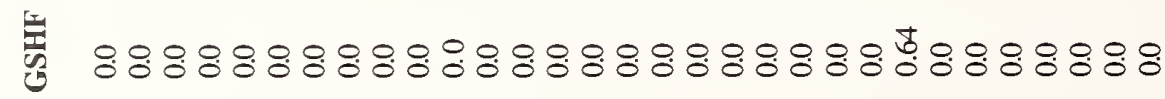

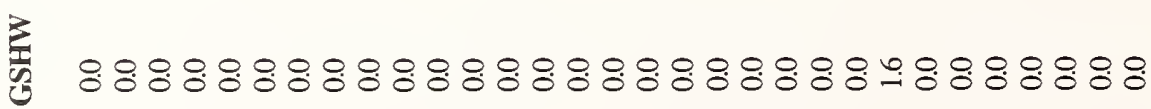

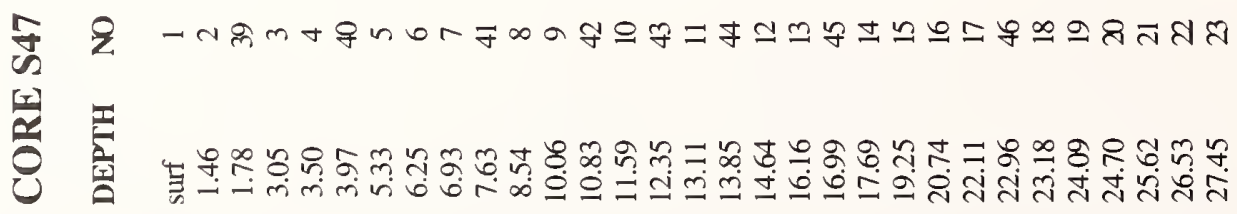


APPENDIX 2.-Continued.

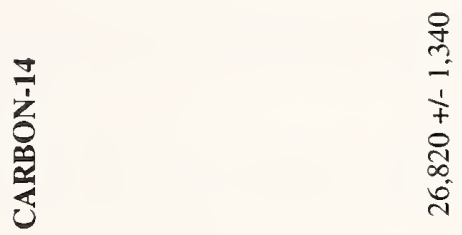

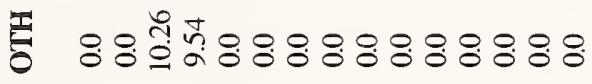

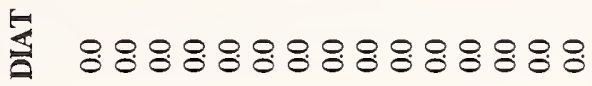

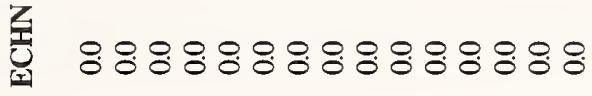

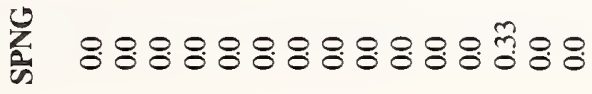

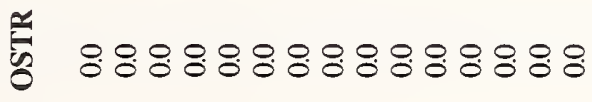

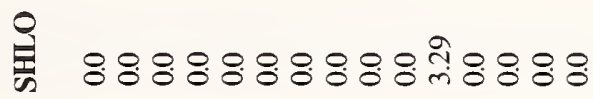

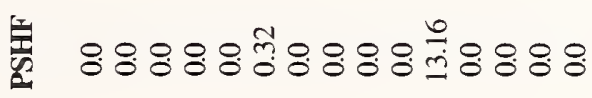

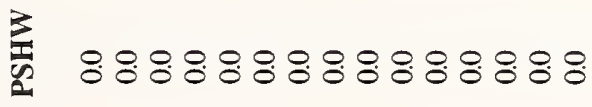

霰

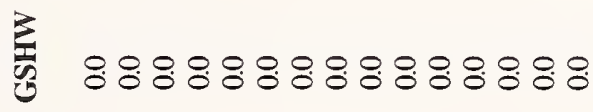

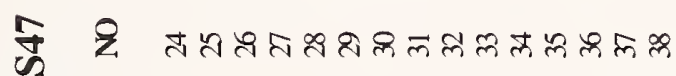

造 
APPENDIX 2.-Continued.

:

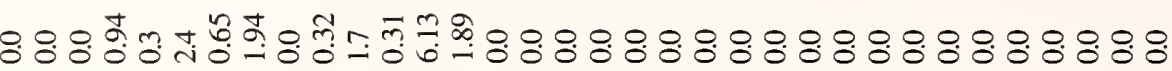

每

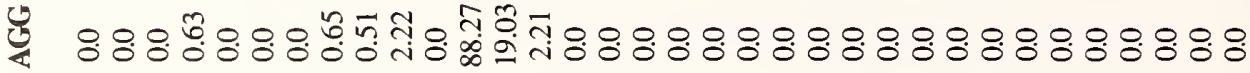

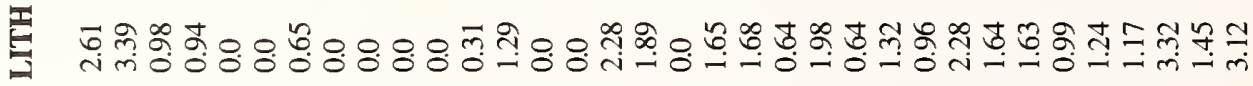

유.

过

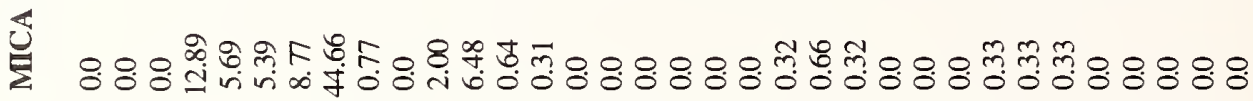

造

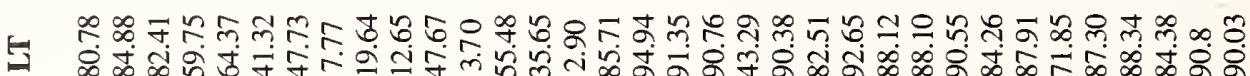

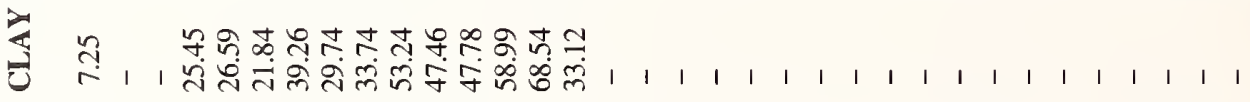

卢

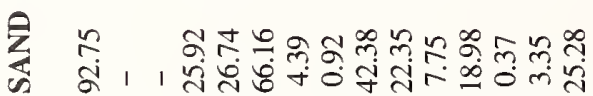

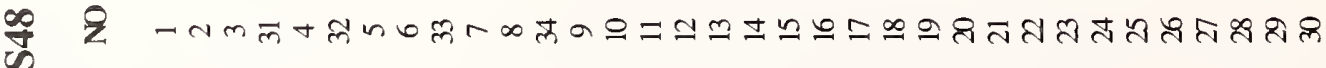

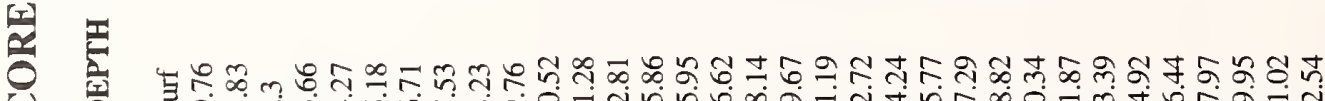

窗 
APPENDIX 2.-Continued.

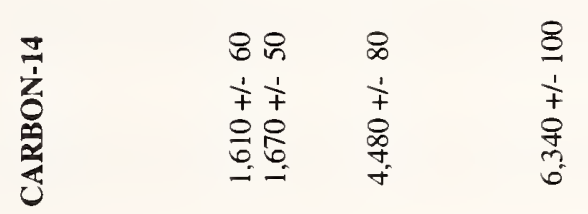

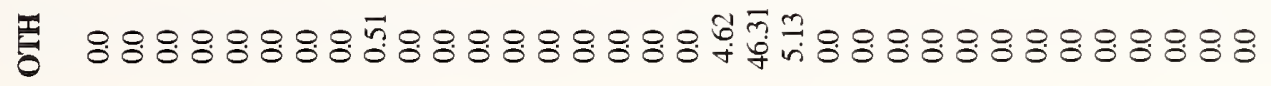

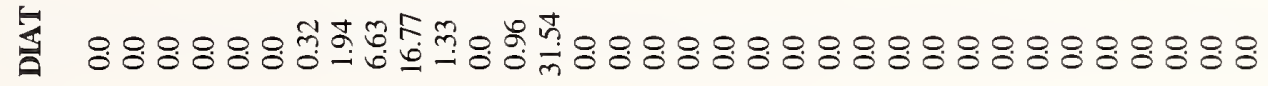

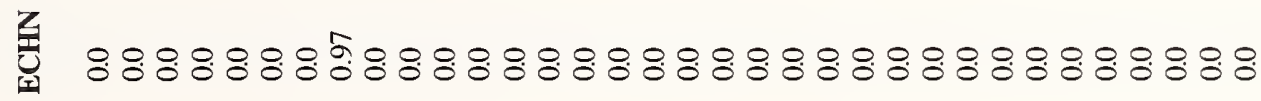

幽

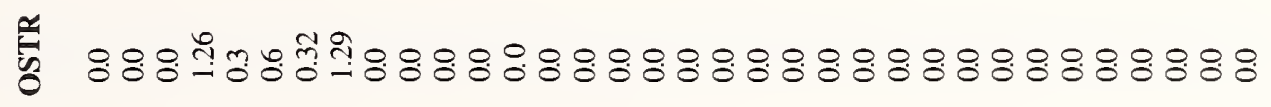

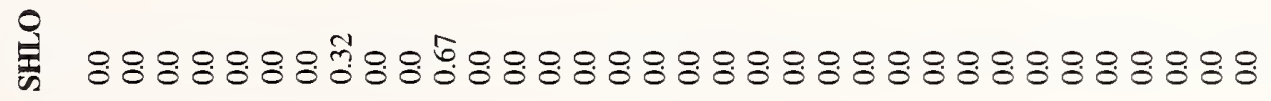

鼻 $8 \%$ 융

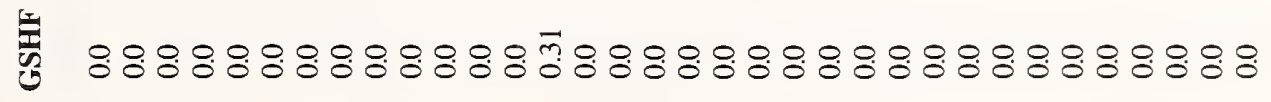

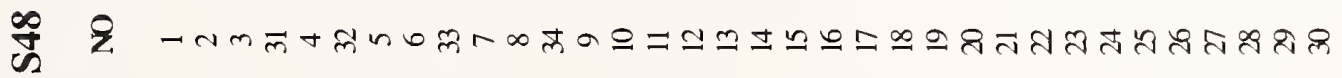

造

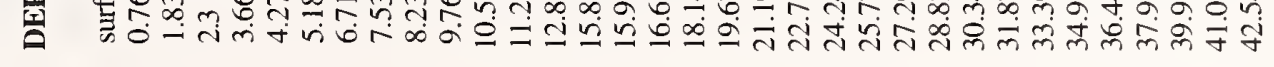


APPENDIX 2.-Continued.

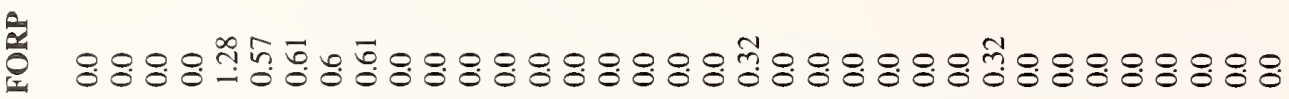

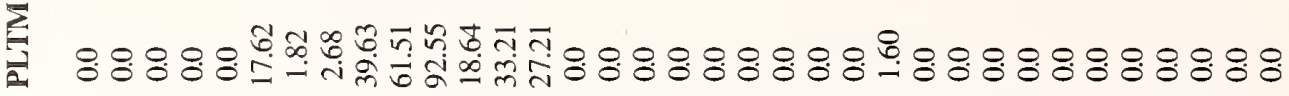

莣 $8.8: 8 \%$

焉

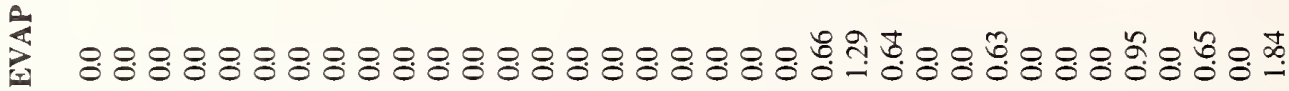

:

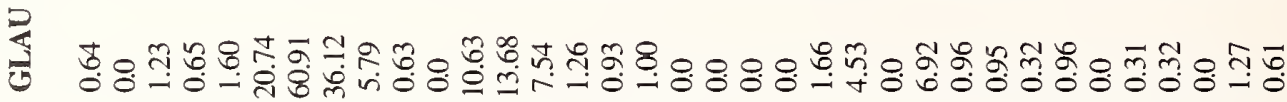

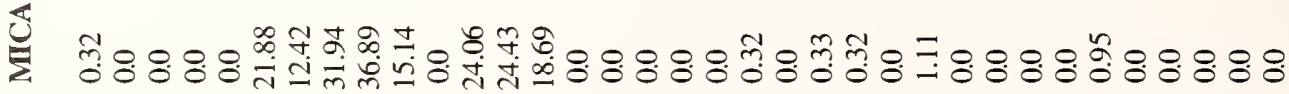

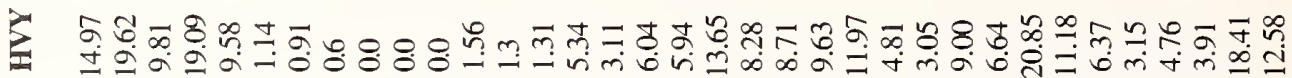

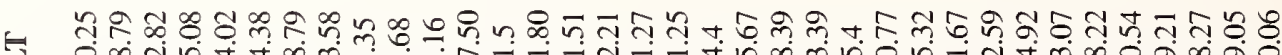

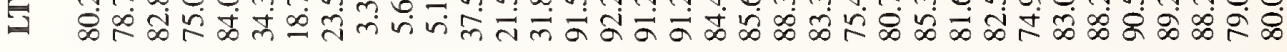

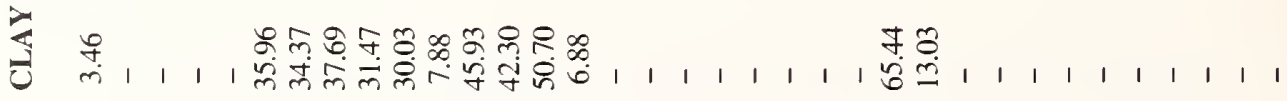

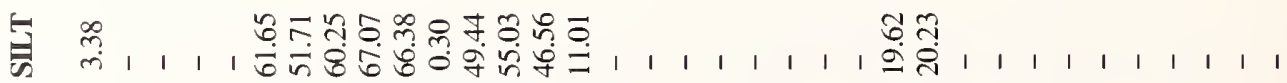

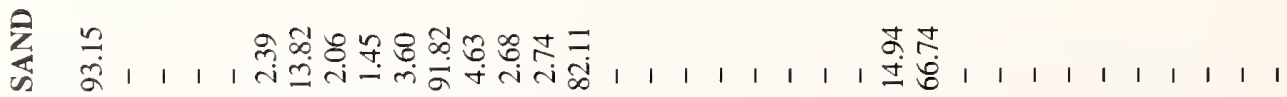

Fे 2 -

U 
APPENDIX 2.-Continued.

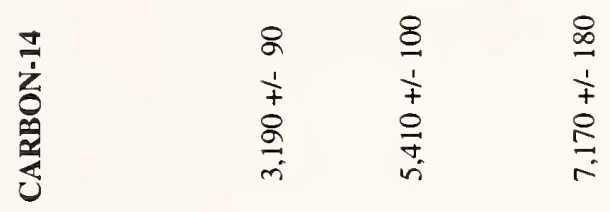

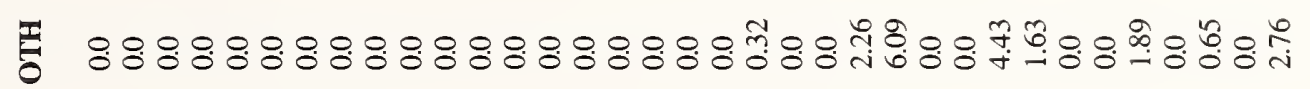

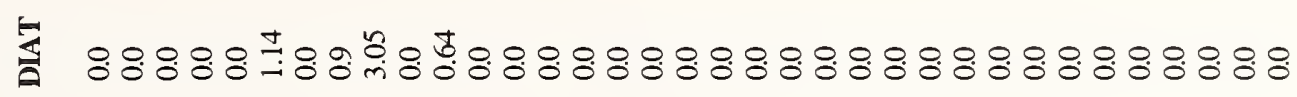

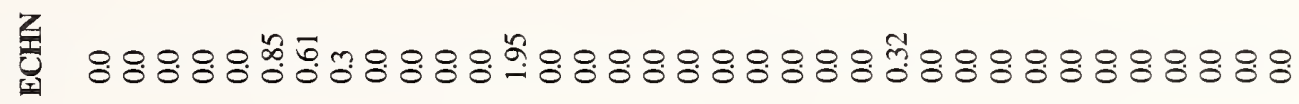

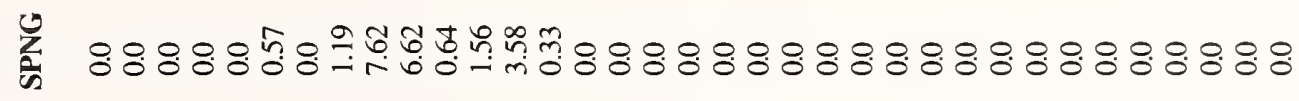

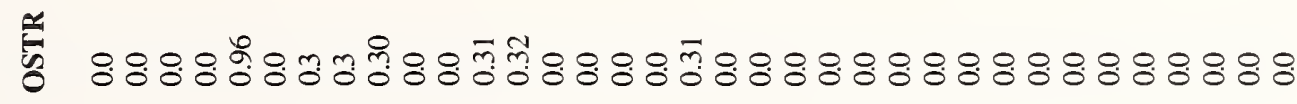

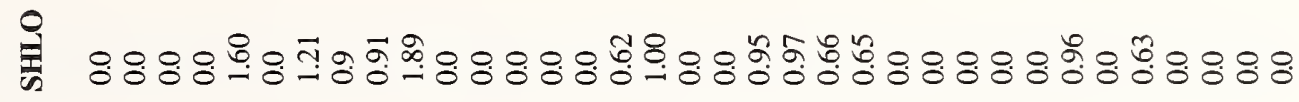

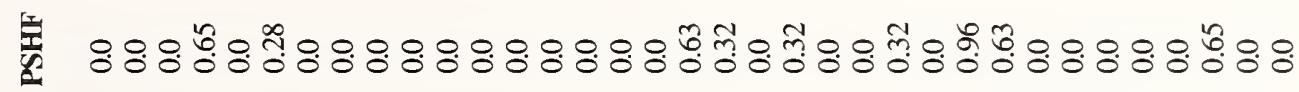

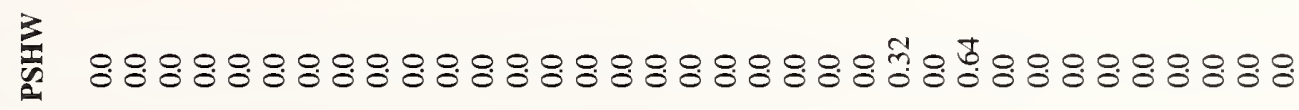

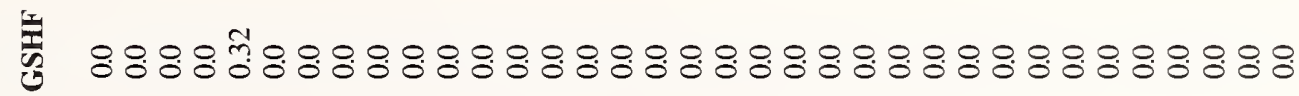

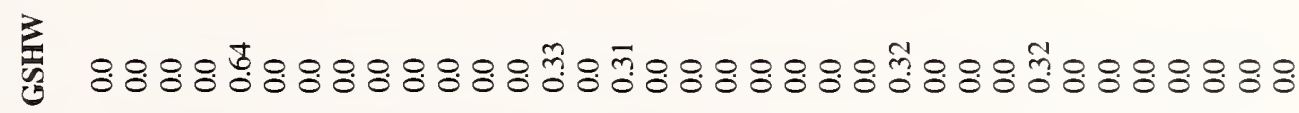

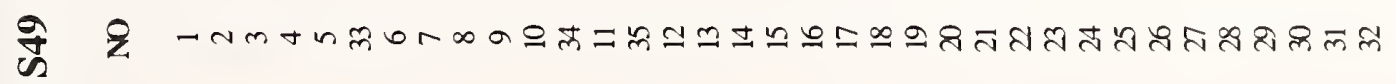

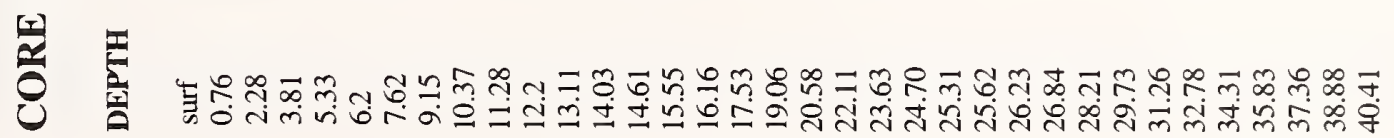


APPENDIX 2.-Continued.

尊

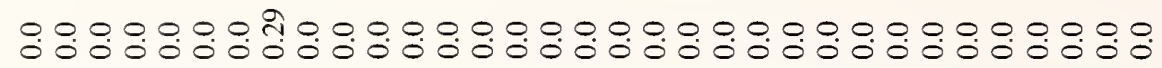

$\underset{\mathrm{E}}{\mathrm{E}}$

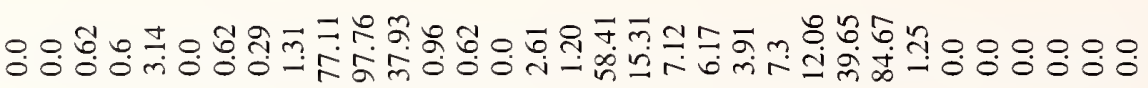

过

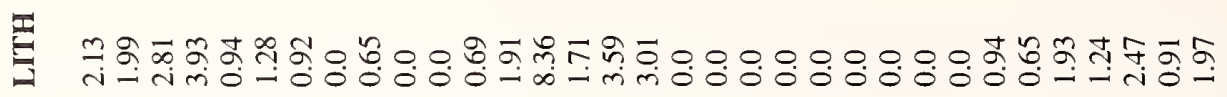

运

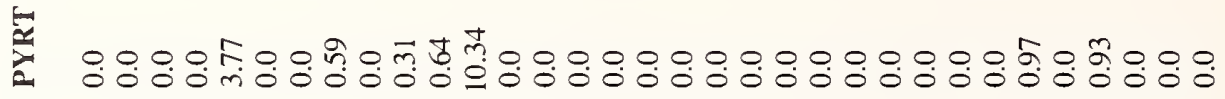

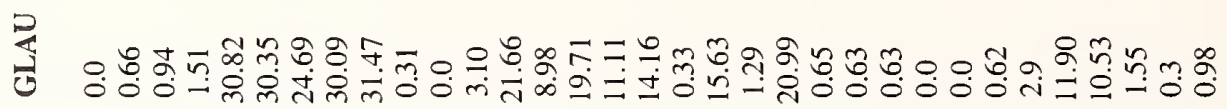

选

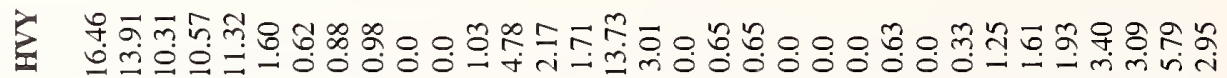

- + 궁의

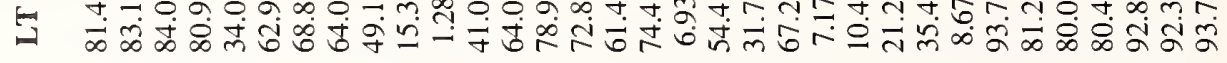

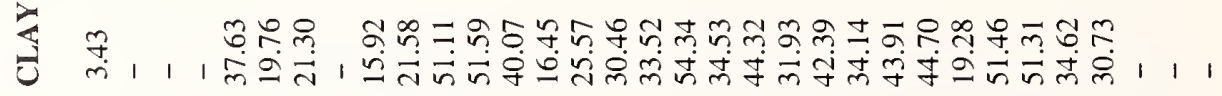

与

察

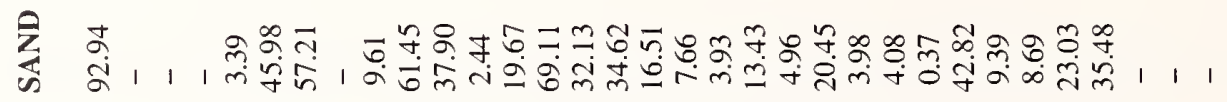

ซึ

重

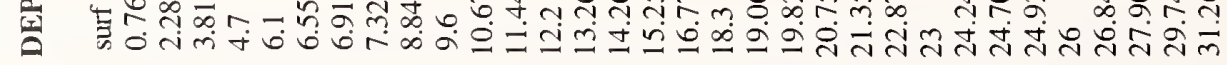


APPENDIX 2.-Continued.

藏 8080080

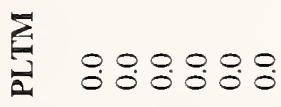

矛 $: 0: 0: 0: 0$

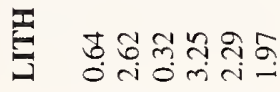

it 0000000

인 8000.000

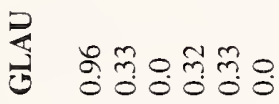

¿

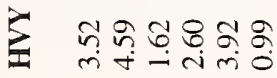

- 구의

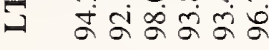

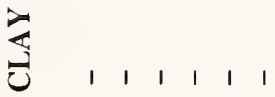

丞11111

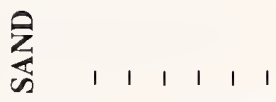

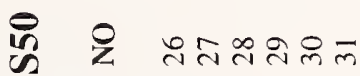

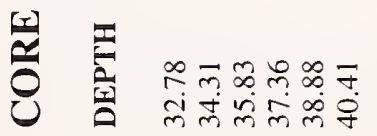




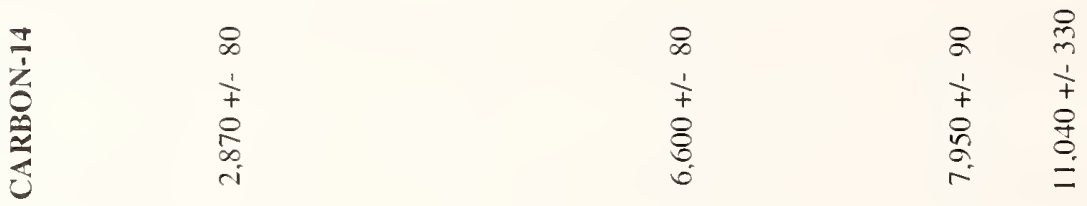

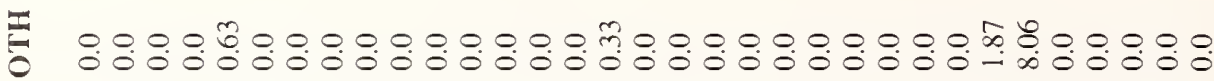

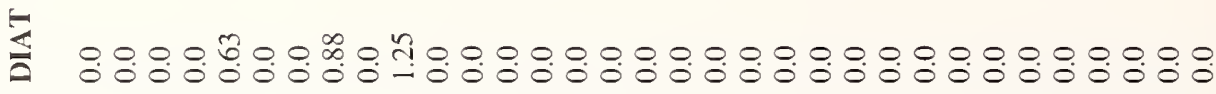

خ

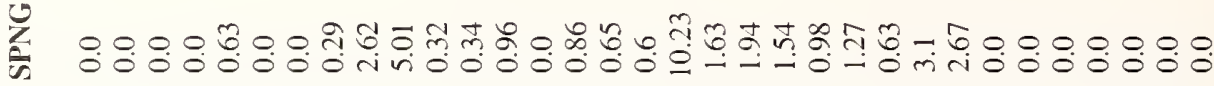

:

基

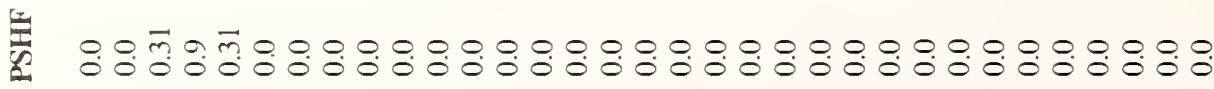

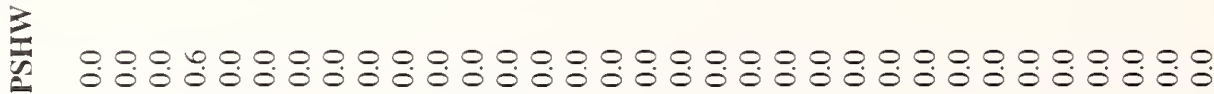

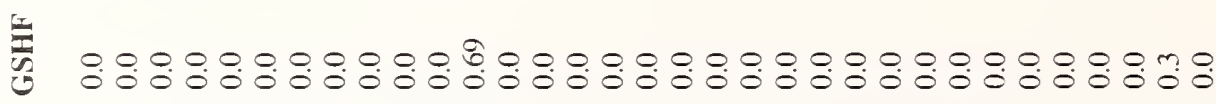

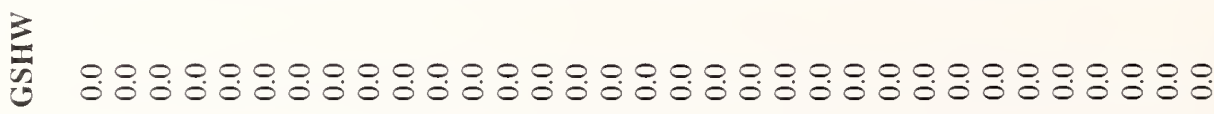

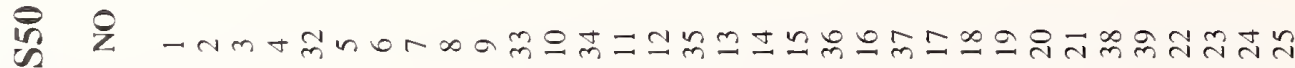

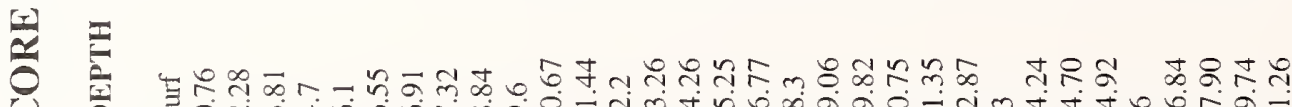

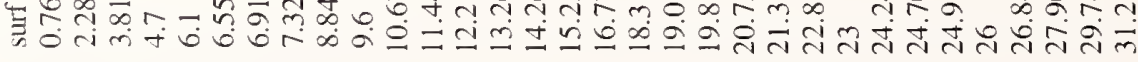


APPENDIX 2.-Continued.

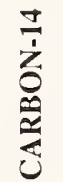

풍 $8: 88: 88$

毫 $8: 8: 8: 8:$

屏 8808880

产 $8: 8: 8: 8$

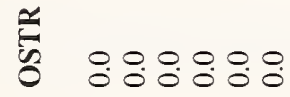

ํㅐำ

善 808880

言 $8888: 8$

衰

产 $8: 88: 80$

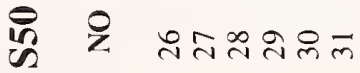

啳 
APPENDIX 2.-Continued.

옹

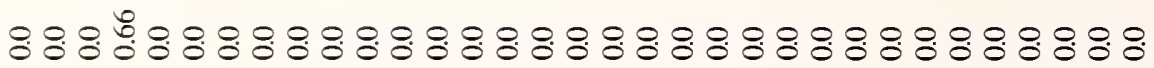

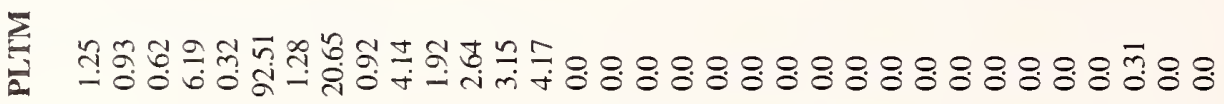

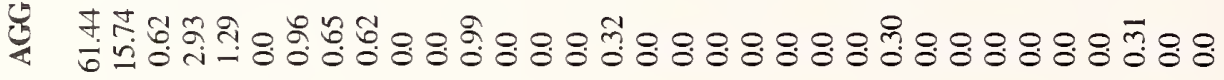

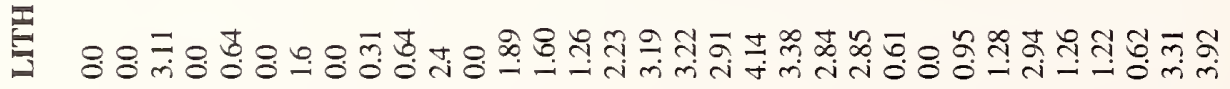

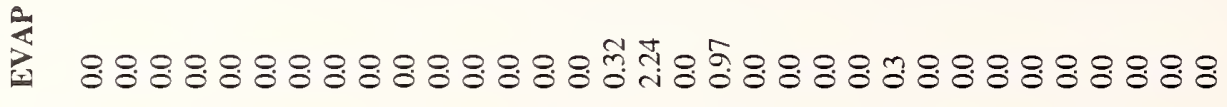

:

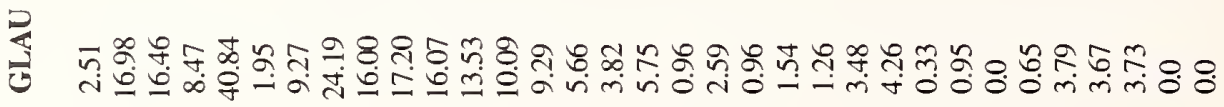

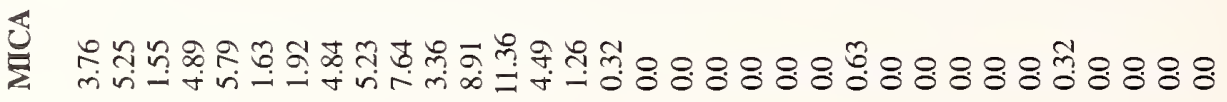

¿

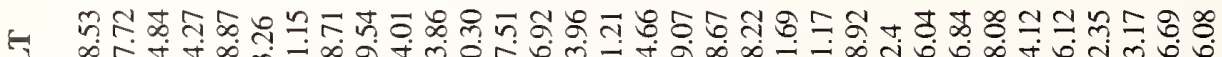

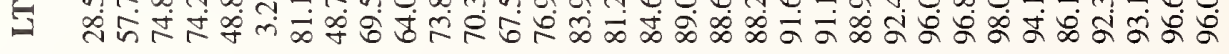

उ

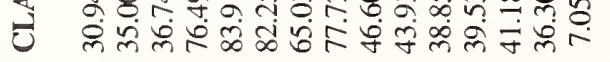

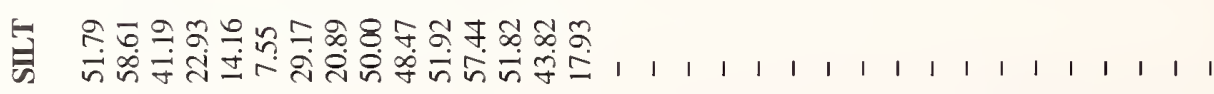

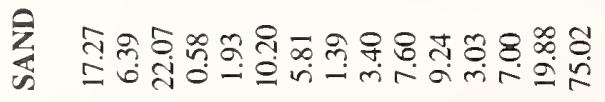

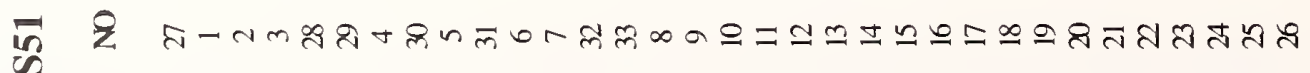


APPENDIX 2.-Continued.

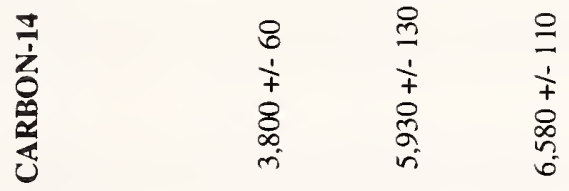

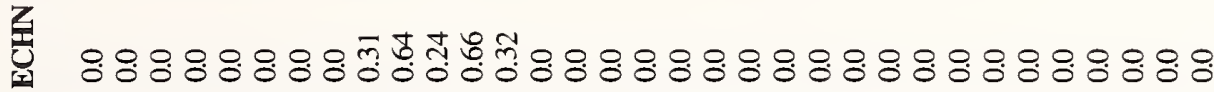

荒

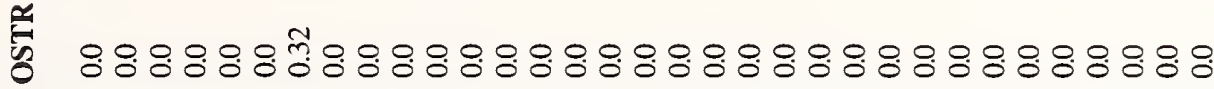

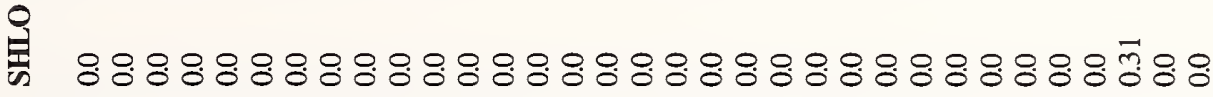

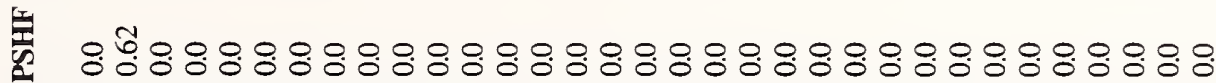

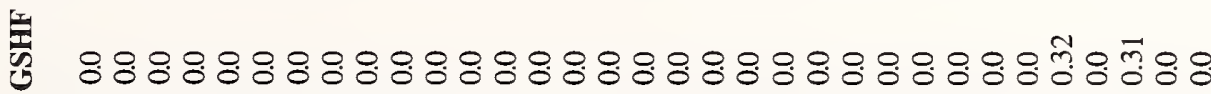

请

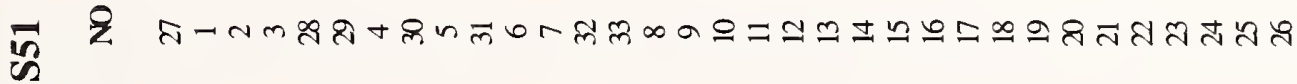

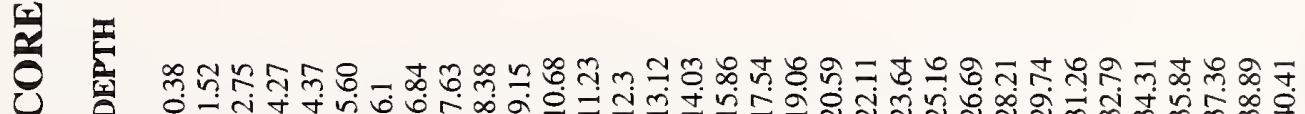


APPENDIX 2.-Continued.

$\frac{1}{2}$

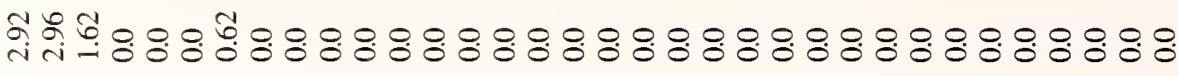

$\sum_{i=1}$

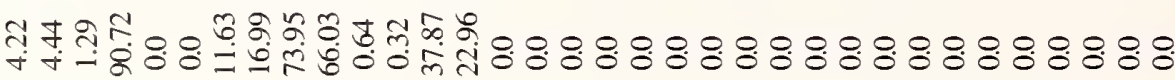

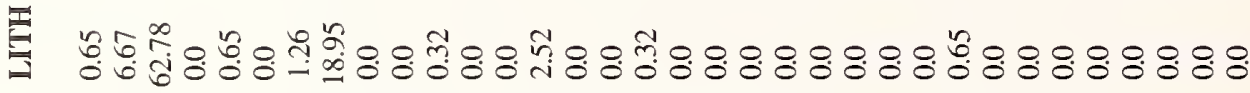

育

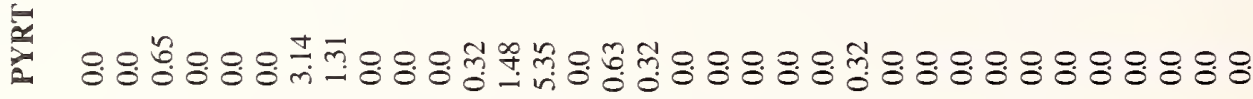

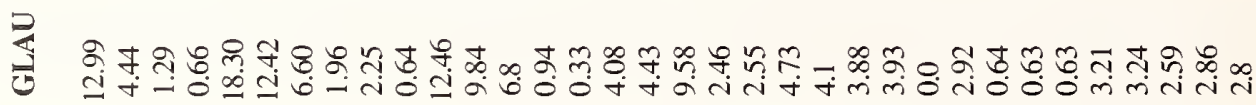

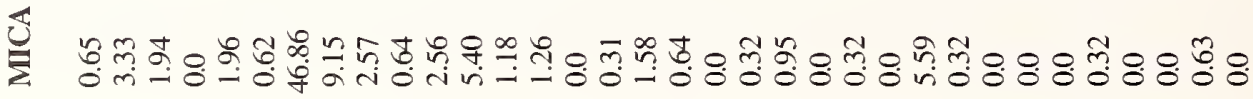

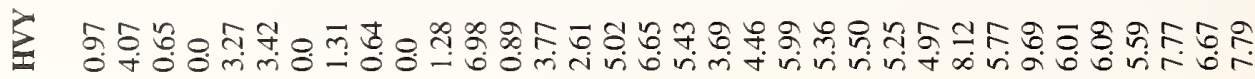

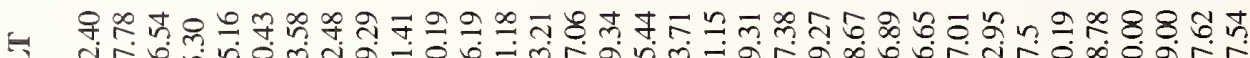

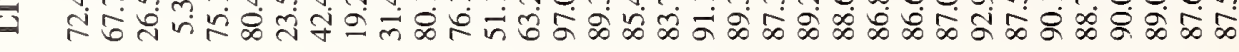

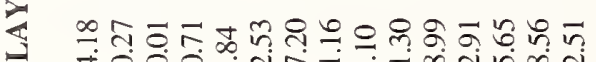

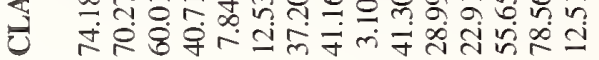

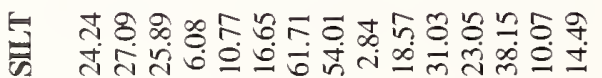

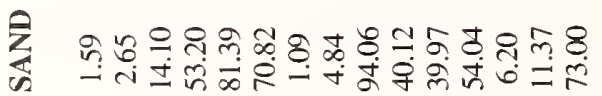

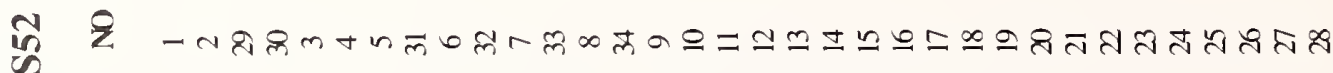

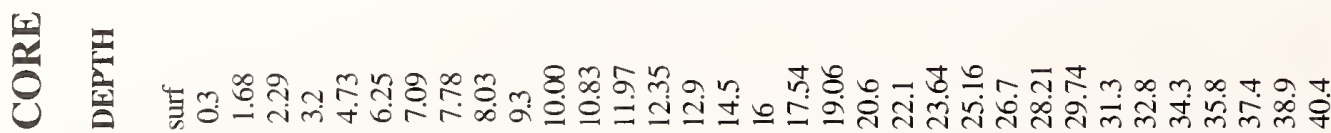


APPENDIX 2.-Continued.

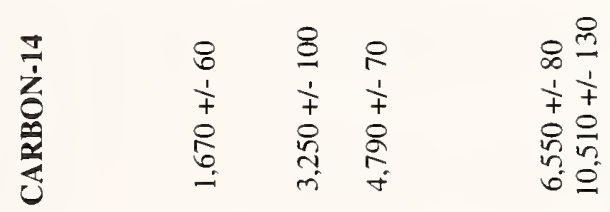

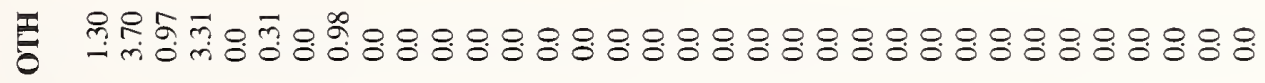

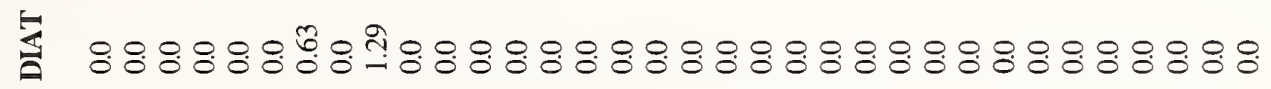

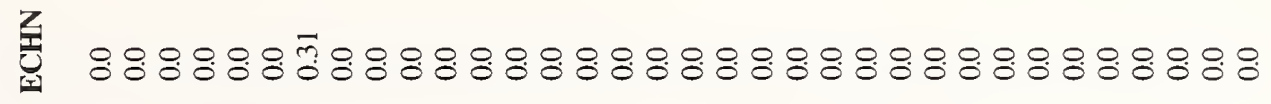

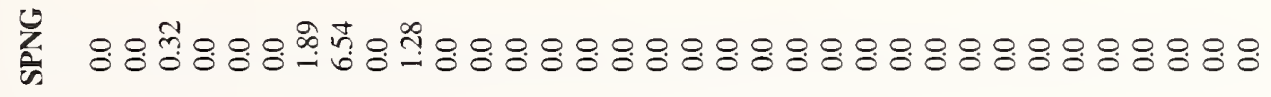

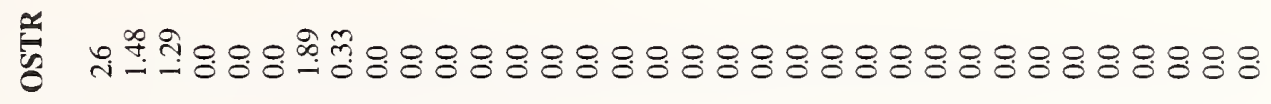

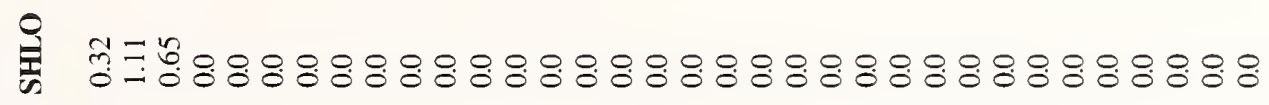

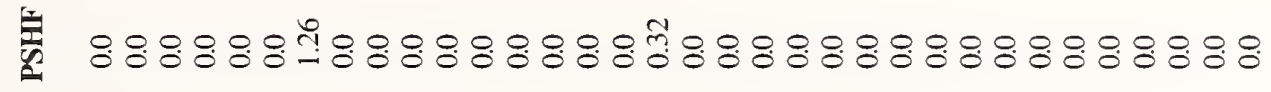

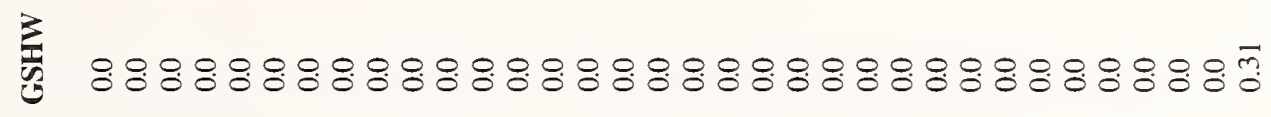

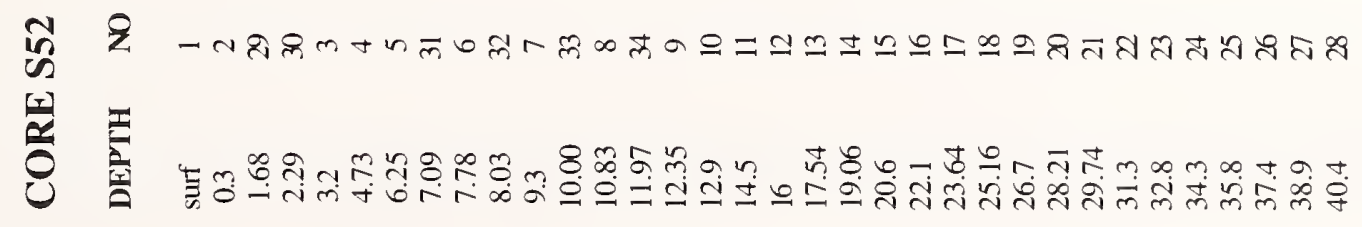


APPENDIX 2.-Continued.

盖

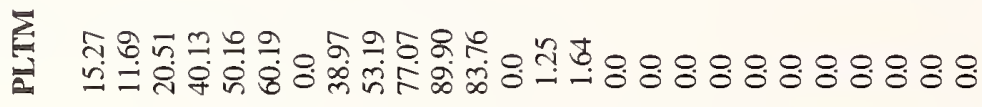

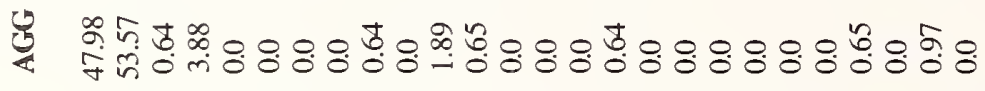

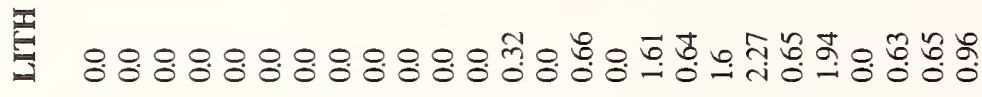

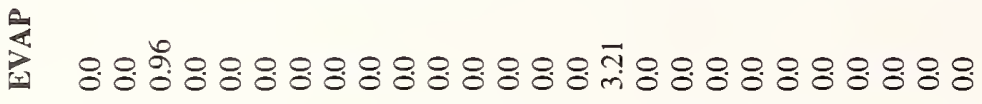

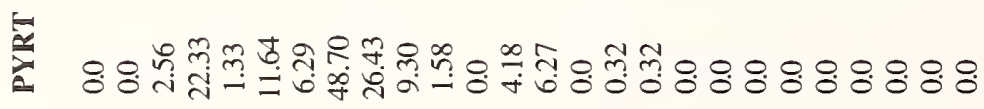

苞

氙 군

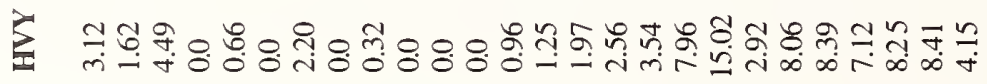

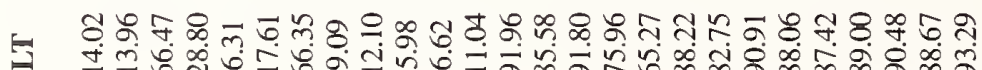

-

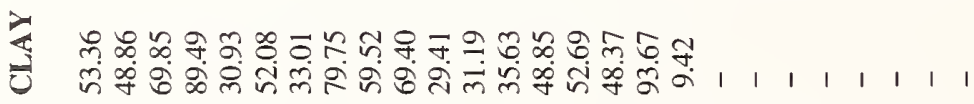

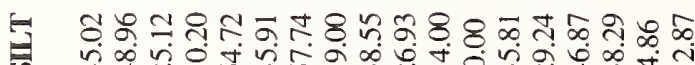

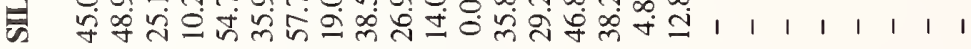

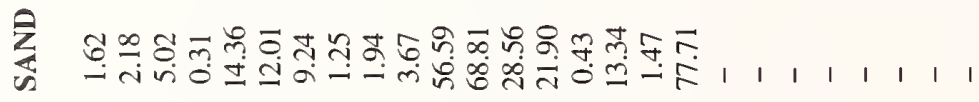

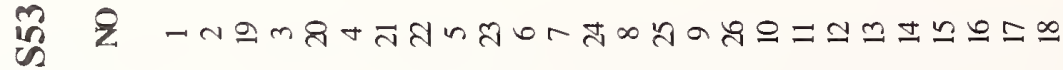

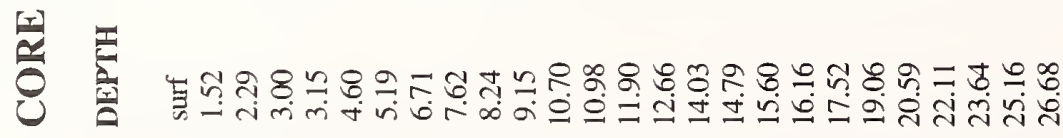


APPENDIX 2.-Continued.

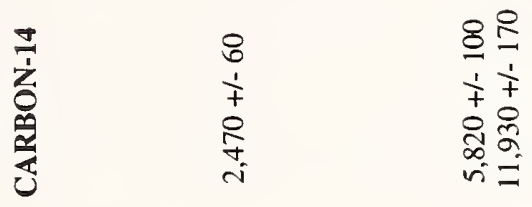

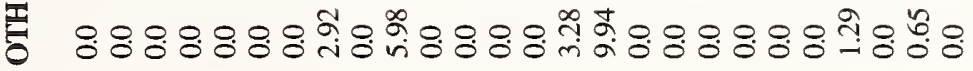

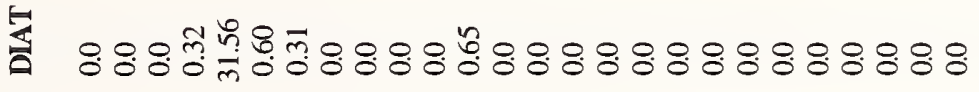

尊 $8: 8: 8: 8: 8: 8: 8: 8: 8: 8: 8: 8: 8: 8:$

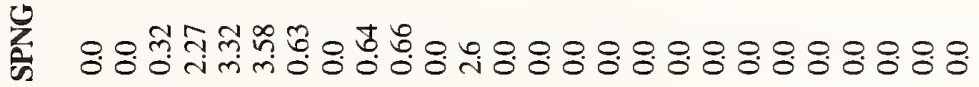

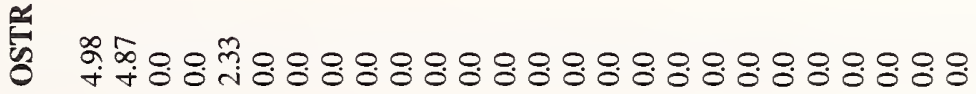

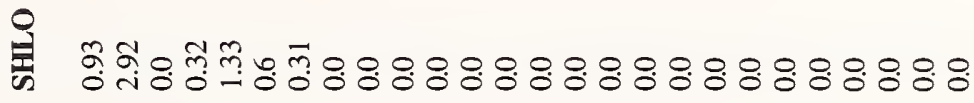

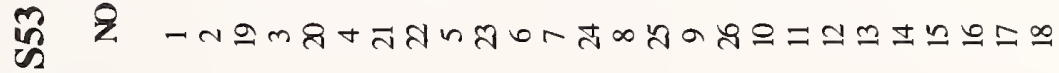

㰻 


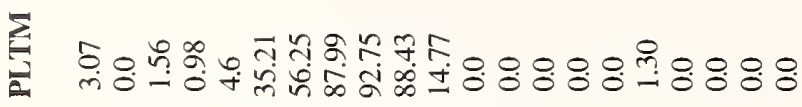

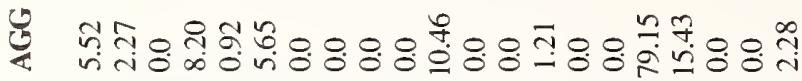

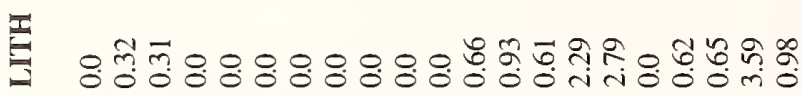

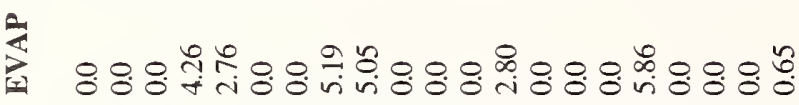

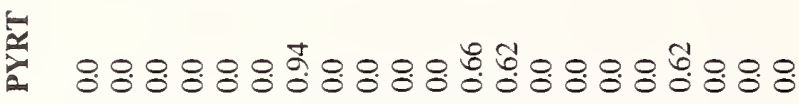

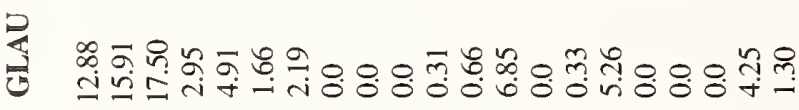

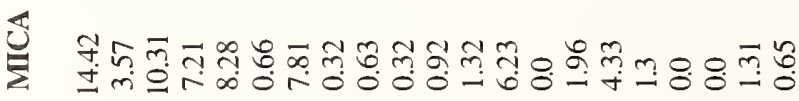

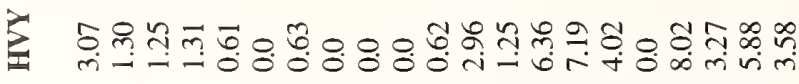

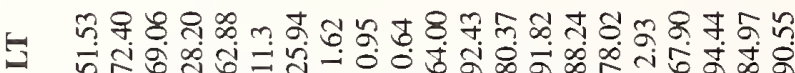

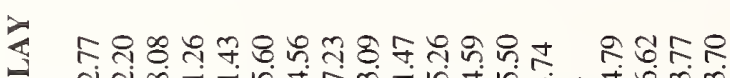

ड पुं

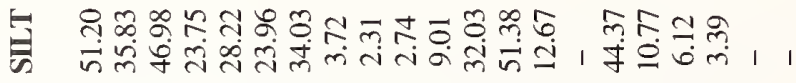

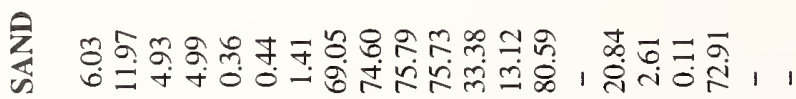

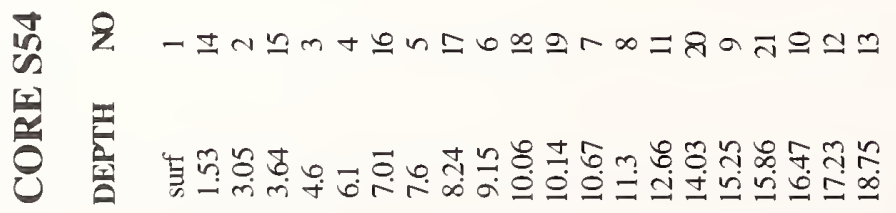


APPENDIX 2.-Continued.

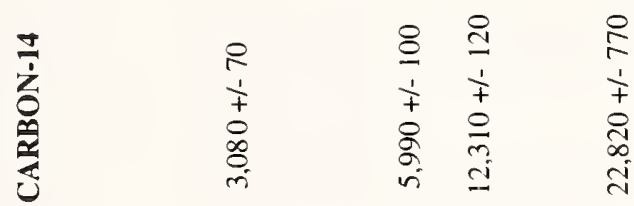

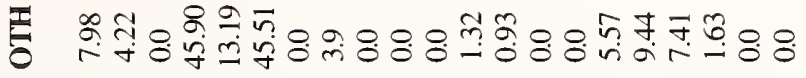

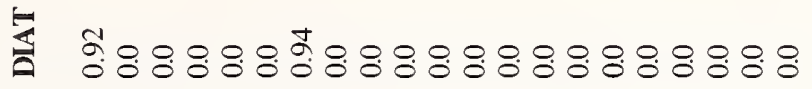

栲

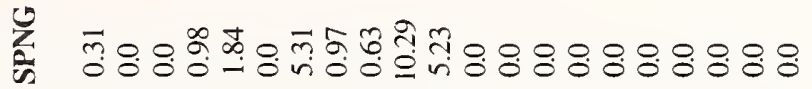

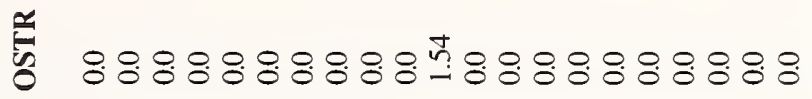

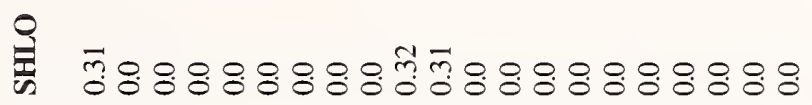

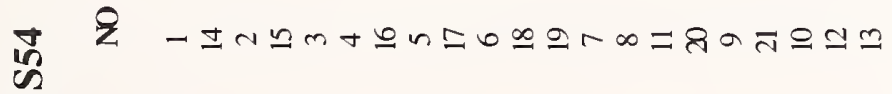

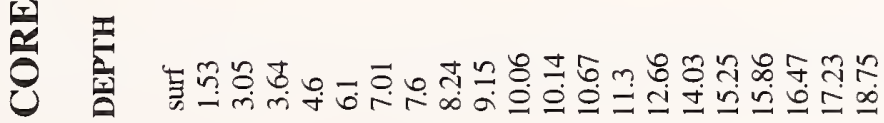


育

岁

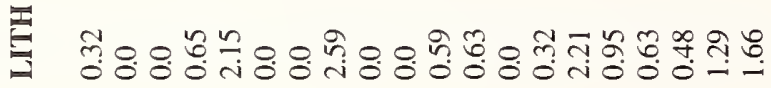

竞

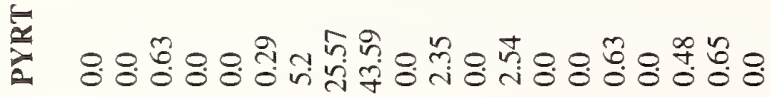

遮

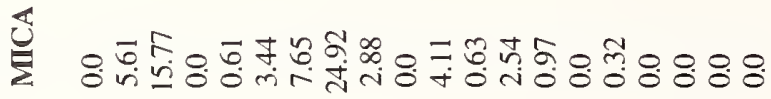

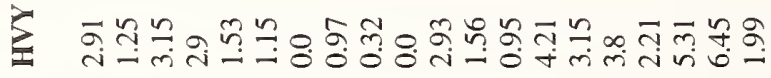

본

กำ

च लंखिं।

둔ำ

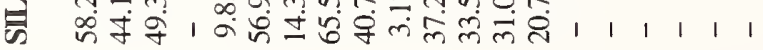

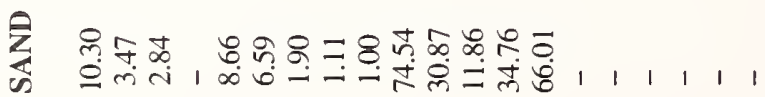

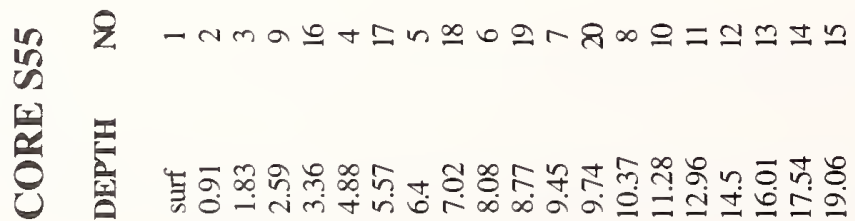


APPENDIX 2.-Continued.

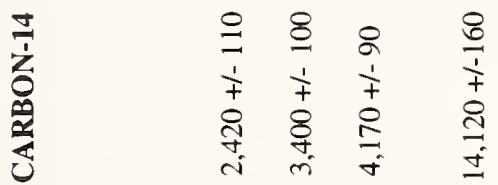

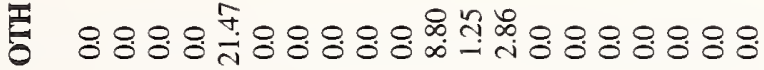

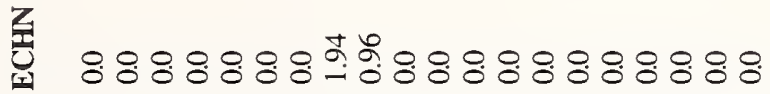

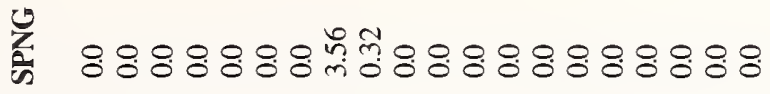

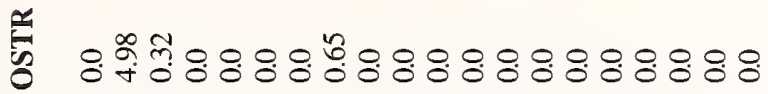

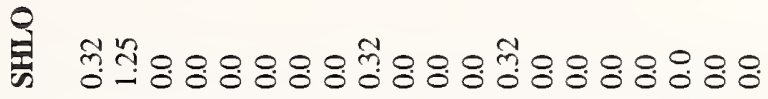

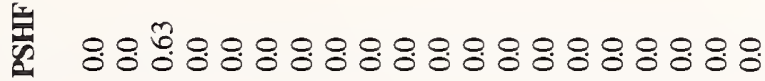

in ஜ

号 产 


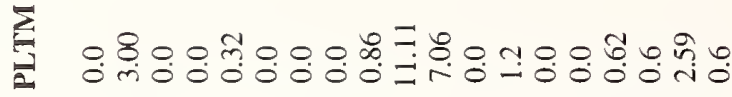

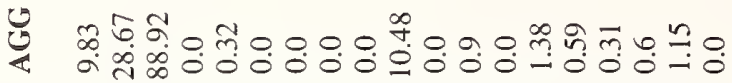

罗

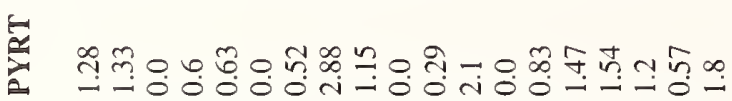

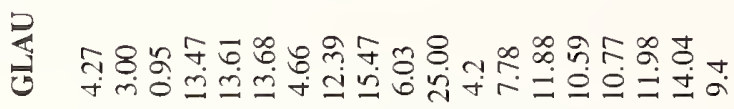

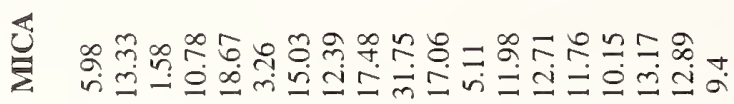

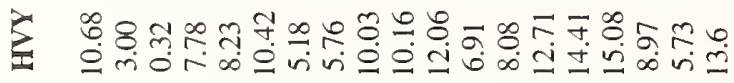

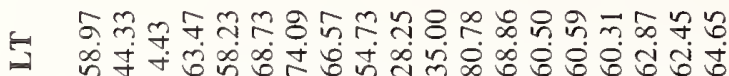

离

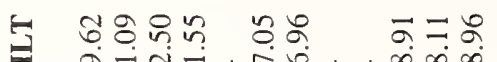

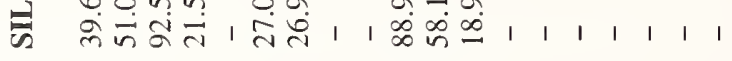

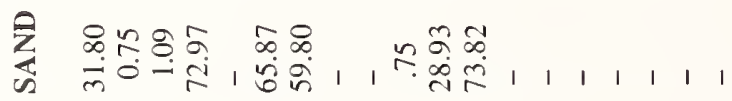

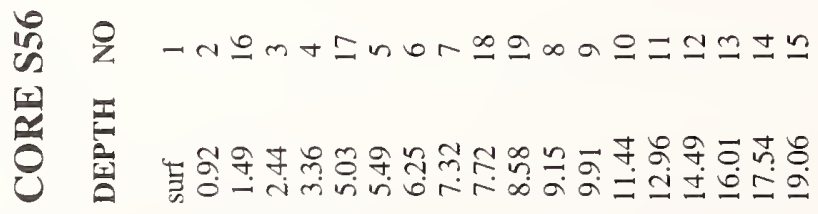


APPENDIX 2.-Continued.

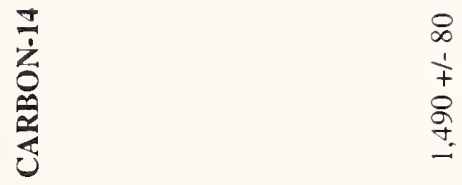

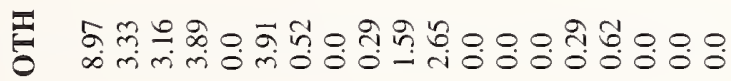

㑑

党

岳

:

通

吕

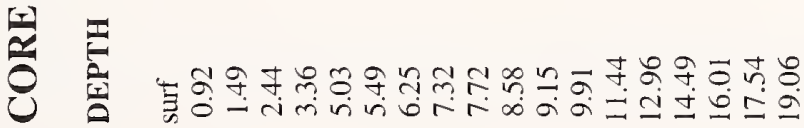


单 :

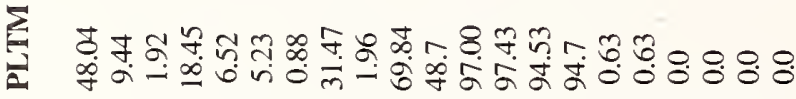

迎

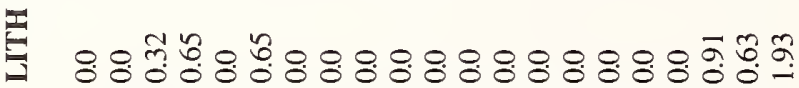

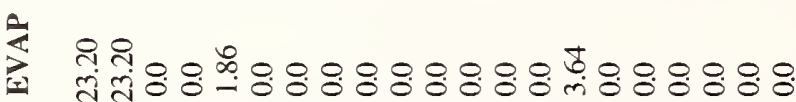

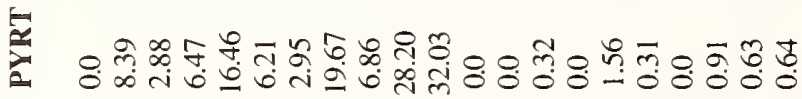

过

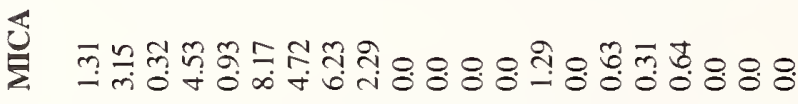

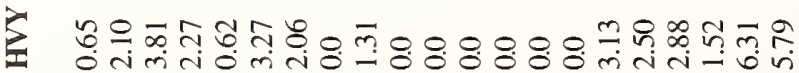

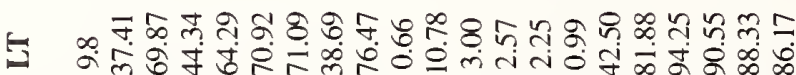

\

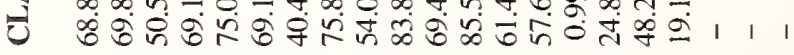

与

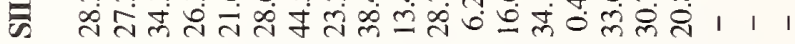

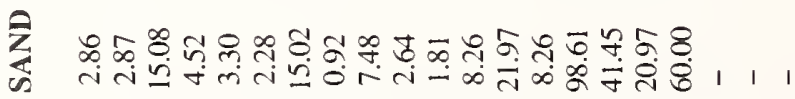

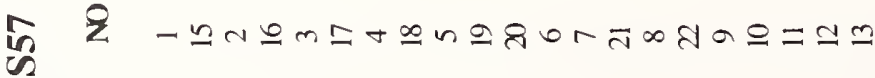

重 
APPENDIX 2.-Continued.

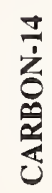

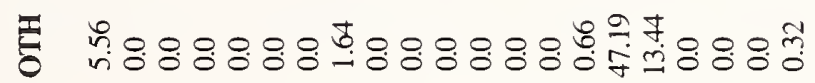

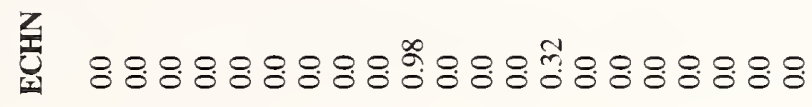

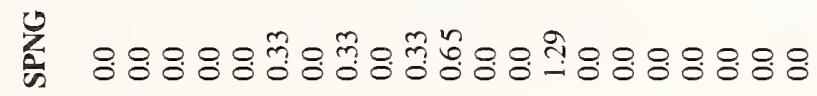

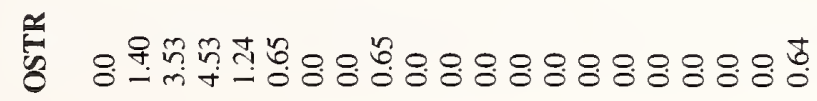

鼻

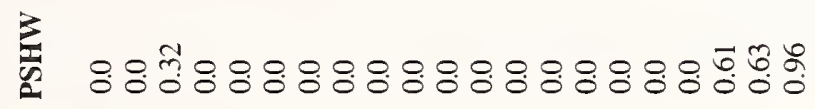

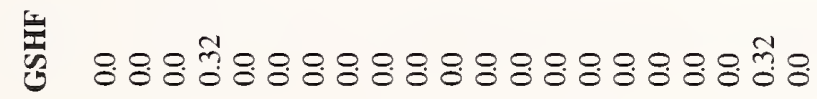

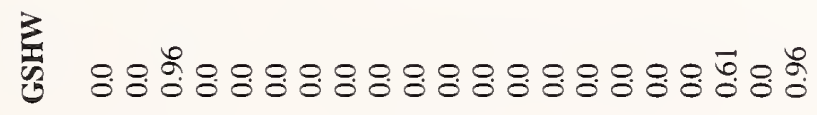

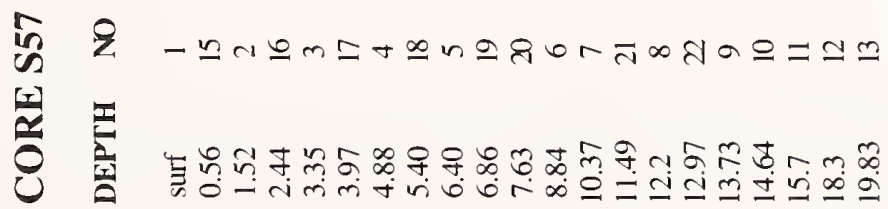




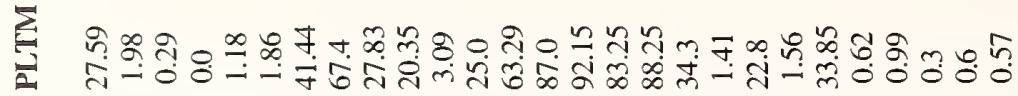

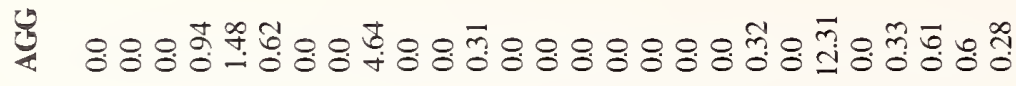

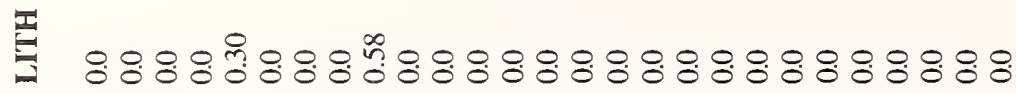

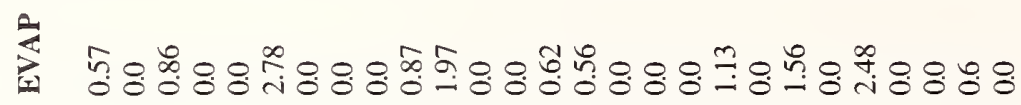

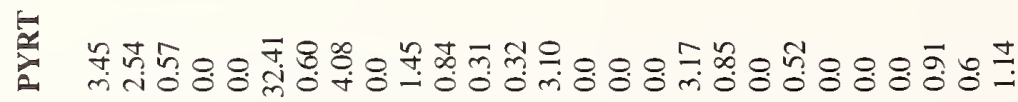

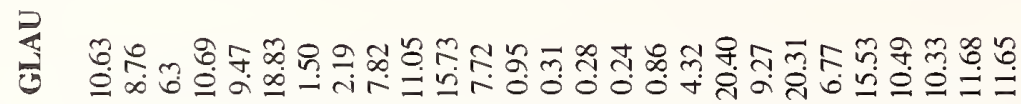

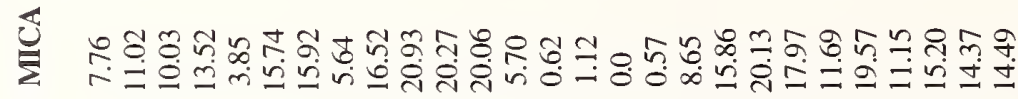

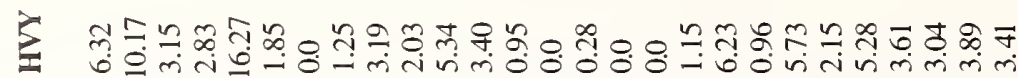

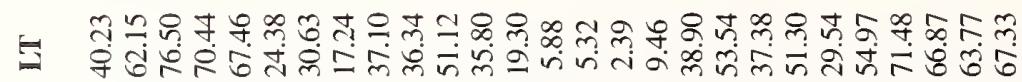

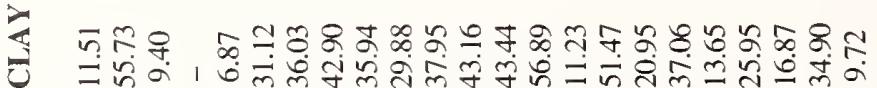

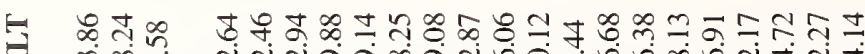

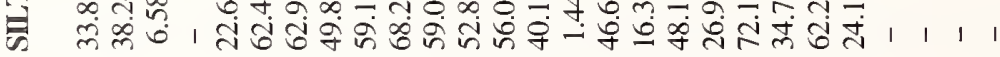

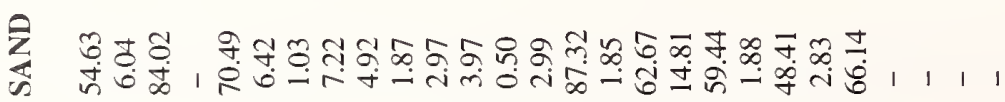

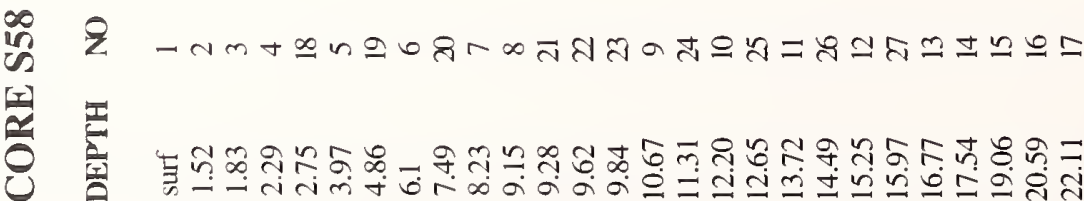


APPENDIX 2.-Continued.

\begin{tabular}{|c|c|c|c|}
\hline 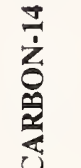 & $\begin{array}{l}8 \\
\infty \\
\frac{1}{+} \\
\stackrel{0}{0} \\
0 \\
0\end{array}$ & $\begin{array}{l}\stackrel{0}{\infty} \\
\frac{1}{+} \\
\stackrel{2}{\circ} \\
\stackrel{2}{F}\end{array}$ & 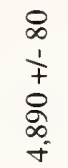 \\
\hline
\end{tabular}

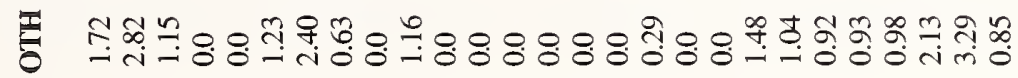

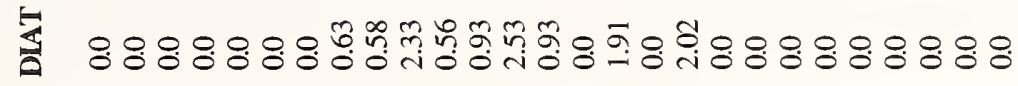

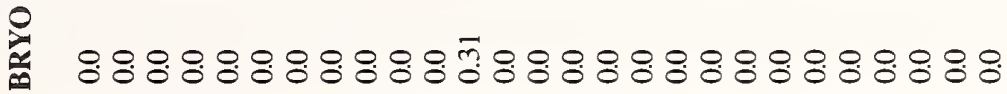

壳

দ̆

\%

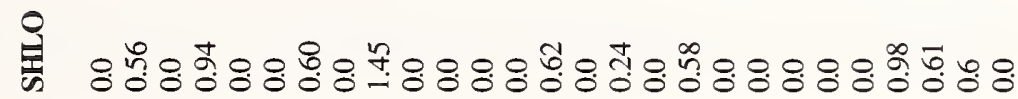

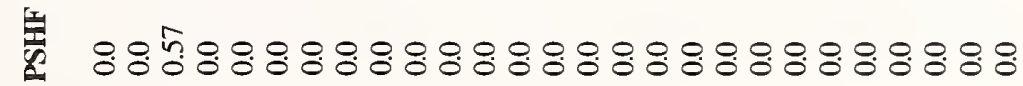

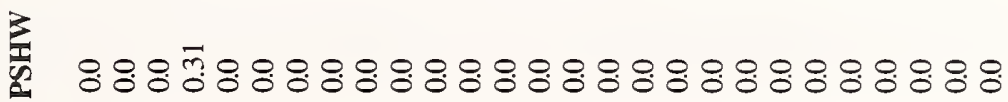

恿

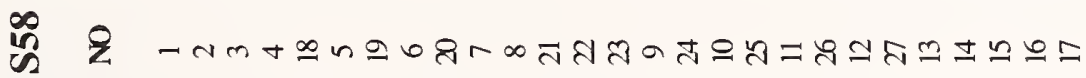

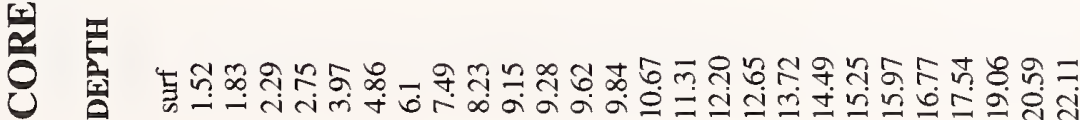


APPENDIX 2.-Continued.

产

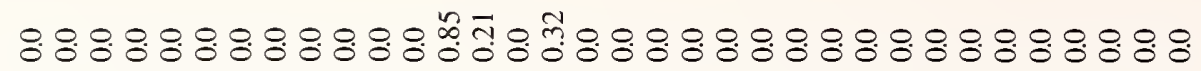

管

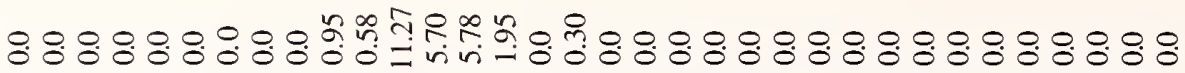

宓

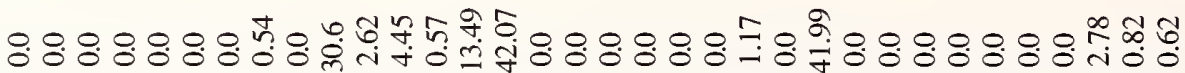

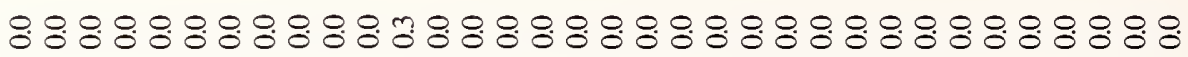

罚

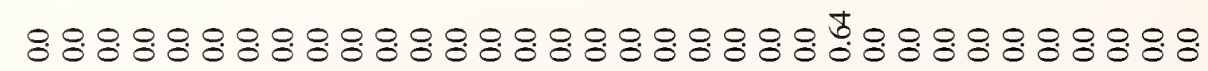
$\sum^{2}$

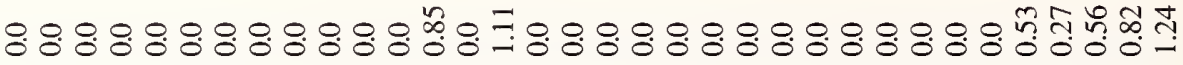

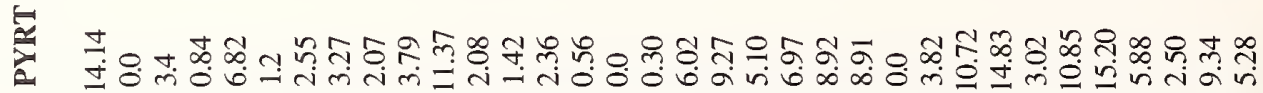

舀

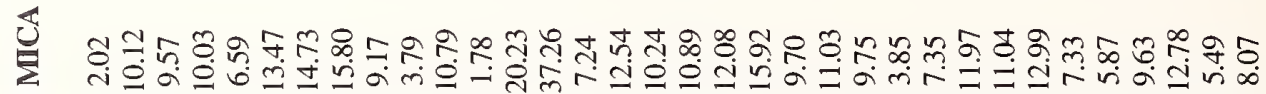

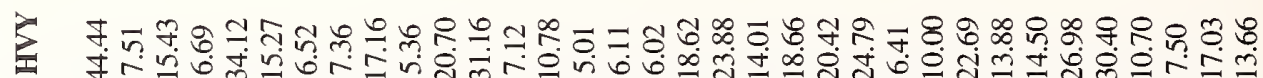

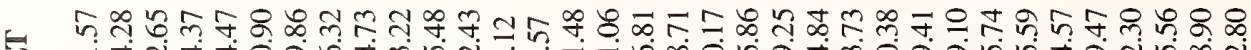

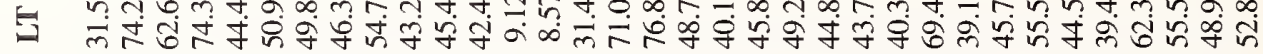

过

है,

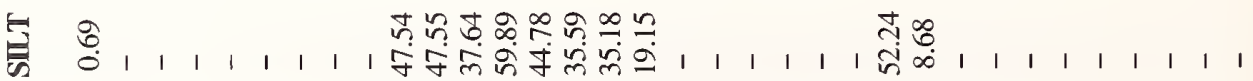

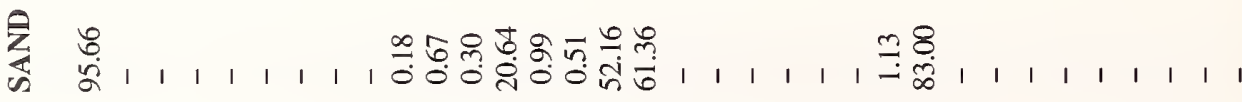

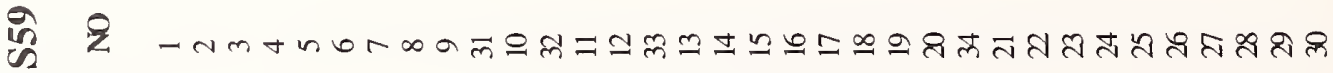

능 들

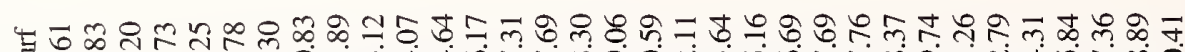

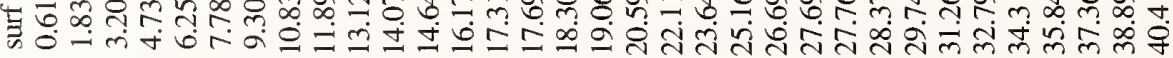


APPENDIX 2,-Continued.

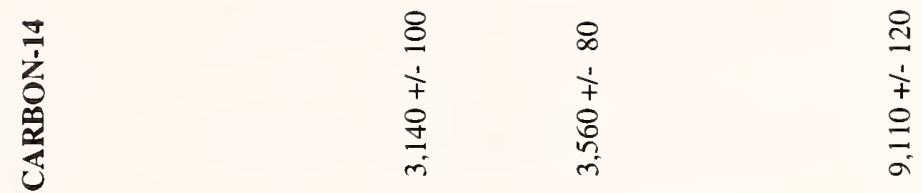

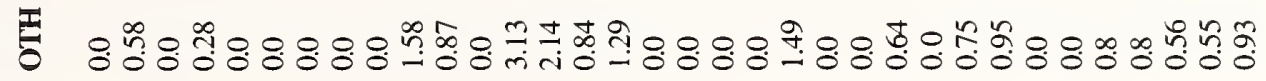

产

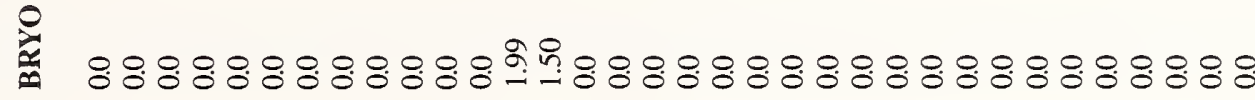

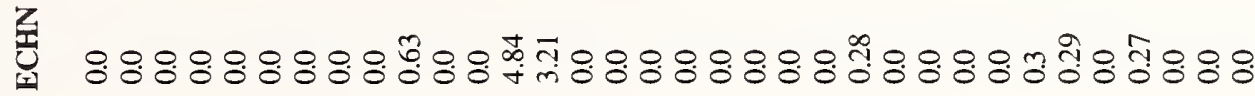

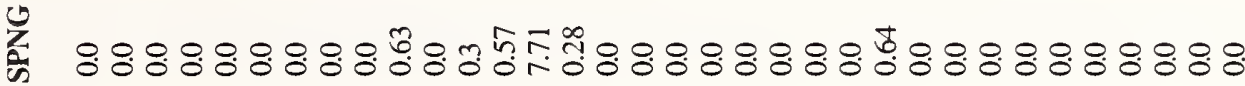

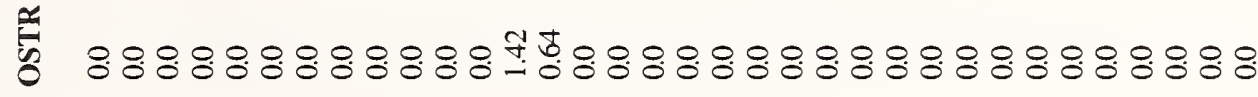

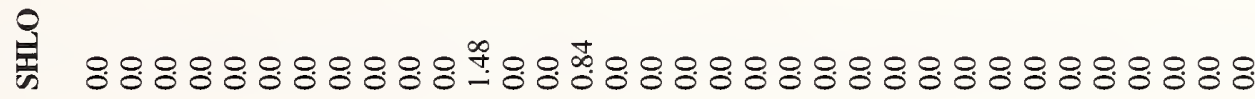

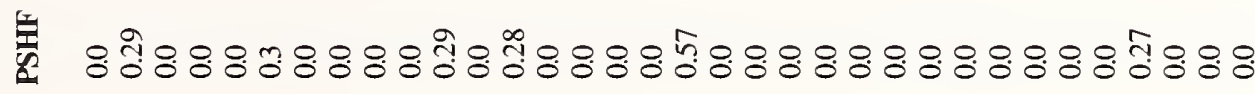

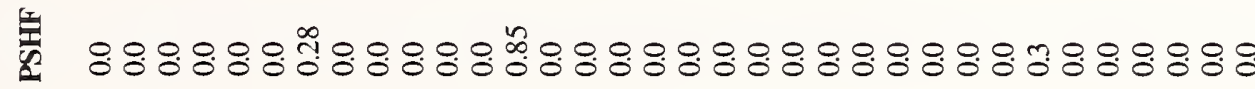

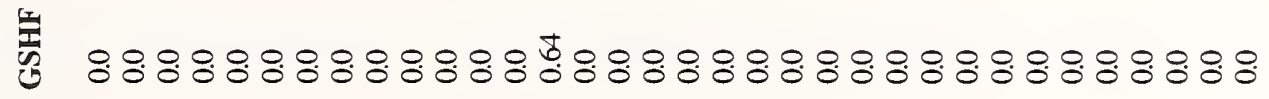

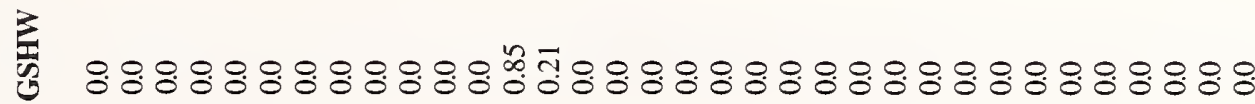

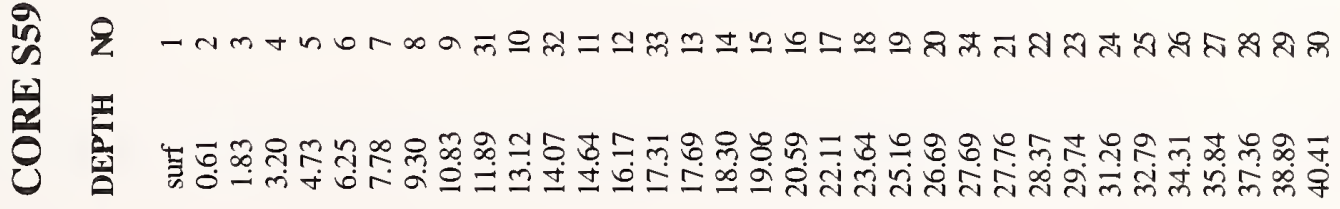


APPENDIX 2.-Continued.

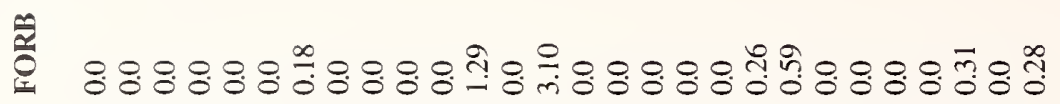

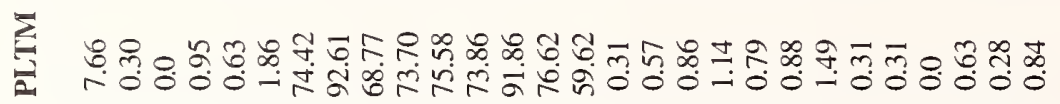

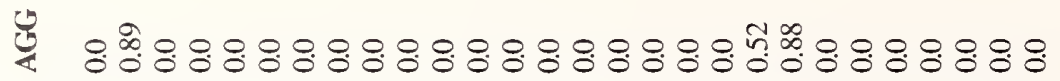

플

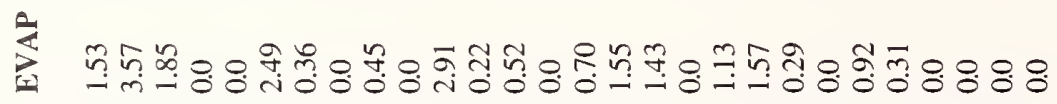

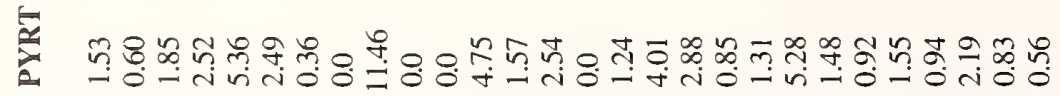

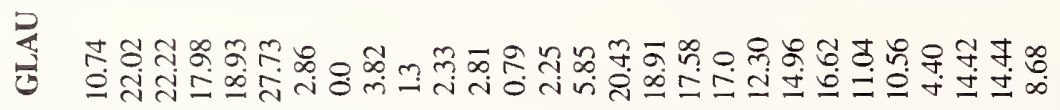

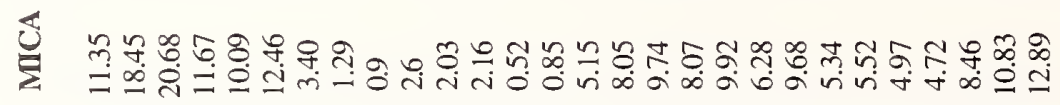

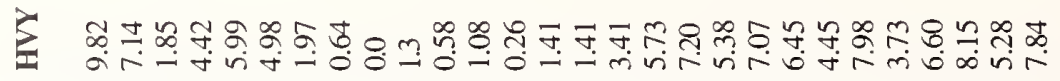

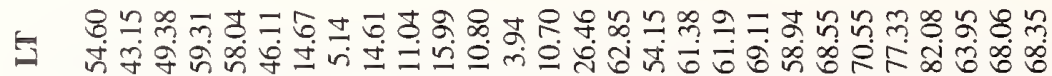

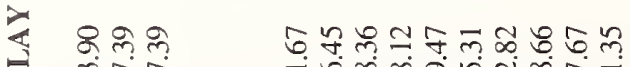

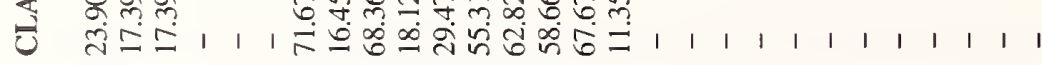

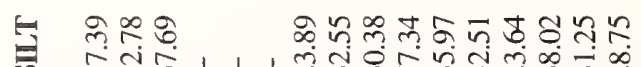

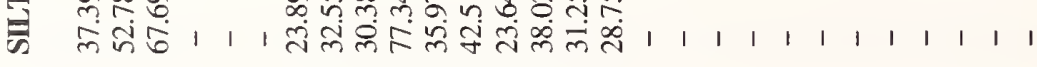

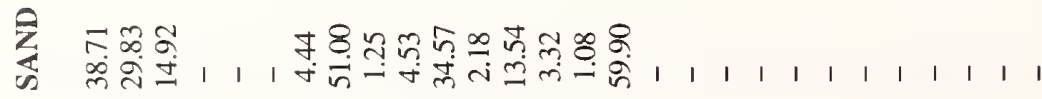

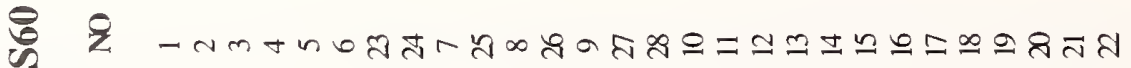

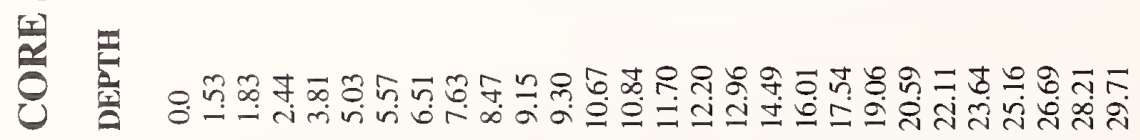


APPENDIX 2.-Continued.

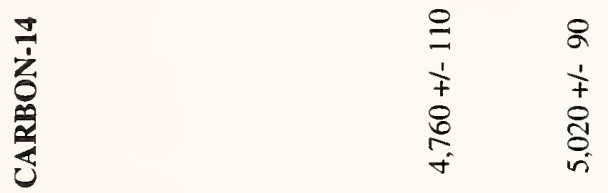

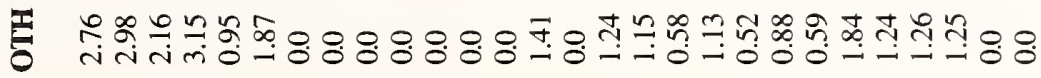

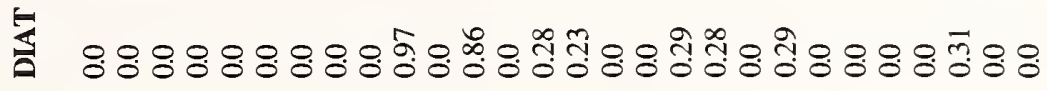

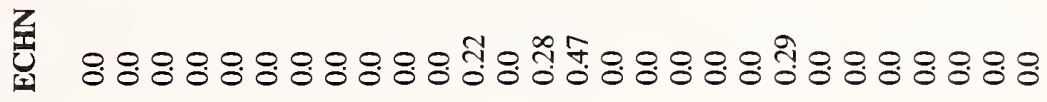

ڤ̆

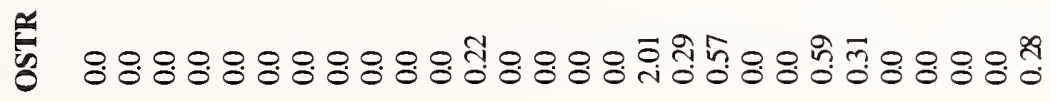

舅

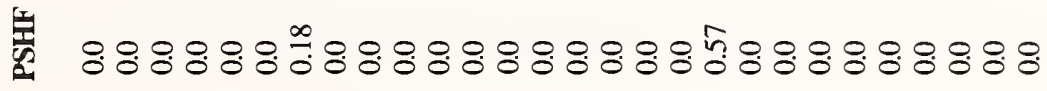

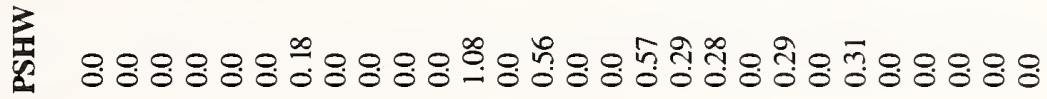

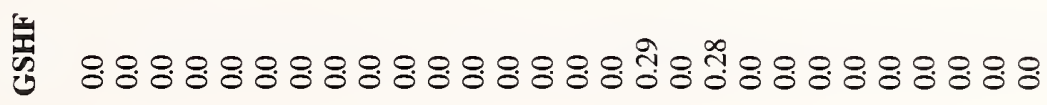

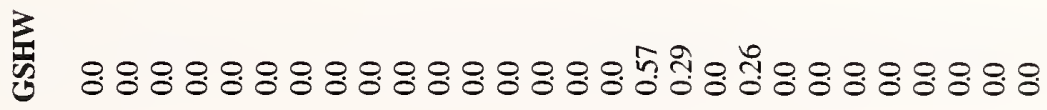

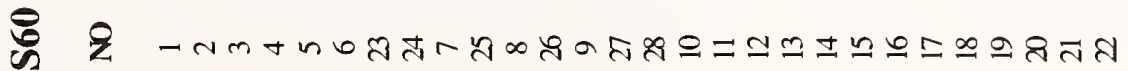

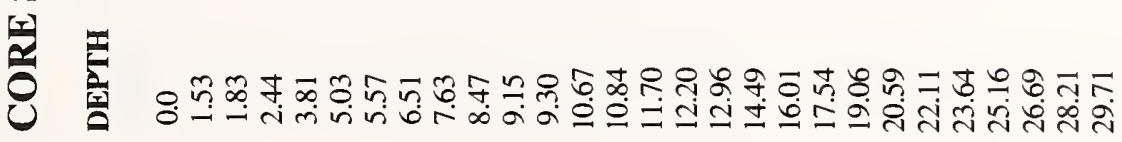


APPENDIX 2.-Continued.

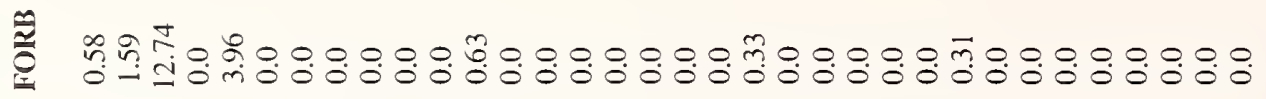

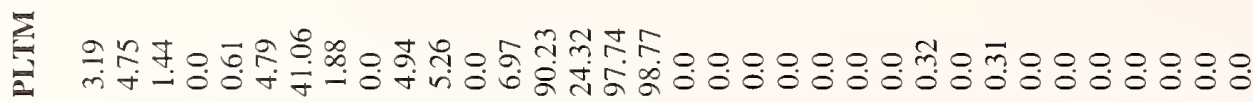

은

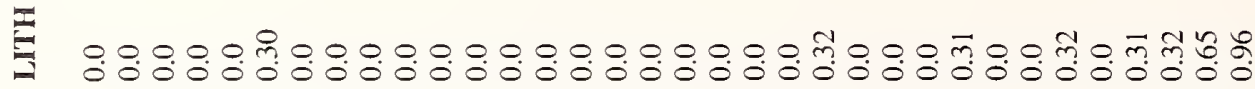

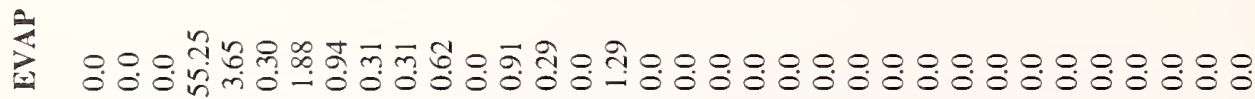

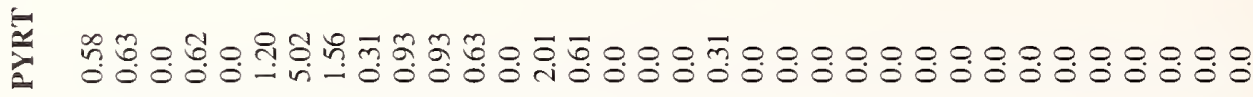

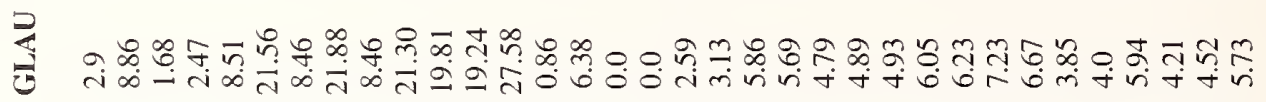

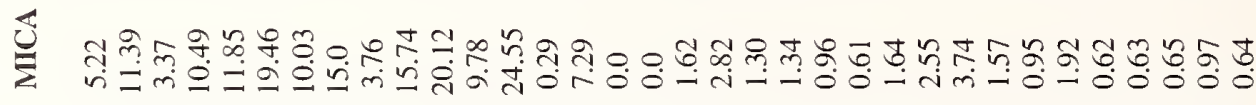

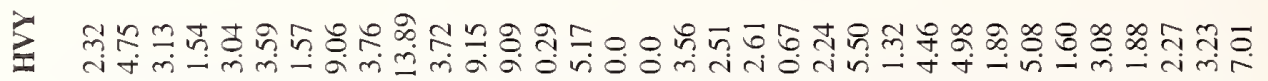

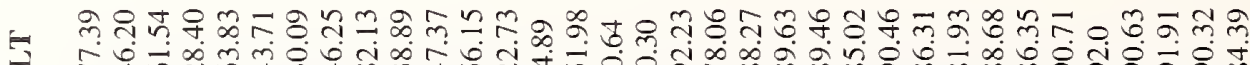

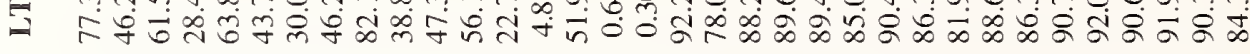

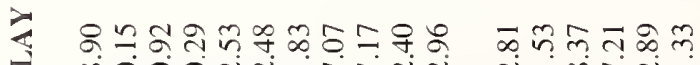

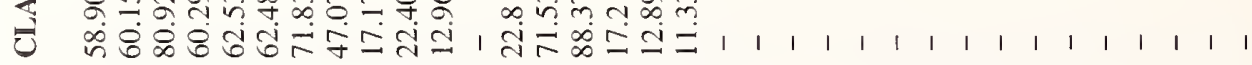

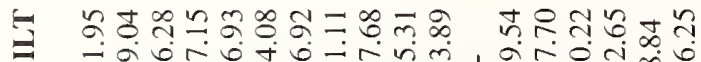

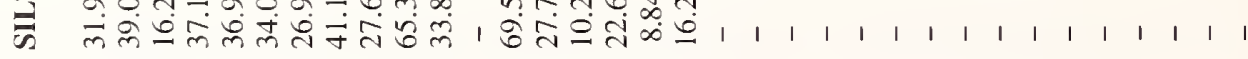

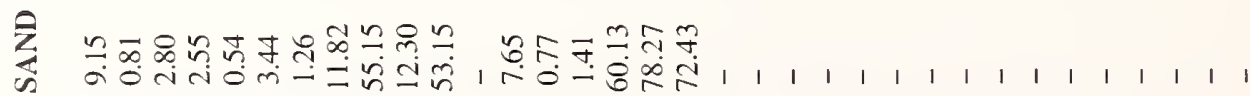

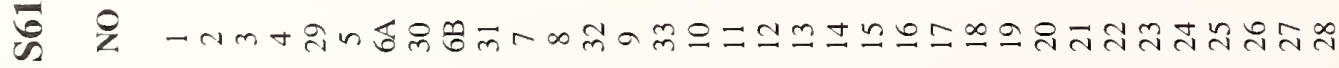

椞 
APPENDIX 2.-Continued.

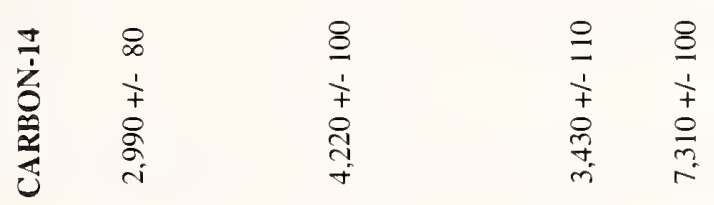

평

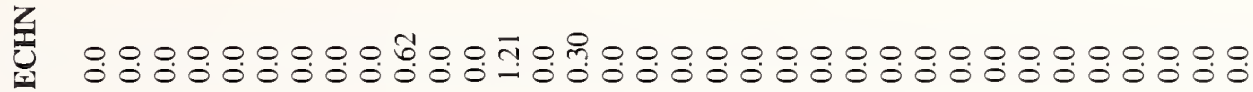

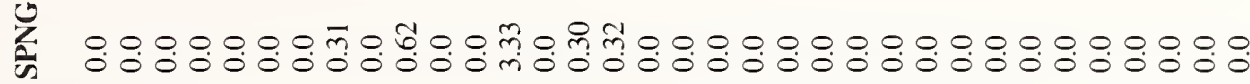

\%

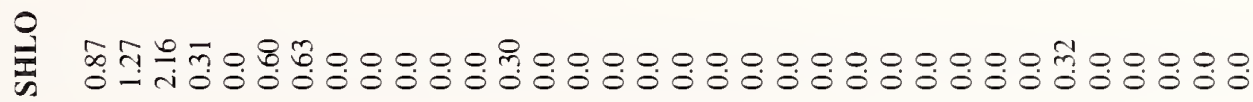

岁:

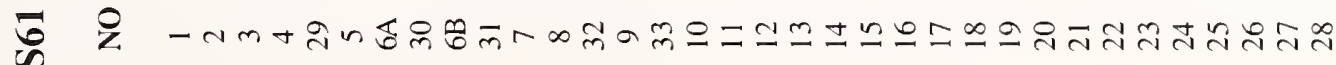

至 
APPENDIX 2.-Continued.

䓪

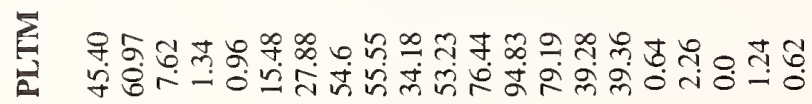

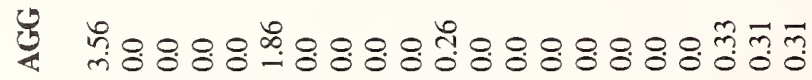

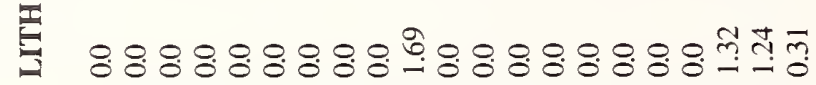

育

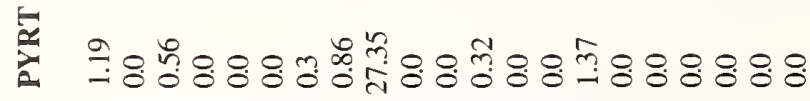

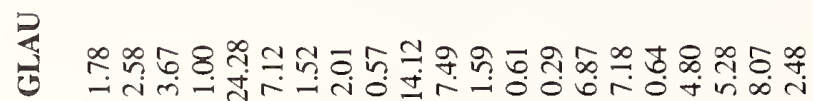

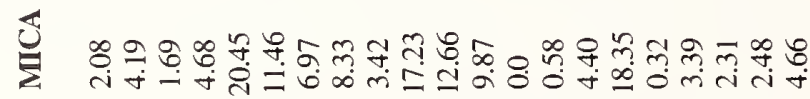

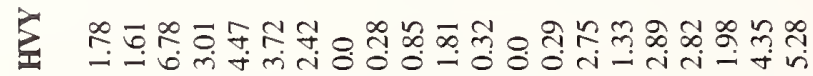

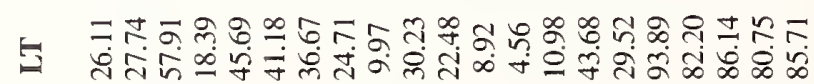

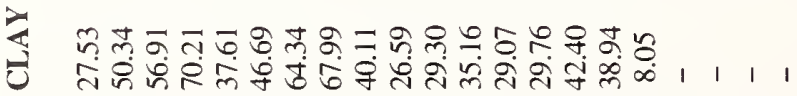

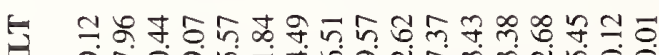

क में

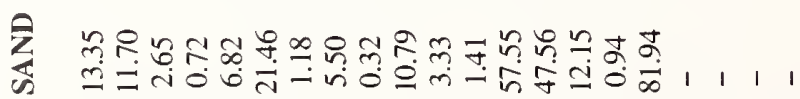

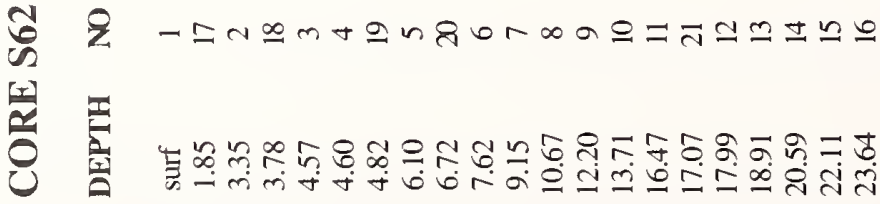


APPENDIX 2.-Continued.

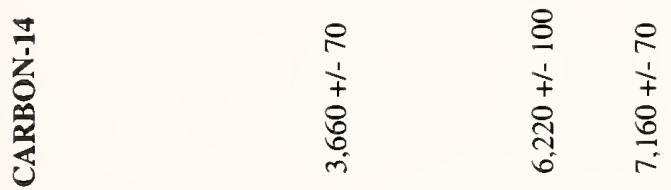

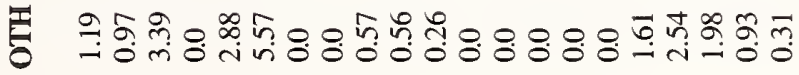

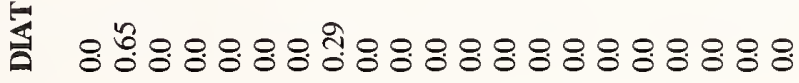

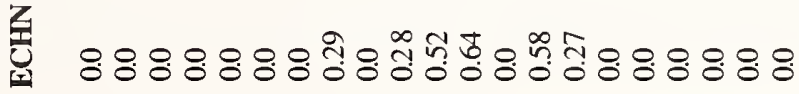

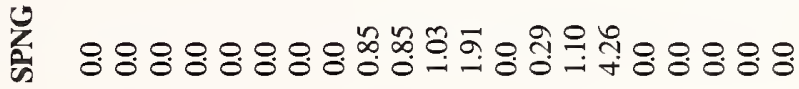

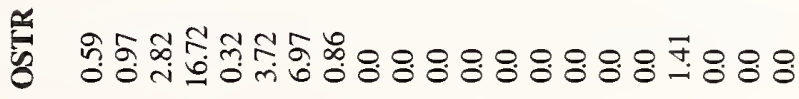

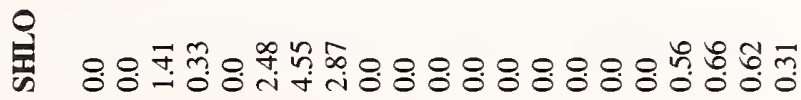

嚅

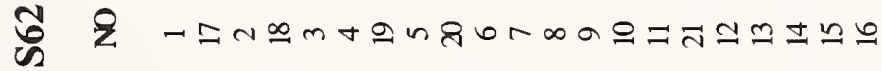

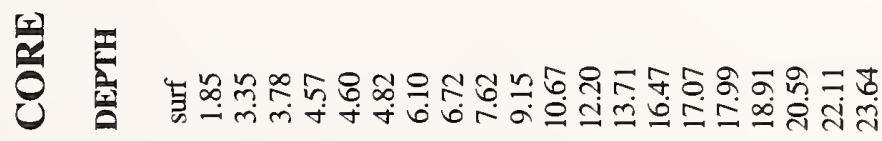


远

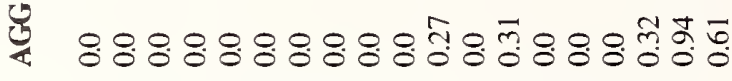

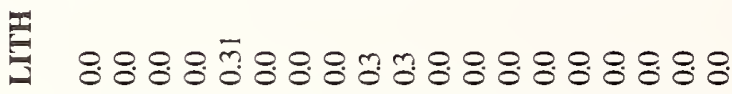

竞

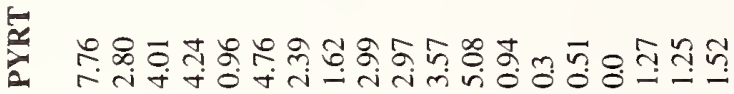

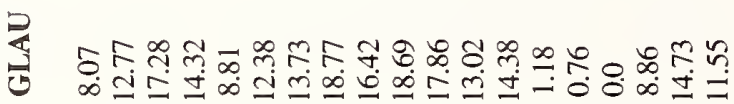

岁

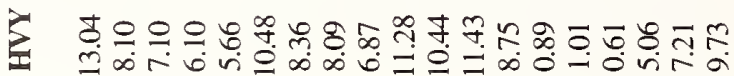

ㄴ

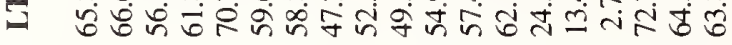

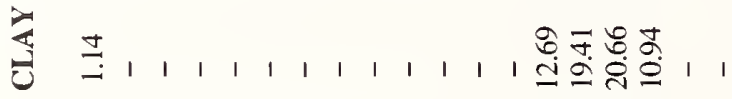

告

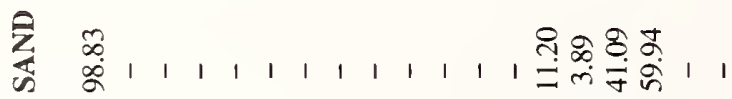

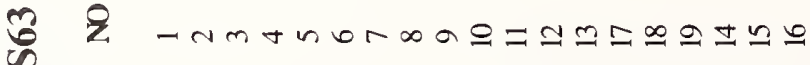

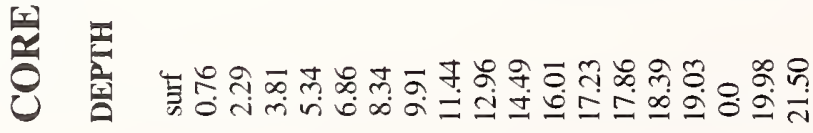


APPENDIX 2.-Continued.

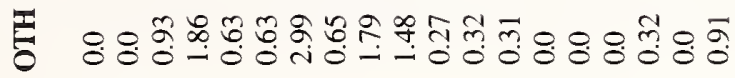

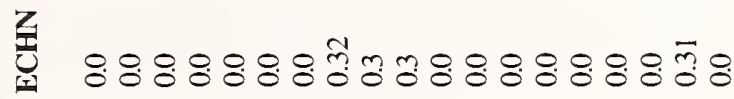

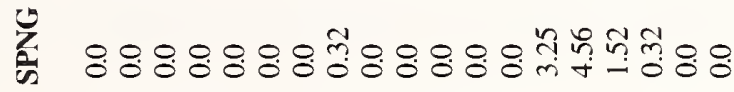

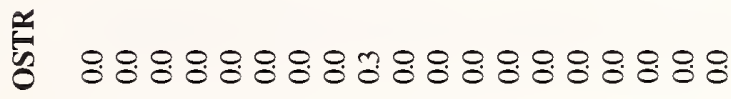

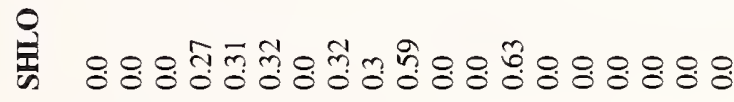

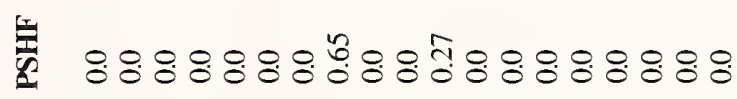

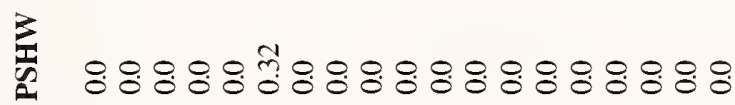

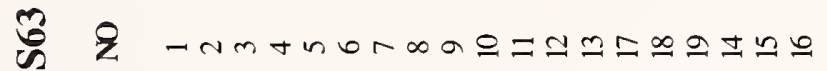

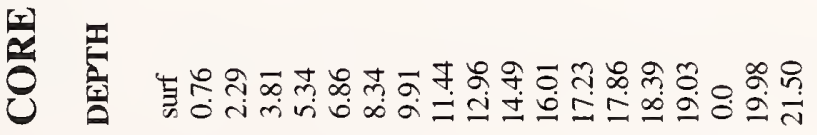


APPENDIX 2.-Continued.

홍

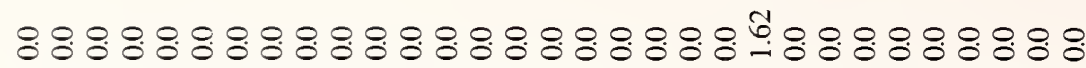

药

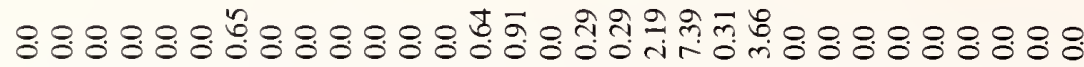

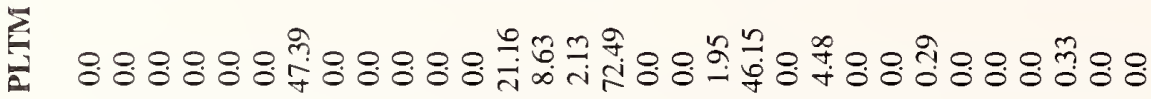

选

至

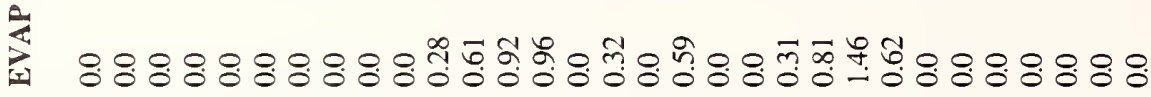

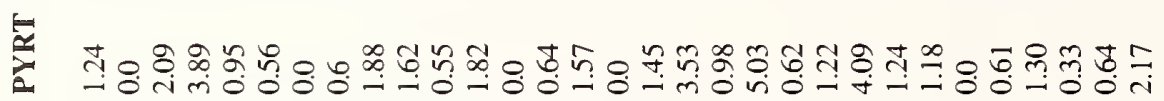

茯

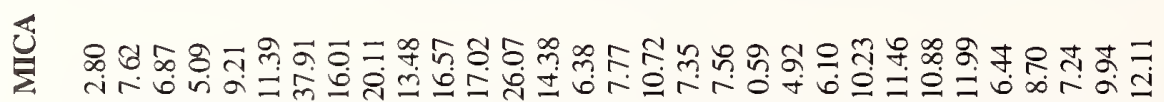

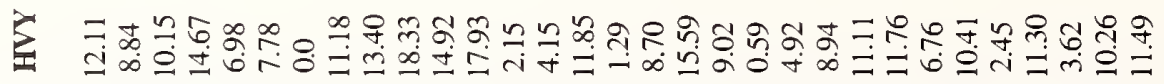

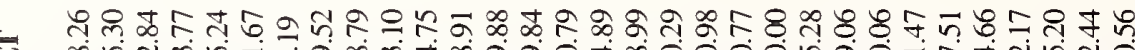

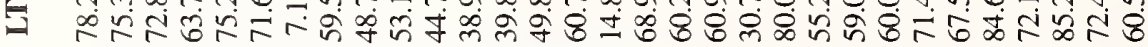

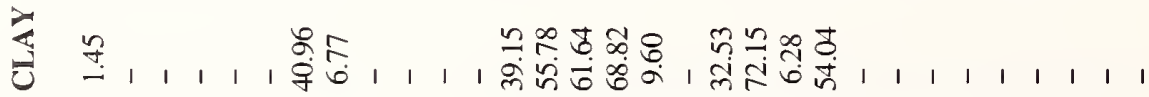

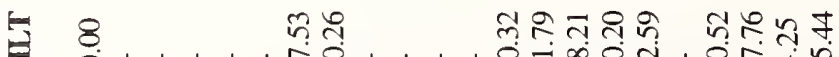

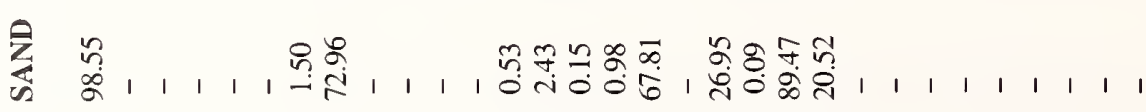

造 Z - 
APPENDLX 2.-Continued.

产 $888: 8$

: $8.8 \% 8$

运 8.8080

윤

연 $8.80 \%$

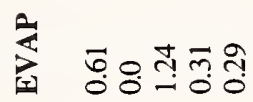

กิบ

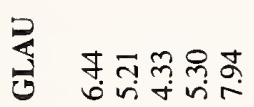

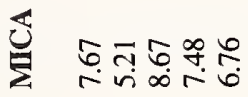

\&

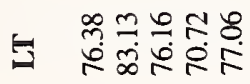

突 111111

岁 111111

点 1111111

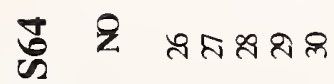

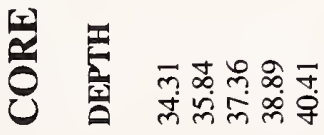


APPENDIX 2.-Continued.

交

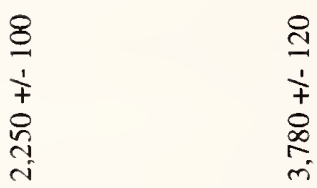

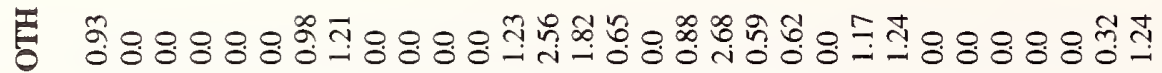

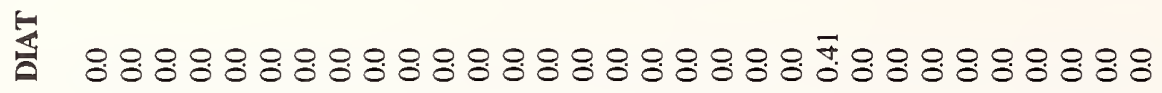

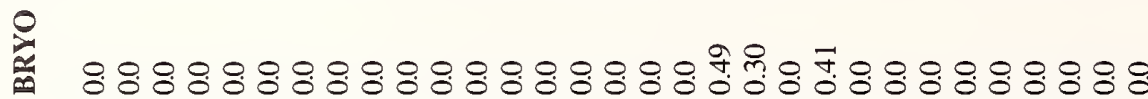

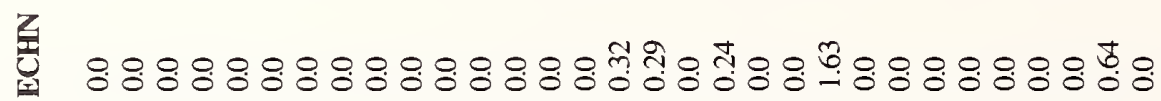

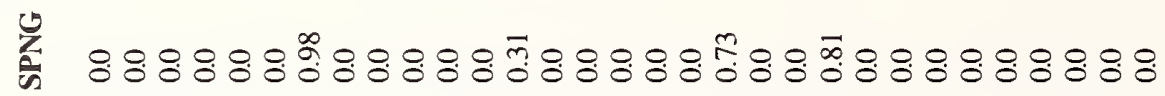

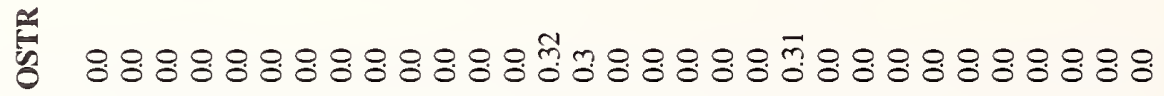

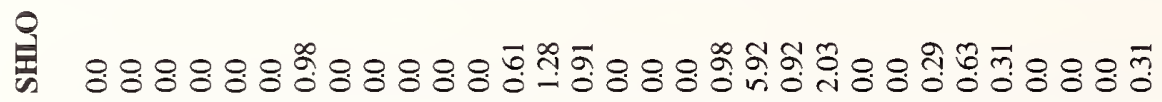

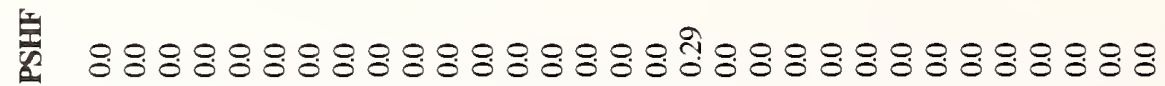

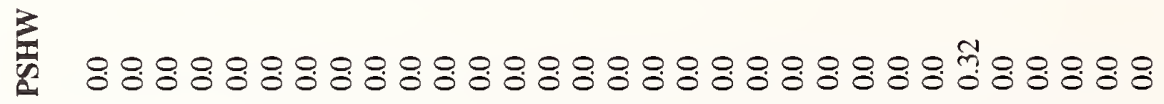

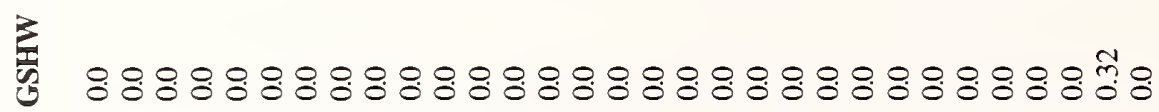

ず

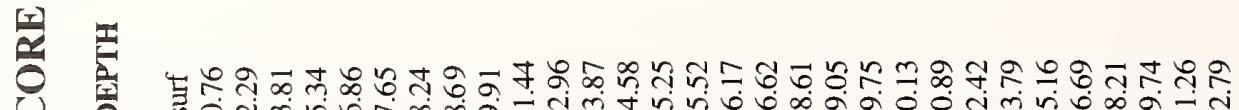

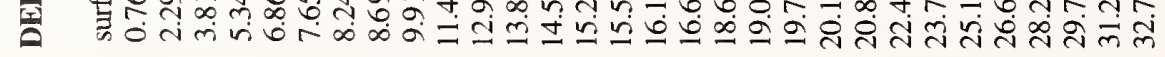


APPENDIX 2.-Continued.

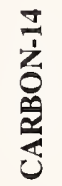

픙 훙형응 경용

谙 88888

荒 $8: 88:$

惫 $8: 8: 8$

产 $8888:$

突 88888

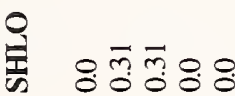

裹 $88: 88$

产 $8: 8: 8$

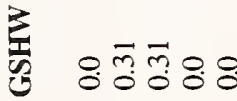

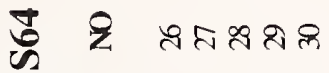

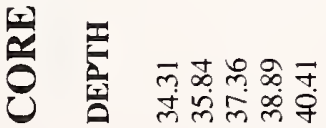


APPENDIX 2.-Continued.

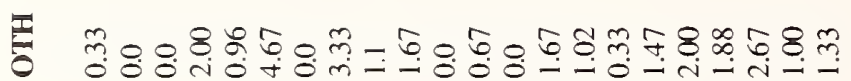

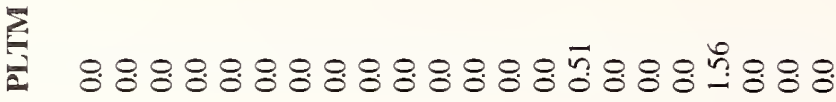

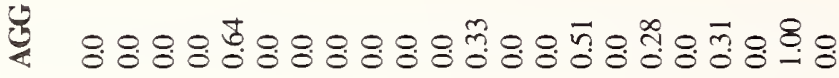

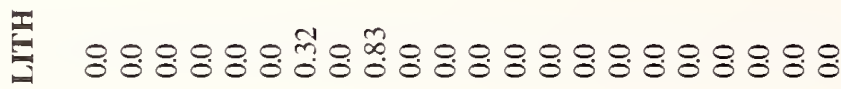

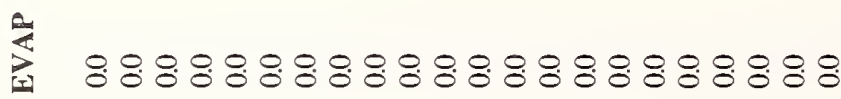

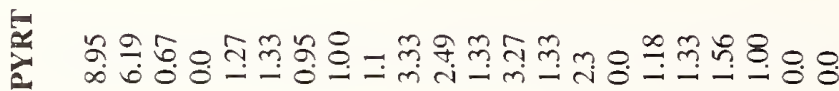

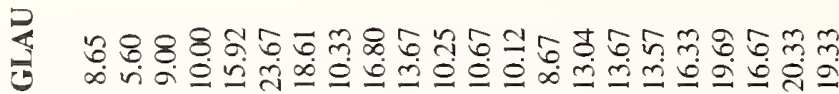

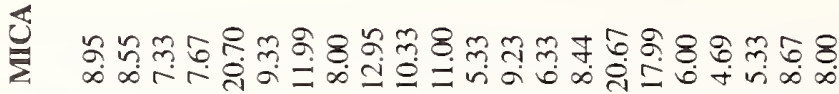

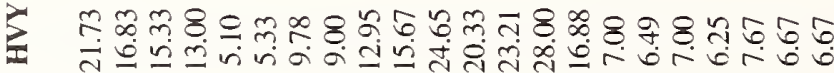

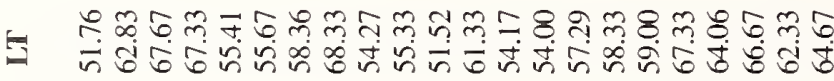

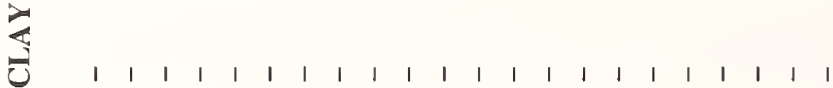

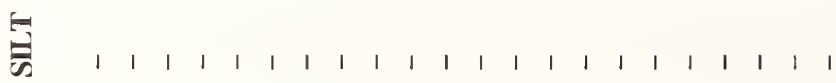

$\hat{z}$
$\vdots$

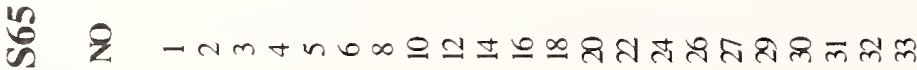

ป 
NUMBER 37

APPENDIX 2.-Continued on following page. 
APPENDIX 2.-Continued.

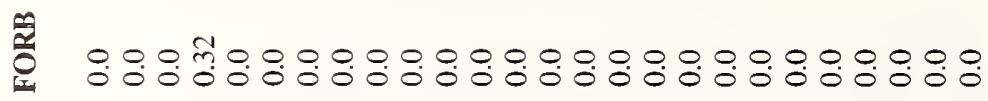

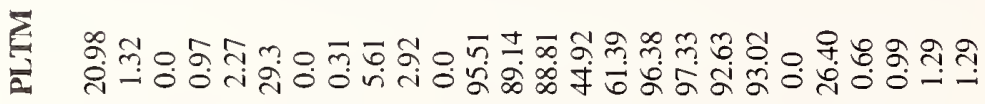

丞

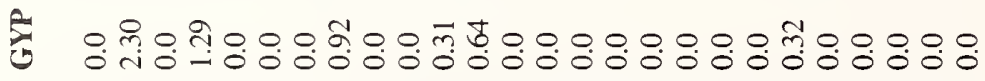

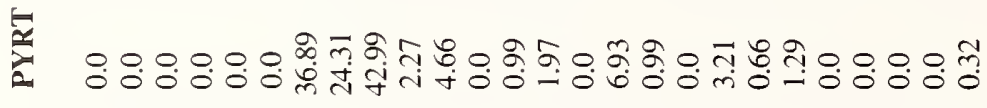

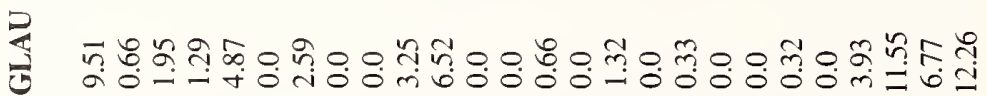

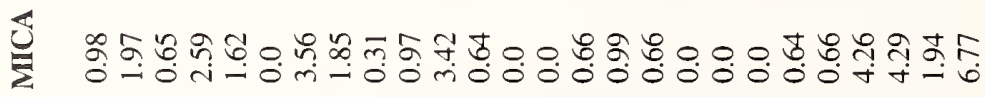

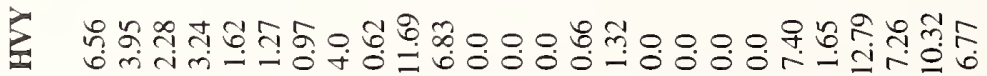

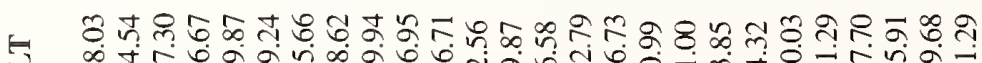

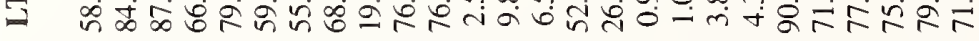

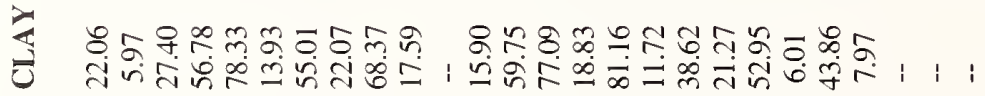

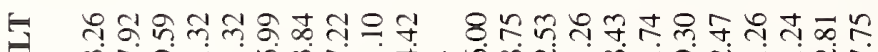

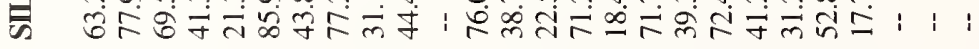

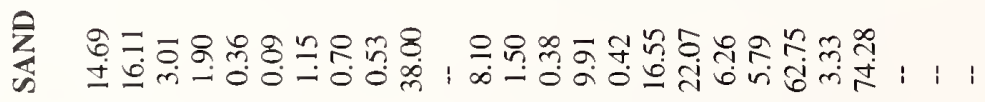

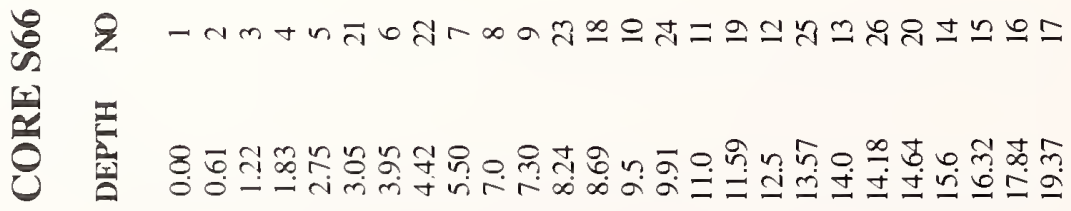


APPENDIX 2.-Continued.

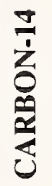

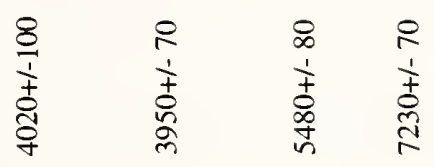

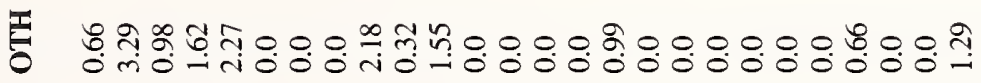

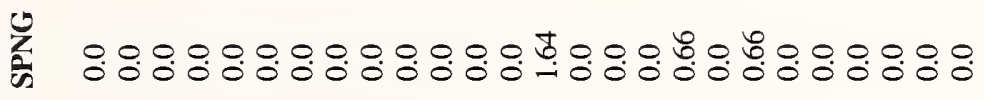

爱 ஃั

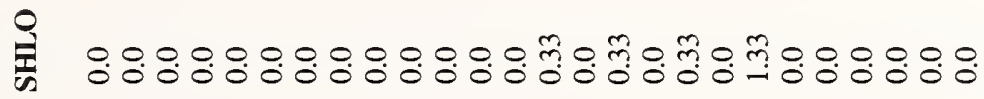

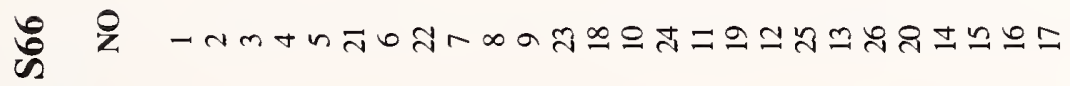

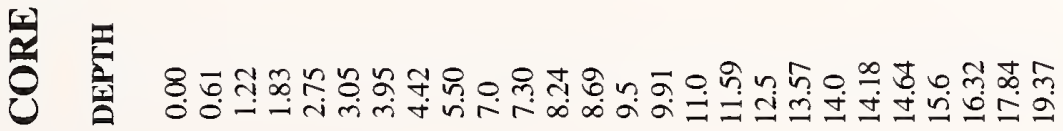


응 $\therefore: 0: 00: 0000080000$

売

鼠

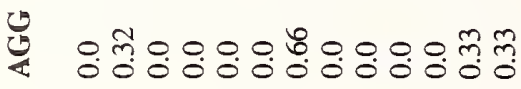

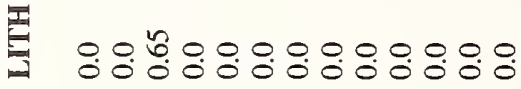

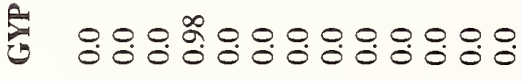

年

节

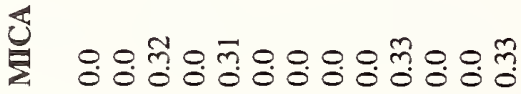

远

ज़ڤ

岂

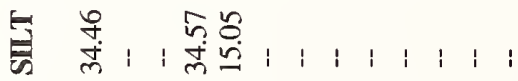

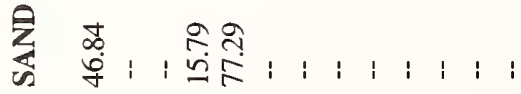

is $2-2 m m+n$ -

仓 
APPENDIX 2.-Continued.

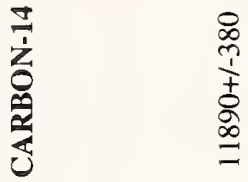

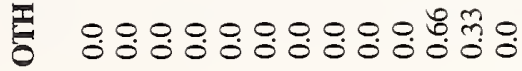

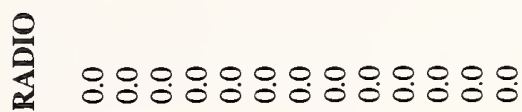

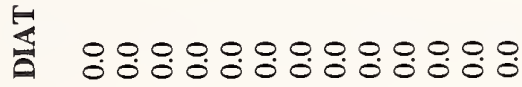

Z

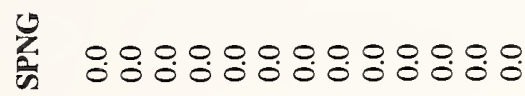

냉

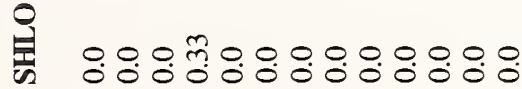

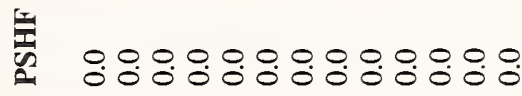

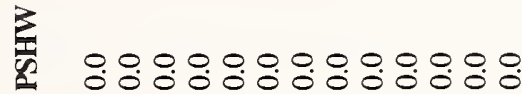

留

婊

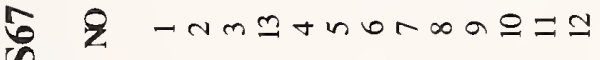

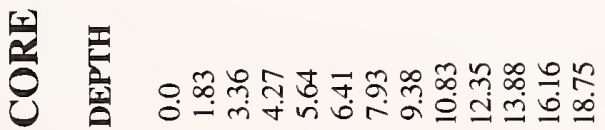


APPENDIX 2.-Continued.

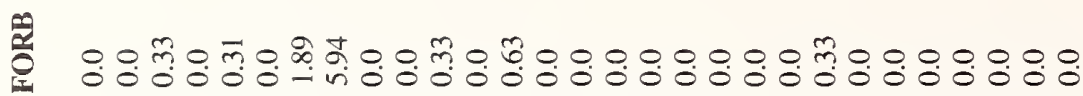

进

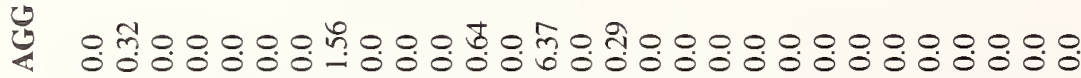

虑

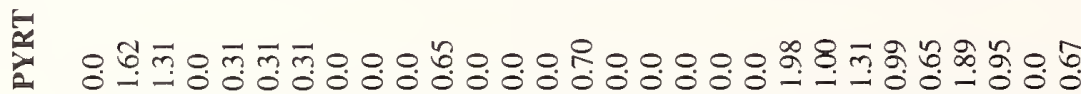

芶

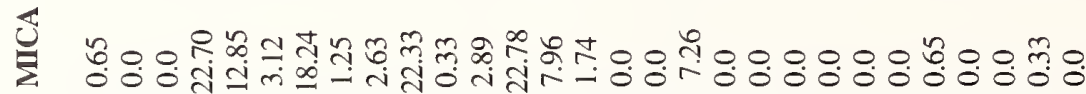

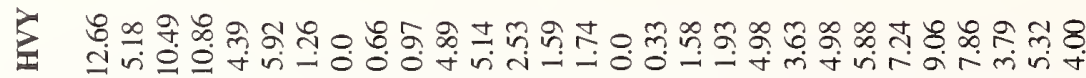

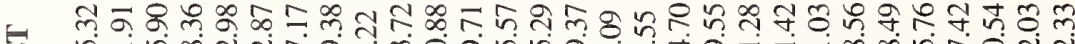

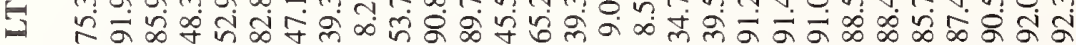

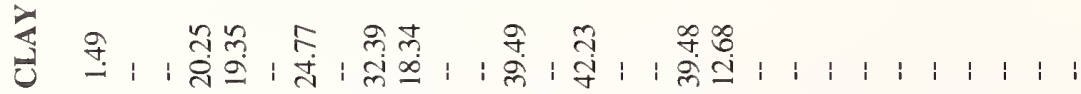

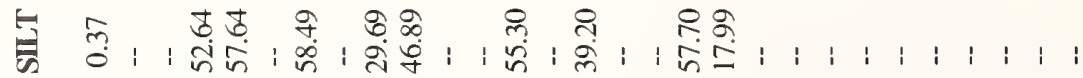

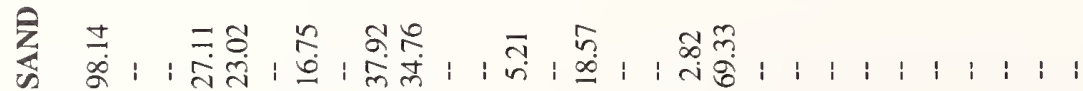

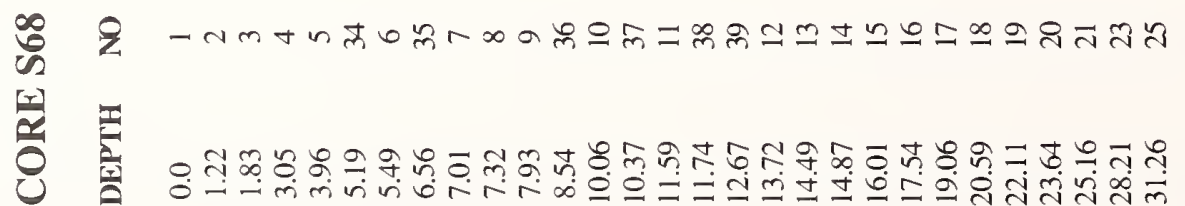


APPENDIX 2.-Continued.

: $0: 00:$

․․․ 8080

연 영융

댕 :8:0:

ํํำ 30 ㅇํㅇ

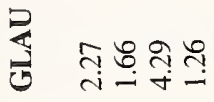

ङ 8008

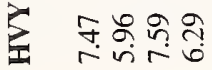

5 항ํㅇㅇํㅇ

= केष्ठ

¿

舅: : :

意: : : :

ஜ ₹ त成

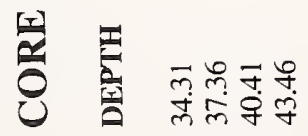


APPENDIX 2.-Continued.

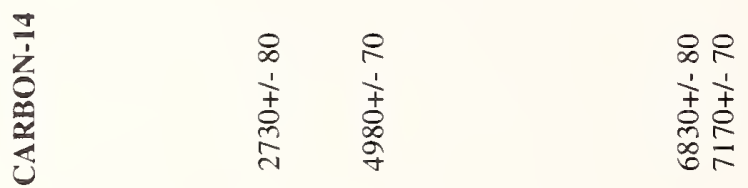

당

运

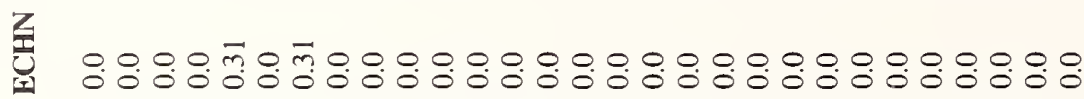

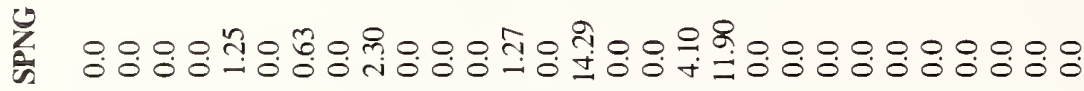

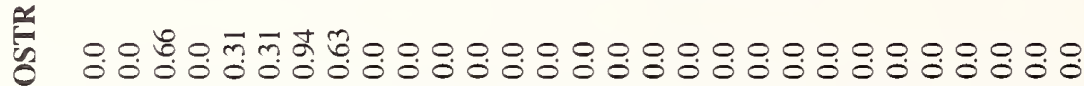

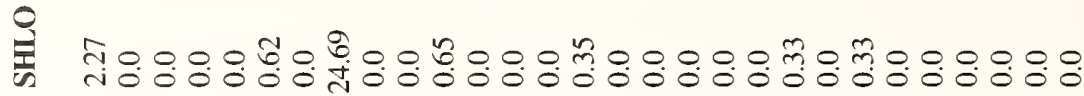

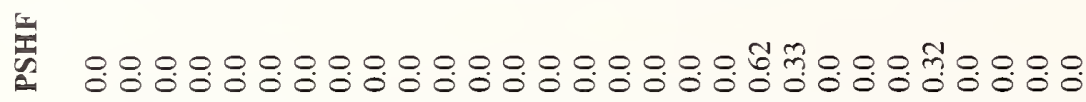

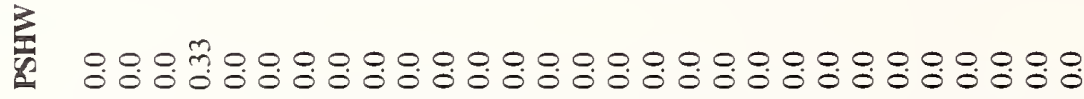

焉

운

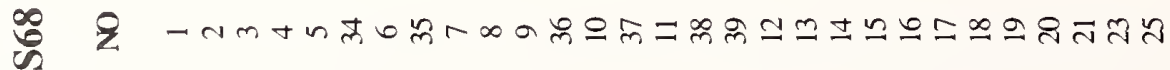

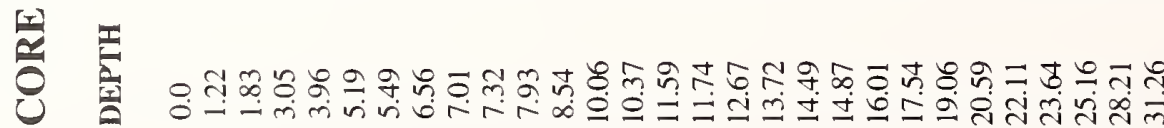


APPENDIX 2.--Continued.

$\frac{⿱ 亠 乂}{2}$

응 융웅우

茪 0.0000

夏 80000

娄 융용

\& 0.800

윤

蹗

융유

엔융유.

인 0.000

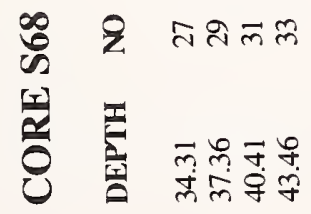


APPENDIX 2.-Continued.

䓵

望

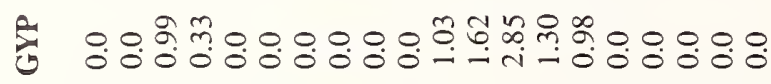

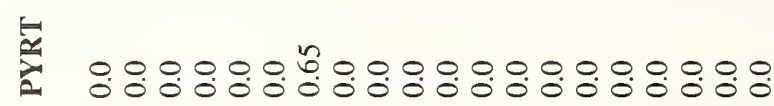

忽

造

空

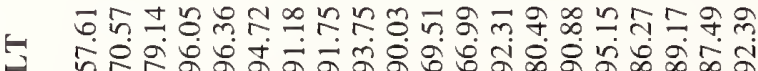

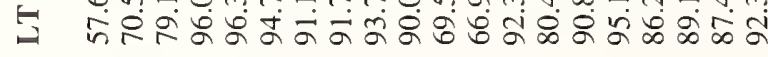

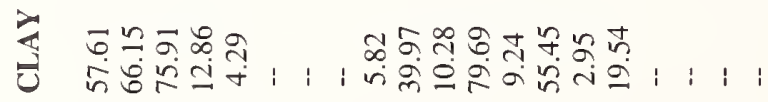

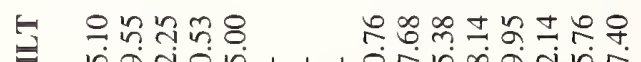

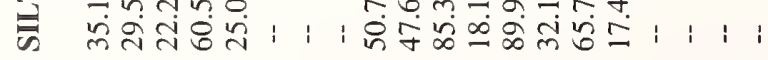

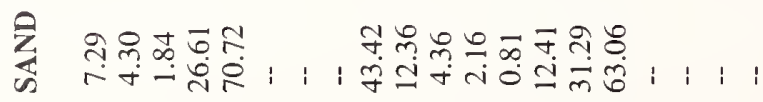

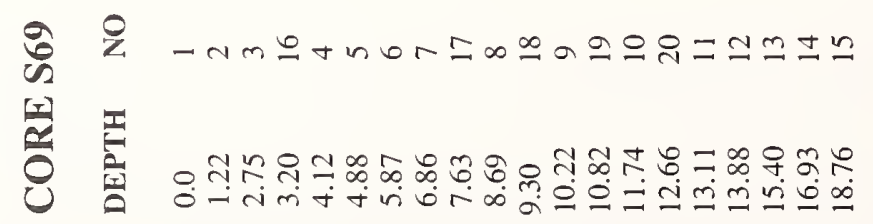


APPENDIX 2.-Continued.

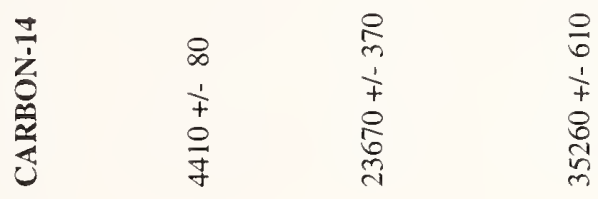

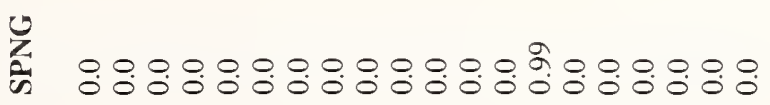

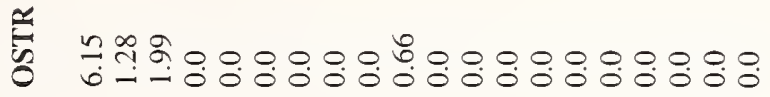

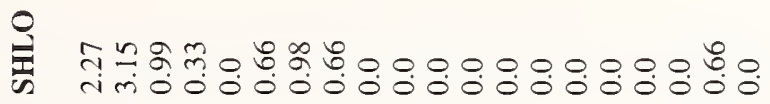

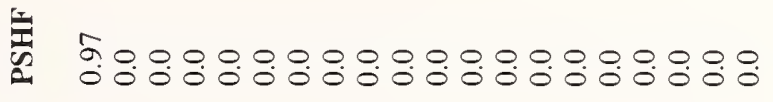

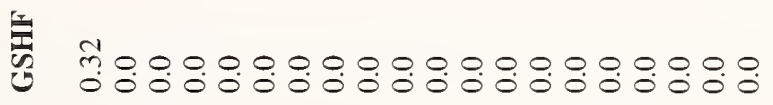

鄯

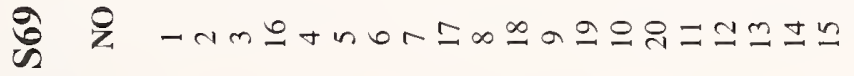

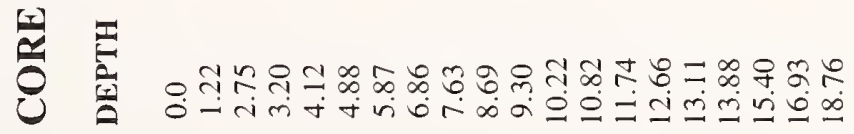


APPENDIX 2.-Continued.

产

绾

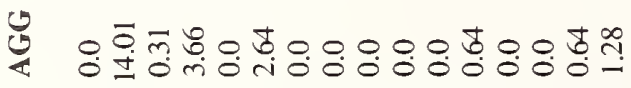

तु

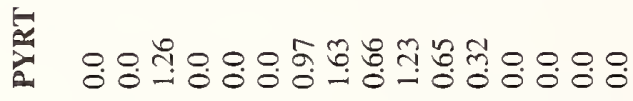

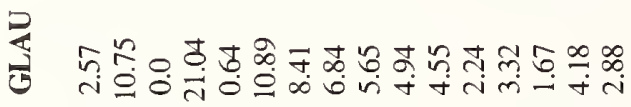

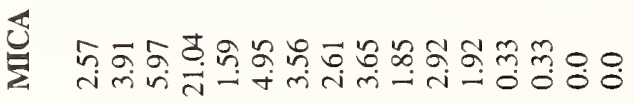

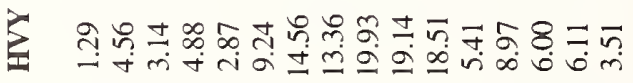

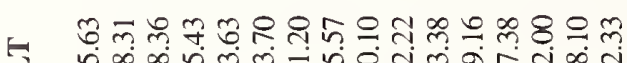

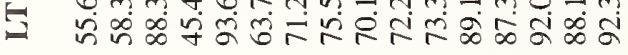

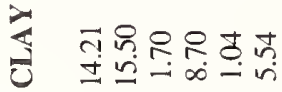

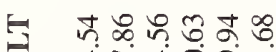

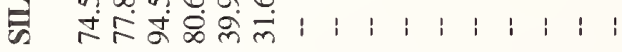

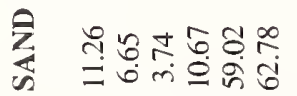

을

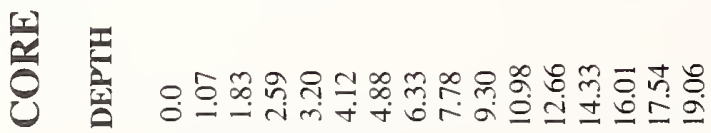


APPENDIX 2.-Continued.

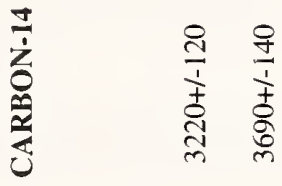

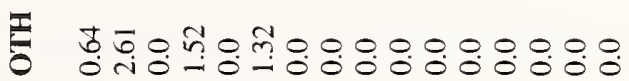

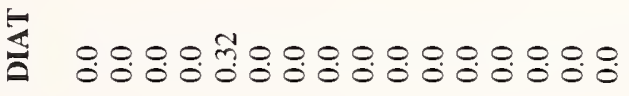

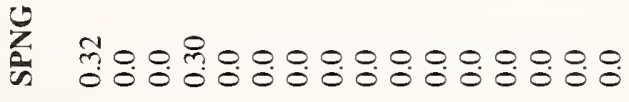

㝵

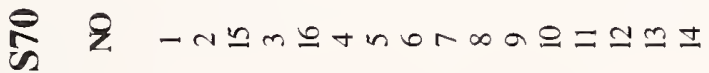

重 
APPENDIX 2.-Continued.

㠃

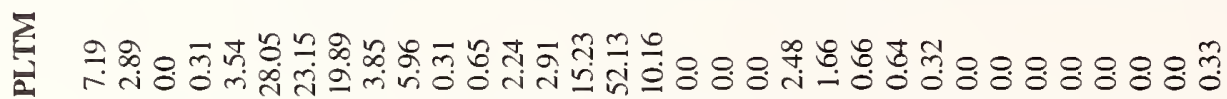

烍 $8: 8.88$.

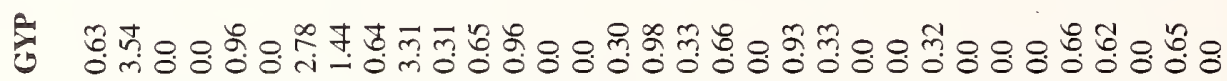

กัน

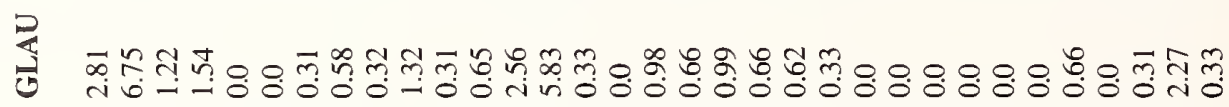

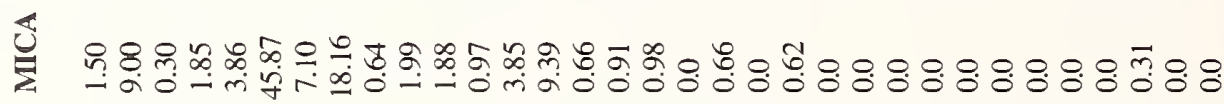

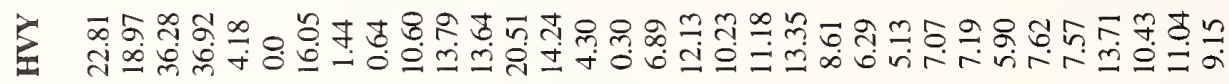

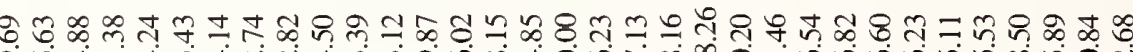

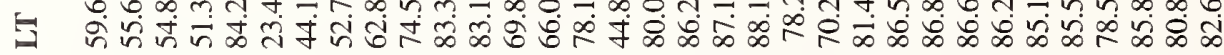

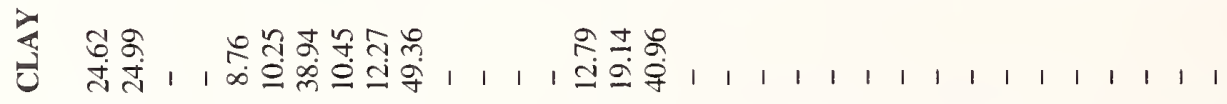

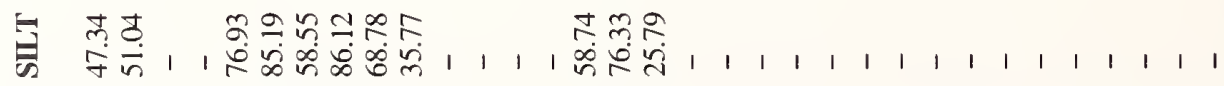

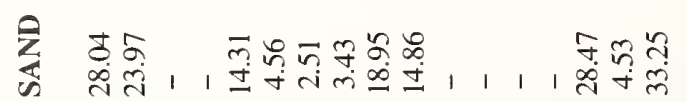

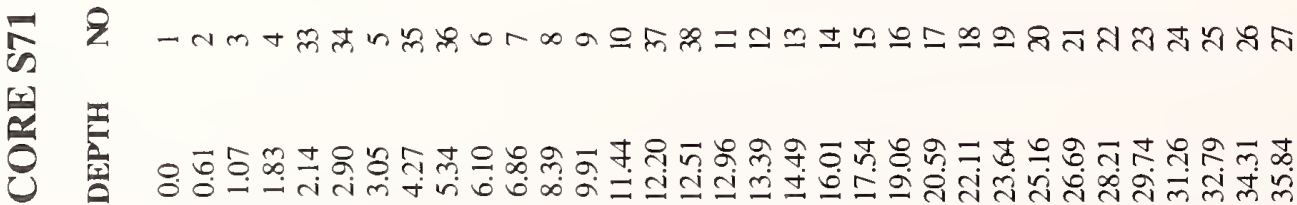


APPENDIX 2.-Continued.

융 융ํํㅇ

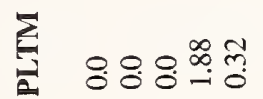

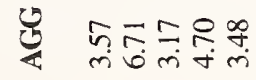

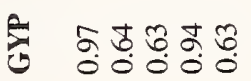

8080.

蛋

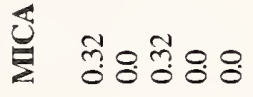

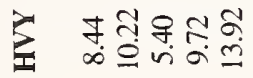

ב.

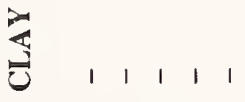

章 111111

密 1111111

त्र ₹ वृष्तल ल

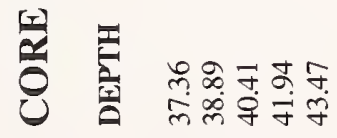


APPENDIX 2.-Continued.

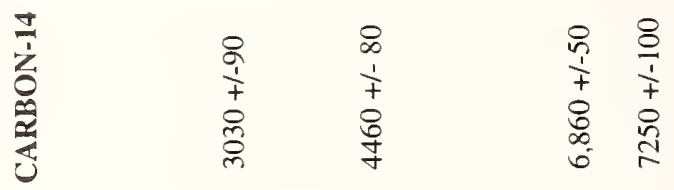

:

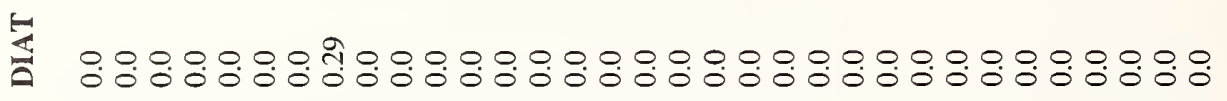

:

蔍

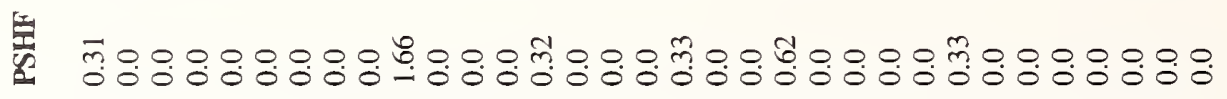

请

贸

荡

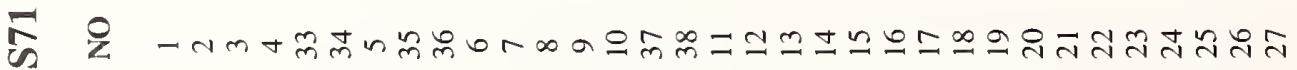

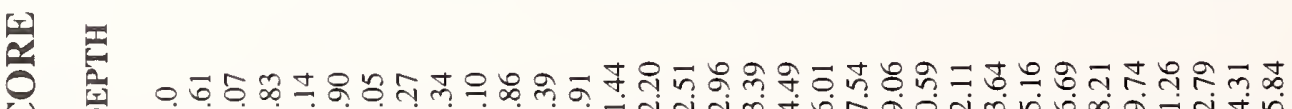


APPENDIX 2.-Continued.

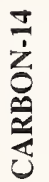

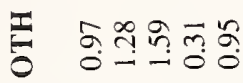

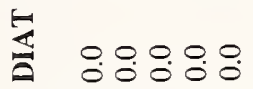

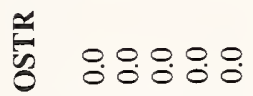

党

온 0.800

逼 8080.8

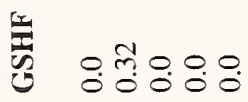

还

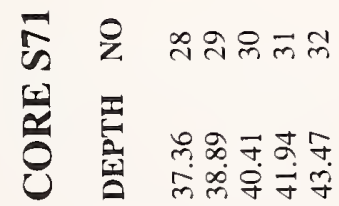


APPENDIX 2.-Continued.

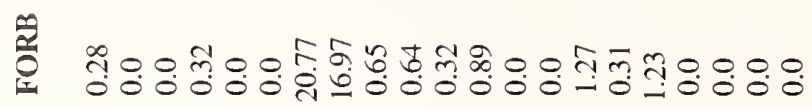

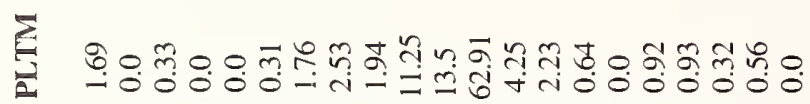

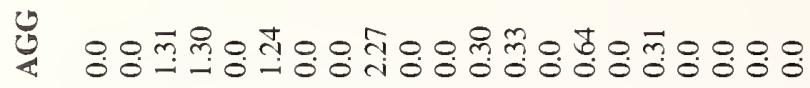

놀

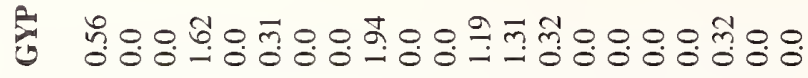

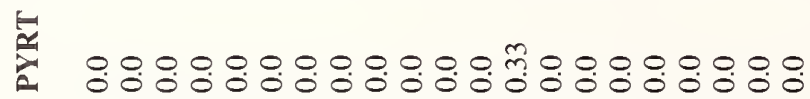

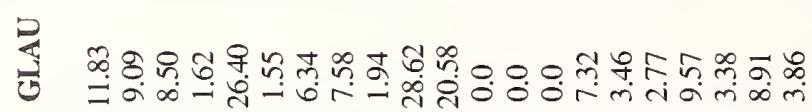

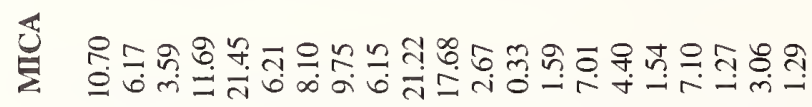

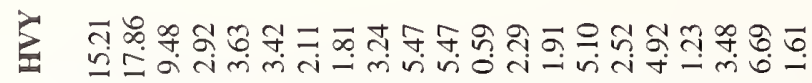

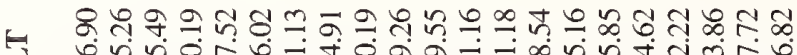

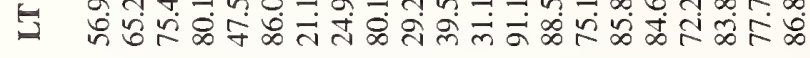

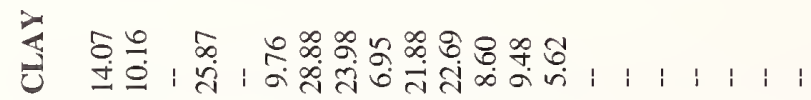

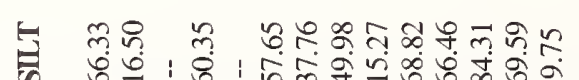

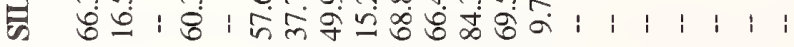

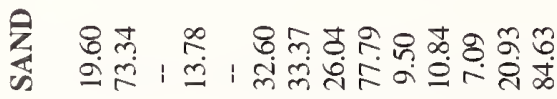

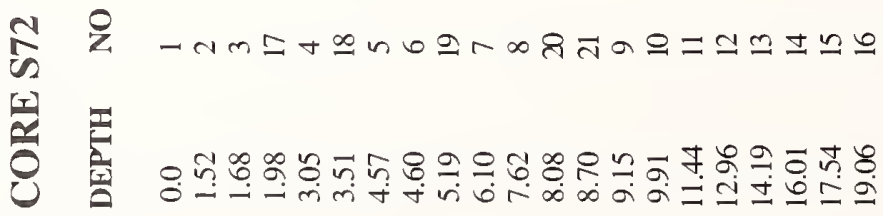


APPENDIX 2.-Continued.

$\frac{\square}{\grave{1}}$

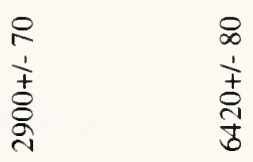

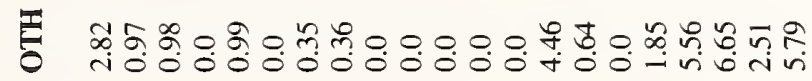

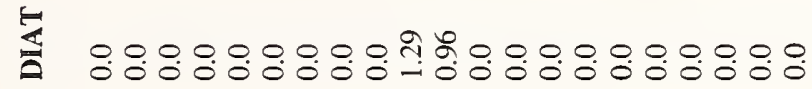

呈

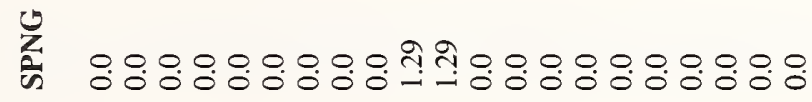

袠

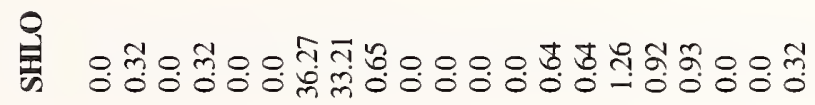

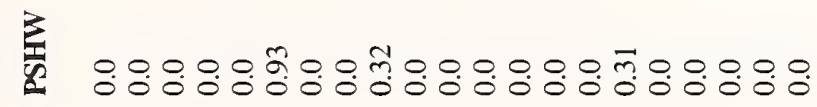

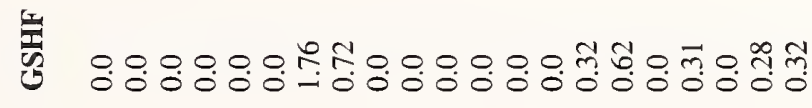

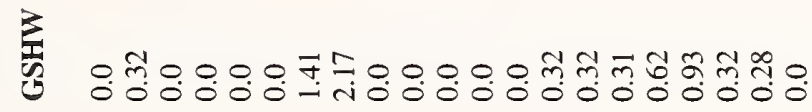

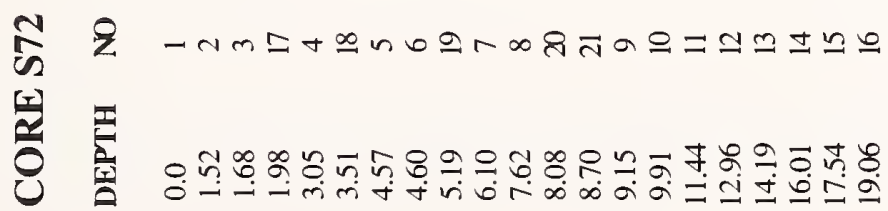


APPENDIX 2.-Continued.

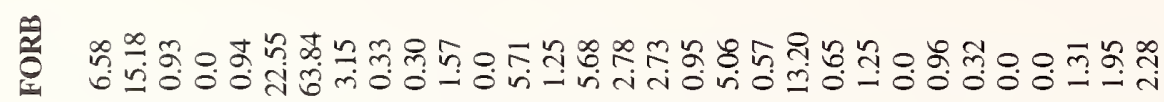

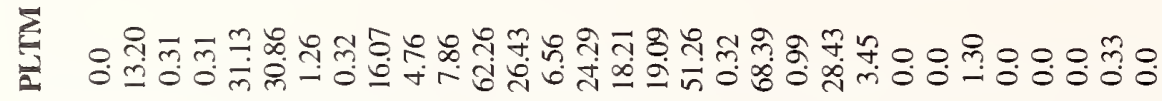

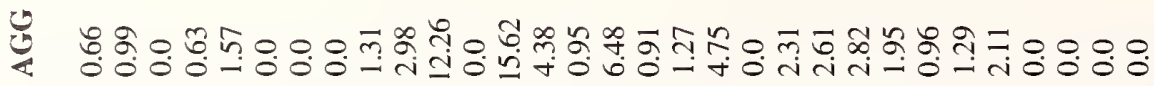

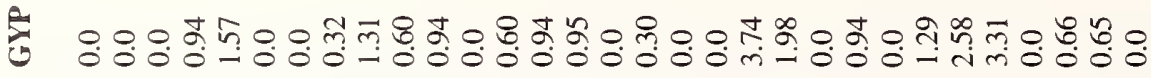

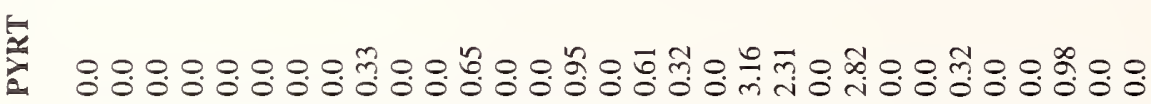

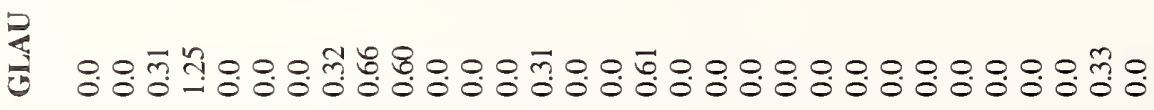

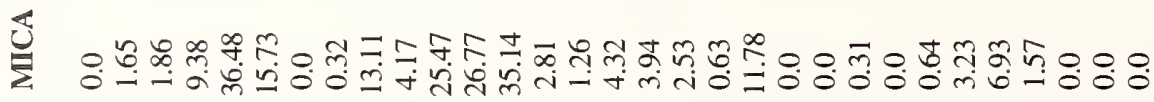

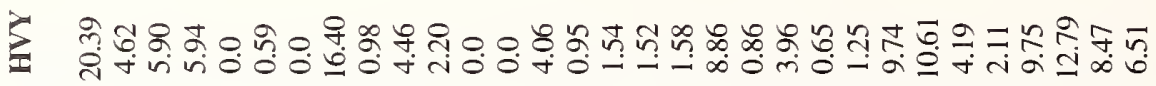

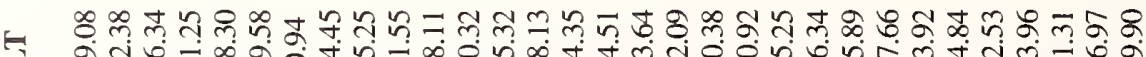

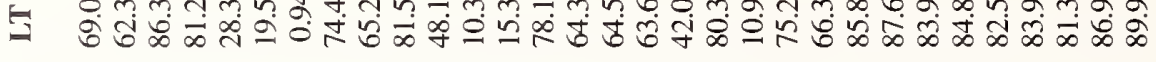

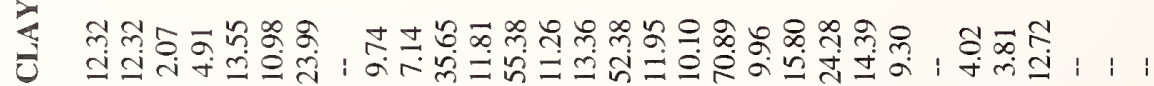

旨

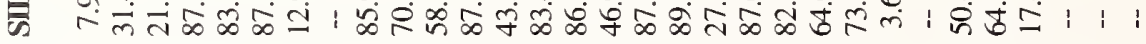

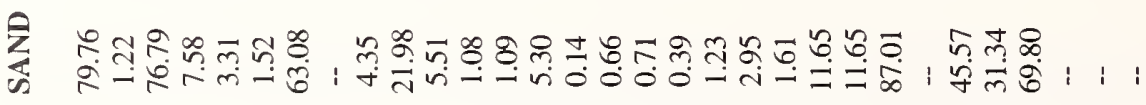

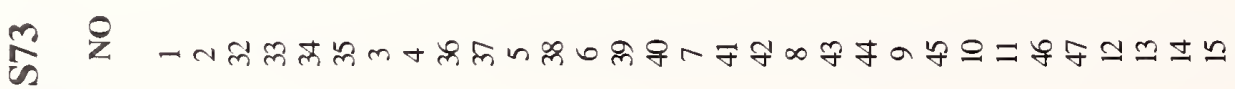

攻 


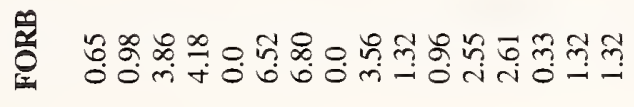

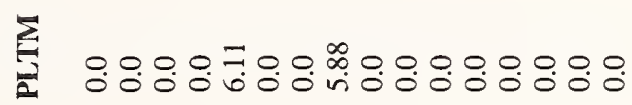

ํำ

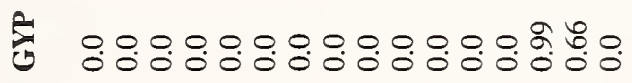

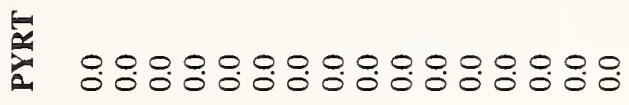

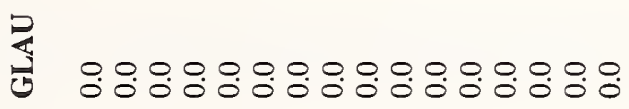

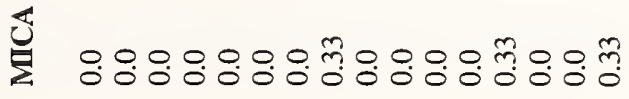

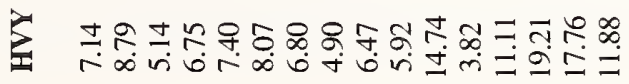

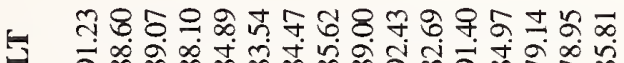

$\begin{array}{lllllllllllllllll}3 & 1 & 1 & 1 & 1 & 1 & 1 & 1 & 1 & 1 & 1 & 1 & 1 & 1 & 1 & 1 & 1\end{array}$

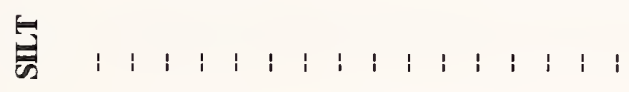

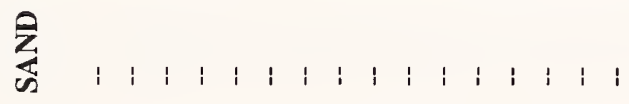

そ) そ

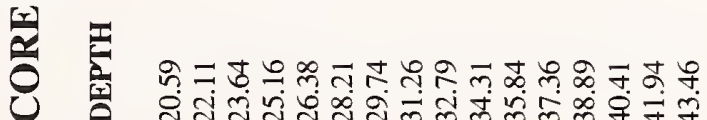


APPENDIX 2.-Continued.

$\frac{ \pm}{\frac{1}{2}}$

8
$\frac{1}{+}$
8
g
n.

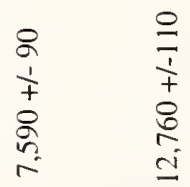

产

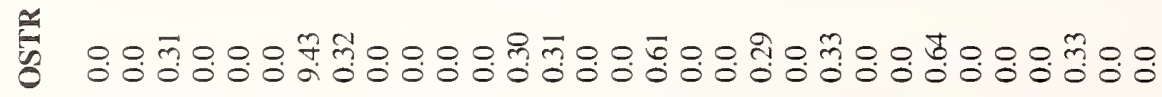

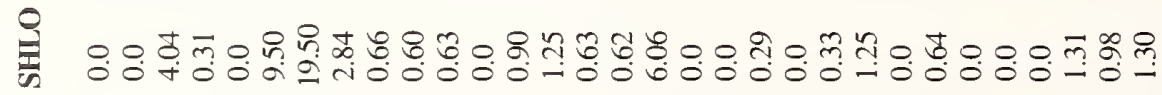

重

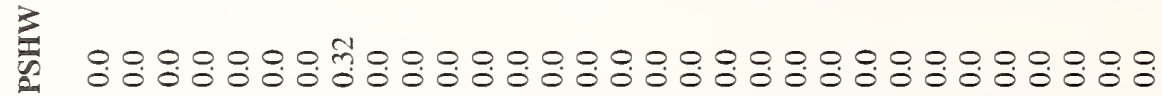

留

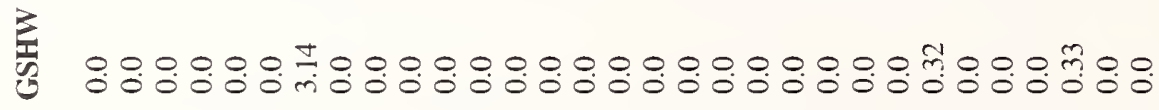

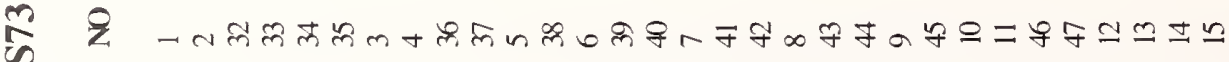

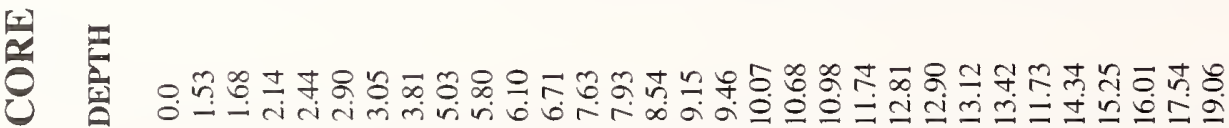


APPENDIX 2.-Continued.

$\frac{J}{2}$
$\frac{1}{0}$
$\frac{0}{0}$

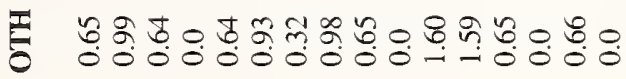

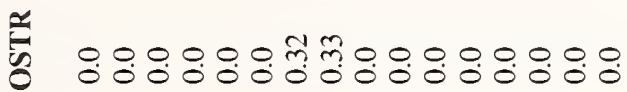

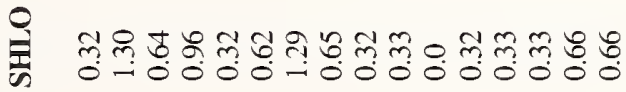

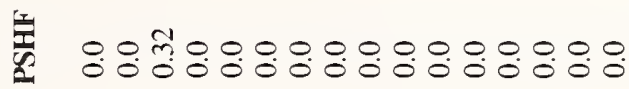

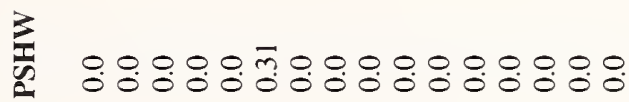

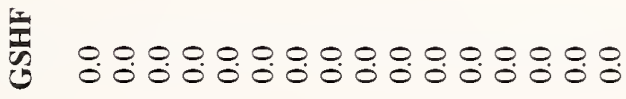

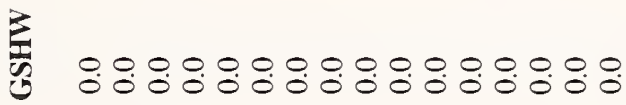

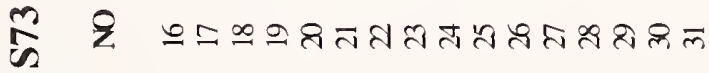

告 


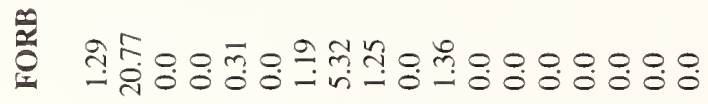

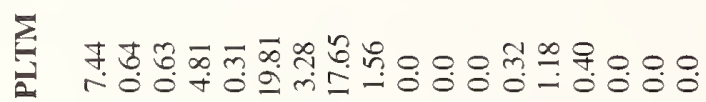

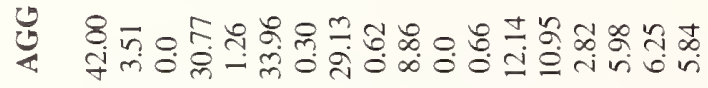

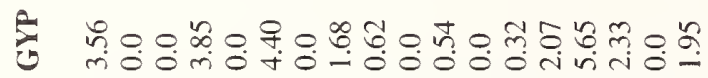

:

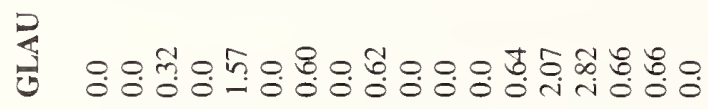

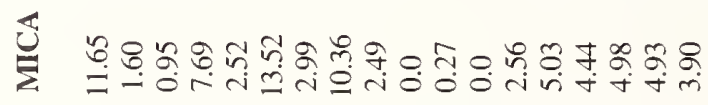

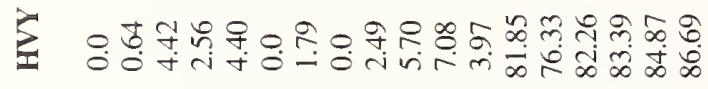

与 m.

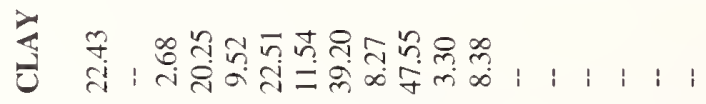

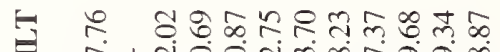

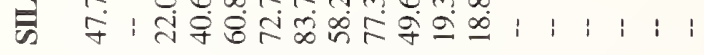

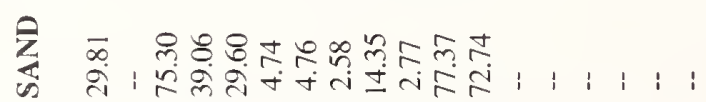

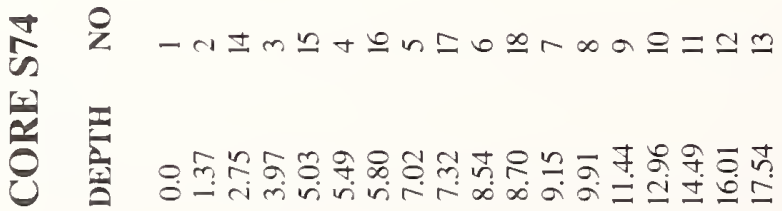




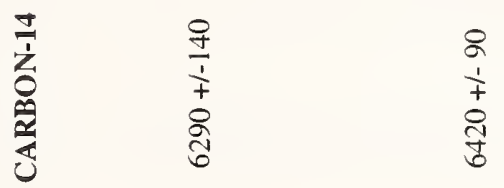

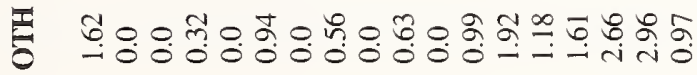

苗

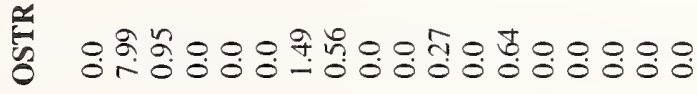

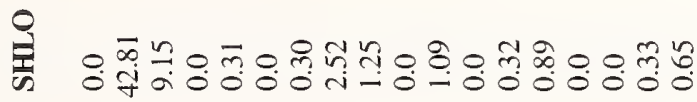

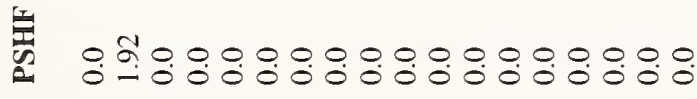

造

I 2 \&

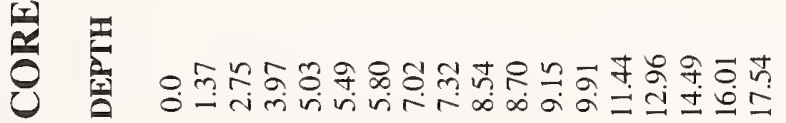


APPENDIX 2.-Continued.

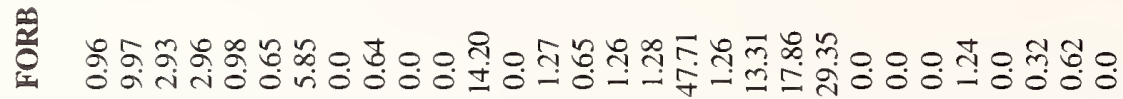

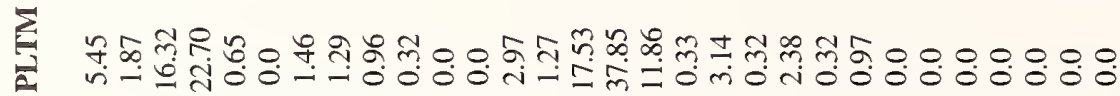

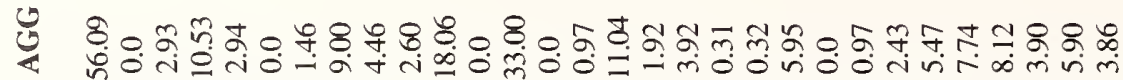

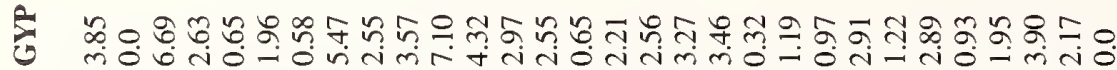

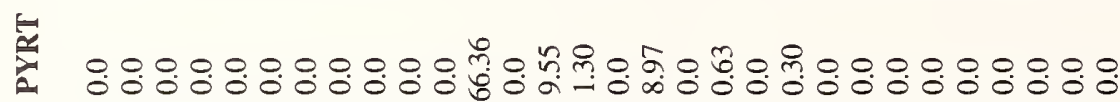

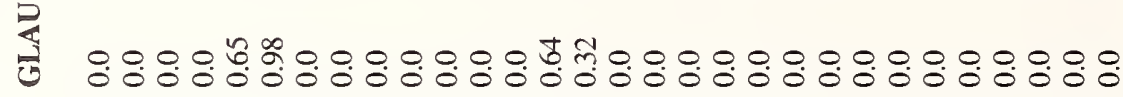

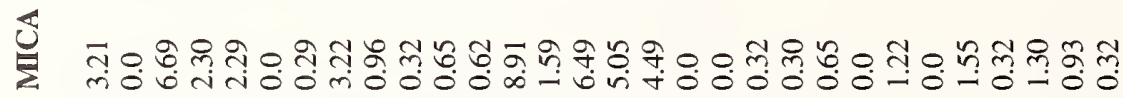

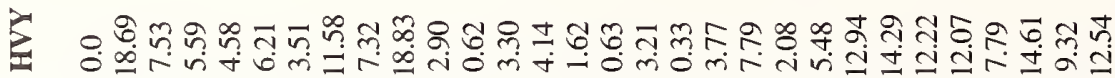

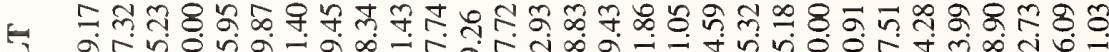

5 बin

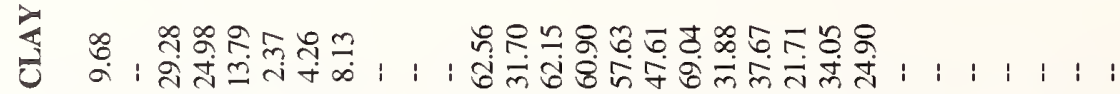

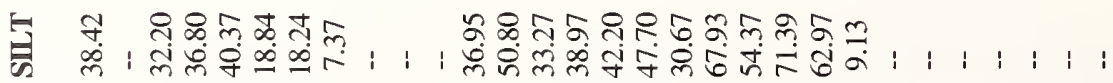

搽

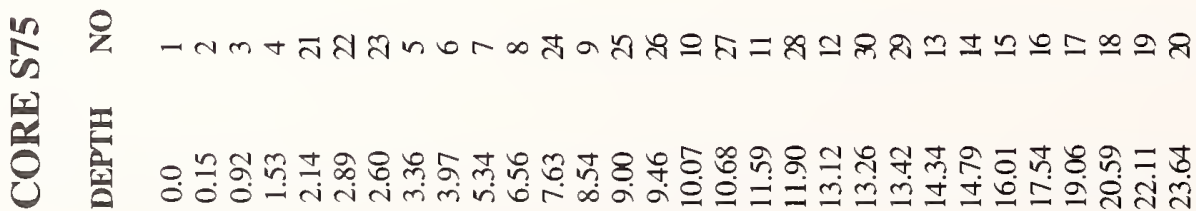


APPENDIX 2.-Continued.

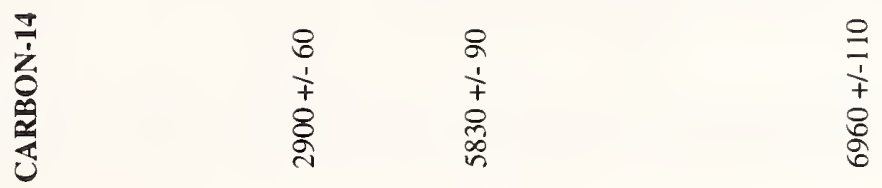

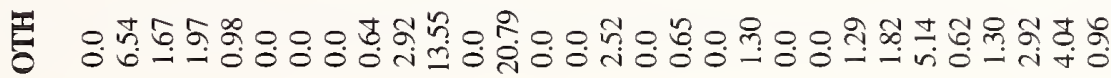

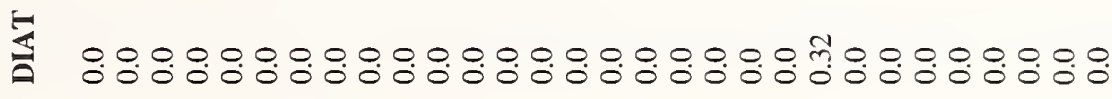

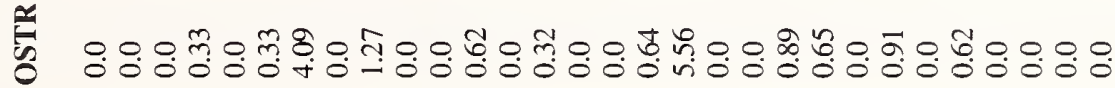

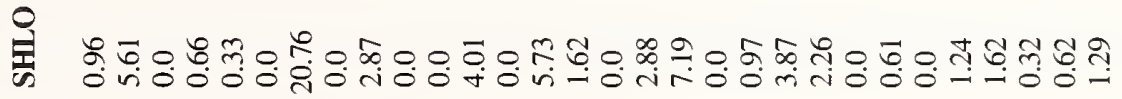

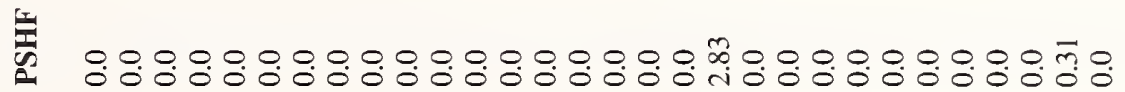

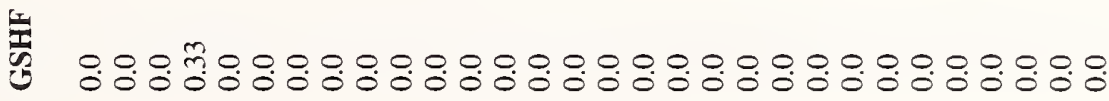

ํㅗㄴ

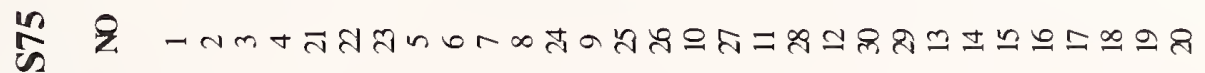

造 
APPENDIX 2.-Continued.

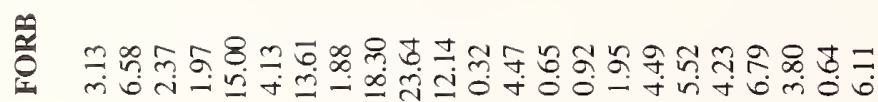

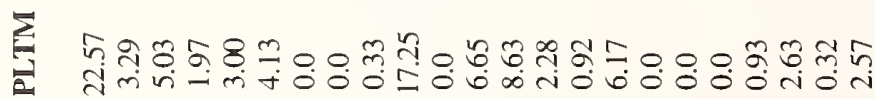

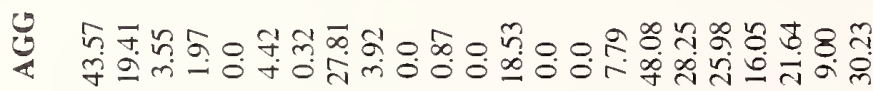

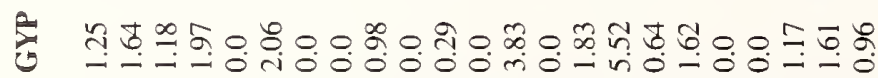

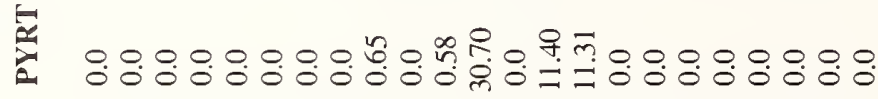

罢

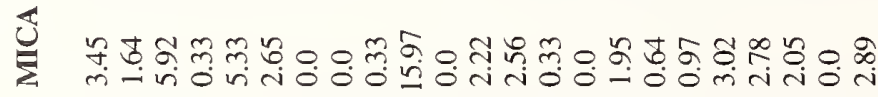

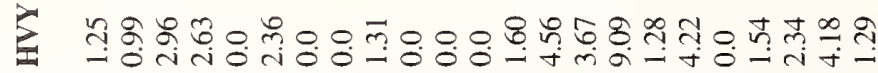

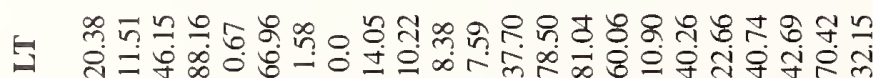

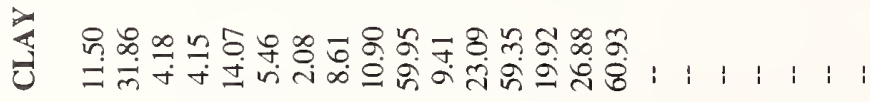

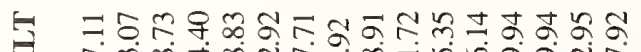

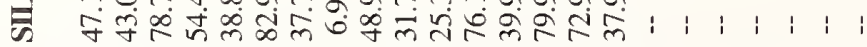

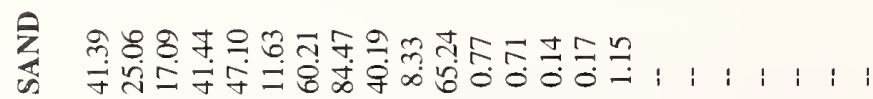

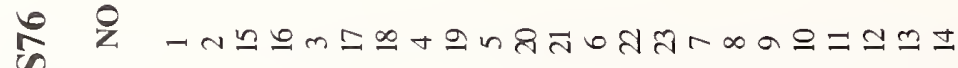

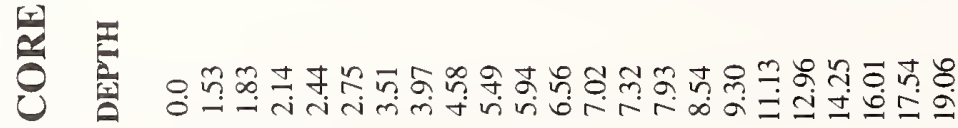


APPENDIX 2.-Continued.

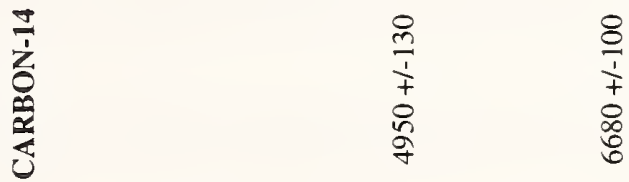

इ

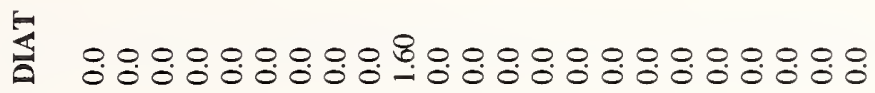

岳

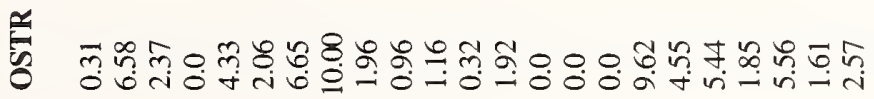

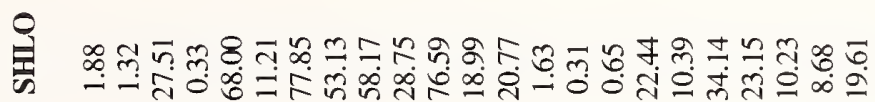

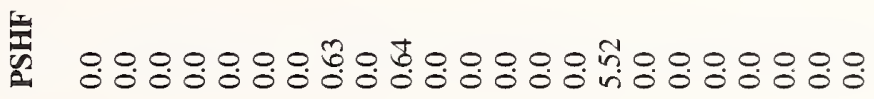

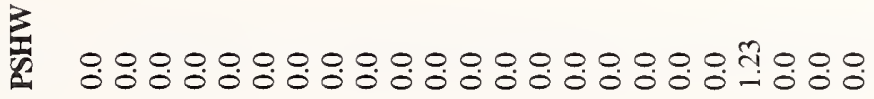

栲

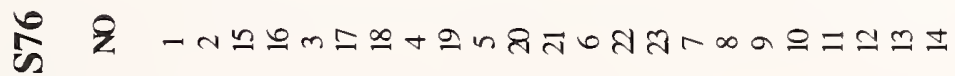

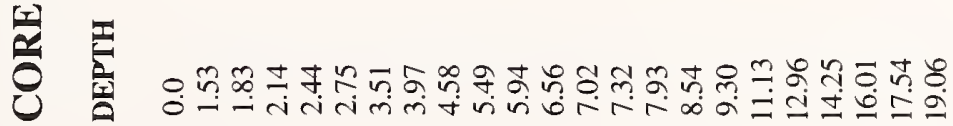


APPENDIX 2.-Continued.

을

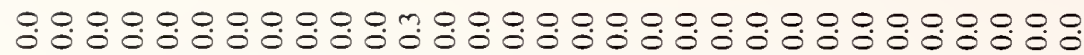

$\frac{1}{0}$

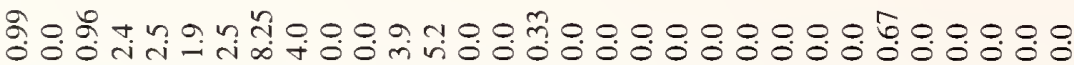

$\sum_{E}$

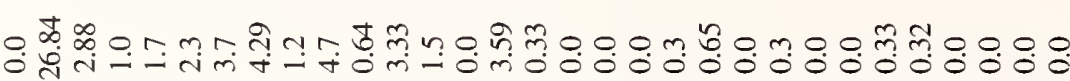

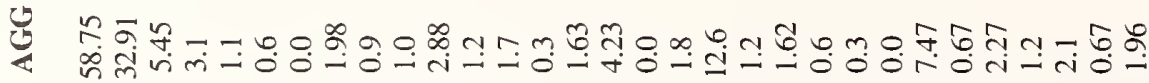

들

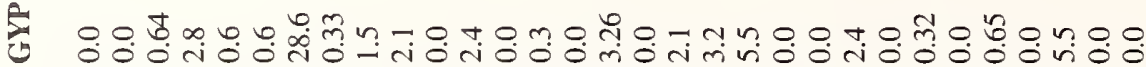

E

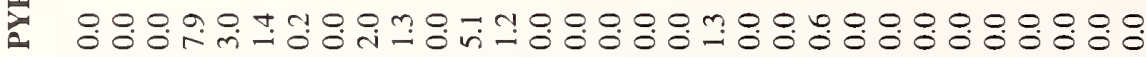

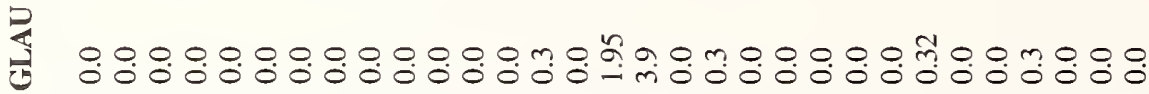

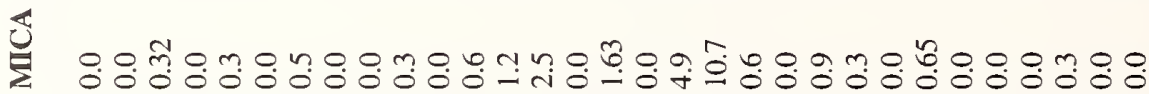

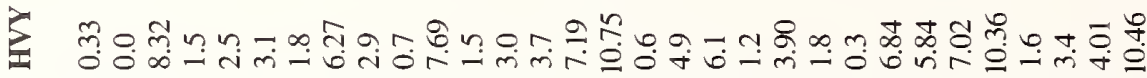

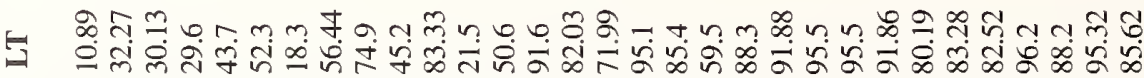

过

F

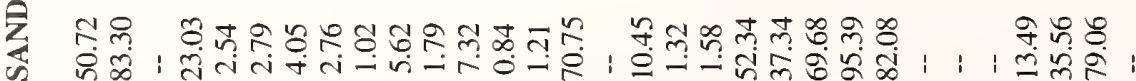

I 是 ー

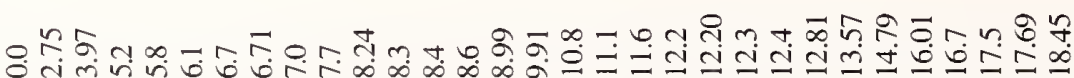


을

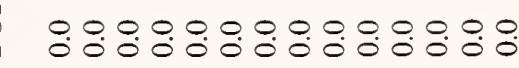

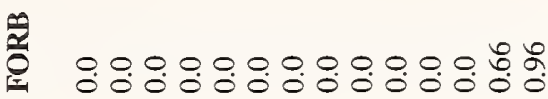

연

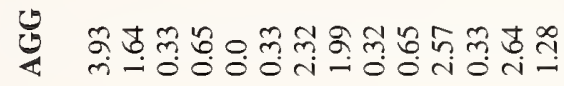

声

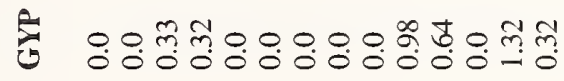

茎

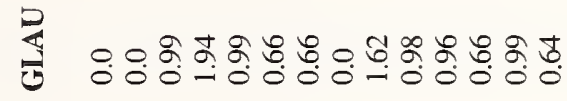

岁

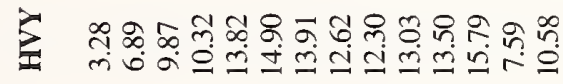

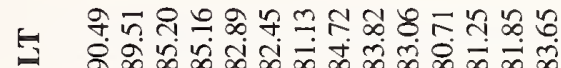

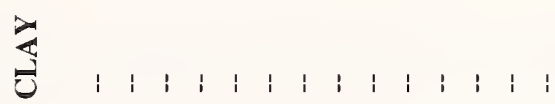

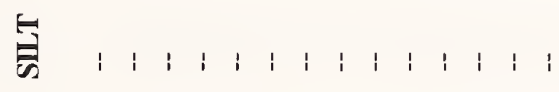

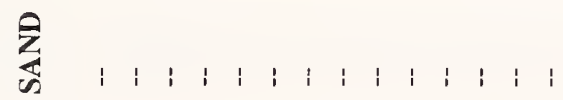

in

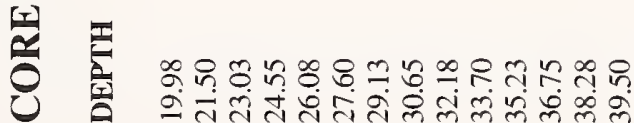


APPENDIX 2.-Continued.

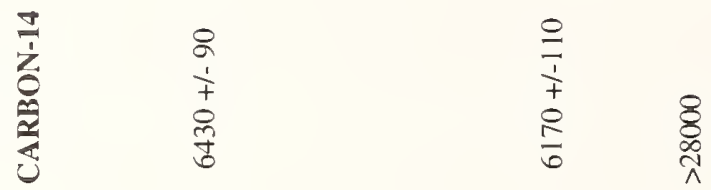

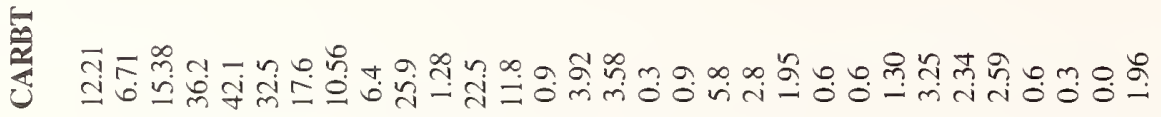

乡

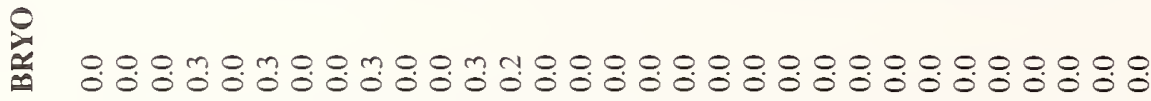

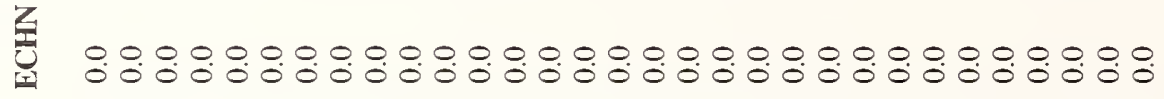

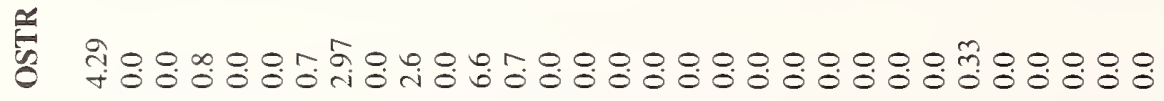

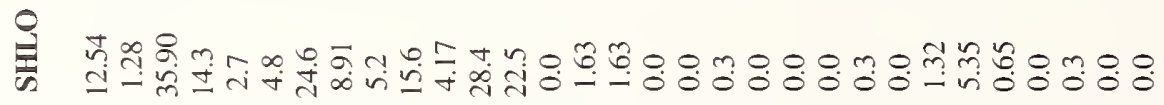

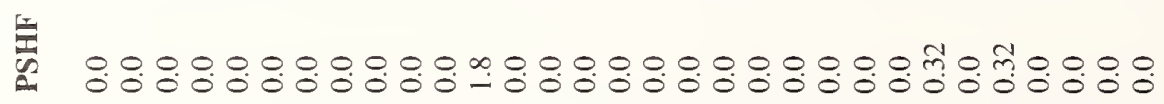

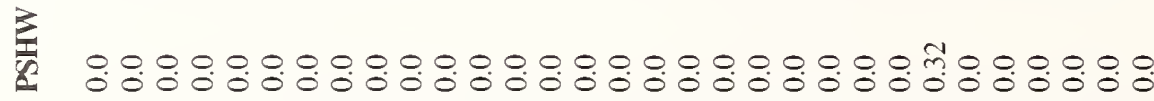

焉

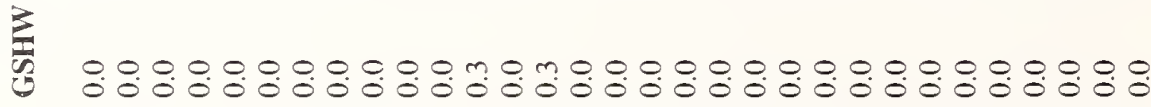

Iิ

署 
APPENDIX 2.-Continued.

$\frac{\square}{3}$

每

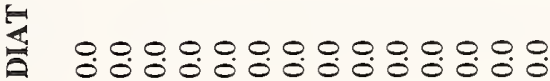

은

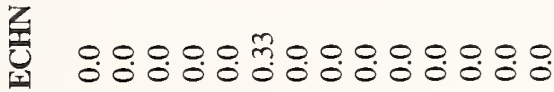

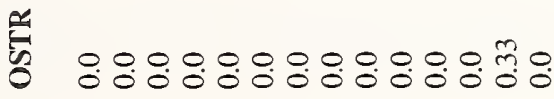

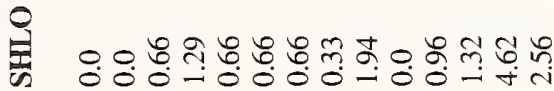

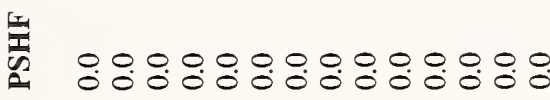

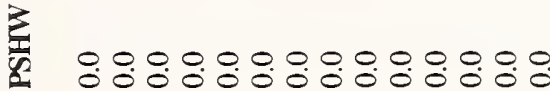

焉

芴

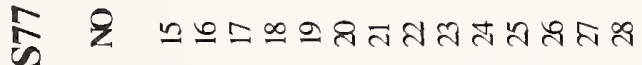

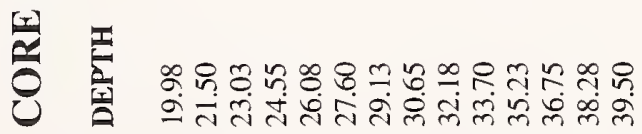


APPENDIX 2.-Continued.

军

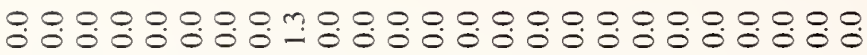

옹

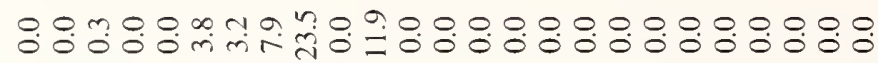

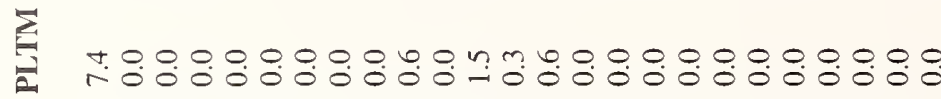

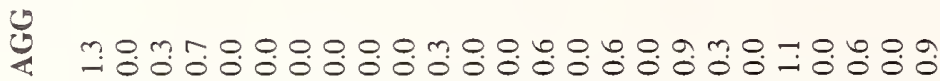

m

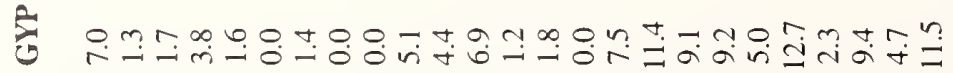

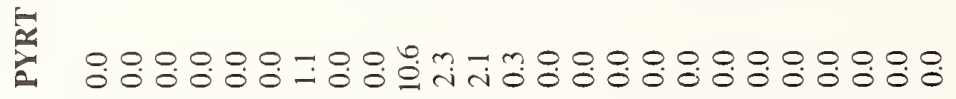

蛋

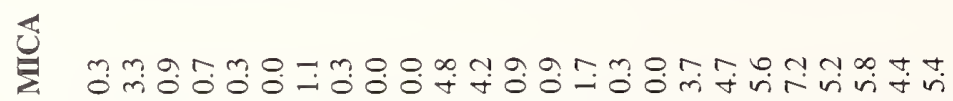

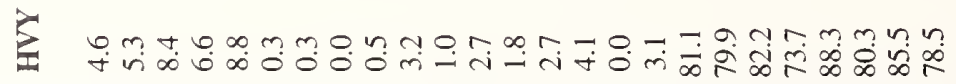

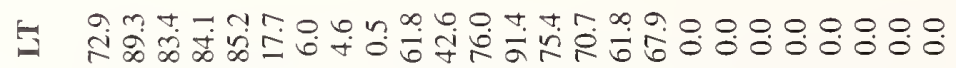

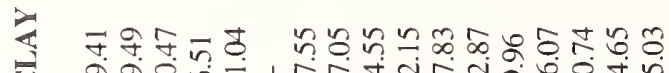

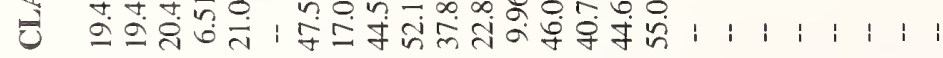

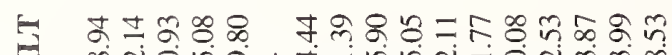

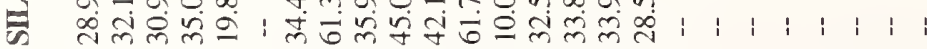

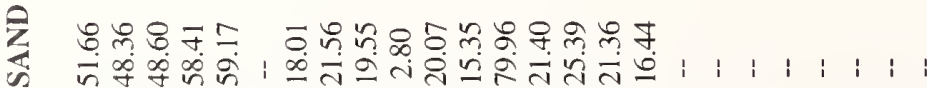

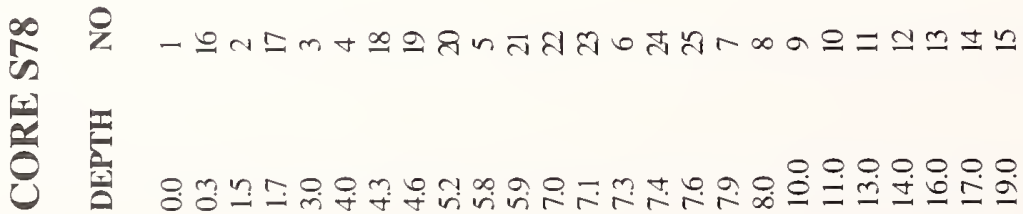


APPENDIX 2.-Continued.

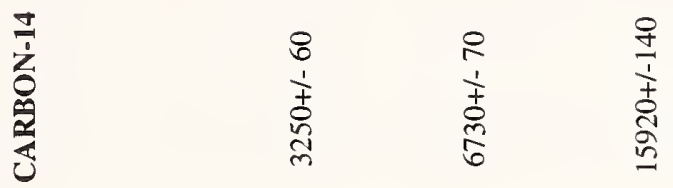

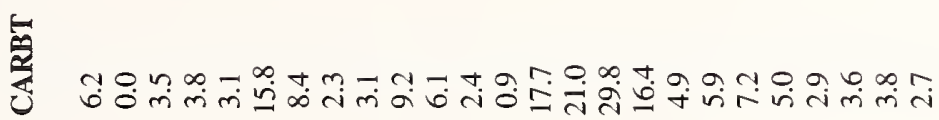

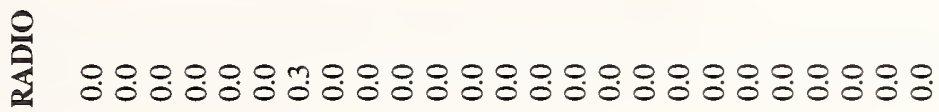

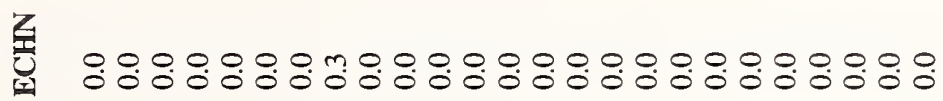

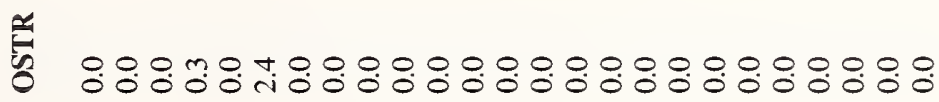

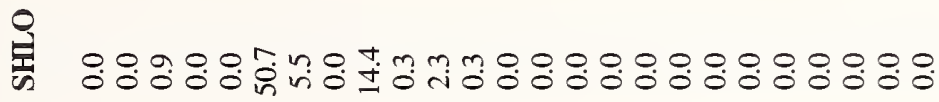

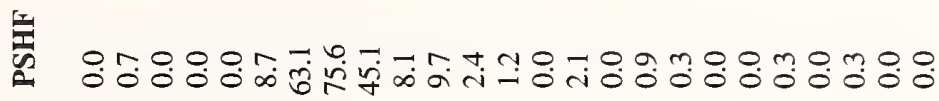

蛋

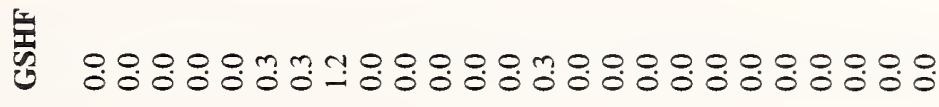

要

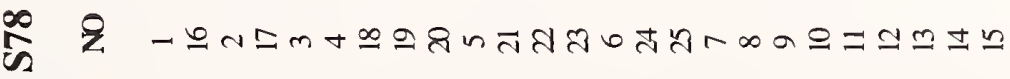

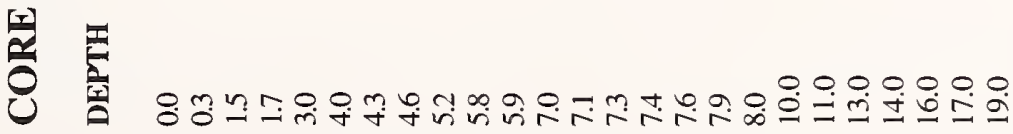


APPENDIX 2.-Continued.

옹

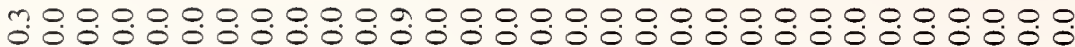

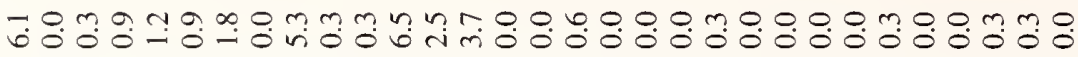

$\sum_{\text {党 }}$

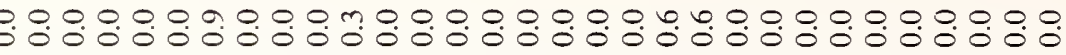

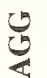

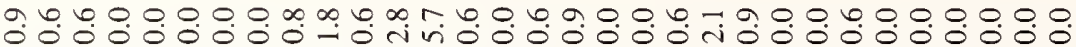

昱

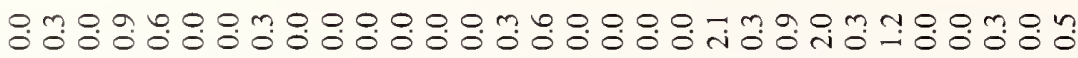

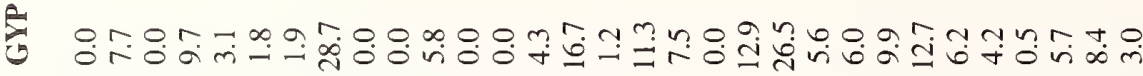

닌

芶

茪

Z

ज

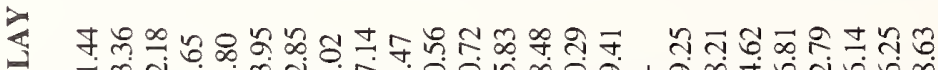

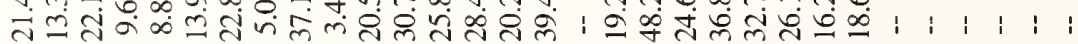

匀

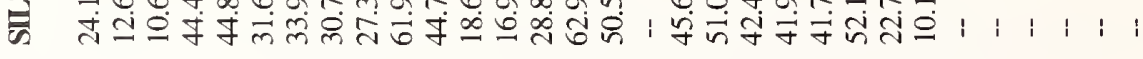

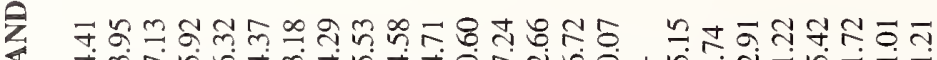

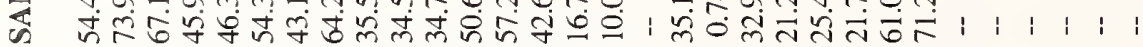

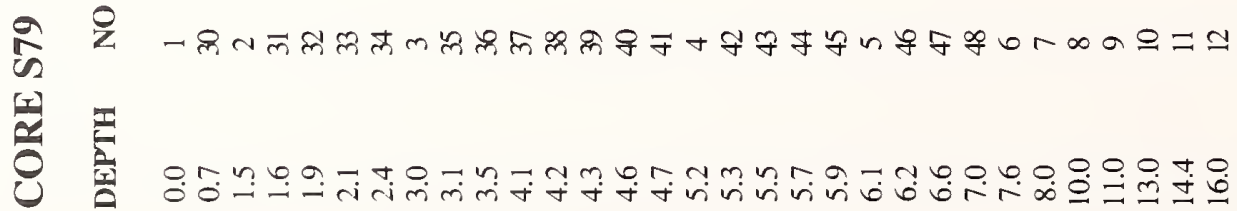


APPENDIX 2.-Continued.

농

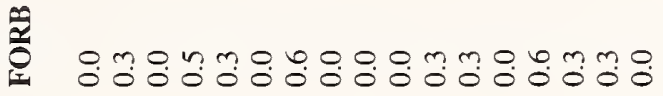

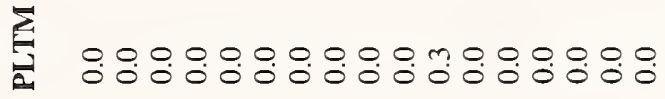

过

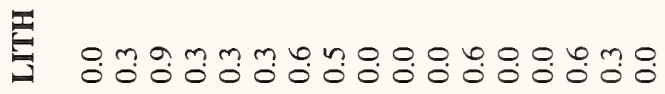

我 mơr

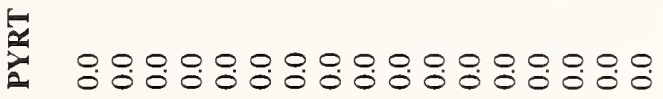

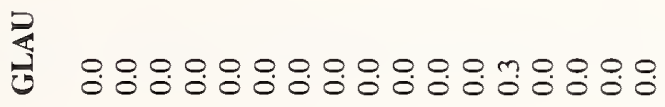

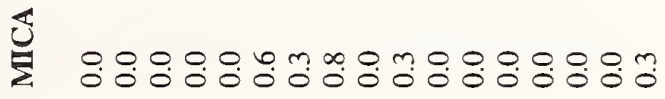

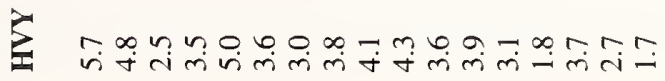

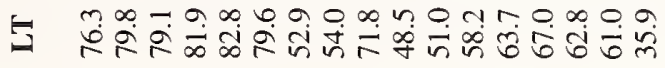

离

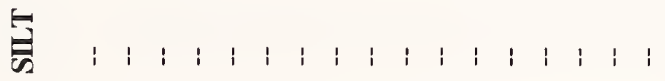

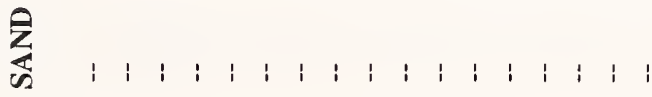

ลี่

造 
APPENDIX 2.-Continued.

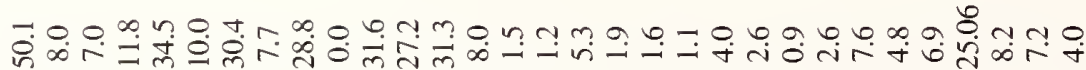

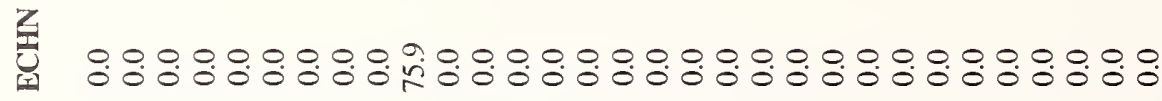

若

素

犂

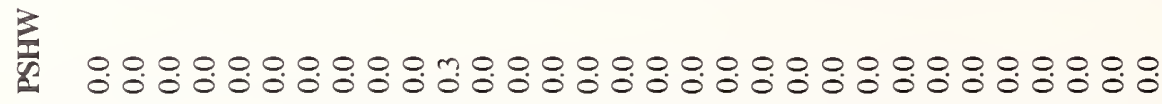

焉

供

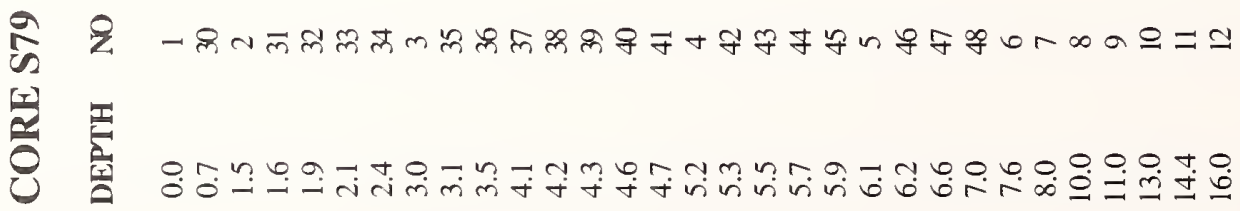


APPENDIX 2,-Continued.

$\frac{ \pm}{3}$

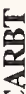

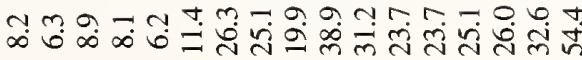

늡

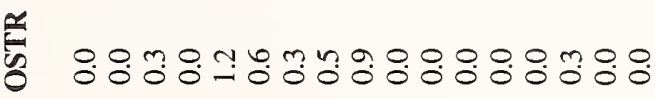

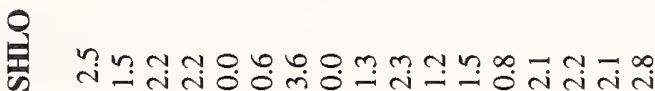

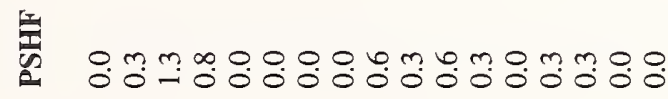

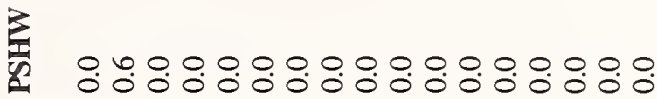

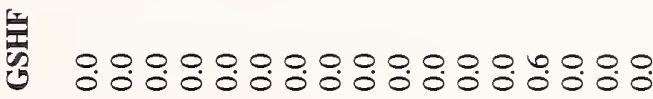

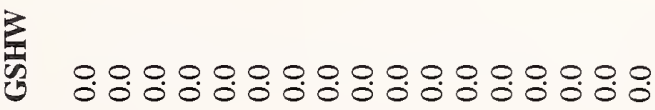

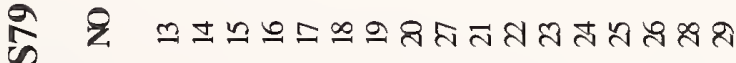

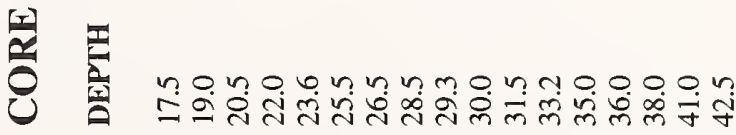


APPENDIX 2.-Continued.

일

n긍

蒏

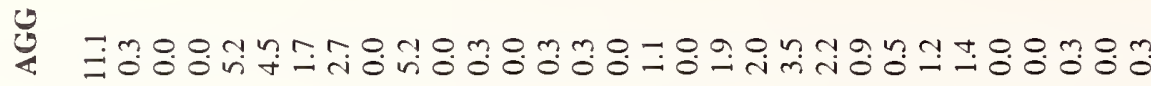

军

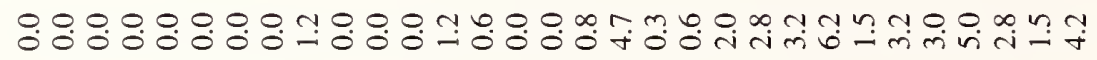

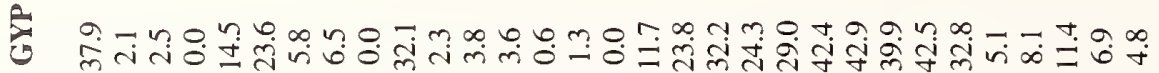

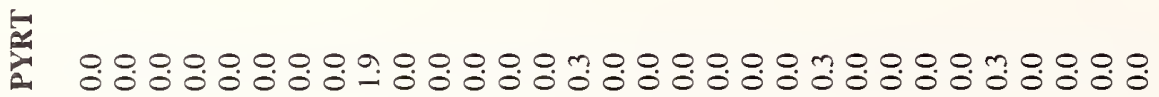

声

茪

Z

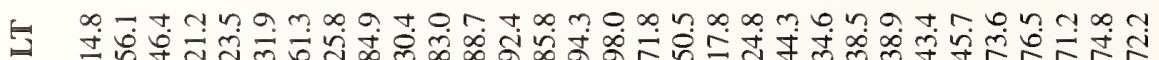

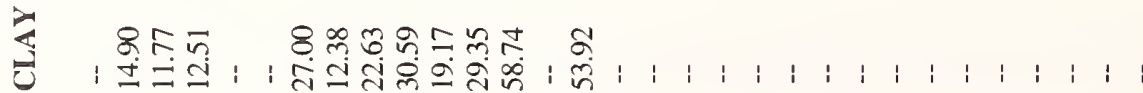

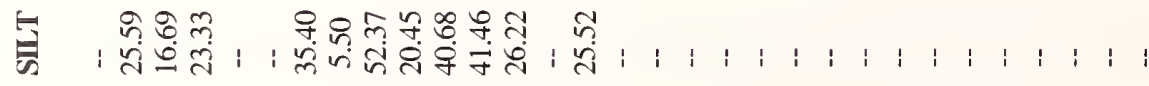

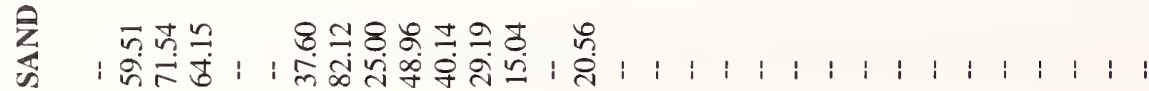

®

U 
APPENDIX 2,-Continued.

: $8: 08: 88: 808$

E 80080080000

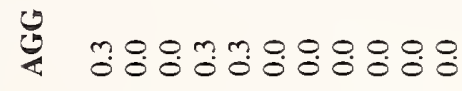

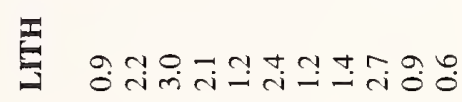

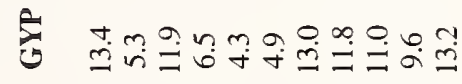

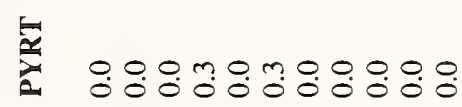

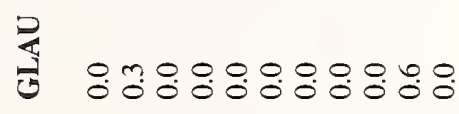

递 00800000009800

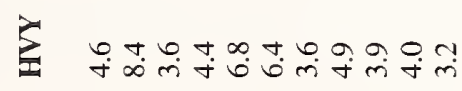

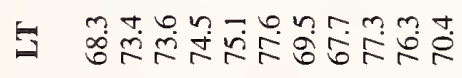

起: : : : : : : : : : : :

实:1:1: : : : : : :

会：：：：：：：：：：：：

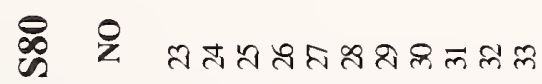

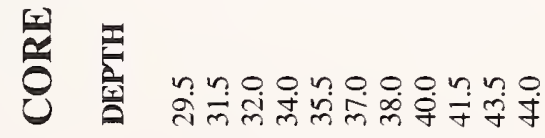


APPENDIX 2.-Continued.

$\begin{array}{lll}\frac{\pi}{1} & 8 & \infty \\ 0 & \frac{1}{1} & \frac{1}{1} \\ 0 & 0 & + \\ 0 & 0 & 0 \\ 0 & 0 & 0\end{array}$

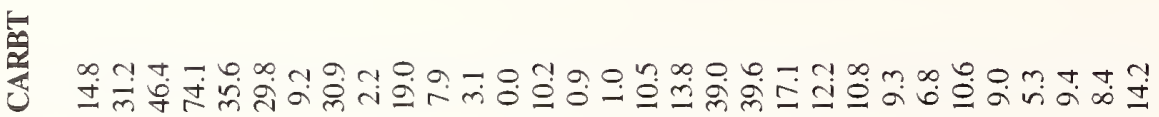

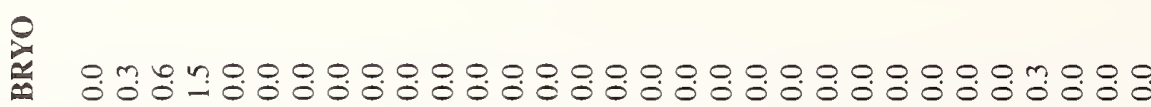

离

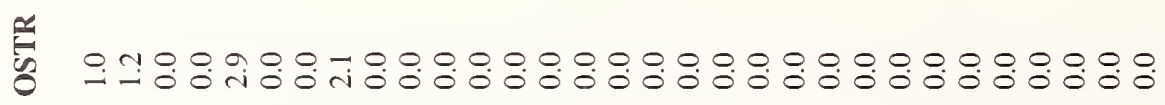

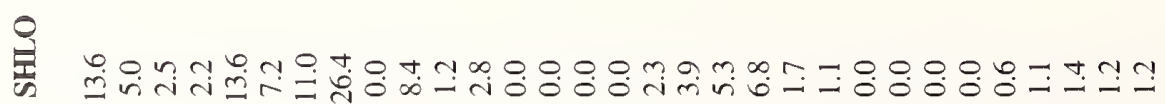

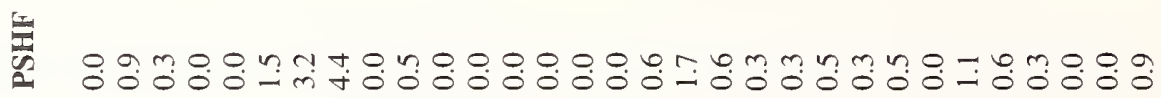

选

造

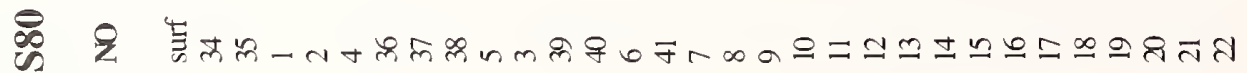

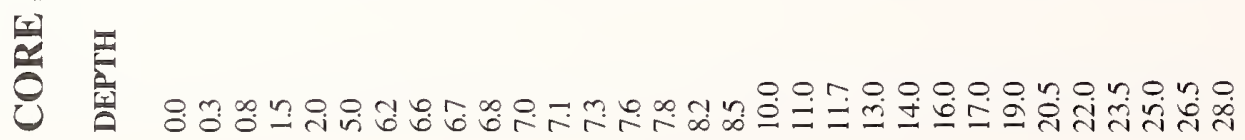


APPENDIX 2.-Continued.

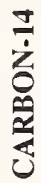

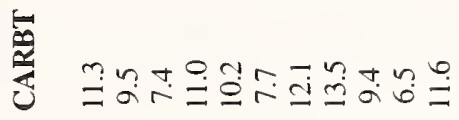

용용용용용

茪

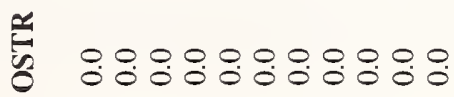

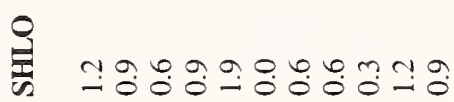

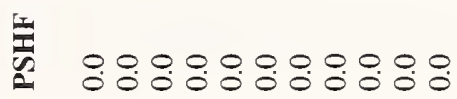

息 용용용용용용요

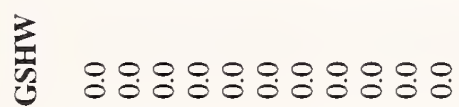

ஜ

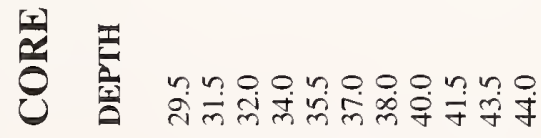


APPENDIX 2.-Continued.

望

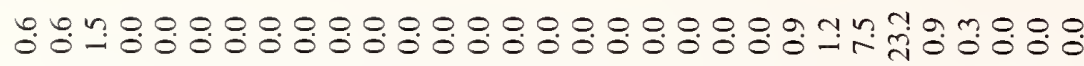

$\sum_{0}$

웅

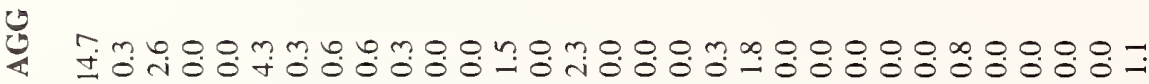

:

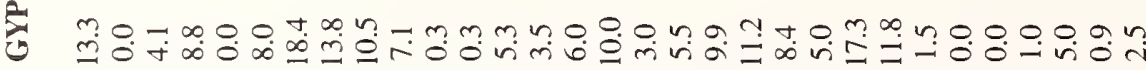

을

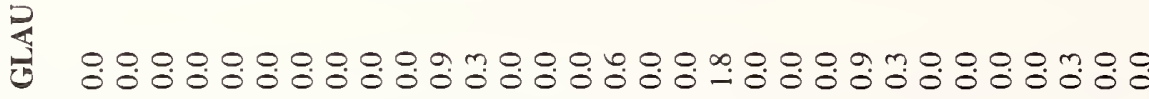

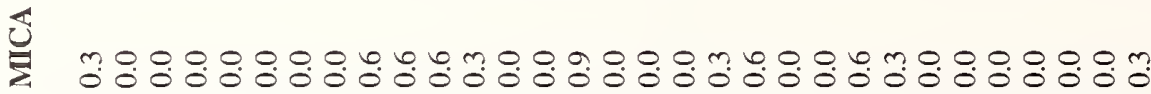

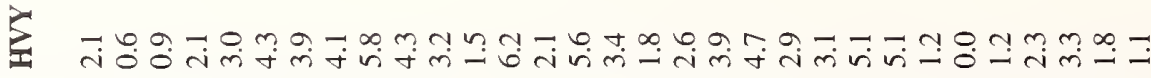

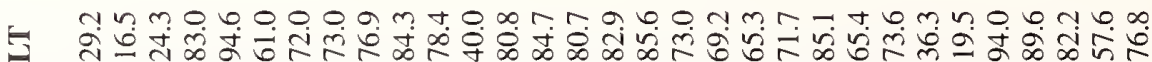

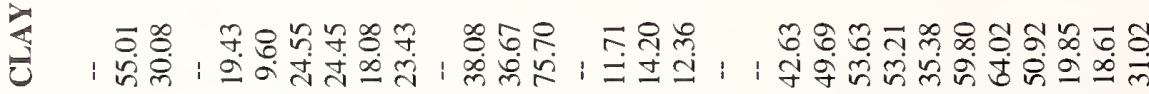

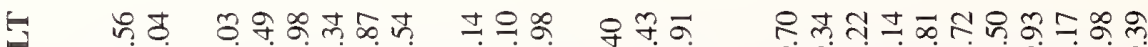

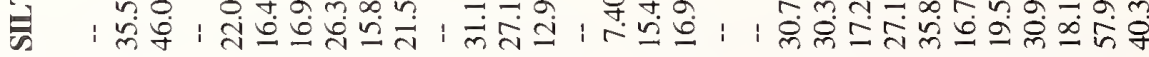

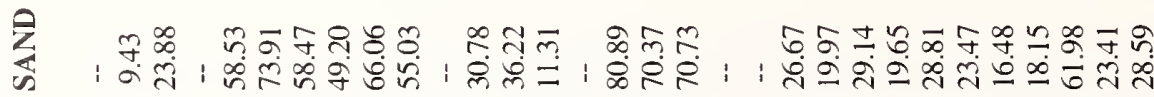

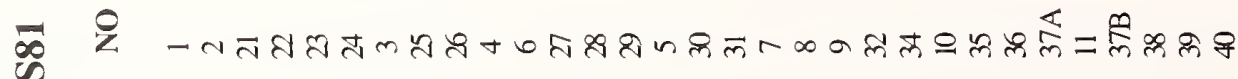

稀

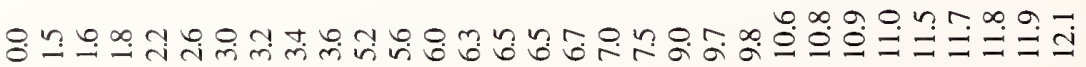


APPENDIX 2.-Continued.

ำ 80000800808080080

늘

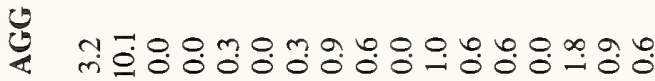

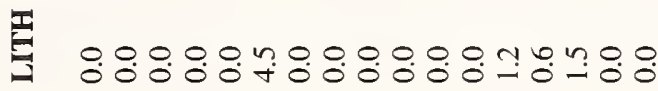

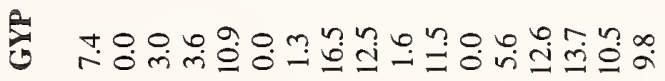

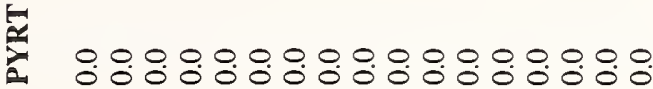

곤

岁 :

齐

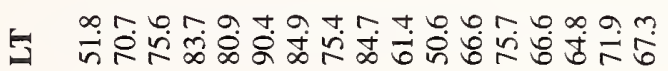

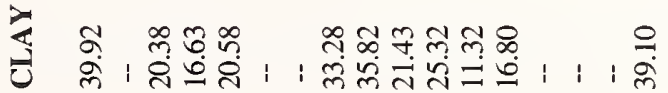

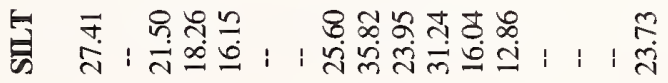

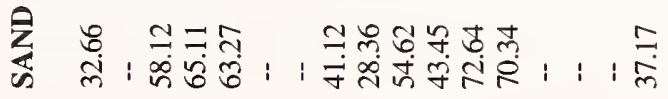

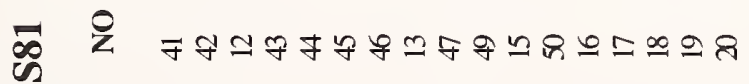

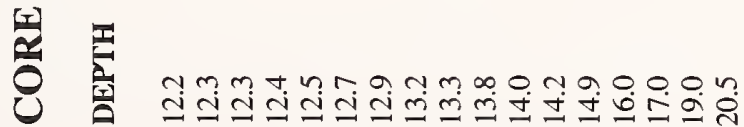


APPENDIX 2.-Continued.

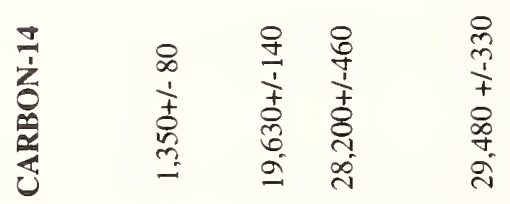

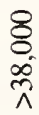

造

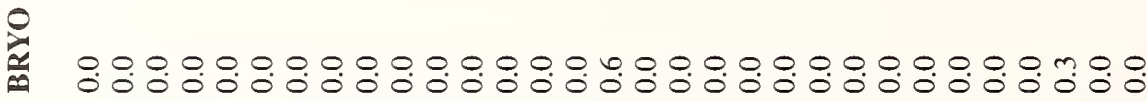

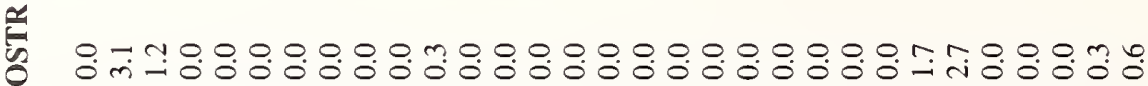

苞

党

क्ष

낭 돈

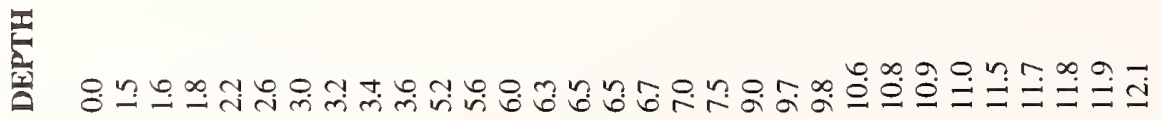


APPENDIX 2.-Continued.

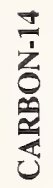

点

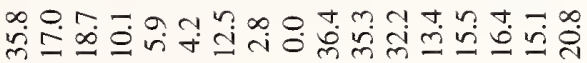

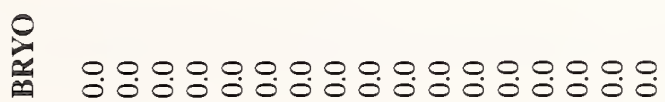

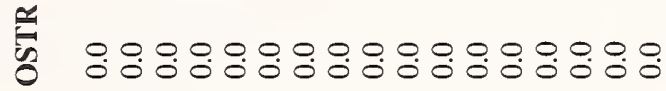

은 영영영영영영영영

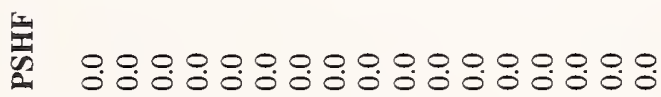

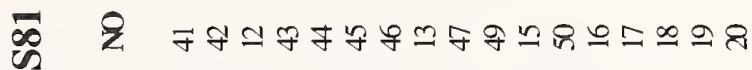

능

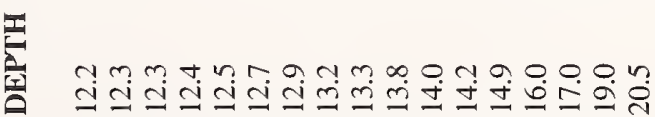


APPENDIX 2.-Continued.

을

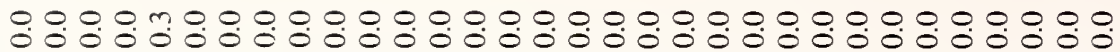

$\frac{m}{2}$

웅영

$\sum_{1}$

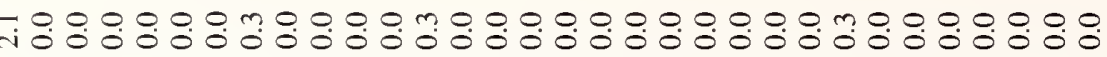

$\underset{4}{4}$

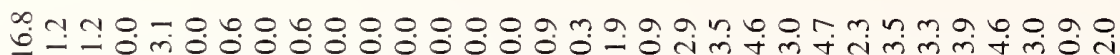

罭

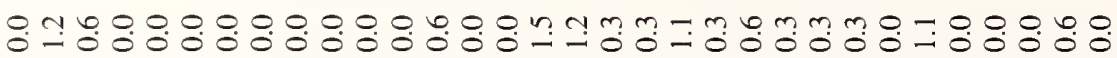

-



苛

茪

li

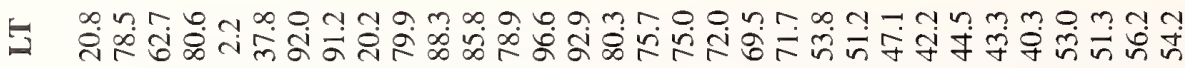

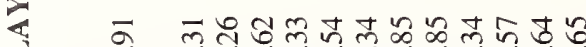

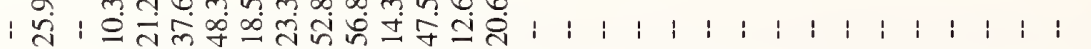

E $\quad$ gे

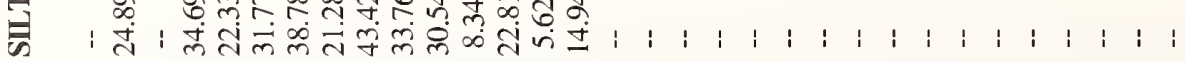

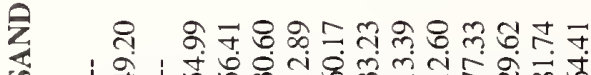

| 守

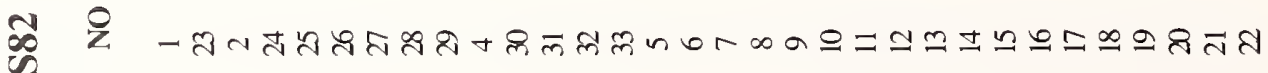


APPENDIX 2.-Continued.

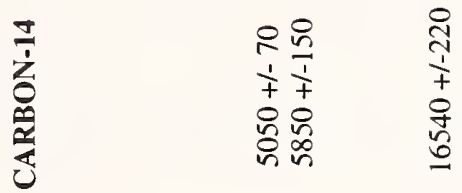

造

能

苟

星 은

委

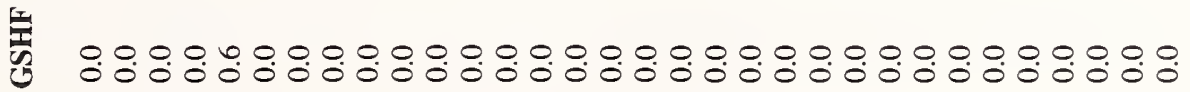

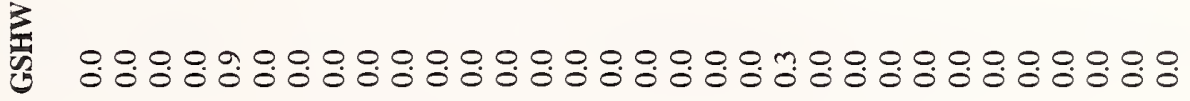

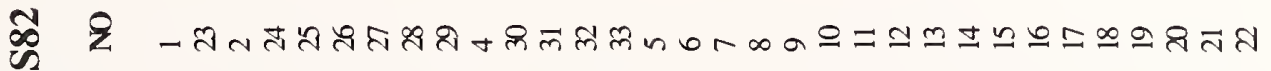

绕 존

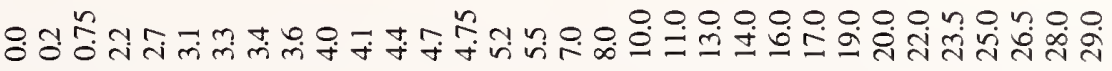


APPENDIX 2.--Continued.

童

H.

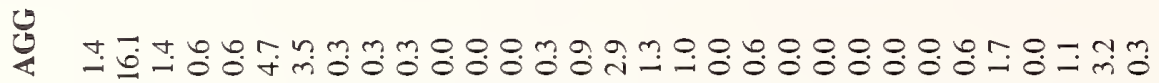

:

发 웅

는

过

通

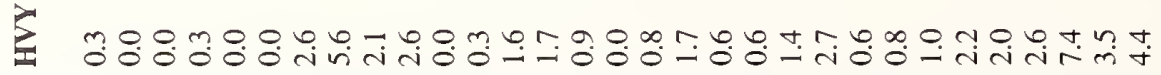

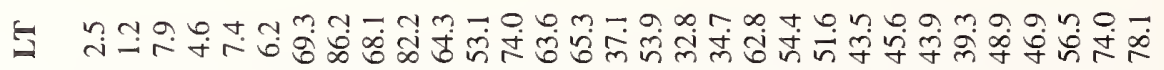

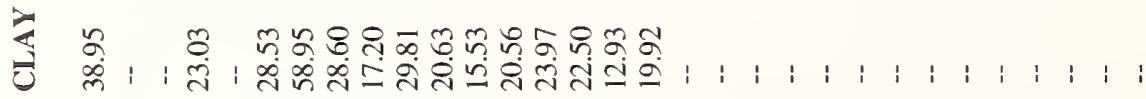

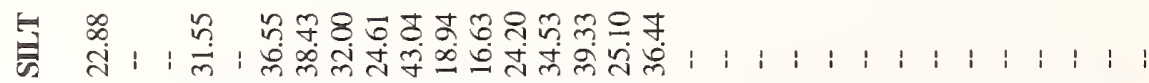

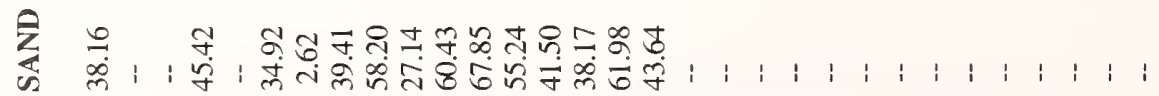

ஜ

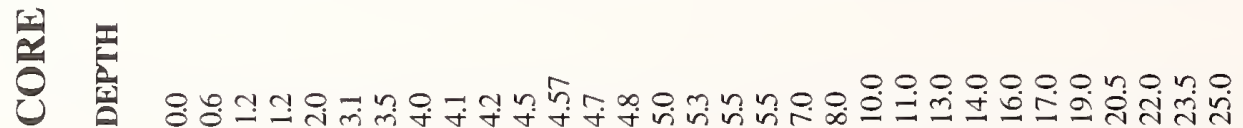


APPENDIX 2.-Continued.

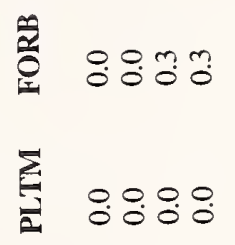

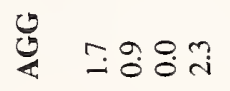

罵

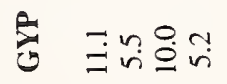

슬 웅웅웅

晃

岁 $\ddot{0} \amalg \amalg \dot{0}$

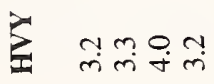

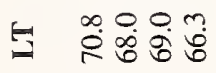

造 $\quad$,

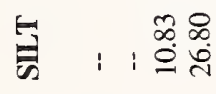

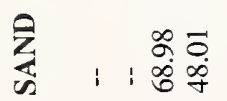

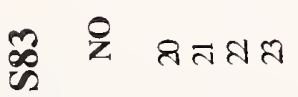

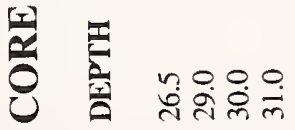




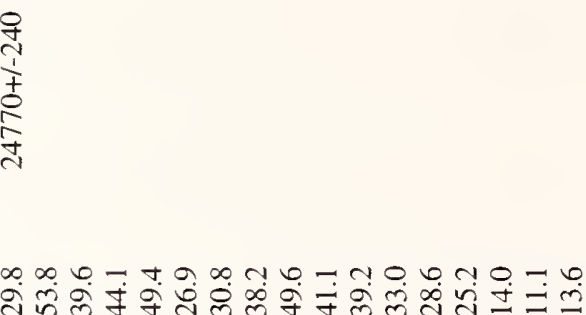

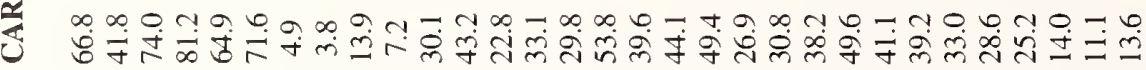

宓

ঋ

严

ڤ

㶾

는

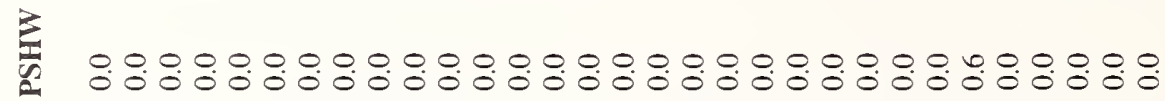

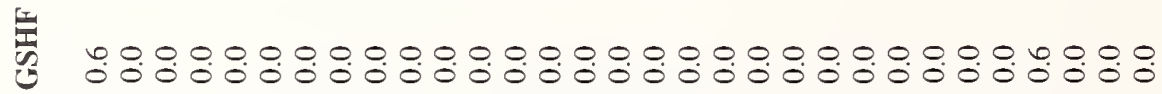

至

๙

\&

논

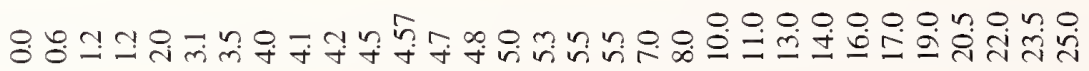


APPENDIX 2.-Continued.

齐

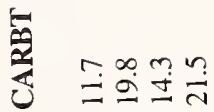

这

을 웅용

否 $0.00 \%$

능 웅유

带

은 융용용

융유

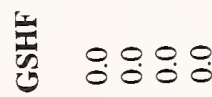

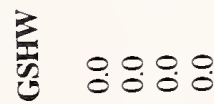

ஜ ஜ ชกฝ๙

乩 
APPENDIX 2.-Continued.

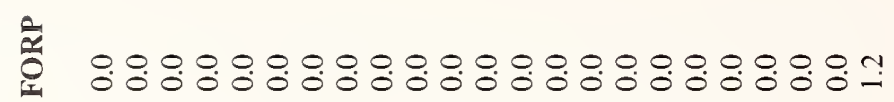

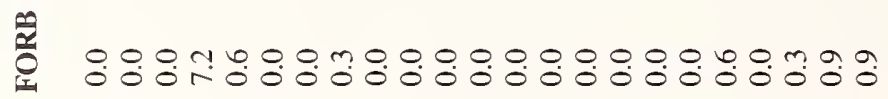

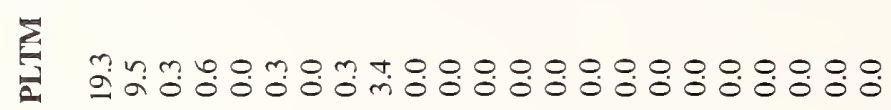

造

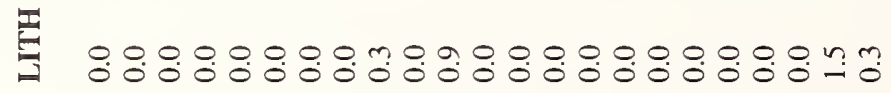

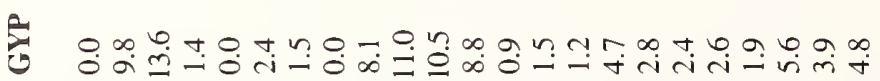

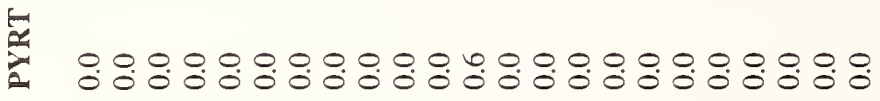

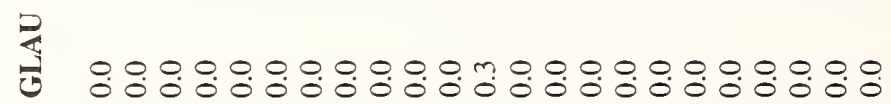

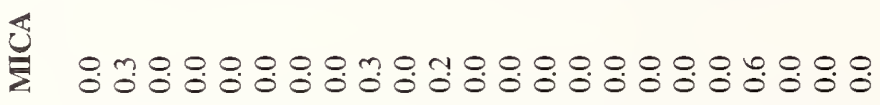

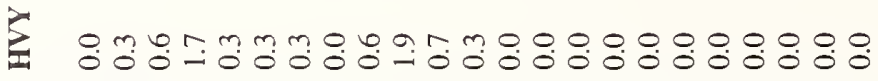

G

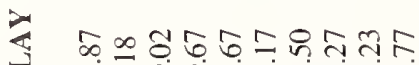

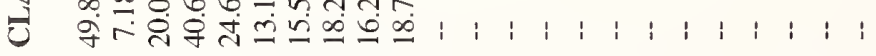

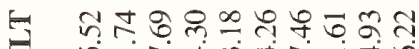

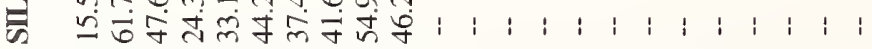

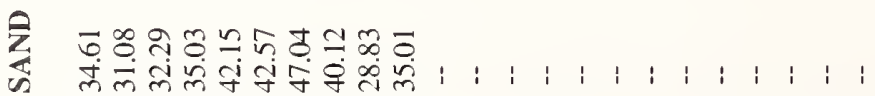

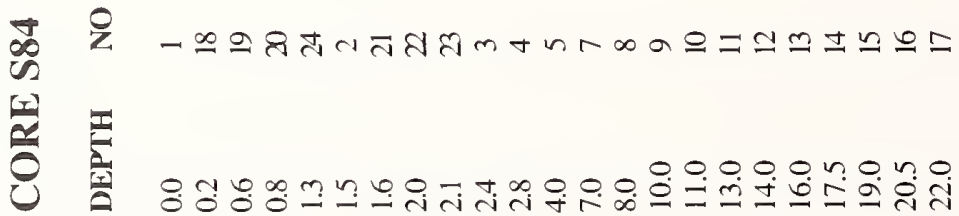


APPENDIX 2.-Continued.

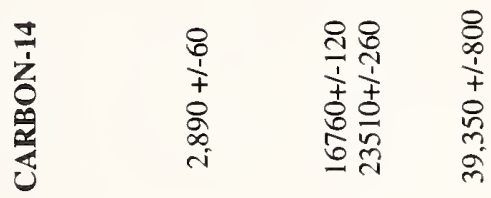

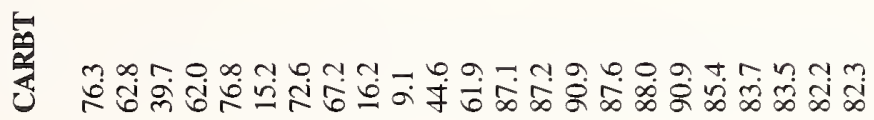

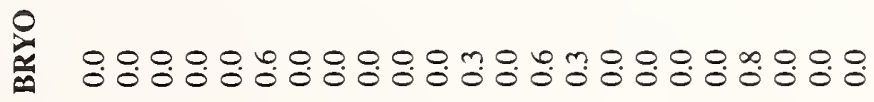

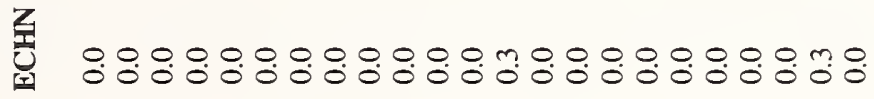

\%

曷

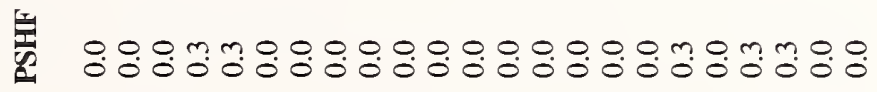

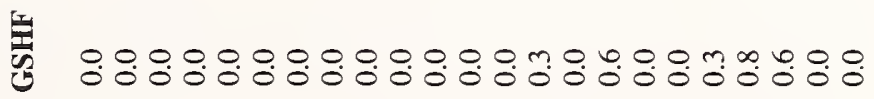

サ

年 
APPENDIX 2.-Continued.

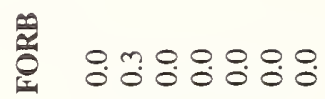

选

壼

J न

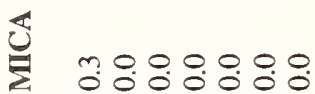

I

-

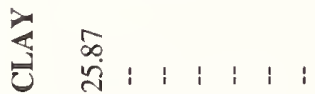

卷 8

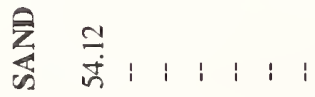

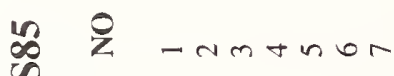

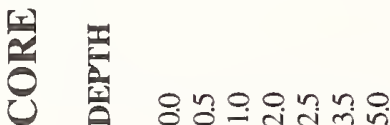


APPENDIX 2.-Continued.

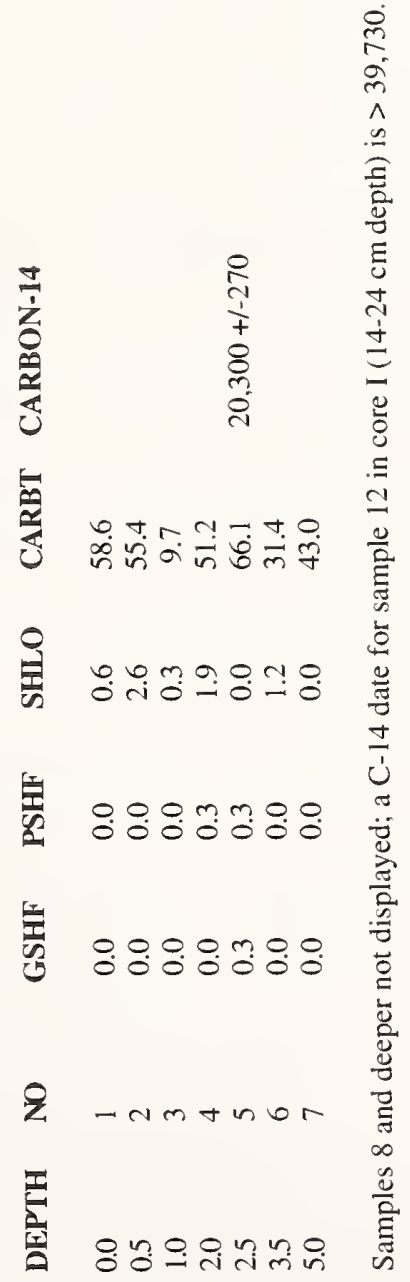




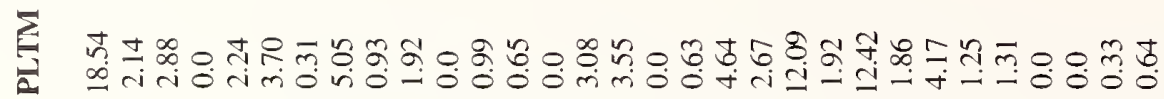

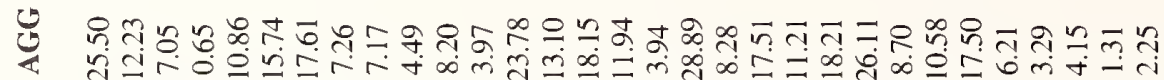

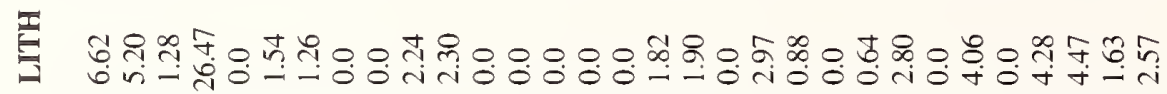

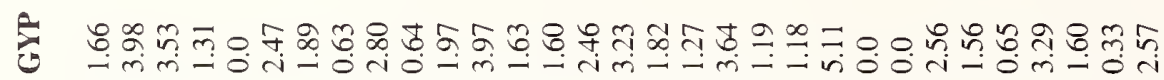

崫

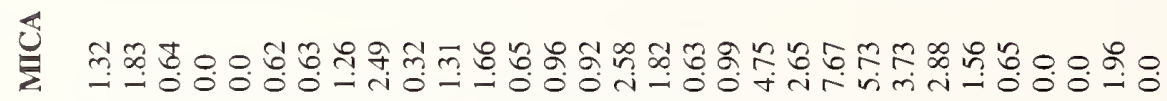

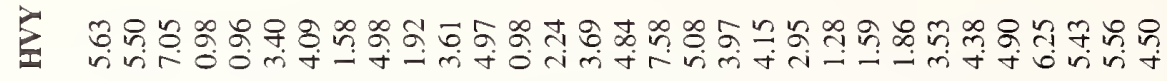

-

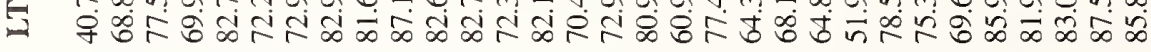

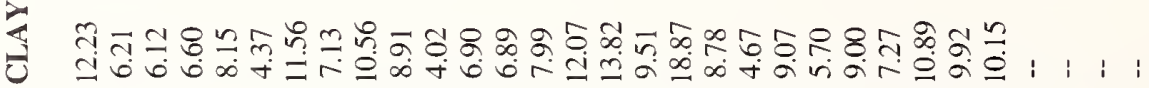

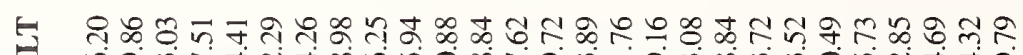

其

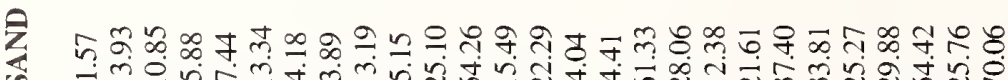

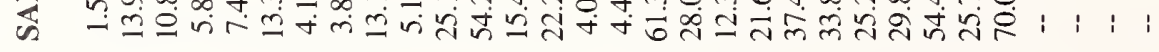

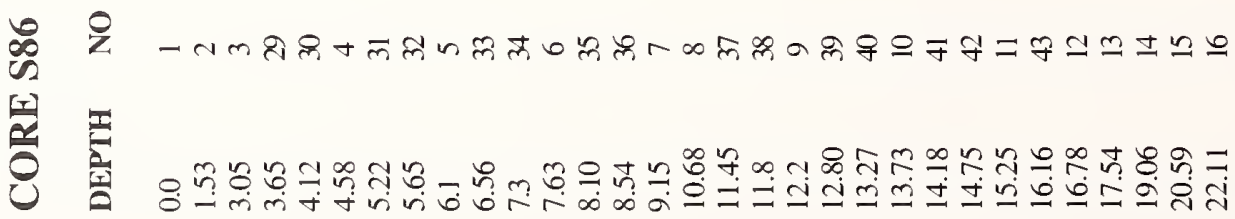


APPENDIX 2.-Continued.

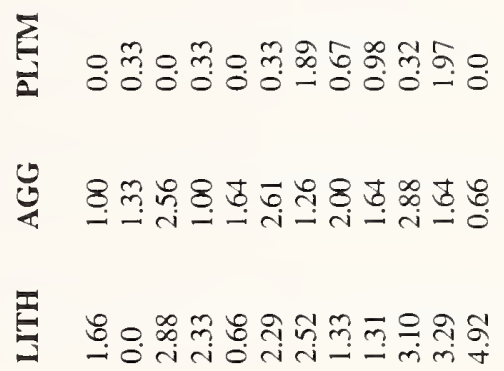

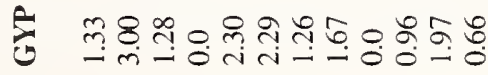

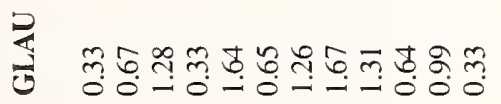

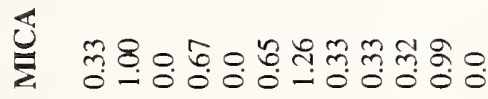

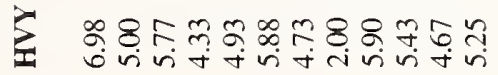

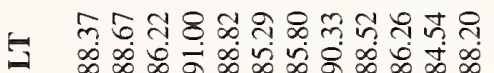

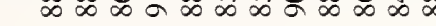

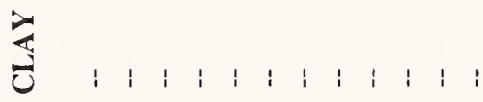

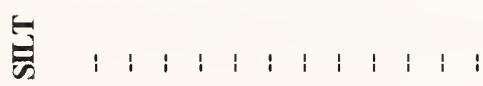

竎: : : : : :

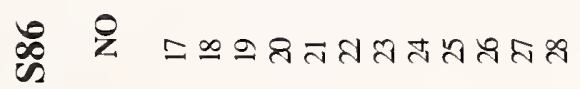

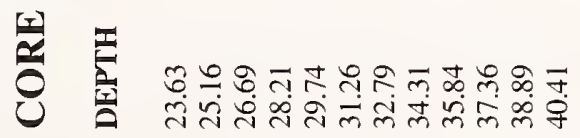


APPENDIX 2.-Continued.

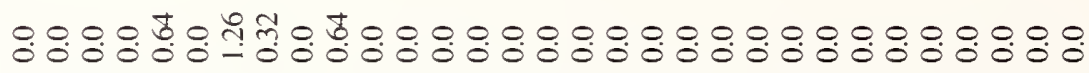

商

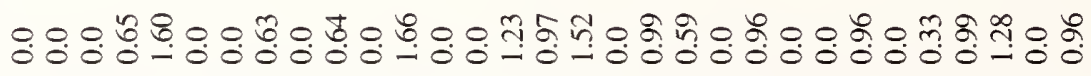

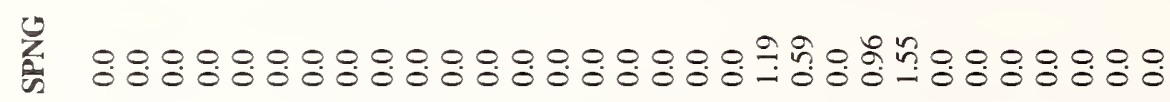

舅

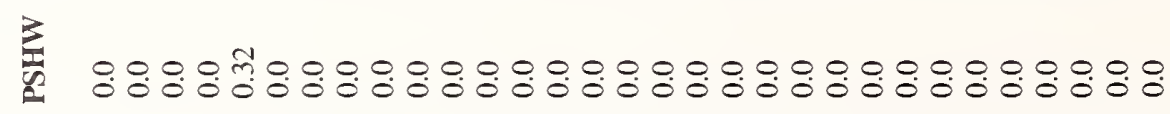

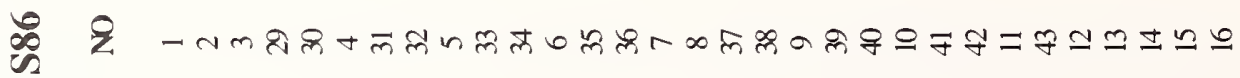

㳣

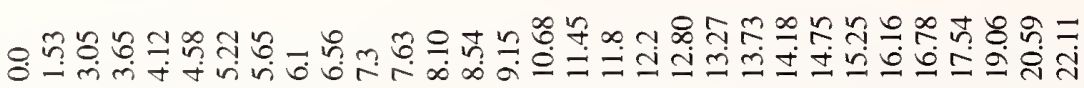


APPENDIX 2.-Continued.

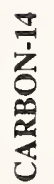

实

8000080008000

$\Rightarrow$

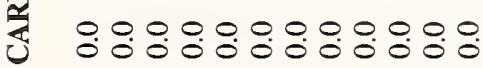

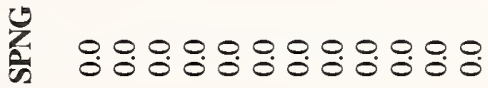

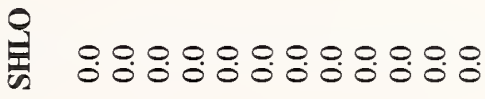

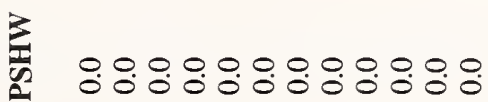

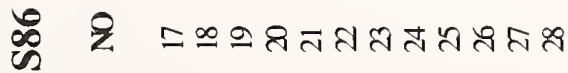

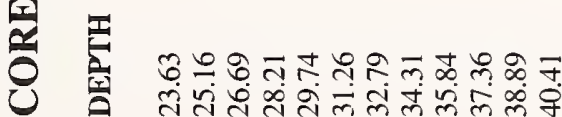




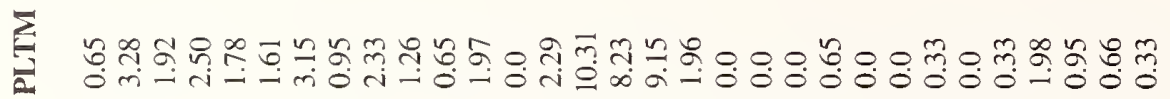

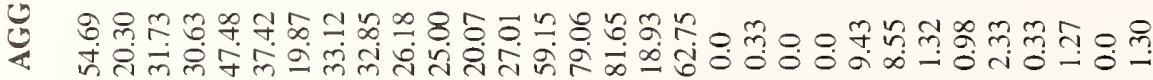

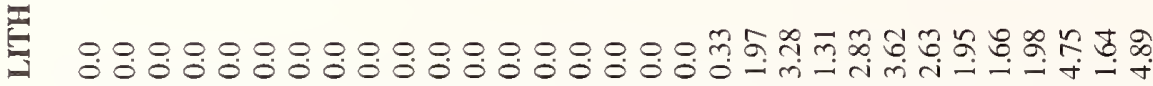

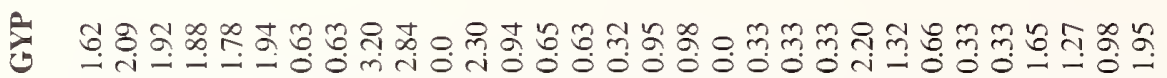

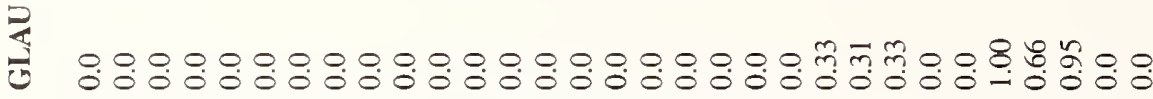

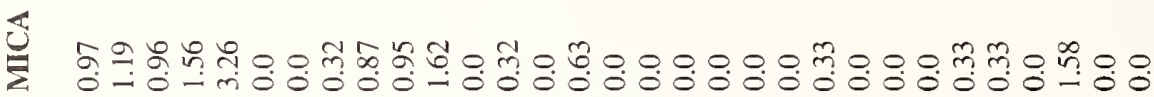

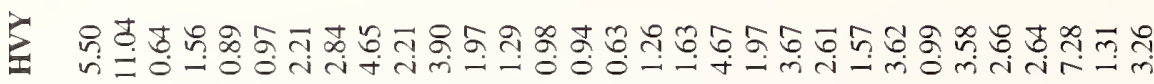

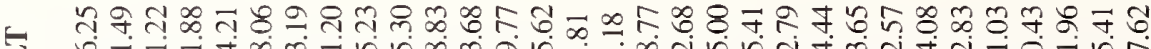

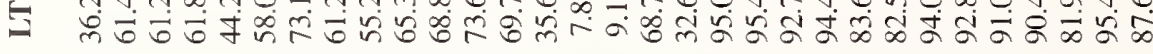

U.

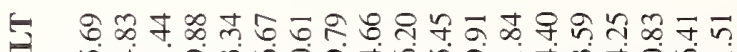

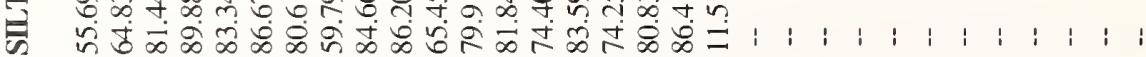

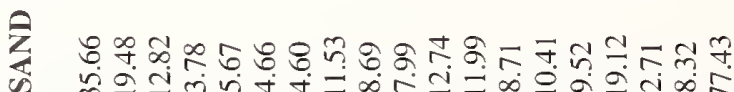

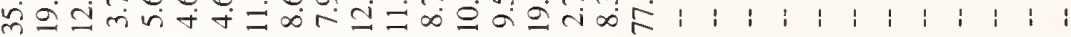

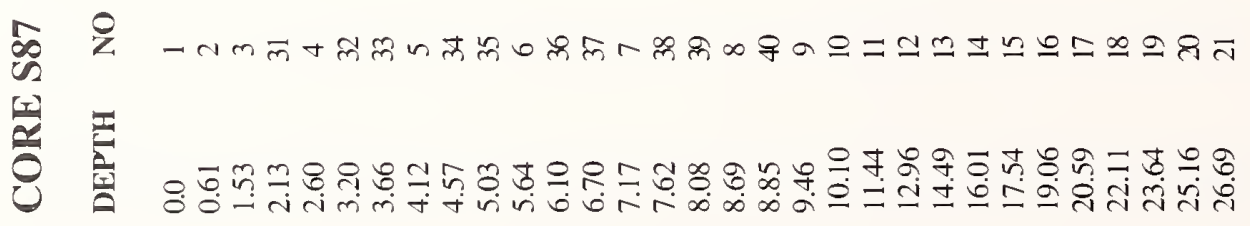


APPENDIX 2.-Continued.

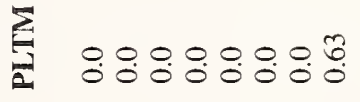

类

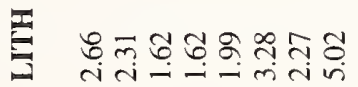

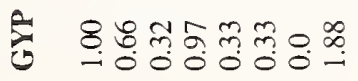

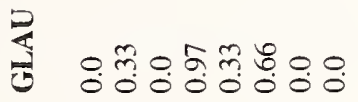

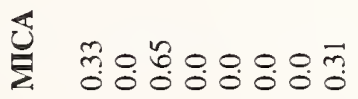

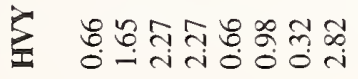

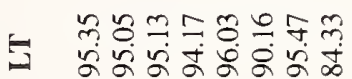

Ð $1: 1: 1: 1:$ :

葛: : : : : : : :

畜: 1: $1: 1: 1:$ :

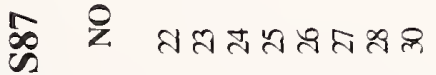

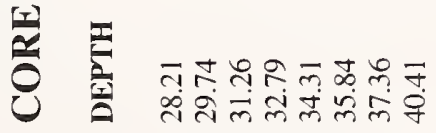


APPENDIX 2.-Continued.

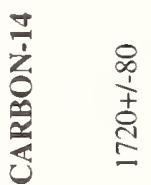

$\frac{\substack{1 \\ \frac{1}{1}}}{8}$

畧

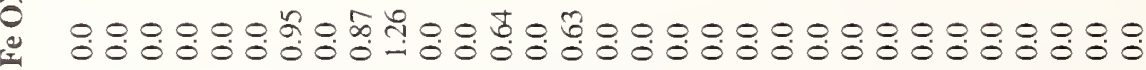

u

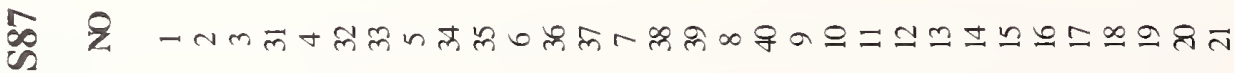

0

문 
APPENDIX 2.-Continued.

$\frac{ \pm}{3}$

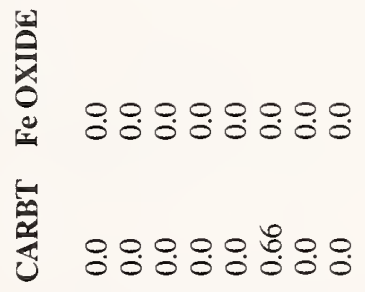

क Z

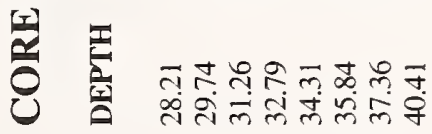




\section{Literature Cited}

Abdel-Kader, A.

1982. Landsat Analysis of the Nile Delta, Egypt. 260 pages. Master's thesis, University of Delaware, Newark, Delaware.

Abdel Wahab, H.S. El Din, and D.J. Stanley

1991. Clay Mineralogy and the Recent Evolution of the North-central Nile Delta, Egypt. Journal of Coastal Research, 7:317-329.

Abu-Zeid, M.M., and D.J. Stanley

1990. Temporal and Spatial Distribution of Clay Minerals in the Late Quaternary Deposits of the Nile Delta, Egypt. Journal of Coastal Research, 5:677-698.

Allen, R., H. Hamroush, and D.J. Stanley

1993. Impact of the Environment on Egyptian Civilization Before the Pharaohs. Analytical Chemistry, 65:32A-43A.

Anastasakis, G.C., and D.J. Stanley

1984. Sapropels and Organic-rich Variants in the Mediterranean: Sequence Development and Classification. In D.V. Stow and D.J.W. Piper, editors, Fine-Grained Sediments: Deep Water Processes. London Geological Society Special Publication, 15:497-510.

1985. Uppermost Sapropel in the Eastern Mediterranean: Sedimentological Approach for Interpreting Paleoceanography and Stagnation. National Geographic Research \& Exploration, 2:179-197.

Arbouille, D., and D.J. Stanley

1991. Late Quaternary Evolution of the Burullus Lagoon Region, North-central Nile Delta, Egypt. Marine Geology, 99:45-66.

Attia, M.

1954. Deposits in the Nile Valley and the Delta. 356 pages. Cairo, Egypt: Geological Survey of Egypt and Government Press.

Bernasconi, M.P., and D.J. Stanley

1994. Molluscan Biofacies and Their Environmental Implications, Nile Delta Lagoons, Egypt. Journal of Coastal Research, 10:440-465.

In press. Molluscan Biofacies off Egypt in the Nile Littoral Cell, Southeastern Mediterranean. Journal of Coastal Research.

Bernasconi, M.P., D.J. Stanley, and I. DiGeronimo

1991. Molluscan Faunas and Paleobathymetry of Holocene Sequences in the Northeastern Nile Delta, Egypt. Marine Geology, 99:29-43.

Biswas, A.K

1993. Land Resources for Sustainable Agricultural Development in Egypt. Ambio, 22: 556-560.

Broussard, M.

1975. Deltas: Models for Exploration. 555 pages. Houston, Texas: Houston Geological Society.

Butzer, K.W.

1976. Early Hydraulic Civilization in Egypt. 134 pages. Chicago, Illinois: University of Chicago Press.

Chen, Z., and D.J. Stanley

1993. Alluvial Stiff Muds (Late Pleistocene) Underlying the Lower Nile Delta Plain, Egypt: Petrology, Stratigraphy and Origin. Journal of Coastal Research, 9:539-576.

Chen, Z., War ne, A.G., and D.J. Stanley

1992. Late Quater nary Evolution of the Nor thwestern Nile Delta between the Rosetta Promontory and Alexandria, Egypt. Journal of Coastal Research, 8:527-561.

Coleman, J.M.

1982. Deltas: Processes of Deposition and Models for Exploration, Second edition, 124 pages. Boston: International Human Resources Development Corporation.

Coleman, J.M., H. Roberts, S. Murray, and M. Salama

1981. Morphology and Dynamic Sedimentology of the Easter n Nile Delta. Marine Geology, 42:301-326.
Coutellier, V., and D.J. Stanley

1987. Late Quaternary Stratigraphy and Paleogeography of the Eastern Nile Delta, Egypt. Marine Geology, 77:257-275.

Dominik, J., and D.J. Stanley

1993. Boron, Beryllium and Sulfur in Holocene Sediments and Peats of the Nile Delta, Egypt: Their Use as Indicators of Salinity and Climate. Chemical Geology, 104:203-216.

Foucault, A., and D.J. Stanley

1989. Late Quaternary Paleoclimatic Oscillations in East Africa Recorded by Heavy Minerals in the Nile Delta. Nature, 339:44-46.

Frihy, O.E.

1988. Nile Delta Shoreline Changes: Aerial Photographic Study of a 28-year Period. Journal of Coastal Research, 4:597-606.

Frihy, O.E., and M.F. Lotfy

1994. Mineralogic Evidence for the Remnant Sebennitic Promontory on the Continental Shelf off the Central Nile Delta. Marine Geology, 117:187-194.

Frihy, O.E., A.A. Moussa, and D.J. Stanley

1995. Abu Quir Bay, A Sediment Sink off the Northwestern Nile Delta, Egypt. Marine Geology, 121:199-211.

Frihy, O.E., and D.J. Stanley

1987. Quartz Grain Surface Textures and Depositional Interpretations, Nile Delta Region, Egypt. Marine Geology, 77:247-255.

1988. Texture and Coarse Fraction Composition of Nile Delta Deposits: Facies Analysis and Stratigraphic Correlation. Journal of African Earth Sciences, 7:237-255.

Gerber, C.D.

1988. Clay Mineralogy and Geochemistry of Nile Delta Sediments, Lake Manzala Area, Northeast Egypt. 177 pages. Master's thesis, The George Washington University, Washington, D.C.

Gerges, M.A., and D.J. Stanley

1985. Assessing Hydrography and Man's Influence on Sediments in the Northern Suez Canal. Marine Geology, 65:325-331.

Goodfriend, G., and D.J. Stanley

1996. Reworking and Discontinuities in Holocene Sedimentation in the Nile Delta: Documentation from Amino Acid Racemization and Stable Isotopes in Mollusk Shells. Marine Geology, 129:271-283.

Gupta, N.

1989. Geochemical and Mineralogical Relations of Holocene Mud-Rich Sediments in the Vicinity of the Extinct Pelusian Branch and Northern Lake Manzala, Northeastern Nile Delta, Egypt. 180 pages. Master's thesis, The George Washington University, Washington, D.C.

Hamroush, H.A., and D.J. Stanley

1991. Paleoclimatic Oscillations in East Africa Interpreted by Analysis of Trace Elements in Nile Delta Sediments. Episodes, 13:264-269.

Howa, H., and D.J. Stanley

1991. Plant-rich Holocene Sequences in the Northern Nile Delta Plain, Egypt: Petrology, Distribution and Depositional Environments. Journal of Coastal Research, 7:1077-1096.

Kay, R., editor

1993. Deltas of the World. 138 pages. New York: American Society of Civil Engineers.

Kerambrun, $\mathrm{P}$.

1986. Coastal Lagoons Along the Southern Mediterranean Coast (Algeria, Egypt, Libya, Morocco, Tunisia) Description and Bibliography. UNESCO Reports in Marine Science, 34: 184 pages.

Kulyk, V.

1987. Holocene Foraminifera of the Eastern Nile Delta, Egypt. 90 pages. 
Master's thesis, The George Washington University, Washington, D.C.

Leroy, $S$.

1992. Palyonological Evidence of Azolla nilotica dec. In Recent Holocene of the Eastern Nile Delta and Palaeoenvironment. Vegetation History and Archaeobotany, 1:43-52.

Loizeau, J.-L., and D.J. Stanley

1993. Petrological-statistical Approach to Interpret Recent and Subrecent Lagoon Subfacies, Idku, Nile Delta of Egypt. Marine Geology, 111:55-81.

1994. Bottom Sediment Patterns Evolving in Polluted Mariut Lake, Nile Delta, Egypt. Journal of Coastal Research, 10:416-439.

Longo, F.

1992. Molluscan Thanatocoenoses of the Nile Delta Lagoons. 213 pages. Master's thesis, Universita degli Studi della Calabria, Italy [in Italian].

Maldonado, A., and D.J. Stanley

1978. Nile Cone Depositional Processes and Patterns in the Late Quaternary. In D.J. Stanley and G. Kelling, editors, Sedimentation in Submarine Canyons, Fans and Trenches, pages 239-257. Stroudsburg, Pennsylvania: Dowden, Hutchinson \& Ross.

Morcos, S., and S. Messieh

1973. Change in the Current Regime in the Suez Canal After Construction of the Aswan High Dam. Nature, 242:38-39.

Pimmel, A., and D.J. Stanley

1989. Verdinized Fecal Pellets as Indicators of Prodelta and Delta-front Deposits in the Nile Delta, Egypt. Marine Geology, 86:339-347.

Posamentier, H.W., and P.R. Vail

1987. Eustatic Controls on Clastic Deposition, 1I: Sequence and Systems Tracts Models. In C.K. Wilgus, B.S. Hastings, H.W. Posamentier, J. van Wagoner, C.K. Ross, editors, Sea Level Changes: An Integrated Approach. Society of Economic Paleontologists and Mineralogists, Special Publication, 42:125-154.

Pugliese, N., and D.J. Stanley

1991. Ostracoda, Depositional Environments and Late Quaternary Evolution of the Eastern Nile Delta, Egypt. Il Quaternario, 4:275-302.

Randazzo, G.

1992. Evolution of the Nile Delta: Interaction of Present and Recent Sedimentation and Holocene Environments in the Deltaic Plain. 197 pages. Master's thesis, Universita di Messina, Messina, Italy.

Schneiderman, J.S.

1995. Detrital Opaque Oxides as Provenance Indicators in Nile River Sediments. Journal of Sedimentary Petrology, A65:668-674.

Shergill, B.S.

1990. Geochemical and Mineralogical Relations of Holocene Sediments along the Damietta Nile and Adjacent Continental Shelf, Nile Delta, Egypt. 287 pages. Master's thesis, The George Washington University, Washington, D.C.

Siegel, F.R., C. Gerber, N. Gupta, D.J. Stanley, and B. Shergill

1995. Geochemistry of Holocene Sediments from the Nile Delta, Egypt: Damietta to Gulf of Tineh. Journal of Coastal Research, 11:415431.

Siegel, F.R., M.L. Slaboda, and D.J. Stanley

1994. Metal Pollution Loading, Manzalah Lagoon, Nile Delta, Egypt: Implications for Aquaculture. Environmental Geology, 23:89-98.

Slaboda, M.L.

1993. Baseline-Contaminant Metal Concentrations in Sediment Cores from Manzalah Lagoon, Nile Delta, Egypt: Implications for Aquaculture. 226 pages. Master's thesis, The George Washington University, Washington, D.C.

Smith, S., and A. Abdel-Kader

1988. Coastal Erosion along the Egyptian Delta. Journal of Coastal Research, 4:245-255.
Stanley, D.J.

1985. Mud Redeposition Processes as a Major Influence on Margin-based Sedimentation. In D.J. Stanley and F.C. Wezel, editors, Geological Evolution of the Mediterranean Basin, pages 377-410. New York: Springer-Verlag.

1986. Mediterranean Deltas and Cones: Introduction and Generalities. Rapports et Procès-Verbaux, Commission International pour l'Exploration Scientifique de la Mer Méditerrannée, 30:61-62.

1988a. Low Sediment Accumulation Rates and Erosion on the Middle and Outer Nile Delta Shelf off Egypt. Marine Geology, 84:111-117.

1988b. Subsidence in the Northeastern Nile Delta: Rapid Rates, Possible Causes, and Consequences. Science, 240:497-500.

1989. Sediment Transport on the Coast and Shelf Between the Nile Delta and lsraeli Margin as Determined by Heavy Minerals. Journal of Coastal Research, 5:813-828.

1990. Recent Subsidence and Northeast Tilting of the Nile Delta, Egypt. Marine Geology, 94:147-154.

1992. Will the Nile Delta Sink into the Sea? National Geographic, 181:xiii.

1993. Harsh Winter in 1992 and Climate Change in the Nile Delta. National Geographic Research \& Exploration, 9:250-256.

Stanley, D.J., V. Arad, Y. Bartov, and F.M. El Bedewy

1994. The Nile Delta: Bibliography of Geological Research. Geological Survey of Israel, Jerusalem, Report GSI/22/93, 169 pages.

Stanley, D.J., D. Arnold, and A.G. Warne

1992. Oldest Pharaonic Site Yet Discovered in the North-central Nile Delta, Egypt. National Geographic Research \& Exploration, 8:264-275.

Stanley, D.J., and Z. Chen

1991. Distinguishing Sand Facies in the Nile Delta, Egypt, by Stained Grain and Compositional Component Analysis. Journal of Coastal Research, 7:863-877.

Stanley, D.J., G.L. Freeland, and H. Sheng

1982. Dispersal of Mediterranean and Suez Bay Sediments in the Suez Canal. Marine Geology, 49:61-79.

Stanley, D.J., and F.H. Hamza

1992. Terrigenous-carbonate Sediment Interface (Late Quaternary) along the Northwestern Margin of the Nile Delta, Egypt. Journal of Coastal Research, 8:153-171.

Stanley, D.J., and A.N. Liyanage

1986. Clay-mineral Variations in the Northeastern Nile Delta as Influences by Depositional Processes. Marine Geology, 73:263-283.

Stanley, D.J., and A. Maldonado

1977. Nile Cone: Late Quaternary Stratigraphy and Sediment Dispersal. Nature, 266:129-135.

1983. Southeastern Mediterranean (Levantine Basin-Nile Cone) Sedimentation and Evolution. National Geographic Society Reports, 15: 609-627.

Stanley, D.J., and H. Sheng

1986. Volcanic Shards from Santorini (Upper Minoan Ash) in the Nile Delta, Egypt. Nature, 360:733-735.

Stanley, D.J., H. Sheng, and Y. Pan

1988. Heavy Minerals and Provenance of Late Quaternary Sands, Eastern Nile Delta. Journal of African Earth Sciences, 7:735-741.

Stanley, D.J., and A.G. Warne

1993a. Nile Delta: Recent Geological Evolution and Human Impact. Science, 260:628-634.

1993b. Sea Level and Initiation of Predynastic Culture in the Nile Delta. Nature, 363:435-438.

1994. Worldwide Initiation of Holocene Marine Deltas: Deceleration of Sea-Level Rise as Principal Factor. Science, 265:228-231.

Stanley, D.J., A.G. Warne, H.R. Davis, M.P. Bernasconi, and Z. Chen

1992. Nile Delta: The Late Quaternary North-central Nile Delta from 
Manzala to Burullus Lagoons, Egypt. National Geographic Research \& Exploration, 8:22-51.

Stanley, D.J., and F.C. Wezel, editors

1985. Geological Evolution of the Mediterranean Basin. 591 pages. New York: Springer-Verlag.

UNDP/UNESCO

1976. Proceedings of Seminar on Nile Delta Sedimentology: Alexandria, Egypt. The Academy of Scientific Research and Technology, 257 pages.

1977. Proceedings of Seminar on Nile Delta Coastal Processes with Special Emphasis on Hydrodynamical Aspects: Alexandria, Egypt. The Academy of Scientific Research and Technology, 624 pages.

1978. Coastal Protection Studies, Project Findings and Recommendations: Paris. UNDP/EGY/73/063, 483 pages.
War ne, A.G., and D.J. Stanley

1993a. Archaeology to Refine Holocene Subsidence Rates Along the Nile Delta Margin, Egypt. Geology, 21:715-718.

1993b. Late Quater nary Evolution of the Northwest Nile Delta and Adjacent Coast in the Alexandria Region, Egypt. Journal of Coastal Research, 9:26-64.

1995. Sea-Level Change as a Critical Factor in Development of Basin Margin Sequences: New Evidence from Late Quaternary Record. Journal of Coastal Research, Special Publication, 17:231-240.

Waterbury, J.

1979. Hydropolitics of the Nile Valley. 301 pages. Syracuse, New York: Syracuse University Press.

Wigley, T.M.L., and S.C.B. Raper

1992. Implications for Climate and Sea Level of Revised 1PCC Emissions Scenarios. Nature, 357:293-300. 


\section{REQUIREMENTS FOR SMITHSONIAN SERIES PUBLICATION}

Manuscripts intended for series publication receive substantive review (conducted by their originating Smithsonian museums or offices) and are submitted to the Smithsonian Institution Press with Form SI-36, which must show the approval of the appropriate authority designated by the sponsoring organizational unit. Requests for special treatment-use of color, foldouts, case-bound covers, etc.-require, on the same form, the added approval of the sponsoring authority.

Review of manuscripts and art by the Press for requirements of series format and style, completeness and clarity of copy, and arrangement of all material, as outlined below, will govern, within the judgment of the Press, acceptance or rejection of manuscripts and art.

Copy must be prepared on typewriter or word processor, double-spaced, on one side of standard white bond paper (not erasable), with $1 \frac{1}{4}$ " margins, submitted as ribbon copy (not carbon or xerox), in loose sheets (not stapled or bound), and accompanied by original art. Minimum acceptable length is 30 pages.

Front matter (preceding the text) should include: title page with only title and author and no other information; abstract page with author, title, series, etc., following the established format; table of contents with indents reflecting the hierarchy of heads in the paper; also, foreword and/or preface, if appropriate.

First page of text should carry the title and author at the top of the page; second page should have only the author's name and professional mailing address, to be used as an unnumbered footnote on the first page of printed text.

Center heads of whatever level should be typed with initial caps of major words, with extra space above and below the head, but no other preparation (such as all caps or underline, except for the underline necessary for generic and specific epithets). Run-in paragraph heads should use period/dashes or colons as necessary.

Tabulations within text (lists of data, often in parallel columns) can be typed on the text page where they occur, but they should not contain rules or numbered table captions.

Formal tables (numbered, with captions, boxheads, stubs, rules) should be submitted as carefully typed, double-spaced copy separate from the text; they will be typeset unless otherwise requested. If camera-copy use is anticipated, do not draw rules on manuscript copy.

Taxonomic keys in natural history papers should use the aligned-couplet form for zoology and may use the multi-level indent form for botany. If cross referencing is required between key and text, do not include page references within the key, but number the keyed-out taxa, using the same numbers with their corresponding heads in the text.

Synonymy in zoology must use the short form (taxon, author, year:page), with full reference at the end of the paper under "Literature Cited." For botany, the long form (taxon, author, abbreviated journal or book title, volume, page, year, with no reference in "Literature Cited") is optional.

Text-reference system (author, year:page used within the text, with full citation in "Literature Cited" at the end of the text) must be used in place of bibliographic footnotes in all Contributions Series and is strongly recommended in the Studies Series: "(Jones, 1910:122)" or "...Jones (1910:122)." If bibliographic footnotes are required, use the short form (author, brief title, page) with the full citation in the bibliography.

Footnotes, when few in number, whether annotative or bibliographic, should be typed on separate sheets and inserted immediately after the text pages on which the references occur. Extensive notes must be gathered together and placed at the end of the text in a notes section.

Bibliography, depending upon use, is termed "Literature Cited," "References," or "Bibliography." Spell out titles of books, articles, journals, and monographic series. For book and article titles use sentence-style capitalization according to the rules of the language employed (exception: capitalize all major words in English). For journal and series titles, capitalize the initial word and all subsequent words except articles, conjunctions, and prepositions. Transliterate languages that use a non-Roman alphabet according to the Library of Congress system. Underline (for italics) titles of journals and series and titles of books that are not part of a series. Use the parentheses/colon system for volume (number):pagination: "10(2):5-9." For alignment and arrangement of elements, follow the format of recent publications in the series for which the manuscript is intended. Guidelines for preparing bibliography may be secured from Series Section, SI Press.

Legends for illustrations must be submitted at the end of the manuscript, with as many legends typed, double-spaced, to a page as convenient.

Illustrations must be submitted as original art (not copies) accompanying, but separate from, the manuscript. Guidelines for preparing art may be secured from the Series Section, SI Press. All types of illustrations (photographs, line drawings, maps, etc.) may be intermixed throughout the printed text. They should be termed Figures and should be numbered consecutively as they will appear in the monograph. If several illustrations are treated as components of a single composite figure, they should be designated by lowercase italic letters on the illustration; also, in the legend and in text references the italic letters (underlined in copy) should be used: "Figure 9b." Illustrations that are intended to follow the printed text may be termed Plates, and any components should be similarly lettered and referenced: "Plate $9 \underline{b}$." Keys to any symbols within an illustation should appear on the art rather than in the legend.

Some points of style: Do not use periods after such abbreviations as "mm, $\mathrm{ft}$, USNM, NNE." Spell out numbers "one" through "nine" in expository text, but use digits in all other cases if possible. Use of the metric system of measurement is preferable; where use of the English system is unavoidable, supply metric equivalents in parentheses. Use the decimal system for precise measurements and relationships, common fractions for approximations. Use day/month/ year sequence for dates: "9 April 1976." For months in tabular listings or data sections, use three-letter abbreviations with no periods: "Jan, Mar, Jun," etc. Omit space between initials of a personal name: "J.B. Jones."

Arrange and paginate sequentially every sheet of manuscript in the following order: (1) title page, (2) abstract, (3) contents, (4) foreword and/or preface, (5) text, (6) appendices, (7) notes section, (8) glossary, (9) bibliography, (10) legends, (11) tables. Index copy may be submitted at page proof stage, but plans for an index should be indicated when the manuscript is submitted. 

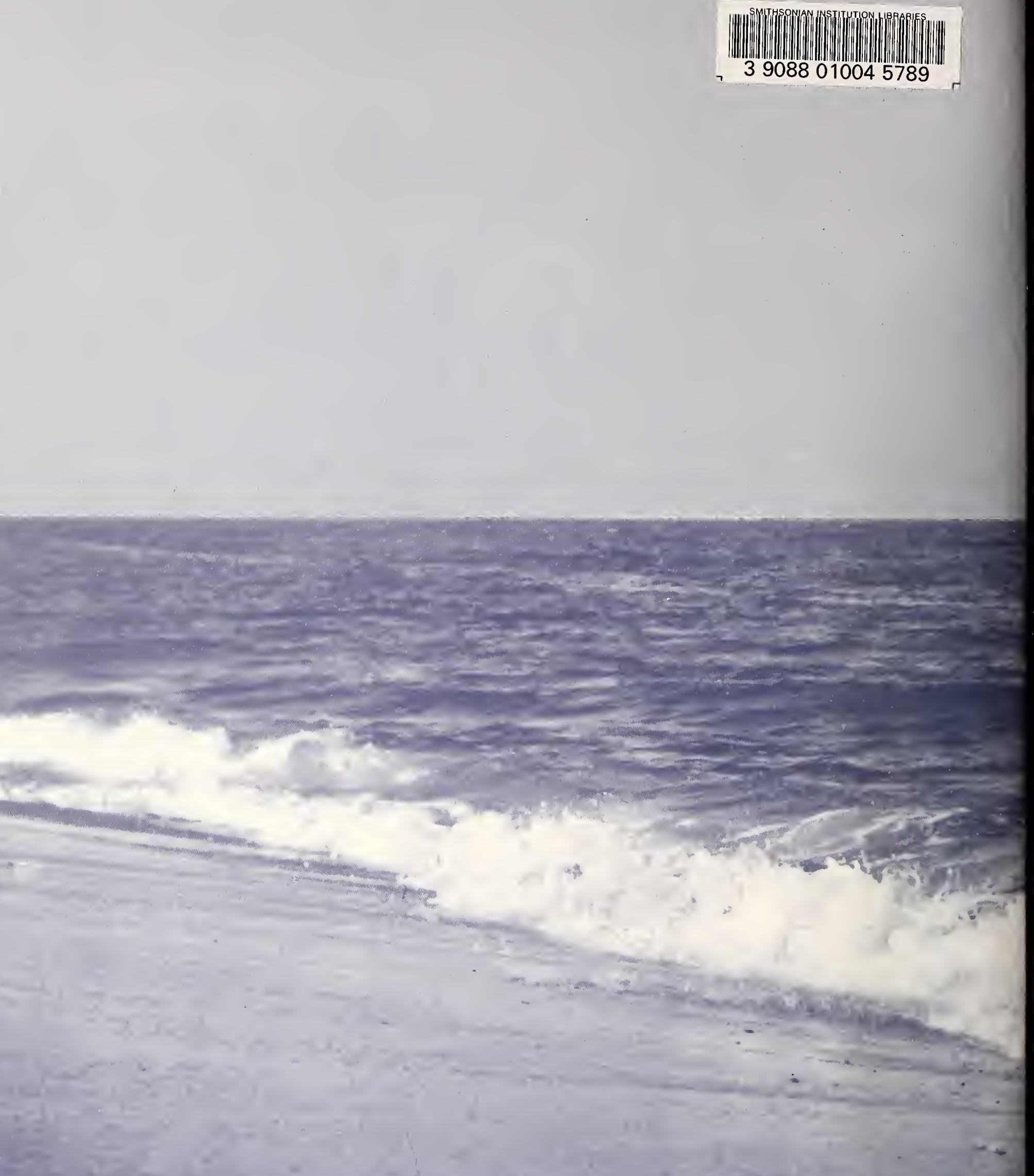